\title{
Partial Material Strength Reduction Factors for ACI 318
}

Tong Zhang, The University of Western Ontario

Supervisor: Bartlett, Michael, The University of Western Ontario

A thesis submitted in partial fulfillment of the requirements for the Master of Engineering Science degree in Civil and Environmental Engineering

(C) Tong Zhang 2017

Follow this and additional works at: https://ir.lib.uwo.ca/etd

Part of the Structural Engineering Commons

\section{Recommended Citation}

Zhang, Tong, "Partial Material Strength Reduction Factors for ACI 318" (2017). Electronic Thesis and Dissertation Repository. 5135.

https://ir.lib.uwo.ca/etd/5135

This Dissertation/Thesis is brought to you for free and open access by Scholarship@Western. It has been accepted for inclusion in Electronic Thesis and Dissertation Repository by an authorized administrator of Scholarship@Western. For more information, please contact wlswadmin@uwo.ca. 


\begin{abstract}
The strength reduction factors, $\phi$, defined in ACI 318-14 for different structural actions and elements lead to inconsistent results. This study proposes partial material strength reduction factors for concrete, $\phi_{c}$, and reinforcing steel, $\phi_{s}$, that yield similar design strengths and more consistent reliability indices. Three structural actions are investigated: moment; shear; and, combined moment and axial force. The first-order, second-moment method is used to compute reliability indices for moment and shear, and Monte Carlo simulation is used for combined moment and axial force. The statistical parameters assumed for the professional factor for shear strength significantly impact the reliability indices. Although no single combination of $\phi_{s}$ and $\phi_{c}$ is the best for these three actions, the recommended partial material strength reduction factors are $\phi_{s}$ of 0.90 and $\phi_{c}$ of 0.60 , or for spirally reinforced columns, 0.70. Alternatively, for shear, the combination with $\phi_{s}$ of 0.80 and $\phi_{c}$ of 0.65 is recommended.

\section{Keywords}

reinforced concrete; partial material strength reduction factors; moment; shear; slabs; beams; columns; design strengths; reliability. 


\section{Acknowledgments}

I would like to express my sincere appreciation to my supervisor, Dr. Michael Bartlett for providing me with the opportunity to do this research. His educational and research experience allowed me to learn various knowledge, his earnestness and preciseness encouraged me to be strict with myself, and his patience made me overcome difficulties.

I also appreciate the colleagues and professors who inspired me.

I thank The University of Western Ontario for providing financial support in the form of the Western Graduate Research Scholarship, Western Graduate Research Assistance, Graduate Research Assistantship, and Graduate Teaching Assistantship. Financial support through Dr. Bartlett's NSERC Discovery Grant is also gratefully acknowledged.

Finally, I am grateful to my family for their encouragement. 


\section{Table of Contents}

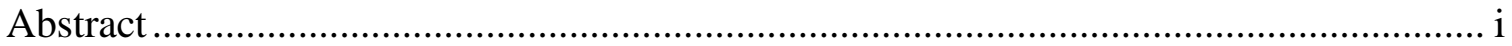

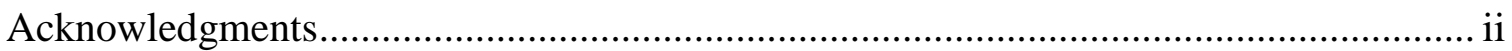

Table of Contents ............................................................................................... ii

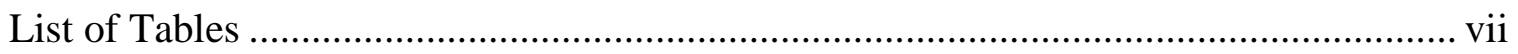

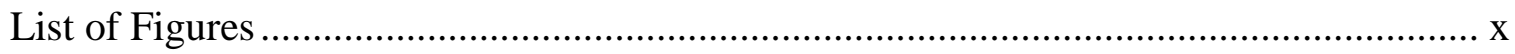

List of Appendices ..................................................................................................

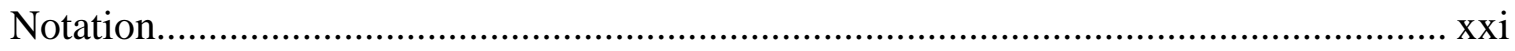

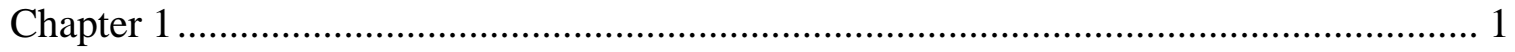

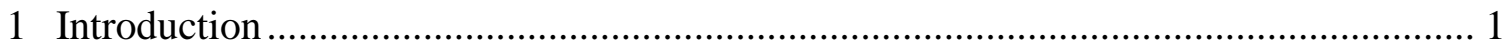

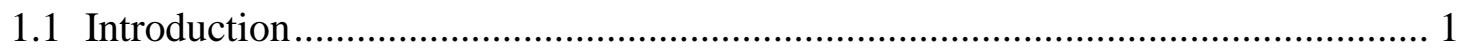

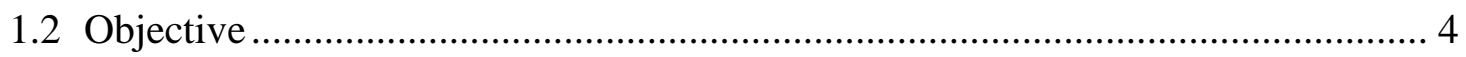

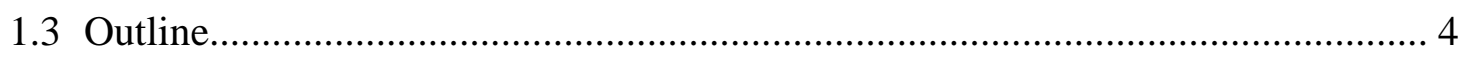

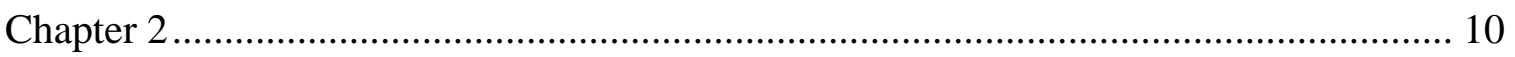

2 Derivation of Partial Material Strength Reduction Factors Based on Design Strengths

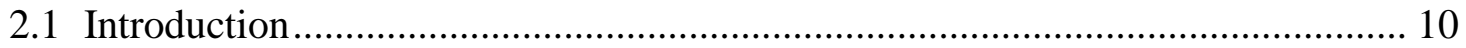

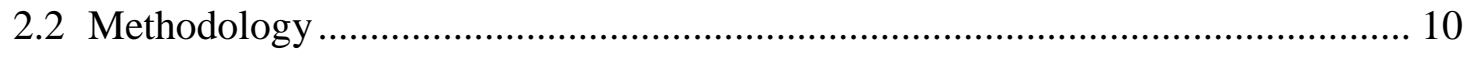

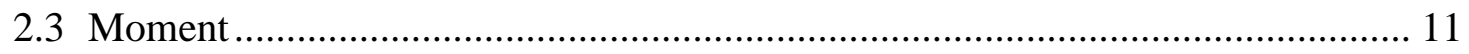

2.3.1 Geometric and Material Properties ..................................................... 11

2.3.2 Design Strength Ratios ............................................................... 12

2.3.3 Recommended Partial Material Strength Reduction Factors .................. 15

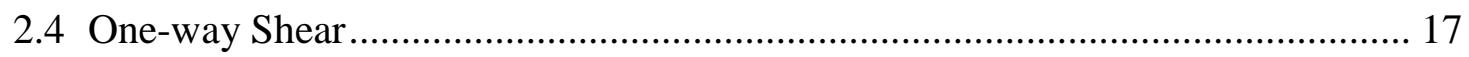

2.4.1 Geometric and Material Properties ................................................. 17

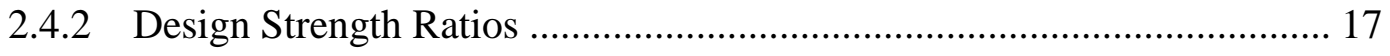

2.4.3 Recommended Partial Material Strength Reduction Factors ................... 19 


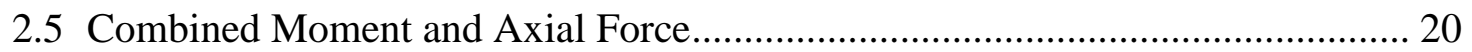

2.5.1 Geometric and Material Properties ……………...................................... 20

2.5.2 Design Strength Ratios ..................................................................... 21

2.5.3 Recommended Partial Material Strength Reduction Factors..................... 28

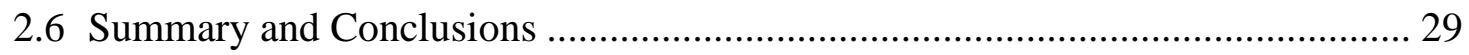

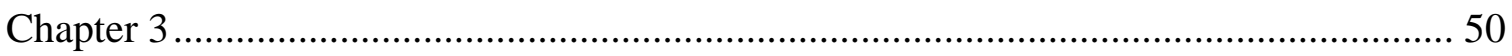

3 Derivation of Partial Material Strength Reduction Factors for Moment or One-way

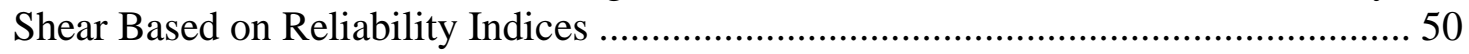

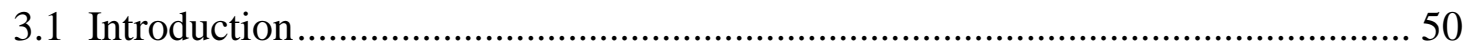

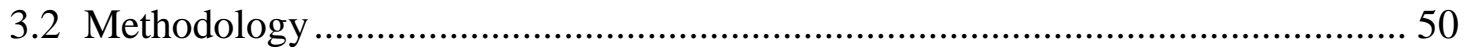

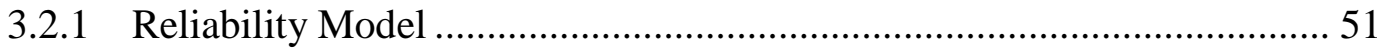

3.2.2 Determination of Statistical Parameters for Resistance and Load Effect. 52

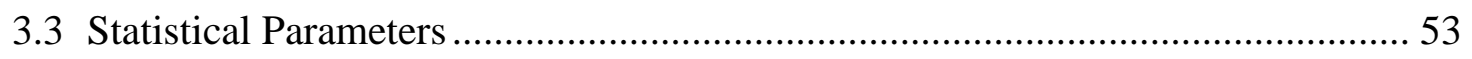

3.3.1 Geometric Properties ……………………....................................... 53

3.3.2 Material Strengths ............................................................................. 53

3.3.3 Professional Factors ............................................................................. 54

3.3.4 Load Effects ................................................................................... 55

3.4 Moment

3.4.1 Assumptions and Design Criteria ………………………………………... 56

3.4.2 Reliability Analyses .......................................................................... 57

3.4.3 Recommended Partial Material Strength Reduction Factors.................... 61

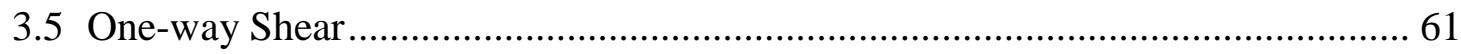

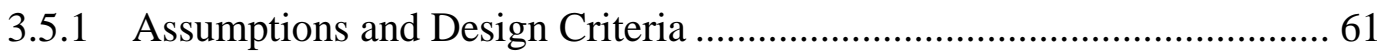

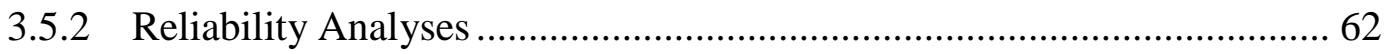

3.5.3 Recommended Partial Material Strength Reduction Factors..................... 65

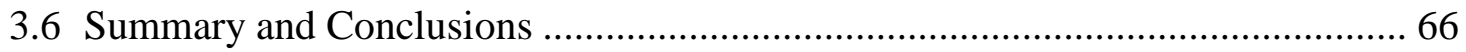


4 Derivation of Partial Material Strength Reduction Factors for Combined Moment and Axial Force Based on Reliability Indices.............................................................. 86

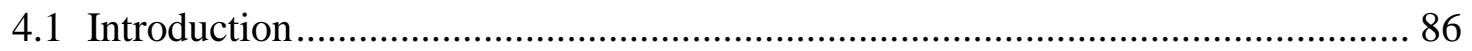

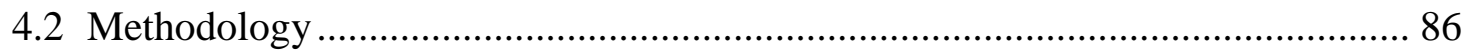

4.3 Assumptions and Design Criteria ............................................................. 87

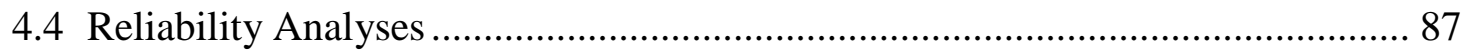

4.5 Recommended Partial Material Strength Reduction Factors ............................. 93

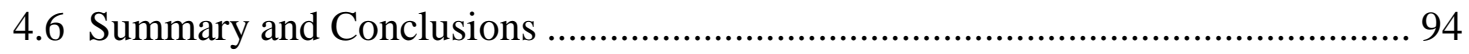

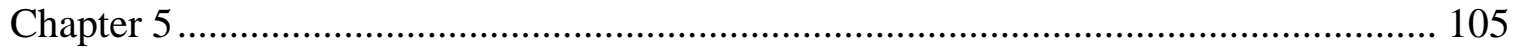

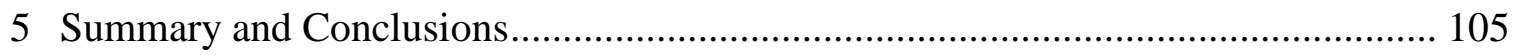

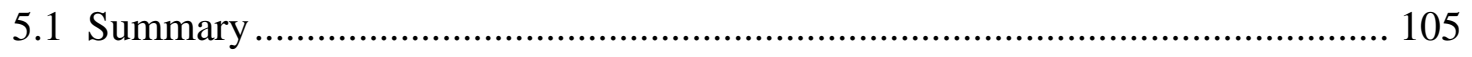

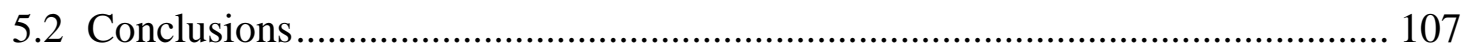

5.3 Suggestions for Future Work ........................................................... 110

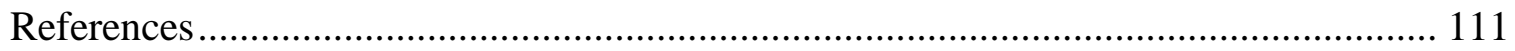

Appendix A-Supplementary Information for Chapter 2 ..................................... 113

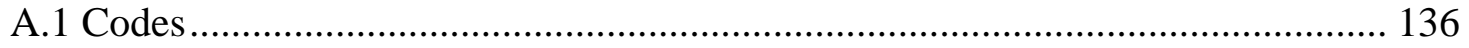

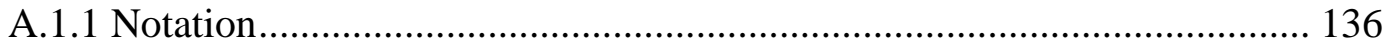

A.1.2 Column Section 1 ............................................................................ 139

A.1.3 Column Section 5 ...................................................................... 153

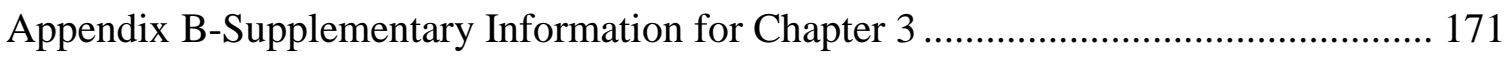

B.1 Supplemtentary Information for Concrete Compressive Strength .................... 171

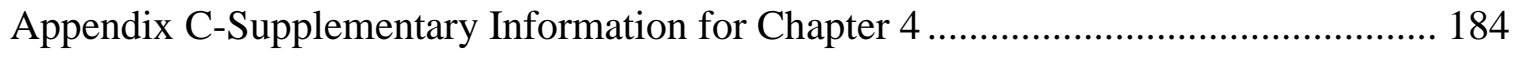

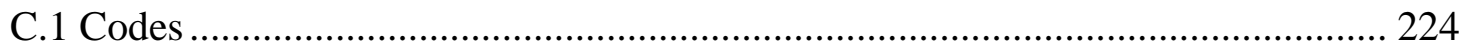

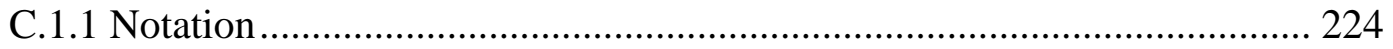

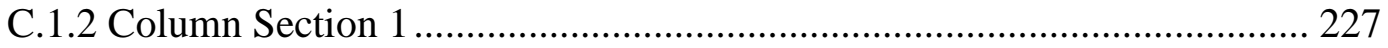


C.1.3 Column Section 5.

Curriculum Vitae ... 


\section{List of Tables}

Table 1.1: Strength reduction factors, $\phi$, in ACI 318-14 6

Table 1.2: Strength reduction factors, $\phi$, for moment, axial force, or combined moment and axial force, in ACI 318-14

Table 2.1: Partial material strength reduction factor combinations

Table 2.2: Means and standard deviations of design flexural strength ratios, $\alpha_{M}$, for $f_{c}{ }^{\prime}=25$

MPa and $\rho=0.003-0.005$ 31

Table 2.3: Means and standard deviations of design flexural strength ratios, $\alpha_{M}$, for $f_{c}^{\prime}=25$

MPa and $\rho=0.006-0.010$ 31

Table 2.4: Means and standard deviations of design flexural strength ratios, $\alpha_{M}$, for $f_{c}^{\prime}=25$

MPa and $\rho=0.011-0.018$ 32

Table 2.5: Means and standard deviations of design flexural strength ratios, $\alpha_{M}$, for $f_{c}^{\prime}=45$ MPa and $\rho=0.003-0.005$ 32

Table 2.6: Means and standard deviations of design flexural strength ratios, $\alpha_{M}$, for $f_{c}{ }^{\prime}=45$

MPa and $\rho=0.006-0.010$ 32

Table 2.7: Means and standard deviations of design flexural strength ratios, $\alpha_{M}$, for $f_{c}^{\prime}=45$

MPa and $\rho=0.011-0.018$

Table 2.8: Means and standard deviations of design shear strength ratios, $\alpha_{V}$, for $f_{c}{ }^{\prime}=25$

$\mathrm{MPa}$ and $\rho_{t}=0.001-0.007$

Table 2.9: Means and standard deviations of design shear strength ratios, $\alpha_{V}$, for $f_{c}{ }^{\prime}=45$

$\mathrm{MPa}$ and $\rho_{t}=0.001-0.010$

Table 2.10: Section properties for columns 34 
Table 2.11: Means and standard deviations of design combined flexural and axial strength ratios, $\alpha_{P M}$, for Column Section 1 and $0 \leq e / h \leq 0.3$.

Table 2.12: Means and standard deviations of design combined flexural and axial strength ratios, $\alpha_{P M}$, for Column Section 1 and $0.3<e / h \leq 1.0$. 34

Table 2.13: Means and standard deviations of design combined flexural and axial strength ratios, $\alpha_{P M}$, for Column Section 1 and $1.0<e / h \leq 10.0$. 35

Table 2.14: Means and standard deviations of design combined flexural and axial strength ratios, $\alpha_{P M}$, for Column Section 1 and $e / h \leq 0$.

Table 2.15: Means and standard deviations of design combined flexural and axial strength ratios, $\alpha_{P M}$, for Column Section 5 and $0 \leq e / h \leq 0.3$.

Table 2.16: Means and standard deviations of design combined flexural and axial strength ratios, $\alpha_{P M}$, for Column Section 5 and $0.3<e / h \leq 1.0$. 36

Table 2.17: Means and standard deviations of design combined flexural and axial strength ratios, $\alpha_{P M}$, for Column Section 5 and $1.0<e / h \leq 10.0$ 36

Table 2.18: Means and standard deviations of design combined flexural and axial strength ratios, $\alpha_{P M}$, for Column Section 5 and $e / h \leq 0$.

Table 3.1: Statistical parameters for geometric properties used in this study. 67

Table 3.2: Statistical parameters for concrete compressive strength used in this study 67

Table 3.3: Statistical parameters for professional factors 68

Table 3.4: Statistical parameters for load effects 69

Table 3.5: Design conditions for moment. 70

Table 3.6: Statistical parameters for moment reliability analysis 
Table 3.7: Means and standard deviations of reliability indices for moment using partial material strength reduction factors, $\beta_{M r}$, for $f_{c}{ }^{\prime}=25 \mathrm{MPa}$ and $\rho=0.003-0.005$

Table 3.8: Means and standard deviations of reliability indices for moment using partial material strength reduction factors, $\beta_{M r}$, for $f_{c}^{\prime}=25 \mathrm{MPa}$ and $\rho=0.006-0.010$ 72

Table 3.9: Means and standard deviations of reliability indices for moment using partial material strength reduction factors, $\beta_{M r}$, for $f_{c}^{\prime}=25 \mathrm{MPa}$ and $\rho=0.011-0.018$ 73

Table 3.10: Design conditions for shear 73

Table 3.11: Statistical parameters for shear reliability analysis 74

Table 3.12: Statistical parameters for professional factor for shear 74

Table 3.13: Means and standard deviations of reliability index ratios for shear, $\beta_{V u} / \beta_{V r}$, for $f_{c}^{\prime}$ $=25 \mathrm{MPa}$ and $\rho_{t}=0.001-0.007$ 75

Table 4.1: Statistical parameters for column reliability analysis 95

Table 4.2: Means, standard deviations, minima and maxima of reliability indices for combined moment and axial force, $\beta_{P M}$, for Column Section 1

Table 4.3: Means, standard deviations, minima and maxima of reliability indices for combined moment and axial force, $\beta_{P M}$, for Column Section 2

Table 4.4: Means, standard deviations, minima and maxima of reliability indices for combined moment and axial force, $\beta_{P M}$, for Column Section 3 98

Table 4.5: Means, standard deviations, minima and maxima of reliability indices for combined moment and axial force, $\beta_{P M}$, for Column Section 4

Table 4.6: Means, standard deviations, minima and maxima of reliability indices for combined moment and axial force, $\beta_{P M}$, for Column Section 5 


\section{List of Figures}

Figure 1.1: Strain distribution and net tensile strain in a nonprestressed member (ACI

Committee 318 2014) 7

Figure 1.2: Variation of $\phi$ with net tensile strain in extreme tension reinforcement, $\varepsilon_{t}$ (ACI Committee 318 2014)

Figure 1.3: Interaction diagrams for a square column 8

Figure 1.4: Interaction diagrams for a circular tied column 8

Figure 1.5: Interaction diagrams for an L-shape wall (Lequesne and Pincheira 2014) .......... 9

Figure 2.1: Design flexural strength ratios, $\alpha_{M}$, for $f_{c}{ }^{\prime}=25 \mathrm{MPa}$ and $\rho=0.003-0.018 \ldots \ldots . .37$

Figure 2.2: Design flexural strength ratios, $\alpha_{M}$, for $f_{c}{ }^{\prime}=45 \mathrm{MPa}$ and $\rho=0.003-0.018 \ldots \ldots . .38$

Figure 2.3: Design shear strength ratios, $\alpha_{V}$, for $f_{c}^{\prime}=25 \mathrm{MPa}$ and $\rho_{t}=0.001-0.007$ : (a) $\phi_{s}=$ $0.80 ;(\mathrm{b}) \phi_{s}=0.85 ;$ (c) $\phi_{s}=0.90 ;$ (d) $\phi_{s}=0.95$ 40

Figure 2.4: Design shear strength ratios, $\alpha_{V}$, for $f_{c}^{\prime}=45 \mathrm{MPa}$ and $\rho_{t}=0.001-0.010$ : (a) $\phi_{s}=$ $0.80 ;(\mathrm{b}) \phi_{s}=0.85 ;$ (c) $\phi_{s}=0.90 ;$ (d) $\phi_{s}=0.95$ 42

Figure 2.5: Five column cross sections: (a) Column Section 1; (b) Column Section 2; (c) Column Section 3; (d) Column Section 4; (e) Column Section 5. 43

Figure 2.6: Circular segments: (a) $a \leq h / 2, \theta \leq \pi / 2$; (b) $a>h / 2, \theta>\pi / 2$ (Wight 2016) 43

Figure 2.7: Design combined flexural and axial strength ratios, $\alpha_{P M}$, for Column Section 1 and Property Combination 1: (a) $e / h>0$; (b) $e / h<0$.

Figure 2.8: Design combined flexural and axial strength ratios, $\alpha_{P M}$, corresponding to ACI 318-14, and $\phi_{s}=0.90$ and $\phi_{c}=0.60$, for Column Section 1: (a) $e / h>0$; (b) $e / h<0$

Figure 2.9: Design combined flexural and axial strength ratios, $\alpha_{P M}$, for Column Section 5 and Property Combination 1: (a) $e / h>0$; (b) $e / h<0$ 
Figure 3.1: Reliability indices for moment, $\beta_{M}$, for $f_{c}^{\prime}=25 \mathrm{MPa}, w_{L} / w_{D}=0.5, \rho=0.003-$

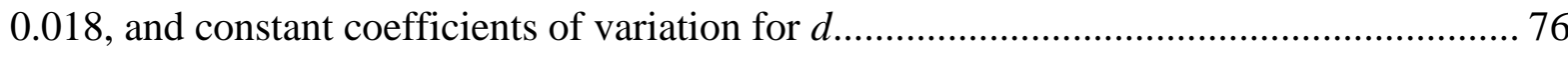

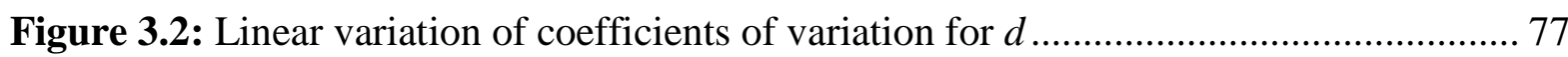

Figure 3.3: Reliability indices for moment, $\beta_{M}$, for $f_{c}{ }^{\prime}=25 \mathrm{MPa}, w_{L} / w_{D}=0.5, \rho=0.003-$

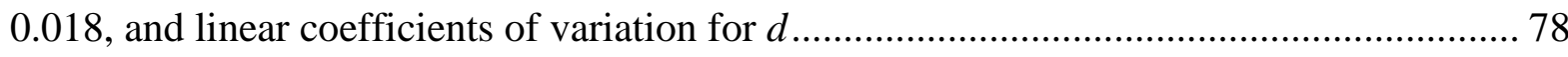

Figure 3.4: Reliability indices for moment, $\beta_{M}$, for $f_{c}{ }^{\prime}=25 \mathrm{MPa}, w_{L} / w_{D}=1.5, \rho=0.003-$ 0.018 , and linear coefficients of variation for $d$.

Figure 3.5: Reliability indices for shear, $\beta_{V}$, for $f_{c}{ }^{\prime}=25 \mathrm{MPa}, w_{L} / w_{D}=0.5$, and $\rho_{t}=0.001-$ 0.007: (a) $\phi_{s}=0.80 ;$ (b) $\phi_{s}=0.85 ;$ (c) $\phi_{s}=0.90 ;$ (d) $\phi_{s}=0.95$ 81

Figure 3.6: Reliability indices for shear, $\beta_{V}$, for $f_{c}^{\prime}=25 \mathrm{MPa}, w_{L} / w_{D}=1.5$, and $\rho_{t}=0.001-$ 0.007: (a) $\phi_{s}=0.80 ;$ (b) $\phi_{s}=0.85 ;$ (c) $\phi_{s}=0.90 ;$ (d) $\phi_{s}=0.95$. 83

Figure 3.7: Reliability indices for shear, $\beta_{V}$, for $f_{c}^{\prime}=25 \mathrm{MPa}, w_{L} / w_{D}=0.5, \rho_{t}=0.001-0.007$, bias coefficient for professional factor $=1.075$, and coefficient of variation for professional factor $=0.10$ : (a) $\phi_{s}=0.80 ;$ (b) $\phi_{s}=0.85 ;$ (c) $\phi_{s}=0.90 ;$ (d) $\phi_{s}=0.95$ 85

Figure 4.1: Reliability indices for combined moment and axial force using strength reduction factors in ACI 318-14, $\beta_{P M u}$, for Column Section 1 and $L / D=0.5$ : (a) $e / h>0$; (b) $e / h<0.101$

Figure 4.2: Reliability indices for combined moment and axial force, $\beta_{P M r}$, corresponding to $\phi_{s}=0.90$ and $\phi_{c}=0.60$, for Column Section 1 and $L / D=0.5$ : (a) $e / h>0$; (b) $e / h<0$ 102

Figure 4.3: Reliability indices for combined moment and axial force, $\beta_{P M}$, for Column Section 1, Property Combination 1, and $L / D=0.5$ : (a) $e / h>0$; (b) $e / h<0$ 103

Figure 4.4: Reliability indices for combined moment and axial force, $\beta_{P M}$, for Column Section 5, Property Combination 1, $L / D=0.5$, and: $(a) e / h>0 ;(b) e / h<0$. 104 


\section{List of Appendices}

Table A.1: Means and standard deviations of design combined flexural and axial strength ratios, $\alpha_{P M}$, for Column Section 2 and $0 \leq e / h \leq 0.4$

Table A.2: Means and standard deviations of design combined flexural and axial strength ratios, $\alpha_{P M}$, for Column Section 2 and $0.4<e / h \leq 1.0$

Table A.3: Means and standard deviations of design combined flexural and axial strength ratios, $\alpha_{P M}$, for Column Section 2 and $1.0<e / h \leq 10.0$

Table A.4: Means and standard deviations of design combined flexural and axial strength ratios, $\alpha_{P M}$, for Column Section 2 and $e / h \leq 0$.

Table A.5: Means and standard deviations of design combined flexural and axial strength ratios, $\alpha_{P M}$, for Column Section 3 and $0 \leq e / h \leq 0.3$

Table A.6: Means and standard deviations of design combined flexural and axial strength ratios, $\alpha_{P M}$, for Column Section 3 and $0.3<e / h \leq 1.0$

Table A.7: Means and standard deviations of design combined flexural and axial strength ratios, $\alpha_{P M}$, for Column Section 3 and $1.0<e / h \leq 10.0$

Table A.8: Means and standard deviations of design combined flexural and axial strength ratios, $\alpha_{P M}$, for Column Section 3 and $e / h \leq 0$.

Table A.9: Means and standard deviations of design combined flexural and axial strength ratios, $\alpha_{P M}$, for Column Section 4 and $0 \leq e / h \leq 0.3$

Table A.10: Means and standard deviations of design combined flexural and axial strength ratios, $\alpha_{P M}$, for Column Section 4 and $0.3<e / h \leq 1.0$

Table A.11: Means and standard deviations of design combined flexural and axial strength ratios, $\alpha_{P M}$, for Column Section 4 and $1.0<e / h \leq 10.0$ 
Table A.12: Means and standard deviations of design combined flexural and axial strength ratios, $\alpha_{P M}$, for Column Section 4 and $e / h \leq 0$. 116

Figure A.1: Design combined flexural and axial strength ratios, $\alpha_{P M}$, for Column Section 1 and Property Combination 2: (a) $e / h>0$; (b) $e / h<0$.

Figure A.2: Design combined flexural and axial strength ratios, $\alpha_{P M}$, for Column Section 1 and Property Combination 3: (a) $e / h>0$; (b) $e / h<0$.

Figure A.3: Design combined flexural and axial strength ratios, $\alpha_{P M}$, for Column Section 1 and Property Combination 4: (a) $e / h>0$; (b) $e / h<0$.

Figure A.4: Design combined flexural and axial strength ratios, $\alpha_{P M}$, for Column Section 1 and Property Combination 5: (a) $e / h>0$; (b) $e / h<0$.

Figure A.5: Design combined flexural and axial strength ratios, $\alpha_{P M}$, for Column Section 1 and Property Combination 6: (a) $e / h>0$; (b) $e / h<0$.

Figure A.6: Design combined flexural and axial strength ratios, $\alpha_{P M}$, for Column Section 1 and Property Combination 7: (a) $e / h>0$; (b) $e / h<0$.

Figure A.7: Design combined flexural and axial strength ratios, $\alpha_{P M}$, for Column Section 1 and Property Combination 8: (a) $e / h>0$; (b) $e / h<0$.

Figure A.8: Design combined flexural and axial strength ratios, $\alpha_{P M}$, for Column Section 2 and Property Combination 1: (a) $e / h>0$; (b) $e / h<0$.

Figure A.9: Design combined flexural and axial strength ratios, $\alpha_{P M}$, for Column Section 2 and Property Combination 2: (a) $e / h>0$; (b) $e / h<0$.

Figure A.10: Design combined flexural and axial strength ratios, $\alpha_{P M}$, for Column Section 2 and Property Combination 3: (a) $e / h>0$; (b) $e / h<0$. 
Figure A.11: Design combined flexural and axial strength ratios, $\alpha_{P M}$, for Column Section 2 and Property Combination 4: (a) $e / h>0$; (b) $e / h<0$

Figure A.12: Design combined flexural and axial strength ratios, $\alpha_{P M}$, for Column Section 2 and Property Combination 5: (a) $e / h>0$; (b) $e / h<0$

Figure A.13: Design combined flexural and axial strength ratios, $\alpha_{P M}$, for Column Section 2 and Property Combination 6: (a) $e / h>0$; (b) $e / h<0$.

Figure A.14: Design combined flexural and axial strength ratios, $\alpha_{P M}$, for Column Section 2 and Property Combination 7: (a) $e / h>0$; (b) $e / h<0$.

Figure A.15: Design combined flexural and axial strength ratios, $\alpha_{P M}$, for Column Section 2 and Property Combination 8: (a) $e / h>0$; (b) $e / h<0$.

Figure A.16: Design combined flexural and axial strength ratios, $\alpha_{P M}$, for Column Section 3 and Property Combination 1: (a) $e / h>0$; (b) $e / h<0$.

Figure A.17: Design combined flexural and axial strength ratios, $\alpha_{P M}$, for Column Section 3 and Property Combination 2: (a) $e / h>0$; (b) $e / h<0$.

Figure A.18: Design combined flexural and axial strength ratios, $\alpha_{P M}$, for Column Section 3 and Property Combination 3: (a) $e / h>0$; (b) $e / h<0$.

Figure A.19: Design combined flexural and axial strength ratios, $\alpha_{P M}$, for Column Section 3 and Property Combination 4: (a) $e / h>0$; (b) $e / h<0$. 126

Figure A.20: Design combined flexural and axial strength ratios, $\alpha_{P M}$, for Column Section 3 and Property Combination 5: (a) $e / h>0$; (b) $e / h<0$. 126

Figure A.21: Design combined flexural and axial strength ratios, $\alpha_{P M}$, for Column Section 3 and Property Combination 6: (a) $e / h>0$; (b) $e / h<0$.

Figure A.22: Design combined flexural and axial strength ratios, $\alpha_{P M}$, for Column Section 3 and Property Combination 7: (a) $e / h>0$; (b) $e / h<0$. 
Figure A.23: Design combined flexural and axial strength ratios, $\alpha_{P M}$, for Column Section 3

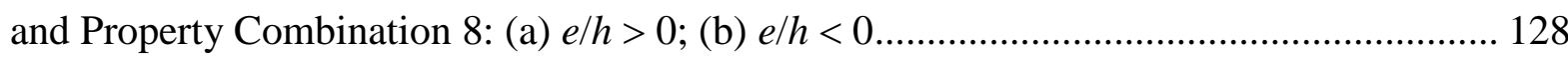

Figure A.24: Design combined flexural and axial strength ratios, $\alpha_{P M}$, for Column Section 4

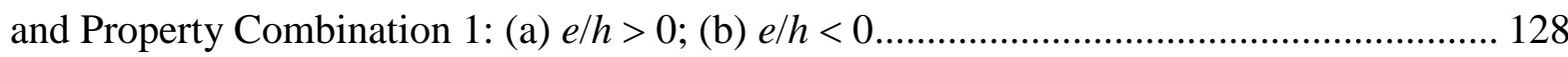

Figure A.25: Design combined flexural and axial strength ratios, $\alpha_{P M}$, for Column Section 4 and Property Combination 2: (a) $e / h>0$; (b) $e / h<0$

Figure A.26: Design combined flexural and axial strength ratios, $\alpha_{P M}$, for Column Section 4 and Property Combination 3: (a) $e / h>0$; (b) $e / h<0$

Figure A.27: Design combined flexural and axial strength ratios, $\alpha_{P M}$, for Column Section 4 and Property Combination 4: (a) $e / h>0$; (b) $e / h<0$

Figure A.28: Design combined flexural and axial strength ratios, $\alpha_{P M}$, for Column Section 4 and Property Combination 5: (a) $e / h>0$; (b) $e / h<0$.

Figure A.29: Design combined flexural and axial strength ratios, $\alpha_{P M}$, for Column Section 4 and Property Combination 6: (a) $e / h>0$; (b) $e / h<0$.

Figure A.30: Design combined flexural and axial strength ratios, $\alpha_{P M}$, for Column Section 4 and Property Combination 7: (a) $e / h>0$; (b) $e / h<0$.

Figure A.31: Design combined flexural and axial strength ratios, $\alpha_{P M}$, for Column Section 4 and Property Combination 8: (a) $e / h>0$; (b) $e / h<0$.

Figure A.32: Design combined flexural and axial strength ratios, $\alpha_{P M}$, for Column Section 5 and Property Combination 2: (a) $e / h>0$; (b) $e / h<0$.

Figure A.33: Design combined flexural and axial strength ratios, $\alpha_{P M}$, for Column Section 5 and Property Combination 3: (a) $e / h>0$; (b) $e / h<0$.

Figure A.34: Design combined flexural and axial strength ratios, $\alpha_{P M}$, for Column Section 5 and Property Combination 4: (a) $e / h>0$; (b) $e / h<0$. 
Figure A.35: Design combined flexural and axial strength ratios, $\alpha_{P M}$, for Column Section 5 and Property Combination 5: (a) $e / h>0$; (b) $e / h<0$

Figure A.36: Design combined flexural and axial strength ratios, $\alpha_{P M}$, for Column Section 5 and Property Combination 6: (a) $e / h>0$; (b) $e / h<0$ 134

Figure A.37: Design combined flexural and axial strength ratios, $\alpha_{P M}$, for Column Section 5 and Property Combination 7: (a) $e / h>0$; (b) $e / h<0$. 135

Figure A.38: Design combined flexural and axial strength ratios, $\alpha_{P M}$, for Column Section 5 and Property Combination 8: (a) $e / h>0$; (b) $e / h<0$. 135

Table B.1: Statistical parameters for geometric properties. 172

Table B.2: Statistical parameters for $F_{1}$ 173

Table B.3: Statistical parameters for $F_{2}$ 173

Table B.4: Statistical parameters for $F_{i-p}$ 173

Table B.5: Statistical parameters for in-situ concrete compressive strength 174

Table B.6: Statistical parameters for $f_{y}=420 \mathrm{MPa}$ 174

Table B.7: Means and standard deviations of reliability indices for moment using partial material strength reduction factors, $\beta_{M r}$, for $f_{c}^{\prime}=45 \mathrm{MPa}$ and $\rho=0.003-0.005$ 175

Table B.8: Means and standard deviations of reliability indices for moment using partial material strength reduction factors, $\beta_{M r}$, for $f_{c}^{\prime}=45 \mathrm{MPa}$ and $\rho=0.006-0.010$ 175

Table B.9: Means and standard deviations of reliability indices for moment using partial material strength reduction factors, $\beta_{M r}$, for $f_{c}^{\prime}=45 \mathrm{MPa}$ and $\rho=0.011-0.018$

Table B.10: Means and standard deviations of reliability index ratios for shear, $\beta_{V u} / \beta_{V r}$, for $f_{c}{ }^{\prime}$ $=45 \mathrm{MPa}$ and $\rho_{t}=0.001-0.010$. 176 
Figure B.1: Reliability indices for moment, $\beta_{M}$, for $f_{c}{ }^{\prime}=45 \mathrm{MPa}, w_{L} / w_{D}=0.5, \rho=0.003-$ 0.018 , and constant coefficients of variation for $d$.

Figure B.2: Reliability indices for moment, $\beta_{M}$, for $f_{c}{ }^{\prime}=45 \mathrm{MPa}, w_{L} / w_{D}=0.5, \rho=0.003-$ 0.018 , and linear coefficients of variation for $d$

Figure B.3: Reliability indices for moment, $\beta_{M}$, for $f_{c}{ }^{\prime}=45 \mathrm{MPa}, w_{L} / w_{D}=1.5, \rho=0.003-$ 0.018 , and linear coefficients of variation for $d$ 179

Figure B.4: Reliability indices for shear, $\beta_{V}$, for $f_{c}{ }^{\prime}=45 \mathrm{MPa}, w_{L} / w_{D}=0.5$, and $\rho_{t}=0.001-$ 0.010: (a) $\phi_{s}=0.80 ;$ (b) $\phi_{s}=0.85 ;$ (c) $\phi_{s}=0.90 ;$ (d) $\phi_{s}=0.95$. 181

Figure B.5: Reliability indices for shear, $\beta_{V}$, for $f_{c}{ }^{\prime}=45 \mathrm{MPa}, w_{L} / w_{D}=1.5$, and $\rho_{t}=0.001-$ 0.010: (a) $\phi_{s}=0.80 ;$ (b) $\phi_{s}=0.85 ;$ (c) $\phi_{s}=0.90 ;$ (d) $\phi_{s}=0.95$ 183

Figure C.1: Reliability indices for combined moment and axial force using strength reduction factors in ACI 318-14, $\beta_{P M u}$, for Column Section 1 and $L / D=1.5$ : (a) $e / h>0$; (b) $e / h<0$ 184

Figure C.2: Reliability indices for combined moment and axial force, $\beta_{P M r}$, corresponding to $\phi_{s}=0.90$ and $\phi_{c}=0.60$, for Column Section 1 and $L / D=1.5$ : (a) $e / h>0$; (b) $e / h<0$

Figure C.3: Reliability indices for combined moment and axial force, $\beta_{P M}$, for Column Section 1, Property Combination 2, and $L / D=0.5$ : (a) $e / h>0$; (b) $e / h<0$ 186

Figure C.4: Reliability indices for combined moment and axial force, $\beta_{P M}$, for Column Section 1, Property Combination 3, and $L / D=0.5$ : (a) $e / h>0$; (b) $e / h<0$

Figure C.5: Reliability indices for combined moment and axial force, $\beta_{P M}$, for Column Section 1, Property Combination 4, and $L / D=0.5$ : (a) $e / h>0$; (b) $e / h<0$ 188 
Figure C.6: Reliability indices for combined moment and axial force, $\beta_{P M}$, for Column Section 1, Property Combination 5, and $L / D=0.5$ : (a) $e / h>0$; (b) $e / h<0$

Figure C.7: Reliability indices for combined moment and axial force, $\beta_{P M}$, for Column Section 1, Property Combination 6, and $L / D=0.5$ : (a) $e / h>0$; (b) $e / h<0$

Figure C.8: Reliability indices for combined moment and axial force, $\beta_{P M}$, for Column Section 1, Property Combination 7, and $L / D=0.5$ : (a) $e / h>0$; (b) $e / h<0$

Figure C.9: Reliability indices for combined moment and axial force, $\beta_{P M}$, for Column Section 1, Property Combination 8 , and $L / D=0.5$ : (a) $e / h>0$; (b) $e / h<0$ 192

Figure C.10: Reliability indices for combined moment and axial force, $\beta_{P M}$, for Column Section 2, Property Combination 1, and $L / D=0.5$ : (a) $e / h>0$; (b) $e / h<0$

Figure C.11: Reliability indices for combined moment and axial force, $\beta_{P M}$, for Column Section 2, Property Combination 2, and $L / D=0.5$ : (a) $e / h>0$; (b) $e / h<0$ 194

Figure C.12: Reliability indices for combined moment and axial force, $\beta_{P M}$, for Column Section 2, Property Combination 3, and $L / D=0.5$ : (a) $e / h>0$; (b) $e / h<0$

Figure C.13: Reliability indices for combined moment and axial force, $\beta_{P M}$, for Column Section 2, Property Combination 4, and $L / D=0.5$ : (a) $e / h>0$; (b) $e / h<0$ 196

Figure C.14: Reliability indices for combined moment and axial force, $\beta_{P M}$, for Column Section 2, Property Combination 5, and $L / D=0.5$ : (a) $e / h>0$; (b) $e / h<0$

Figure C.15: Reliability indices for combined moment and axial force, $\beta_{P M}$, for Column Section 2, Property Combination 6, and $L / D=0.5$ : (a) $e / h>0$; (b) $e / h<0$ 198

Figure C.16: Reliability indices for combined moment and axial force, $\beta_{P M}$, for Column Section 2, Property Combination 7, and $L / D=0.5$ : (a) $e / h>0$; (b) $e / h<0$

Figure C.17: Reliability indices for combined moment and axial force, $\beta_{P M}$, for Column Section 2, Property Combination 8 , and $L / D=0.5$ : (a) $e / h>0$; (b) $e / h<0$ 
Figure C.18: Reliability indices for combined moment and axial force, $\beta_{P M}$, for Column Section 3, Property Combination 1 , and $L / D=0.5$ : (a) $e / h>0$; (b) $e / h<0$ 201

Figure C.19: Reliability indices for combined moment and axial force, $\beta_{P M}$, for Column Section 3, Property Combination 2, and $L / D=0.5$ : (a) $e / h>0$; (b) $e / h<0$ 202

Figure C.20: Reliability indices for combined moment and axial force, $\beta_{P M}$, for Column Section 3, Property Combination 3, and $L / D=0.5$ : (a) $e / h>0$; (b) $e / h<0$

Figure C.21: Reliability indices for combined moment and axial force, $\beta_{P M}$, for Column Section 3, Property Combination 4, and $L / D=0.5$ : (a) $e / h>0$; (b) $e / h<0$ 204

Figure C.22: Reliability indices for combined moment and axial force, $\beta_{P M}$, for Column Section 3, Property Combination 5, and $L / D=0.5$ : (a) $e / h>0$; (b) $e / h<0$ 205

Figure C.23: Reliability indices for combined moment and axial force, $\beta_{P M}$, for Column Section 3, Property Combination 6, and $L / D=0.5$ : (a) $e / h>0$; (b) $e / h<0$ 206

Figure C.24: Reliability indices for combined moment and axial force, $\beta_{P M}$, for Column Section 3, Property Combination 7, and $L / D=0.5$ : (a) $e / h>0$; (b) $e / h<0$ 207

Figure C.25: Reliability indices for combined moment and axial force, $\beta_{P M}$, for Column Section 3, Property Combination 8, and $L / D=0.5$ : (a) $e / h>0$; (b) $e / h<0$ 208

Figure C.26: Reliability indices for combined moment and axial force, $\beta_{P M}$, for Column Section 4, Property Combination 1, and $L / D=0.5$ : (a) $e / h>0$; (b) $e / h<0$

Figure C.27: Reliability indices for combined moment and axial force, $\beta_{P M}$, for Column Section 4, Property Combination 2, and $L / D=0.5$ : (a) $e / h>0$; (b) $e / h<0$ 210

Figure C.28: Reliability indices for combined moment and axial force, $\beta_{P M}$, for Column Section 4, Property Combination 3, and $L / D=0.5$ : (a) $e / h>0$; (b) $e / h<0$

Figure C.29: Reliability indices for combined moment and axial force, $\beta_{P M}$, for Column Section 4, Property Combination 4, and $L / D=0.5$ : (a) $e / h>0$; (b) $e / h<0$ 
Figure C.30: Reliability indices for combined moment and axial force, $\beta_{P M}$, for Column Section 4, Property Combination 5, and $L / D=0.5$ : (a) $e / h>0$; (b) $e / h<0$ 213

Figure C.31: Reliability indices for combined moment and axial force, $\beta_{P M}$, for Column Section 4, Property Combination 6, and $L / D=0.5$ : (a) $e / h>0$; (b) $e / h<0$ 214

Figure C.32: Reliability indices for combined moment and axial force, $\beta_{P M}$, for Column Section 4, Property Combination 7, and $L / D=0.5$ : (a) $e / h>0$; (b) $e / h<0$ 215

Figure C.33: Reliability indices for combined moment and axial force, $\beta_{P M}$, for Column Section 4, Property Combination 8 , and $L / D=0.5$ : (a) $e / h>0$; (b) $e / h<0$ 216

Figure C.34: Reliability indices for combined moment and axial force, $\beta_{P M}$, for Column Section 5, Property Combination 2, and $L / D=0.5$ : (a) $e / h>0$; (b) $e / h<0$ 217

Figure C.35: Reliability indices for combined moment and axial force, $\beta_{P M}$, for Column Section 5, Property Combination 3, and $L / D=0.5$ : (a) $e / h>0$; (b) $e / h<0$ 218

Figure C.36: Reliability indices for combined moment and axial force, $\beta_{P M}$, for Column Section 5, Property Combination 4, and $L / D=0.5$ : (a) $e / h>0$; (b) $e / h<0$

Figure C.37: Reliability indices for combined moment and axial force, $\beta_{P M}$, for Column Section 5, Property Combination 5, and $L / D=0.5$ : (a) $e / h>0$; (b) $e / h<0$ 220

Figure C.38: Reliability indices for combined moment and axial force, $\beta_{P M}$, for Column Section 5, Property Combination 6, and $L / D=0.5$ : (a) $e / h>0$; (b) $e / h<0$.... 221

Figure C.39: Reliability indices for combined moment and axial force, $\beta_{P M}$, for Column Section 5, Property Combination 7, and $L / D=0.5$ : (a) $e / h>0$; (b) $e / h<0$

Figure C.40: Reliability indices for combined moment and axial force, $\beta_{P M}$, for Column Section 5, Property Combination 8, and $L / D=0.5$ : (a) $e / h>0$; (b) $e / h<0$ 


\section{Notation}

$a$

$a_{r}$

$a_{v}$

A

$A_{g}$

$A_{i}$

$A_{s}$

$A_{s i}$

$A_{s t}$

$A_{v}$

$A \bar{y}$

b

$b_{w}$

$B_{i}$

$c$

$c_{i}$

$C_{c}$

$C_{i j}$

$C_{r c}$

$d$

$d_{i}$

$d_{t}$

D

$D_{i}$

depth of equivalent rectangular stress block in ACI 318-14

depth of equivalent rectangular stress block for partial material strength reduction factors format

shear span, equal to distance from center of concentrated load to either: (a) face of support for continuous or cantilevered members, or (b) center of support for simply supported members

area of compression segment of circular section

gross area of section

structural load

area of nonprestressed longitudinal tension reinforcement

area of the $i$ th layer of reinforcement

total area of nonprestressed longitudinal reinforcement

area of shear reinforcement within spacing $s$

moment of compression segment about center of circular section

width of compression face of member

web width

modeling parameter

distance from extreme compression fiber to neutral axis

influence coefficient

nominal compressive force in concrete

name of strength reduction factor combination

factored compressive force in concrete for partial material strength reduction factors format

effective depth, equal to distance from extreme compression fiber to centroid of longitudinal tension reinforcement

$d_{i} \quad$ distance from extreme compression fiber to the $i$ th layer of reinforcement, where $i=1$ refers to the reinforcement located furthest

distance from extreme compression fiber to extreme layer of tension steel

effect of service dead load

simulated value of dead load 


\begin{tabular}{|c|c|}
\hline$e$ & eccentricity \\
\hline$e_{i}$ & simulated value of eccentricity \\
\hline$e_{r}$ & $\begin{array}{l}\text { design eccentricity for partial material strength reduction factors format, equal } \\
\text { to } M_{r} / P_{r}\end{array}$ \\
\hline$e_{\text {rbal }}$ & $\begin{array}{l}\text { eccentricity corresponding to balanced failure for partial material strength } \\
\text { reduction factors format }\end{array}$ \\
\hline$\left(e_{r b a l}\right)_{\max }$ & $\begin{array}{l}\text { maximum eccentricity corresponding to balanced failure for partial material } \\
\text { strength reduction factors format }\end{array}$ \\
\hline$\left(e_{r b a l}\right)_{\min }$ & $\begin{array}{l}\text { minimum eccentricity corresponding to balanced failure for partial material } \\
\text { strength reduction factors format }\end{array}$ \\
\hline$e_{u}$ & design eccentricity for ACI 318-14, equal to $\phi M_{n} / \phi P_{n}$ \\
\hline$e_{u b a l}$ & eccentricity corresponding to balanced failure for ACI 318-14 \\
\hline$\left(e_{u b a l}\right)_{\max }$ & maximum eccentricity corresponding to balanced failure for ACI 318-14 \\
\hline$\left(e_{u b a l}\right)_{\min }$ & minimum eccentricity corresponding to balanced failure for ACI 318-14 \\
\hline$E_{s}$ & modulus of elasticity of reinforcement \\
\hline$f(\bullet)$ & function of resistance or load effect in limit state function \\
\hline$f_{c}^{\prime}$ & specified compressive strength of concrete \\
\hline$f_{c}^{*}$ & reduced compressive strength of concrete \\
\hline$f_{c, i-p}$ & in-place compressive strength of concrete \\
\hline$f_{s}$ & stress in reinforcement at service loads \\
\hline$f_{s}^{*}$ & reduced stress in reinforcement \\
\hline$f_{s i}$ & stress in the $i$ th layer of reinforcement \\
\hline$f_{y}$ & specified yield strength for nonprestressed reinforcement \\
\hline$f_{y}^{*}$ & reduced yield strength for nonprestressed reinforcement \\
\hline$f_{y t}$ & specified yield strength of transverse reinforcement \\
\hline$F_{i-p}$ & factor to account for variation of in-place strength \\
\hline$F_{r}$ & factor to account for rate-of-loading effects \\
\hline$F_{r s i}$ & $\begin{array}{l}\text { factored force in the } i \text { th layer of reinforcement for partial material strength } \\
\text { reduction factors format }\end{array}$ \\
\hline$F_{\text {si }}$ & nominal force in the $i$ th layer of reinforcement \\
\hline$F_{1}$ & ratio of mean 28-day control cylinder strength to specified 28-day strength \\
\hline
\end{tabular}




\begin{tabular}{|c|c|}
\hline$F_{2}$ & ratio of mean in-place strength at 28 days to mean 28 -day cylinder strength \\
\hline$g(\bullet)$ & limit state function \\
\hline$G$ & geometric property \\
\hline$h$ & overall thickness, height, or depth of member \\
\hline$l$ & span length of member \\
\hline$L$ & effect of service live load \\
\hline$L_{i}$ & simulated value of live load \\
\hline$M$ & material strength property \\
\hline$M_{i}$ & simulated value of flexural strength \\
\hline$M_{n}$ & nominal flexural strength at section \\
\hline$M_{r}$ & design flexural strength for partial material strength reduction factors format \\
\hline$M_{u}$ & factored moment at section \\
\hline$n$ & number of samples \\
\hline$P$ & professional factor \\
\hline$P_{f}$ & probability of failure \\
\hline$P_{i}$ & simulated value of axial strength \\
\hline$P_{n}$ & nominal axial strength of member \\
\hline$P_{n, \max }$ & maximum nominal axial compressive strength of member \\
\hline$P_{n t}$ & nominal axial tensile strength of member \\
\hline $\operatorname{Pr}$ & design axial strength for partial material strength reduction factors format \\
\hline$P_{r, \max }$ & $\begin{array}{l}\text { maximum design axial compressive strength for partial material strength } \\
\text { reduction factors format }\end{array}$ \\
\hline$P_{r t}$ & $\begin{array}{l}\text { design axial tensile strength for partial material strength reduction factors } \\
\text { format }\end{array}$ \\
\hline$P_{u}$ & factored axial force \\
\hline$Q$ & $\begin{array}{l}\text { load effect in limit state function, mean }=\bar{Q} \text {, standard deviation }=\sigma_{Q} \text {, } \\
\text { coefficient of variation }=V_{Q}\end{array}$ \\
\hline$Q_{i}$ & load effect for the $i$ th type load \\
\hline$Q_{M}$ & load effect in limit state function for flexural member \\
\hline$Q_{V}$ & load effect in limit state function for member resisting shear force \\
\hline$R$ & resistance in limit state function, mean $=\bar{R}$, standard deviation $=\sigma$ \\
\hline
\end{tabular}


coefficient of variation $=V_{R}$

$\begin{array}{ll}R_{M} & \text { resistance in limit state function for flexural member } \\ R_{V} & \text { resistance in limit state function for member resisting shear force } \\ S & \text { center-to-center spacing of transverse reinforcement } \\ S_{n} & \text { nominal strength } \\ T_{D} & \text { factor to account for transformation from dead load to dead load effect } \\ T_{D i} & \text { simulated value of transformation from dead load to dead load effect } \\ T_{L} & \text { factor to account for transformation from live load to live load effect } \\ T_{L i} & \text { simulated value of transformation from live load to live load effect } \\ U & \text { required strength computed using factored load combinations } \\ V & \text { coefficient of variation } \\ V_{c} & \text { nominal shear strength provided by concrete } \\ V_{n} & \text { nominal shear strength } \\ V_{r} & \text { design shear strength for partial material strength reduction factors format } \\ V_{r c} & \text { design shear strength provided by concrete for partial material strength } \\ & \text { reduction factors format } \\ V_{r s} & \text { design shear strength provided by shear reinforcement for partial material }\end{array}$ strength reduction factors format

$V_{s} \quad$ nominal shear strength provided by shear reinforcement

$V_{u} \quad$ factored shear force at section

$w_{D} \quad$ specified dead load per unit length

$w_{L} \quad$ specified live load per unit length

$X_{i} \quad$ resistance or load variable, mean $=\bar{X}_{i}$, standard deviation $=\sigma_{X_{i}}$

$X_{i}^{*} \quad$ resistance or load variable at linearizing point

$Z \quad$ ratio of strain in extreme tension layer of reinforcement to yield strain

$Z \quad$ limit state function, mean $=\bar{Z}$, standard deviation $=\sigma_{Z}$

$\alpha_{M} \quad$ design flexural strength ratio, equal to design flexural strength obtained using strength reduction factors in ACI 318-14 to that obtained using partial material strength reduction factors

$\alpha_{P M} \quad$ design combined flexural and axial strength ratio, equal to design combined flexural and axial strength obtained using strength reduction factors in ACI 
318-14 to that obtained using partial material strength reduction factors

$\alpha_{V} \quad$ design shear strength ratio, equal to design shear strength obtained using strength reduction factors in ACI 318-14 to that obtained using partial material strength reduction factors

$\beta \quad$ reliability index

$\beta_{M} \quad$ reliability index for moment

$\beta_{M r} \quad$ reliability index for moment obtained using partial material strength reduction factors

$\beta_{M u} \quad$ reliability index for moment obtained using strength reduction factors in ACI 318-14

$\beta_{P M} \quad$ reliability index for combined moment and axial force

$\beta_{P M r} \quad$ reliability index for combined moment and axial force obtained using partial material strength reduction factors

$\beta_{P M u} \quad$ reliability index for combined moment and axial force obtained using strength reduction factors in ACI 318-14

$\beta_{V} \quad$ reliability index for shear

$\beta_{V r} \quad$ reliability index for shear obtained using partial material strength reduction factors

$\beta_{V u} \quad$ reliability index for shear obtained using strength reduction factors in ACI 31814

$\beta_{1} \quad$ factor relating depth of equivalent rectangular compressive stress block to depth of neutral axis ratio of distance between outer layers of reinforcement in column to overall column depth

$\delta \quad$ bias coefficient

$\varepsilon_{c u} \quad$ maximum usable strain at extreme concrete compression fiber

$\varepsilon_{s i} \quad$ strain in the $i$ th layer of reinforcement, where $i=1$ refers to the reinforcement located furthest from extreme compression fiber

$\varepsilon_{t} \quad$ net tensile strain in extreme layer of longitudinal tension reinforcement at nominal strength

$\varepsilon_{t y} \quad$ yield strain in extreme layer of longitudinal tension reinforcement 


$$
\begin{array}{ll}
\varepsilon_{y} & \text { yield strain of reinforcement, equal to } f_{y} / E_{s} \\
\theta & \text { angle used to calculate compression segment of circular column } \\
\lambda & \text { modification factor to account for reduced shear strength of lightweight } \\
& \text { concrete } \\
\rho & \text { longitudinal reinforcement ratio, equal to ratio of } A_{s} \text { to } b d \\
\rho_{g} & \text { total reinforcement ratio, equal to ratio of total longitudinal reinforcement area } \\
\rho_{t} & \text { to cross-sectional area of column } \\
& \text { transverse reinforcement ratio, equal to ratio of area of distributed transverse } \\
\sigma & \text { reinforcement to gross concrete area perpendicular to that reinforcement } \\
\phi & \text { standard deviation } \\
\phi c & \text { partial material strength reduction factor for concrete } \\
\phi_{s} & \text { partial material strength reduction factor for reinforcing steel } \\
\phi M_{n} & \text { design flexural strength in ACI 318-14 } \\
\phi P_{n} & \text { design axial strength in ACI 318-14 } \\
\phi P_{n, \text { max }} & \text { maximum design axial compressive strength in ACI 318-14 } \\
\phi P_{n t} & \text { design axial tensile strength in ACI 318-14 } \\
\phi V_{n} & \text { design shear strength in ACI 318-14 } \\
\Phi(\bullet) & \text { cumulative distribution function of standard normal distribution }
\end{array}
$$




\section{Chapter 1}

\section{Introduction}

\subsection{Introduction}

In the current Building Code Requirements for Structural Concrete (ACI 318-14) and Commentary (ACI 318R-14) (ACI Committee 318 2014), the basic requirement for strength design is

$$
\phi S_{n} \geq U
$$

where: $\phi$ is the strength reduction factor; $S_{n}$ is the nominal strength; and, $U$ is the required strength computed using the factored load combinations. The overall strength reduction factor, $\phi$, accounts for "the probability of understrength due to variations of in-place material strengths and dimensions, the effect of simplifying assumptions in the design equations, the degree of ductility, potential failure mode of the member, the required reliability, and significance of failure and existence of alternative load paths for the member in the structure" (ACI Committee 318 2014).

For members resisting moment, axial force, combined moment and axial force, or shear, Table 1.1 shows strength reduction factors, $\phi$, defined in Chapter 21 of ACI 318-14. The strength reduction factor for shear equals 0.75, while for moment, axial force, or combined moment and axial force, the strength reduction factor ranges from 0.65 to 0.90 . The additional requirements shown in Table 1.2, which is identical to Table 21.2.2 in ACI 318-14, need to be applied to determine the exact value for a specific combination of moment and axial force for spirally reinforced or tied columns. The strength reduction factor in these cases depends on the net tensile strain in the extreme layer of longitudinal reinforcement, $\varepsilon_{t}$, which is determined assuming a linear strain distribution and a strain in the extreme compression fiber of 0.003 at nominal strength. This is shown in Figure 1.1, which is identical to Fig. R21.2.2a in ACI 318R-14. If $\varepsilon_{t}$ is greater than or equal to 0.005 , the section is defined as tension-controlled, or if it is less than or equal to $\varepsilon_{t y}$, the section is compression-controlled, where $\varepsilon_{t y}$ is the yield strain in the extreme tension layer of 
reinforcement, equal to $f_{y} / E_{s}$ for deformed reinforcement, $f_{y}$ is the specified yield strength for nonprestressed reinforcement, and $E_{s}$ is the modulus of elasticity of reinforcement. Between the two limits of 0.005 and $\varepsilon_{t y}$, a transition occurs between the strength reduction factor for moment for lightly reinforced sections, 0.90 , and that for axial force combined with moment, 0.65 or 0.75 for tied or spirally reinforced columns, respectively. This is shown in Figure 1.2, which is identical to Fig. R21.2.2b in ACI 318R-14.

The overall strength reduction factor presented in ACI 318-14, $\phi$, has some shortcomings that have been identified by others, as follows:

1. For a member subjected to combined moment and axial force, an odd variation happens within the transition region (e.g., Gamble 1998, 2015). Figure 1.3, which is similar to figures generated by Gamble (2015) shows the interaction diagrams for a $325 \mathrm{~mm}$ square column with eight bars distributed equally in four faces. The ratio of the distance between the outer layers of reinforcement in a column to the overall column depth, $\gamma$, is 0.6 and the ratio of total reinforcement area to the cross-sectional area of column, $\rho_{g}$, is 0.01 . The specified compressive strength of concrete, $f_{c}^{\prime}$, and $f_{y}$ are $25 \mathrm{MPa}$ and $420 \mathrm{MPa}$, respectively. The interaction diagram derived using the strength reduction factors in ACI 318-14 shows an inconsistency in the transition region compared to the nominal strength interaction diagram. If partial material strength reduction factors are applied, e.g. $f_{c}{ }^{*}=0.6 f_{c}^{\prime}$ and $f_{y}{ }^{*}=0.9 f_{y}\left(f_{s}{ }^{*}=0.9 f_{s}\right)$ recommended by Gamble (2015), the inconsistency disappears, where $f_{c}^{*}$ is the reduced compressive strength of concrete, $f_{y}^{*}$ is the reduced yield strength for nonprestressed reinforcement, $f_{s}$ is the stress in reinforcement at service loads, and $f_{s}^{*}$ is the reduced stress in reinforcement.

Similarly, Figure 1.4 is generated for a $325 \mathrm{~mm}$ diameter circular column with eight evenly distributed bars and ties. Similar to the square column, the current ACI 318-14 strength reduction factors create an awkward transition that is eliminated when the partial reduced material strengths are used (Gamble 2015). 
2. Figure 1.5, originally created by Lequesne and Pincheira (2014), shows the design interaction diagram obtained using ACI 318-11 strength reduction factors which are identical to those for ACI 318-14, for an L-shaped wall section. In this case and for other sections with wide flanges, the results are again unreasonable: on the right side of point $\mathrm{B}$, the flexural and axial strengths increase simultaneously with the increasing eccentricity when the compression zone stress block extends into the web and $\varepsilon_{t y} \leq \varepsilon_{t} \leq 0.005$. The reason for the increasing design axial strength, $\phi P_{n}$, with the increasing eccentricity is that $\phi$ increases at a proportionally higher rate than the nominal axial strength, $P_{n}$, decreases. This results in non-unique moment capacities for one axial strength level between points $\mathrm{A}$ and $\mathrm{B}$ as shown (Lequesne and Pincheira 2014).

3. For members subjected to shear, the statistical parameters for professional factor have significant changed. The professional factor is defined as a value observed experimentally divided by the value predicted using the actual geometric and material properties and so quantities the accuracy of an equation for resistance. For example, the bias coefficient and coefficient of variation of the professional factor reported by Somo and Hong (2006) are equal to 1.47 and 0.36, respectively, for beams with stirrups and shear span-to-depth ratio larger than or equal to 2 . In the original calibration of the ACI strength reduction factors, values of 1.09 and 0.12 were adopted by Israel et al. (1987). Similar values of 1.075 and 0.10 were recommended by Nowak and Szerszen (2003). These changes may significantly affect the reliability, so the strength reduction factor needs to be reevaluated. Specifically the higher bias coefficient reported by Somo and Hong (2006) will increase the reliability and so permit use of a greater strength reduction factor. The higher coefficient of variation, however, has the opposite effect.

4. A single overall value of $\phi$ cannot clarify the contributions of concrete and reinforcing steel, and variabilities of their strengths, so partial material strength reduction factors may yield advantages for reinforced concrete (Israel et al. 1987). 
It is noteworthy that Canadian Standard CSA-A23.3 "Design of Concrete Structures" (CSA 2014) has used partial resistance factors for the concrete and steel material strengths since 1984 .

\subsection{Objective}

The objective of this study is to select partial material strength reduction factors for concrete and reinforcing steel that yield similar design strengths to those obtained using the current ACI 318-14 provisions. Similarly, the reliability indices corresponding to the proposed strength reduction factors should be similar to or more appropriate than those corresponding to the current provisions.

Three structural actions acting on nonprestressed members shall be investigated: moment; one-way shear; and, combined moment and axial force. For members subjected to moment, the full range of flexural reinforcement ratios, corresponding to those in twoway slabs, one-way slabs and beams shall be investigated. Similarly, for members subjected to one-way shear, a realistic range of shear reinforcement ratios shall be investigated. For members subjected to combined moment and axial force, realistic total reinforcement ratios and reinforcement arrangements in the cross sections shall be investigated. Design strengths will be compared based on the current and partial material strength reduction factors. Statistical parameters related to the reliability index calculation will be collected from the literature. The first-order, second-moment (FOSM) reliability analysis method will be applied for members subjected to moment or shear. Monte Carlo simulation will be used to determine the reliability of members subjected to combined moment and axial force because of the complexity of the equations necessary to generate interaction diagrams.

\subsection{Outline}

In Chapter 2, potential partial material strength reduction factors that yield similar design strengths as the current ACI 318-14 provisions are investigated. For members subjected to moment, the design strengths of singly reinforced two-way slabs, one-way slabs and beams corresponding to ACI 318-14 and the partial material strength reduction factors 
are calculated for different concrete compressive strengths, $f_{c}{ }^{\prime}$, and ratios of nonprestressed longitudinal tension reinforcement, $\rho$. For members subjected to one-way shear, the design strengths of beams with different transverse reinforcement ratios, $\rho_{t}$, and $f_{c}^{\prime}$ are studied. For members subjected to combined moment and axial force, five column sections are investigated: square section with three bars in each face; square section with three bars in two end faces only; square section with three bars in two side faces only; circular section with eight evenly distributed bars and ties; and, circular section with eight evenly distributed bars and spiral reinforcement. For each column section, different $\gamma, f_{c}^{\prime}$, and $\rho_{g}$ are studied. For each structural action, appropriate partial material strength reduction factors are proposed.

Chapter 3 presents the reliability model and the first-order, second-moment (FOSM) method. It then summarizes statistical parameters for geometric properties, material strengths, professional factors and load effects collected from the literature. The calculated reliability indices for members subjected to moment or one-way shear are presented for the different geometric and material properties, and two live-to-dead load ratios. Partial material strength reduction factors are then proposed based on the reliability analyses and the results obtained in Chapter 2.

Chapter 4 presents the reliability analyses for columns conducted by Monte Carlo simulation. Different geometric and material properties, and two live-to-dead load ratios are investigated. The applied moment and axial force are assumed perfectly correlated and reliability indices are computed for a range of specific eccentricities. Again, appropriate partial material strength reduction factors are proposed based on the reliability analyses and the results obtained in Chapter 2.

Chapter 5 presents the summary, conclusions, and suggestions for future work.

Appendices A, B and C present supplementary tables, figures and Matlab (Version R2016b; The Mathworks, Inc. 2016) codes that complement the material presented in Chapters 2, 3 and 4, respectively. 
Table 1.1: Strength reduction factors, $\phi$, in ACI 318-14

\begin{tabular}{cc}
\hline Action or structural element & $\phi$ \\
\hline $\begin{array}{c}\text { Moment, axial force, or } \\
\text { combined moment and axial force } \\
\text { Shear }\end{array}$ & 0.65 to 0.90 \\
\hline
\end{tabular}

Table 1.2: Strength reduction factors, $\phi$, for moment, axial force, or combined moment and axial force, in ACI 318-14

\begin{tabular}{cccc}
\hline & & \multicolumn{2}{c}{$\phi$} \\
\cline { 3 - 4 } Net tensile strain $\varepsilon_{t}$ & Classification & \multicolumn{2}{c}{ Type of transverse reinforcement } \\
\cline { 3 - 4 }$\varepsilon_{t} \leq \varepsilon_{t y}$ & $\begin{array}{c}\text { Compression- } \\
\text { controlled }\end{array}$ & 0.75 & Other \\
$\varepsilon_{t y}<\varepsilon_{t}<0.005$ & Transition & $0.75+0.15 \frac{\left(\varepsilon_{t}-\varepsilon_{t y}\right)}{\left(0.005-\varepsilon_{t y}\right)}$ & 0.65 \\
$\varepsilon_{t} \geq 0.005$ & $\begin{array}{c}\text { Tension- } \\
\text { controlled }\end{array}$ & 0.90 & 0.90 \\
\hline
\end{tabular}




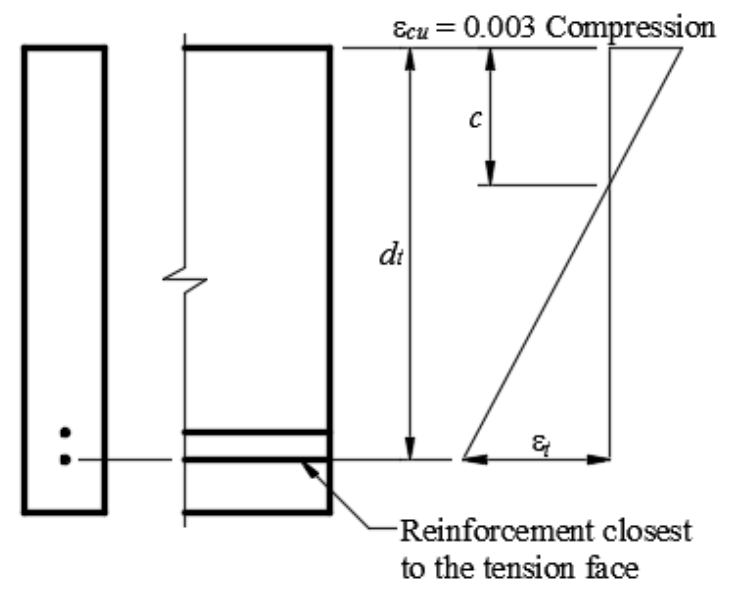

Figure 1.1: Strain distribution and net tensile strain in a nonprestressed member (ACI Committee 318 2014)

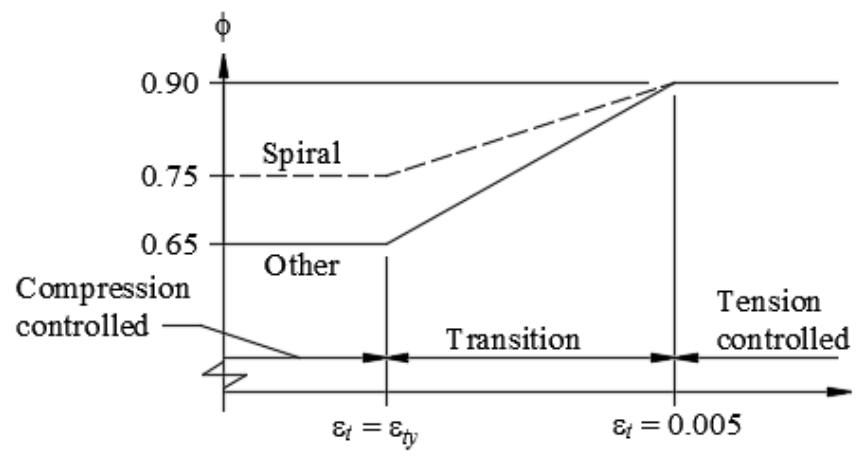

Figure 1.2: Variation of $\phi$ with net tensile strain in extreme tension reinforcement, $\varepsilon_{t}$ (ACI Committee 318 2014) 


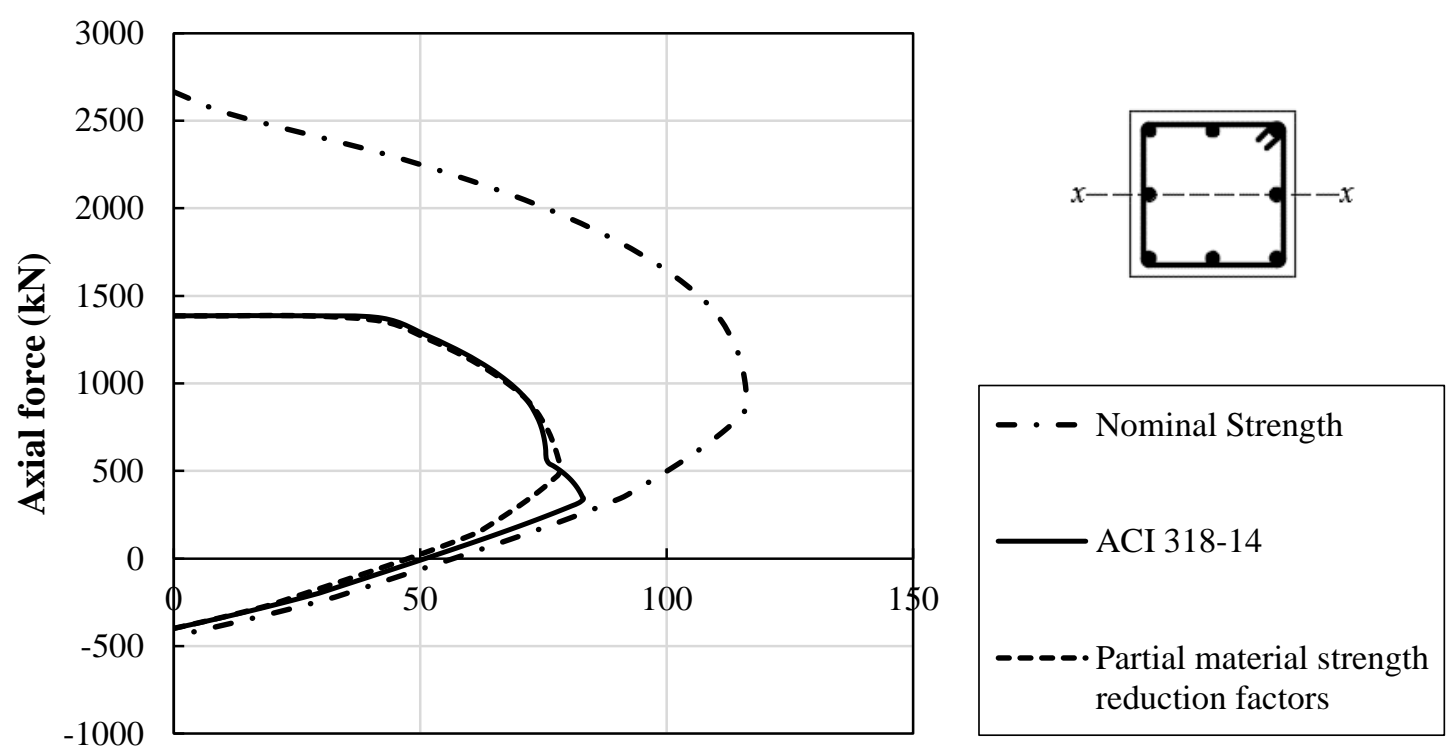

Moment (kN·m)

Figure 1.3: Interaction diagrams for a square column
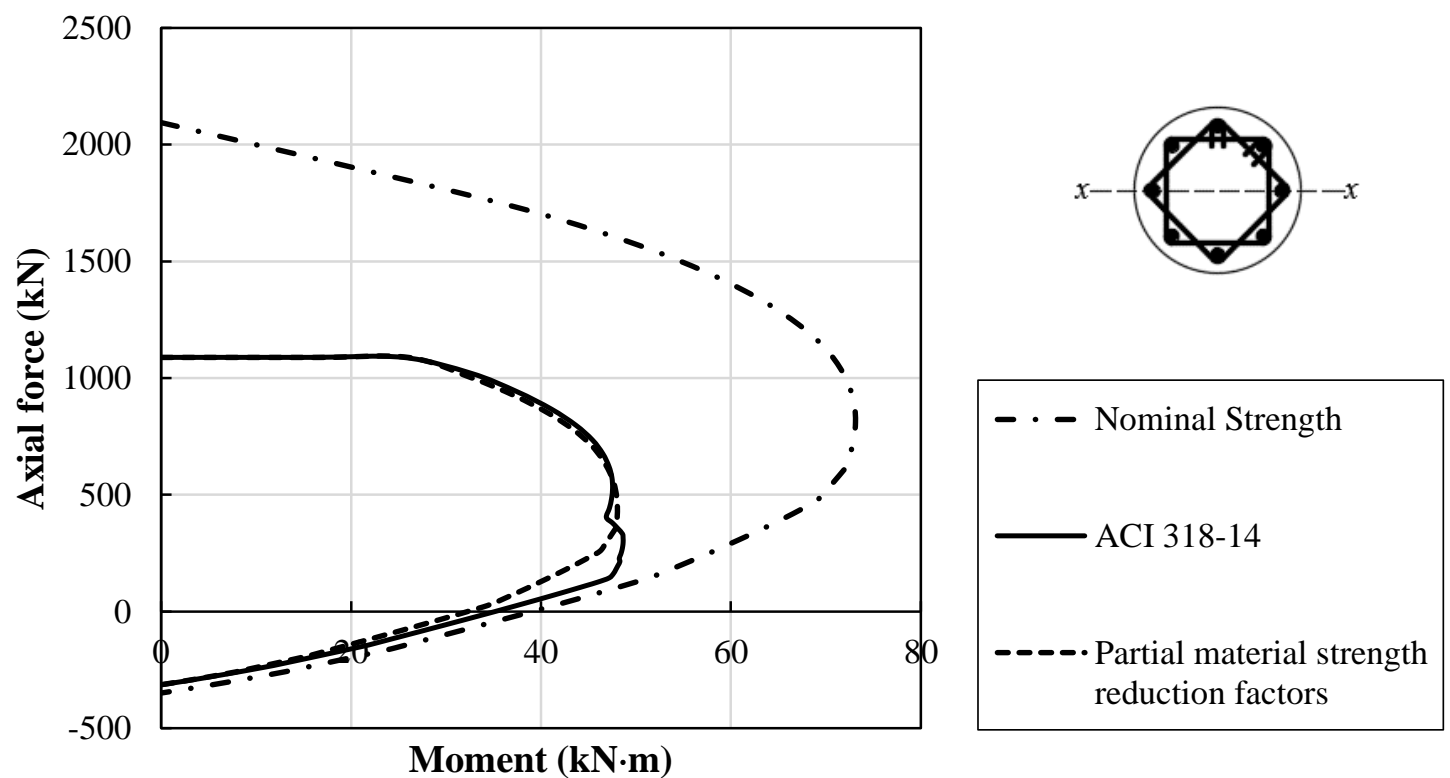

Figure 1.4: Interaction diagrams for a circular tied column 


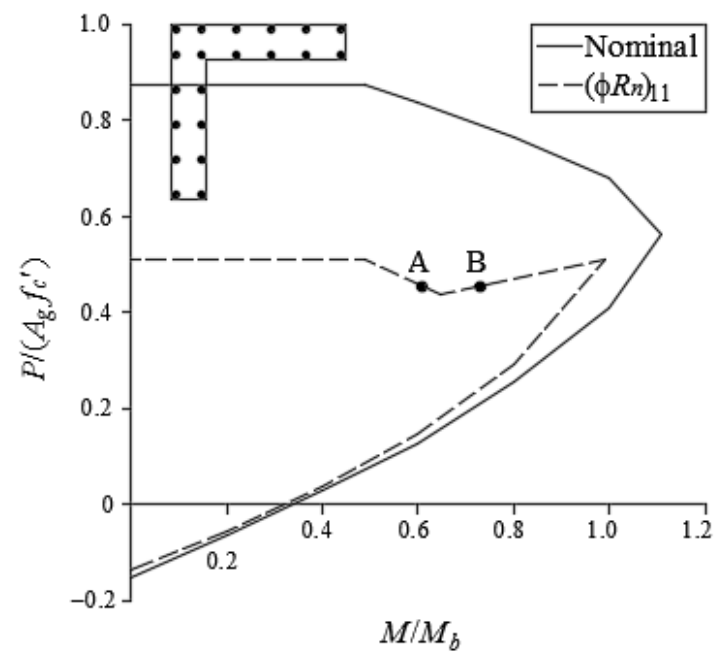

Figure 1.5: Interaction diagrams for an L-shape wall (Lequesne and Pincheira 2014) 


\section{Chapter 2}

\section{Derivation of Partial Material Strength Reduction Factors Based on Design Strengths}

\subsection{Introduction}

Chapter 21 of Building Code Requirements for Structural Concrete (ACI 318-14) and Commentary (ACI 318R-14) (ACI Committee 318 2014) specifies an overall strength reduction factor, $\phi$, for reinforced concrete elements, based on the structural action being resisted. The overall objective of this thesis is to propose partial material strength reduction factors for concrete and reinforcing steel that are independent of the structural action.

The objective of this chapter is to identify suitable partial material strength reduction factors that best duplicate the design strengths obtained using the current ACI 318-14 provisions. The preliminary results obtained in this chapter indicate the potential ranges of the best partial material strength reduction factors, and the final decision will be made after conducting reliability analyses presented in Chapters 3 and 4.

\subsection{Methodology}

This chapter develops partial material strength reduction factors for cross sections resisting three structural actions: moment; one-way shear; and, combined moment and axial force. For each action, design strengths are computed using ACI 318-14 and various partial material strength reduction factors, $\phi_{c}$ for concrete and $\phi_{s}$ for reinforcing steel. Table 2.1 shows the sixteen partial material strength reduction factor combinations considered in this study. The $C_{i j}$ notation shown represents a particular combination, where $i$ is the $i$-th value of $\phi_{s}$ and $j$ is the $j$-th value of $\phi_{c}$.

The calculations are conducted using Microsoft Excel (Version 2013; Microsoft 2013) and Matlab (Version R2016b; The Mathworks, Inc. 2016) to compute the design strength ratio, which is defined as the design strength obtained using the strength reduction factor in ACI 318-14 to that obtained using a particular pair of partial material strength 
reduction factors. Design strength ratios greater than 1 represent cases where the ACI 318-14 design strengths exceed those computed using the proposed values, and so indicate that the proposed values are more conservative. For this investigation, the best combination of partial material strength reduction factors will give design strengths that most closely approximate those obtained using the current ACI 318-14 provisions. This corresponds to the mean design strength ratio approaching 1 with the least standard deviation. Reliability analyses based on these preliminary results will be presented in Chapters 3 and 4.

\subsection{Moment}

This section presents proposed partial material strength reduction factors that most closely approximate the design flexural strengths obtained using the ACI 318-14 criteria. The ranges of geometric and material parameters are quantified and the design flexural strength equations corresponding to the current ACI 318-14 and the partial material strength reduction factors formats are presented. Typical design flexural strength ratios, $\alpha_{M}$, for each combination of partial material strength reduction factors are presented, and the means and standard deviations for each combination are quantified. The sensitivities of the design flexural strength ratios to the partial material strength reduction factors, for various geometric and material properties are investigated. The best factor combinations are recommended.

\subsubsection{Geometric and Material Properties}

The investigation of moment is limited to rectangular singly reinforced cross sections designed based on ACI 318-14 with a specified reinforcement yield strength, $f_{y}$, of 420 $\mathrm{MPa}$ and specified concrete compressive strengths, $f_{c}{ }^{\prime}$, of 25 and $45 \mathrm{MPa}$. These material strengths represent the range of strengths commonly used in flexural members. Three ranges of reinforcement ratio are investigated: 0.003 to 0.005 , which is representative of two-way slabs; 0.006 to 0.010 , which is representative of one-way slabs; and, 0.011 to 0.018, which is representative of beams. The reasons for selecting these three ranges are: they reflect typical reinforcement ratio ranges for slabs and beams; minimum and maximum reinforcement ratio limits are satisfactory in all cases; and, the upper limit of 
the studied range of beams is defined by the maximum reinforcement ratio for a beam with $f_{c}{ }^{\prime}$ of $25 \mathrm{MPa}$. The same maximum reinforcement ratio is used for beams with $f_{c}{ }^{\prime}$ of $45 \mathrm{MPa}$. One layer of reinforcing steel is assumed.

\subsubsection{Design Strength Ratios}

Design flexural strength equations corresponding to the current ACI 318-14 and partial material strength reduction factors formats are defined in this section. The equations and definitions below refer to ACI 318-14, MacGregor and Bartlett (2000), and Wight (2016).

In ACI 318-14, the depth of the equivalent rectangular stress block, $a$, is computed as (Wight 2016)

$$
a=\frac{A_{s} f_{y}}{0.85 f_{c}^{\prime} b}
$$

where: $A_{s}$ is the area of the nonprestressed longitudinal tension reinforcement; $f_{y}$ is the specified yield strength for nonprestressed reinforcement; $f_{c}{ }^{\prime}$ is the specified compressive strength of concrete; and, $b$ is the width of the compression face of the member. The design flexural strength, $\phi M_{n}$, is (Wight 2016)

$$
\phi M_{n}=\phi A_{s} f_{y}\left(d-\frac{a}{2}\right)
$$

where: $\phi$ is the strength reduction factor in ACI $318-14 ; M_{n}$ is the nominal flexural strength at a section; and, $d$ is the distance from the extreme compression fiber to the centroid of the longitudinal tension reinforcement.

For the proposed method, the depth of the equivalent rectangular stress block, $a_{r}$, based on the partial material strength reduction factors format presented in MacGregor and Bartlett (2000), is assumed to be

$$
a_{r}=\frac{\phi_{s} A_{s} f_{y}}{0.85 \phi_{c} f_{c}^{\prime} b}
$$


where: $\phi_{s}$ is the strength reduction factor for reinforcing steel; and, $\phi_{c}$ is the strength reduction factor for concrete. The design flexural strength for partial material strength reduction factors method, $M_{r}$, is (MacGregor and Bartlett 2000)

$$
M_{r}=\phi_{s} A_{s} f_{y}\left(d-\frac{a_{r}}{2}\right)
$$

For the longitudinal reinforcement ratio, $\rho$, defined as (ACI Committee 318 2014)

$$
\rho=\frac{A_{s}}{b d}
$$

the design flexural strength ratio, $\alpha_{M}$, is

$$
\alpha_{M}=\frac{\phi M_{n}}{M_{r}}=\frac{\phi\left(1-\frac{\rho}{1.7} \cdot \frac{f_{y}}{f_{c}^{\prime}}\right)}{\phi_{s}\left(1-\frac{\phi_{s} \rho}{1.7 \phi_{c}} \cdot \frac{f_{y}}{f_{c}^{\prime}}\right)}
$$

For each strength reduction factor combination, design flexural strength ratios, $\alpha_{M}$, were calculated with respect to longitudinal reinforcement ratios, $\rho$. Also, each calculation was done twice because two specified compressive strengths of concrete, $f_{c}$ ', were studied. In particular, for $f_{c}^{\prime}$ of $25 \mathrm{MPa}$, the strength reduction factors in ACI 318-14, $\phi$, are not always equal to 0.90 in the range of reinforcement ratios studied, e.g., $\phi$ is equal to 0.86 for $\rho$ of 0.017 and $\phi$ is equal to 0.83 for $\rho$ of 0.018 . As mentioned in Chapter 1, when the section is tension-controlled, i.e. the net tensile strain in the extreme layer of longitudinal tension reinforcement at nominal strength, $\varepsilon_{t}$, is larger than or equal to 0.005 , $\phi$ is equal to 0.90 . Moreover, $\phi$ less than 0.90 implies that $\varepsilon_{t}$ is less than 0.005 , but at least 0.004 (minimum requirement, used to control the upper limit of longitudinal reinforcement ratio) and the section is in the transition region between the tension-controlled and compression-controlled regions (ACI Committee 318 2014). The equation to compute the net tensile strain in the extreme layer of longitudinal tension reinforcement at nominal strength, $\varepsilon_{t}$, is (Wight 2016) 


$$
\varepsilon_{t}=\left(\frac{d_{t}-c}{c}\right) \varepsilon_{c u}
$$

where: $d_{t}$ is the distance from the extreme compression fiber to the extreme layer of tension steel; $\varepsilon_{c u}$ is the maximum usable strain at the extreme concrete compression fiber, assumed equal to 0.003 in $\mathrm{ACI} 318-14$; and, $c$ is the distance from the extreme compression fiber to the neutral axis given by (ACI Committee 318 2014)

$$
c=\frac{a}{\beta_{1}}
$$

where $\beta_{1}$ is the factor relating the depth of the equivalent rectangular compressive stress block to the depth of the neutral axis. It is computed as (ACI Committee 318 2014)

For $17.5 \mathrm{MPa} \leq f_{c}^{\prime} \leq 28 \mathrm{MPa}$,

$$
\beta_{1}=0.85
$$

For $28 \mathrm{MPa}<f_{c}^{\prime}<56 \mathrm{MPa}$,

$$
\beta_{1}=0.85-\frac{0.05\left(f_{c}{ }^{\prime}-28\right)}{7}
$$

For $f_{c}{ }^{\prime} \geq 56 \mathrm{MPa}$,

$$
\beta_{1}=0.65
$$

In this study, one layer of reinforcing steel is assumed, so $d_{t}$ equals $d$. From Equations [2.1], [2.7] and [2.8], $\varepsilon_{t}$ can be computed as

$$
\varepsilon_{t}=0.003 \times \frac{0.85 \beta_{1} f_{c}^{\prime}}{\rho f_{y}}-0.003
$$

According to Equation [2.12], increasing the longitudinal reinforcement ratio, $\rho$, reduces $\varepsilon_{t}$, and when $\varepsilon_{t}$ is less than $0.005, \phi$ also reduces. Similarly, when $f_{c}^{\prime}$ increases, $\varepsilon_{t}$ 
increases, so all of the $\phi$ values equal 0.90 for $f_{c}^{\prime}$ of $45 \mathrm{MPa}$ for the range of reinforcement ratios studied.

The relationships between the design flexural strength ratios, $\alpha_{M}$, and the longitudinal reinforcement ratios, $\rho$, for all ranges of reinforcement ratio are summarized in Figure 2.1 for $f_{c}^{\prime}$ of $25 \mathrm{MPa}$ and Figure 2.2 for $f_{c}^{\prime}$ of $45 \mathrm{MPa}$. For each partial material strength reduction factor combination, when $\phi$ equals $0.90, \alpha_{M}$ increases as $\rho$ increases. This occurs because the impact of $\phi_{c}$ on the lever arm between the resultant tension and compression forces increases as the reinforcement ratio and associated stress block depth increase. $\alpha_{M}$ decreases when $\phi$ is less than 0.90 , because the gradual decrease of $\phi$ leads to the reduction of the design flexural strength in ACI 318-14, $\phi M_{n}$. In both figures, four families of trend lines correspond to the four $\phi_{s}$ values, while the differences within each family are defined by the four $\phi_{c}$ values. Therefore, the design flexural strength ratio, $\alpha_{M}$, is more sensitive to $\phi_{s}$ than to $\phi_{c}$. The influence of the reinforcement ratios is small when sections are in the tension-controlled region ( $\phi$ of 0.90$)$, but relatively large changes of $\alpha_{M}$ occur in the transition region. Comparing Figures 2.1 and 2.2, the influence of $f_{c}^{\prime}$ is small, but $f_{c}^{\prime}$ affects the dispersion of each family: the data are more concentrated for a given $\phi_{s}$ value when $f_{c}^{\prime}$ is $45 \mathrm{MPa}$.

\subsubsection{Recommended Partial Material Strength Reduction Factors}

For $f_{c}^{\prime}$ of $25 \mathrm{MPa}$, the means and standard deviations of $\alpha_{M}$ for the three ranges of longitudinal reinforcement ratio are summarized in Tables 2.2-2.4. Regardless of the reinforcement ratios, as $\phi_{c}$ increases from 0.60 to 0.75 , the mean of $\alpha_{M}$ decreases and as $\phi_{s}$ increases from 0.80 to 0.95 , the mean of $\alpha_{M}$ also reduces, but more markedly. This demonstrates $\alpha_{M}$ is more sensitive to $\phi_{s}$, because the design flexural strength is affected more by $\phi_{s}$ than by $\phi_{c}$. This is also evident from the results shown in Figure 2.1.

The standard deviation of $\alpha_{M}$, reduces for increased $\phi_{c}$ values for reinforcement ratios ranging from 0.003 to 0.005 and from 0.006 to 0.010 , respectively. An opposite trend occurs for reinforcement ratios ranging from 0.011 to 0.018 because the strength reduction factor in ACI 318-14, $\phi$, is not always equal to 0.90 , e.g., $\phi$ equals 0.86 for $\rho$ of 
0.017 and $\phi$ equals 0.83 for $\rho$ of 0.018 . This is evident in Figure 2.1: the slopes become flatter with the increase of $\phi_{c}$ when $\phi$ equals 0.90 , so the variations of $\alpha_{M}$ become smaller. When $\phi_{s}$ increases, the standard deviation of $\alpha_{M}$ increases for the first two reinforcement ratio ranges, but an opposite trend still occurs for reinforcement ratios ranging from 0.011 to 0.018 .

The principle for selecting the best partial material strength reduction factors is to find the combinations where the mean design strength ratios approach 1 (or perhaps a value slightly larger than 1 to make the proposed design strengths slightly conservative) and the standard deviations are the least. When reinforcement ratios range from 0.003 to 0.005 , the best combination is $\phi_{s}$ of 0.90 and $\phi_{c}$ of 0.75 . When they range from 0.006 to 0.010 , the best combination is again $\phi_{s}$ of 0.90 and $\phi_{c}$ of 0.75 . And for beams with reinforcement ratios from 0.011 to 0.018 , the best combination is $\phi_{s}$ of 0.95 and $\phi_{c}$ of 0.65 , but the combination with $\phi_{s}$ of 0.90 and $\phi_{c}$ of 0.75 is nearly optimal. These also can be realized by inspection of Equation [2.6]: when $\phi$ equals $0.90, \phi_{s}$ and $\phi_{c}$ should be located on opposite sides of 0.90 to achieve the similar design strengths. Moreover, the design strength is more sensitive to $\phi_{s}$ in the tension-controlled region, so if $\phi_{s}$ equals $0.90, \phi_{c}$ should approach 0.90 and the closest value, 0.75 , is the best. When the section is in the transition region, $\phi$ reduces and the influence of the concrete strength increases, so $\phi_{c}$ tends to reduce.

For $f_{c}^{\prime}$ of $45 \mathrm{MPa}$, the means and standard deviations of $\alpha_{M}$ are summarized in Tables 2.5-2.7. The mean has the similar trend to that observed previously for $f_{c}^{\prime}$ of $25 \mathrm{MPa}$. The variation of the standard deviations, for all reinforcement ratio ranges is similar to that observed for $f_{c}^{\prime}$ of $25 \mathrm{MPa}$ and reinforcement ratios ranging from 0.003 to 0.005 and from 0.006 to 0.010 . The reason is that, for $f_{c}^{\prime}$ of $45 \mathrm{MPa}$, the strength reduction factor in ACI 318-14, $\phi$, is always equal to 0.90 in the range of reinforcement ratios studied. The best combination is therefore again $\phi_{s}$ of 0.90 and $\phi_{c}$ of 0.75 .

In summary, if the section is tension-controlled with $\phi$ of 0.90 , the best partial material strength reduction factor combination is $\phi_{s}$ of 0.90 and $\phi_{c}$ of 0.75 . If the section is in the 
transition region between the tension-controlled and compression-controlled regions, the combination with $\phi_{s}$ of 0.95 and $\phi_{c}$ of 0.65 is the best. Actually, all of the combinations in the family with $\phi_{s}$ of 0.90 are potentially suitable. For lower $\phi_{c}$ values, although the standard deviations of $\alpha_{M}$ are larger, they also yield higher means.

\subsection{One-way Shear}

This section presents partial material strength reduction factors that most closely approximate the design shear strengths obtained using the ACI 318-14 criteria. The analysis process is similar to that previously presented for moment.

\subsubsection{Geometric and Material Properties}

The investigation of one-way shear is limited to rectangular beams designed in accordance with ACI 318-14 with stirrups perpendicular to the longitudinal axes of the beams. The specified yield strength of transverse reinforcement, $f_{y t}$, equals $420 \mathrm{MPa}$ and the two grades of normalweight concrete represent the range of commonly used strengths, $f_{c}^{\prime}$, of 25 and $45 \mathrm{MPa}$. The ranges of stirrup ratio, $\rho_{t}$, investigated are from 0.001 to 0.007 for $f_{c}^{\prime}$ of $25 \mathrm{MPa}$ and from 0.001 to 0.010 for $f_{c}^{\prime}$ of $45 \mathrm{MPa}$. The selected ranges represent the ranges of minimum to maximum transverse reinforcement ratios permitted by ACI 318-14. Maximum stirrup spacing criteria are not always satisfied for some of the transverse reinforcement ratios, because the bar size is assumed fixed and the change of transverse reinforcement ratios is controlled by the spacing.

\subsubsection{Design Strength Ratios}

Design shear strength equations corresponding to the current ACI 318-14 and partial material strength reduction factors formats are defined in this section. The equations and definitions below refer to ACI 318-14, MacGregor and Bartlett (2000), and Wight (2016).

In ACI 318-14, for nonprestressed members without axial force, the nominal shear strength provided by concrete, $V_{c}$, is 


$$
V_{c}=0.17 \lambda \sqrt{f_{c}^{\prime}} b_{w} d
$$

where: $\lambda$ is the modification factor to account for the reduced shear strength of lightweight concrete, and equals 1.0 for normalweight concrete; and, $b_{w}$ is the web width. The nominal shear strength provided by shear reinforcement, $V_{s}$, is (ACI Committee 318 2014)

$$
V_{s}=\frac{A_{v} f_{y t} d}{s}
$$

where $s, A_{v}$ and $f_{y t}$ are the center-to-center spacing, the area within spacing $s$, and the specified yield strength, respectively, of the transverse reinforcement. The design oneway shear strength at a cross section, $\phi V_{n}$, is (ACI Committee 318 2014)

$$
\phi V_{n}=\phi\left(V_{c}+V_{s}\right)
$$

where $V_{n}$ is the nominal one-way shear strength, and $\phi$ equals 0.75 for members resisting shear.

For the proposed method, the design shear strength provided by concrete, $V_{r c}$, based on the partial material strength reduction factors format presented in MacGregor and Bartlett (2000), is assumed to be

$$
V_{r c}=0.17 \lambda \phi_{c} \sqrt{f_{c}^{\prime}} b_{w} d
$$

The design shear strength provided by shear reinforcement, $V_{r s}$, is (MacGregor and Bartlett 2000)

$$
V_{r s}=\frac{\phi_{s} A_{v} f_{y t} d}{s}
$$

The design one-way shear strength, $V_{r}$, is therefore (MacGregor and Bartlett 2000)

$$
V_{r}=V_{r c}+V_{r s}
$$


For the transverse reinforcement ratio, $\rho_{t}$, defined as (ACI Committee 318 2014)

$$
\rho_{t}=\frac{A_{v}}{b_{w} s}
$$

the design shear strength ratio, $\alpha_{V}$, is

$$
\alpha_{V}=\frac{\phi V_{n}}{V_{r}}=\frac{\phi\left(0.17 \lambda \sqrt{f_{c}^{\prime}}+\rho_{t} f_{y t}\right)}{0.17 \lambda \phi_{c} \sqrt{f_{c}^{\prime}}+\phi_{s} \rho_{t} f_{y t}}
$$

For each partial material strength reduction factor combination, the design shear strength ratios, $\alpha_{V}$, were calculated with respect to transverse reinforcement ratios, $\rho_{t}$. Again, each calculation was conducted twice because two $f_{c}^{\prime}$ values were investigated.

The relationships between the design shear strength ratios, $\alpha_{V}$, and the transverse reinforcement ratios, $\rho_{t}$, are summarized in Figure 2.3 for $f_{c}^{\prime}$ of $25 \mathrm{MPa}$ and Figure 2.4 for $f_{c}{ }^{\prime}$ of $45 \mathrm{MPa}$. For each partial material strength reduction factor combination, the design shear strength ratio, $\alpha_{V}$, declines as the transverse reinforcement ratio, $\rho_{t}$, increases. However, the sensitivity of $\alpha_{V}$ to $\rho_{t}$ becomes small for increased $\rho_{t}$. As for sections resisting moment, four families of trend lines correspond to the four $\phi_{s}$ values, and the differences within each family are defined by the four $\phi_{c}$ values. The four families overlap, however, so have been shown in separate figures. The variation of $\alpha_{V}$ is greatest for low $\rho_{t}$, low $\phi_{c}$, and high $\phi_{s}$ : as $\rho_{t}$ or $\phi_{c}$ increases or $\phi_{s}$ decreases, the sensitivities of $\alpha_{V}$ to changes of these parameters reduce. Comparing Figures 2.3 and 2.4, the influence of $f_{c}^{\prime}$ on $\alpha_{V}$ is small.

\subsubsection{Recommended Partial Material Strength Reduction Factors}

For $f_{c}{ }^{\prime}$ of $25 \mathrm{MPa}$ and $\rho_{t}$ of $0.001-0.007$, the means and standard deviations of $\alpha_{V}$ are summarized in Table 2.8. As $\phi_{c}$ increases from 0.60 to 0.75 , the mean of $\alpha_{V}$ decreases and as $\phi_{s}$ increases from 0.80 to 0.95 , the mean of $\alpha_{V}$ also reduces. The magnitude of the proposed design shear strength is more sensitive to $\phi_{s}$ than to $\phi_{c}$, but the difference is less than that for flexural members. This is why the four families of trend lines defined for 
each $\phi_{s}$ in Figure 2.3 tend to overlap. The standard deviation of $\alpha_{V}$ reduces for increased $\phi_{c}$ values, and increases for increased $\phi_{s}$ values. This is consistent with a previous observation concerning Figure 2.3: the variation of $\alpha_{V}$ is greatest for low $\phi_{c}$ and high $\phi_{s}$, because as $\phi_{c}$ increases or $\phi_{s}$ decreases, the sensitivities of $\alpha_{V}$ to these parameters reduce.

The best strength reduction factor combination has a mean design strength ratio approaching 1 with the least standard deviation. The best partial material strength reduction factor combination is $\phi_{s}$ of 0.80 and $\phi_{c}$ of 0.65 . Moreover, combinations with $\phi_{s}$ of 0.80 and $\phi_{c}$ of 0.70 , and $\phi_{s}$ of 0.85 and $\phi_{c}$ of 0.60 are close to optimal. These also can be realized by inspection of Equation [2.20]: when $\phi$ equals $0.75, \phi_{s}$ and $\phi_{c}$ should be located on opposite sides of 0.75 to achieve the similar design strengths.

For $f_{c}^{\prime}$ of $45 \mathrm{MPa}$, the means and standard deviations are summarized in Table 2.9. The values have the similar trends to those discussed previously for $f_{c}^{\prime}$ of $25 \mathrm{MPa}$. The best partial material strength reduction factor combination remains $\phi_{s}$ of 0.80 and $\phi_{c}$ of 0.65 . Again, combinations with $\phi_{s}$ of 0.80 and $\phi_{c}$ of 0.70 , and $\phi_{s}$ of 0.85 and $\phi_{c}$ of 0.60 are close to optimal.

\subsection{Combined Moment and Axial Force}

This section proposes partial material strength reduction factors that most closely approximate the combined flexural and axial strengths obtained using the ACI 318-14 design criteria. The analysis process is similar to those previously presented for moment and shear. Five column cross sections and eight property combinations for each cross section are investigated. The ratios of the design strengths corresponding to ACI 318-14 to those corresponding to various combinations of partial material strength reduction factors are calculated with respect to specific $e / h$ (eccentricity/overall column depth) values.

\subsubsection{Geometric and Material Properties}

The investigation of combined moment and axial force is limited to the following five column cross sections, as shown in Figure 2.5: 
1. Square section with three bars in each face.

2. Square section with three bars in two end faces only.

3. Square section with three bars in two side faces only.

4. Tied circular section with eight bars evenly distributed around the perimeter.

5. Spirally reinforced circular section with eight bars evenly distributed around the perimeter.

In all cases, the bending is assumed applied about a horizontal axis, $x-x$.

For each cross section, eight property combinations designed based on ACI 318-14 shown in Table 2.10 are investigated to account for varying steel location, steel area, and concrete strength. The values of $\gamma$, the ratios of the distance between the outer layers of reinforcement to the overall column depth, of 0.6 and 0.9 are considered as they bound commonly used values. The widths and overall depths corresponding to these two $\gamma$ values are 325 and $1300 \mathrm{~mm}$, respectively, to achieve a $65 \mathrm{~mm}$ distance from the outer reinforcement layer to the adjacent column face. Total reinforcement ratios, $\rho_{g}$, of 0.01 and 0.04 are investigated because these are the lower and upper limits, respectively, in columns containing lap splices (ACI Committee 318 2014). Specified concrete compressive strengths, $f_{c}^{\prime}$, of 25 and $45 \mathrm{MPa}$ are investigated. In all cases, the reinforcement yield strength, $f_{y}$, equals $420 \mathrm{MPa}$. These material strengths are identical to those investigated previously for moment and shear.

\subsubsection{Design Strength Ratios}

Design combined flexural and axial strength equations corresponding to the current $\mathrm{ACI}$ 318-14 and partial material strength reduction factors formats are defined in this section. The equations and definitions below refer to ACI 318-14, MacGregor and Bartlett (2000), and Wight (2016).

Setting the strain in the extreme tension layer of steel, $\varepsilon_{s 1}$, equal to $Z \varepsilon_{y}$, where $\varepsilon_{y}$ is the yield strain of reinforcement, and $Z$ is the ratio of the strain in the extreme tension layer of steel to the yield strain, the distance from the extreme compression fiber to the neutral axis, $c$, is (Wight 2016) 


$$
c=\left(\frac{0.003}{0.003-Z \varepsilon_{y}}\right) d_{1}
$$

where $d_{1}$ is the distance from the extreme compression fiber to the reinforcement located furthest from the extreme compression fiber. The depth of the equivalent rectangular stress block, $a$, is computed as (Wight 2016)

$$
a=\beta_{1} c
$$

and must not be greater than the section depth, $h$. The strain in the ith layer of reinforcement, $\varepsilon_{s i}$, is (Wight 2016)

$$
\varepsilon_{s i}=\left(\frac{c-d_{i}}{c}\right) 0.003
$$

where $d_{i}$ is the distance from the extreme compression fiber to the $i$ th layer of reinforcement. Tensile strains, stresses and forces are taken to be negative quantities. The stress in the $i$ th layer of reinforcement, $f_{s i}$, is (Wight 2016)

$$
f_{s i}=\varepsilon_{s i} E_{s} \text { but }-f_{y} \leq f_{s i} \leq f_{y}
$$

where $E_{s}$ is the modulus of elasticity of reinforcement.

In ACI 318-14, the nominal compressive force in concrete, $C_{c}$, is (Wight 2016)

$$
C_{c}=0.85 f_{c}^{\prime} a b
$$

If $a$ is less than $d_{i}$, the nominal force in the $i$ th layer of reinforcement, $F_{s i}$, is (Wight 2016)

$$
F_{s i}=f_{s i} A_{s i}
$$

where $A_{s i}$ is the area of the $i$ th layer of reinforcement. If $a$ is greater than $d_{i}$, it is necessary to account for the concrete displaced by the steel (Wight 2016):

$$
F_{s i}=\left(f_{s i}-0.85 f_{c}^{\prime}\right) A_{s i}
$$


The design axial strength, $\phi P_{n}$, is (Wight 2016)

$$
\phi P_{n}=\phi\left(C_{c}+\sum_{i=1}^{n} F_{s i}\right)
$$

where $P_{n}$ is the nominal axial strength of a member.

The design flexural strength, $\phi M_{n}$, is (Wight 2016)

$$
\phi M_{n}=\phi\left(C_{c}\left(\frac{h}{2}-\frac{a}{2}\right)+\sum_{i=1}^{n} F_{s i}\left(\frac{h}{2}-d_{i}\right)\right)
$$

To account for accidental eccentricities, the maximum design axial compressive strength, $\phi P_{n, \max }$, for tied columns is (Wight 2016)

$$
\phi P_{n, \max }=0.80 \phi\left(0.85 f_{c}^{\prime}\left(A_{g}-A_{s t}\right)+f_{y} A_{s t}\right)
$$

and for spirally reinforced columns is (Wight 2016)

$$
\phi P_{n, \max }=0.85 \phi\left(0.85 f_{c}^{\prime}\left(A_{g}-A_{s t}\right)+f_{y} A_{s t}\right)
$$

where: $P_{n, \max }$ is the maximum nominal axial compressive strength of a member; $A_{g}$ is the gross area of the section; and, $A_{s t}$ is the total area of the nonprestressed longitudinal reinforcement. The design axial tensile strength, $\phi P_{n t}$, is (Wight 2016)

$$
\phi P_{n t}=-\phi f_{y} A_{s t}
$$

where $P_{n t}$ is the nominal axial tensile strength of a member.

For the proposed method, the factored compressive force in concrete, $C_{r c}$, based on the partial material strength reduction factors format presented in MacGregor and Bartlett (2000), is assumed to be

$$
C_{r c}=0.85 \phi_{c} f_{c}^{\prime} a b
$$


If $a$ is less than $d_{i}$, the factored force in the $i$ th layer of reinforcement, $F_{r s i}$, is (MacGregor and Bartlett 2000)

$$
F_{r s i}=\phi_{s} f_{s i} A_{s i}
$$

If $a$ is greater than $d_{i}$, it is assumed to be (MacGregor and Bartlett 2000)

$$
F_{r s i}=\left(\phi_{s} f_{s i}-0.85 \phi_{c} f_{c}^{\prime}\right) A_{s i}
$$

The design axial strength, $P_{r}$, is (MacGregor and Bartlett 2000)

$$
P_{r}=C_{r c}+\sum_{i=1}^{n} F_{r s i}
$$

The design flexural strength, $M_{r}$, is (MacGregor and Bartlett 2000)

$$
M_{r}=C_{r c}\left(\frac{h}{2}-\frac{a}{2}\right)+\sum_{i=1}^{n} F_{r s i}\left(\frac{h}{2}-d_{i}\right)
$$

To account for accidental eccentricities, the maximum design axial compressive strength, $P_{r, m a x}$, for tied columns is assumed to be (MacGregor and Bartlett 2000)

$$
P_{r, \max }=0.80\left(0.85 \phi_{c} f_{c}^{\prime}\left(A_{g}-A_{s t}\right)+\phi_{s} f_{y} A_{s t}\right)
$$

and for spirally reinforced columns is assumed to be (MacGregor and Bartlett 2000)

$$
P_{r, \max }=0.85\left(0.85 \phi_{c} f_{c}^{\prime}\left(A_{g}-A_{s t}\right)+\phi_{s} f_{y} A_{s t}\right)
$$

The design axial tensile strength, $P_{r t}$, is (MacGregor and Bartlett 2000)

$$
P_{r t}=-\phi_{s} f_{y} A_{s t}
$$

The design eccentricity for ACI 318-14, $e_{u}$, equals $\phi M_{n} / \phi P_{n}$, and for the proposed method, $e_{r}$, equals $M_{r} / P_{r}$. For a specific value of $e / h$, ratio $\alpha_{P M}$, of design combined 
flexural and axial strengths computed using the strength reduction factor in ACI 318-14 and the partial material strength reduction factors is (Hong and Zhou 1999)

$$
\alpha_{P M}=\frac{\sqrt{\left(\phi P_{n}\right)^{2}+\left(\frac{\phi M_{n}}{h}\right)^{2}}}{\sqrt{P_{r}^{2}+\left(\frac{M_{r}}{h}\right)^{2}}}=\frac{\left|\phi P_{n}\right| \sqrt{1+\left(e_{u} / h\right)^{2}}}{\left|P_{r}\right| \sqrt{1+\left(e_{r} / h\right)^{2}}}
$$

Values of $\alpha_{P M}$ greater than 1 represent cases where the design strengths computed using the current ACI 318-14 criteria exceed, and are therefore unconservative with respect to, those computed using the partial material strength reduction factors criteria.

For circular columns, the strain-compatibility solution described above can also be used. The only differences are the area of the compression segment of the circular section, $A$, and the moment of this area about the center of the column, $A \bar{y}$, which are (Wight 2016)

$$
\begin{gathered}
A=h^{2}\left(\frac{\theta-\sin \theta \cos \theta}{4}\right) \\
A \bar{y}=h^{3}\left(\frac{\sin ^{3} \theta}{12}\right)
\end{gathered}
$$

where angle $\theta$, defined in Figure 2.6 (which is similar to Fig. 11-20 in Wight (2016)), is expressed in radians. If $a \leq h / 2$, which corresponds to $\theta \leq \pi / 2$ (Wight 2016),

$$
\theta=\cos ^{-1}\left(\frac{h / 2-a}{h / 2}\right)
$$

If $a>h / 2$, which corresponds to $\theta>\pi / 2$ (Wight 2016),

$$
\theta=\pi-\cos ^{-1}\left(\frac{a-h / 2}{h / 2}\right)
$$

To compare design strengths for ACI 318-14 and the proposed method, the calculation of $\alpha_{P M}$ is based on identical $e_{u} / h$ and $e_{r} / h$ values of 0 (compression only), 0.1, 0.2, 0.3, 0.4, 
$0.5,0.6,0.7,0.8,0.9,1.0,2.0,3.0,4.0,5.0,6.0,7.0,8.0,9.0,10.0,-10.0,-5.0,-1.0$, $-0.5,-0.1$ and 0 (tension only). Interpolation in Matlab (Version R2016b; The Mathworks, Inc. 2016) was used to obtain $\phi P_{n}, P_{r}$ and $\alpha_{P M}$ values corresponding to these specific e/h values. The Matlab (Version R2016b; The Mathworks, Inc. 2016) codes for Column Section 1 (square section with three bars in each face) and Column Section 5 (spirally reinforced circular section with eight bars evenly distributed around the perimeter), two representative cross sections, are presented in Appendix A.

For Column Section 1 (square section with three bars in each face), the relationships between $\alpha_{P M}$, and the specific $e / h$ for Property Combination 1 ( $\gamma$ of $0.6, f_{c}^{\prime}$ of $25 \mathrm{MPa}$, and $\rho_{g}$ of 0.01 ) are shown in Figure 2.7a for $e / h>0$. The dotted vertical line on the figure shows $e_{\text {ubal }} / h$ of 0.42 , where $e$ corresponds to the balanced failure for ACI 318-14. The solid vertical lines show the range of $e$ values corresponding to the balanced failures for the sixteen partial material strength reduction factor combinations, from $\left(e_{\text {rbal }} / h\right)_{\min }$ of 0.43 to $\left(e_{\text {rbal }} / h\right)_{\max }$ of 0.50 . The horizontal axis has a $\log$ scale to separate the data corresponding to the lower $e / h$ values, which are the most common cases in short columns. When $e / h>0$, for each partial material strength reduction factor combination, $\alpha_{P M}$ reduces as $e / h$ increases until the balance point is reached. The large increase of $\alpha_{P M}$ between $e / h \approx 0.4$ and $e / h \approx 0.8$ is due to the increase of $\phi$ in ACI 318-14 from 0.65 to 0.90 . As the eccentricity increases, columns tend to be tension-controlled and $\alpha_{P M}$ declines slightly to a stable level. In the compression-controlled region, four distinct families of the lines correspond to the four $\phi_{c}$ values, and the slight differences within each family are defined by the four $\phi_{s}$ values, which indicates the significance of the concrete strength. As the eccentricity increases, the steel strength becomes more influential so the four families of the lines are defined by the four $\phi_{s}$ values, and the differences within each family are defined by the four $\phi_{c}$ values.

When $e / h<0$, Figure $2.7 \mathrm{~b}$ shows that $\alpha_{P M}$ increases slightly as the absolute value of $e / h$ increases. For the cross sections subjected to tension, the trend lines are also much more dependent on $\phi_{s}$, because the tensile strength of concrete is ignored. Figures 
corresponding to the other property combinations are shown in Figures A.1-A.7 of Appendix A.

Figures 2.8a and 2.8b show $\alpha_{P M}$ values for the eight property combinations for Column Section 1 (square section with three bars in each face) for $e / h>0$ and $e / h<0$, respectively. The partial material strength reduction factors correspond to $\phi_{s}$ of 0.90 and $\phi_{c}$ of 0.60 . For $e / h$ approximately ranging from 0.1 to 0.38 , all of the eight property combinations for both of the ACI 318-14 and partial material strength reduction factors criteria do not reach the minimum value of $e_{u b a l} / h$ or $e_{r b a l} / h$, so they are compressioncontrolled. In this region, the influence of $\gamma$ is very small, because of the small applied eccentricity. The influence of $f_{c}{ }^{\prime}$ is moderate, but $\rho_{g}$ makes a big difference. For $e / h$ approximately ranging from 0.38 to 1.3 , some reach the balance point, but others do not. For $e / h$ approximately ranging from 1.3 to 10 , all of the eight property combinations for both of the ACI 318-14 and partial material strength reduction factors criteria equal or exceed the maximum value of $e_{u b a l} / h$ or $e_{r b a l} / h$, so they are in the transition or tensioncontrolled regions. The two lines corresponding to Property Combination 2 ( $\gamma$ of $0.6, f_{c}^{\prime}$ of $25 \mathrm{MPa}$, and $\rho_{g}$ of 0.04 ) and Property Combination 4 ( $\gamma$ of $0.6, f_{c}^{\prime}$ of $45 \mathrm{MPa}$, and $\rho_{g}$ of 0.04), differ from the others shown because they remain in the transition region until $e / h$ equals 10: in other words, $\varepsilon_{t}$ equal to 0.005 occurs when $e / h \geq 10$. For $e / h$ less than 0 , the sections are subjected to tension. In the tension-controlled region, with the increase of $e / h, \gamma$ tends to become more significant, and followed by $\rho_{g}$ and $f_{c}^{\prime}$.

For Column Sections 2, 3 and 4, the $\alpha_{P M}$ values are similar to those for Column Section 1. The results are shown in Figures A.8-A.31 of Appendix A. For Column Section 5 (spirally reinforced circular section with eight bars evenly distributed around the perimeter), the relationships between $\alpha_{P M}$, and the specific $e / h$ for Property Combination $1\left(\gamma\right.$ of $0.6, f_{c}^{\prime}$ of $25 \mathrm{MPa}$, and $\rho_{g}$ of 0.01$)$ are shown in Figures 2.9a and 2.9b, for $e / h>0$ and $e / h<0$, respectively. In the compression-controlled region, $\alpha_{P M}$ increases markedly compared to the values for the tied columns, because $\phi$ in ACI 318-14 for spirally reinforced columns equals 0.75 instead of 0.65 . Figures corresponding to the other property combinations are shown in Figures A.32-A.38. 


\subsubsection{Recommended Partial Material Strength Reduction Factors}

For Column Section 1 (square section with three bars in each face), the minimum and maximum values of $e / h$ for all eight property combinations corresponding to the ACI 318-14 and partial material strength reduction factors criteria at the balance point are 0.383 and 1.361, respectively. It is therefore decided to categorize the data in four $e / h$ ranges: (1) $0 \leq e / h \leq 0.3$, corresponding to compression-controlled failures; (2) $0.3<e / h$ $\leq 1.0$, an intermediate range; (3) $1.0<e / h \leq 10.0$, corresponding to data below the balanced point on interaction diagrams, in the tension-controlled region or the transition region; and, (4) $e / h \leq 0$, corresponding to axial tension. For the eight property combinations, the means and standard deviations of $\alpha_{P M}$ are summarized in Tables 2.112.14. As $\phi_{c}$ increases from 0.60 to 0.75 , the mean of $\alpha_{P M}$ decreases. Similarly as $\phi_{s}$ increases from 0.80 to 0.95 , the mean of $\alpha_{P M}$ also decreases. The magnitude of the design strength ratio is more sensitive to $\phi_{c}$ than to $\phi_{s}$ in Range (1), the compression-controlled region, as shown in Table 2.11, while the reverse happens in Ranges (3) and (4), as shown in Tables 2.13 and 2.14. All the means are less than 1 in the compressioncontrolled region, indicating any of the partial material strength reduction factor combination considered yields higher design strengths and so is less conservative compared with ACI 318-14. The standard deviation of $\alpha_{P M}$, reduces with increased $\phi_{c}$ values and increases with increased $\phi_{s}$ values in Ranges (1) and (4). In Ranges (2) and (3), the standard deviation of $\alpha_{P M}$ decreases with increased $\phi_{s}$ and $\phi_{c}$.

The best partial material strength reduction factor combination corresponds to that having a mean design strength ratio approaching 1 with the least standard deviation. In the compression-controlled region, the family of partial material strength reduction factors with $\phi_{c}$ of 0.60 is the best and, at greater eccentricities, the family of partial material strength reduction factors with $\phi_{s}$ of 0.90 is the best. To further investigate which combination of partial material strength reduction factors is appropriate, reliability analyses will be presented in Chapter 4 . 
For Column Sections 2, 3 and 4, the results are similar to Column Section 1 and the results are shown in Tables A.1-A.12 of Appendix A. And the boundary for $e / h$ between Ranges (1) and (2) is 0.4 for Column Section 2.

For Column Section 5 (spirally reinforced circular section with eight bars evenly distributed around the perimeter), the results are shown in Tables 2.15-2.18: In the compression-controlled region, the family of partial material strength reduction factors with $\phi_{c}$ of 0.70 is the best and, at greater eccentricities, both the families with $\phi_{s}$ of 0.90 and 0.95 are the best. Alternatively an overall factor could be applied to the factored resistance of spirally reinforced columns to account for the advantage of confinement. The magnitude of this factor should be between $(0.75 / 0.65=) 1.15$ and $(0.90 / 0.90=) 1$. However, if a single overall factor is adopted, the associated best partial material strength reduction factors for the compression-controlled and tension-controlled regions will be different. As the different $\phi$ factors for tied and spirally reinforced columns in ACI 31814 impact mostly the region of compression-controlled failure, it may be better to apply a unique $\phi_{c}$ value to spirally reinforced columns. To further investigate which combination of partial material strength reduction factors is appropriate, reliability analyses will be presented in Chapter 4.

\subsection{Summary and Conclusions}

This chapter has presented the calculation of design strength ratios for cross sections subjected to moment, one-way shear, and combined moment and axial force. The best partial material strength reduction factors that best duplicate the design strengths obtained using the current ACI 318-14 provisions are proposed. The geometric and material properties of the designed sections represent commonly used values and meet most of the requirements in ACI 318-14. The preliminary results obtained in this chapter will be referred for reliability analyses presented in Chapters 3 and 4.

For members subjected to moment, the best partial material strength reduction factor combination is $\phi_{s}$ of 0.90 and $\phi_{c}$ of 0.75 if the section is tension-controlled. If the section is in the transition region between the tension-controlled and compression-controlled regions, the combination with $\phi_{s}$ of 0.95 and $\phi_{c}$ of 0.65 is the best, but the $\phi_{s}$ of 0.95 is an 
extreme value, so maybe not satisfactory for reliability analyses. Actually, all of the combinations in the family with $\phi_{s}$ of 0.90 are potentially suitable. For lower $\phi_{c}$ values, although the standard deviations of $\alpha_{M}$ are larger, they also yield higher means.

For members resisting one-way shear, the best strength reduction factor combination is $\phi_{s}$ of 0.80 and $\phi_{c}$ of 0.65 . Combinations with $\phi_{s}$ of 0.80 and $\phi_{c}$ of 0.70 , and $\phi_{s}$ of 0.85 and $\phi_{c}$ of 0.60 are close to optimal. These results are only based on the design strength calculations, so they just duplicate the design strengths corresponding to ACI 318-14. As mentioned in Chapter 1, however, for members subjected to shear, the statistical parameters for the professional factor have changed significantly, so the reliability indices may be unsuitable for both ACI 318-14 and the partial material strength reduction factors, and lead to different preferred partial material strength reduction factors.

For members resisting combined moment and axial force, $\phi_{s}$ of 0.90 is the best for the tension-controlled region and $\phi_{c}$ of 0.60 is the best for the compression-controlled region for tied columns. For spirally reinforced columns, $\phi_{c}$ of 0.70 is preferred and both the families with $\phi_{s}$ of 0.90 and 0.95 seem reasonable.

The results obtained in this chapter are only based on the design strengths. However, if the reliability indices for the current ACI 318-14 criteria are not suitable, the reliability indices for the partial material strength reduction factors just duplicating the design strengths of the current criteria may be also unsatisfactory. In that case, the results obtained in this chapter will be less useful and the best partial material strength reduction factors will be determined only based on the reliability analyses.

Therefore, the final selection of the best partial material strength reduction factors will be made based on the reliability analyses presented in Chapters 3 and 4 . 
Table 2.1: Partial material strength reduction factor combinations

\begin{tabular}{ccccc}
\hline & \multicolumn{4}{c}{$\phi_{c}$} \\
\cline { 2 - 5 }$\phi_{s}$ & 0.60 & 0.65 & 0.70 & 0.75 \\
\hline 0.80 & $C_{11}$ & $C_{12}$ & $C_{13}$ & $C_{14}$ \\
0.85 & $C_{21}$ & $C_{22}$ & $C_{23}$ & $C_{24}$ \\
0.90 & $C_{31}$ & $C_{32}$ & $C_{33}$ & $C_{34}$ \\
0.95 & $C_{41}$ & $C_{42}$ & $C_{43}$ & $C_{44}$ \\
\hline
\end{tabular}

Table 2.2: Means and standard deviations of design flexural strength ratios, $\alpha_{M}$, for $f_{c}^{\prime}=$ $25 \mathrm{MPa}$ and $\rho=0.003-0.005$

\begin{tabular}{ccccccccc}
\hline & \multicolumn{8}{c}{$\phi_{c}$} \\
\cline { 2 - 10 }$\phi_{s}$ & \multicolumn{2}{c}{0.60} & \multicolumn{2}{c}{0.65} & \multicolumn{2}{c}{0.70} & \multicolumn{2}{c}{0.75} \\
\cline { 2 - 10 } 0.80 & 1.141 & 0.004 & 1.136 & 0.003 & 1.132 & 0.002 & 1.128 & 0.001 \\
0.85 & 1.077 & 0.005 & 1.072 & 0.004 & 1.068 & 0.002 & 1.065 & 0.002 \\
0.90 & 1.021 & 0.006 & 1.016 & 0.004 & 1.012 & 0.003 & 1.008 & 0.002 \\
0.95 & 0.971 & 0.006 & 0.966 & 0.005 & 0.962 & 0.004 & 0.958 & 0.003 \\
\hline
\end{tabular}

Note: $\sigma$, standard deviation.

Table 2.3: Means and standard deviations of design flexural strength ratios, $\alpha_{M}$, for $f_{c}^{\prime}=$ $25 \mathrm{MPa}$ and $\rho=0.006-0.010$

\begin{tabular}{ccccccccc}
\hline & \multicolumn{8}{c}{$\phi_{c}$} \\
\cline { 2 - 9 }$\phi_{s}$ & \multicolumn{2}{c}{0.60} & \multicolumn{2}{c}{0.65} & \multicolumn{2}{c}{0.70} & \multicolumn{2}{c}{0.75} \\
\cline { 2 - 10 } & Mean & $\sigma$ & Mean & $\sigma$ & Mean & $\sigma$ & Mean & $\sigma$ \\
\hline 0.80 & 1.158 & 0.007 & 1.148 & 0.005 & 1.139 & 0.003 & 1.131 & 0.001 \\
0.85 & 1.098 & 0.009 & 1.088 & 0.006 & 1.079 & 0.004 & 1.071 & 0.003 \\
0.90 & 1.045 & 0.010 & 1.034 & 0.008 & 1.025 & 0.006 & 1.018 & 0.004 \\
0.95 & 0.998 & 0.011 & 0.987 & 0.009 & 0.977 & 0.007 & 0.970 & 0.005 \\
\hline
\end{tabular}


Table 2.4: Means and standard deviations of design flexural strength ratios, $\alpha_{M}$, for $f_{c}^{\prime}=$ $25 \mathrm{MPa}$ and $\rho=0.011-0.018$

\begin{tabular}{ccccccccc}
\hline & \multicolumn{8}{c}{$\phi_{c}$} \\
\cline { 2 - 10 }$\phi_{s}$ & \multicolumn{2}{c}{0.60} & \multicolumn{2}{c}{0.65} & \multicolumn{2}{c}{0.70} & \multicolumn{2}{c}{0.75} \\
\cline { 2 - 10 } & Mean & $\sigma$ & Mean & $\sigma$ & Mean & $\sigma$ & Mean & $\sigma$ \\
\hline 0.80 & 1.174 & 0.027 & 1.153 & 0.029 & 1.135 & 0.030 & 1.121 & 0.032 \\
0.85 & 1.121 & 0.024 & 1.100 & 0.026 & 1.082 & 0.027 & 1.067 & 0.029 \\
0.90 & 1.075 & 0.022 & 1.053 & 0.023 & 1.035 & 0.025 & 1.019 & 0.026 \\
0.95 & 1.035 & 0.021 & 1.012 & 0.021 & 0.993 & 0.022 & 0.977 & 0.024 \\
\hline
\end{tabular}

Table 2.5: Means and standard deviations of design flexural strength ratios, $\alpha_{M}$, for $f_{c}{ }^{\prime}=$ $45 \mathrm{MPa}$ and $\rho=0.003-0.005$

\begin{tabular}{ccccccccc}
\hline & \multicolumn{10}{c}{$\phi_{c}$} \\
\cline { 2 - 10 }$\phi_{s}$ & \multicolumn{2}{c}{0.60} & \multicolumn{2}{c}{0.65} & \multicolumn{2}{c}{0.70} & \multicolumn{2}{c}{0.75} \\
\cline { 2 - 10 } & Mean & $\sigma$ & Mean & $\sigma$ & Mean & $\sigma$ & Mean & $\sigma$ \\
\hline 0.80 & 1.133 & 0.002 & 1.131 & 0.002 & 1.129 & 0.001 & 1.127 & 0.000 \\
0.85 & 1.069 & 0.003 & 1.066 & 0.002 & 1.064 & 0.001 & 1.062 & 0.001 \\
0.90 & 1.011 & 0.003 & 1.009 & 0.002 & 1.006 & 0.002 & 1.005 & 0.001 \\
0.95 & 0.960 & 0.003 & 0.957 & 0.003 & 0.955 & 0.002 & 0.953 & 0.001 \\
\hline
\end{tabular}

Table 2.6: Means and standard deviations of design flexural strength ratios, $\alpha_{M}$, for $f_{c}^{\prime}=$ $45 \mathrm{MPa}$ and $\rho=0.006-0.010$

\begin{tabular}{cccccccccc}
\hline & \multicolumn{10}{c}{$\phi_{c}$} \\
\cline { 2 - 10 }$\phi_{s}$ & \multicolumn{2}{c}{0.60} & \multicolumn{2}{c}{0.65} & \multicolumn{2}{c}{0.70} & \multicolumn{2}{c}{0.75} \\
\cline { 2 - 10 } & Mean & $\sigma$ & Mean & $\sigma$ & Mean & $\sigma$ & Mean & $\sigma$ \\
\hline 0.80 & 1.143 & 0.004 & 1.137 & 0.003 & 1.132 & 0.002 & 1.128 & 0.001 \\
0.85 & 1.080 & 0.004 & 1.074 & 0.003 & 1.069 & 0.002 & 1.065 & 0.001 \\
0.90 & 1.024 & 0.005 & 1.018 & 0.004 & 1.013 & 0.003 & 1.009 & 0.002 \\
0.95 & 0.974 & 0.006 & 0.968 & 0.004 & 0.963 & 0.003 & 0.959 & 0.002 \\
\hline
\end{tabular}


Table 2.7: Means and standard deviations of design flexural strength ratios, $\alpha_{M}$, for $f_{c}{ }^{\prime}=$ $45 \mathrm{MPa}$ and $\rho=0.011-0.018$

\begin{tabular}{ccccccccc}
\hline & \multicolumn{10}{c}{$\phi_{c}$} \\
\cline { 2 - 10 }$\phi_{s}$ & \multicolumn{2}{c}{0.60} & \multicolumn{2}{c}{0.65} & \multicolumn{2}{c}{0.70} & \multicolumn{2}{c}{0.75} \\
\cline { 2 - 10 } & Mean & $\sigma$ & Mean & $\sigma$ & Mean & $\sigma$ & Mean & $\sigma$ \\
\hline 0.80 & 1.159 & 0.006 & 1.148 & 0.004 & 1.139 & 0.003 & 1.132 & 0.001 \\
0.85 & 1.099 & 0.008 & 1.088 & 0.005 & 1.079 & 0.004 & 1.071 & 0.002 \\
0.90 & 1.045 & 0.009 & 1.035 & 0.007 & 1.025 & 0.005 & 1.018 & 0.003 \\
0.95 & 0.998 & 0.010 & 0.987 & 0.008 & 0.978 & 0.006 & 0.970 & 0.004 \\
\hline
\end{tabular}

Table 2.8: Means and standard deviations of design shear strength ratios, $\alpha_{V}$, for $f_{c}{ }^{\prime}=25$

$\mathrm{MPa}$ and $\rho_{t}=0.001-0.007$

\begin{tabular}{ccccccccc}
\hline & \multicolumn{10}{c}{$\phi_{c}$} \\
\cline { 2 - 10 }$\phi_{s}$ & \multicolumn{2}{c}{0.60} & \multicolumn{2}{c}{0.65} & \multicolumn{2}{c}{0.70} & \multicolumn{2}{c}{0.75} \\
\cline { 2 - 10 } & Mean & $\sigma$ & Mean & $\sigma$ & Mean & $\sigma$ & Mean & $\sigma$ \\
\hline 0.80 & 1.038 & 0.047 & 1.011 & 0.033 & 0.985 & 0.021 & 0.961 & 0.010 \\
0.85 & 0.997 & 0.055 & 0.971 & 0.041 & 0.947 & 0.029 & 0.924 & 0.018 \\
0.90 & 0.958 & 0.061 & 0.934 & 0.048 & 0.912 & 0.036 & 0.891 & 0.026 \\
0.95 & 0.923 & 0.066 & 0.900 & 0.054 & 0.879 & 0.042 & 0.860 & 0.032 \\
\hline
\end{tabular}

Table 2.9: Means and standard deviations of design shear strength ratios, $\alpha_{V}$, for $f_{c}{ }^{\prime}=45$

$\mathrm{MPa}$ and $\rho_{t}=0.001-0.010$

\begin{tabular}{cccccccccc}
\hline & \multicolumn{8}{c}{$\phi_{c}$} \\
\cline { 2 - 10 }$\phi_{s}$ & \multicolumn{2}{c}{0.60} & \multicolumn{2}{c}{0.65} & \multicolumn{2}{c}{0.70} & \multicolumn{2}{c}{0.75} \\
\cline { 2 - 10 } & Mean & $\sigma$ & Mean & $\sigma$ & Mean & $\sigma$ & Mean & $\sigma$ \\
\hline 0.80 & 1.039 & 0.050 & 1.011 & 0.035 & 0.985 & 0.022 & 0.961 & 0.010 \\
0.85 & 0.997 & 0.058 & 0.971 & 0.044 & 0.947 & 0.031 & 0.924 & 0.019 \\
0.90 & 0.959 & 0.065 & 0.935 & 0.051 & 0.912 & 0.039 & 0.891 & 0.027 \\
0.95 & 0.924 & 0.071 & 0.901 & 0.058 & 0.880 & 0.045 & 0.860 & 0.034 \\
\hline
\end{tabular}


Table 2.10: Section properties for columns

\begin{tabular}{ccccccc}
\hline Category & $\gamma$ & $b(\mathrm{~mm})$ & $h(\mathrm{~mm})$ & $f_{c}{ }^{\prime}(\mathrm{MPa})$ & $f_{y}(\mathrm{MPa})$ & $\rho_{g}$ \\
\hline 1 & 0.6 & 325 & 325 & 25 & 420 & 0.01 \\
2 & 0.6 & 325 & 325 & 25 & 420 & 0.04 \\
3 & 0.6 & 325 & 325 & 45 & 420 & 0.01 \\
4 & 0.6 & 325 & 325 & 45 & 420 & 0.04 \\
5 & 0.9 & 1300 & 1300 & 25 & 420 & 0.01 \\
6 & 0.9 & 1300 & 1300 & 25 & 420 & 0.04 \\
7 & 0.9 & 1300 & 1300 & 45 & 420 & 0.01 \\
8 & 0.9 & 1300 & 1300 & 45 & 420 & 0.04 \\
\hline
\end{tabular}

Table 2.11: Means and standard deviations of design combined flexural and axial strength ratios, $\alpha_{P M}$, for Column Section 1 and $0 \leq e / h \leq 0.3$

\begin{tabular}{ccccccccc}
\hline & \multicolumn{8}{c}{$\phi_{c}$} \\
\cline { 2 - 9 }$\phi_{s}$ & \multicolumn{2}{c}{0.60} & \multicolumn{2}{c}{0.65} & \multicolumn{2}{c}{0.70} & \multicolumn{2}{c}{0.75} \\
\cline { 2 - 10 } 0.80 & 0.997 & 0.041 & 0.943 & 0.027 & 0.895 & 0.017 & 0.852 & 0.008 \\
0.85 & 0.979 & 0.049 & 0.926 & 0.035 & 0.879 & 0.024 & 0.837 & 0.014 \\
0.90 & 0.961 & 0.057 & 0.910 & 0.042 & 0.865 & 0.031 & 0.824 & 0.021 \\
0.95 & 0.943 & 0.063 & 0.894 & 0.049 & 0.850 & 0.037 & 0.811 & 0.027 \\
\hline
\end{tabular}

Table 2.12: Means and standard deviations of design combined flexural and axial strength ratios, $\alpha_{P M}$, for Column Section 1 and $0.3<e / h \leq 1.0$

\begin{tabular}{ccccccccc}
\hline & \multicolumn{10}{c}{$\phi_{c}$} \\
\cline { 2 - 10 }$\phi_{s}$ & \multicolumn{2}{c}{0.60} & \multicolumn{2}{c}{0.65} & \multicolumn{2}{c}{0.70} & \multicolumn{2}{c}{0.75} \\
\cline { 2 - 10 } 0.80 & 1.073 & 0.136 & 1.036 & 0.134 & 1.004 & 0.134 & 0.975 & 0.135 \\
0.85 & 1.037 & 0.130 & 1.002 & 0.128 & 0.970 & 0.126 & 0.941 & 0.126 \\
0.90 & 1.005 & 0.126 & 0.970 & 0.122 & 0.939 & 0.120 & 0.911 & 0.119 \\
0.95 & 0.975 & 0.122 & 0.941 & 0.118 & 0.911 & 0.115 & 0.884 & 0.113 \\
\hline
\end{tabular}


Table 2.13: Means and standard deviations of design combined flexural and axial strength ratios, $\alpha_{P M}$, for Column Section 1 and $1.0<e / h \leq 10.0$

\begin{tabular}{ccccccccc}
\hline & \multicolumn{8}{c}{$\phi_{c}$} \\
\cline { 2 - 10 }$\phi_{s}$ & \multicolumn{2}{c}{0.60} & \multicolumn{2}{c}{0.65} & \multicolumn{2}{c}{0.70} & \multicolumn{2}{c}{0.75} \\
\cline { 2 - 10 } 0.80 & 1.157 & 0.044 & 1.142 & 0.041 & 1.129 & 0.041 & 1.117 & 0.042 \\
0.85 & 1.099 & 0.043 & 1.085 & 0.040 & 1.073 & 0.039 & 1.061 & 0.039 \\
0.90 & 1.047 & 0.043 & 1.034 & 0.040 & 1.022 & 0.038 & 1.011 & 0.037 \\
0.95 & 1.000 & 0.044 & 0.988 & 0.040 & 0.977 & 0.037 & 0.966 & 0.035 \\
\hline
\end{tabular}

Table 2.14: Means and standard deviations of design combined flexural and axial strength ratios, $\alpha_{P M}$, for Column Section 1 and $e / h \leq 0$

\begin{tabular}{ccccccccc}
\hline & \multicolumn{10}{c}{$\phi_{c}$} \\
\cline { 2 - 9 }$\phi_{s}$ & \multicolumn{2}{c}{0.60} & \multicolumn{2}{c}{0.65} & \multicolumn{2}{c}{0.70} & \multicolumn{2}{c}{0.75} \\
\cline { 2 - 10 } 0 Mean & $\sigma$ & Mean & $\sigma$ & Mean & $\sigma$ & Mean & $\sigma$ \\
\hline 0.80 & 1.142 & 0.022 & 1.136 & 0.016 & 1.131 & 0.011 & 1.126 & 0.010 \\
0.85 & 1.078 & 0.025 & 1.073 & 0.019 & 1.068 & 0.014 & 1.064 & 0.010 \\
0.90 & 1.022 & 0.028 & 1.017 & 0.022 & 1.012 & 0.017 & 1.008 & 0.012 \\
0.95 & 0.972 & 0.031 & 0.967 & 0.025 & 0.962 & 0.020 & 0.958 & 0.015 \\
\hline
\end{tabular}

Table 2.15: Means and standard deviations of design combined flexural and axial strength ratios, $\alpha_{P M}$, for Column Section 5 and $0 \leq e / h \leq 0.3$

\begin{tabular}{ccccccccc}
\hline & \multicolumn{8}{c}{$\phi_{c}$} \\
\cline { 2 - 10 }$\phi_{s}$ & \multicolumn{2}{c}{0.60} & \multicolumn{2}{c}{0.65} & \multicolumn{2}{c}{0.70} & \multicolumn{2}{c}{0.75} \\
\cline { 2 - 10 } & Mean & $\sigma$ & Mean & $\sigma$ & Mean & $\sigma$ & Mean & $\sigma$ \\
\hline 0.80 & 1.151 & 0.046 & 1.089 & 0.031 & 1.033 & 0.019 & 0.983 & 0.009 \\
0.85 & 1.129 & 0.055 & 1.069 & 0.040 & 1.015 & 0.027 & 0.966 & 0.016 \\
0.90 & 1.109 & 0.064 & 1.050 & 0.048 & 0.998 & 0.035 & 0.950 & 0.024 \\
0.95 & 1.089 & 0.072 & 1.032 & 0.055 & 0.981 & 0.042 & 0.935 & 0.030 \\
\hline
\end{tabular}


Table 2.16: Means and standard deviations of design combined flexural and axial strength ratios, $\alpha_{P M}$, for Column Section 5 and $0.3<e / h \leq 1.0$

\begin{tabular}{ccccccccc}
\hline & \multicolumn{8}{c}{$\phi_{c}$} \\
\cline { 2 - 10 }$\phi_{s}$ & \multicolumn{2}{c}{0.60} & \multicolumn{2}{c}{0.65} & \multicolumn{2}{c}{0.70} & \multicolumn{2}{c}{0.75} \\
\cline { 2 - 10 } 0.80 & 1.162 & 0.075 & 1.122 & 0.073 & 1.086 & 0.073 & 1.053 & 0.074 \\
0.85 & 1.123 & 0.073 & 1.084 & 0.070 & 1.049 & 0.069 & 1.018 & 0.068 \\
0.90 & 1.088 & 0.073 & 1.050 & 0.068 & 1.016 & 0.066 & 0.986 & 0.065 \\
0.95 & 1.055 & 0.072 & 1.019 & 0.067 & 0.986 & 0.064 & 0.957 & 0.062 \\
\hline
\end{tabular}

Table 2.17: Means and standard deviations of design combined flexural and axial strength ratios, $\alpha_{P M}$, for Column Section 5 and $1.0<e / h \leq 10.0$

\begin{tabular}{ccccccccc}
\hline & \multicolumn{10}{c}{$\phi_{c}$} \\
\cline { 2 - 9 }$\phi_{s}$ & \multicolumn{2}{c}{0.60} & \multicolumn{2}{c}{0.65} & \multicolumn{2}{c}{0.70} & \multicolumn{2}{c}{0.75} \\
\cline { 2 - 10 } 0 Mean & $\sigma$ & Mean & $\sigma$ & Mean & $\sigma$ & Mean & $\sigma$ \\
\hline 0.80 & 1.163 & 0.036 & 1.146 & 0.037 & 1.129 & 0.039 & 1.114 & 0.042 \\
0.85 & 1.108 & 0.033 & 1.091 & 0.034 & 1.075 & 0.035 & 1.061 & 0.037 \\
0.90 & 1.058 & 0.031 & 1.041 & 0.031 & 1.027 & 0.032 & 1.013 & 0.034 \\
0.95 & 1.013 & 0.029 & 0.997 & 0.029 & 0.983 & 0.030 & 0.970 & 0.031 \\
\hline
\end{tabular}

Table 2.18: Means and standard deviations of design combined flexural and axial strength ratios, $\alpha_{P M}$, for Column Section 5 and $e / h \leq 0$

\begin{tabular}{ccccccccc}
\hline & \multicolumn{8}{c}{$\phi_{c}$} \\
\cline { 2 - 10 }$\phi_{s}$ & \multicolumn{2}{c}{0.60} & \multicolumn{2}{c}{0.65} & \multicolumn{2}{c}{0.70} & \multicolumn{2}{c}{0.75} \\
\cline { 2 - 10 } & Mean & $\sigma$ & Mean & $\sigma$ & Mean & $\sigma$ & Mean & $\sigma$ \\
\hline 0.80 & 1.149 & 0.026 & 1.141 & 0.020 & 1.133 & 0.018 & 1.125 & 0.018 \\
0.85 & 1.088 & 0.029 & 1.080 & 0.023 & 1.072 & 0.019 & 1.065 & 0.017 \\
0.90 & 1.033 & 0.031 & 1.025 & 0.025 & 1.018 & 0.020 & 1.011 & 0.017 \\
0.95 & 0.984 & 0.034 & 0.976 & 0.028 & 0.970 & 0.023 & 0.963 & 0.019 \\
\hline
\end{tabular}




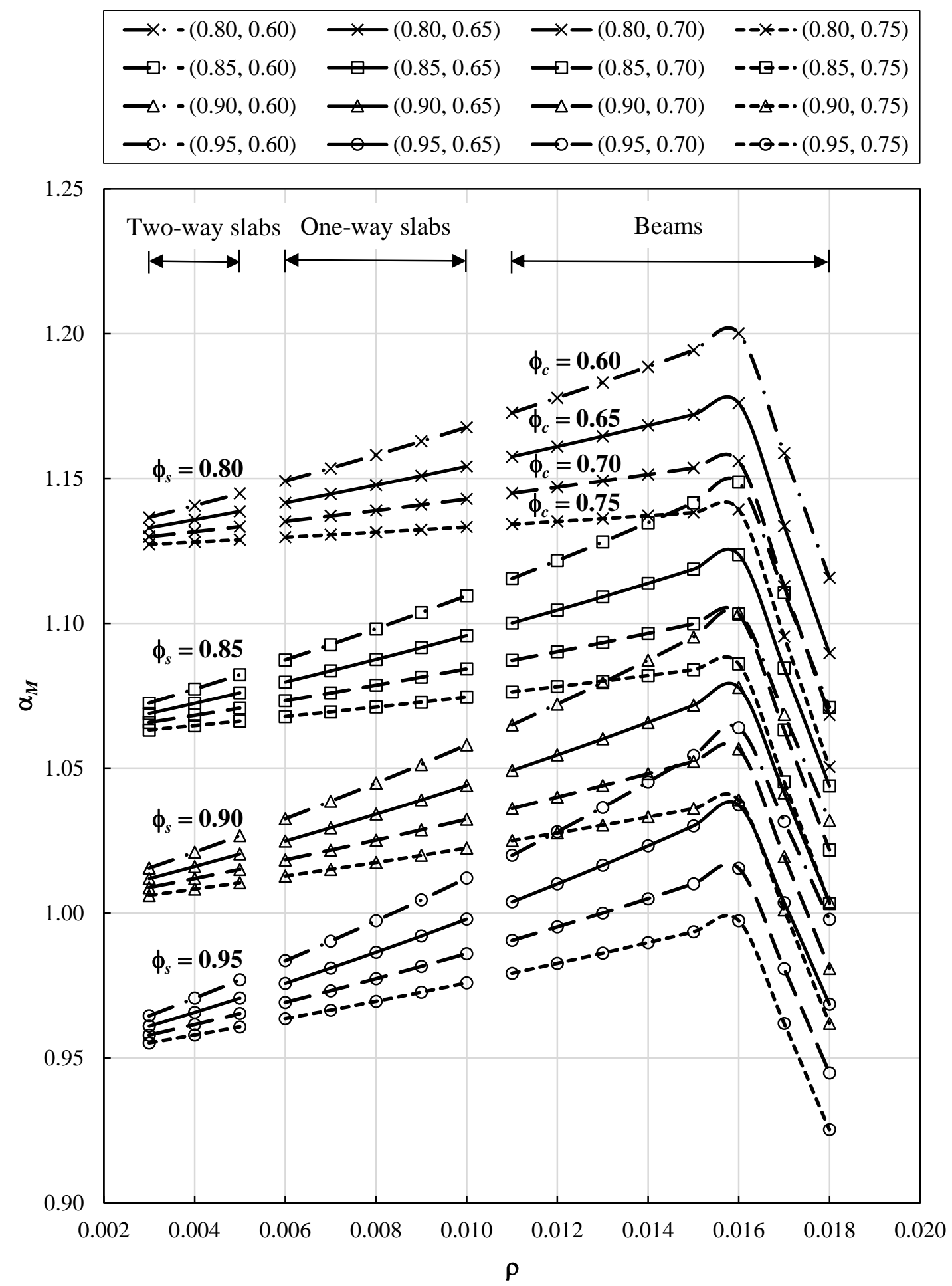

Figure 2.1: Design flexural strength ratios, $\alpha_{M}$, for $f_{c}^{\prime}=25 \mathrm{MPa}$ and $\rho=0.003-0.018$ 


$$
\begin{aligned}
& \longrightarrow \times \cdot-(0.80,0.60) \quad \longrightarrow(0.80,0.65) \quad \longrightarrow \times-(0.80,0.70) \quad--x-\cdot(0.80,0.75) \\
& \square \cdot-(0.85,0.60) \quad \square(0.85,0.65) \quad \square-(0.85,0.70) \quad-\square-\cdot(0.85,0.75) \\
& \rightarrow \cdot-(0.90,0.60) \quad \longrightarrow(0.90,0.65) \quad \longrightarrow-(0.90,0.70) \quad--\Delta-\cdot(0.90,0.75) \\
& \longrightarrow \cdot(0.95,0.60) \quad-(0.95,0.65) \quad \longrightarrow(0.95,0.70) \quad-\rightarrow \cdot(0.95,0.75)
\end{aligned}
$$

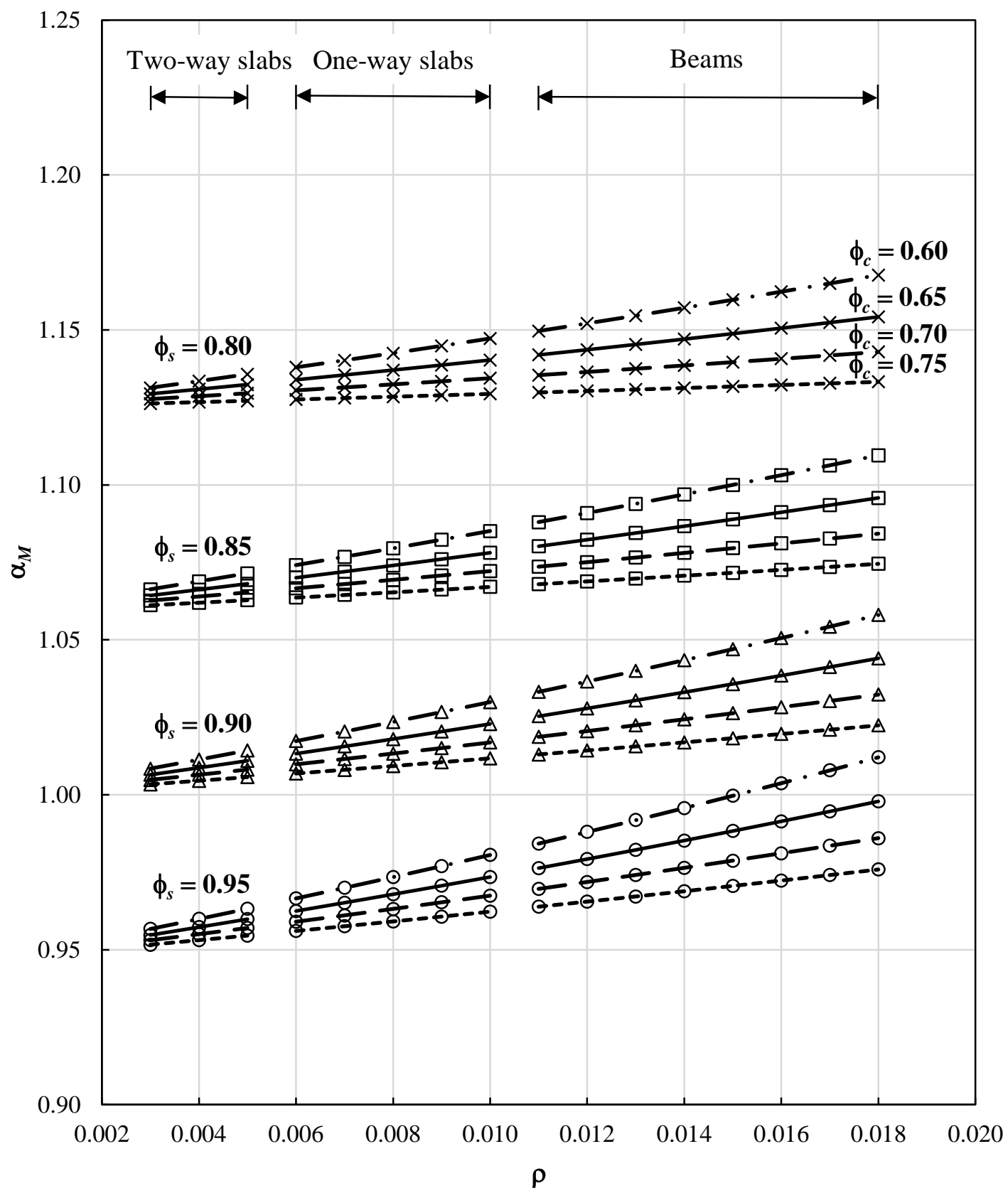

Figure 2.2: Design flexural strength ratios, $\alpha_{M}$, for $f_{c}^{\prime}=45 \mathrm{MPa}$ and $\rho=0.003-0.018$ 


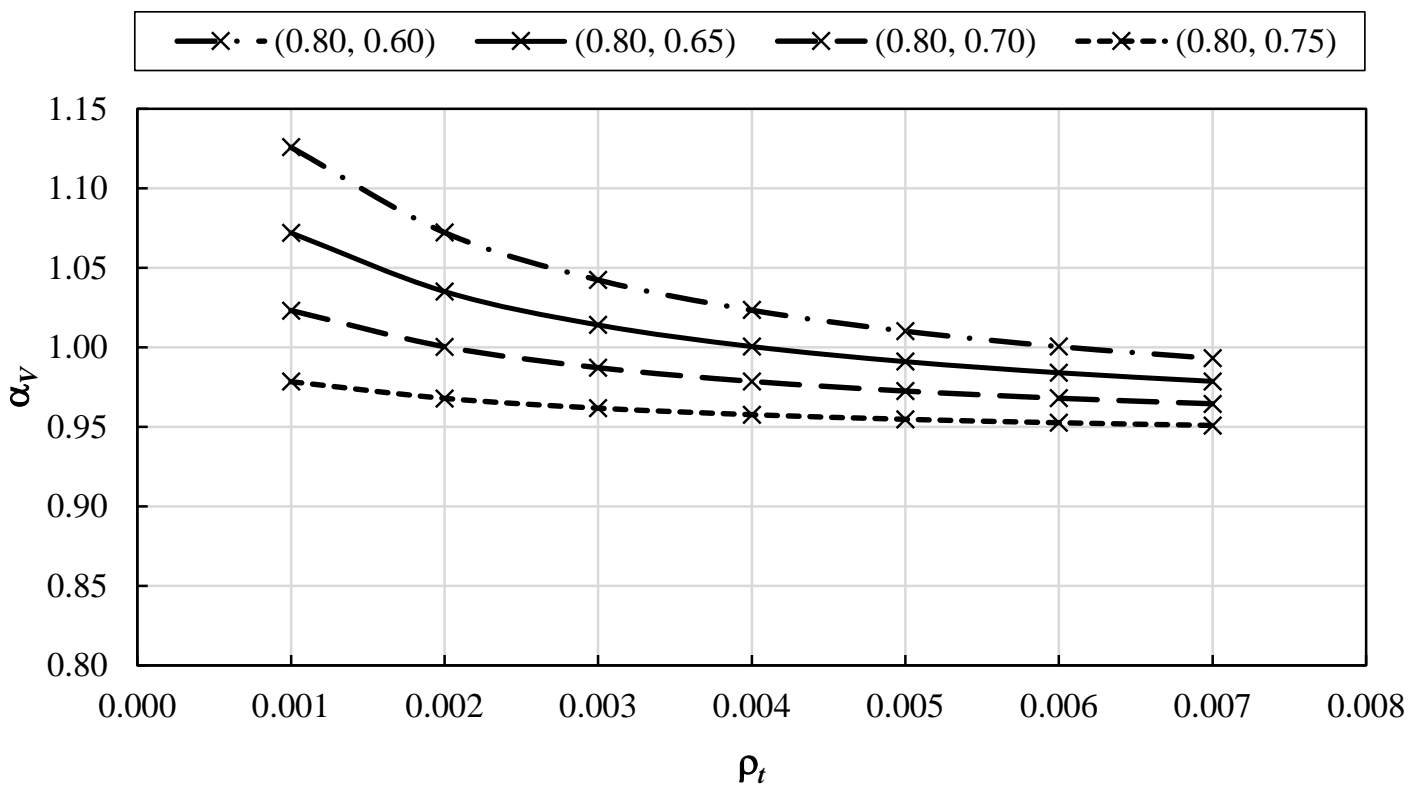

(a)

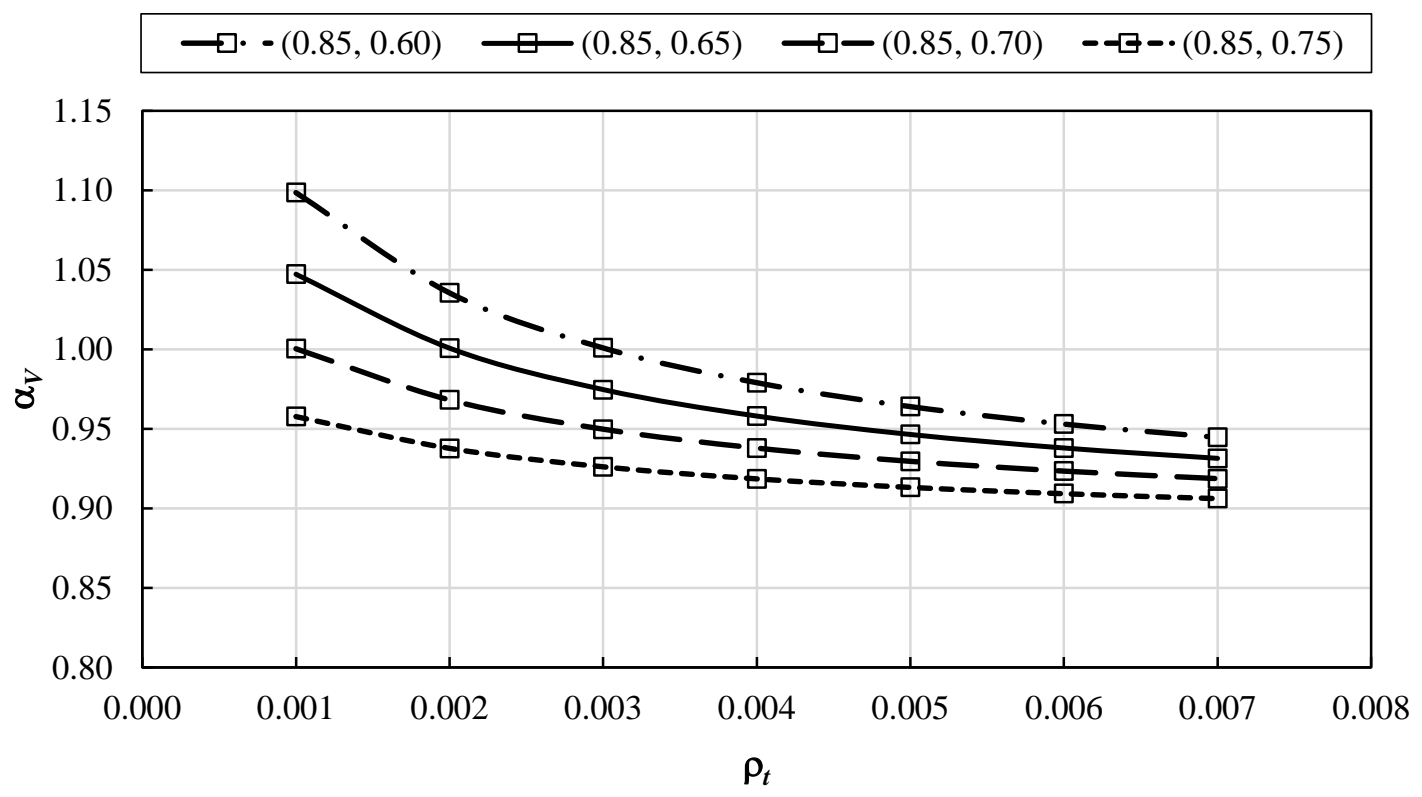

(b) 


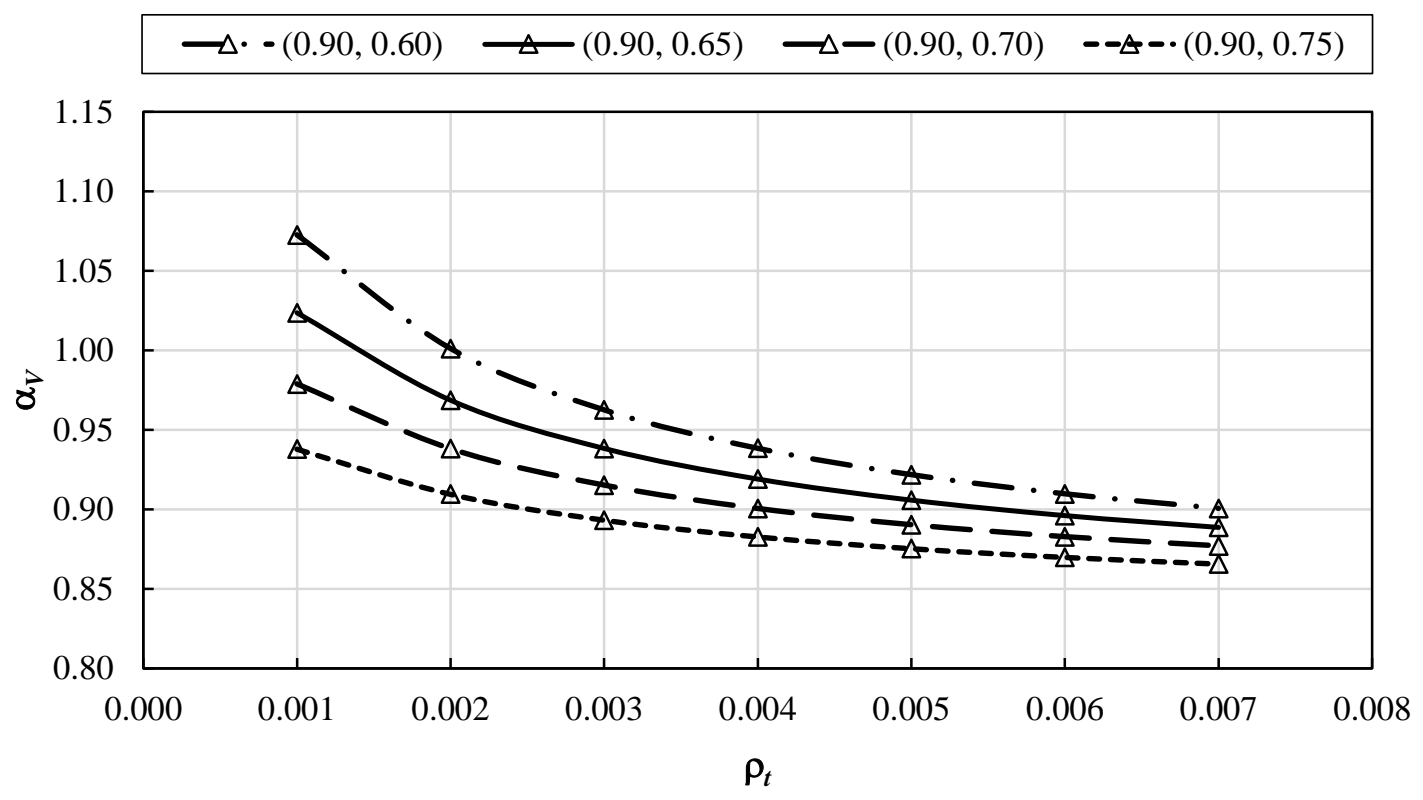

(c)

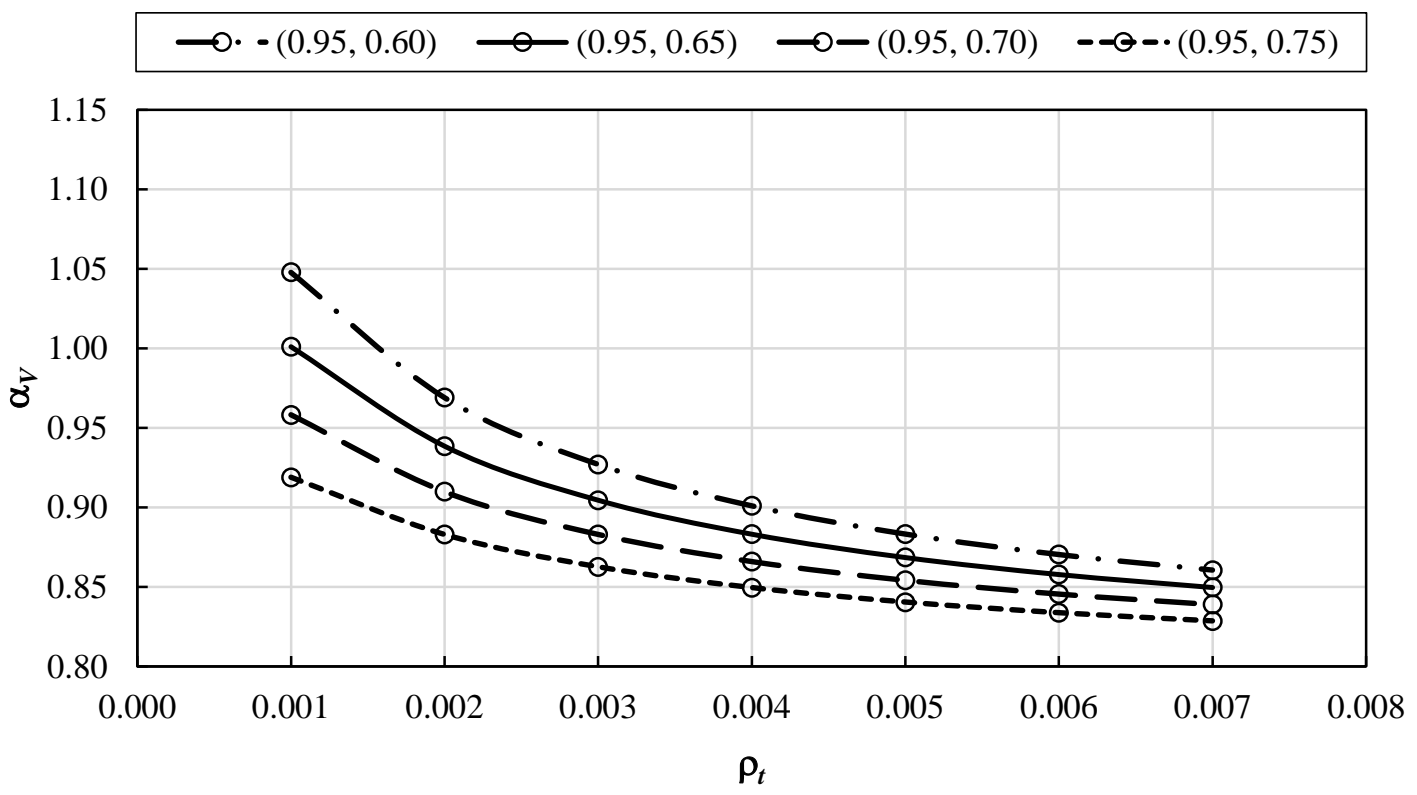

(d)

Figure 2.3: Design shear strength ratios, $\alpha_{V}$, for $f_{c}^{\prime}=25 \mathrm{MPa}$ and $\rho_{t}=0.001-0.007$ : (a) $\phi_{s}$

$$
=0.80 ;(\mathrm{b}) \phi_{s}=0.85 ; \text { (c) } \phi_{s}=0.90 ; \text { (d) } \phi_{s}=0.95
$$




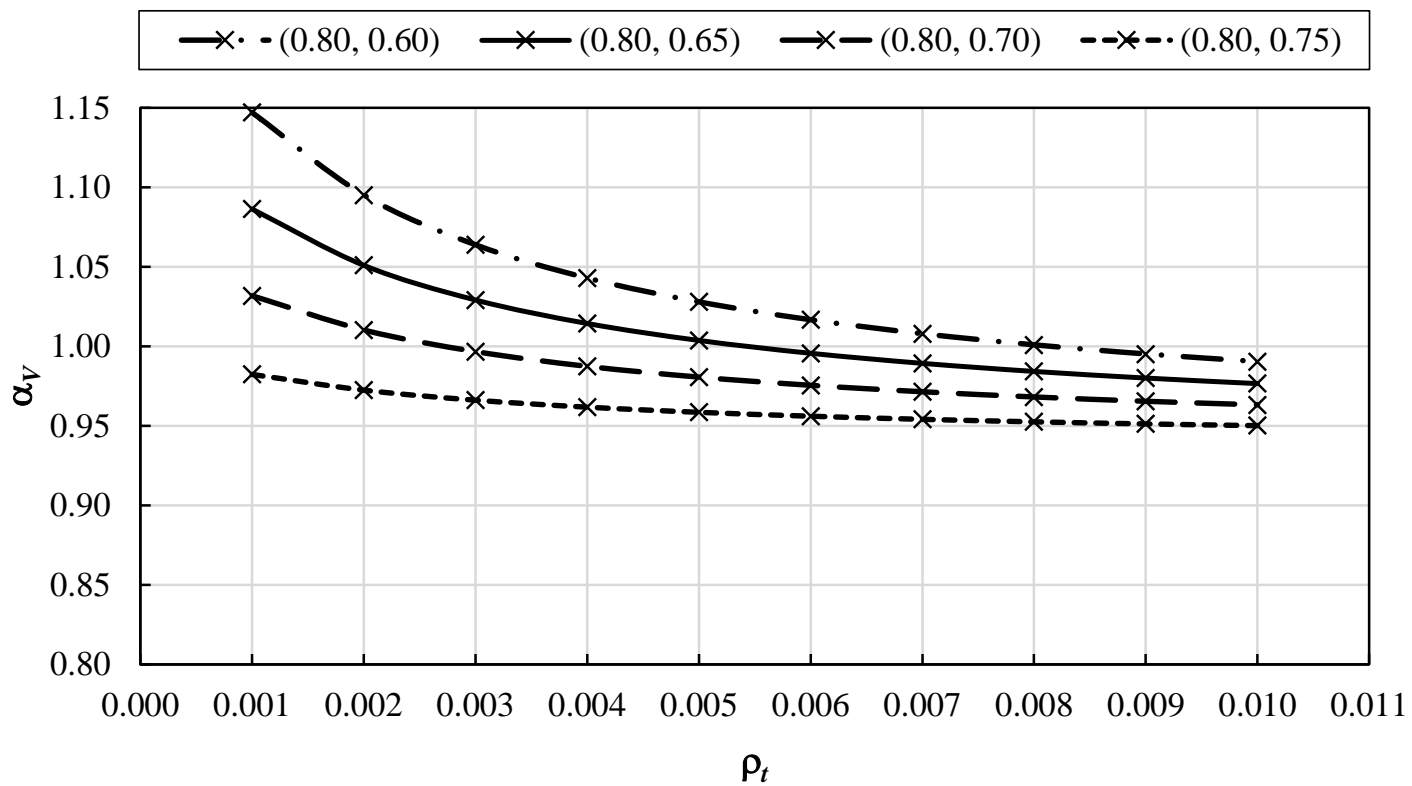

(a)

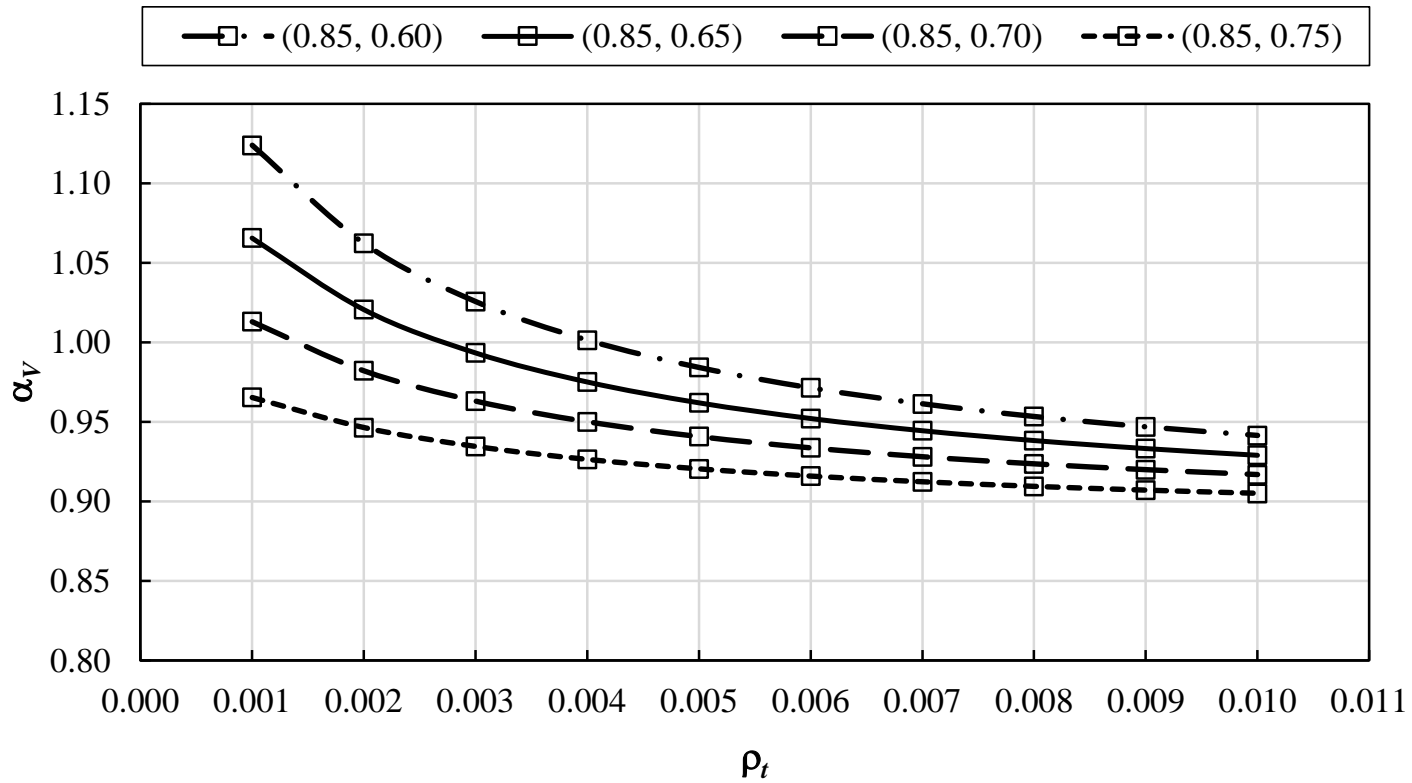

(b) 


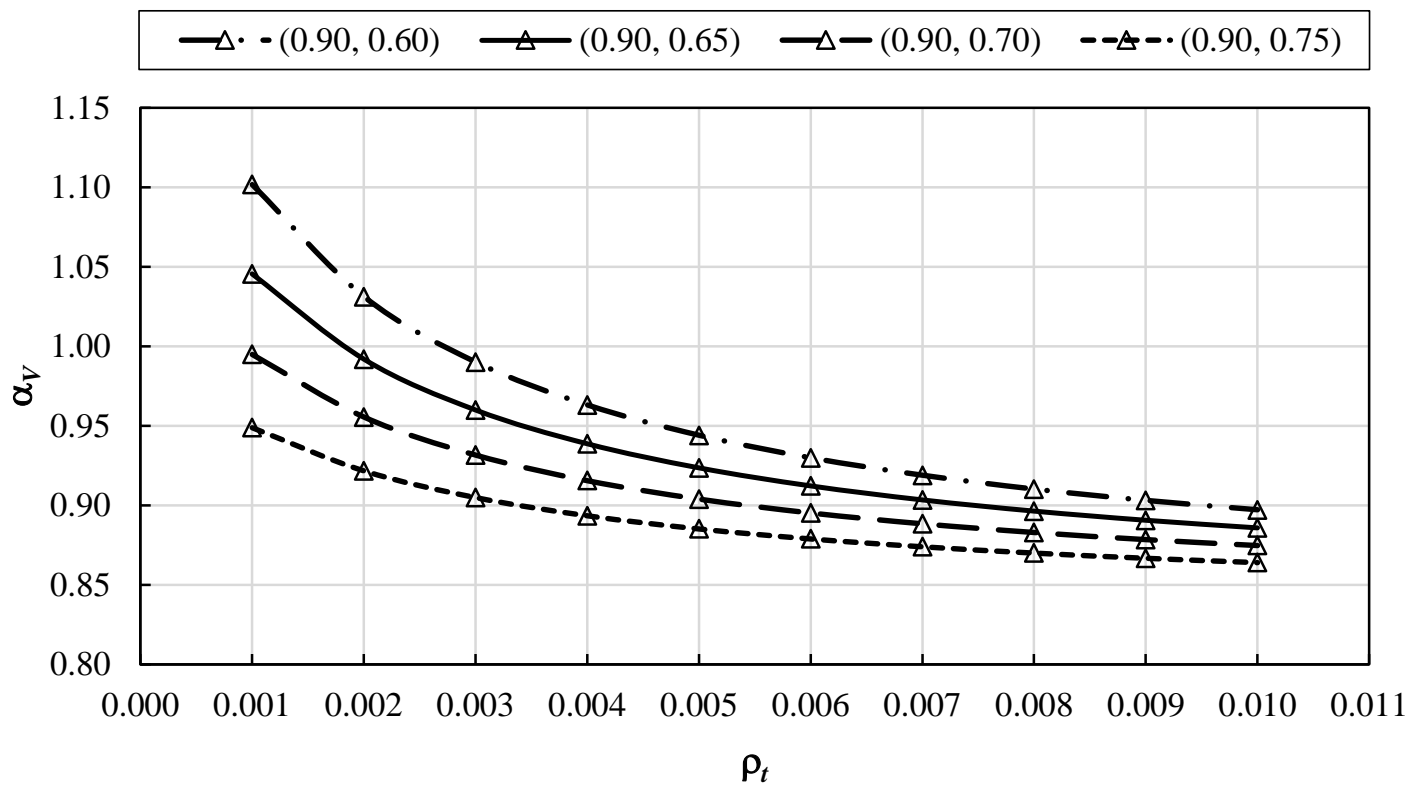

(c)

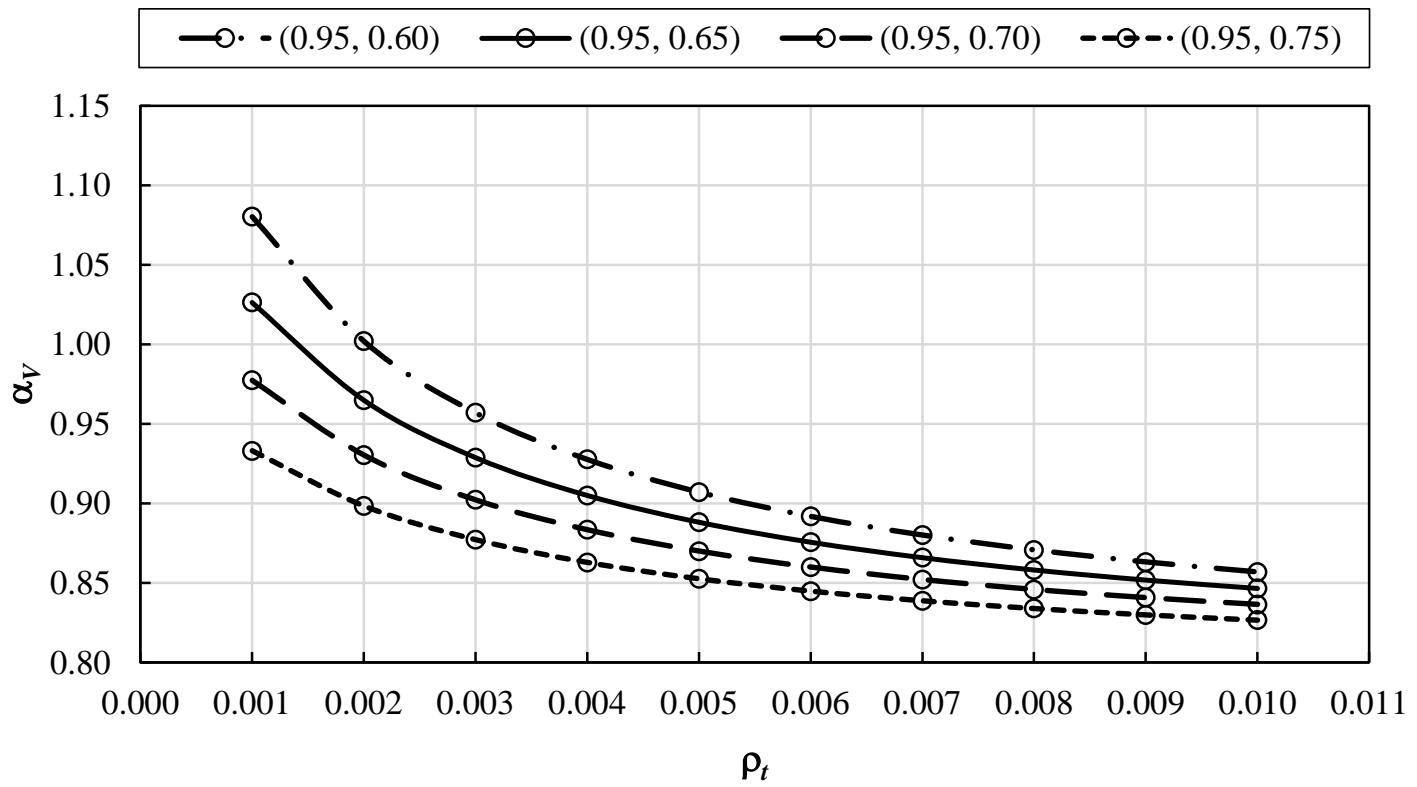

(d)

Figure 2.4: Design shear strength ratios, $\alpha_{V}$, for $f_{c}^{\prime}=45 \mathrm{MPa}$ and $\rho_{t}=0.001-0.010$ : (a) $\phi_{s}$

$$
=0.80 ; \text { (b) } \phi_{s}=0.85 ; \text { (c) } \phi_{s}=0.90 ; \text { (d) } \phi_{s}=0.95
$$




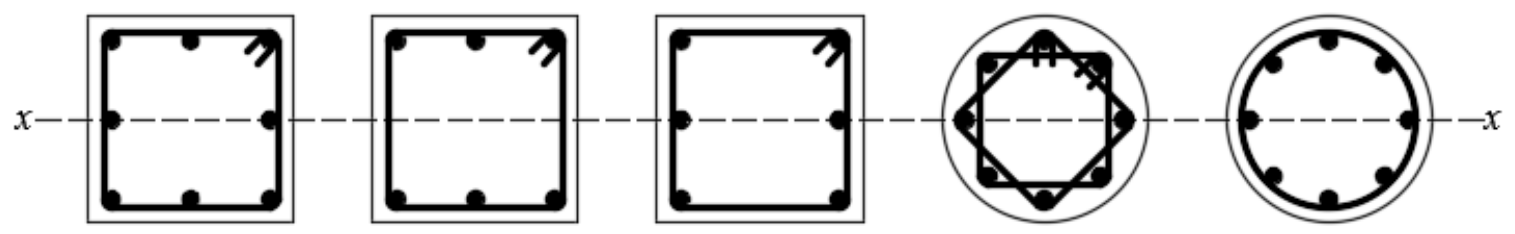

(a)

(b)

(c)

(d)

(e)

Figure 2.5: Five column cross sections: (a) Column Section 1; (b) Column Section 2; (c) Column Section 3; (d) Column Section 4; (e) Column Section 5

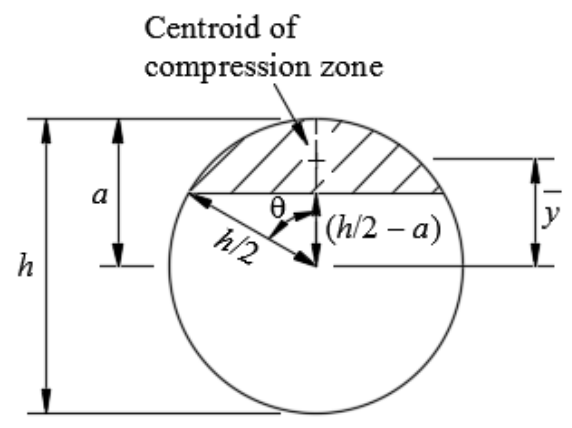

(a)

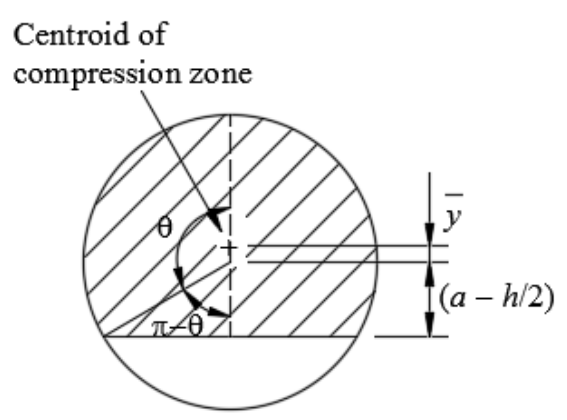

(b)

Figure 2.6: Circular segments: (a) $a \leq h / 2, \theta \leq \pi / 2$; (b) $a>h / 2, \theta>\pi / 2$ (Wight 2016) 


\begin{tabular}{|c|c|c|c|}
\hline$\longrightarrow \times \cdot-(0.80,0.60)$ & $\longrightarrow(0.80,0.65)$ & $\rightarrow x-(0.80,0.70)$ & $--×-\cdot(0.80,0.75)$ \\
\hline$\square \cdot-(0.85,0.60)$ & $\square(0.85,0.65)$ & $\square-(0.85,0.70)$ & $\cdot(0.85,0.75)$ \\
\hline$-\Delta \cdot-(0.90,0.60)$ & $\triangle(0.90,0.65)$ & $\longrightarrow-(0.90,0.70)$ & $--\Delta-\cdot(0.90,0.75)$ \\
\hline$\longrightarrow \cdot-(0.95,0.60)$ & $\multimap(0.95,0.65)$ & $\longrightarrow-(0.95,0.70)$ & $--\Theta-\cdot(0.95,0.75)$ \\
\hline ----eubal/h & (erbal/h)max & $\longrightarrow(\mathrm{erbal} / \mathrm{h}) \mathrm{min}$ & \\
\hline
\end{tabular}

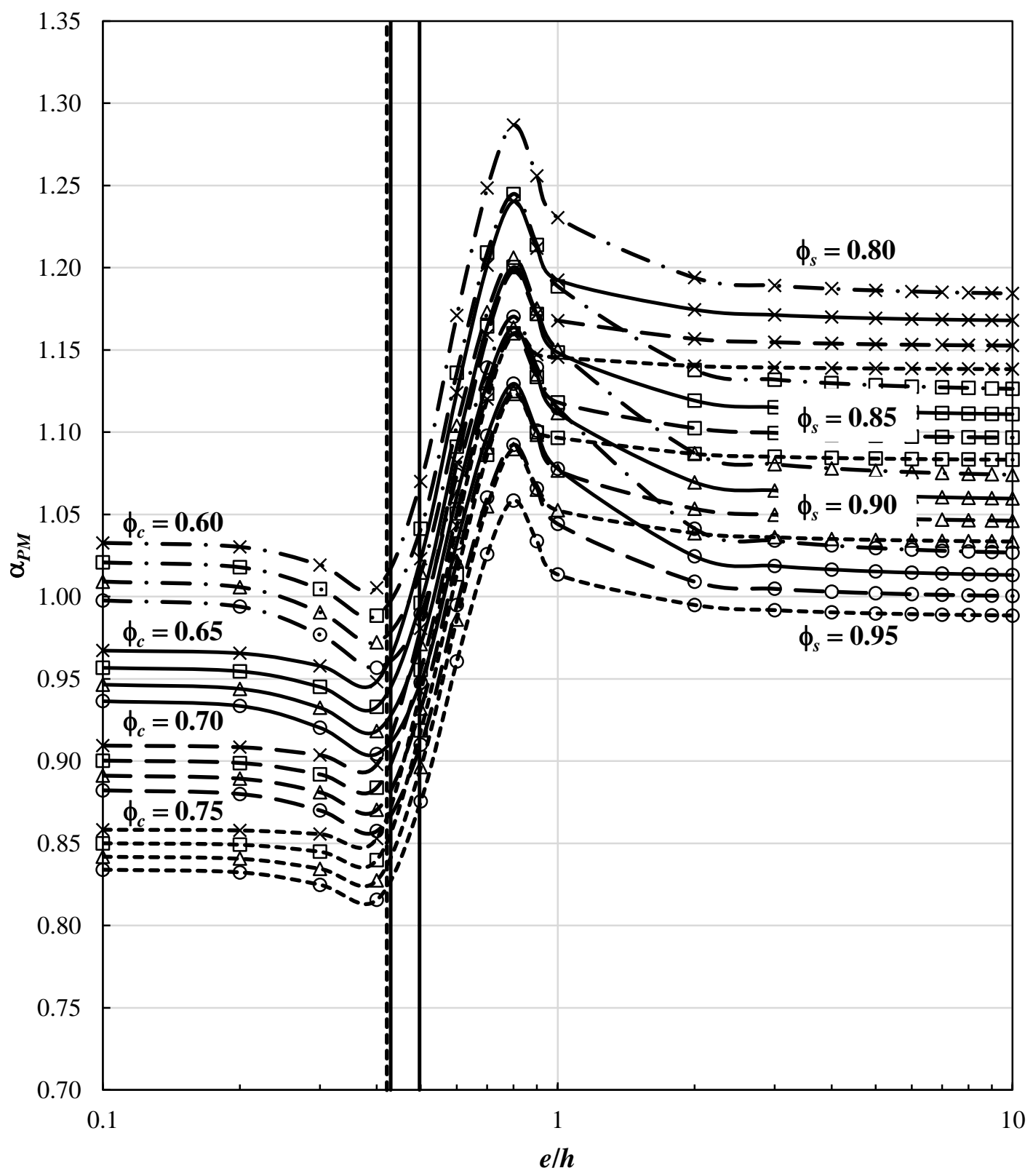

(a) 


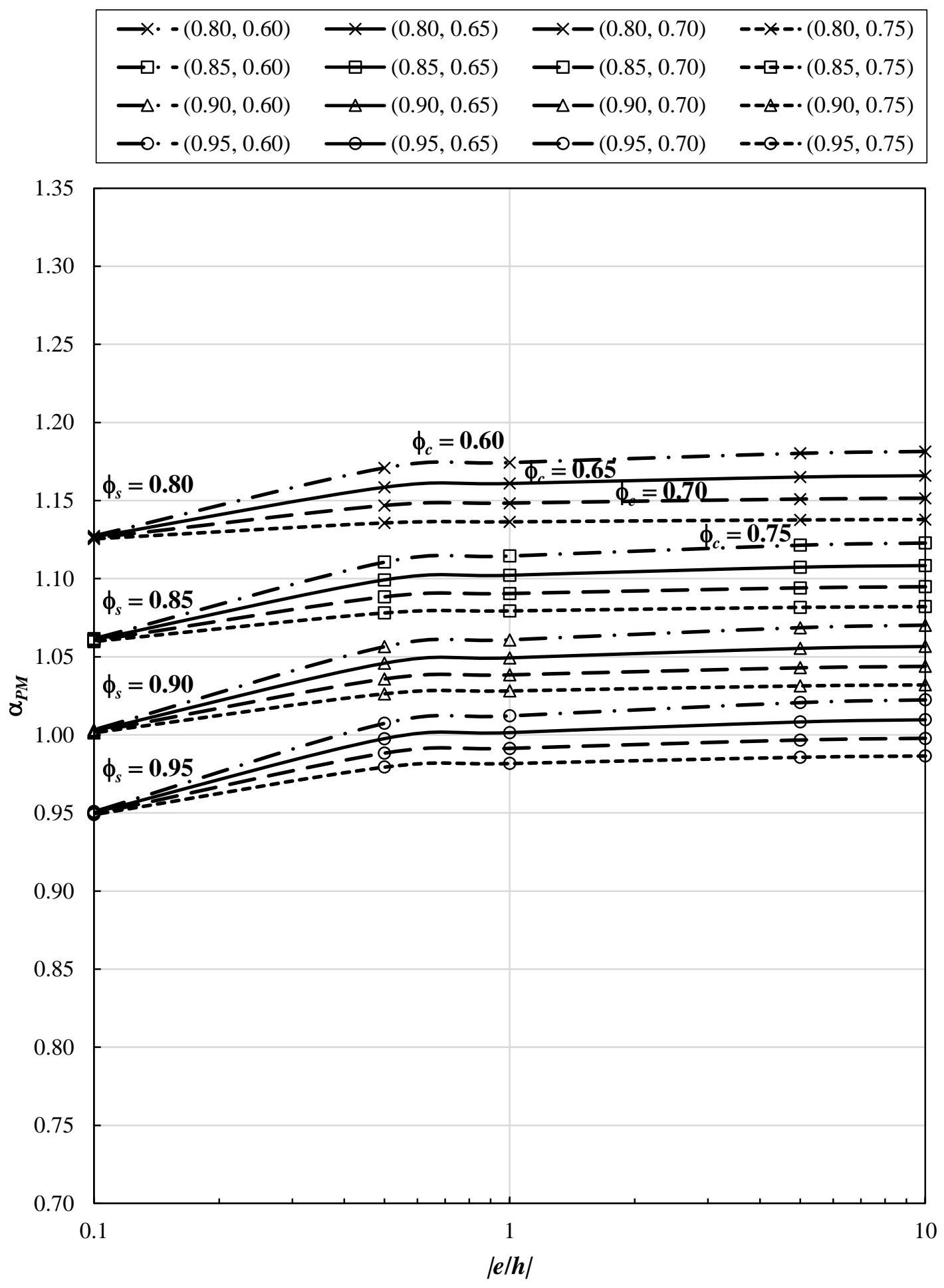

(b)

Figure 2.7: Design combined flexural and axial strength ratios, $\alpha_{P M}$, for Column Section 1 and Property Combination 1: (a) $e / h>0$; (b) $e / h<0$ 

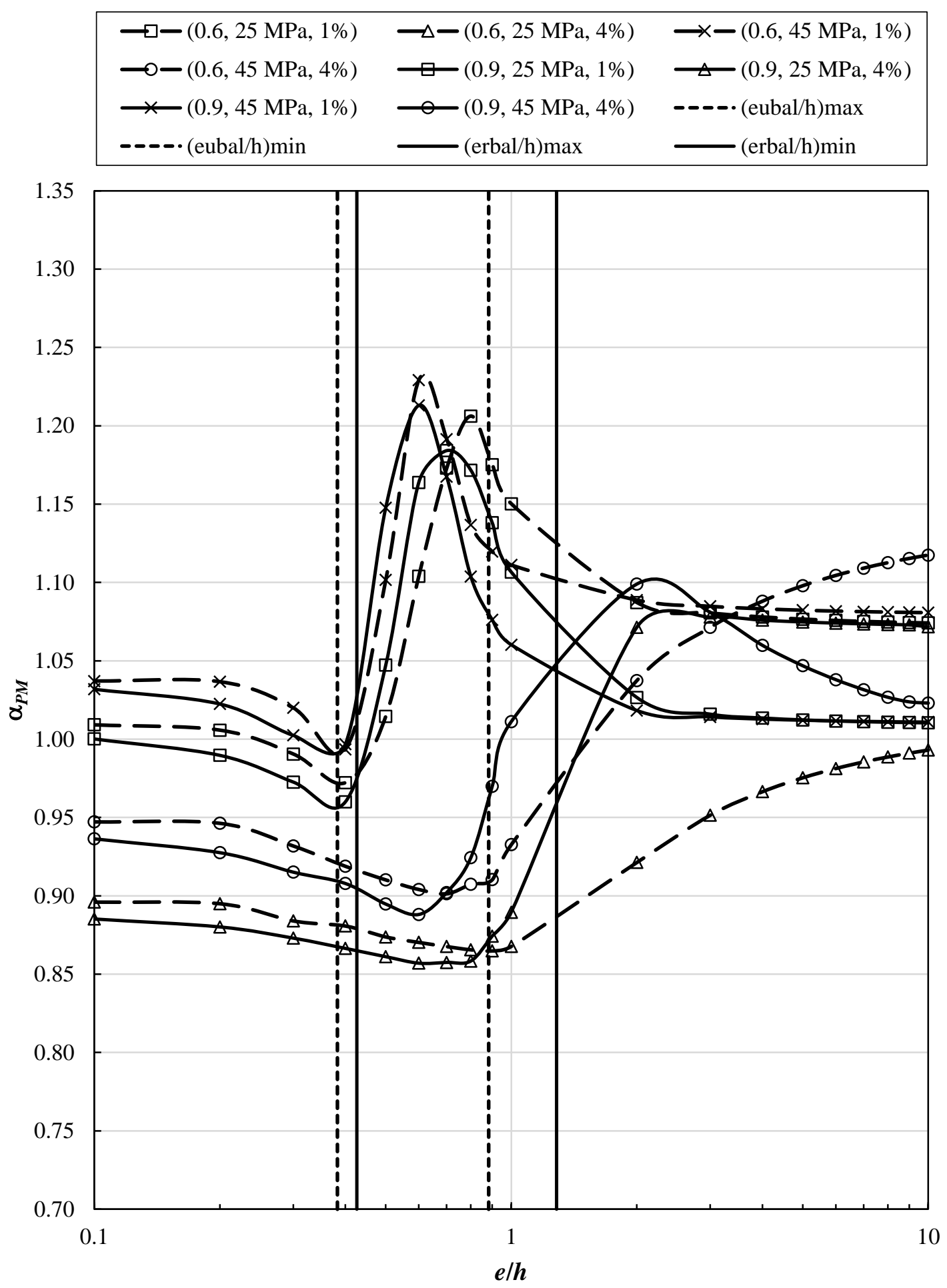

(a) 

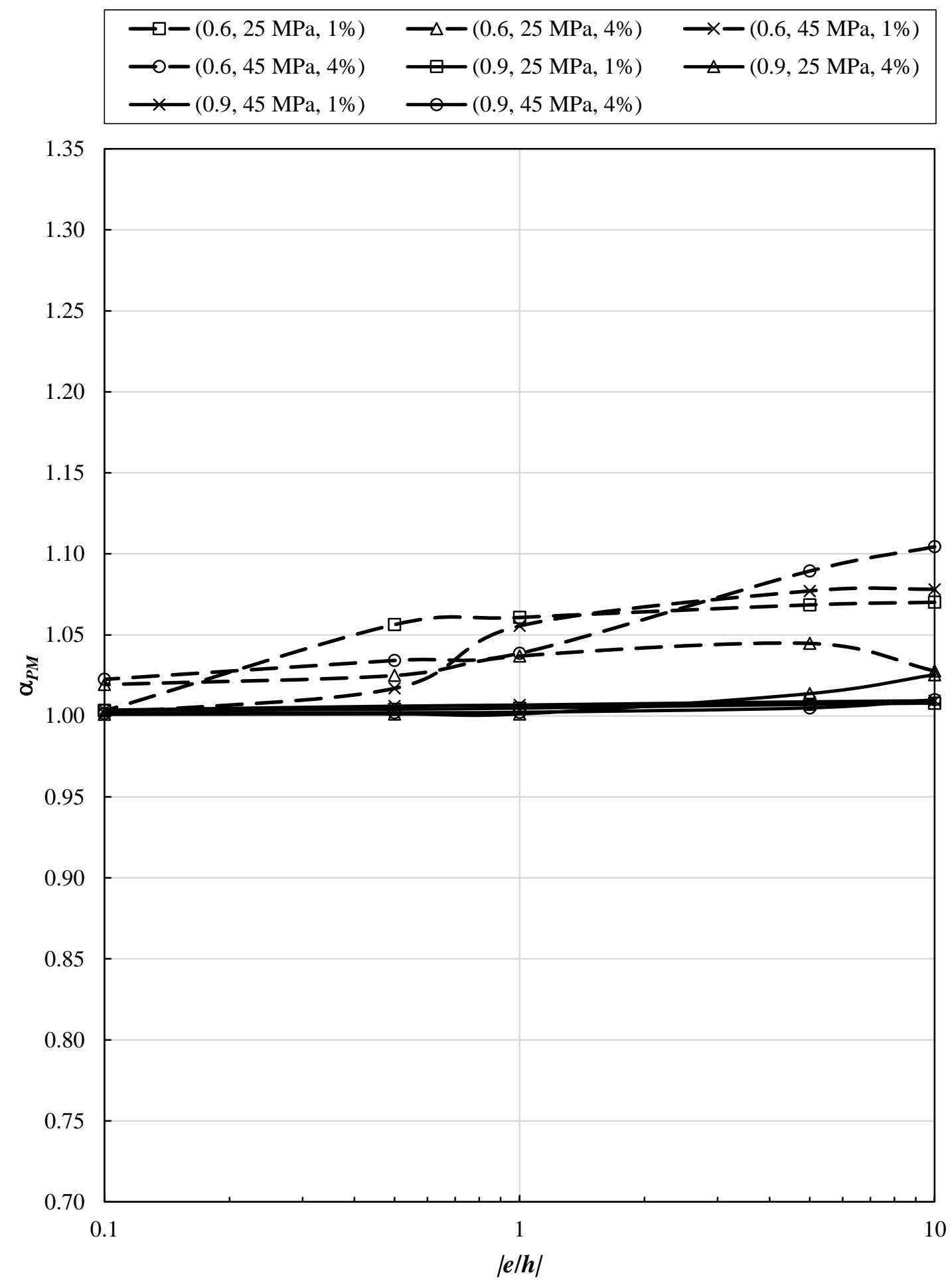

(b)

Figure 2.8: Design combined flexural and axial strength ratios, $\alpha_{P M}$, corresponding to ACI 318-14, and $\phi_{s}=0.90$ and $\phi_{c}=0.60$, for Column Section 1: (a) $e / h>0$; (b) $e / h<0$ 


\begin{tabular}{|c|c|c|c|}
\hline$\longrightarrow \times \cdot-(0.80,0.60)$ & $\longrightarrow(0.80,0.65)$ & $\rightarrow x-(0.80,0.70)$ & $--×-\cdot(0.80,0.75)$ \\
\hline$\square \cdot-(0.85,0.60)$ & $\square(0.85,0.65)$ & $\square-(0.85,0.70)$ & $\cdot(0.85,0.75)$ \\
\hline$-\Delta \cdot-(0.90,0.60)$ & $\triangle(0.90,0.65)$ & $\longrightarrow-(0.90,0.70)$ & $--\Delta-\cdot(0.90,0.75)$ \\
\hline$\longrightarrow \cdot-(0.95,0.60)$ & $\multimap(0.95,0.65)$ & $\longrightarrow-(0.95,0.70)$ & $--\Theta-\cdot(0.95,0.75)$ \\
\hline ----eubal/h & (erbal/h)max & $\longrightarrow(\mathrm{erbal} / \mathrm{h}) \mathrm{min}$ & \\
\hline
\end{tabular}

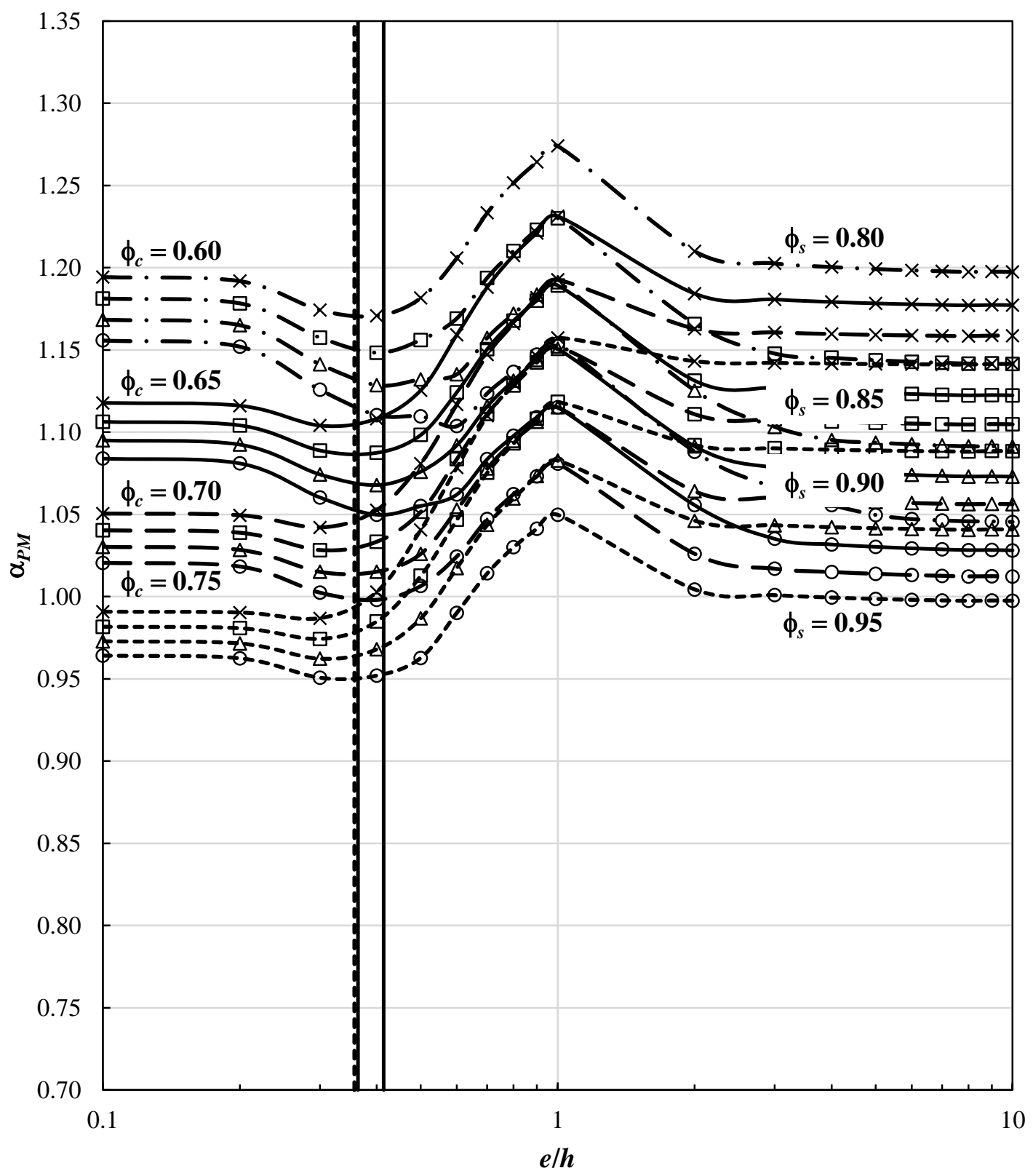

(a) 


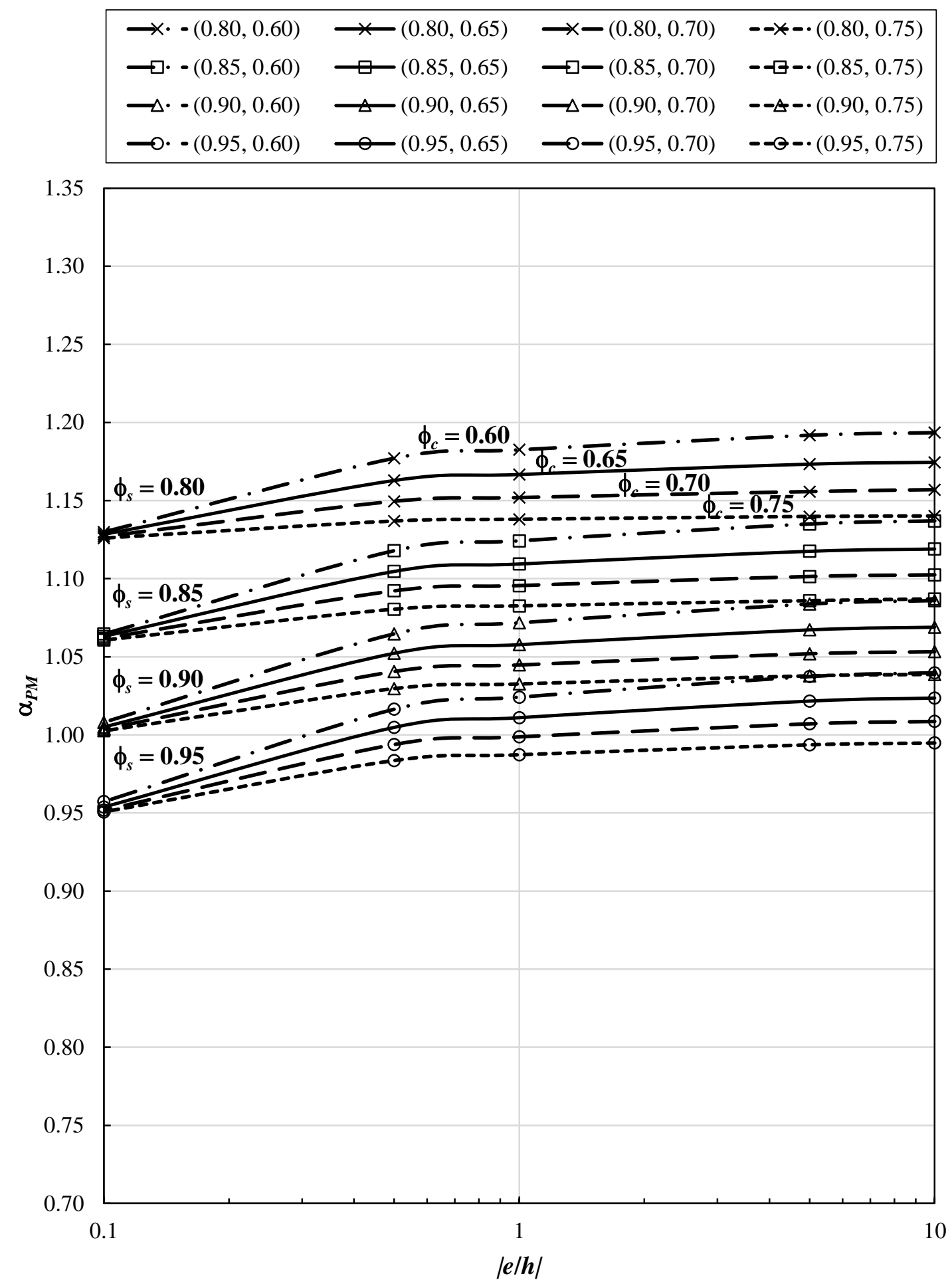

(b)

Figure 2.9: Design combined flexural and axial strength ratios, $\alpha_{P M}$, for Column Section 5 and Property Combination 1: (a) $e / h>0$; (b) $e / h<0$ 


\section{Chapter 3}

\section{Derivation of Partial Material Strength Reduction Factors for Moment or One-way Shear Based on Reliability Indices}

\subsection{Introduction}

In Chapter 2, the partial material strength reduction factor combinations were recommended for moment, one-way shear, and combined moment and axial force based on similar design strengths obtained using ACI 318-14 (ACI Committee 318 2014) and the partial material strength reduction factors method. This simple calibration to the ACI 318-14 design strength criteria may not give the best results, however, if the ACI 318-14 criteria yield unsatisfactory reliabilities. This chapter presents the probability-based analyses to obtain reliability indices for moment and one-way shear based on ACI 318-14 and the proposed method. The range of geometric and material properties investigated are identical to those presented in Chapter 2.

The objective of this chapter is to determine appropriate partial material strength reduction factor combinations that approximate reliability indices obtained using the current ACI 318-14 provisions. A second objective is to quantify the ranges of reliability.

\subsection{Methodology}

Microsoft Excel (Version 2013; Microsoft 2013) is used to compute the reliability indices. The first-order, second-moment (FOSM) method is applied for simply supported members resisting moment or one-way shear. Statistical parameters to quantify the resistances and load effects are obtained from the literature. For the investigation presented in this chapter, the best combination of partial material strength reduction factors will give reliability indices that most closely approximate those obtained using the current ACI 318-14 provisions, if the range of reliability indices corresponding to ACI 318-14 is satisfactory. However, if the range of reliability indices is unsuitable, correction will be applied. 


\subsubsection{Reliability Model}

The limit state function, $Z$, also denoted as $g(\bullet)$, related to the resistance, $R$, and the load effect, $Q$, is defined as (e.g., Ellingwood et al. 1980)

$$
Z=g(R, Q)=\frac{R}{Q}
$$

Failure corresponds to $Z<1$ or $\ln Z<0$.

The resistance is assumed to be represented by the following product model originally proposed by Galambos and Ravindra (1977):

$$
R=G M P
$$

where: $G$ is a geometric property; $M$ is a material strength property; and, $P$ is the professional factor.

The load effect for the $i$ th type load, $Q_{i}$, quantifies the structural demand and is expressed as (Ellingwood et al. 1980)

$$
Q_{i}=c_{i} B_{i} A_{i}
$$

where: $c_{i}$ is an influence coefficient; $B_{i}$ is a modelling parameter; and, $A_{i}$ is the structural load itself. Ellingwood et al. (1980) assumed that "the transformation from load to load effect is linear, and $c_{i}, B_{i}$ and $A_{i}$ are statistically independent." $B_{i}$ accounts for "the load model which transforms the actual spatially and temporally varying load into a statically equivalent uniformly distributed load", and $c_{i}$ reflects "the analysis which transforms the equivalent uniformly distributed load to a load effect".

For the limit state function defined by Equation [3.1], the first-order, second-moment reliability index, $\beta$, can be computed as (e.g., Ellingwood et al. 1980)

$$
\beta=\frac{\ln (\bar{R} / \bar{Q})}{\sqrt{V_{R}^{2}+V_{Q}^{2}}}
$$


where: $\bar{R}$ is the mean resistance; $\bar{Q}$ is the mean load effect; $V_{R}$ is the coefficient of variation of the resistance; and, $V_{Q}$ is the coefficient of variation of the load effect. To determine $\beta$, these four values must be calculated first before the reliability index can be computed.

\subsubsection{Determination of Statistical Parameters for Resistance and Load Effect}

The reliability analyses for members resisting moment or shear are based on the Taylor Series expansion to compute the resistance and load effect statistical parameters. This method is described by Ellingwood et al. (1980). The general form of the resistance or the load effect is

$$
R\{\text { or } Q\}=f\left(X_{1}, X_{2}, \ldots X_{n}\right)
$$

where $f(\bullet)$ is the function of resistance or load effect in the limit state function, and $X_{i}$ is the resistance or load variable, characterized by its first and second moments. The resistance and the load effect must be linearized at some point for the reliability analysis. The linearization, based on the Taylor Series expansion is (Ellingwood et al. 1980)

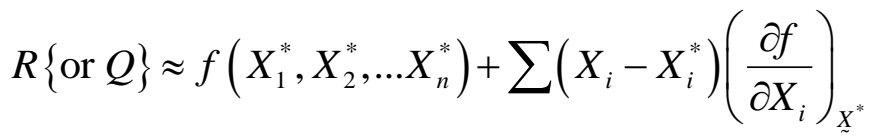

where $\left(X_{1}^{*}, X_{2}^{*}, \ldots X_{n}^{*}\right)$ is the linearizing point, taken as the means of the variables in this study. In other words, $\left(X_{1}^{*}, X_{2}^{*}, \ldots X_{n}^{*}\right)=\left(\bar{X}_{1}, \bar{X}_{2}, \ldots \bar{X}_{n}\right)$. Assuming the variables are statistically independent, the mean and standard deviation of $R$ or $Q, \bar{R}$ or $\bar{Q}$, and $\sigma_{R}$ or $\sigma_{Q}$, respectively, are approximated by (Ellingwood et al. 1980)

$$
\begin{gathered}
\bar{R}\{\text { or } \bar{Q}\} \approx f\left(\bar{X}_{1}, \bar{X}_{2}, \ldots \bar{X}_{n}\right) \\
\sigma_{R}\left\{\text { or } \sigma_{Q}\right\} \approx\left[\sum\left(\frac{\partial f}{\partial X_{i}}\right)_{\bar{X}_{i}}^{2} \sigma_{X_{i}}^{2}\right]^{1 / 2}
\end{gathered}
$$


where $\bar{X}_{i}$ and $\sigma_{X_{i}}$ are the mean and standard deviation of the resistance or load variable, respectively.

\subsection{Statistical Parameters}

This section presents the statistical parameters obtained from the literature for use in the reliability analyses.

\subsubsection{Geometric Properties}

The geometric properties include the width, $b$, and the height, $h$, of the concrete cross section, the effective depth, $d$, of the flexural reinforcement and the area of the reinforcement, $A_{s}$. Table B.1 in Appendix B summarizes the absolute values, means and standard deviations, $\sigma$, for concrete geometric properties reported in the literature. They are due to measurement errors in the construction process and are controlled by specified tolerances and so are absolute values in $\mathrm{mm}$. Table 3.1 shows the values used in this study selected from Table B.1. The statistical parameters for $b$ value for beams and $b$ and $h$ values for columns used in this study are derived from Ellingwood et al. (1980) and Mirza and MacGregor (1979). The standard deviation of $d$ is assumed to be $1 / 2$ of the tolerance specified in Table 26.6.2.1a in ACI 318-14: this is consistent with the common approximation that the total tolerance range equals four standard deviations. The bias

coefficient, $\delta$, and coefficient of variation, $V$, for the area of the reinforcement, $A_{s}$, is obtained from Nowak and Szerszen (2003).

\subsubsection{Material Strengths}

The equation used by Bartlett (2007) to characterize the concrete compressive strength is:

$$
M=f_{c, i-p} / f_{c}^{\prime}=F_{1} F_{2} F_{i-p} F_{r}
$$

where: $f_{c, i-p}$ is the in-place compressive strength of the concrete; $f_{c}^{\prime}$ is the 28 -day specified strength; $F_{1}$ is a parameter representing the ratio of the mean 28-day control cylinder strength to the specified 28-day strength; $F_{2}$ is the ratio of the mean in-place strength at 28 days to the mean 28-day cylinder strength; $F_{i-p}$ accounts for the variation of the in- 
place strength; and, $F_{r}$ accounts for rate-of-loading effects (Bartlett 2007). It is assumed that $F_{1}, F_{2}, F_{i-p}$ and $F_{r}$ are statistically independent.

The statistical parameters for $F_{1}, F_{2}, F_{i-p}$ obtained from the literature are shown in Table B.2-B.4 and the corresponding demonstration is also presented in Appendix B. Table B.5 shows statistical parameters for in-situ concrete compressive strength reported by Ellingwood et al. (1980). A summary of the statistical parameters for cast-in-place concrete used in the present study is shown in Table 3.2. Bartlett (2007) computed $F_{r}$ as 0.88 for dead plus live load combination for $f_{c}^{\prime}$ from 20 to $35 \mathrm{MPa}$ with assumption of 1 hour loading duration for live loads. The coefficients of variation for $F_{1}, F_{2}$ and $F_{i-p}$ are relatively large compared to $F_{r}$, so the coefficient of variation for $F_{r}$ is ignored (Bartlett 2007). The resulting statistical parameters for concrete compressive strength, adopted in the present study are a bias coefficient of 1.15 and a coefficient of variation of 0.211 .

Table B.6 shows statistical parameters for reinforcement with yield strength, $f_{y}$, of 420 MPa. Nowak and Szerszen (2003) recommended a bias coefficient of 1.145 and a coefficient of variation of 0.05 , which implies better control of yield strength than that reported by Ellingwood et al. (1980). The statistical parameters, a bias coefficient of 1.125 and a coefficient of variation of 0.098, reported by Ellingwood et al. (1980) have been adopted for the present study, because they are more conservative.

\subsubsection{Professional Factors}

Table 3.3 presents statistical parameters for professional factors from several sources. Somo and Hong (2006) explored the professional factor for shear strength based on 1146 beam tests reported in the literature. They categorized the results by the presence of stirrups and shear span-to-depth ratios, $a_{v} / d$. Their dataset includes data from Kani et al. (1979) for shallow beams tested at a very young age. Therefore, Somo and Hong (2006) also reanalyzed a reduced dataset that excludes Kani's beam tests. Collins (2001) studied the professional factor for shear based on two datasets: one containing 776 beam tests and the other 413 beam tests. The larger dataset contains a much higher proportion of beams with depths less than $350 \mathrm{~mm}$, more prestressed beams, and more beams without stirrups. In addition, at least $98 \%$ of the data in each dataset are for beams subjected to point 
loads, whereas in practice, in buildings, it is more common to have beams subjected to uniformly distributed loads (Collins 2001). In a previous calibration of the ACI strength reduction factors, values of 1.09 and 0.12 were adopted by Israel et al. (1987).

Nowak and Szerszen (2003) investigated the bias coefficients and coefficients of variation for different structural members combining results reported by Ellingwood et al. (1980) with "engineering judgement".

In the present investigation, for beams subjected to shear, beams with stirrups and shear span-to-depth ratios, $a_{v} / d$, greater than 2 are of interest, so a bias coefficient of 1.47 and a coefficient of variation of 0.36 reported by Somo and Hong (2006) are used. For members subjected to other structural actions, statistical parameters presented by Nowak and Szerszen (2003) are used.

\subsubsection{Load Effects}

Based on Table 5.3.1 in ACI 318-14, the load combination investigated is:

$$
U=1.2 D+1.6 L
$$

where: $U$ is the required strength computed using the factored load combinations; $D$ is the effect of the service dead load; and, $L$ is the effect of the service live load.

The statistical parameters pertaining to the dead load effect and the 50-year maximum live load effect are shown in Table 3.4. For dead load, Ellingwood et al. (1980) assumed all construction materials have the same bias coefficients and coefficients of variation. Szerszen and Nowak (2003) concluded that the statistical parameters for cast-in-place and precast members were similar as shown. A bias coefficient of 1.05 and a coefficient of variation of 0.10 reported by Ellingwood et al. (1980) have been used in this study.

For live load, Table 3.4 shows that Israel et al. (1987) assumed a bias coefficient of 1.00 and a coefficient of variation of 0.25 when A58.1-1982 (ANSI 1982) live load reductions were used and these values are used in this study. Szerszen and Nowak (2003) and Bartlett et al. (2003) selected different parameters based on their literature review and assumptions, as shown. In particular, Bartlett et al. (2003) accounted for the 
transformation from the load to the load effect separately which is the impact of the influence coefficient, $c_{i}$, and the modeling parameter, $B_{i}$, shown in Equation [3.3]. Denote the transformations from the dead load and live load to the dead load and live load effect by $T_{D}$ and $T_{L}$, respectively. In contrast, this transformation is already included in the live load effects reported by Israel et al. (1987), and Szerszen and Nowak (2003). In these cases, $T_{L}$ has a bias coefficient of 1.0 and a coefficient of variation of 0 . The parameters reported by Bartlett et al. (2003) are based on the 1995 NBCC (NRCC 1995) live load reduction factors, so they are not suitable for the calibration in this study. The parameters reported by Israel et al. (1987) have been used.

\subsection{Moment}

This section presents reliability indices for moment derived using the partial material strength reduction factors for comparison with those computed based on the ACI 318-14 criteria. The design of the three cross sections representative of two-way slabs, one-way slabs and beams are quantified and assumptions applied in analyses are presented. The means and standard deviations of the reliability indices are quantified. The sensitivities of the reliability indices to the partial material strength reduction factor combinations for various geometric and material properties are investigated. The best factor combinations are recommended. The factor combinations deemed "best" not only give reliability indices that are similar to those obtained using the ACI 318-14 strength reduction factors but also yield acceptable absolute reliability index values. In other words, if the reliability indices corresponding to the ACI 318-14 strength reduction factors are excessively high or low, partial material strength reduction factor combinations are proposed that yield more satisfactory values.

\subsubsection{Assumptions and Design Criteria}

The reliability analysis of moment is based on the first-order, second-moment (FOSM) method (Ellingwood et al. 1980). Nominal values of geometric properties, material strengths and live-to-dead load ratios are selected that simulate practical values and are consistent with the requirements in ACI 318-14, as shown in Table 3.5. The design of the rectangular singly reinforced sections is based on Equations [2.1] to [2.5], reinforcement 
limits, minimum thickness limits, specified concrete cover requirements and material strength requirements specified in ACI 318-14. The ranges of reinforcement ratio investigated are consistent with those in Chapter 2, specifically 0.003 to 0.005 for twoway slabs, 0.006 to 0.010 for one-way slabs and 0.011 to 0.018 for beams. The material strengths also correspond to those investigated in Chapter 2 with $f_{c}{ }^{\prime}$ of 25 or $45 \mathrm{MPa}$ and $f_{y}$ of $420 \mathrm{MPa}$.

The typical specified live-to-dead load ratio, $w_{L} / w_{D}$, for flexural members ranges from 0.5 to 1.5 (Ellingwood et al. 1980), so the present study investigates ratios within this range. The specified dead and live loads are determined to exactly achieve the design flexural strength, $\phi M_{n}$ or $M_{r}$. Live load reduction factors due to tributary area are neglected. If they are considered, the range of typical $w_{L} / w_{D}$ ratios reduces slightly, but the reliability indices computed for a specific $w_{L} / w_{D}$ value are correct. Simply supported members are assumed, so the maximum factored moment at mid-span is computed as

$$
M_{u}=\frac{\left(1.2 w_{D}+1.6 w_{L}\right) l^{2}}{8}
$$

where: $w_{D}$ is the specified dead load per unit length; $w_{L}$ is the specified live load per unit length; and, $l$ is the span length of a member.

\subsubsection{Reliability Analyses}

As mentioned previously, the limit state function is $Z=R / Q$. For flexural members, the flexural resistance, $R_{M}$, is

$$
R_{M}=P A_{s} f_{y}\left(d-\frac{A_{s} f_{y}}{1.7 f_{c}^{\prime} b}\right)
$$

where $P$ is the professional factor. The load effect for flexural members, $Q_{M}$, is

$$
Q_{M}=\frac{\left(w_{D} T_{D}+w_{L} T_{L}\right) l^{2}}{8}
$$


where: $T_{D}$ is the factor that accounts for the transformation from the dead load to the dead load effect; and, $T_{L}$ is the factor that accounts for the transformation from the live load to the live load effect.

The statistical parameters used for reliability analyses for moment summarized from Section 3.3 are shown in Table 3.6. The slab widths (1 m unit length) and member span lengths are assumed deterministic. The statistical parameters for area and line loads are assumed identical. $T_{D}$ and $T_{L}$ are already included in the selected parameters for $w_{D}$ and $w_{L}$ reported by Ellingwood et al. (1980) and Israel et al. (1987), so bias coefficients of 1.0 and coefficients of variation of 0 are assumed.

The reliability analysis process is as follows:

1. Calculate the design flexural strength $\phi M_{n}$ using Equations [2.1] and [2.2] or $M_{r}$ using Equations [2.3] and [2.4].

2. Calculate $w_{D}$ and $w_{L}$ by equating the design flexural strength, $\phi M_{n}$ or $M_{r}$, to the factored moment, $M_{u}$, with the given load ratio, $w_{L} / w_{D}$, based on Equation [3.11], obtaining

$$
w_{D}=\frac{8 \phi M_{n}\left\{\text { or } M_{r}\right\}}{\left[1.2+1.6\left(w_{L} / w_{D}\right)\right] l^{2}}
$$

and

$$
w_{L}=\frac{8\left(w_{L} / w_{D}\right) \phi M_{n}\left\{\text { or } M_{r}\right\}}{\left[1.2+1.6\left(w_{L} / w_{D}\right)\right] l^{2}}
$$

3. Calculate the means and coefficients of variation of the resistances and the load effects using Equations [3.7], [3.8], [3.12] and [3.13].

4. Calculate the reliability index, $\beta_{M u}$ or $\beta_{M r}$, using Equation [3.4]. Here, $\beta_{M u}$ is the reliability index for moment obtained using the strength reduction factors in ACI 318- 
14 and $\beta_{M r}$ is the reliability index for moment obtained using the partial material strength reduction factors.

5. Summarize the results.

The variations of reliability indices for moment, $\beta_{M}$, with respect to longitudinal reinforcement ratios, $\rho$, for $w_{L} / w_{D}$ of 0.5 are shown in Figure 3.1 for $f_{c}^{\prime}$ of $25 \mathrm{MPa}$. Very similar results for $f_{c}^{\prime}$ of $45 \mathrm{MPa}$ are shown in Figure B.1 of Appendix B. Clearly, an abrupt discontinuity occurs between the reinforcement ratios of 0.010 and 0.011 , which represent the upper and lower limits, respectively, of the ranges for one-way slabs and beams. A smaller discontinuity occurs between $\rho$ of 0.005 and 0.006 , which represent the upper and lower limits, respectively, of the ranges for two-way slabs and one-way slabs. In reality, the reliability index variation should not show such discontinuities. The discontinuities are due to the different statistical parameters adopted for the geometric properties of two-way slabs, one-way slabs and beams.

To find reasons for these discontinuities, the design conditions and statistical parameters used for the three representative cross sections in Tables 3.5 and 3.6 were critically reviewed. The nominal values of $b, h, d$, and $l$ differ, and the reliability index is sensitive to the statistical parameters for $b$ and, particularly, $d$. In reality, any variation of parameters should be gradual instead of abrupt. The analyses were therefore repeated using values of coefficient of variation for $d, V_{d}$, that vary linearly with the reinforcement ratios, as shown in Figure 3.2. The bias coefficients equal 1 for all ranges, so don't need to be modified. The coefficients of variation for $d$ are the standard deviations shown in Table 3.6 divided by the mean values, and are roughly $0.031,0.028$ and 0.015 for the three ranges of reinforcement ratio. The linear transition was therefore assumed to start at $\rho$ of 0.004 , an intermediate value for two-way slabs, and to end at $\rho$ of 0.016 , which corresponds approximately to the tension-controlled limit of the section with $f_{c}^{\prime}$ of 25 MPa. This variation of the coefficients of variation is reasonable because, as $d$ increases, the coefficient of variation decreases if the standard deviation of $d$ remains constant. 
The recalculated results are shown in Figures 3.3 and 3.4 for $f_{c}{ }^{\prime}$ of $25 \mathrm{MPa}$ and $w_{L} / w_{D}$ of 0.5 and 1.5, respectively. The discontinuities in Figure 3.1 are corrected and the trend lines are continuous. Similar relationships for $f_{c}^{\prime}$ of $45 \mathrm{MPa}$ are shown in Figures B.2 and B. 3 of Appendix B. Moreover, $\beta_{M}$ increases for increased $\rho$, and this is desirable because increasing $\rho$ causes a flexural failure to be less ductile.

The abrupt increase of slope for reliability indices corresponding to ACI 318-14 strength reduction factors in Figure 3.3 for $\rho$ greater than 0.016 is caused by the reduction of $\phi$ for sections that are not tension-controlled. The reason is identical to that abrupt decrease occurring in Figure 2.1 of Chapter 2. The four families of trend lines shown correspond to the four $\phi_{s}$ values, while the differences within each family are due to the four $\phi_{c}$ values. Therefore, the reliability index for moment obtained using the partial material strength reduction factors, $\beta_{M r}$, is more sensitive to $\phi_{s}$ than to $\phi_{c}$. The slopes shown for lower $\phi_{c}$ values are steeper than those for higher values, but the differences are small. Comparing Figures 3.3 and 3.4, the influence of $w_{L} / w_{D}$ is not large, affecting only the dispersion of the trend lines, and the lines with the higher load ratio are more concentrated. As shown in Figures B.2 and B.3, the influence of increasing $f_{c}{ }^{\prime}$ to $45 \mathrm{MPa}$ is small, affecting the dispersion in each family: the reliability indices are more concentrated for a typical $\phi_{s}$. In other words, the reliability indices for a typical $\phi_{s}$ are less sensitive to $\phi_{c}$ for $f_{c}^{\prime}$ of $45 \mathrm{MPa}$.

According to ASCE 7-10 (ASCE 2010), for a 50-year service period, normal buildings with Risk Category II should exhibit a reliability index of: "3.0 if the failure is not sudden and does not lead to widespread progression of damage; 3.5 if the failure is either sudden or leads to widespread progression of damage; and, 4.0 if the failure is sudden and results in widespread progression of damage". Therefore, the desired reliability index ranges are 3.0 for slabs and 3.0 to 3.5 for beams. The reliability indices, $\beta_{M u}$, calculated corresponding to the strength reduction factors from ACI 318-14 for $f_{c}^{\prime}$ of $25 \mathrm{MPa}$ and $w_{L} / w_{D}$ of 0.5 , range from 3.15 for two-way slabs to 3.86 for beams as shown in Figure 3.3. Similarly, the range is 3.05-3.60 for $w_{L} / w_{D}$ of 1.5 as shown in Figure 3.4. For $f_{c}^{\prime}$ of $45 \mathrm{MPa}$, the ranges are 3.14-3.28 and 3.04-3.14 as shown in Figures B.2 and B.3 for 
$w_{L} / w_{D}$ of 0.5 and 1.5 , respectively. The lower strength reduction factors, $\phi$, less than 0.90 , apply for beams with $f_{c}^{\prime}$ of $25 \mathrm{MPa}$ with reinforcement ratios higher than 0.016. $\beta_{M u}$ is too conservative for these sections. Hence, partial material strength reduction factor combinations should be selected to correspond to reliability indices that satisfy ASCE 710 instead of simply duplicating the reliability levels achieved using ACI 318-14.

\subsubsection{Recommended Partial Material Strength Reduction Factors}

Based on Figures 3.3, 3.4, B.2, and B.3, the best partial material strength reduction factor combinations have $\phi_{s}$ of 0.90 . The influence of $\phi_{c}$ is not as large. The means and standard deviations of the reliability indices for moment, $\beta_{M r}$, for the three ranges of longitudinal reinforcement ratio are summarized in Tables 3.7-3.9 for $f_{c}^{\prime}$ of $25 \mathrm{MPa}$ and in Tables B.7-B.9 of Appendix B for $f_{c}^{\prime}$ of $45 \mathrm{MPa}$. The mean reliability index decreases as $\phi_{s}$ or $\phi_{c}$ increases, and the standard deviation of reliability index increases as $\phi_{s}$ increases and decreases as $\phi_{c}$ increases. For $\phi_{s}$ of 0.90 and $\phi_{c}$ of 0.75 , the mean reliability indices computed based on the two load ratios and the two $f_{c}^{\prime}$ values are approximately 3.14 , 3.22 , and 3.36 for $\rho$ of 0.003 to $0.005,0.006$ to 0.010 , and 0.011 to 0.018 , respectively. Thus this combination yields an appropriate range of reliability indices. Again, as noted previously in Chapter 2, the adoption of $\phi_{s}$ of 0.90 is desirable, and if a lower $\phi_{c}$ is chosen, the reliability index will increase and tend to be conservative, but the standard deviation will also increase.

\subsection{One-way Shear}

This section compares reliability indices for one-way shear corresponding to the partial material strength reduction factors with those corresponding to the existing ACI 318-14 criteria. The first-order, second-moment (FOSM) analysis procedure is again adopted.

\subsubsection{Assumptions and Design Criteria}

Similar to moment, the reliability analysis of shear is based on the FOSM method (Ellingwood et al. 1980). Nominal values of geometric properties, material strengths and live-to-dead load ratios are selected that simulate practical values and are consistent with 
the requirements in ACI 318-14, as shown in Table 3.10. The design of the beams is based on Equations [2.13] to [2.19], shear reinforcement limits, minimum thickness limits, specified concrete cover requirements and material strength requirements specified in ACI 318-14. The stirrup yield strength, $f_{y t}$, is $420 \mathrm{MPa}$ and the transverse reinforcement ratio, $\rho_{t}$, ranges from 0.001 to 0.007 for $f_{c}{ }^{\prime}$ of $25 \mathrm{MPa}$ and from 0.001 to 0.010 for $f_{c}^{\prime}$ of $45 \mathrm{MPa}$ to represent the ranges permitted by ACI 318-14. However, maximum stirrup spacing criteria are not always satisfied for some of the transverse reinforcement ratios, because the stirrup size is assumed to be a No.3 (9.5 mm diameter) bar and the change of transverse reinforcement ratios is controlled by the spacing.

The typical specified live-to-dead load ratio is identical to that assumed for moment, ranging from 0.5 to 1.5 . The specified dead and live loads are determined to exactly achieve the design shear strength, $\phi V_{n}$ or $V_{r}$. Tributary-area-based live load reduction factors are again neglected. Simply supported members are assumed, so the maximum factored shear force can be computed as

$$
V_{u}=\frac{\left(1.2 w_{D}+1.6 w_{L}\right) l}{2}
$$

\subsubsection{Reliability Analyses}

For members resisting shear force, the resistance, $R_{V}$, is

$$
R_{V}=P\left(0.17 \lambda \sqrt{f_{c}^{\prime}} b_{w} d+\frac{A_{v} f_{y t} d}{s}\right)
$$

where: $\lambda$ is the modification factor to account for the reduced shear strength of lightweight concrete, and equals 1.0 for normalweight concrete; $b_{w}$ is the web width; $s$ is the center-to-center spacing of the transverse reinforcement; and, $A_{v}$ is the area within spacing $s$ of the transverse reinforcement.

The load effect for members resisting shear force, $Q_{V}$, is 


$$
Q_{V}=\frac{\left(w_{D} T_{D}+w_{L} T_{L}\right) l}{2}
$$

The statistical parameters for reliability analyses obtained from the literature are shown in Table 3.11. The stirrup spacing, $s$, length of the beam, $l$, and modification factor, $\lambda$, are assumed deterministic. The professional factor has statistical parameters recommended by Somo and Hong (2006), with a relatively high bias coefficient, 1.47, and a relatively high coefficient of variation, 0.36. The statistical parameters for area and line loads are assumed identical. Similar to moment, $T_{D}$ and $T_{L}$ are already included in the selected parameters for $w_{D}$ and $w_{L}$ reported by Ellingwood et al. (1980) and Israel et al. (1987), so bias coefficients of 1.0 and coefficients of variation of 0 are assumed.

The reliability analysis process is as follows:

1. Calculate the design shear strength $\phi V_{n}$ using Equations [2.13], [2.14] and [2.15] or $V_{r}$ using Equations [2.16], [2.17] and [2.18].

2. Calculate $w_{D}$ and $w_{L}$ by equating the design shear strength, $\phi V_{n}$ or $V_{r}$, to the factored shear force, $V_{u}$, with the given load ratio, $w_{L} / w_{D}$, based on Equation [3.16], obtaining

$$
w_{D}=\frac{2 \phi V_{n}\left\{\text { or } V_{r}\right\}}{\left[1.2+1.6\left(w_{L} / w_{D}\right)\right] l}
$$

and

$$
w_{L}=\frac{2\left(w_{L} / w_{D}\right) \phi V_{n}\left\{\text { or } V_{r}\right\}}{\left[1.2+1.6\left(w_{L} / w_{D}\right)\right] l}
$$

3. Calculate the means and coefficients of variation of the resistances and the load effects using Equations [3.7], [3.8], [3.17] and [3.18].

4. Calculate the reliability index, $\beta_{V u}$ or $\beta_{V r}$, using Equation [3.4], where $\beta_{V u}$ is the reliability index for shear obtained using the strength reduction factors in ACI 318-14 
and $\beta_{V r}$ is the reliability index for shear obtained using the partial material strength reduction factors.

5. Summarize the results.

The reliability indices for shear, $\beta_{V}$, were calculated with respect to transverse reinforcement ratios, $\rho_{t}$, as shown in Figures 3.5 and 3.6 for $f_{c}{ }^{\prime}$ of $25 \mathrm{MPa}$ and $w_{L} / w_{D}$ of 0.5 and 1.5, respectively. Similar results were obtained for $f_{c}^{\prime}$ of $45 \mathrm{MPa}$ as shown in Figures B.4 and B.5 of Appendix B. The reliability index for shear corresponding to ACI 318-14, $\beta_{V u}$, increases as $\rho_{t}$ increases. As the transverse reinforcement ratio increases, however, the failure of the reinforced member becomes more ductile, so this trend may not be particularly desirable. In contrast, the variation of $\beta_{V r}$ can decrease with the increased $\rho_{t}$, depending on the various $\phi_{s}$ and $\phi_{c}$ values. Again, the four $\phi_{s}$ values create distinct families of $\beta_{V r}$ values that are not as diverse as those for moment, and are shown in separate figures. Differences within each family are due to the different $\phi_{c}$ values. Comparing Figures 3.5 and B.4, the influence of $f_{c}^{\prime}$ is slight. Comparing Figures 3.5 and 3.6, the higher $w_{L} / w_{D}$ value yields slightly higher reliability indices.

According to ASCE 7-10, for a 50-year service period, normal buildings with Risk Category II should exhibit a reliability range of 3.0 to 3.5 for beams, which is achieved by the best strength reduction factor combination for moment. However, the reliability indices shown in Figures 3.5, 3.6, B.4 and B.5 for shear, range from 2.65 to 2.82, and 2.20 to 3.11 computed for the ACI 318-14 criteria and the various partial material strength reduction factor combinations, respectively. In other words, the safety level for moment is markedly greater than that for shear. This is undesirable, because a shear failure is less ductile than a flexural failure and so has a greater target reliability index.

The reliability indices for shear are sensitive to the statistical parameters assumed for the professional factor. For example, if a bias coefficient of 1.075 and a coefficient of variation of 0.10 are assumed for the professional factor, as recommended by Nowak and Szerszen (2003), the reliability index corresponding to the ACI 318-14 criteria ranges from 4.27 to 4.39 for $f_{c}^{\prime}$ of $25 \mathrm{MPa}$ and $w_{L} / w_{D}$ of 0.5 as shown in Figure 3.7. The 
reliability indices corresponding to the various partial material strength reduction factor combinations range from 3.21 to 4.99 . It is therefore necessary to review the basis for the various statistical parameters for the professional factor reported in the literature.

Nowak and Szerszen (2003) recommended statistical parameters for professional factor by modifying slightly the values recommended in Ellingwood et al. (1980) based on their "engineering judgement". The database used by Ellingwood et al. (1980) contains 62 test beams with stirrups and 96 beams with no stirrups. The database analyzed by Somo and Hong (2006) contains 419 test beams with stirrups and 727 beams with no stirrups, and the total 1146 test beams, includes 878 beams with $h \geq 300 \mathrm{~mm}$ and $f_{c}^{\prime} \geq 20 \mathrm{MPa}$. Collins (2001) computed professional factors using two databases, one with 413 test results and the other with 776 test results. He observed that the larger database contains much higher number of beams with $h \leq 350 \mathrm{~mm}$, prestressed beams, and beams without stirrups, and so it is less representative of realistic concrete construction than the smaller database. The three sets of statistical parameters for the professional factor are shown in Table 3.12. The parameters reported by Somo and Hong (2006) are most comprehensively presented, e.g., the parameters are classified by $a_{v} / d$ and the presence of stirrups and consider prestressed members separately. The parameters recommended by Nowak and Szerszen (2003) have the lowest bias coefficient which is conservative but also the lowest coefficient of variation which is unconservative. Somo and Hong (2006) analyzed the largest number of beams, including the database assembled by Bentz (2000) which is the source of 413 test beams for the study of Collins (2001). Therefore, the parameters for professional factor reported by Somo and Hong (2006) are likely the most appropriate.

\subsubsection{Recommended Partial Material Strength Reduction Factors}

Although the reliability indices calculated by applying parameters from Somo and Hong (2006) are lower than the desirable values, the influence of the statistical parameters for the professional factor is consistent for both ACI 318-14 and the partial material strength reduction factors. In other words, the reliability indices increase or decrease consistently in both cases when different professional factor parameters are chosen. Therefore, the reliability indices calculated for ACI 318-14 are assumed adequate and the best partial 
material strength reduction factor combinations are selected as those yielding a mean reliability index ratio, $\beta_{V u} / \beta_{V r}$, of 1 with the least standard deviation. The means and standard deviations for $\beta_{V u} / \beta_{V r}$ are summarized in Table 3.13 for $f_{c}^{\prime}$ of $25 \mathrm{MPa}$ and similar results are shown in Table B.10 for $f_{c}^{\prime}$ of $45 \mathrm{MPa}$. The mean increases as $\phi_{s}$ or $\phi_{c}$ increases. The standard deviation increases as $\phi_{s}$ increases and decreases as $\phi_{c}$ increases. The best partial material strength reduction factor combination is $\phi_{s}$ of 0.80 and $\phi_{c}$ of 0.65 . Moreover, combinations with $\phi_{s}$ of 0.80 and $\phi_{c}$ of 0.70 , and $\phi_{s}$ of 0.85 and $\phi_{c}$ of 0.60 are close to optimal. These results are identical to those reported for shear in Chapter 2.

\subsection{Summary and Conclusions}

This chapter has presented statistical parameters collection and reliability analyses for members subject to moment and one-way shear using the FOSM method.

For members subjected to moment, the best partial material strength reduction factor combination is $\phi_{s}$ of 0.90 and $\phi_{c}$ of 0.75 . Similar to Chapter 2, the family with $\phi_{s}$ of 0.90 is most desirable. If a lower $\phi_{c}$ value is chosen, the reliability index will increase, and tend to exceed the target values, but the standard deviation will also increase.

For members subjected to one-way shear, the statistical parameters for the professional factor markedly influence the reliability indices. Based on the parameters reported by Somo and Hong (2006), reliability indices corresponding to ACI 318-14 and the partial material strength reduction factors both yield low values, whereas much higher values occur when parameters reported by Nowak and Szerszen (2003) are used. Therefore, because the professional factor has the similar impacts on the reliability indices for ACI 318-14 and the partial material strength reduction factors criteria, and assuming the reliability indices calculated for ACI 318-14 are adequate, partial material strength reduction factors are selected that yield reliability indices that approximate those derived using the ACI 318-14 provisions. The best partial material strength reduction factor combination is $\phi_{s}$ of 0.80 and $\phi_{c}$ of 0.65 . Moreover, combinations with $\phi_{s}$ of 0.80 and $\phi_{c}$ of 0.70 , and $\phi_{s}$ of 0.85 and $\phi_{c}$ of 0.60 are close to optimal, which are identical to those recommended in Chapter 2. 
Table 3.1: Statistical parameters for geometric properties used in this study

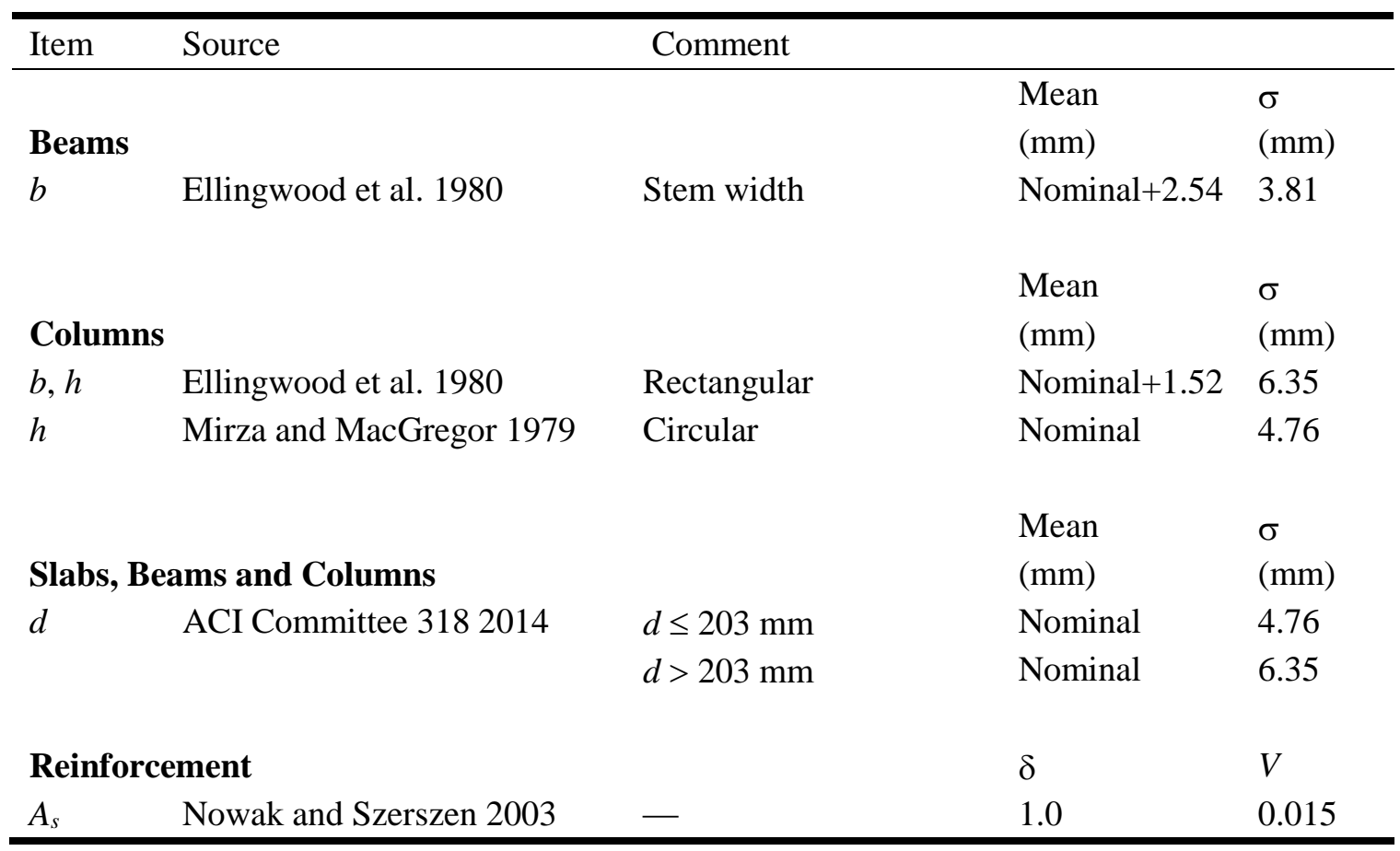

Table 3.2: Statistical parameters for concrete compressive strength used in this study

\begin{tabular}{lllll}
\hline Item & Source & Comment & $\delta$ & $V$ \\
\hline$F_{1}$ & Bartlett 2007 & Cast-in-place concrete & 1.27 & 0.122 \\
$F_{2}$ & Bartlett 2007 & Cast-in-place concrete & 1.03 & 0.113 \\
$F_{i-p}$ & Bartlett and MacGregor 1999 & Cast-in-place concrete & 1.0 & 0.130 \\
$F_{r}$ & Bartlett 2007 & 1 hour live load loading & 0.88 & 0 \\
Overall & & & 1.15 & 0.211 \\
\hline
\end{tabular}


Table 3.3: Statistical parameters for professional factors

\begin{tabular}{|c|c|c|c|}
\hline Source & Comment & $\delta$ & $V$ \\
\hline \multicolumn{4}{|l|}{ Shear } \\
\hline \multirow[t]{16}{*}{ Somo and Hong 2006} & Beams without stirrups & & \\
\hline & All $a_{v} / d$ values & 2.17 & 0.75 \\
\hline & $a_{v} / d \geq 2$ & 1.74 & 0.47 \\
\hline & $a_{v} / d<2$ & 4.86 & 0.53 \\
\hline & Beams with stirrups & & \\
\hline & All $a_{v} / d$ values & 1.51 & 0.37 \\
\hline & $a_{v} / d \geq 2$ & 1.47 & 0.36 \\
\hline & $a_{v} / d<2$ & 1.79 & 0.35 \\
\hline & All Beams & & \\
\hline & All $a_{v} / d$ values & 1.92 & 0.71 \\
\hline & $a_{v} / d \geq 2$ & 1.64 & 0.45 \\
\hline & $a_{v} / d<2$ & 3.96 & 0.66 \\
\hline & Without Kani's beams & & \\
\hline & All $a_{v} / d$ values & 1.75 & 0.60 \\
\hline & $a_{v} / d \geq 2$ & 1.58 & 0.46 \\
\hline & $a_{v} / d<2$ & 3.22 & 0.59 \\
\hline \multirow[t]{2}{*}{ Collins 2001} & 776 beams dataset & 1.30 & 0.278 \\
\hline & 413 beams dataset & 1.19 & 0.339 \\
\hline Israel et al. 1987 & Beam, shear & 1.09 & 0.12 \\
\hline Nowak and Szerszen 2003 & Beam, shear & 1.075 & 0.10 \\
\hline \multicolumn{4}{|l|}{ Others } \\
\hline \multirow[t]{4}{*}{ Nowak and Szerszen 2003} & Beam, flexure & 1.02 & 0.06 \\
\hline & Slab & 1.02 & 0.06 \\
\hline & Column, tied & 1.00 & 0.08 \\
\hline & Column, spiral & 1.05 & 0.06 \\
\hline
\end{tabular}


Table 3.4: Statistical parameters for load effects

\begin{tabular}{|c|c|c|c|c|}
\hline Item & Source & Comment & $\delta$ & $V$ \\
\hline Dead & Ellingwood et al. 1980 & All construction materials & 1.05 & 0.10 \\
\hline \multirow[t]{2}{*}{ load } & Szerszen and Nowak 2003 & Cast-in-place concrete & 1.05 & 0.10 \\
\hline & & Precast concrete & 1.03 & 0.08 \\
\hline \multirow{5}{*}{$\begin{array}{l}\text { Live } \\
\text { load }\end{array}$} & Israel et al. 1987 & A58.1-1982 live load & 1.00 & 0.25 \\
\hline & & reductions are used & & \\
\hline & Szerszen and Nowak 2003 & 50 year maximum load & 1.00 & 0.18 \\
\hline & Bartlett et al. 2003 & $\begin{array}{l}50 \text { year maximum load, } \\
1995 \text { NBCC live load } \\
\text { reductions are used }\end{array}$ & 0.900 & 0.170 \\
\hline & & Transformation to load effect & 1.000 & 0.206 \\
\hline
\end{tabular}


Table 3.5: Design conditions for moment

\begin{tabular}{|c|c|c|c|}
\hline Section & Item & Nominal value & Unit \\
\hline & \multicolumn{3}{|c|}{ Geometric properties } \\
\hline \multirow[t]{4}{*}{ Two-way slabs } & $b$ & 1000 (unit width) & $\mathrm{mm}$ \\
\hline & $h$ & 200 & $\mathrm{~mm}$ \\
\hline & $d$ & 155 & $\mathrm{~mm}$ \\
\hline & $l$ & 6 & $\mathrm{~m}$ \\
\hline \multirow[t]{4}{*}{ One-way slabs } & $b$ & 1000 (unit width) & $\mathrm{mm}$ \\
\hline & $h$ & 200 & $\mathrm{~mm}$ \\
\hline & $d$ & 170 & $\mathrm{~mm}$ \\
\hline & $l$ & 4 & $\mathrm{~m}$ \\
\hline \multirow[t]{8}{*}{ Beams } & $b$ & 300 & $\mathrm{~mm}$ \\
\hline & $h$ & 500 & $\mathrm{~mm}$ \\
\hline & $d$ & 435 & $\mathrm{~mm}$ \\
\hline & $l$ & 8 & $\mathrm{~m}$ \\
\hline & \multicolumn{3}{|c|}{ Material strengths } \\
\hline & $f_{c}^{\prime}$ & 25 and 45 & $\mathrm{MPa}$ \\
\hline & $f_{y}$ & 420 & $\mathrm{MPa}$ \\
\hline & \multicolumn{3}{|c|}{ Load ratios } \\
\hline Two-way slabs & $w_{L} / w_{D}$ & 0.5 and 1.5 & \\
\hline One-way slabs & $w_{L} / w_{D}$ & 0.5 and 1.5 & \\
\hline Beams & $w_{L} / w_{D}$ & 0.5 and 1.5 & \\
\hline
\end{tabular}


Table 3.6: Statistical parameters for moment reliability analysis

\begin{tabular}{|c|c|c|c|c|}
\hline Item & Source & Comment & & \\
\hline \multicolumn{3}{|c|}{ Geometric properties } & Mean & $\sigma$ \\
\hline \multicolumn{3}{|c|}{ Two-way slabs } & $(\mathrm{mm})$ & $(\mathrm{mm})$ \\
\hline$b$ & - & Assumed deterministic & Nominal & 0 \\
\hline$d$ & ACI Committee 3182014 & $d \leq 203 \mathrm{~mm}$ & Nominal & 4.76 \\
\hline \multirow[t]{2}{*}{$l$} & - & Assumed deterministic & Nominal & 0 \\
\hline & & & Mean & $\sigma$ \\
\hline \multicolumn{3}{|c|}{ One-way slabs } & $(\mathrm{mm})$ & $(\mathrm{mm})$ \\
\hline$b$ & - & Assumed deterministic & Nominal & 0 \\
\hline$d$ & ACI Committee 3182014 & $d \leq 203 \mathrm{~mm}$ & Nominal & 4.76 \\
\hline \multirow[t]{2}{*}{$l$} & - & Assumed deterministic & Nominal & 0 \\
\hline & & & Mean & $\sigma$ \\
\hline \multicolumn{2}{|c|}{ Beams } & & $(\mathrm{mm})$ & $(\mathrm{mm})$ \\
\hline$b$ & Ellingwood et al. 1980 & Stem width & Nominal+2.54 & 3.81 \\
\hline$d$ & ACI Committee 3182014 & $d>203 \mathrm{~mm}$ & Nominal & 6.35 \\
\hline$l$ & - & Assumed deterministic & Nominal & 0 \\
\hline \multicolumn{2}{|c|}{ Reinforcement } & & $\delta$ & $V$ \\
\hline$A_{s}$ & Nowak and Szerszen 2003 & - & 1.0 & 0.015 \\
\hline \multicolumn{2}{|c|}{ Material Strengths } & & $\delta$ & $V$ \\
\hline \multirow[t]{2}{*}{$f_{c}^{\prime}$} & Bartlett 2007 & Cast-in-place concrete & 1.15 & 0.211 \\
\hline & Bartlett and MacGregor 1999 & & & \\
\hline$f_{y}$ & Ellingwood et al. 1980 & - & 1.125 & 0.098 \\
\hline \multicolumn{2}{|c|}{ Professional factor } & & $\delta$ & $V$ \\
\hline$P$ & Nowak and Szerszen 2003 & Beam, flexure, and slab & 1.02 & 0.06 \\
\hline \multicolumn{2}{|c|}{ Load effects } & & $\delta$ & $V$ \\
\hline$w_{D} T_{D}$ & Ellingwood et al. 1980 & All construction materials & 1.05 & 0.10 \\
\hline$w_{L} T_{L}$ & Israel et al. 1987 & $\begin{array}{l}\text { A58.1-1982 live load } \\
\text { reductions are used }\end{array}$ & 1.00 & 0.25 \\
\hline
\end{tabular}


Table 3.7: Means and standard deviations of reliability indices for moment using partial material strength reduction factors, $\beta_{M r}$, for $f_{c}^{\prime}=25 \mathrm{MPa}$ and $\rho=0.003-0.005$

\begin{tabular}{cccccccccc}
\hline & & \multicolumn{10}{c}{$\phi_{c}$} \\
\cline { 3 - 10 }$w_{L} / w_{D}$ & $\phi_{s}$ & Mean & $\sigma$ & Mean & $\sigma$ & Mean & $\sigma$ & Mean & $\sigma$ \\
\cline { 2 - 10 } 0.5 & 0.80 & 4.002 & 0.039 & 3.975 & 0.032 & 3.951 & 0.026 & 3.931 & 0.021 \\
& 0.85 & 3.639 & 0.044 & 3.610 & 0.036 & 3.585 & 0.030 & 3.564 & 0.024 \\
& 0.90 & 3.299 & 0.048 & 3.268 & 0.040 & 3.242 & 0.033 & 3.219 & 0.027 \\
& 0.95 & 2.978 & 0.053 & 2.945 & 0.044 & 2.917 & 0.037 & 2.893 & 0.031 \\
& & & & & & & & & \\
1.5 & 0.80 & 3.743 & 0.029 & 3.720 & 0.023 & 3.701 & 0.018 & 3.685 & 0.014 \\
& 0.85 & 3.446 & 0.033 & 3.422 & 0.027 & 3.402 & 0.022 & 3.384 & 0.017 \\
& 0.90 & 3.167 & 0.038 & 3.142 & 0.031 & 3.120 & 0.025 & 3.102 & 0.020 \\
& 0.95 & 2.904 & 0.042 & 2.878 & 0.035 & 2.855 & 0.028 & 2.835 & 0.023 \\
\hline
\end{tabular}

Table 3.8: Means and standard deviations of reliability indices for moment using partial material strength reduction factors, $\beta_{M r}$, for $f_{c}^{\prime}=25 \mathrm{MPa}$ and $\rho=0.006-0.010$

\begin{tabular}{cccccccccc}
\hline & & \multicolumn{10}{c}{$\phi_{c}$} \\
\cline { 3 - 10 }$w_{L} / w_{D}$ & $\phi_{s}$ & Mean & $\sigma$ & Mean & $\sigma$ & Mean & $\sigma$ & Mean & $\sigma$ \\
\cline { 3 - 10 } 0.5 & 0.80 & 4.172 & 0.069 & 4.114 & 0.055 & 4.064 & 0.044 & 4.021 & 0.035 \\
& 0.85 & 3.829 & 0.077 & 3.767 & 0.063 & 3.713 & 0.051 & 3.668 & 0.041 \\
& 0.90 & 3.509 & 0.086 & 3.442 & 0.071 & 3.385 & 0.058 & 3.336 & 0.047 \\
& 0.95 & 3.209 & 0.095 & 3.137 & 0.079 & 3.077 & 0.065 & 3.025 & 0.053 \\
& & & & & & & & & \\
1.5 & 0.80 & 3.869 & 0.051 & 3.821 & 0.040 & 3.781 & 0.031 & 3.746 & 0.024 \\
& 0.85 & 3.589 & 0.058 & 3.538 & 0.047 & 3.495 & 0.037 & 3.458 & 0.029 \\
& 0.90 & 3.328 & 0.066 & 3.274 & 0.054 & 3.228 & 0.043 & 3.188 & 0.035 \\
& 0.95 & 3.084 & 0.074 & 3.026 & 0.061 & 2.977 & 0.050 & 2.935 & 0.040 \\
\hline
\end{tabular}


Table 3.9: Means and standard deviations of reliability indices for moment using partial material strength reduction factors, $\beta_{M r}$, for $f_{c}^{\prime}=25 \mathrm{MPa}$ and $\rho=0.011-0.018$

\begin{tabular}{ccccccccccc}
\hline & & \multicolumn{10}{c}{$\phi_{c}$} \\
\cline { 3 - 10 }$w_{L} / w_{D}$ & $\phi_{s}$ & Mean & $\sigma$ & Mean & $\sigma$ & Mean & $\sigma$ & Mean & $\sigma$ \\
\cline { 2 - 10 } 0.5 & 0.80 & 4.453 & 0.098 & 4.334 & 0.073 & 4.233 & 0.053 & 4.148 & 0.036 \\
& 0.85 & 4.153 & 0.118 & 4.025 & 0.090 & 3.917 & 0.068 & 3.825 & 0.049 \\
& 0.90 & 3.878 & 0.138 & 3.740 & 0.108 & 3.624 & 0.084 & 3.525 & 0.063 \\
& 0.95 & 3.625 & 0.159 & 3.477 & 0.127 & 3.353 & 0.100 & 3.247 & 0.078 \\
& & & & & & & & & \\
1.5 & 0.80 & 4.081 & 0.076 & 3.985 & 0.056 & 3.904 & 0.040 & 3.834 & 0.026 \\
& 0.85 & 3.839 & 0.092 & 3.735 & 0.070 & 3.648 & 0.052 & 3.573 & 0.037 \\
& 0.90 & 3.616 & 0.109 & 3.504 & 0.085 & 3.411 & 0.065 & 3.331 & 0.049 \\
& 0.95 & 3.411 & 0.126 & 3.291 & 0.100 & 3.191 & 0.079 & 3.106 & 0.061 \\
\hline
\end{tabular}

Table 3.10: Design conditions for shear

\begin{tabular}{llll}
\hline Section & Item & Nominal value & Unit \\
\hline \multirow{3}{*}{ Beams } & Geometric properties & \\
& $b$ & 300 & $\mathrm{~mm}$ \\
$h$ & 500 & $\mathrm{~mm}$ \\
& $d$ & 435 & $\mathrm{~mm}$ \\
$l$ & 8 & $\mathrm{~m}$ \\
$A_{v}$ & $71 \times 2$ & $\mathrm{~mm}^{2}$ \\
& & \\
Material Strengths & & $\mathrm{MPa}$ \\
$f_{c}^{\prime}$ & 25 and 45 & $\mathrm{MPa}$ \\
$f_{y t}$ & 420 & \\
$\lambda$ & 1.0 & \\
& & \\
& Load ratios & & \\
& $w_{L} / w_{D}$ & 0.5 and 1.5 & \\
\hline
\end{tabular}


Table 3.11: Statistical parameters for shear reliability analysis

\begin{tabular}{|c|c|c|c|c|}
\hline Item & Source & Comment & & \\
\hline \multicolumn{3}{|c|}{ Geometric properties } & Mean & $\sigma$ \\
\hline \multicolumn{3}{|c|}{ Beams } & $(\mathrm{mm})$ & $(\mathrm{mm})$ \\
\hline$b_{w}$ & Ellingwood et al. 1980 & Stem width & Nominal +2.54 & 3.81 \\
\hline$d$ & ACI Committee 3182014 & $d>203 \mathrm{~mm}$ & Nominal & 6.35 \\
\hline$s$ & - & Assumed deterministic & Nominal & 0 \\
\hline$l$ & - & Assumed deterministic & Nominal & 0 \\
\hline \multicolumn{3}{|c|}{ Reinforcement } & $\delta$ & $V$ \\
\hline$A_{v}$ & Nowak and Szerszen 2003 & - & 1.0 & 0.015 \\
\hline \multicolumn{3}{|c|}{ Material Strengths } & $\delta$ & $V$ \\
\hline \multirow[t]{2}{*}{$f_{c}^{\prime}$} & Bartlett 2007 & Cast-in-place concrete & 1.15 & 0.211 \\
\hline & Bartlett and MacGregor 1999 & & & \\
\hline$f_{y t}$ & Ellingwood et al. 1980 & - & 1.125 & 0.098 \\
\hline$\lambda$ & - & Assumed deterministic & 1 & 0 \\
\hline \multicolumn{3}{|c|}{ Professional factor } & $\delta$ & $V$ \\
\hline$P$ & Somo and Hong 2006 & Beams with stirrups, $a_{v} / d \geq 2$ & 1.47 & 0.36 \\
\hline \multicolumn{3}{|c|}{ Load effects } & $\delta$ & $V$ \\
\hline$w_{D} T_{D}$ & Ellingwood et al. 1980 & All construction materials & 1.05 & 0.10 \\
\hline$w_{L} T_{L}$ & Israel et al. 1987 & $\begin{array}{l}\text { A58.1-1982 live load } \\
\text { reductions are used }\end{array}$ & 1.00 & 0.25 \\
\hline
\end{tabular}

Table 3.12: Statistical parameters for professional factor for shear

\begin{tabular}{llll}
\hline Source & Comment & $\delta$ & $V$ \\
\hline Nowak and Szerszen 2003 & Beams, shear & 1.075 & 0.10 \\
Somo and Hong 2006 & Beams with stirrups, $a_{v} / d \geq 2$ & 1.47 & 0.36 \\
Collins 2001 & 413 beams dataset & 1.19 & 0.339 \\
\hline
\end{tabular}


Table 3.13: Means and standard deviations of reliability index ratios for shear, $\beta_{v_{u}} / \beta_{V_{r}}$, for $f_{c}^{\prime}=25 \mathrm{MPa}$ and $\rho_{t}=0.001-0.007$

\begin{tabular}{cccccccccc}
\hline & & \multicolumn{10}{c}{$\phi_{c}$} \\
\cline { 3 - 10 }$w_{L} / w_{D}$ & $\phi_{s}$ & Mean & $\sigma$ & Mean & $\sigma$ & Mean & $\sigma$ & Mean & $\sigma$ \\
\hline & 0.60 & 0.967 & 0.039 & 0.991 & 0.030 & 1.015 & 0.021 & 1.041 & 0.010 \\
& 0.80 & 0.05 & 0.70 & \\
& 0.85 & 1.007 & 0.051 & 1.031 & 0.042 & 1.057 & 0.032 & 1.083 & 0.022 \\
& 0.90 & 1.048 & 0.063 & 1.074 & 0.054 & 1.100 & 0.044 & 1.128 & 0.034 \\
& 0.95 & 1.091 & 0.076 & 1.118 & 0.067 & 1.146 & 0.057 & 1.174 & 0.047 \\
& & & & & & & & & \\
1.5 & 0.80 & 0.969 & 0.037 & 0.991 & 0.028 & 1.014 & 0.019 & 1.037 & 0.010 \\
& 0.85 & 1.006 & 0.047 & 1.029 & 0.038 & 1.052 & 0.029 & 1.076 & 0.020 \\
& 0.90 & 1.044 & 0.058 & 1.067 & 0.049 & 1.091 & 0.040 & 1.116 & 0.030 \\
& 0.95 & 1.083 & 0.069 & 1.107 & 0.060 & 1.132 & 0.051 & 1.158 & 0.042 \\
\hline
\end{tabular}




$$
\begin{aligned}
& \rightarrow x-(0.80,0.60) \quad \longrightarrow(0.80,0.65) \quad \longrightarrow x-(0.80,0.70) \quad--x-\cdot(0.80,0.75) \\
& \square \cdot-(0.85,0.60) \quad \square(0.85,0.65) \quad \rightarrow-(0.85,0.70) \quad--\square-\cdot(0.85,0.75) \\
& \rightarrow \cdot-(0.90,0.60) \quad \longrightarrow(0.90,0.65) \quad \longrightarrow-(0.90,0.70) \quad--\Delta-\cdot(0.90,0.75) \\
& \longrightarrow \cdot(0.95,0.60) \quad 0(0.95,0.65) \quad \longrightarrow(0.95,0.70) \quad-\Theta-\cdot(0.95,0.75)
\end{aligned}
$$

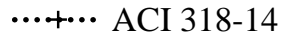

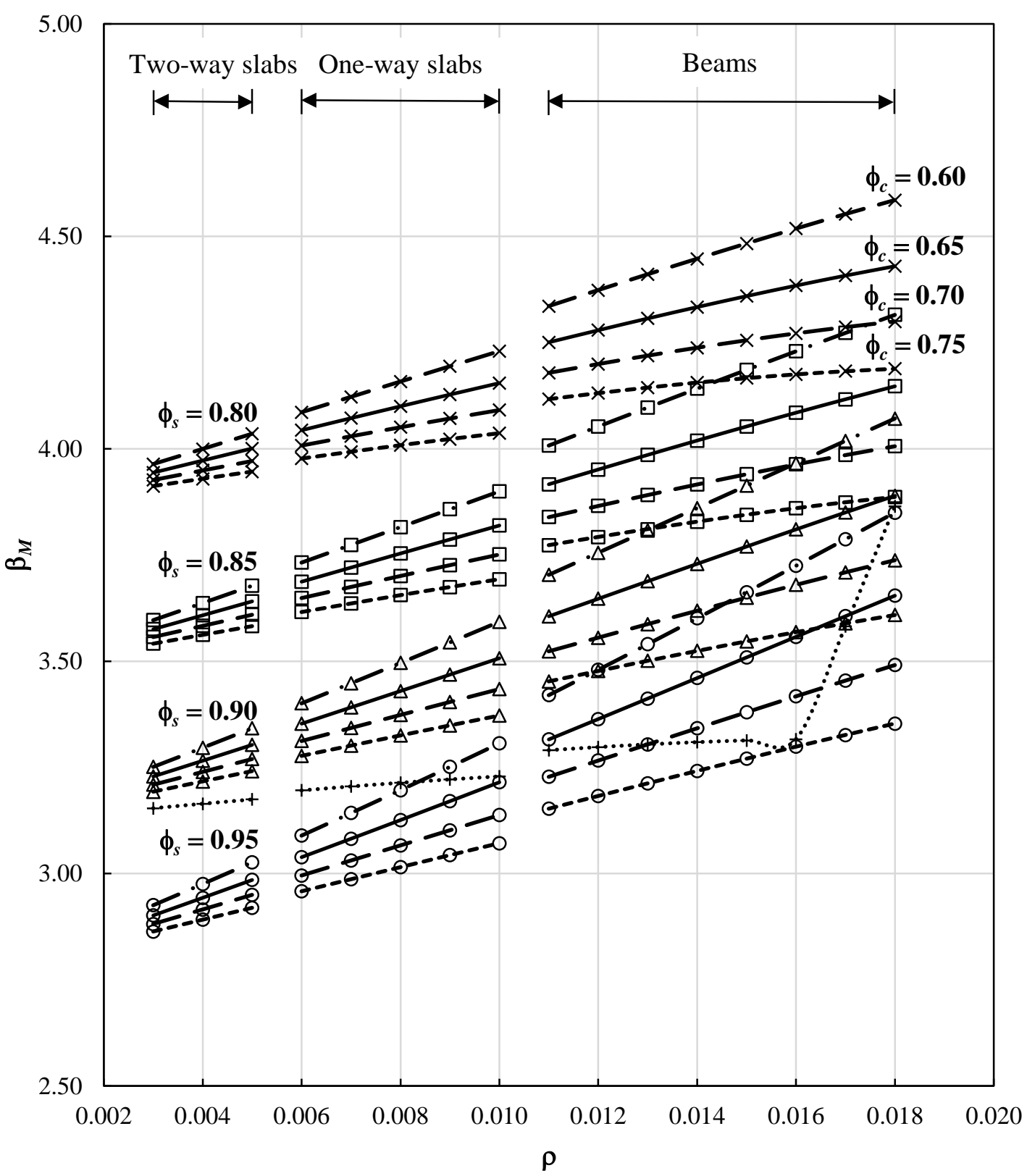

Figure 3.1: Reliability indices for moment, $\beta_{M}$, for $f_{c}^{\prime}=25 \mathrm{MPa}, w_{L} / w_{D}=0.5, \rho=0.003-$ 0.018 , and constant coefficients of variation for $d$ 


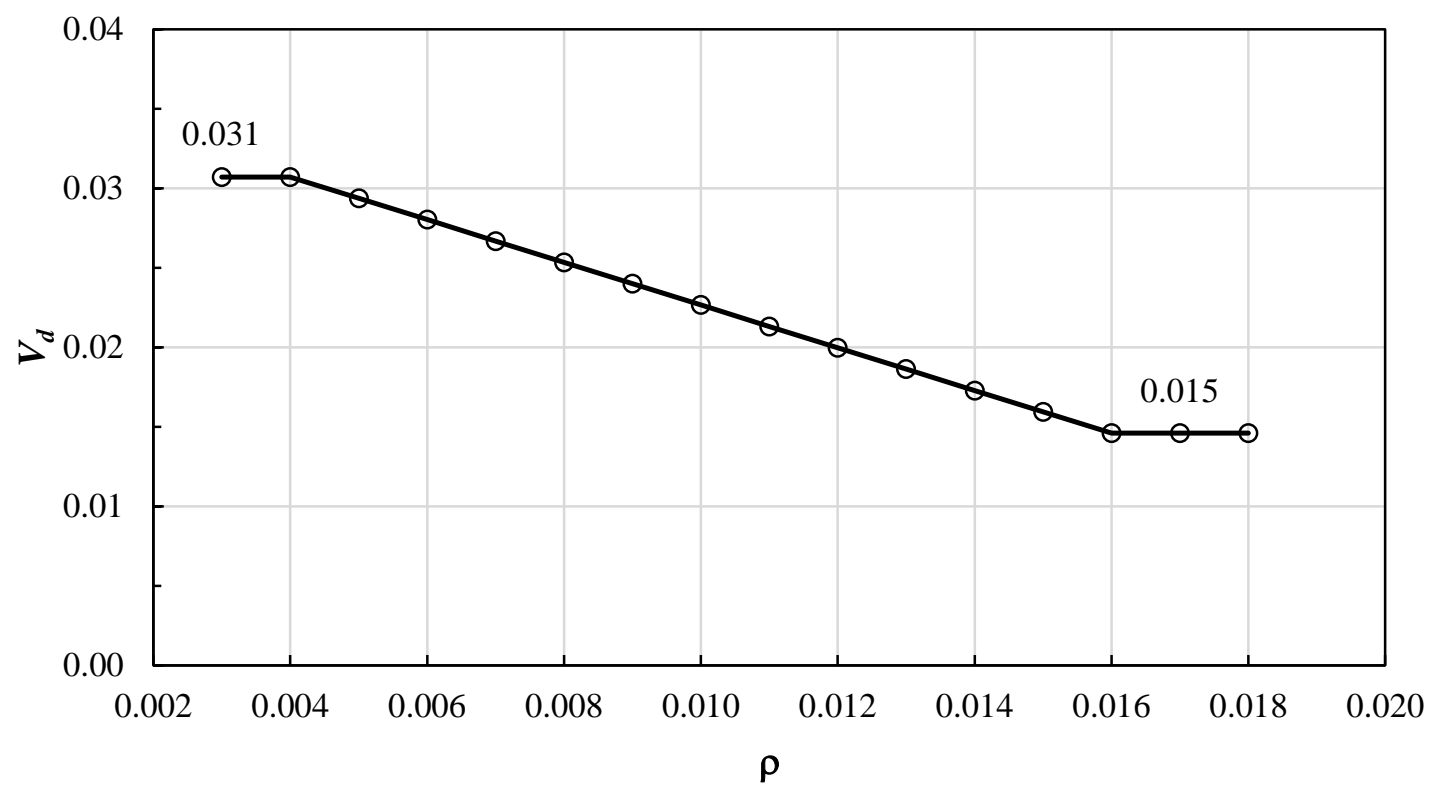

Figure 3.2: Linear variation of coefficients of variation for $d$ 


$$
\begin{aligned}
& \rightarrow \times \cdot-(0.80,0.60) \quad \longrightarrow(0.80,0.65) \quad \longrightarrow \times-(0.80,0.70) \quad--x-\cdot(0.80,0.75) \\
& \square \cdot-(0.85,0.60) \quad \square(0.85,0.65) \quad \square-(0.85,0.70) \quad-\square-\cdot(0.85,0.75) \\
& \rightarrow \cdot-(0.90,0.60) \quad \longrightarrow(0.90,0.65) \quad \longrightarrow-(0.90,0.70) \quad--\Delta-\cdot(0.90,0.75) \\
& \longrightarrow(0.95,0.60) \quad-(0.95,0.65) \quad \longrightarrow(0.95,0.70) \quad--\Theta-\cdot(0.95,0.75)
\end{aligned}
$$

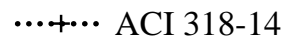

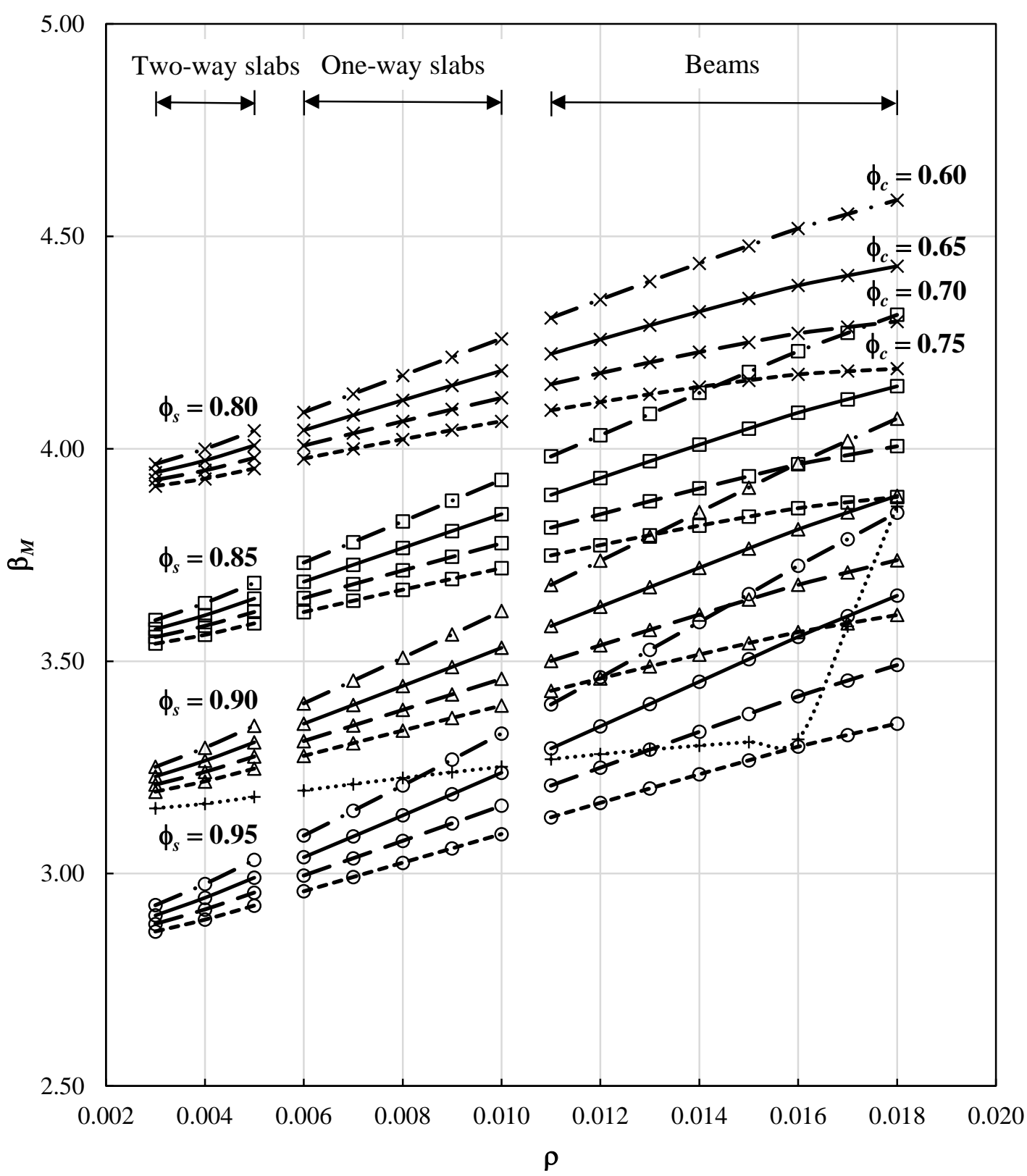

Figure 3.3: Reliability indices for moment, $\beta_{M}$, for $f_{c}^{\prime}=25 \mathrm{MPa}, w_{L} / w_{D}=0.5, \rho=0.003-$ 0.018 , and linear coefficients of variation for $d$ 


$$
\begin{aligned}
& \longrightarrow \times \cdot-(0.80,0.60) \quad \longrightarrow(0.80,0.65) \quad \longrightarrow \times-(0.80,0.70) \quad--x-\cdot(0.80,0.75) \\
& \square \cdot-(0.85,0.60) \quad \square(0.85,0.65) \quad \rightarrow-(0.85,0.70) \quad--\square-\cdot(0.85,0.75) \\
& \rightarrow \cdot-(0.90,0.60) \quad \longrightarrow(0.90,0.65) \quad \longrightarrow-(0.90,0.70) \quad--\Delta-\cdot(0.90,0.75) \\
& \longrightarrow(0.95,0.60) \quad-(0.95,0.65) \quad \longrightarrow(0.95,0.70) \quad-\Theta-\cdot(0.95,0.75)
\end{aligned}
$$

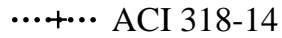

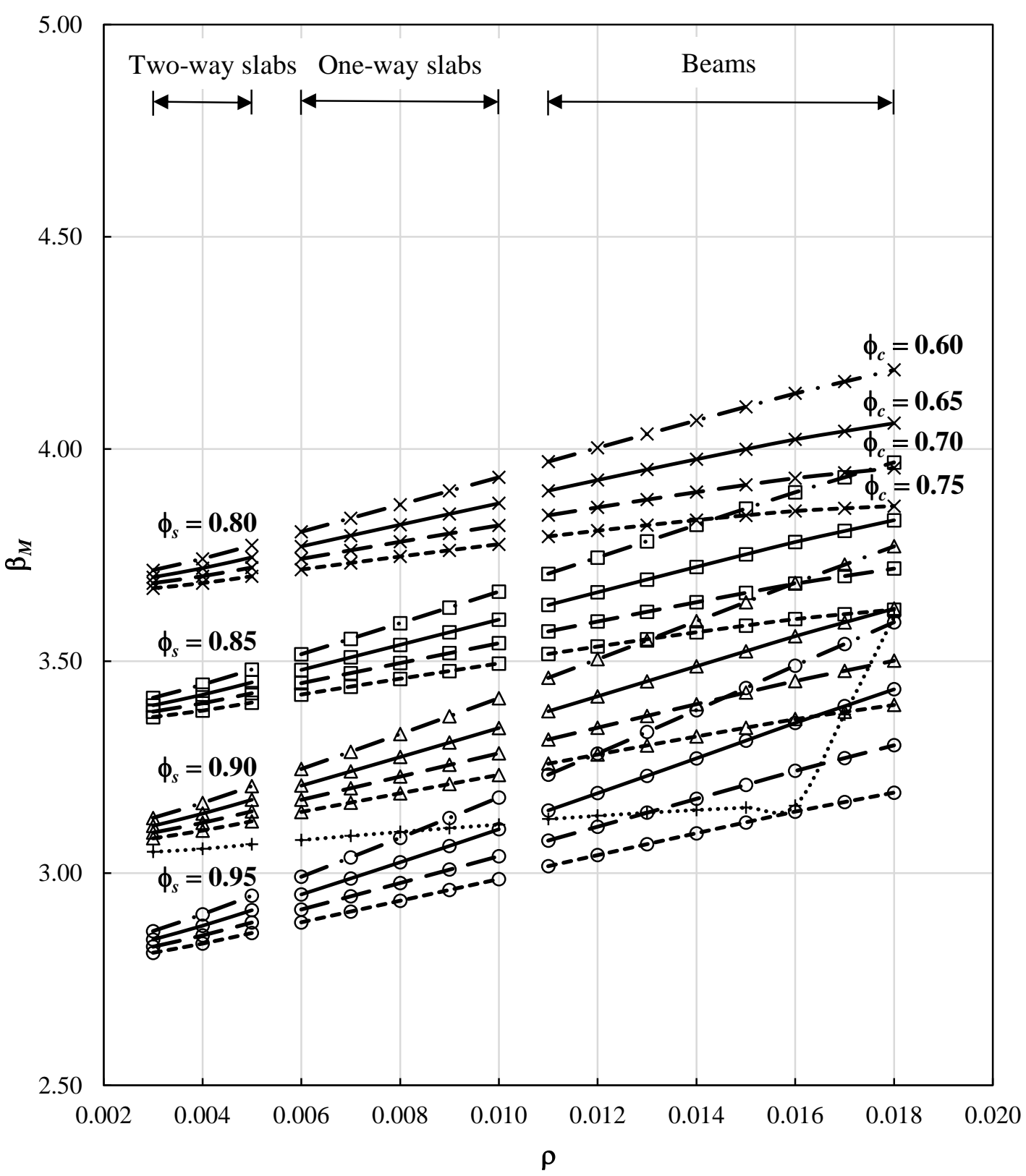

Figure 3.4: Reliability indices for moment, $\beta_{M}$, for $f_{c}^{\prime}=25 \mathrm{MPa}, w_{L} / w_{D}=1.5, \rho=0.003-$ 0.018 , and linear coefficients of variation for $d$ 


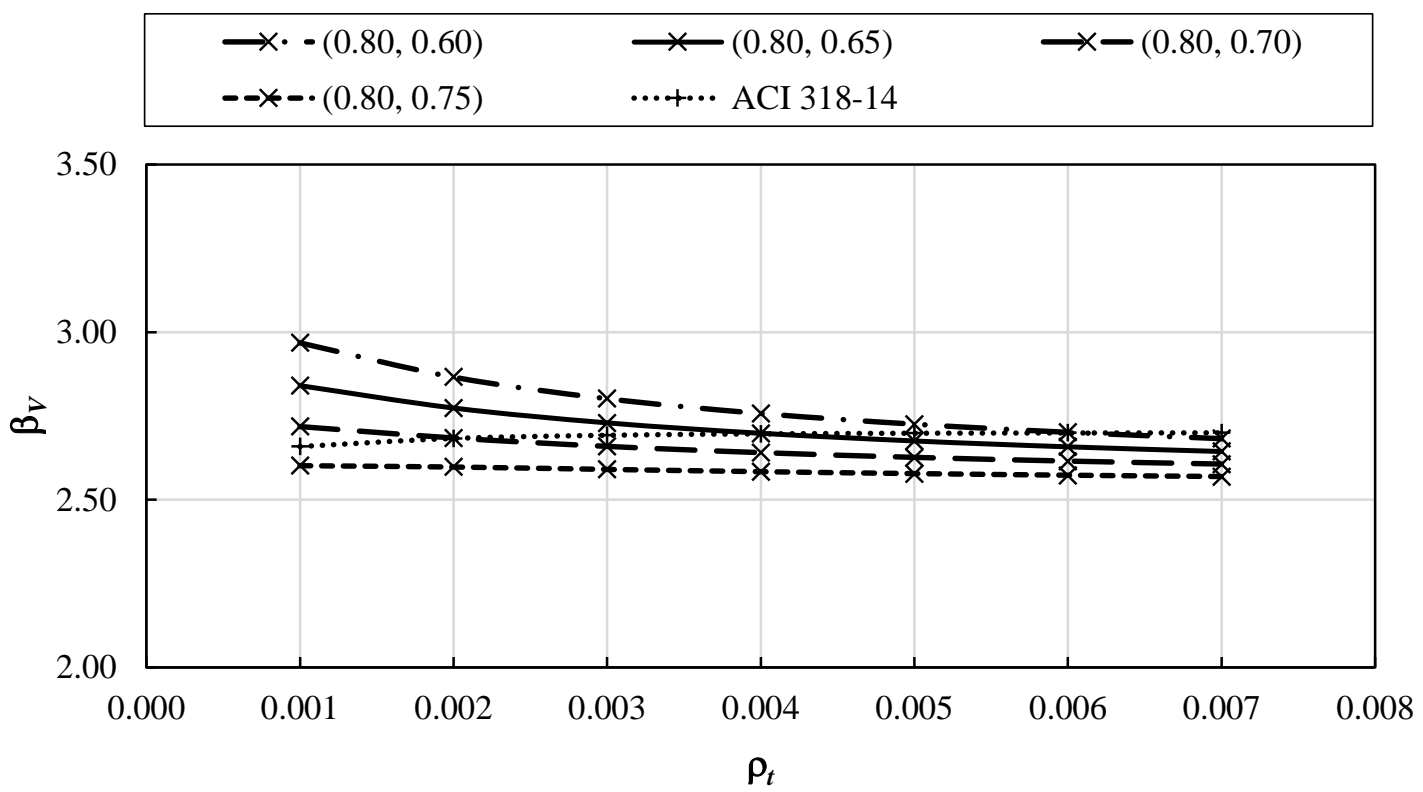

(a)

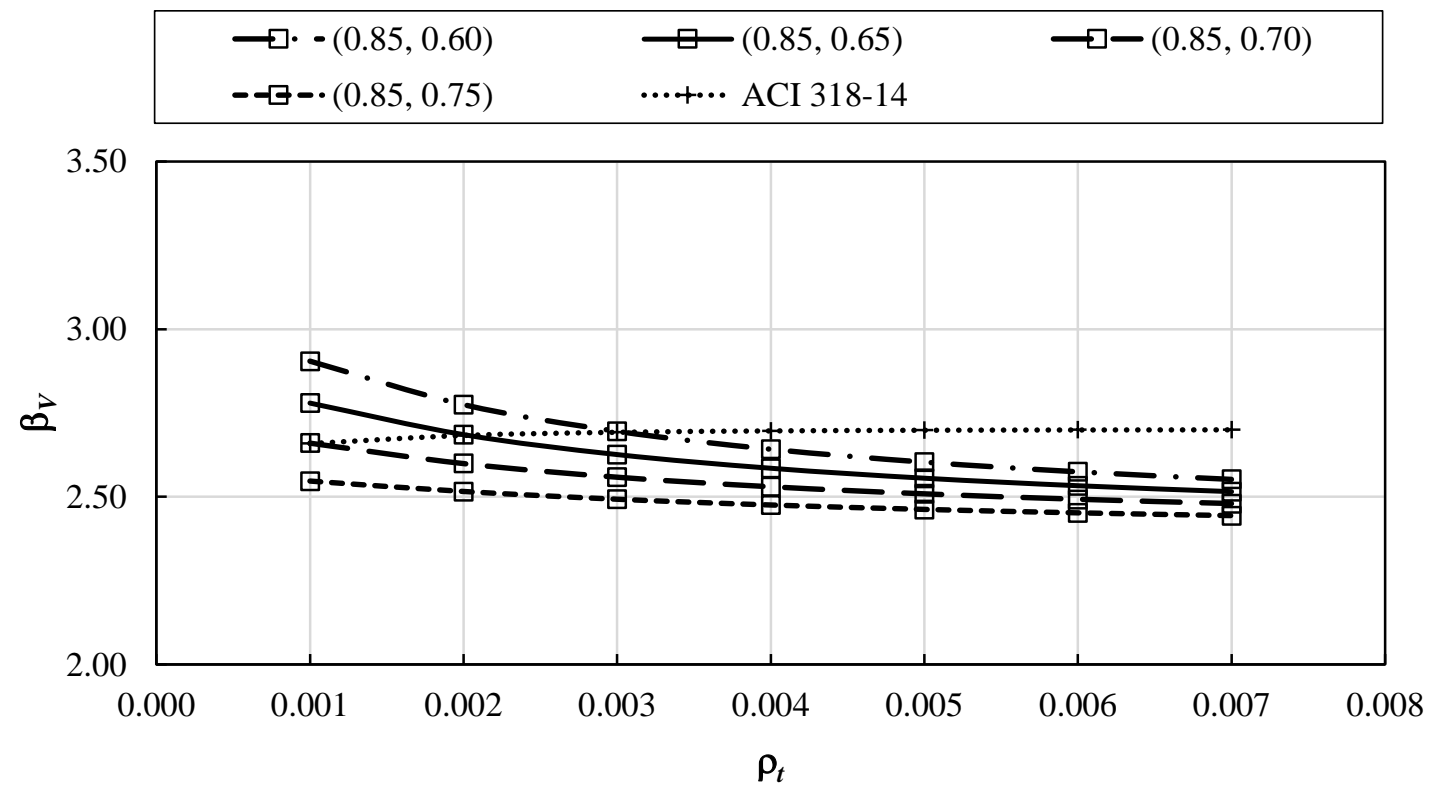

(b) 


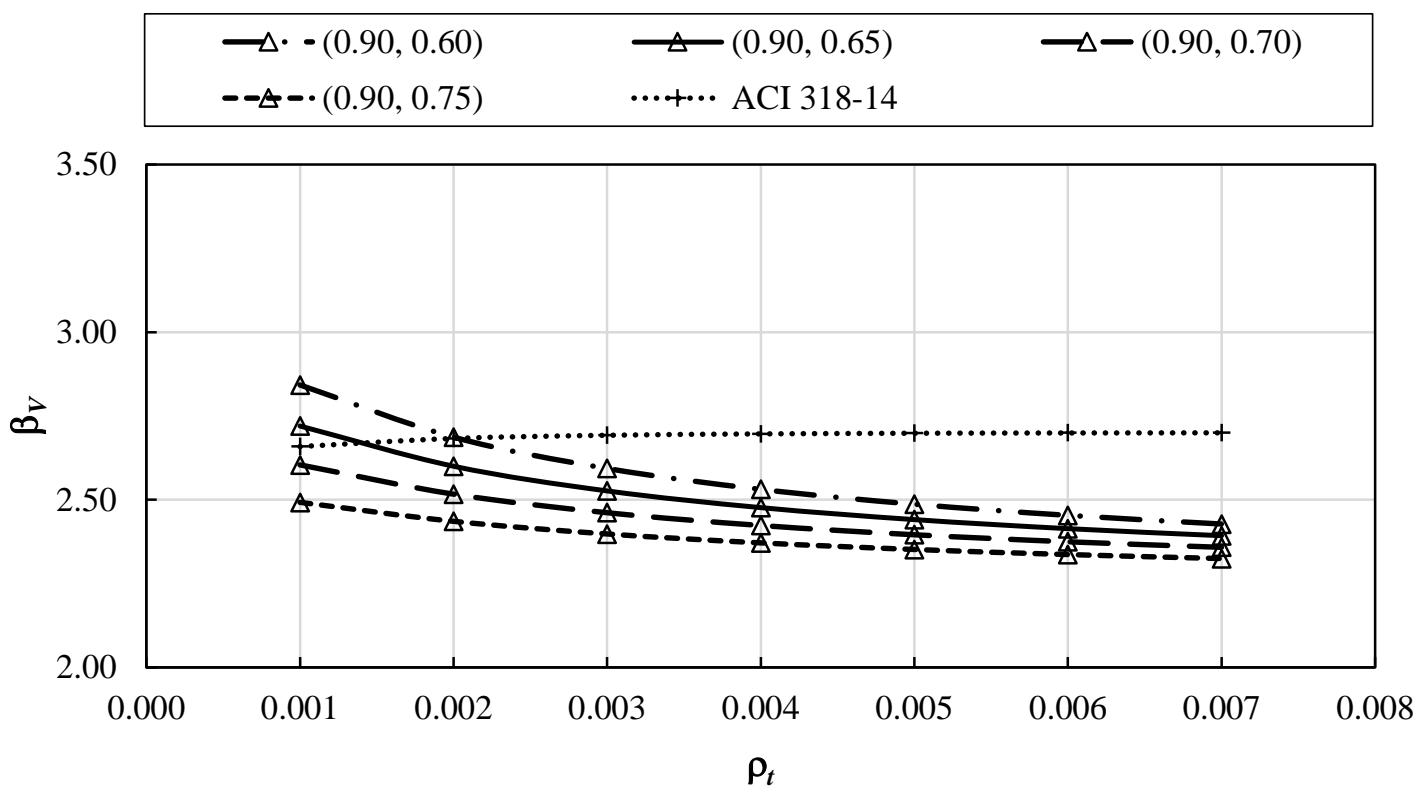

(c)

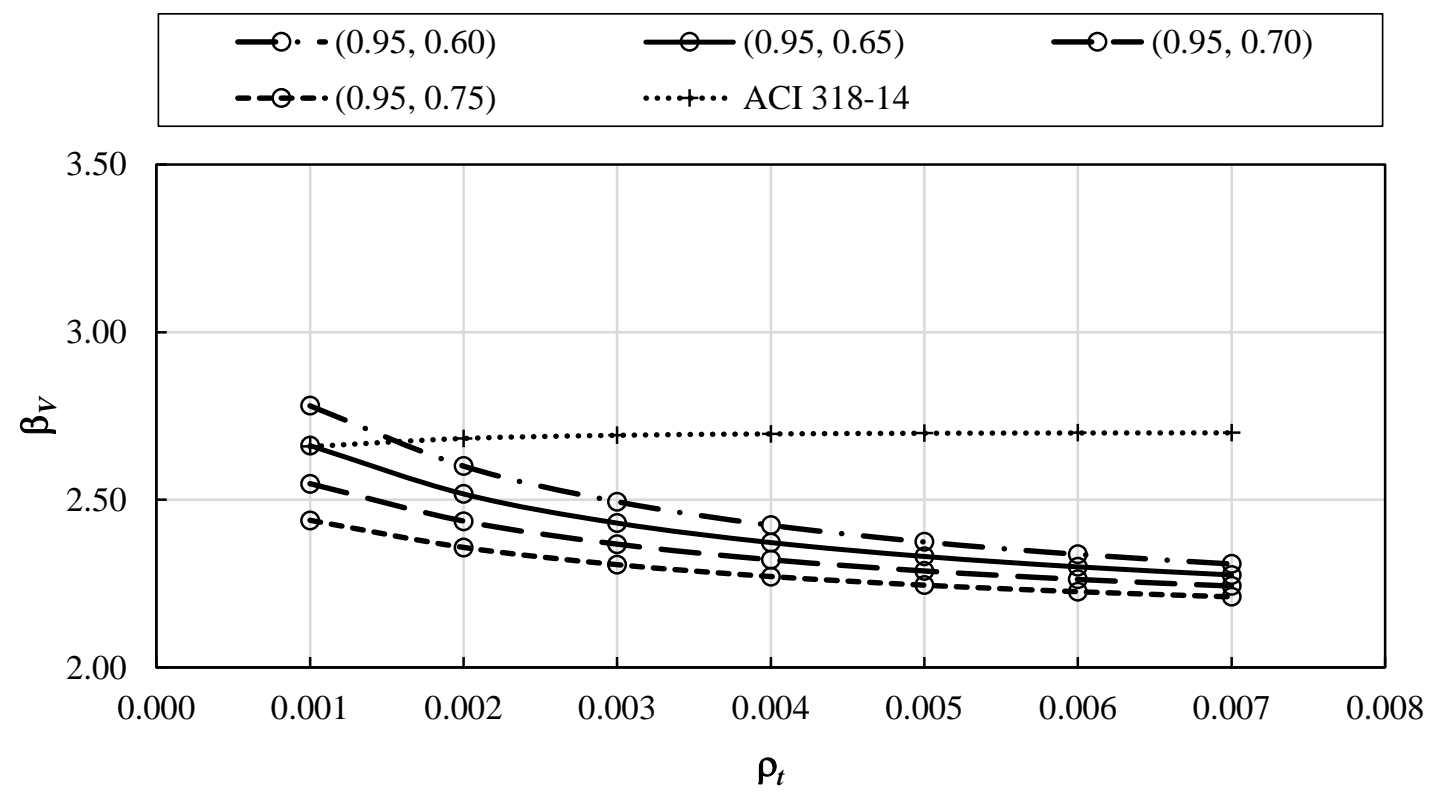

(d)

Figure 3.5: Reliability indices for shear, $\beta_{V}$, for $f_{c}^{\prime}=25 \mathrm{MPa}, w_{L} / w_{D}=0.5$, and $\rho_{t}=$ 0.001-0.007: (a) $\phi_{s}=0.80 ;$ (b) $\phi_{s}=0.85 ;$ (c) $\phi_{s}=0.90 ;$ (d) $\phi_{s}=0.95$ 


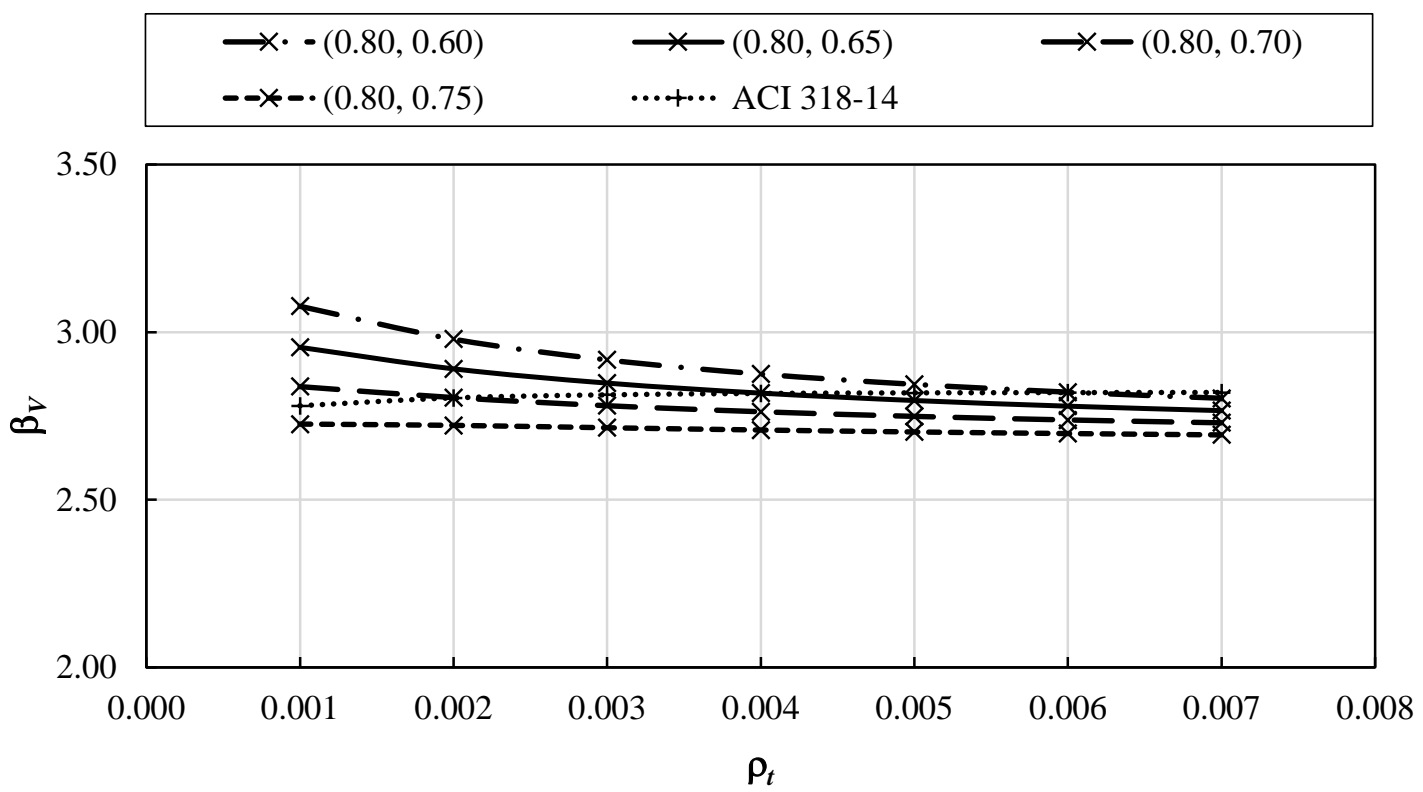

(a)

$\begin{array}{ll}\square \cdot-(0.85,0.60) & \square-(0.85,0.65) \\ -\square-\cdot(0.85,0.75) & \cdots+\cdots \text { ACI 318-14 }\end{array}$

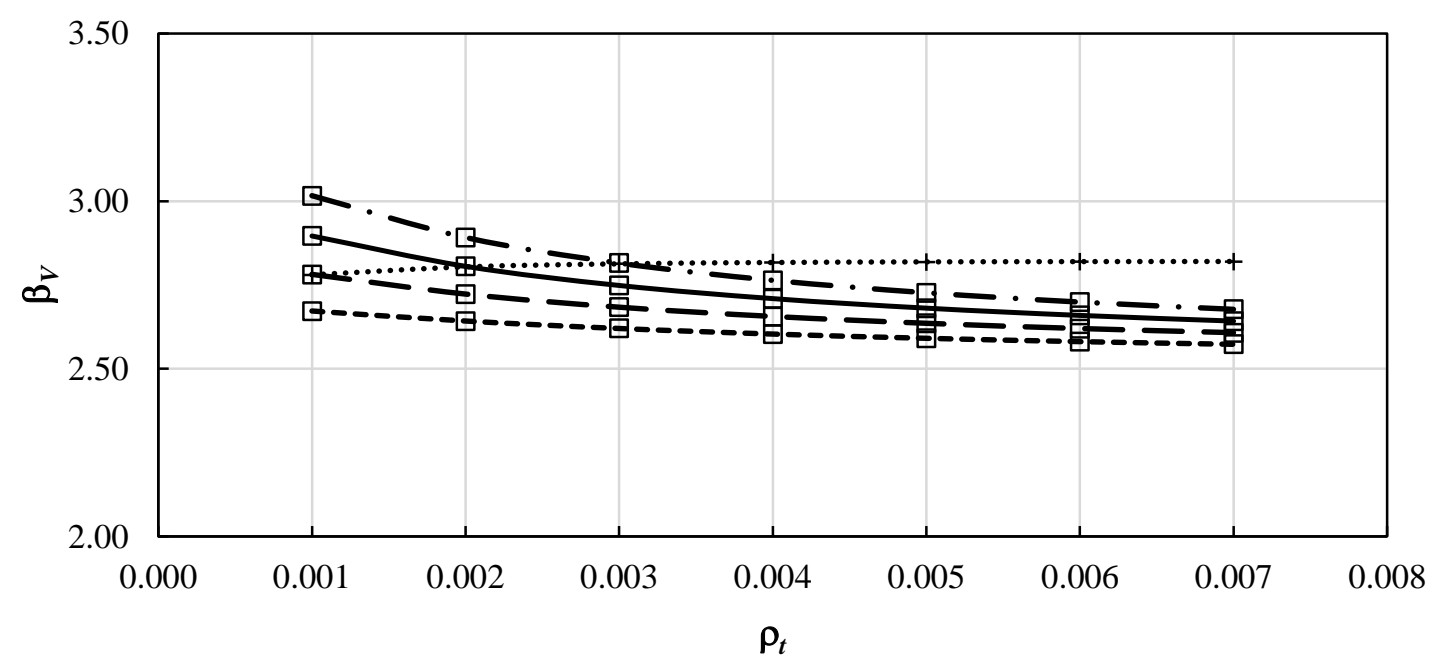

(b) 


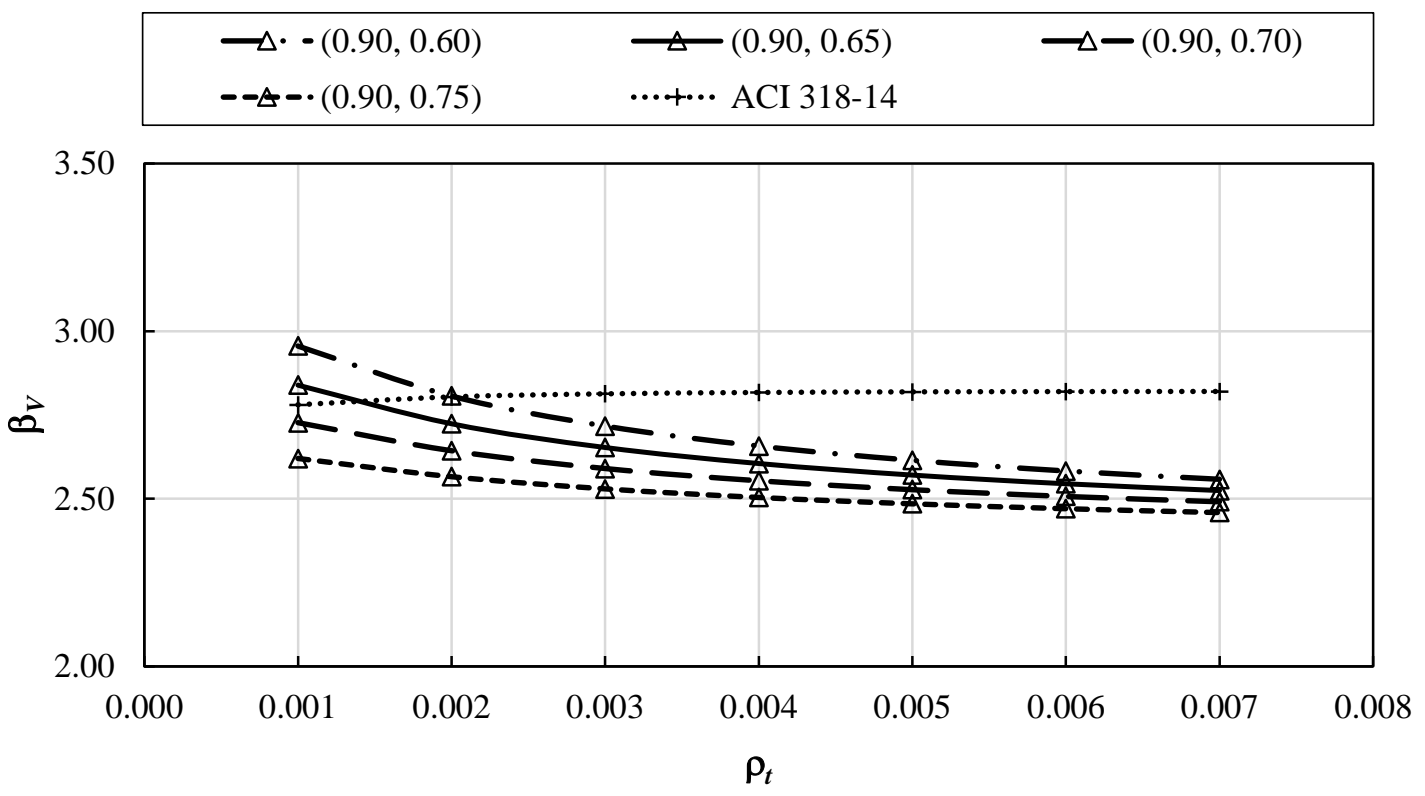

(c)

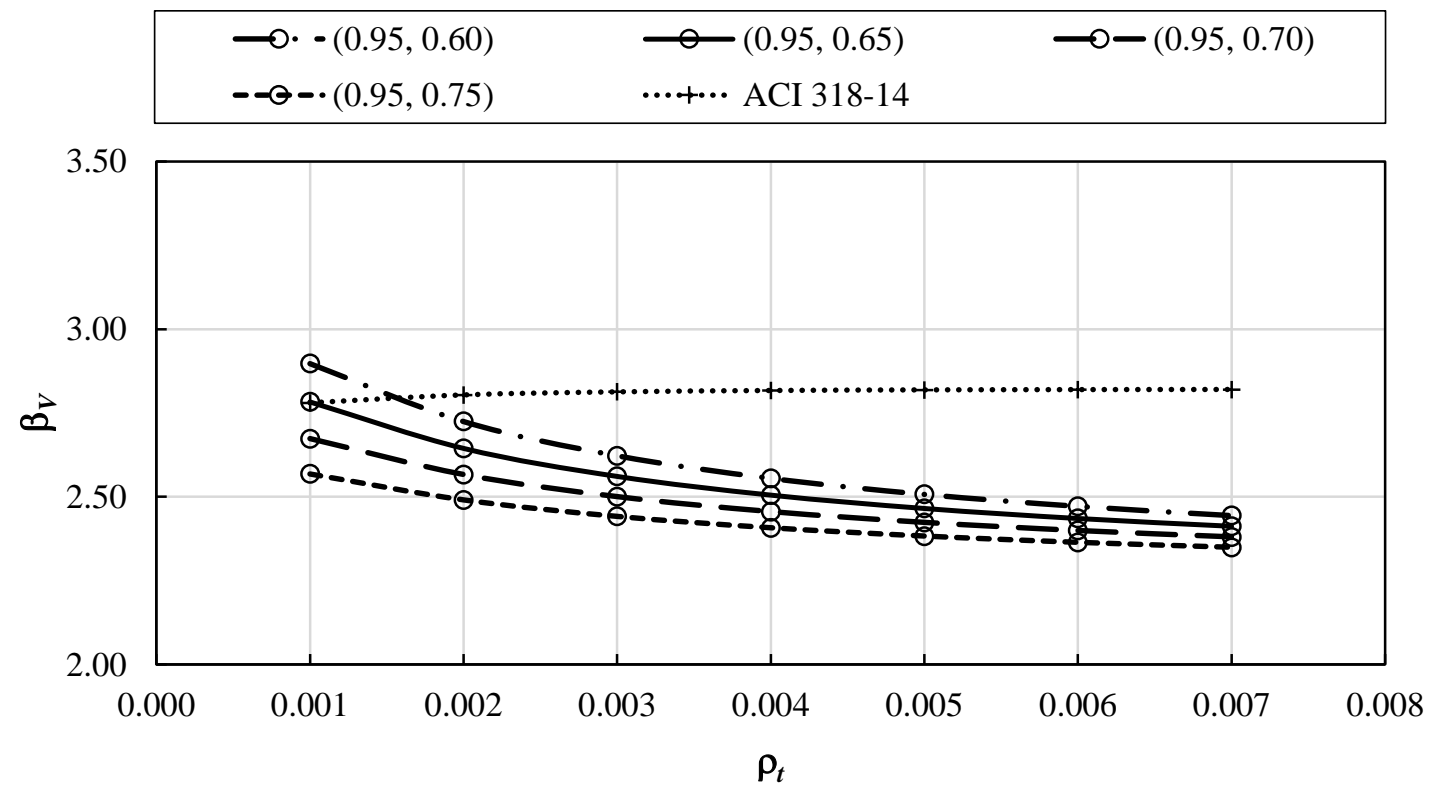

(d)

Figure 3.6: Reliability indices for shear, $\beta_{V}$, for $f_{c}^{\prime}=25 \mathrm{MPa}, w_{L} / w_{D}=1.5$, and $\rho_{t}=$ 0.001-0.007: (a) $\phi_{s}=0.80$; (b) $\phi_{s}=0.85$; (c) $\phi_{s}=0.90$; (d) $\phi_{s}=0.95$ 


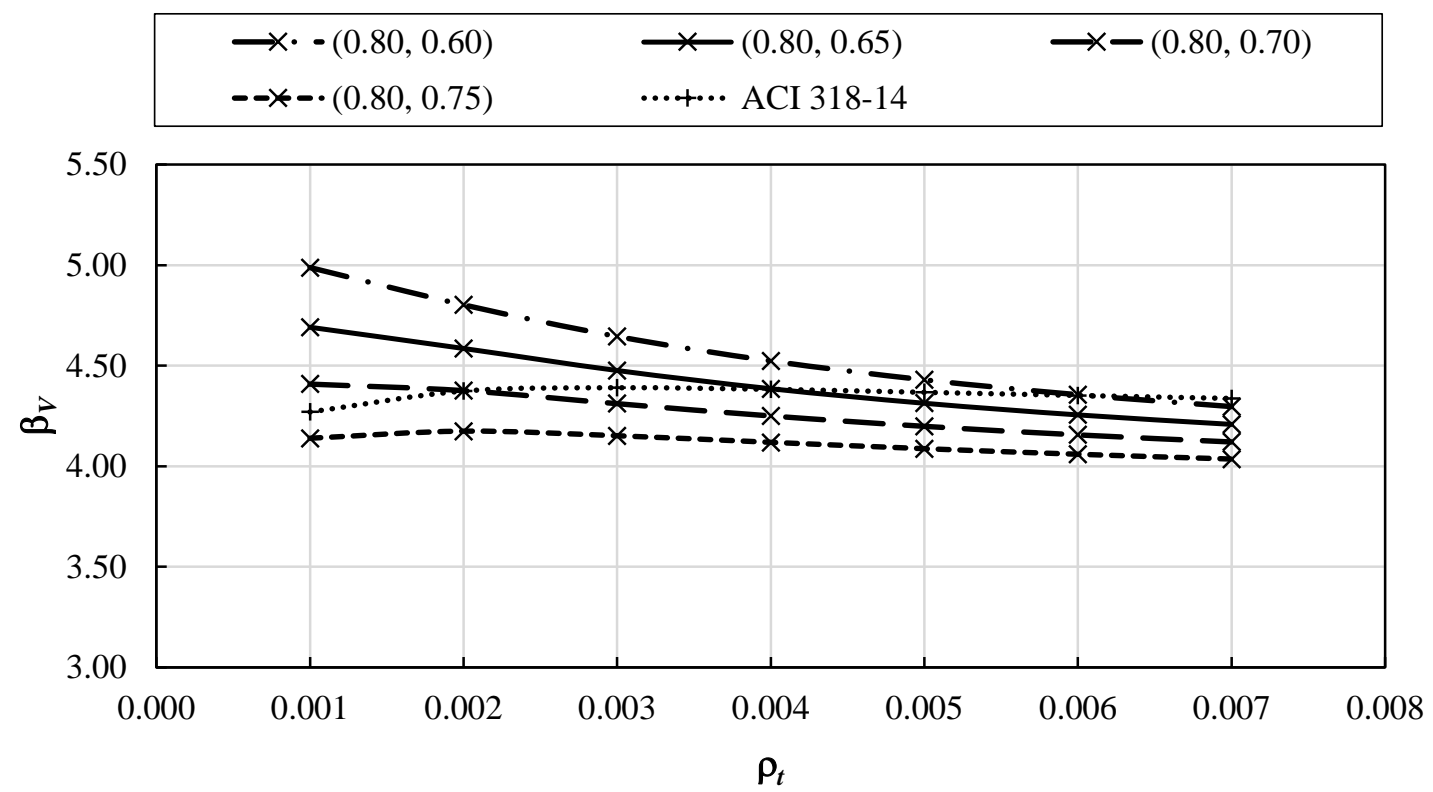

(a)

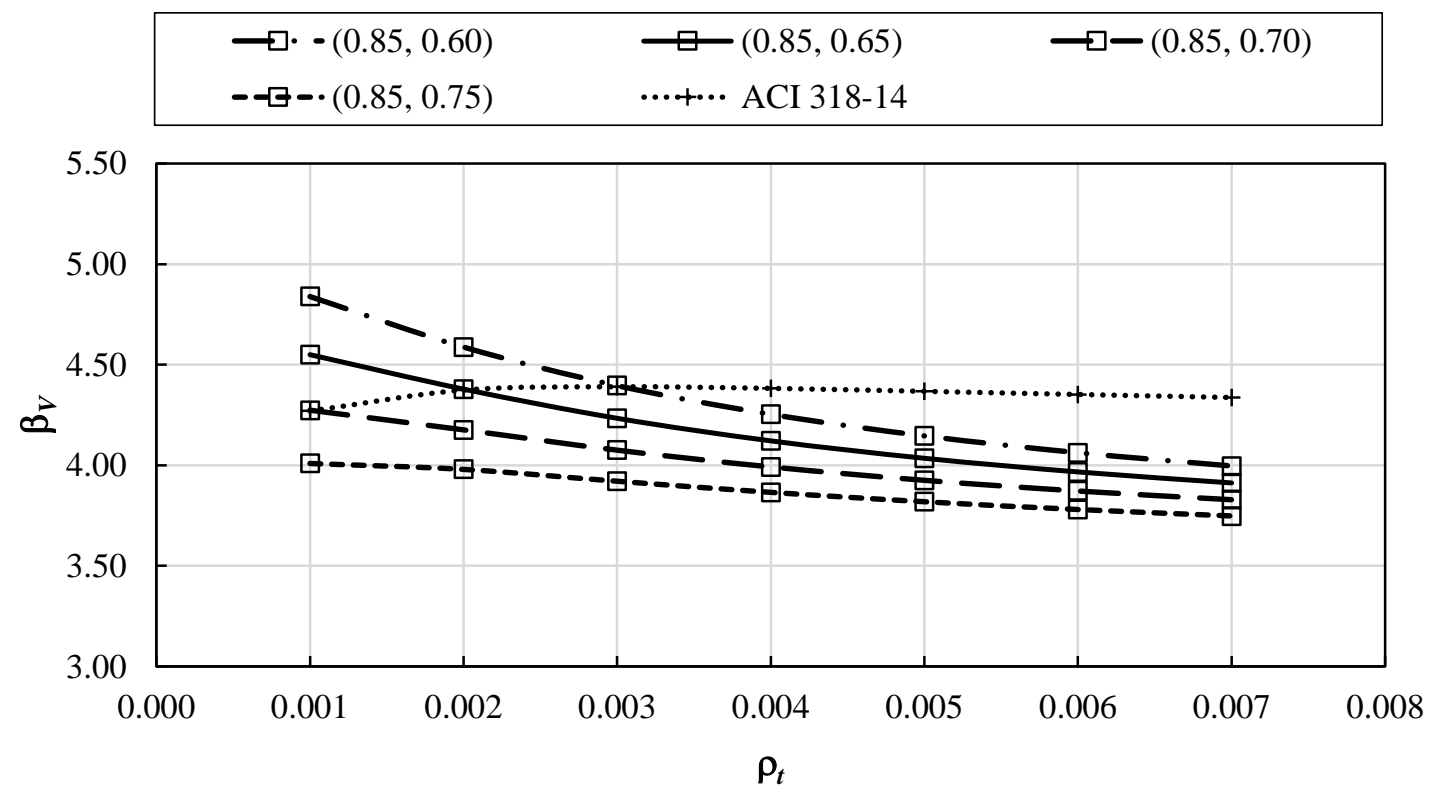

(b) 


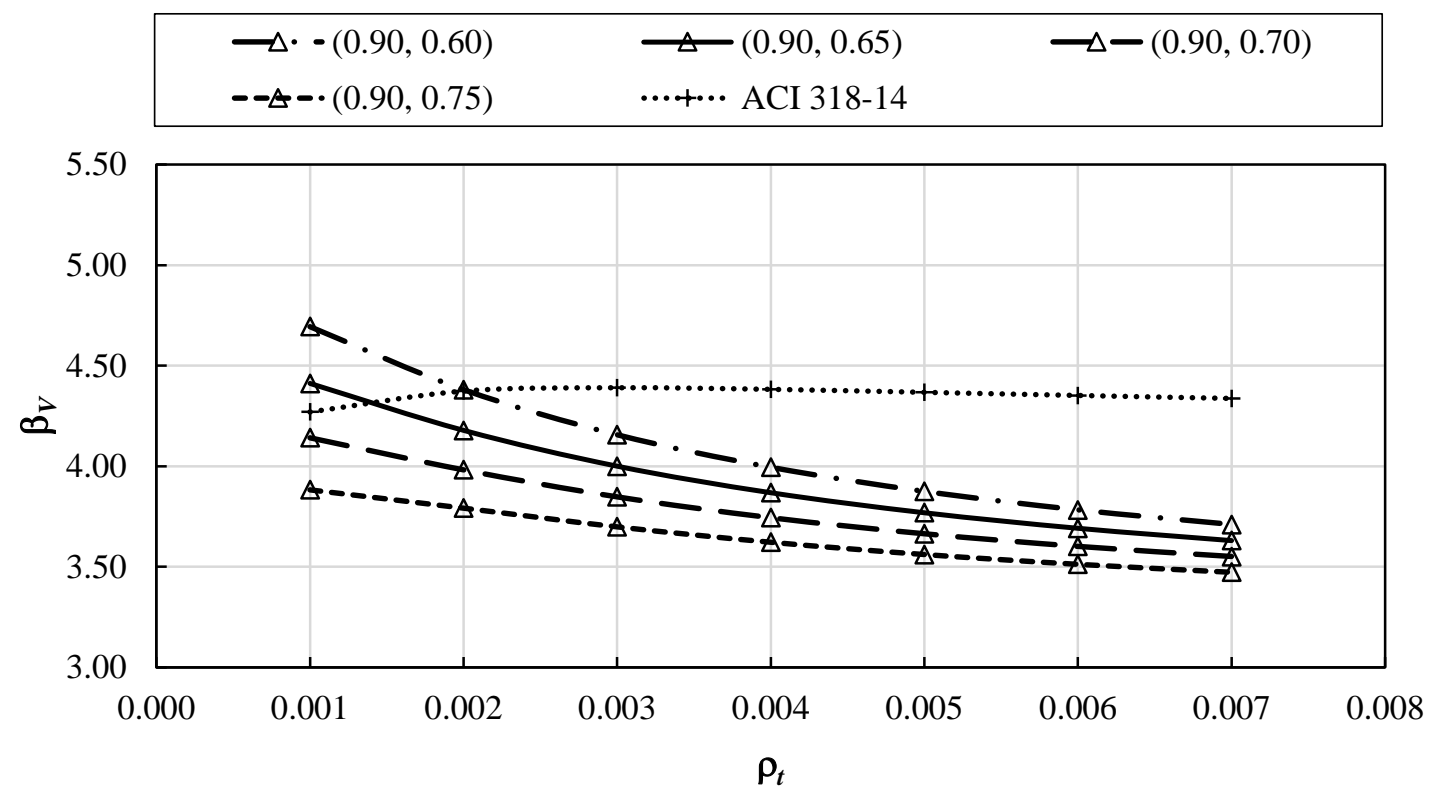

(c)

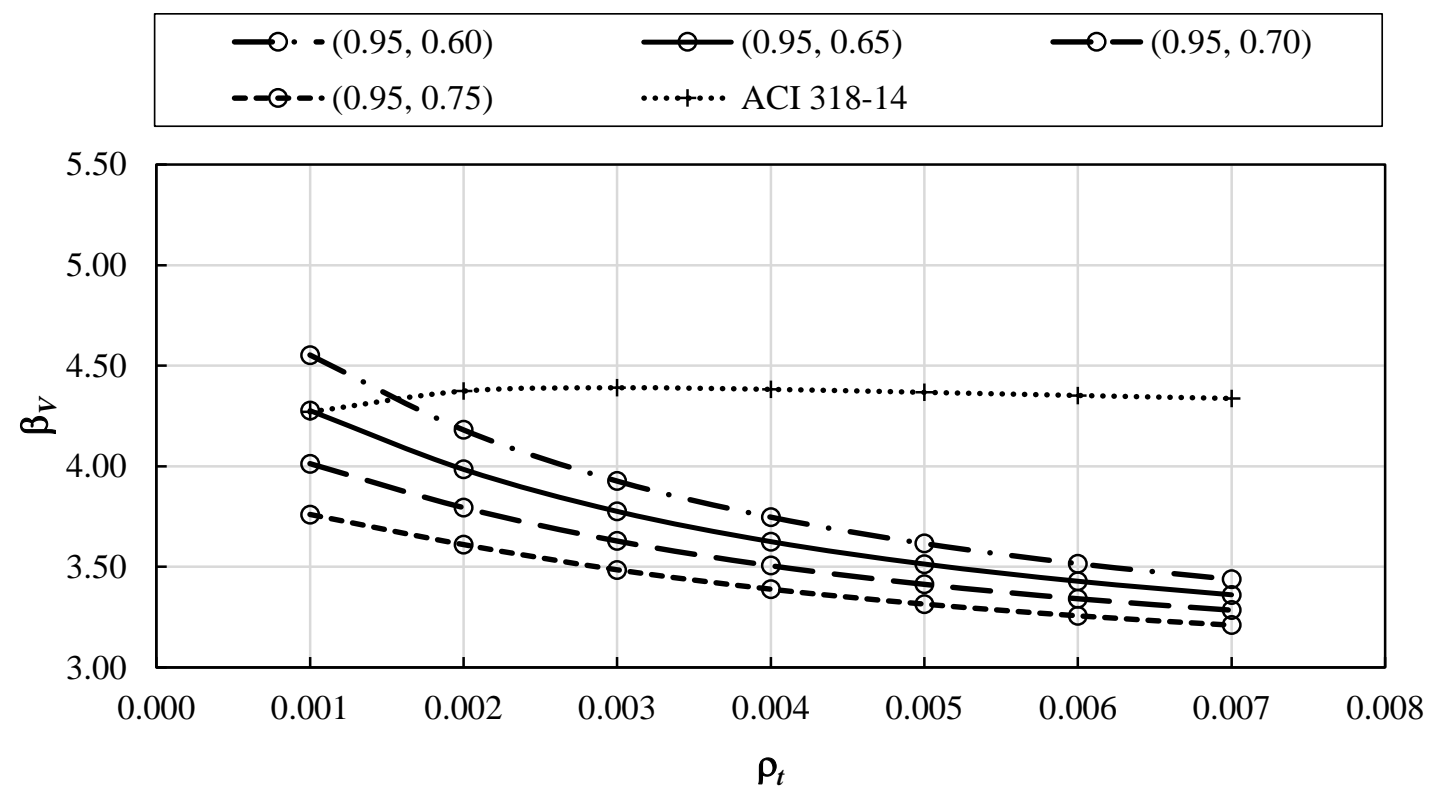

(d)

Figure 3.7: Reliability indices for shear, $\beta_{V}$, for $f_{c}^{\prime}=25 \mathrm{MPa}, w_{L} / w_{D}=0.5, \rho_{t}=0.001-$ 0.007, bias coefficient for professional factor $=1.075$, and coefficient of variation for professional factor $=0.10$ : (a) $\phi_{s}=0.80$; (b) $\phi_{s}=0.85$; (c) $\phi_{s}=0.90$; (d) $\phi_{s}=0.95$ 


\section{Chapter 4}

\section{Derivation of Partial Material Strength Reduction Factors for Combined Moment and Axial Force Based on Reliability Indices}

\subsection{Introduction}

In Chapter 3, the reliability indices presented for moment and one-way shear are based on the first-order, second-moment (FOSM) method. The equations to generate interaction diagrams for combined moment and axial force presented in Chapter 2 are more complicated, however, so a different analysis method, Monte Carlo simulation, is necessary. This chapter presents the reliability analyses for combined moment and axial force to obtain reliability indices based on ACI 318-14 (ACI Committee 318 2014) and the proposed partial material strength reduction factors. The eight geometric and material property combinations of the five column cross sections considered are identical to those presented in Chapter 2.

The objective of this chapter is to select appropriate partial material strength reduction factor combinations that approximately duplicate reliability indices obtained using the current provisions, but may be more uniform for a range of $\gamma, f_{c}{ }^{\prime}, \rho_{g}$ and $e / h$ values, which are the ratio of the distance between the outer layers of reinforcement in a column to the overall column depth, the specified compressive strength of concrete, the total reinforcement ratio, and the eccentricity-to-column depth ratio, respectively.

\subsection{Methodology}

The simulation is conducted using Monte Carlo techniques (e.g., Hong 2015) which are powerful reliability analysis tools. The basic procedure is to generate $n$ sets of random variables and then run the analysis $n$ times to simulate the performance (Hong 2015). The transformations from standard uniform random variables or standard normal random variables to normal, lognormal and Gumbel distributed random variables are derived from Hong (2015). 
The simulation is run $10^{6}$ times for each case using Matlab (Version R2016b; The Mathworks, Inc. 2016) to compute the reliability indices. By simulating $10^{6}$ times, the reliability indices are not sensitive to a single simulation, so the results tend to be constant.

The means, standard deviations, minimum and maximum values of the reliability indices for ACI 318-14 and each partial material strength reduction factor combination are quantified. The sensitivities of the reliability indices to the partial material strength reduction factor combinations for various geometric and material properties are investigated, and the best partial material strength reduction factor combinations are proposed.

\subsection{Assumptions and Design Criteria}

Nominal values of geometric and material properties investigated in this chapter are those presented previously in Table 2.10. Again, $\gamma$ of 0.6 and $0.9, \rho_{g}$ of 0.01 and $0.04, f_{c}^{\prime}$ of 25 and $45 \mathrm{MPa}$, and the specified yield strength of reinforcement, $f_{y}$, of $420 \mathrm{MPa}$ are considered. The live-to-dead load ratios, $L / D$, are assumed to be identical to those adopted previously for moment and shear, of 0.5 and 1.5. The specified dead and live loads are determined to exactly achieve the design strengths. Live load reduction factors due to tributary area are neglected. If they are considered, the range of typical $L / D$ ratios reduces slightly, but the reliability indices computed for a specific $L / D$ value are correct. The applied axial load and moment are assumed perfectly correlated.

\subsection{Reliability Analyses}

The limit state function is $Z=g(X)=R / Q$, where $R$ is the resistance and $Q$ is the load effect. For short columns, the limit state function at a given eccentricity, $e_{i}=M_{i} / P_{i}$, is (Israel et al. 1987)

$$
g(X)=\sqrt{P_{i}^{2}+\left(\frac{P_{i} e_{i}}{h}\right)^{2}} / \sqrt{\left(D_{i} T_{D i}+L_{i} T_{L i}\right)^{2}+\left(\frac{\left(D_{i} T_{D i}+L_{i} T_{L i}\right) e_{i}}{h}\right)^{2}}
$$


where $e_{i}, P_{i}, M_{i}, D_{i}, L_{i}, T_{D i}$, and $T_{L i}$, are the simulated values of the eccentricity, axial strength, flexural strength, dead load, live load, transformation from the dead load to the dead load effect, and transformation from the live load to the live load effect, respectively. The professional factor is included in $P_{i}$ and $M_{i}$. In Equation [4.1], $h$ is the nominal column depth (Hong and Zhou 1999). The eccentricity of the applied load effect, $e_{i}$, is equal to the nominal value, $e$, because the axial load and moment are assumed to be perfectly correlated, with identical bias coefficients and coefficients of variation. The $e / h$ values investigated are identical to those in Chapter 2, that is, 0 (compression only), 0.1 , $0.2,0.3,0.4,0.5,0.6,0.7,0.8,0.9,1.0,2.0,3.0,4.0,5.0,6.0,7.0,8.0,9.0,10.0,-10.0$, $-5.0,-1.0,-0.5,-0.1$ and 0 (tension only).

The statistical parameters used in the current reliability analysis were obtained from the literature and are shown in Table 4.1. The statistical parameters for column width, $b$, depth, $h$, and effective depth, $d$, depend on measurement errors in the construction process and the parameters for $d$ are controlled by specified tolerances in ACI 318-14. The standard deviation of $d$ is assumed to be $1 / 2$ of the tolerance specified in Table 26.6.2.1a in ACI 318-14: this is consistent with the common approximation that the total tolerance range equals four standard deviations. The modulus of elasticity of reinforcement, $E_{S}$, is assumed deterministic. The statistical parameters for area loads, point loads, and moments are assumed identical. The transformation from the dead load to the dead load effect, $T_{D}$, and the transformation from the live load to the live load effect, $T_{L}$, are already included in the selected parameters for $D$ and $L$ reported by Ellingwood et al. (1980) and Israel et al. (1987), so bias coefficients of 1.0 and coefficients of variation of 0 are assumed.

The reliability analysis process is as follows:

1. Calculate the design axial strength, $\phi P_{n}$ or $P_{r}$, and the associated design flexural strength, $\phi M_{n}$ or $M_{r}$, for a range of $Z$ values, using Equations [2.21] to [2.45] to generate interaction diagrams. Here, $Z$ is the ratio of the strain in the extreme tension layer of reinforcement to the yield strain. Interpolate for the specific $e / h$ values to 
obtain corresponding design axial strength, $\phi P_{n}$ or $P_{r}$. (This step was done in Chapter 2.)

2. Calculate nominal loads, $D$ and $L$, by equating the design axial strength, $\phi P_{n}$ or $P_{r}$, to the factored axial force from ACI 318-14, $P_{u}=1.2 D+1.6 L$, for the given load ratio, $L / D$. The associated equations are:

$$
D=\frac{\phi P_{n}\left\{\text { or } P_{r}\right\}}{[1.2+1.6(L / D)]}
$$

and

$$
L=\frac{(L / D) \phi P_{n}\left\{\text { or } P_{r}\right\}}{[1.2+1.6(L / D)]}
$$

3. Calculate the resistance: generate $10^{6}$ sets of random variables using the statistical parameters shown in Table 4.1, and run the simulation to derive $10^{6}$ distinct interaction diagrams by using Equations [2.21] to [2.45] with strength reduction factors equal to 1 and accounting for the professional factor.

4. Calculate the load effects: generate $10^{6}$ sets of random variables for load effects at each specified eccentricity using the statistical parameters shown in Table 4.1.

5. Interpolate on each of the $10^{6}$ interaction diagrams to determine the value of the limit state function, Equation [4.1], at each specified eccentricity.

6. Calculate the number of failures and compute the associated reliability index for combined moment and axial force, $\beta_{P M u}$ or $\beta_{P M r}$, using $\beta=-\Phi^{-1}\left(P_{f}\right)$ (Hong 2015). Here, $\beta_{P M u}$ is the reliability index for combined moment and axial force obtained using the strength reduction factors in ACI 318-14, $\beta_{P M r}$ is the reliability index for combined moment and axial force obtained using the partial material strength reduction factors, $\Phi(\bullet)$ is the cumulative distribution function of the standard normal distribution, and $P_{f}$ is the probability of failure. 
7. Summarize the results.

The Matlab (Version R2016b; The Mathworks, Inc. 2016) codes used to conduct the process for Column Section 1 (square section with three bars in each face) and Column Section 5 (spirally reinforced circular section with eight bars evenly distributed around the perimeter), two representative cross sections, are presented in Appendix C.

To save time and avoid unnecessary calculations, only four pairs of partial material strength reduction factors are analyzed for tied columns, combinations with $\phi_{s}$ of 0.85 and 0.90 , and $\phi_{c}$ of 0.60 and 0.65 .

For Column Section 1 (square section with three bars in each face), the reliability indices for combined moment and axial force corresponding to ACI 318-14, $\beta_{P M u}$, with respect to $e / h$ are shown in Figures $4.1 \mathrm{a}$ and $4.1 \mathrm{~b}$ for the eight property combinations with $L / D$ of 0.5 for $e / h>0$ and $e / h<0$, respectively. The vertical dotted lines show the range of $e / h$ at the balance point. For $e / h$ approximately ranging from 0.1 to 0.38 , all of the eight property combinations do not reach the minimum value of $e_{u b a l} / h$, where $e$ corresponds to the balanced failure for ACI 318-14, so they are compression-controlled. In this region, similar to the trend of design combined flexural and axial strength ratio, $\alpha_{P M}$, described in Chapter 2, the most influential property is $\rho_{g}$. For $\rho_{g}$ of $4 \%, \beta_{P M u}$ reaches 4.06 , and for $\rho_{g}$ of $1 \%, \beta_{P M u}$ reaches 2.44 , causing inconsistent reliability indices with varying $e / h$. Varying $f_{c}^{\prime}$ causes small changes for lower $\rho_{g}$, but large changes for higher $\rho_{g}$. And again, the least influential parameter is $\gamma$. For $e / h$ approximately ranging from 0.38 to 0.88 , some of the cases shown reach the balance point, but others do not. For e/h approximately ranging from 0.88 to 10 , all of the eight property combinations equal or exceed the maximum value of $e_{u b a l} / h$, so they are in the transition or tension-controlled regions. The two lines corresponding to Property Combination $2\left(\gamma\right.$ of $0.6, f_{c}{ }^{\prime}$ of $25 \mathrm{MPa}$, and $\rho_{g}$ of 0.04$)$ and Property Combination 4 ( $\gamma$ of $0.6, f_{c}^{\prime}$ of $45 \mathrm{MPa}$, and $\rho_{g}$ of 0.04), differ from the others shown because they remain in the transition region until $e / h$ equals 10. For $e / h$ less than 0 shown in Figure $4.1 \mathrm{~b}$, the sections are subjected to tension. In the tension-controlled region, the influences of $\gamma, f_{c}{ }^{\prime}$ and $\rho_{g}$ on $\beta_{P M u}$ become small. Results for $L / D$ of 1.5 are shown in Figure C.1 of Appendix C. The influence of $L / D$ is small. 
For the partial material strength reduction factors $\phi_{s}$ of 0.90 and $\phi_{c}$ of $0.60, \beta_{P M r}$ values for Column Section 1 are shown in Figures $4.2 \mathrm{a}$ and $4.2 \mathrm{~b}$ for the eight property combinations with $L / D$ of 0.5 for $e / h>0$ and $e / h<0$, respectively. These $\beta_{P M r}$ values are relatively uniform, ranging from 2.75 to 3.40 compared to the range of 2.44 to 4.06 for the current ACI 318-14 criteria shown in Figures 4.1a and 4.1b. This indicates an advantage of using partial material strength reduction factors. In the compression-controlled region, the influence of $\gamma$ is very small and influences of $f_{c}{ }^{\prime}$ and $\rho$ are smaller than those shown in Figure 4.1a. In the tension-controlled region, $\gamma$ has a more significant impact on $\beta_{P M r}$ for increased $e / h$ values. Results for $L / D$ of 1.5 are shown in Figure C. 2 of Appendix C. The influence of $L / D$ is small.

According to ASCE 7-10 (ASCE 2010), for a 50-year service period, normal buildings with Risk Category II should exhibit a reliability index range of 3.0 to 4.0 for columns. Figures $4.3 \mathrm{a}$ and $4.3 \mathrm{~b}$ show the reliability indices for combined moment and axial force, $\beta_{P M}$, for Column Section 1, Property Combination 1 ( $\gamma$ of $0.6, f_{c}{ }^{\prime}$ of $25 \mathrm{MPa}$, and $\rho_{g}$ of $0.01)$ and $L / D$ of 0.5 for $e / h>0$ and $e / h<0$, respectively. The dotted vertical line in Figure 4.3a shows $e_{u b a l} / h$ of 0.42 , the $e$ corresponding to the balanced failure for ACI 318-14. The two solid vertical lines represent the range of $e$ values corresponding to the balanced failures for the sixteen partial material strength reduction factor combinations (although there are only four combinations shown in the figure), from $\left(e_{r b a l} / h\right)_{\min }$ of 0.43 to $\left(e_{r b a l} / h\right)_{\max }$ of 0.50 . When $e / h>0$, the reliability index corresponding to ACI 318-14 decreases abruptly for eccentricities greater than that corresponding to the balance point, because the strength reduction factor in ACI 318-14, $\phi$, increases from 0.65 to 0.90 for tied columns. In the compression-controlled region, two families of lines are defined by the two $\phi_{c}$ values, and the differences within each family are due to the two $\phi_{s}$ values. As the eccentricity increases, the influence of steel strength becomes more significant than that of the concrete strength, so the two families are defined by the two $\phi_{s}$ values. When $e / h<0, \beta_{P M}$ increases slightly as the absolute value of $e / h$ increases. In this case, the influence of $\phi_{s}$ on $\beta_{P M r}$ is greater because the tensile strength of concrete is negligible and does not contribute to the strength. 
Results for the other seven property combinations and $L / D$ of 0.5 are shown in Figures C.3-C.9 of Appendix C. In the compression-controlled region where the impact of $\phi_{c}$ is greatest, $\beta_{P M r}$ corresponding to the partial material strength reduction factor combinations with $\phi_{c}$ of 0.60 approaches $\beta_{P M u}$ when $\rho_{g}$ equals 0.01 . When $\beta_{P M u}$ is too conservative, i.e. for $\rho_{g}$ of $0.04, \beta_{P M r}$ values for $\phi_{c}$ of 0.60 still fall in an appropriate range. In the tensioncontrolled region where $\phi_{S}$ is more influential, $\beta_{P M r}$ corresponding to the combinations with $\phi_{s}$ of 0.90 approaches $\beta_{P M u}$.

Values of $\beta_{P M}$ for Column Sections 2, 3 and 4 and $L / D$ of 0.5 are shown in Figures C.10C.33 of Appendix C. The reliability indices, impacts of $\phi_{s}$ and $\phi_{c}$, and the best partial material strength reduction factors are similar to those shown for Column Section 1.

For Column Section 5 (spirally reinforced circular section with eight bars evenly distributed around the perimeter), the $\phi$ values range from 0.75 to 0.90 . The four partial material strength reduction factor combinations analyzed for the tied columns are therefore not appropriate. The analysis results for Column Section 5 presented in Chapter 2 indicate that: when the failure is compression-controlled, the partial material strength reduction factor combinations with $\phi_{c}$ of 0.70 are the best; and, when the failure is tension-controlled, the combinations with $\phi_{s}$ of 0.90 and 0.95 are the best. Therefore, two combinations with $\phi_{s}$ of 0.90 and $\phi_{c}$ of 0.70 , and $\phi_{s}$ of 0.95 and $\phi_{c}$ of 0.70 are investigated.

Figures 4.4a and 4.4b show $\beta_{P M}$ for Column Section 5, Property Combination 1 ( $\gamma$ of 0.6, $f_{c}^{\prime}$ of $25 \mathrm{MPa}$, and $\rho_{g}$ of 0.01$)$ and $L / D$ of 0.5 for $e / h>0$ and $e / h<0$, respectively. The reliability index corresponding to ACI 318-14 decreases less abruptly as the eccentricity increases beyond that at the balance point compared with that shown in Figure 4.3a. In this case, the difference between the two $\phi$ values is $(0.90-0.75=) 0.15$, which is markedly smaller than $(0.90-0.65=) 0.25$ for the tied column. For the other seven property combinations and $L / D$ of 0.5 , the ranges of reliability index are shown in Figures C.34-C.40 of Appendix C. The influences of geometric and material properties, load ratios, and partial material strength reduction factors are similar to those for Column 
Section 1. Inspection of these figures indicates that the reliability indices corresponding to the combination with $\phi_{s}$ of 0.90 and $\phi_{c}$ of 0.70 are less variable and slightly conservative compared to those corresponding to the ACI 318-14 criteria.

\subsection{Recommended Partial Material Strength Reduction Factors}

For Column Section 1 (square section with three bars in each face), based on Figures 4.3, and C.3-C.9, the best partial material strength reduction factor combination is $\phi_{s}$ of 0.90 and $\phi_{c}$ of 0.60 . Table 4.2 shows the means, standard deviations, minima and maxima of the reliability indices based on the eight property combinations and the two $L / D$ ratios. The category of the four ranges of $e / h$ is same with that presented in Chapter 2 . The combination with $\phi_{s}$ of 0.90 and $\phi_{c}$ of 0.60 is the best: for any range of $e / h$, the minimum reliability indices are not smaller than those obtained using ACI 318-14; the means and maxima are not excessively conservative; and, the standard deviations are relatively small. Adopting this combination of partial material strength reduction factors yields reliability indices that are bounded by those obtained using the ACI 318-14 criteria. The proposed partial material strength reduction factors yield reliability indices that are neither unnecessary large to cause the strength to be excessive nor excessively low to make the column unsafe. Similar results corresponding to Column Sections 2, 3 and 4 are shown in Tables 4.3-4.5, respectively, indicating that the longitudinal reinforcement arrangement is not a significant factor.

For Column Section 5 (spirally reinforced circular section with eight bars evenly distributed around the perimeter), the means, standard deviations, minima and maxima of the reliability indices are shown in Table 4.6. The combination with $\phi_{s}$ of 0.90 and $\phi_{c}$ of 0.70 is the best.

Alternatively, the maximum axial compressive strengths defined in ACI 318-14 are limited to 0.80 and 0.85 of the axial compressive strengths at zero eccentricity for tied and spirally reinforced columns, respectively, and they are approximate axial strengths at $e / h$ of 0.10 and 0.05 , respectively (ACI Committee 318 2014). These values can be 
reviewed and the excessively high reliability indices for columns with the higher reinforcement ratio may reduce.

\subsection{Summary and Conclusions}

This chapter has presented the reliability indices obtained using Monte Carlo simulation for five column cross sections and eight geometric and material property combinations for each cross section. Two live-to-dead load ratios are considered.

When the section is compression-controlled, the reliability index corresponding to ACI 318-14 is very sensitive to the reinforcement ratio, $\rho_{g}$, because the coefficient of variation of $f_{y}$ is markedly less than that of $f_{c}^{\prime}$. When the reinforcement ratio increases, the reliability index also increases (Israel et al. 1987). When the section is tension-controlled, the influence of $\gamma$ becomes greater for $\beta_{P M r}$, while less for $\beta_{P M u}$. The influence of $f_{c}^{\prime}$ on the reliability index is larger in the compression-controlled region than in the tensioncontrolled region. For the partial material strength reduction factors, $\phi_{s}$ is more influential in the tension-controlled region, while $\phi_{c}$ is more influential in the compressioncontrolled region. The $L / D$ ratio has negligible effects on the computed reliability indices.

For columns with tied reinforcement, the best partial material strength reduction factor combination is $\phi_{s}$ of 0.90 and $\phi_{c}$ of 0.60 , which is also identical to the values obtained in Chapter 2. The four tied column cross sections investigated yield the similar results, indicating that the longitudinal reinforcement arrangement is not a significant factor.

For columns with spiral reinforcement, the best partial material strength reduction factor combination is $\phi_{s}$ of 0.90 and $\phi_{c}$ of 0.70 .

Therefore, $\phi_{s}$ of 0.90 is the best for both tied and spirally reinforced columns. A unique $\phi_{c}$ value is appropriate for spirally reinforced columns to account for the advantages of confinement that mainly impact the compression-controlled region. 
Table 4.1: Statistical parameters for column reliability analysis

\begin{tabular}{|c|c|c|c|c|c|}
\hline Item & Source & Comment & Distribution & & \\
\hline \multicolumn{4}{|c|}{ Geometric properties } & Mean & $\sigma$ \\
\hline \multicolumn{4}{|c|}{ Columns } & $(\mathrm{mm})$ & $(\mathrm{mm})$ \\
\hline$b$ & Ellingwood et al. 1980 & Rectangular & Normal & Nominal +1.52 & 6.35 \\
\hline \multirow[t]{2}{*}{$h$} & Ellingwood et al. 1980 & Rectangular & Normal & Nominal+1.52 & 6.35 \\
\hline & $\begin{array}{l}\text { Mirza and MacGregor } \\
1979\end{array}$ & Circular & Normal & Nominal & 4.76 \\
\hline \multirow[t]{2}{*}{$d$} & ACI Committee 3182014 & $d \leq 203 \mathrm{~mm}$ & Normal & Nominal & 4.76 \\
\hline & & $d>203 \mathrm{~mm}$ & Normal & Nominal & 6.35 \\
\hline \multicolumn{2}{|c|}{ Reinforcement } & & & $\delta$ & $V$ \\
\hline$A_{s}$ & Nowak and Szerszen 2003 & - & Normal & 1.0 & 0.015 \\
\hline \multicolumn{2}{|c|}{ Material strengths } & & & $\delta$ & $V$ \\
\hline \multirow[t]{2}{*}{$f_{c}^{\prime}$} & Bartlett 2007 & Cast-in-place & Normal & 1.15 & 0.211 \\
\hline & Bartlett and MacGregor 1999 & & & & \\
\hline$f_{y}$ & Ellingwood et al. 1980 & - & Lognormal & 1.125 & 0.098 \\
\hline$E_{s}$ & - & - & Deterministic & 1 & 0 \\
\hline \multicolumn{3}{|c|}{ Professional factor } & & $\delta$ & $V$ \\
\hline \multirow[t]{2}{*}{$P$} & Nowak and Szerszen 2003 & Tied & Normal & 1.00 & 0.08 \\
\hline & & Spiral & Normal & 1.05 & 0.06 \\
\hline \multicolumn{2}{|c|}{ Load effects } & & & $\delta$ & $V$ \\
\hline$D T_{D}$ & Ellingwood et al. 1980 & All materials & Normal & 1.05 & 0.10 \\
\hline$L T_{L}$ & Israel et al. 1987 & $\begin{array}{l}\text { A58.1-1982 } \\
\text { live load } \\
\text { reductions }\end{array}$ & Gumbel & 1.00 & 0.25 \\
\hline
\end{tabular}


Table 4.2: Means, standard deviations, minima and maxima of reliability indices for combined moment and axial force, $\beta_{P M}$, for Column Section 1

\begin{tabular}{ccccccc}
\hline \multirow{2}{*}{$e / h \quad$} & Statistical & ACI 318- & $\phi_{s}=0.85$, & $\phi_{s}=0.85$, & $\phi_{s}=0.90$, & $\phi_{s}=0.90$, \\
\hline $0 \leq e / h \leq 0.3$ & parameter & 14 & $\phi_{c}=0.60$ & $\phi_{c}=0.65$ & $\phi_{c}=0.60$ & $\phi_{c}=0.65$ \\
\hline \multirow{5}{*}{$0.3<e / h \leq 1.0$} & Mean & 3.234 & 3.140 & 2.981 & 3.080 & 2.920 \\
& $\sigma$ & 0.350 & 0.188 & 0.194 & 0.153 & 0.158 \\
& Min & 2.737 & 2.836 & 2.676 & 2.827 & 2.660 \\
& Max & 3.983 & 3.507 & 3.359 & 3.390 & 3.220 \\
& & & & & & \\
& Mean & 3.176 & 3.291 & 3.165 & 3.169 & 3.040 \\
& $\sigma$ & 0.492 & 0.130 & 0.134 & 0.111 & 0.108 \\
& Min & 2.442 & 2.959 & 2.812 & 2.906 & 2.757 \\
& Max & 4.056 & 3.562 & 3.458 & 3.405 & 3.261 \\
& & & & & & \\
& Mean & 2.825 & 3.263 & 3.207 & 3.037 & 2.982 \\
& $\sigma$ & 0.192 & 0.187 & 0.162 & 0.184 & 0.159 \\
& Min & 2.646 & 2.943 & 2.944 & 2.727 & 2.719 \\
& Max & 3.614 & 3.614 & 3.489 & 3.363 & 3.254 \\
& & & & & & \\
& & & & & & \\
& Mean & 2.742 & 3.096 & 3.075 & 2.844 & 2.820 \\
& $\sigma$ & 0.077 & 0.162 & 0.141 & 0.159 & 0.133 \\
& Min & 2.627 & 2.882 & 2.887 & 2.657 & 2.654 \\
& Max & 3.086 & 3.521 & 3.395 & 3.258 & 3.165 \\
\hline
\end{tabular}


Table 4.3: Means, standard deviations, minima and maxima of reliability indices for combined moment and axial force, $\beta_{P M}$, for Column Section 2

\begin{tabular}{ccccccc}
\hline & Statistical & ACI 318- & $\phi_{s}=0.85$, & $\phi_{s}=0.85$, & $\phi_{s}=0.90$, & $\phi_{s}=0.90$, \\
$e / h$ & parameter & 14 & $\phi_{c}=0.60$ & $\phi_{c}=0.65$ & $\phi_{c}=0.60$ & $\phi_{c}=0.65$ \\
\hline $0 \leq e / h \leq 0.4$ & Mean & 3.303 & 3.177 & 3.020 & 3.107 & 2.951 \\
& $\sigma$ & 0.353 & 0.189 & 0.192 & 0.150 & 0.159 \\
& Min & 2.769 & 2.861 & 2.692 & 2.842 & 2.661 \\
& Max & 4.013 & 3.548 & 3.403 & 3.404 & 3.262 \\
$0.4<e / h \leq 1.0$ & & & & & & \\
& Mean & 3.263 & 3.313 & 3.202 & 3.167 & 3.051 \\
& $\sigma$ & 0.520 & 0.140 & 0.135 & 0.131 & 0.119 \\
& Min & 2.570 & 2.999 & 2.861 & 2.823 & 2.798 \\
& Max & 4.224 & 3.581 & 3.445 & 3.437 & 3.278 \\
& & & & & & \\
& Mean & 2.772 & 3.153 & 3.125 & 2.906 & 2.877 \\
& $\sigma$ & 0.108 & 0.176 & 0.153 & 0.171 & 0.147 \\
& Min & 2.666 & 2.906 & 2.899 & 2.677 & 2.676 \\
& Max & 3.492 & 3.530 & 3.444 & 3.294 & 3.205 \\
& & & & & & \\
& Mean & 2.729 & 3.071 & 3.061 & 2.815 & 2.800 \\
& $\sigma$ & 0.060 & 0.145 & 0.134 & 0.136 & 0.118 \\
& Min & 2.620 & 2.878 & 2.879 & 2.643 & 2.645 \\
& Max & 2.823 & 3.489 & 3.414 & 3.233 & 3.164 \\
\hline
\end{tabular}


Table 4.4: Means, standard deviations, minima and maxima of reliability indices for combined moment and axial force, $\beta_{P M}$, for Column Section 3

\begin{tabular}{ccccccc}
\hline \multirow{2}{*}{$e / h \quad$} & Statistical & ACI 318- & $\phi_{s}=0.85$, & $\phi_{s}=0.85$, & $\phi_{s}=0.90$, & $\phi_{s}=0.90$, \\
\hline $0 \leq e / h \leq 0.3$ & parameter & 14 & $\phi_{c}=0.60$ & $\phi_{c}=0.65$ & $\phi_{c}=0.60$ & $\phi_{c}=0.65$ \\
\hline \multirow{5}{*}{$0.3<e / h \leq 1.0$} & Mean & 3.218 & 3.132 & 2.971 & 3.069 & 2.911 \\
& $\sigma$ & 0.349 & 0.188 & 0.194 & 0.152 & 0.158 \\
& Min & 2.729 & 2.845 & 2.663 & 2.813 & 2.655 \\
& Max & 3.976 & 3.501 & 3.341 & 3.374 & 3.214 \\
& & & & & & \\
& Mean & 3.123 & 3.281 & 3.154 & 3.168 & 3.036 \\
& $\sigma$ & 0.488 & 0.125 & 0.134 & 0.106 & 0.107 \\
& Min & 2.382 & 2.956 & 2.780 & 2.904 & 2.748 \\
& Max & 4.038 & 3.600 & 3.486 & 3.411 & 3.285 \\
& & & & & & \\
& Mean & 2.837 & 3.296 & 3.229 & 3.082 & 3.016 \\
& $\sigma$ & 0.197 & 0.186 & 0.159 & 0.188 & 0.159 \\
& Min & 2.644 & 2.951 & 2.939 & 2.735 & 2.725 \\
& Max & 3.583 & 3.612 & 3.539 & 3.432 & 3.296 \\
& & & & & & \\
& & & & & & \\
& Mean & 2.740 & 3.115 & 3.087 & 2.865 & 2.836 \\
& $\sigma$ & 0.092 & 0.174 & 0.147 & 0.176 & 0.144 \\
& Min & 2.579 & 2.884 & 2.882 & 2.658 & 2.655 \\
& Max & 3.166 & 3.551 & 3.445 & 3.373 & 3.224 \\
\hline
\end{tabular}


Table 4.5: Means, standard deviations, minima and maxima of reliability indices for combined moment and axial force, $\beta_{P M}$, for Column Section 4

\begin{tabular}{ccccccc}
\hline \multirow{2}{*}{$e / h$} & Statistical & ACI 318- & $\phi_{s}=0.85$, & $\phi_{s}=0.85$, & $\phi_{s}=0.90$, & $\phi_{s}=0.90$, \\
\hline $0 \leq e / h \leq 0.3$ & parameter & 14 & $\phi_{c}=0.60$ & $\phi_{c}=0.65$ & $\phi_{c}=0.60$ & $\phi_{c}=0.65$ \\
\hline \multirow{5}{*}{$0.3<e / h \leq 1.0$} & Mean & 3.202 & 3.110 & 2.952 & 3.051 & 2.889 \\
& $\sigma$ & 0.342 & 0.184 & 0.190 & 0.154 & 0.158 \\
& Min & 2.688 & 2.775 & 2.613 & 2.773 & 2.591 \\
& Max & 3.921 & 3.494 & 3.332 & 3.398 & 3.203 \\
& & & & & & \\
& Mean & 3.133 & 3.289 & 3.161 & 3.168 & 3.037 \\
& $\sigma$ & 0.455 & 0.128 & 0.133 & 0.107 & 0.105 \\
& Min & 2.490 & 2.941 & 2.792 & 2.890 & 2.737 \\
& Max & 3.957 & 3.556 & 3.423 & 3.408 & 3.249 \\
& & & & & & \\
& Mean & 2.901 & 3.297 & 3.230 & 3.091 & 3.020 \\
& $\sigma$ & 0.258 & 0.149 & 0.139 & 0.136 & 0.122 \\
& Min & 2.621 & 3.051 & 3.031 & 2.846 & 2.822 \\
& Max & 3.556 & 3.559 & 3.474 & 3.339 & 3.244 \\
& & & & & & \\
& Mean & 2.784 & 3.162 & 3.127 & 2.918 & 2.883 \\
& $\sigma$ & 0.148 & 0.180 & 0.157 & 0.182 & 0.154 \\
& Min & 2.638 & 2.909 & 2.899 & 2.677 & 2.670 \\
& Max & 3.465 & 3.544 & 3.446 & 3.343 & 3.243 \\
\hline
\end{tabular}


Table 4.6: Means, standard deviations, minima and maxima of reliability indices for combined moment and axial force, $\beta_{P M}$, for Column Section 5

\begin{tabular}{ccccc}
\hline & Statistical & & $\phi_{s}=0.90$, & $\phi_{s}=0.95$, \\
$e / h$ & parameter & ACI 318-14 & $\phi_{c}=0.70$ & $\phi_{c}=0.70$ \\
\hline $0 \leq e / h \leq 0.3$ & Mean & 2.974 & 2.946 & 2.889 \\
& $\sigma$ & 0.325 & 0.211 & 0.179 \\
& Min & 2.464 & 2.587 & 2.564 \\
$0.3<e / h \leq 1.0$ & Max & 3.650 & 3.392 & 3.254 \\
& & & & \\
& Mean & 3.147 & 3.192 & 3.074 \\
& $\sigma$ & 0.291 & 0.158 & 0.130 \\
& Min & 2.730 & 2.781 & 2.723 \\
& Max & 3.788 & 3.526 & 3.335 \\
& & & & \\
& Mean & 3.188 & 3.310 & 3.107 \\
$e / h \leq 0$ & $\sigma$ & 0.184 & 0.176 & 0.154 \\
& Min & 2.888 & 3.059 & 2.876 \\
& Max & 3.562 & 3.628 & 3.395 \\
& & & \\
& Mean & 3.133 & 3.217 & 2.980 \\
& $\sigma$ & 0.160 & 0.185 & 0.174 \\
& Min & 2.931 & 2.957 & 2.739 \\
& Max & 3.589 & 3.562 & 3.352 \\
\hline
\end{tabular}



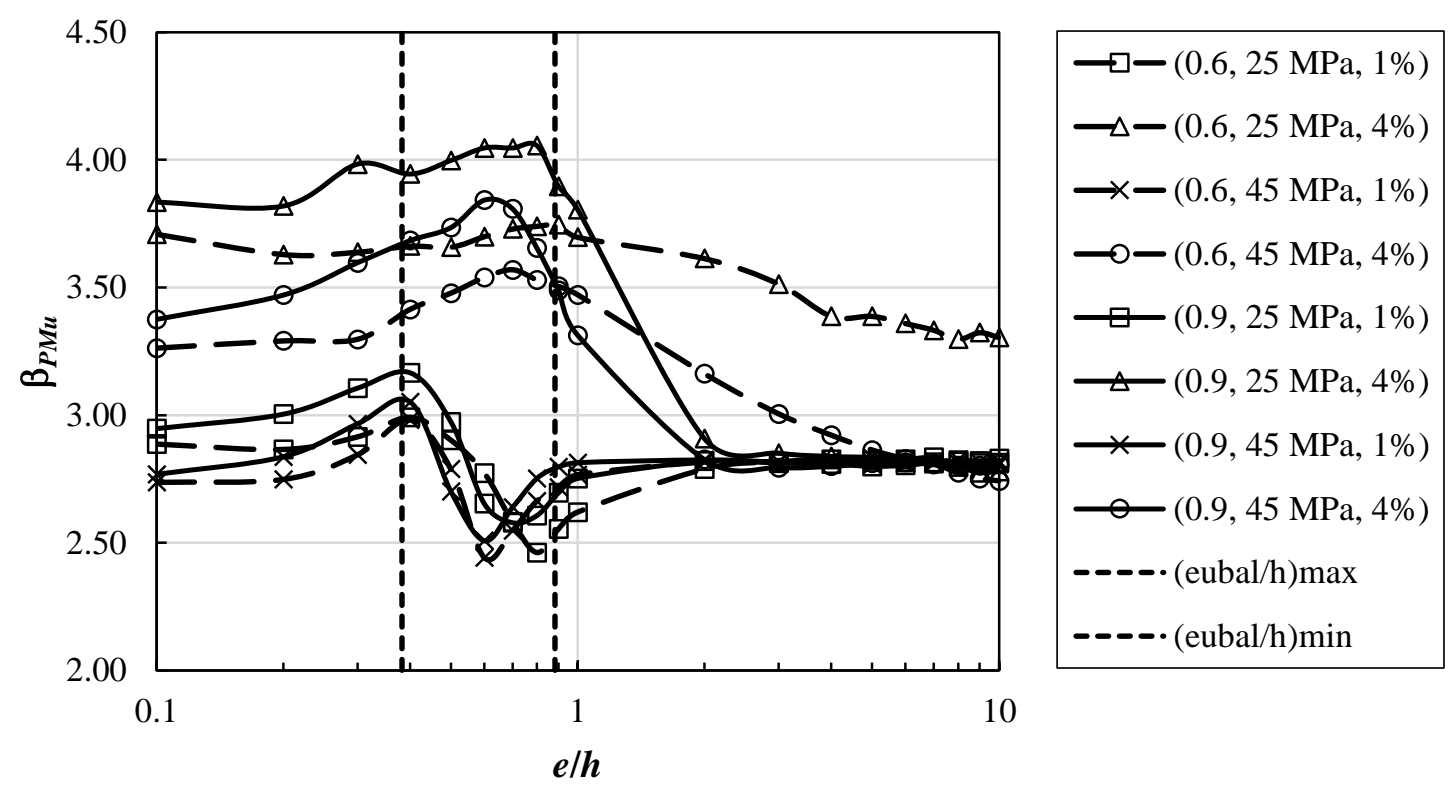

(a)

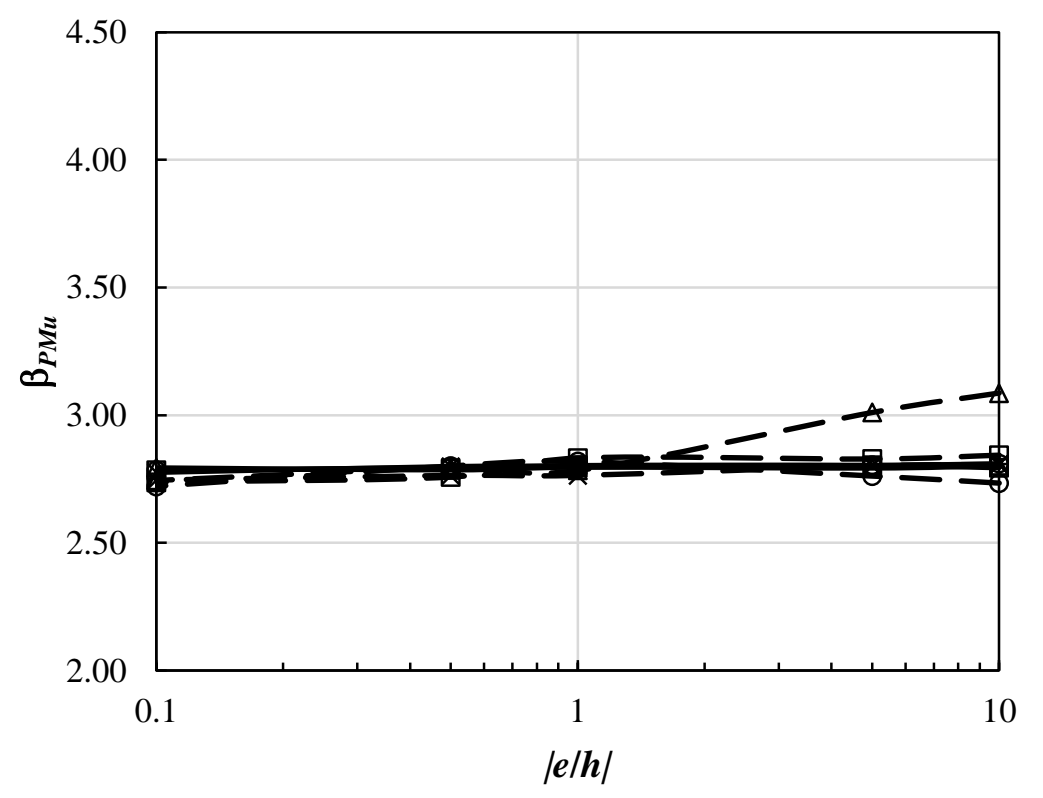

$$
\begin{aligned}
& \longrightarrow-(0.6,25 \mathrm{MPa}, 1 \%) \\
& \rightarrow-(0.6,25 \mathrm{MPa}, 4 \%) \\
& \rightarrow-(0.6,45 \mathrm{MPa}, 1 \%) \\
& \longrightarrow-(0.6,45 \mathrm{MPa}, 4 \%) \\
& \longrightarrow-(0.9,25 \mathrm{MPa}, 1 \%) \\
& \longrightarrow-(0.9,25 \mathrm{MPa}, 4 \%) \\
& \rightarrow-(0.9,45 \mathrm{MPa}, 1 \%) \\
& \longrightarrow-(0.9,45 \mathrm{MPa}, 4 \%)
\end{aligned}
$$

(b)

Figure 4.1: Reliability indices for combined moment and axial force using strength reduction factors in ACI 318-14, $\beta_{P M u}$, for Column Section 1 and $L / D=0.5$ : (a) $e / h>0$; (b) $e / h<0$ 


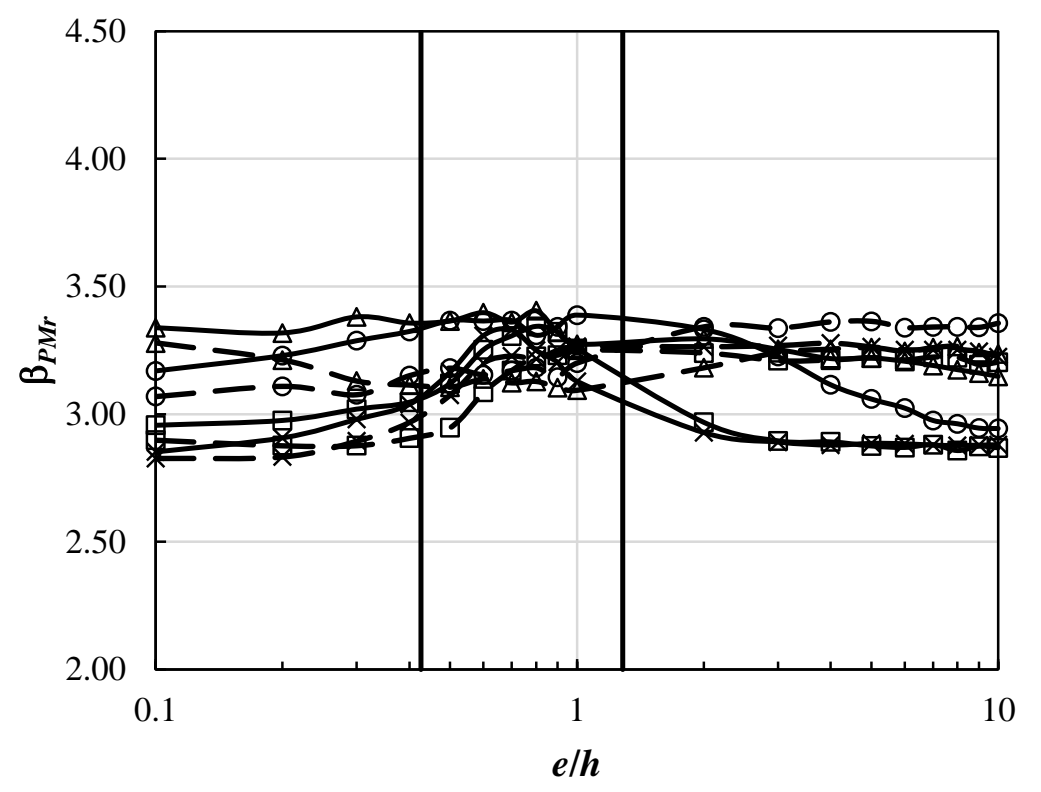

$$
\begin{aligned}
& \longrightarrow \text { (0.6, } 25 \mathrm{MPa}, 1 \%) \\
& \longrightarrow-(0.6,25 \mathrm{MPa}, 4 \%) \\
& \longrightarrow-(0.6,45 \mathrm{MPa}, 1 \%) \\
& \longrightarrow-(0.6,45 \mathrm{MPa}, 4 \%) \\
& \square(0.9,25 \mathrm{MPa}, 1 \%) \\
& \triangle(0.9,25 \mathrm{MPa}, 4 \%) \\
& \longrightarrow(0.9,45 \mathrm{MPa}, 1 \%) \\
& \text { - }(0.9,45 \mathrm{MPa}, 4 \%) \\
& \text { (erbal/h)max }
\end{aligned}
$$

(a)

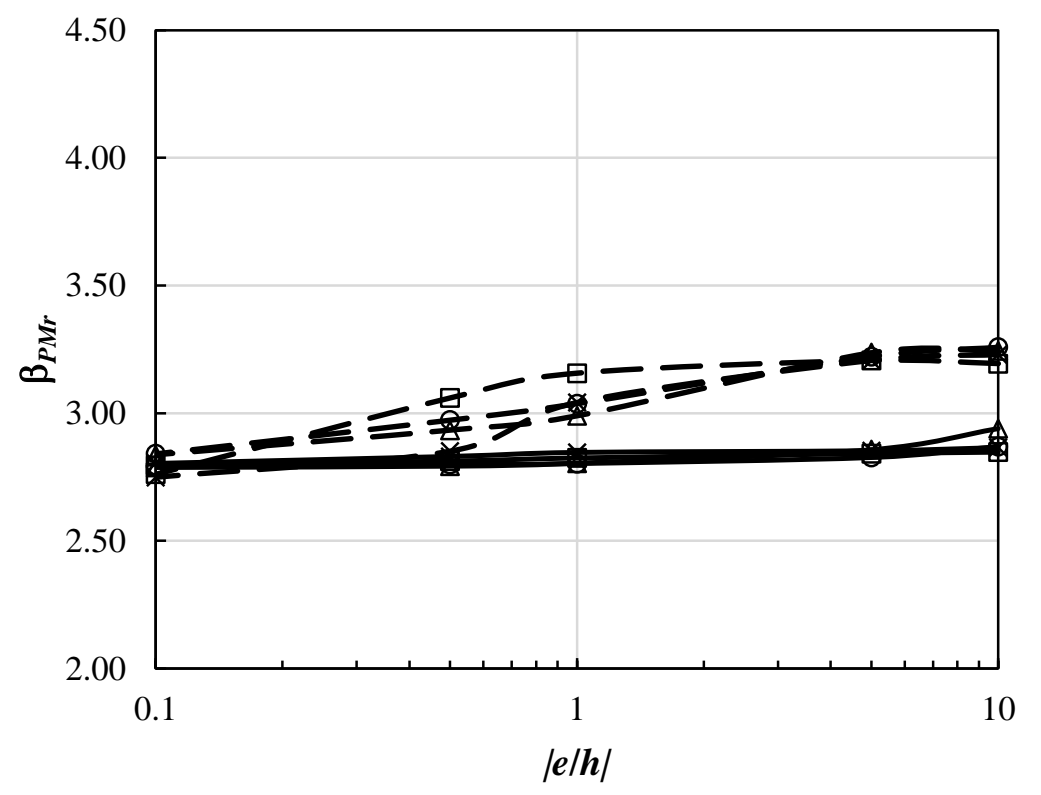

$$
\begin{aligned}
& \longrightarrow-(0.6,25 \mathrm{MPa}, 1 \%) \\
& \rightarrow-(0.6,25 \mathrm{MPa}, 4 \%) \\
& \rightarrow-(0.6,45 \mathrm{MPa}, 1 \%) \\
& \longrightarrow-(0.6,45 \mathrm{MPa}, 4 \%) \\
& \longrightarrow-(0.9,25 \mathrm{MPa}, 1 \%) \\
& \longrightarrow-(0.9,25 \mathrm{MPa}, 4 \%) \\
& \rightarrow-(0.9,45 \mathrm{MPa}, 1 \%) \\
& \longrightarrow-(0.9,45 \mathrm{MPa}, 4 \%)
\end{aligned}
$$

(b)

Figure 4.2: Reliability indices for combined moment and axial force, $\beta_{P M r}$, corresponding to $\phi_{s}=0.90$ and $\phi_{c}=0.60$, for Column Section 1 and $L / D=0.5$ : (a) $e / h>0$;

(b) $e / h<0$ 

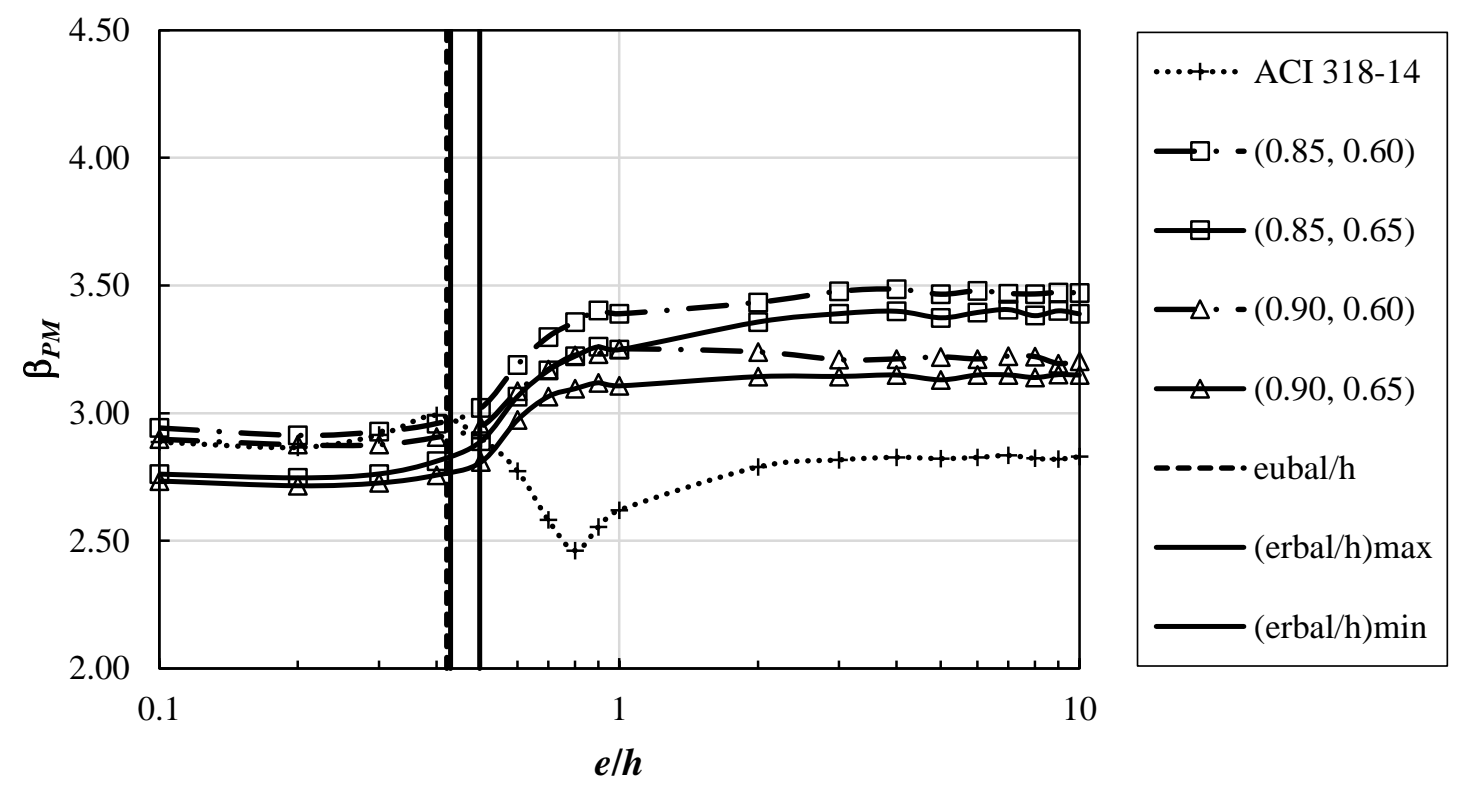

(a)
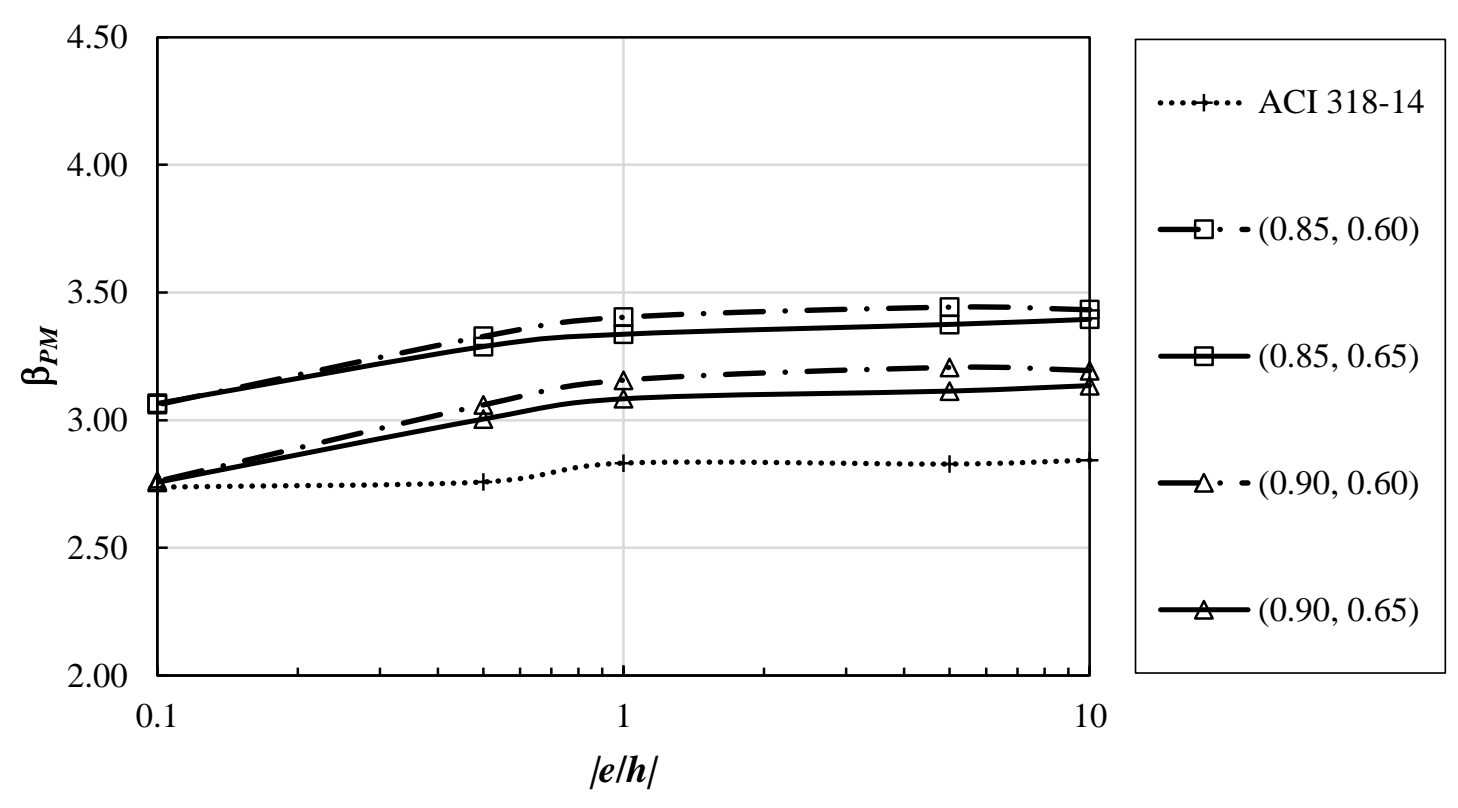

(b)

Figure 4.3: Reliability indices for combined moment and axial force, $\beta_{P M}$, for Column Section 1, Property Combination 1, and $L / D=0.5$ : (a) $e / h>0$; (b) $e / h<0$ 

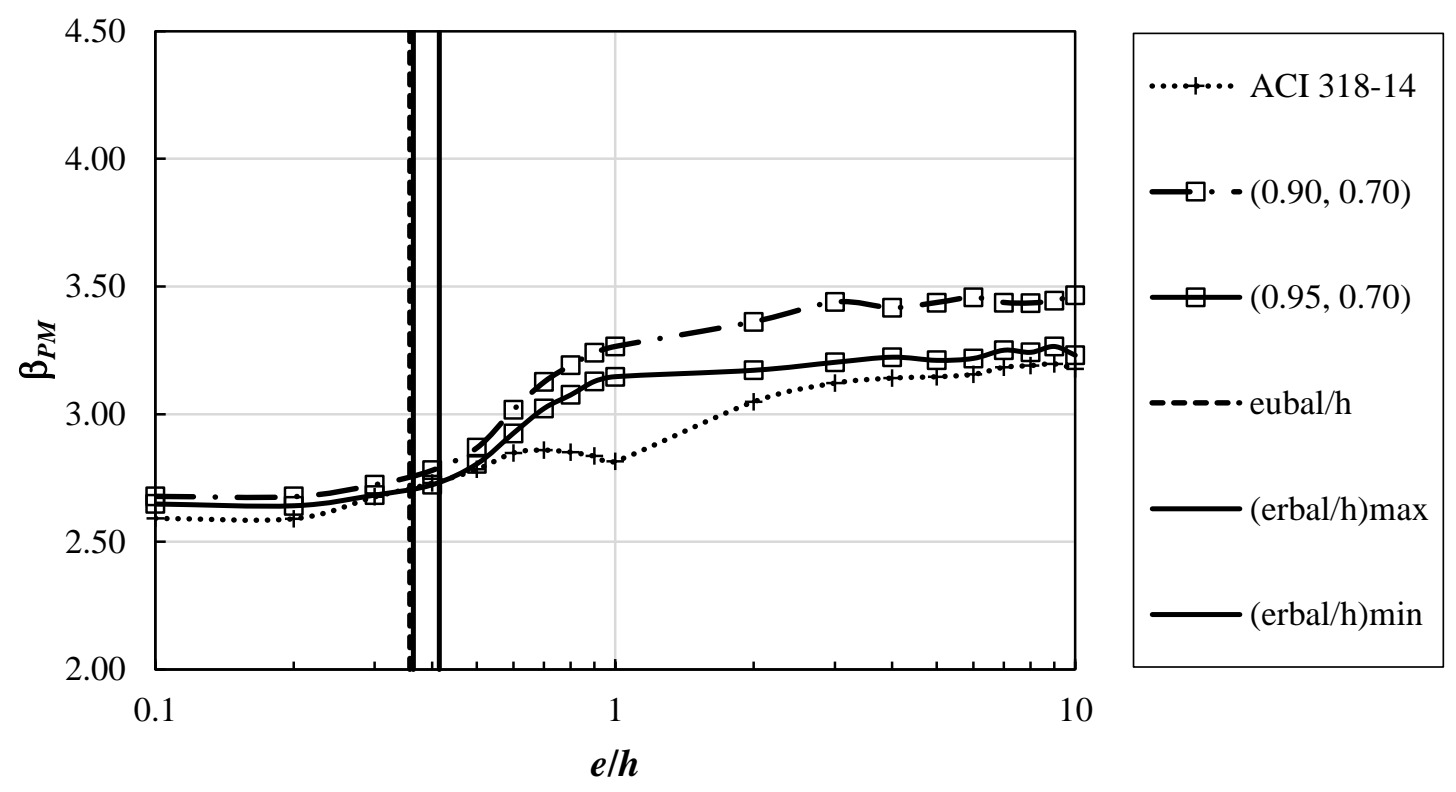

(a)
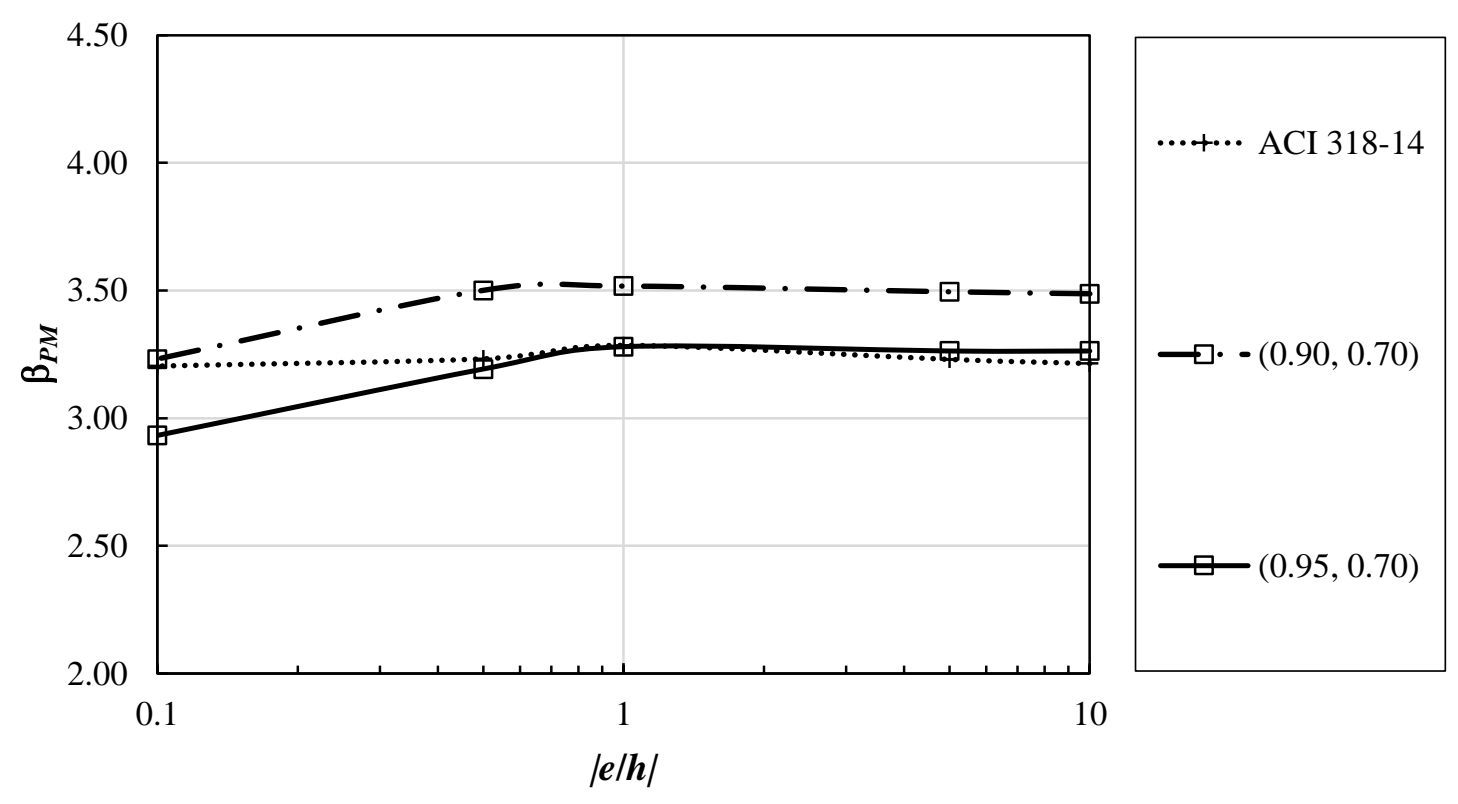

(b)

Figure 4.4: Reliability indices for combined moment and axial force, $\beta_{P M}$, for Column Section 5, Property Combination $1, L / D=0.5$, and: $(a) e / h>0 ;(b) e / h<0$ 


\section{Chapter 5}

\section{Summary and Conclusions}

This chapter presents a summary of the work conducted in this study, lists the conclusions and recommends some suggestions for future work.

\subsection{Summary}

ACI 318-14 (ACI Committee 318 2014) defines an overall strength reduction factor to account for the probability of understrength. However, it leads to some unsatisfactory results, particularly inconsistencies in the transition region of the interaction diagram, between the compression-controlled and tension controlled regions (e.g., Gamble 1998, 2015), and so non-unique moment capacities for one axial strength level for sections with wide flanges (Lequesne and Pincheira 2014). The statistical parameters that quantify the professional factor for shear since previous calibrations (Israel et al. 1987; Nowak and Szerszen 2003) have also changed markedly (Somo and Hong 2006). Therefore, the present study proposes partial material strength reduction factors for concrete, $\phi_{c}$, and reinforcing steel, $\phi_{s}$, that yield similar design strengths and more consistent reliability indices compared to those based on the strength reduction factors, $\phi$, in ACI 318-14. Three structural actions acting on non-prestressed members are investigated: moment; one-way shear; and, combined moment and axial force.

The comparison of design strengths is presented in Chapter 2. For members subjected to moment, singly reinforced sections with concrete compressive strengths, $f_{c}{ }^{\prime}$, of 25 and 45 $\mathrm{MPa}$, reinforcement yield strength, $f_{y}$, of $420 \mathrm{MPa}$, and reinforcement ratio, $\rho$, ranging from 0.003 to 0.018 are investigated. For members subjected to shear, rectangular beam sections with the same material strengths and ranges of transverse reinforcement ratio, $\rho_{t}$, from 0.001 to 0.007 for $f_{c}^{\prime}$ of $25 \mathrm{MPa}$ and 0.001 to 0.010 for $f_{c}^{\prime}$ of $45 \mathrm{MPa}$ are studied. For members subjected to combined moment and axial force, five column cross sections including square section with three bars in each face, square section with three bars in two end faces only, square section with three bars in two side faces only, tied circular 
section with eight bars evenly distributed around the perimeter, and spirally reinforced circular section with eight bars evenly distributed around the perimeter are investigated. For each column section, eight geometric and material property combinations are investigated, specifically, the ratios of the distance between the outer layers of reinforcement to the overall column depth, $\gamma$, of 0.6 and $0.9, f_{c}{ }^{\prime}$ of 25 and $45 \mathrm{MPa}, f_{y}$ of $420 \mathrm{MPa}$ and total reinforcement ratios, $\rho_{g}$, of 0.01 and 0.04 . The design strengths of each section are calculated using ACI 318-14 and the partial material strength reduction factors. Then design strength ratios, defined as the design strength obtained using ACI 318-14 to that obtained using a particular pair of partial material strength reduction factors, are calculated. The sensitivities of the design strength ratios to the geometric and material properties and the partial material strength reduction factors are analyzed, and the best partial material strength reduction factor combinations are proposed.

The reliability analyses for members resisting moment and shear are presented in Chapter 3. The reliability model and the first-order, second-moment (FOSM) method are described. Statistical parameters for geometric properties, material strengths, professional factors and load effects collected from the literature are summarized and those used for the subsequent reliability analyses are listed. The reliability indices are calculated for the different geometric and material properties, and two live-to-dead load ratios, $w_{L} / w_{D}$, of 0.5 and 1.5 for varying $\rho$ and $\rho_{t}$ for moment and shear, respectively, using Microsoft Excel (Version 2013; Microsoft 2013). The means and standard deviations of the reliability indices for each partial material strength reduction factor combination are quantified. The sensitivities of the reliability indices to the geometric and material properties, partial material strength reduction factors, load ratios, and statistical parameters are analyzed, and the best partial material strength reduction factor combinations are proposed.

The reliability analyses for members resisting combined moment and axial force are presented in Chapter 4. The analyses are conducted using Monte Carlo simulation (Hong 2015) because the equations to generate interaction diagrams are relatively complicated. The sections and various geometric and material properties investigated are identical to those for design strength calculations in Chapter 2. Two live-to-dead load ratios, 0.5 and 
1.5 are again investigated. The applied moment and axial force are assumed perfectly correlated and reliability indices are computed for a range of specific eccentricities. To save time and avoid unnecessary calculations, only four pairs of partial material strength reduction factors are analyzed for tied columns, combinations with $\phi_{s}$ of 0.85 and 0.90 , and $\phi_{c}$ of 0.60 and 0.65 . For spirally reinforced columns, two pairs of partial material strength reduction factors are analyzed, combinations with $\phi_{s}$ of 0.90 and 0.95 , and $\phi_{c}$ of 0.70. The simulation is run $10^{6}$ times for each case using Matlab (Version R2016b; The Mathworks, Inc. 2016). The means, standard deviations, minima and maxima for typical reliability indices for each combination are quantified. The sensitivities of the reliability indices to the partial material strength reduction factor combinations are investigated for various geometric and material properties, and the best combinations are proposed.

\subsection{Conclusions}

The following conclusions pertain to the design strength analysis results:

1. The design flexural strength ratio, $\alpha_{M}$, which is defined as the design flexural strength obtained using the strength reduction factor in ACI 318-14 to that obtained using a particular pair of partial material strength reduction factors, is sensitive to $\phi_{s}$ and relatively insensitive to $\phi_{c}$. In the tension-controlled sections, the combination with $\phi_{s}$ of 0.90 and $\phi_{c}$ of 0.75 is the best. If the section is in the transition region, the combination with $\phi_{s}$ of 0.95 and $\phi_{c}$ of 0.65 is the best. And any combination with $\phi_{s}$ of 0.90 is satisfactory for moment. The results are insensitive to $f_{c}^{\prime}$.

2. The design shear strength ratio, $\alpha_{V}$, is also sensitive to $\phi_{s}$ and relatively insensitive to $\phi_{c}$, but the influences of these two factors are not as distinct as they are for moment. The best partial material strength reduction factor combination is $\phi_{s}$ of 0.80 and $\phi_{c}$ of 0.65 . Combinations with $\phi_{s}$ of 0.80 and $\phi_{c}$ of 0.70 , and $\phi_{s}$ of 0.85 and $\phi_{c}$ of 0.60 are close to optimal. The influence of $f_{c}^{\prime}$ is again slight.

3. For tied columns, in the compression-controlled region, the design combined flexural and axial strength ratio, $\alpha_{P M}$, is sensitive to $\phi_{c}$, and $\phi_{c}$ of 0.60 is the best. The 
influences of $\gamma, f_{c}^{\prime}, \rho_{g}$ are very small, moderate, and relatively large, respectively. In the tension-controlled region, $\alpha_{P M}$ is sensitive to $\phi_{s}$, and $\phi_{s}$ of 0.90 is the best. The influence of $\gamma$ becomes more significant, that of $\rho_{g}$ is moderate, and that of $f_{c}^{\prime}$ is limited. The $\alpha_{P M}$ value varies markedly in the transition region where failure mode changes from compression-induced to tension-initiated, because $\phi$ in ACI 318-14 increases from 0.65 to 0.90 .

4. For spirally reinforced circular columns, $\alpha_{P M}$ increases markedly in the compressioncontrolled region compared to those for tied columns, which is due to the strength reduction factor in ACI 318-14, $\phi$, being 0.75 for spirally reinforced columns instead of 0.65 . In the compression-controlled region, $\phi_{c}$ of 0.70 is the best and in the tensioncontrolled region, $\phi_{s}$ of both 0.90 and 0.95 are the best.

The following conclusions pertain to the reliability analysis results:

5. The reliability index for members subjected to moment corresponding to partial material strength reduction factors, $\beta_{M r}$, is more sensitive to $\phi_{s}$ than to $\phi_{c}$. The best partial material strength reduction factor combination is $\phi_{s}$ of 0.90 and $\phi_{c}$ of 0.75 . As for the design strength comparison, any combination with $\phi_{s}$ of 0.90 is satisfactory. The influences of $f_{c}^{\prime}$ and $w_{L} / w_{D}$ are small.

6. The reliability index for members subjected to one-way shear corresponding to partial material strength reduction factors, $\beta_{V r}$, is more sensitive to $\phi_{s}$ than to $\phi_{c}$, but the differences are not as large as they are for moment. The best partial material strength reduction factor combination is $\phi_{s}$ of 0.80 and $\phi_{c}$ of 0.65 . Moreover, combinations with $\phi_{s}$ of 0.80 and $\phi_{c}$ of 0.70 , and $\phi_{s}$ of 0.85 and $\phi_{c}$ of 0.60 are close to optimal, which are identical to the results based on the design strengths. Again, the influences of $f_{c}^{\prime}$ and $w_{L} / w_{D}$ are small.

7. The reliability indices for one-way shear range from 2.65 to 2.82 , and 2.20 to 3.11 computed for the ACI 318-14 criteria and the various partial material strength reduction factor combinations, respectively. These ranges are markedly lower than 
those for moment, which is not desirable because shear failures are less ductile than flexural failures. The reliability indices for shear are very sensitive, however, to the statistical parameters assumed for the professional factor. If the statistical parameters assumed previously by Nowak and Szerszen (2003) are adopted, the reliability indices for shear increase markedly. However, the statistical parameters reported by Somo and Hong (2006) are more appropriate because they are based on larger sample sizes, classification of parameters by $a_{v} / d$ and the presence of stirrups, and considering prestressed members separately. The influence of the statistical parameters for the professional factor selected is consistent for both ACI 318-14 and the partial material strength reduction factors criteria.

8. For tied columns, in the compression-controlled region, the reliability index for members subjected to combined moment and axial force corresponding to partial material strength reduction factors, $\beta_{P M r}$, is sensitive to $\phi_{c}$, and $\phi_{c}$ of 0.60 is the best. In the tension-controlled region, $\beta_{P M r}$ is sensitive to $\phi_{s}$, and $\phi_{s}$ of 0.90 is the best. For ACI 318-14, $\beta_{P M u}$ varies markedly with $\rho_{g}$. For the combination with $\phi_{s}$ of 0.90 and $\phi_{c}$ of 0.60 , however, the $\beta_{P M r}$ values are more consistent. The geometric and material properties and load ratios do not appreciably affect these results. The results are also essentially identical for the four tied column sections investigated, which indicates that the reinforcement arrangement is not a significant factor.

9. For spirally reinforced columns, the influences of material and geometric properties, load ratios, and partial material strength reduction factors are similar to those for tied columns. The best combination is $\phi_{s}$ of 0.90 and $\phi_{c}$ of 0.70 . Therefore, $\phi_{s}$ of 0.90 is the best for both tied and spirally reinforced columns, while a unique $\phi_{c}$ value is appropriate for spirally reinforced columns to account for the advantages of confinement that mainly impact the compression-controlled region.

10. Although no single combination of $\phi_{s}$ and $\phi_{c}$ is the best for members resisting moment, shear, or combined moment and axial force, the recommended partial material strength reduction factors are $\phi_{s}$ of 0.90 and $\phi_{c}$ of 0.60 for slabs and beams subjected to moment, beams subjected to one-way shear, and tied columns, or $\phi_{c}$ of 
0.70 for spirally reinforced columns. Alternatively, for shear, the combination with $\phi_{s}$ of 0.80 and $\phi_{c}$ of 0.65 is recommended.

\subsection{Suggestions for Future Work}

1. The oldest statistical parameters used in this study trace back to 1979. Control of the construction process and material quality may have since improved. Research to determine more current statistical parameters for the geometric properties, material strengths, professional factors, and load effects should be carried out and the recommended partial material strength reduction factors should be reviewed based on these new parameters.

2. The structural actions investigated in this study are moment, one-way shear, and combined moment and axial force. Other actions or structural elements, such as twoway shear, torsion, bearing, brackets and corbels, should be investigated in the future.

3. The reliability index for one-way shear is very sensitive to the statistical parameters used to quantify the professional factor. The basic equations for one-way shear strength in ACI 318 have not changed for more than five decades (Belarbi et al. 2017). Significant changes have occurred in other codes, and the deficiencies of the current provisions include: (1) ignoring the size effect in the calculation of the shear strength resisted by concrete, $V_{c}$; (2) ignoring the presence of shear reinforcement in the computation of $V_{c} ;(3)$ assuming the angle of diagonal compression is fixed at $45^{\circ}$ irrespective of the amount of reinforcement; and other factors (Belarbi et al. 2017). The provisions for one-way shear should be improved and new statistical parameters for the professional factor should be derived, based on these new criteria.

4. The maximum axial compressive strengths defined in ACI 318-14 are limited to 0.80 and 0.85 of the axial compressive strengths at zero eccentricity for tied and spirally reinforced columns, respectively, and they are approximate axial strengths at $e / h$ of 0.10 and 0.05 , respectively (ACI Committee 318 2014). These values should be reviewed and reliability analyses should be conducted. 


\section{References}

American Concrete Institute (ACI) Committee 318. 2014. Building code requirements for structural concrete (ACI 318-14) and commentary (ACI 318R-14). American Concrete Institute (ACI), Farmington Hills, MI.

American National Standards Institute (ANSI). 1982. Minimum design loads for buildings and other structures (ANSI A58.1-1982). American National Standards Institute, New York, N.Y.

American Society of Civil Engineers (ASCE). 2010. Minimum design loads for buildings and other structures. American Society of Civil Engineers (ASCE) 7, Reston, VA.

Bartlett, F.M. 2007. Canadian Standards Association standard A23.3-04 resistance factor for concrete in compression. Canadian Journal of Civil Engineering, 34: 10291037.

Bartlett, F.M., and MacGregor, J.G. 1999. Variation of in-place concrete strength in structures. ACI Materials Journal, 96(2):261-270.

Bartlett, F.M., Hong, H.P., and Zhou, W. 2003. Load factor calibration for the proposed 2005 edition of the National Building Code of Canada: statistics of loads and load effects. Canada Journal of Civil Engineering, 30: 429-439.

Belarbi, A., Kuchma, D.A., and Sanders, D.H. 2017. Proposals for new one-way shear equations for the 318 building code. Concrete International, 39(9): 29-32.

Bentz, E.C. 2000. Sectional analysis of reinforced concrete members. Ph.D. thesis, Graduate Department of Civil Engineering, University of Toronto, Toronto, ON.

Canadian Standards Association (CSA). 2014. Design of concrete structures. CSA Standard A23.3-14. Canadian Standards Association (CSA), Mississauga, ON.

Collins, M.P. 2001. Evaluation of shear design procedures for concrete structures. A report prepared for the CSA Technical Committee on reinforced concrete design. Toronto, ON.

Ellingwood, B., Galambos, T.V., MacGregor, J.G., and Cornell, C.A. 1980. Development of a probability based load criterion for American National Standard A58. NBS Special Publication 577, U.S. Department of Commerce, National Bureau of Standards, Washington, D.C.

Galambos, T.V., and Ravindra, M.K. 1977. The basis for load and resistance factor design criteria of steel building structures. Canadian Journal of Civil Engineering, 4: $178-189$.

Gamble, W.L. 1998. $\phi$-factors and other anomalies. Concrete International, 20(8): 56-58.

Gamble, W.L. 2015. Phi-factors revisited. Concrete International, 37(12): 35-38.

Hong, H.P. 2015. Risk analysis and decision making in engineering (CEE 4458A). University of Western Ontario, London, ON. 
Hong, H.P., and Zhou, W. 1999. Reliability evaluation of RC columns. Journal of Structural Engineering, 125(7): 784-790.

Israel, M., Ellingwood, B., and Corotis, R. 1987. Reliability-based code formulations for reinforced concrete buildings. Journal of Structural Engineering, 113(10): 22352252.

Kani, M.W., Huggins, M.W., and Wittkopp, R.R. 1979. Kani on shear in reinforced concrete. Department of Civil Engineering, University of Toronto, Toronto, ON.

Lequesne, R.D., and Pincheira, J.A. 2014. Proposed revisions to the strength-reduction factor for axially loaded members. Concrete International, 36(9): 43-49.

MacGregor, J.G., and Bartlett, F.M. 2000. Reinforced concrete: mechanics and design. 1st Canadian ed. Prentice Hall Canada Inc., Scarborough, ON.

Microsoft. 2013. Microsoft Excel (Version 2013) [Computer software]. Microsoft, U.S.

Mirza, S.A., and MacGregor, J.G. 1979. Variations in dimensions of reinforced concrete members. Journal of the Structural Division, ASCE, 105(ST4): 751-766.

National Research Council of Canada (NRCC). 1995. National Building Code of Canada (NBCC). Institute for Research in Construction, National Research Council of Canada (NRCC), Ottawa, ON.

Nowak, A.S., and Szerszen, M.M. 2003. Calibration of design code for buildings (ACI 318): part 1-statistical models for resistance. ACI Structural Journal, 100(3): 377382.

Somo, S., and Hong, H.P. 2006. Modeling error analysis of shear predicting models for RC beams. Structural Safety, 28: 217-230.

Szerszen, M.M., and Nowak, A.S. 2003. Calibration of design code for buildings (ACI 318): part 2-reliability analysis and resistance factors. ACI Structural Journal, 100(3): 383-391.

The Mathworks, Inc. 2016. Matlab (Version R2016b) [Computer software]. The Mathworks, Inc., U.S.

Wight, J.K. 2016. Reinforced concrete: mechanics and design. 7th ed. Pearson Education, Inc., Hoboken, N.J. 


\section{Appendix A-Supplementary Information for Chapter 2}

Table A.1: Means and standard deviations of design combined flexural and axial strength ratios, $\alpha_{P M}$, for Column Section 2 and $0 \leq e / h \leq 0.4$

\begin{tabular}{ccccccccc}
\hline & \multicolumn{8}{c}{$\phi_{c}$} \\
\cline { 2 - 9 }$\phi_{s}$ & \multicolumn{2}{c}{0.60} & \multicolumn{2}{c}{0.65} & \multicolumn{2}{c}{0.70} & \multicolumn{2}{c}{0.75} \\
\cline { 2 - 10 } $0.80 a n$ & $\sigma$ & Mean & $\sigma$ & Mean & $\sigma$ & Mean & $\sigma$ \\
\hline 0.85 & 0.989 & 0.040 & 0.938 & 0.027 & 0.892 & 0.017 & 0.850 & 0.008 \\
0.90 & 0.969 & 0.048 & 0.919 & 0.035 & 0.875 & 0.024 & 0.834 & 0.014 \\
0.95 & 0.950 & 0.056 & 0.902 & 0.042 & 0.858 & 0.030 & 0.819 & 0.021 \\
\hline
\end{tabular}

Table A.2: Means and standard deviations of design combined flexural and axial strength ratios, $\alpha_{P M}$, for Column Section 2 and $0.4<e / h \leq 1.0$

\begin{tabular}{cccccccccc}
\hline & \multicolumn{8}{c}{$\phi_{c}$} \\
\cline { 2 - 10 }$\phi_{s}$ & \multicolumn{2}{c}{0.60} & \multicolumn{2}{c}{0.65} & \multicolumn{2}{c}{0.70} & \multicolumn{2}{c}{0.75} \\
\cline { 2 - 10 } & Mean & $\sigma$ & Mean & $\sigma$ & Mean & $\sigma$ & Mean & $\sigma$ \\
\hline 0.80 & 1.056 & 0.133 & 1.027 & 0.135 & 1.001 & 0.138 & 0.977 & 0.140 \\
0.85 & 1.016 & 0.123 & 0.987 & 0.125 & 0.962 & 0.128 & 0.939 & 0.130 \\
0.90 & 0.979 & 0.116 & 0.952 & 0.117 & 0.927 & 0.119 & 0.905 & 0.121 \\
0.95 & 0.946 & 0.109 & 0.919 & 0.110 & 0.895 & 0.111 & 0.874 & 0.113 \\
\hline
\end{tabular}

Table A.3: Means and standard deviations of design combined flexural and axial strength ratios, $\alpha_{P M}$, for Column Section 2 and $1.0<e / h \leq 10.0$

\begin{tabular}{ccccccccc}
\hline & \multicolumn{10}{c}{$\phi_{c}$} \\
\cline { 2 - 10 }$\phi_{s}$ & \multicolumn{2}{c}{0.60} & \multicolumn{2}{c}{0.65} & \multicolumn{2}{c}{0.70} & \multicolumn{2}{c}{0.75} \\
\cline { 2 - 10 } & Mean & $\sigma$ & Mean & $\sigma$ & Mean & $\sigma$ & Mean & $\sigma$ \\
\hline 0.80 & 1.147 & 0.033 & 1.139 & 0.028 & 1.133 & 0.024 & 1.126 & 0.022 \\
0.85 & 1.085 & 0.035 & 1.078 & 0.030 & 1.071 & 0.026 & 1.065 & 0.023 \\
0.90 & 1.029 & 0.036 & 1.022 & 0.031 & 1.016 & 0.027 & 1.011 & 0.024 \\
0.95 & 0.979 & 0.038 & 0.973 & 0.033 & 0.967 & 0.029 & 0.962 & 0.025 \\
\hline
\end{tabular}


Table A.4: Means and standard deviations of design combined flexural and axial strength ratios, $\alpha_{P M}$, for Column Section 2 and $e / h \leq 0$

\begin{tabular}{cccccccccc}
\hline & \multicolumn{10}{c}{$\phi_{c}$} \\
\cline { 2 - 10 }$\phi_{s}$ & \multicolumn{2}{c}{0.60} & \multicolumn{2}{c}{0.65} & \multicolumn{2}{c}{0.70} & \multicolumn{2}{c}{0.75} \\
\cline { 2 - 10 } & Mean & $\sigma$ & Mean & $\sigma$ & Mean & $\sigma$ & Mean & $\sigma$ \\
\hline 0.80 & 1.140 & 0.020 & 1.136 & 0.014 & 1.132 & 0.009 & 1.128 & 0.004 \\
0.85 & 1.076 & 0.022 & 1.072 & 0.017 & 1.068 & 0.013 & 1.065 & 0.008 \\
0.90 & 1.018 & 0.025 & 1.015 & 0.020 & 1.012 & 0.015 & 1.008 & 0.011 \\
0.95 & 0.967 & 0.026 & 0.964 & 0.022 & 0.961 & 0.018 & 0.958 & 0.014 \\
\hline
\end{tabular}

Table A.5: Means and standard deviations of design combined flexural and axial strength ratios, $\alpha_{P M}$, for Column Section 3 and $0 \leq e / h \leq 0.3$

\begin{tabular}{ccccccccc}
\hline & \multicolumn{8}{c}{$\phi_{c}$} \\
\cline { 2 - 9 }$\phi_{s}$ & \multicolumn{2}{c}{0.60} & \multicolumn{2}{c}{0.65} & \multicolumn{2}{c}{0.70} & \multicolumn{2}{c}{0.75} \\
\cline { 2 - 10 } & Mean & $\sigma$ & Mean & $\sigma$ & Mean & $\sigma$ & Mean & $\sigma$ \\
\hline 0.80 & 0.999 & 0.041 & 0.944 & 0.027 & 0.896 & 0.016 & 0.852 & 0.007 \\
0.85 & 0.981 & 0.049 & 0.928 & 0.035 & 0.880 & 0.024 & 0.838 & 0.014 \\
0.90 & 0.963 & 0.056 & 0.912 & 0.042 & 0.866 & 0.031 & 0.825 & 0.021 \\
0.95 & 0.946 & 0.063 & 0.896 & 0.049 & 0.852 & 0.037 & 0.812 & 0.027 \\
\hline
\end{tabular}

Table A.6: Means and standard deviations of design combined flexural and axial strength ratios, $\alpha_{P M}$, for Column Section 3 and $0.3<e / h \leq 1.0$

\begin{tabular}{ccccccccc}
\hline & \multicolumn{8}{c}{$\phi_{c}$} \\
\cline { 2 - 10 }$\phi_{s}$ & \multicolumn{2}{c}{0.60} & \multicolumn{2}{c}{0.65} & \multicolumn{2}{c}{0.70} & \multicolumn{2}{c}{0.75} \\
\cline { 2 - 10 } & Mean & $\sigma$ & Mean & $\sigma$ & Mean & $\sigma$ & Mean & $\sigma$ \\
\hline 0.80 & 1.086 & 0.139 & 1.047 & 0.135 & 1.012 & 0.133 & 0.982 & 0.133 \\
0.85 & 1.051 & 0.134 & 1.013 & 0.130 & 0.979 & 0.127 & 0.949 & 0.125 \\
0.90 & 1.019 & 0.131 & 0.982 & 0.126 & 0.949 & 0.122 & 0.920 & 0.120 \\
0.95 & 0.990 & 0.128 & 0.954 & 0.122 & 0.922 & 0.118 & 0.893 & 0.115 \\
\hline
\end{tabular}


Table A.7: Means and standard deviations of design combined flexural and axial strength ratios, $\alpha_{P M}$, for Column Section 3 and $1.0<e / h \leq 10.0$

\begin{tabular}{cccccccccc}
\hline & \multicolumn{10}{c}{$\phi_{c}$} \\
\cline { 2 - 10 }$\phi_{s}$ & \multicolumn{2}{c}{0.60} & \multicolumn{2}{c}{0.65} & \multicolumn{2}{c}{0.70} & \multicolumn{2}{c}{0.75} \\
\cline { 2 - 10 } & Mean & $\sigma$ & Mean & $\sigma$ & Mean & $\sigma$ & Mean & $\sigma$ \\
\hline 0.80 & 1.161 & 0.045 & 1.144 & 0.044 & 1.128 & 0.044 & 1.113 & 0.046 \\
0.85 & 1.105 & 0.044 & 1.089 & 0.042 & 1.073 & 0.041 & 1.060 & 0.042 \\
0.90 & 1.055 & 0.044 & 1.039 & 0.041 & 1.025 & 0.039 & 1.012 & 0.039 \\
0.95 & 1.009 & 0.045 & 0.994 & 0.041 & 0.981 & 0.038 & 0.968 & 0.037 \\
\hline
\end{tabular}

Table A.8: Means and standard deviations of design combined flexural and axial strength ratios, $\alpha_{P M}$, for Column Section 3 and $e / h \leq 0$

\begin{tabular}{ccccccccc}
\hline & \multicolumn{8}{c}{$\phi_{c}$} \\
\cline { 2 - 10 }$\phi_{s}$ & \multicolumn{2}{c}{0.60} & \multicolumn{2}{c}{0.65} & \multicolumn{2}{c}{0.70} & \multicolumn{2}{c}{0.75} \\
\cline { 2 - 10 } & Mean & $\sigma$ & Mean & $\sigma$ & Mean & $\sigma$ & Mean & $\sigma$ \\
\hline 0.80 & 1.145 & 0.028 & 1.138 & 0.021 & 1.132 & 0.017 & 1.126 & 0.015 \\
0.85 & 1.083 & 0.032 & 1.076 & 0.025 & 1.070 & 0.019 & 1.065 & 0.015 \\
0.90 & 1.027 & 0.035 & 1.021 & 0.028 & 1.015 & 0.022 & 1.010 & 0.017 \\
0.95 & 0.977 & 0.038 & 0.971 & 0.031 & 0.966 & 0.025 & 0.961 & 0.020 \\
\hline
\end{tabular}

Table A.9: Means and standard deviations of design combined flexural and axial strength ratios, $\alpha_{P M}$, for Column Section 4 and $0 \leq e / h \leq 0.3$

\begin{tabular}{ccccccccc}
\hline & \multicolumn{8}{c}{$\phi_{c}$} \\
\cline { 2 - 9 }$\phi_{s}$ & \multicolumn{2}{c}{0.60} & \multicolumn{2}{c}{0.65} & \multicolumn{2}{c}{0.70} & \multicolumn{2}{c}{0.75} \\
\cline { 2 - 10 } & Mean & $\sigma$ & Mean & $\sigma$ & Mean & $\sigma$ & Mean & $\sigma$ \\
\hline 0.80 & 0.998 & 0.040 & 0.943 & 0.027 & 0.895 & 0.016 & 0.852 & 0.007 \\
0.85 & 0.979 & 0.048 & 0.926 & 0.034 & 0.880 & 0.023 & 0.837 & 0.014 \\
0.90 & 0.961 & 0.055 & 0.910 & 0.042 & 0.865 & 0.030 & 0.824 & 0.020 \\
0.95 & 0.944 & 0.062 & 0.894 & 0.048 & 0.850 & 0.036 & 0.811 & 0.026 \\
\hline
\end{tabular}


Table A.10: Means and standard deviations of design combined flexural and axial strength ratios, $\alpha_{P M}$, for Column Section 4 and $0.3<e / h \leq 1.0$

\begin{tabular}{ccccccccc}
\hline & \multicolumn{8}{c}{$\phi_{c}$} \\
\cline { 2 - 9 }$\phi_{s}$ & \multicolumn{2}{c}{0.60} & \multicolumn{2}{c}{0.65} & \multicolumn{2}{c}{0.70} & \multicolumn{2}{c}{0.75} \\
\cline { 2 - 10 } 0 Mean & $\sigma$ & Mean & $\sigma$ & Mean & $\sigma$ & Mean & $\sigma$ \\
\hline 0.80 & 1.084 & 0.129 & 1.047 & 0.127 & 1.013 & 0.126 & 0.983 & 0.126 \\
0.85 & 1.048 & 0.124 & 1.012 & 0.121 & 0.980 & 0.119 & 0.950 & 0.118 \\
0.90 & 1.015 & 0.120 & 0.980 & 0.116 & 0.949 & 0.114 & 0.920 & 0.112 \\
0.95 & 0.984 & 0.116 & 0.951 & 0.112 & 0.920 & 0.109 & 0.893 & 0.108 \\
\hline
\end{tabular}

Table A.11: Means and standard deviations of design combined flexural and axial strength ratios, $\alpha_{P M}$, for Column Section 4 and $1.0<e / h \leq 10.0$

\begin{tabular}{ccccccccc}
\hline & \multicolumn{8}{c}{$\phi_{c}$} \\
\cline { 2 - 9 }$\phi_{s}$ & \multicolumn{2}{c}{0.60} & \multicolumn{2}{c}{0.65} & \multicolumn{2}{c}{0.70} & \multicolumn{2}{c}{0.75} \\
\cline { 2 - 10 } 0 Mean & $\sigma$ & Mean & $\sigma$ & Mean & $\sigma$ & Mean & $\sigma$ \\
\hline 0.80 & 1.145 & 0.065 & 1.128 & 0.067 & 1.112 & 0.069 & 1.097 & 0.071 \\
0.85 & 1.090 & 0.060 & 1.074 & 0.062 & 1.059 & 0.064 & 1.045 & 0.065 \\
0.90 & 1.041 & 0.055 & 1.025 & 0.057 & 1.011 & 0.059 & 0.998 & 0.060 \\
0.95 & 0.997 & 0.051 & 0.981 & 0.053 & 0.968 & 0.055 & 0.955 & 0.056 \\
\hline
\end{tabular}

Table A.12: Means and standard deviations of design combined flexural and axial strength ratios, $\alpha_{P M}$, for Column Section 4 and $e / h \leq 0$

\begin{tabular}{ccccccccc}
\hline & \multicolumn{8}{c}{$\phi_{c}$} \\
\cline { 2 - 10 }$\phi_{s}$ & \multicolumn{2}{c}{0.60} & \multicolumn{2}{c}{0.65} & \multicolumn{2}{c}{0.70} & \multicolumn{2}{c}{0.75} \\
\cline { 2 - 10 } & Mean & $\sigma$ & Mean & $\sigma$ & Mean & $\sigma$ & Mean & $\sigma$ \\
\hline 0.80 & 1.145 & 0.032 & 1.136 & 0.030 & 1.128 & 0.030 & 1.121 & 0.032 \\
0.85 & 1.084 & 0.033 & 1.076 & 0.030 & 1.068 & 0.028 & 1.061 & 0.028 \\
0.90 & 1.029 & 0.034 & 1.021 & 0.030 & 1.014 & 0.028 & 1.008 & 0.027 \\
0.95 & 0.980 & 0.036 & 0.972 & 0.031 & 0.966 & 0.028 & 0.959 & 0.026 \\
\hline
\end{tabular}




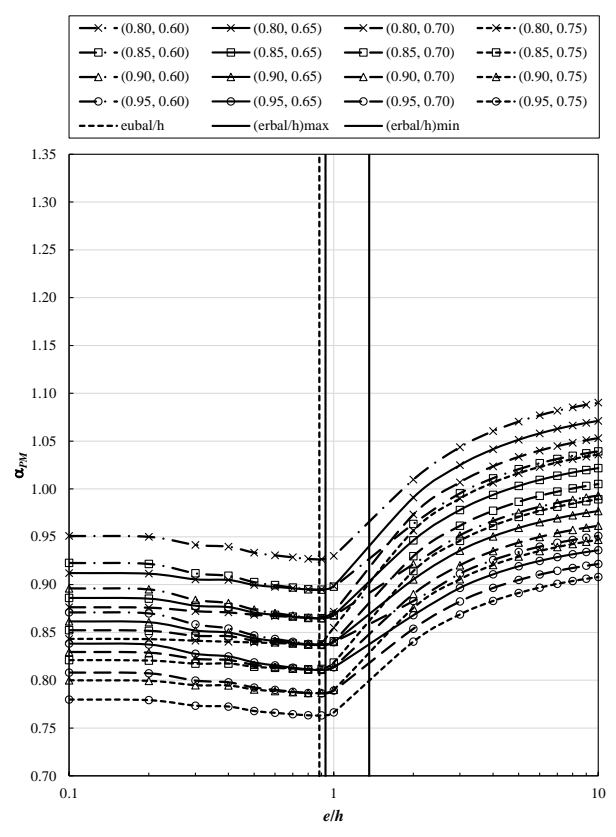

(a)

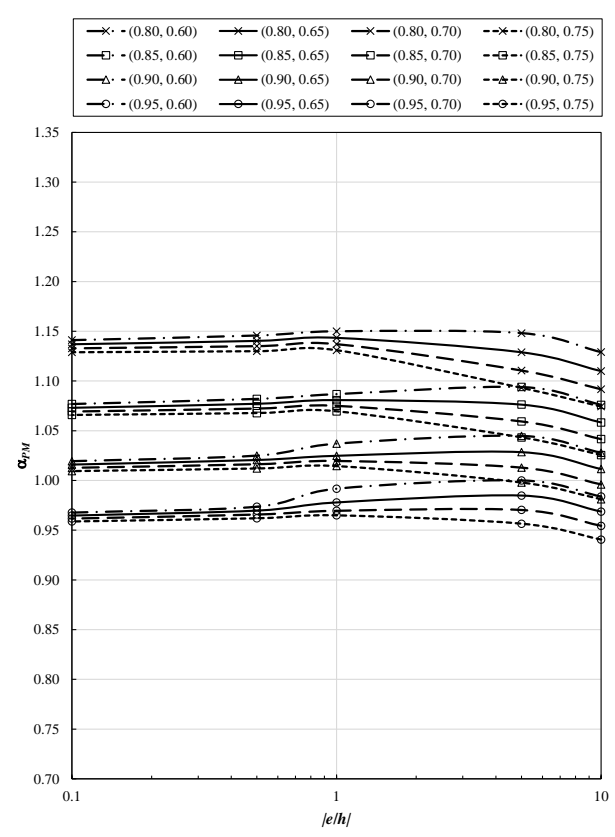

(b)

Figure A.1: Design combined flexural and axial strength ratios, $\alpha_{P M}$, for Column Section 1 and Property Combination 2: (a) $e / h>0$; (b) $e / h<0$

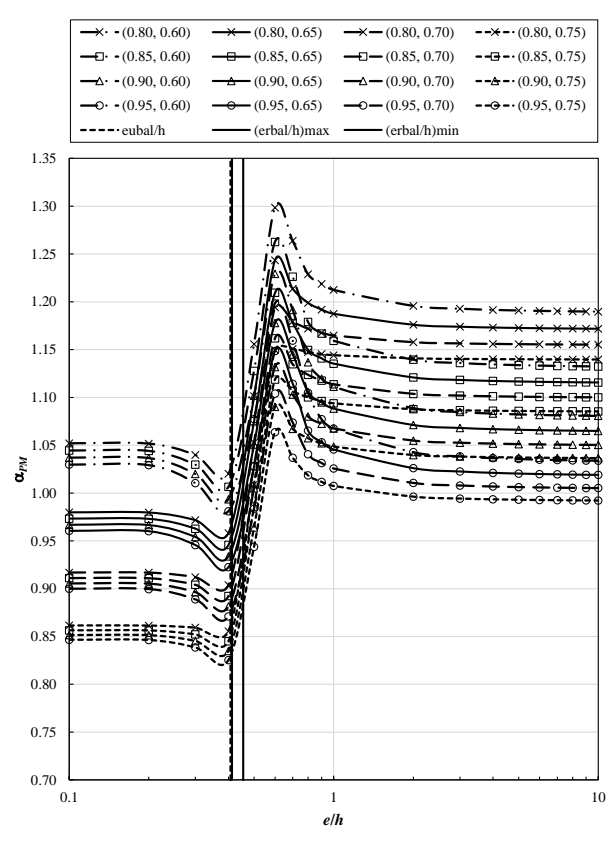

(a)

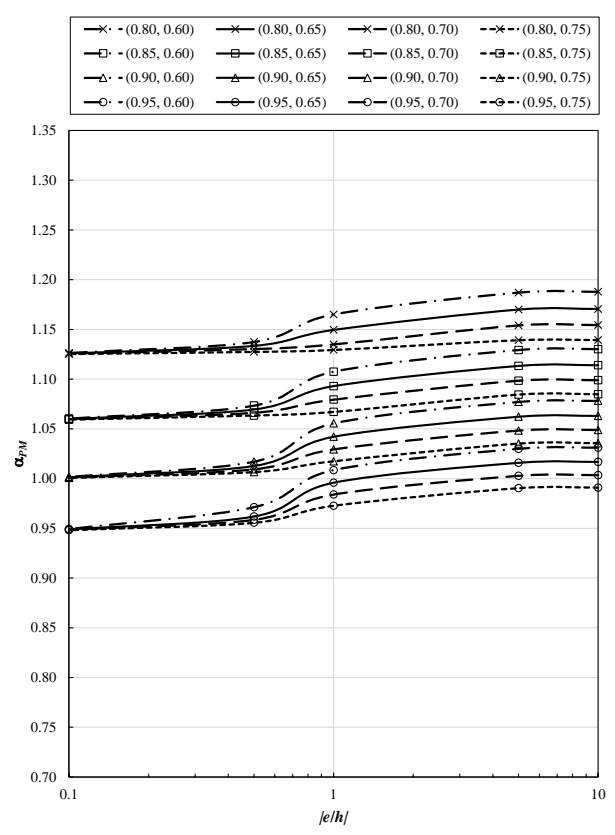

(b)

Figure A.2: Design combined flexural and axial strength ratios, $\alpha_{P M}$, for Column Section 1 and Property Combination 3: (a) $e / h>0$; (b) $e / h<0$ 


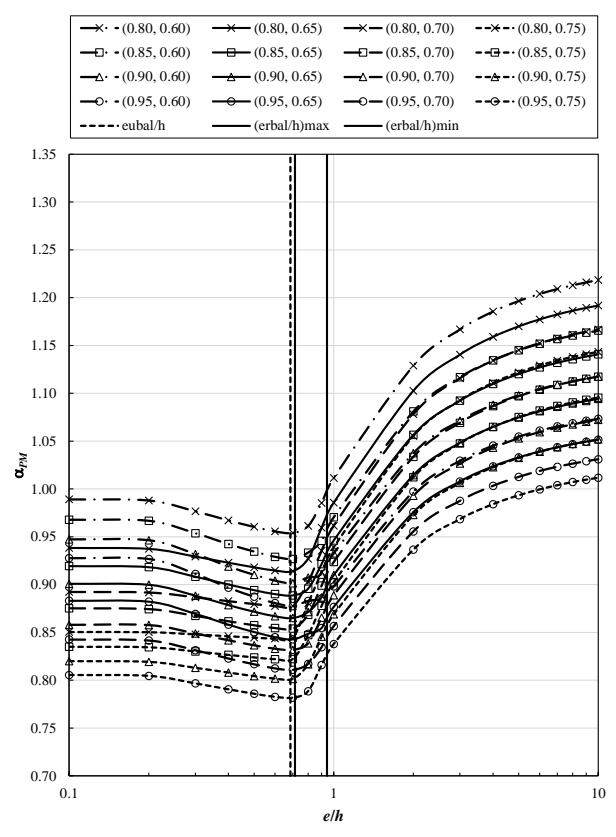

(a)

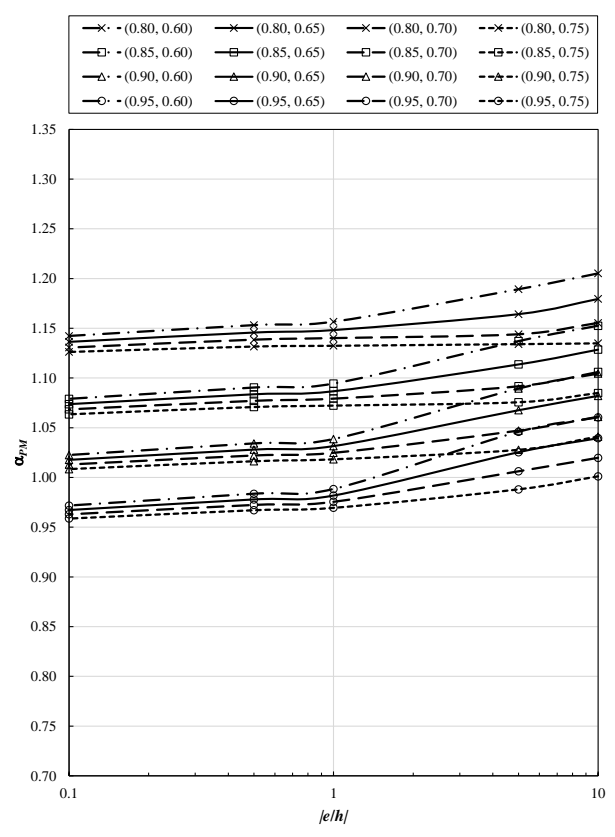

(b)

Figure A.3: Design combined flexural and axial strength ratios, $\alpha_{P M}$, for Column Section 1 and Property Combination 4: (a) $e / h>0$; (b) $e / h<0$

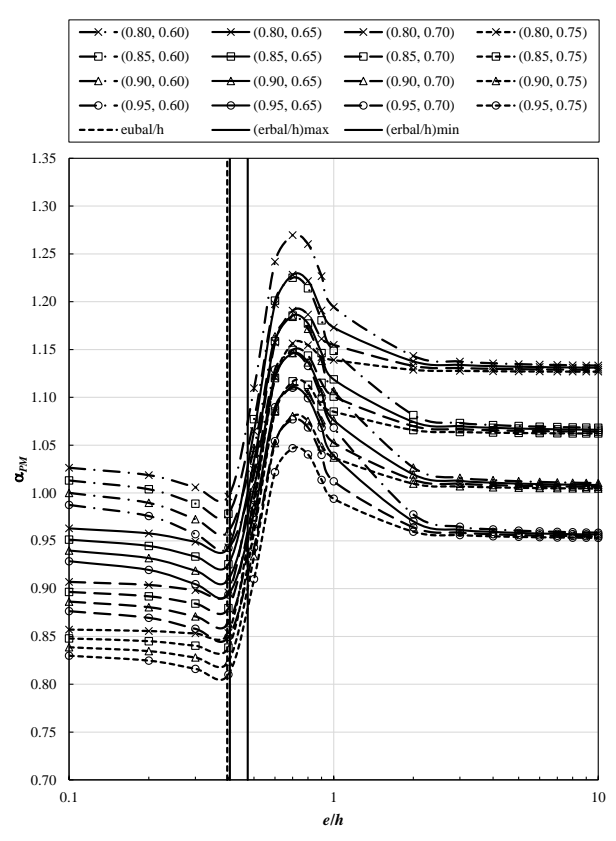

(a)

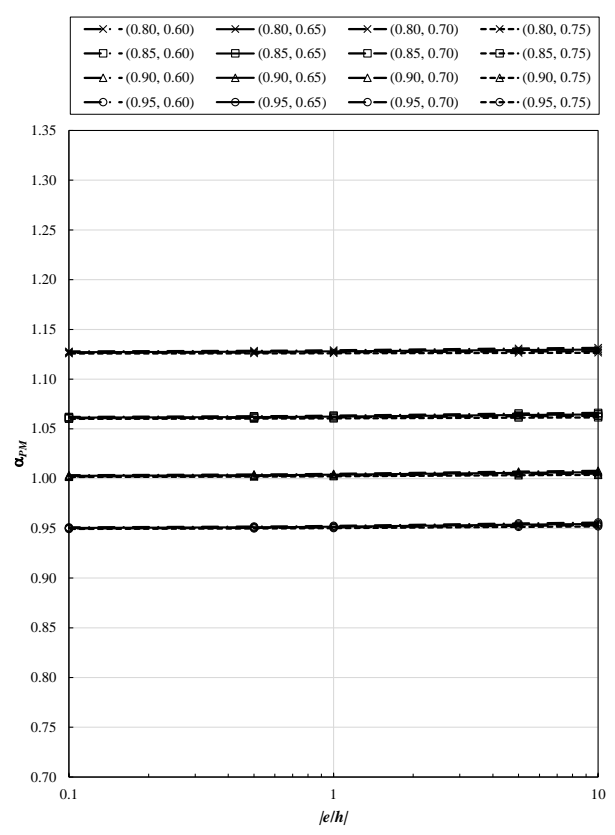

(b)

Figure A.4: Design combined flexural and axial strength ratios, $\alpha_{P M}$, for Column Section 1 and Property Combination 5: (a) $e / h>0$; (b) $e / h<0$ 


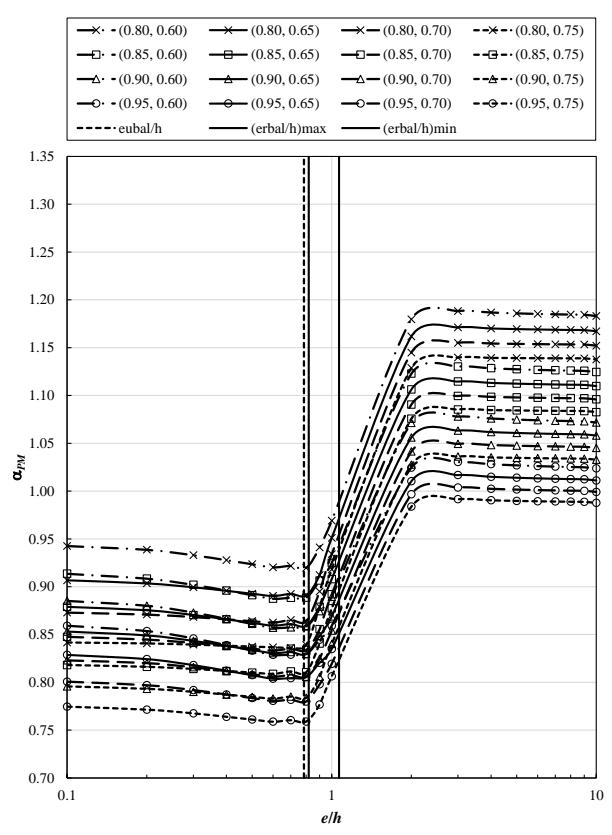

(a)

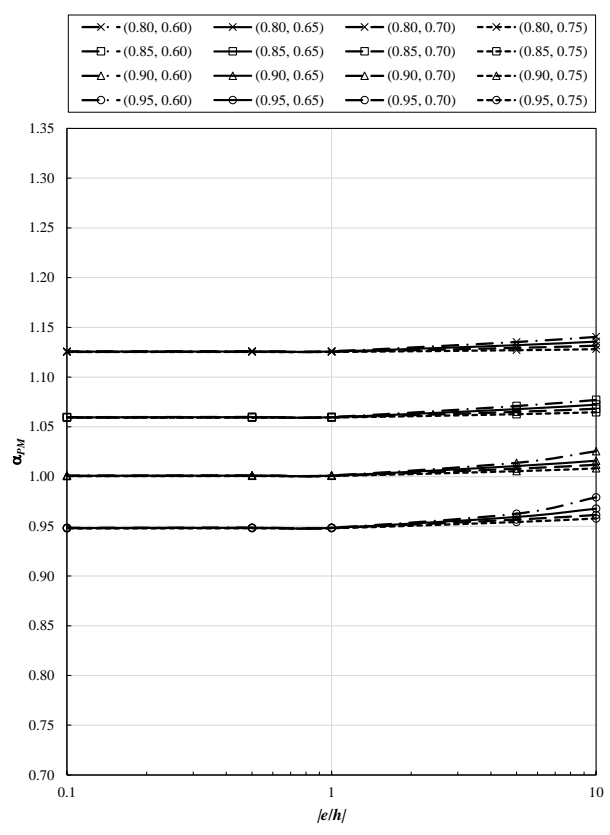

(b)

Figure A.5: Design combined flexural and axial strength ratios, $\alpha_{P M}$, for Column Section 1 and Property Combination 6: (a) $e / h>0$; (b) $e / h<0$

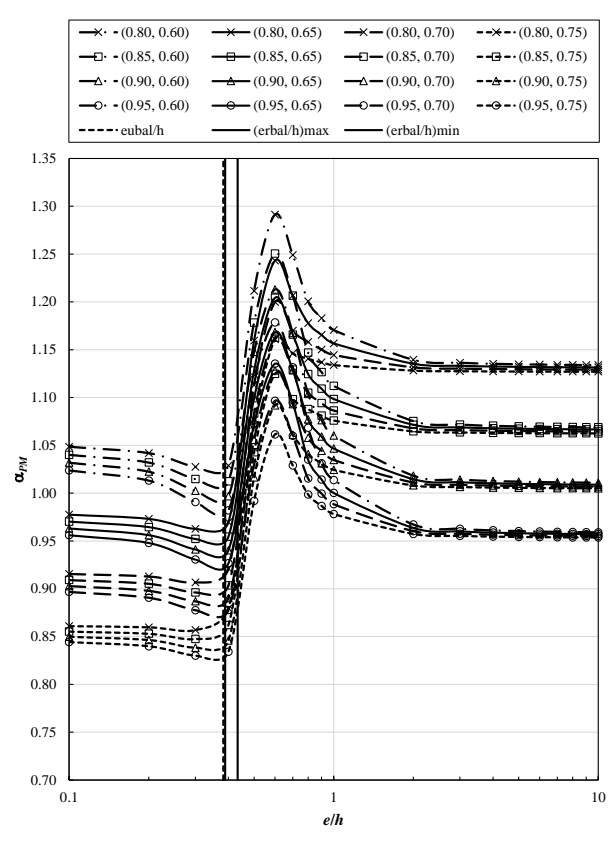

(a)

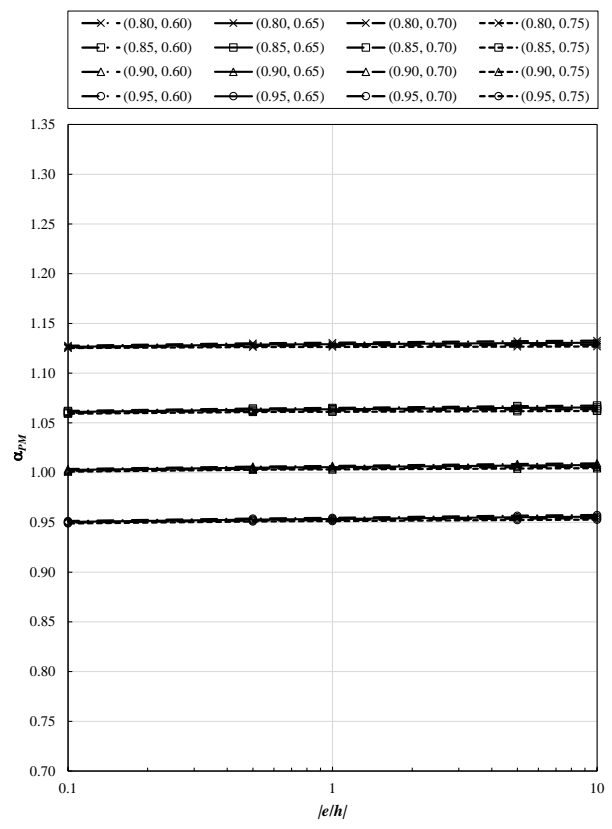

(b)

Figure A.6: Design combined flexural and axial strength ratios, $\alpha_{P M}$, for Column Section 1 and Property Combination 7: (a) $e / h>0$; (b) $e / h<0$ 


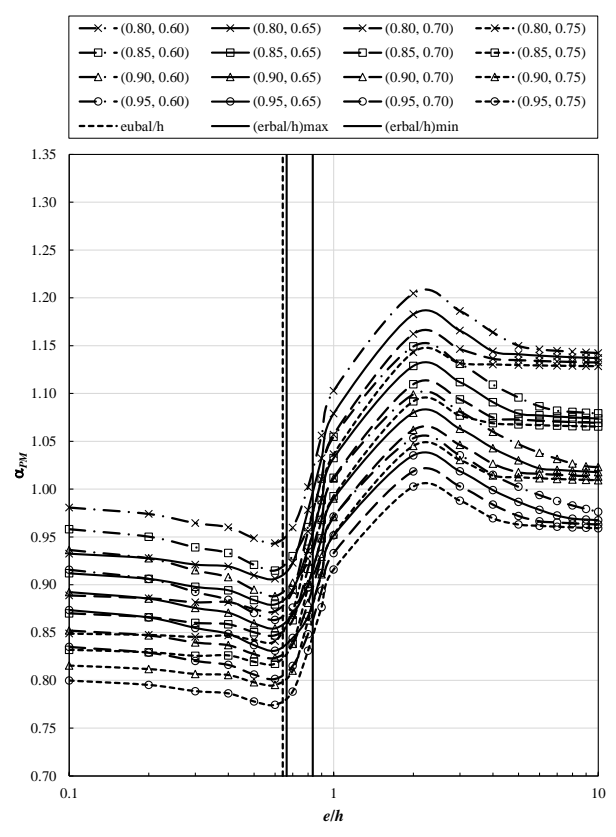

(a)

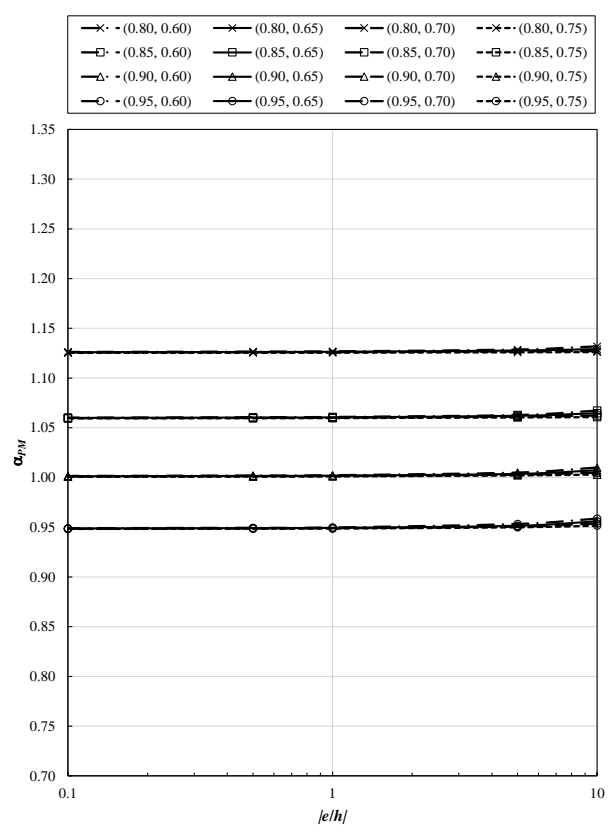

(b)

Figure A.7: Design combined flexural and axial strength ratios, $\alpha_{P M}$, for Column Section 1 and Property Combination 8: (a) $e / h>0$; (b) $e / h<0$

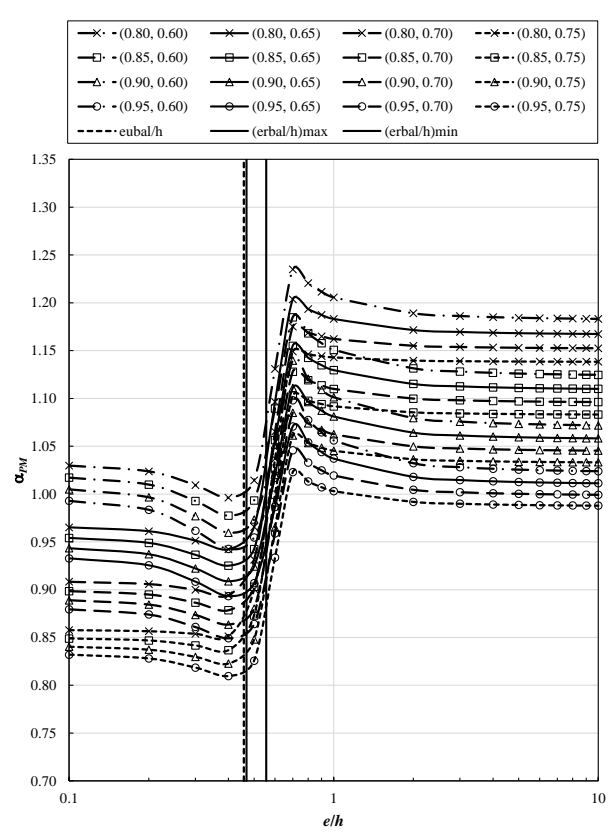

(a)

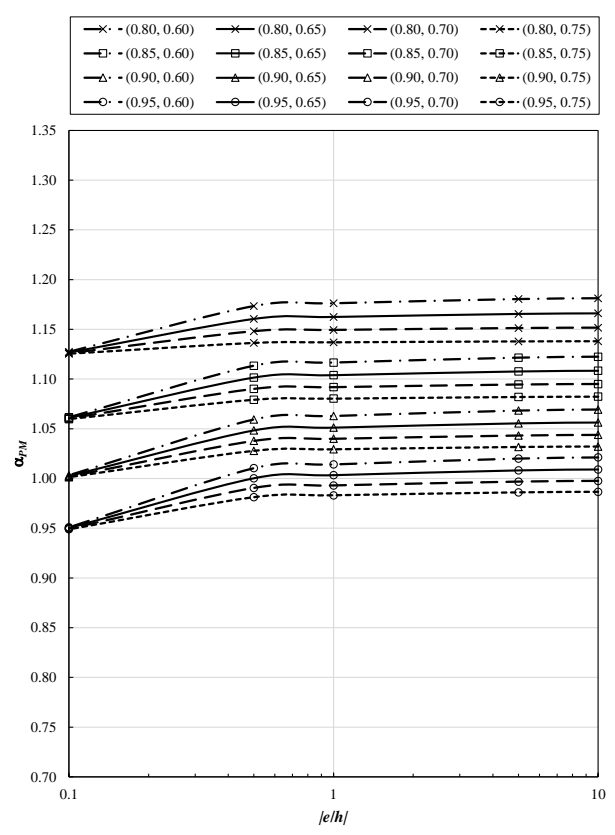

(b)

Figure A.8: Design combined flexural and axial strength ratios, $\alpha_{P M}$, for Column Section 2 and Property Combination 1: (a) $e / h>0$; (b) $e / h<0$ 


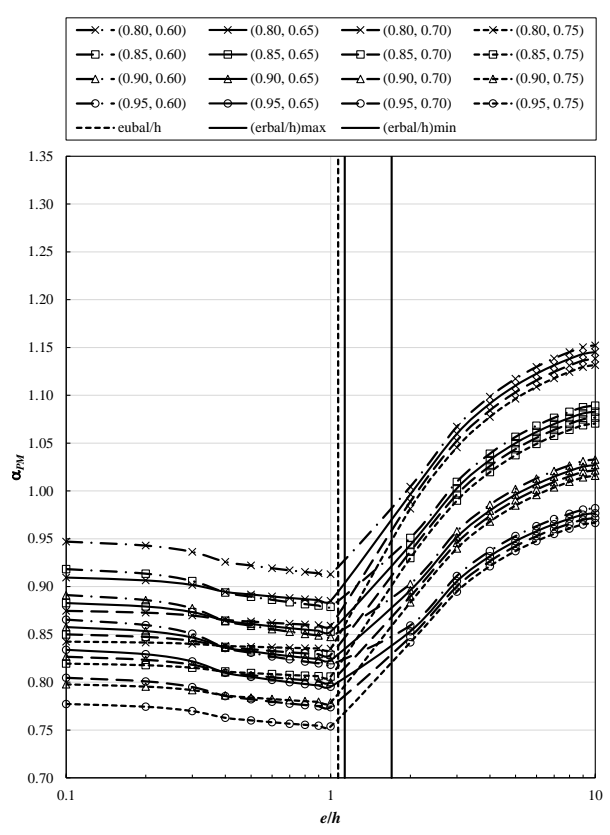

(a)

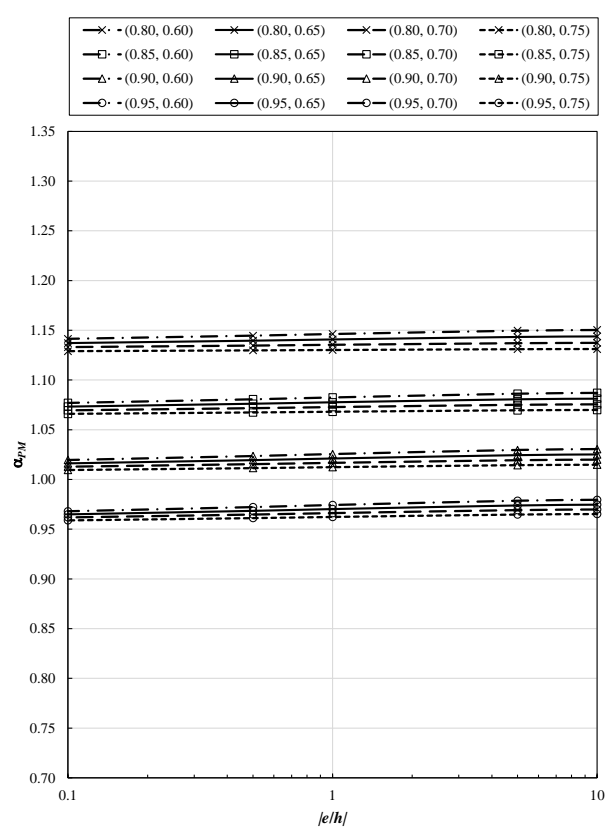

(b)

Figure A.9: Design combined flexural and axial strength ratios, $\alpha_{P M}$, for Column Section 2 and Property Combination 2: (a) $e / h>0$; (b) $e / h<0$

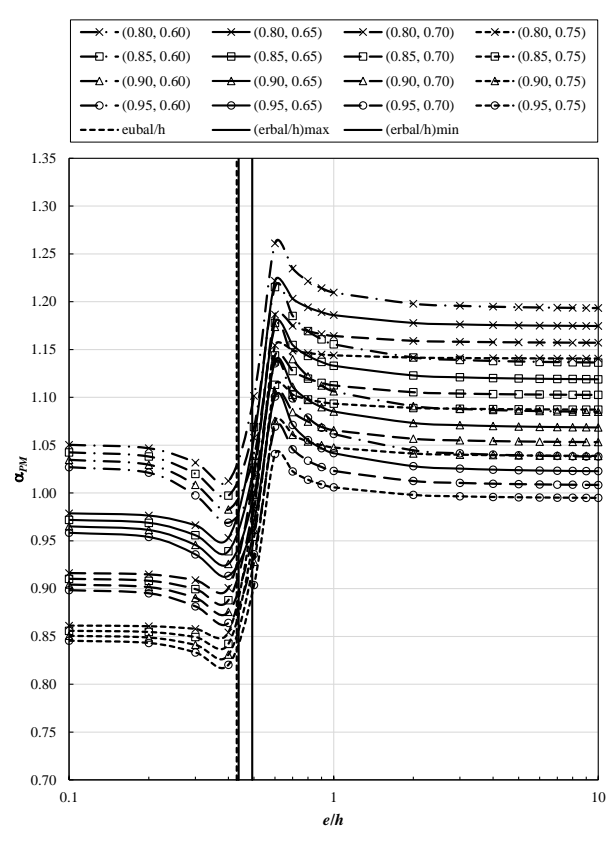

(a)

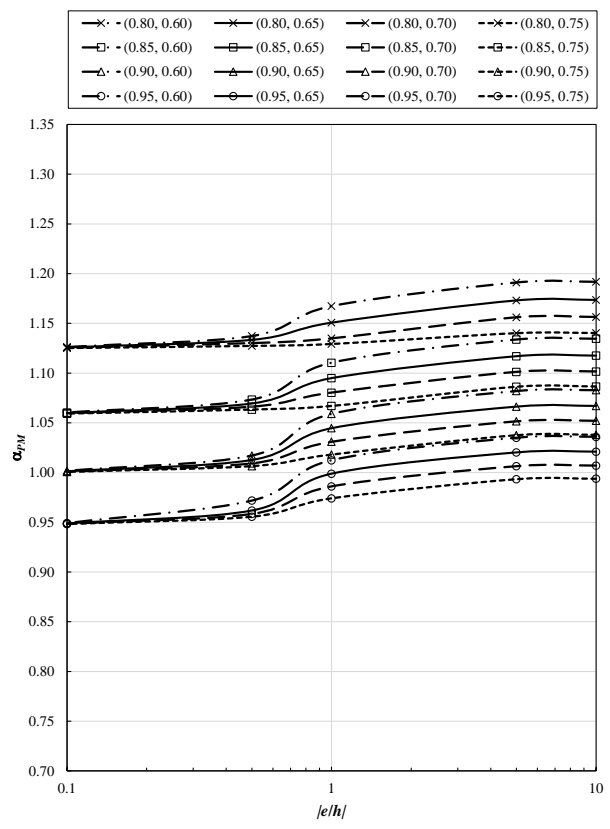

(b)

Figure A.10: Design combined flexural and axial strength ratios, $\alpha_{P M}$, for Column Section 2 and Property Combination 3: (a) $e / h>0$; (b) $e / h<0$ 


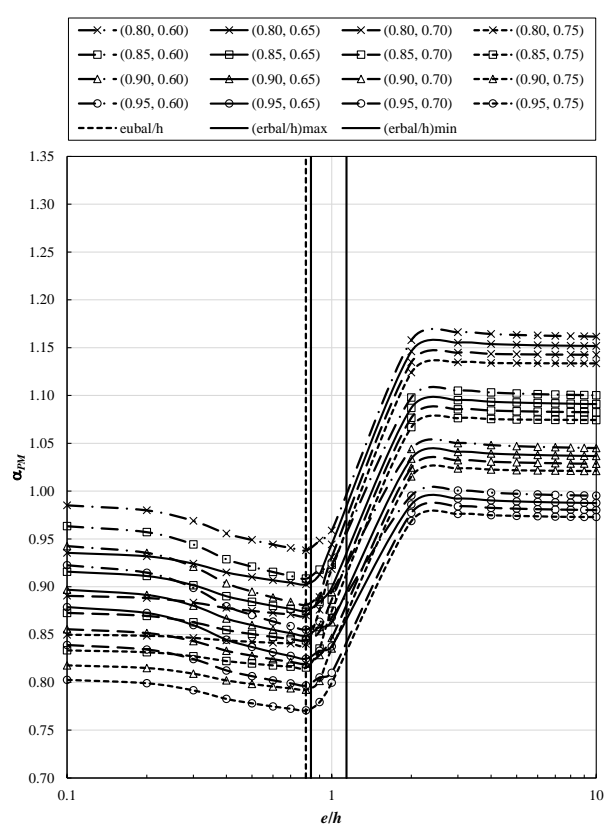

(a)

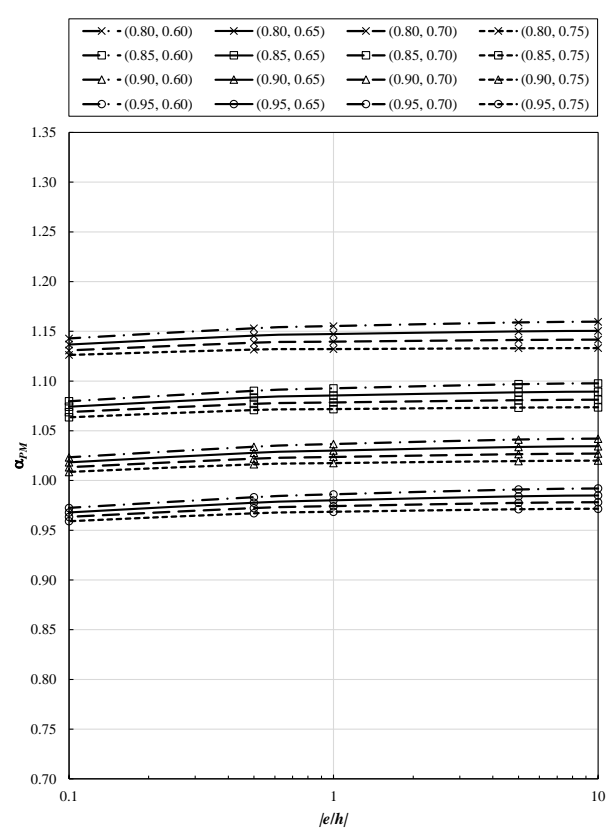

(b)

Figure A.11: Design combined flexural and axial strength ratios, $\alpha_{P M}$, for Column Section 2 and Property Combination 4: (a) $e / h>0$; (b) $e / h<0$

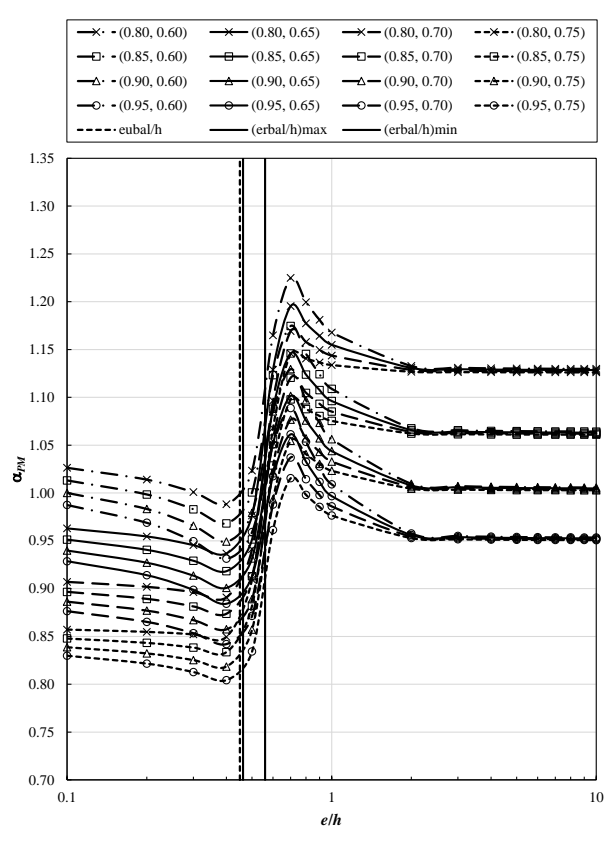

(a)

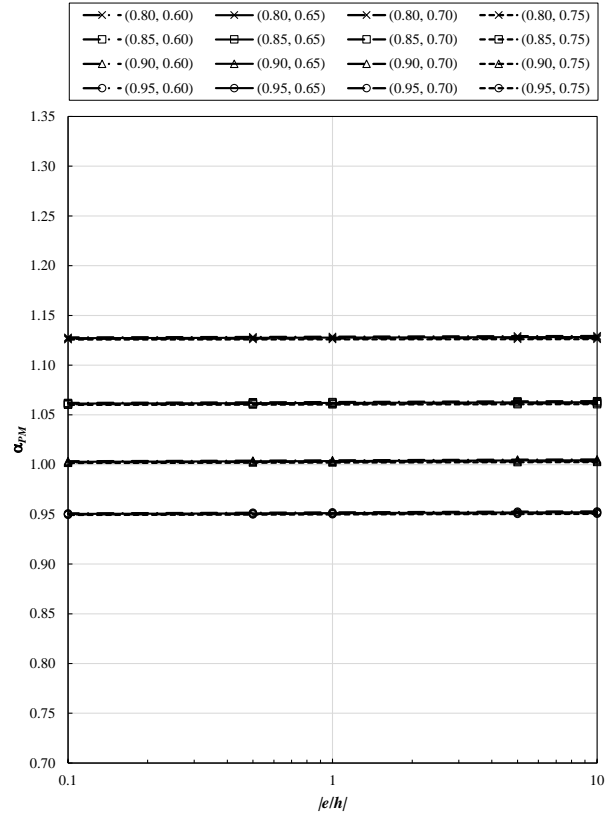

(b)

Figure A.12: Design combined flexural and axial strength ratios, $\alpha_{P M}$, for Column Section 2 and Property Combination 5: (a) $e / h>0$; (b) $e / h<0$ 


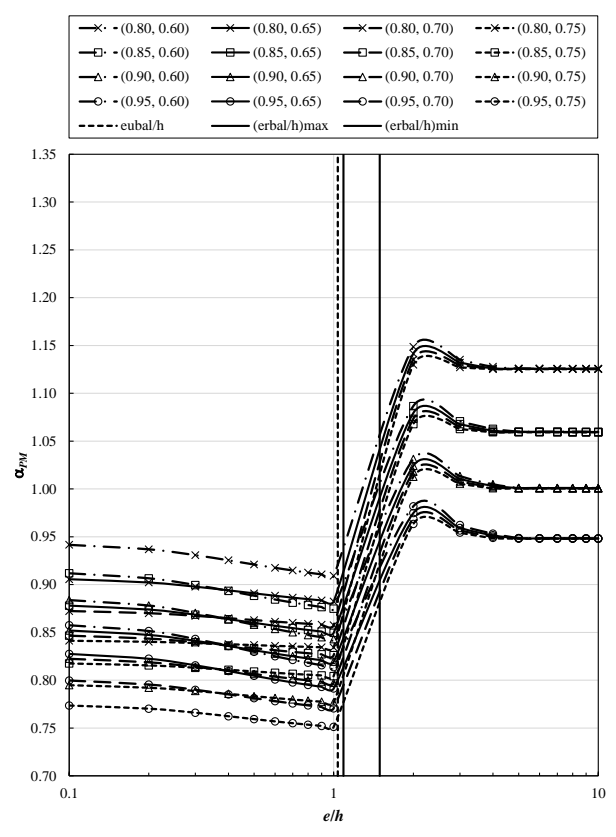

(a)

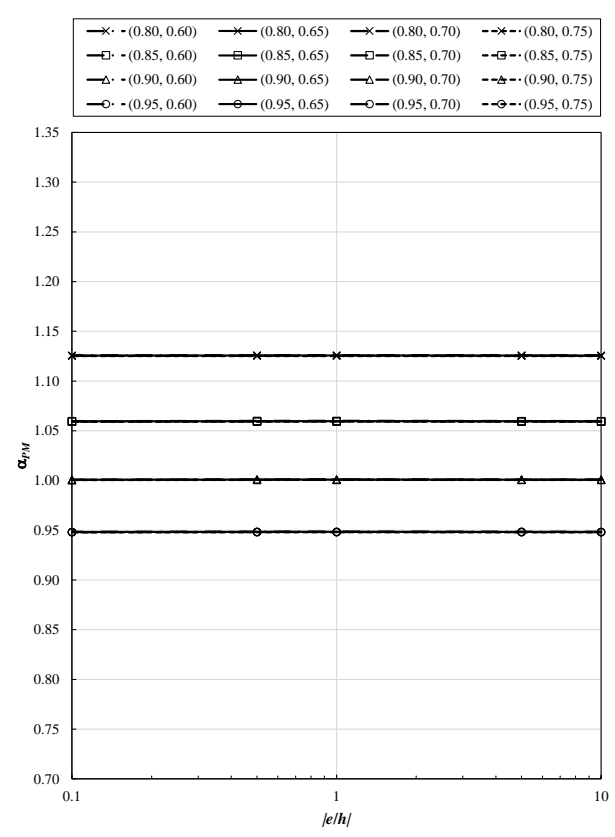

(b)

Figure A.13: Design combined flexural and axial strength ratios, $\alpha_{P M}$, for Column Section 2 and Property Combination 6: (a) $e / h>0$; (b) $e / h<0$

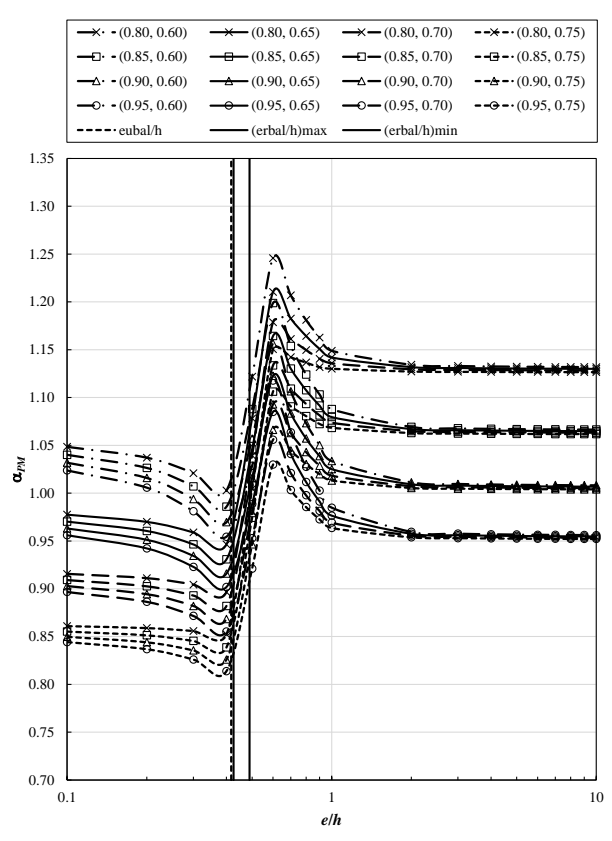

(a)

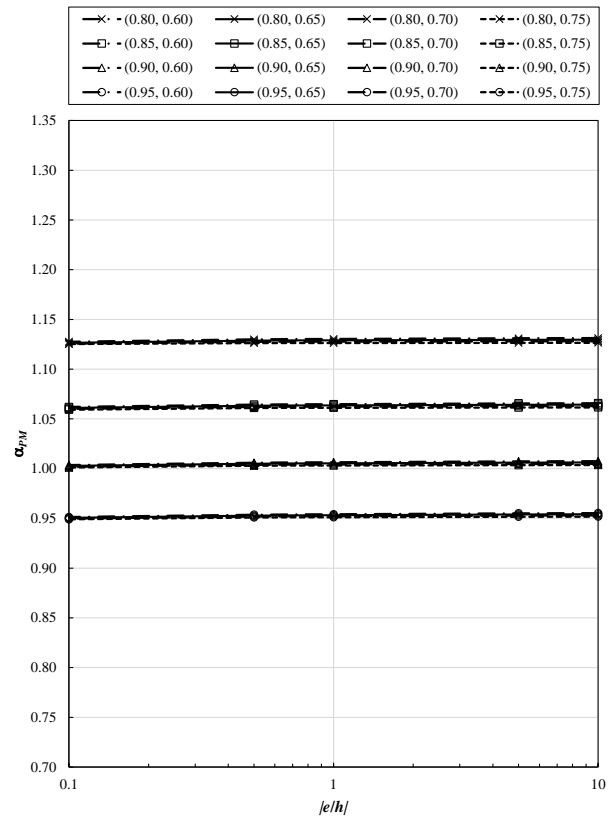

(b)

Figure A.14: Design combined flexural and axial strength ratios, $\alpha_{P M}$, for Column Section 2 and Property Combination 7: (a) $e / h>0$; (b) $e / h<0$ 


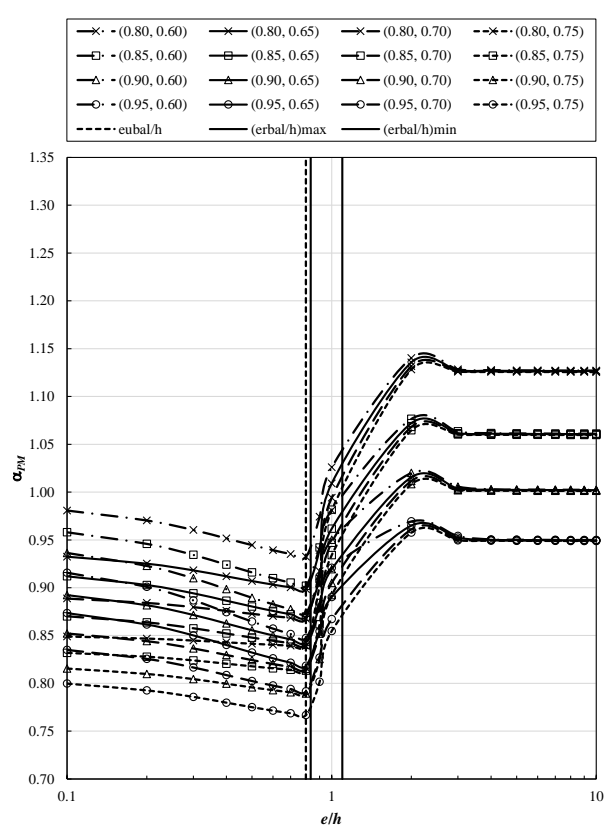

(a)

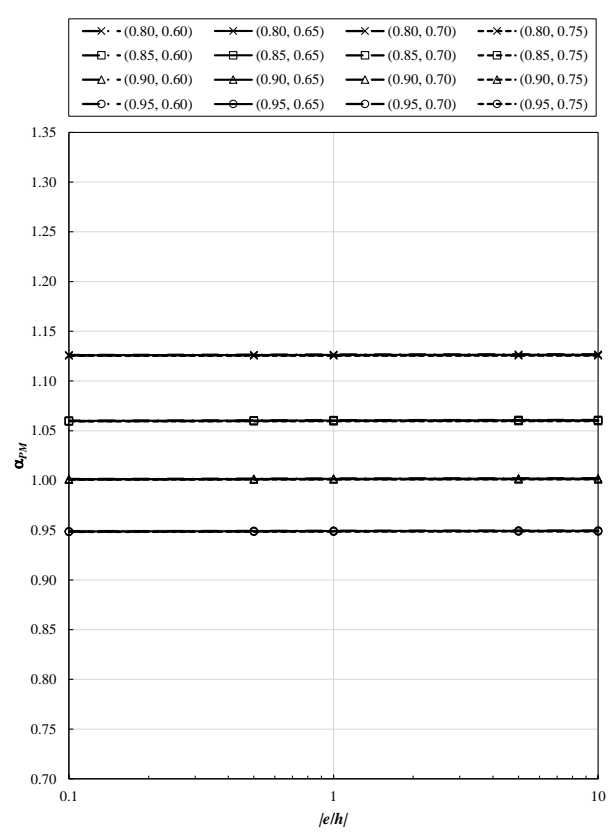

(b)

Figure A.15: Design combined flexural and axial strength ratios, $\alpha_{P M}$, for Column Section 2 and Property Combination 8: (a) $e / h>0$; (b) $e / h<0$

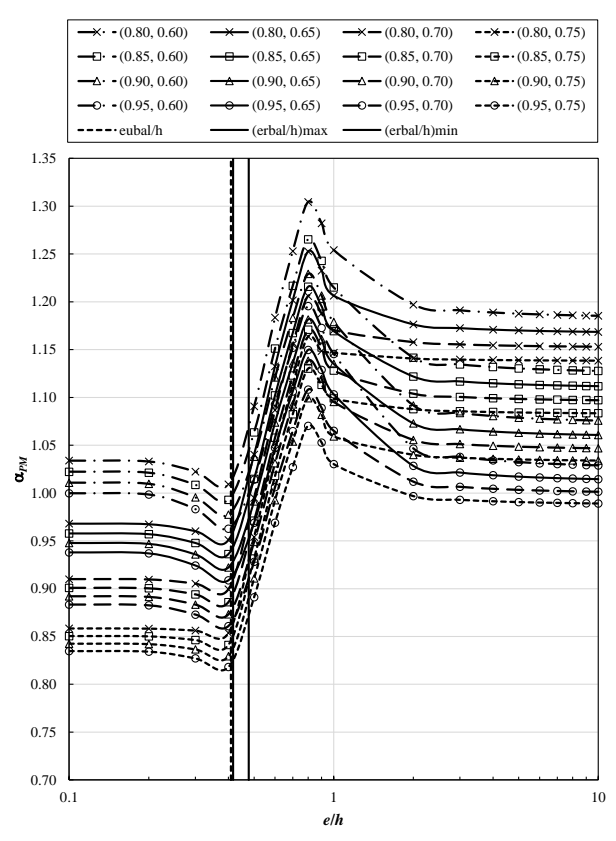

(a)

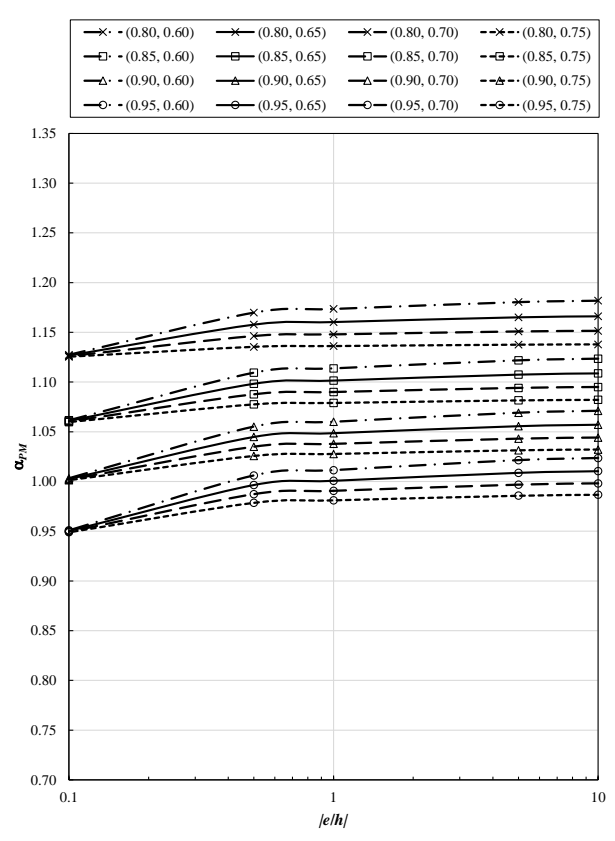

(b)

Figure A.16: Design combined flexural and axial strength ratios, $\alpha_{P M}$, for Column Section 3 and Property Combination 1: (a) $e / h>0$; (b) $e / h<0$ 


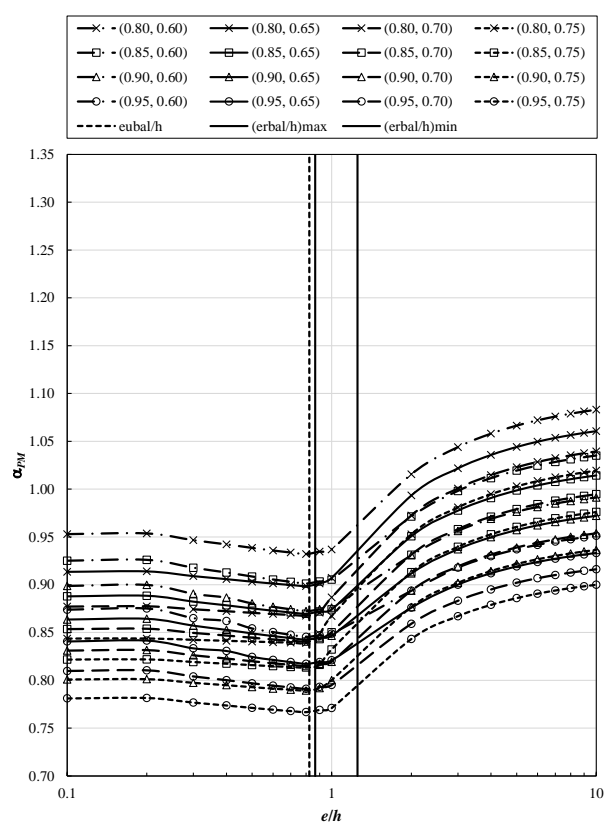

(a)

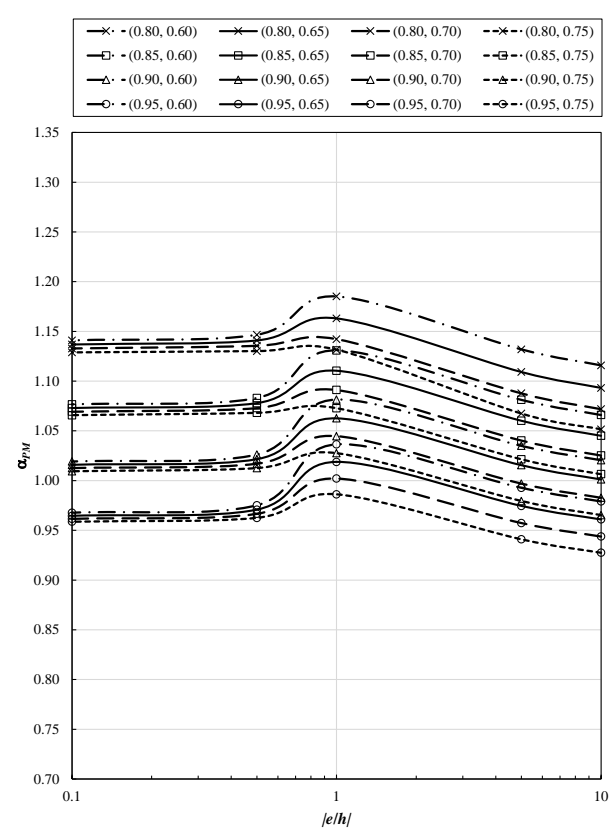

(b)

Figure A.17: Design combined flexural and axial strength ratios, $\alpha_{P M}$, for Column Section 3 and Property Combination 2: (a) $e / h>0$; (b) $e / h<0$

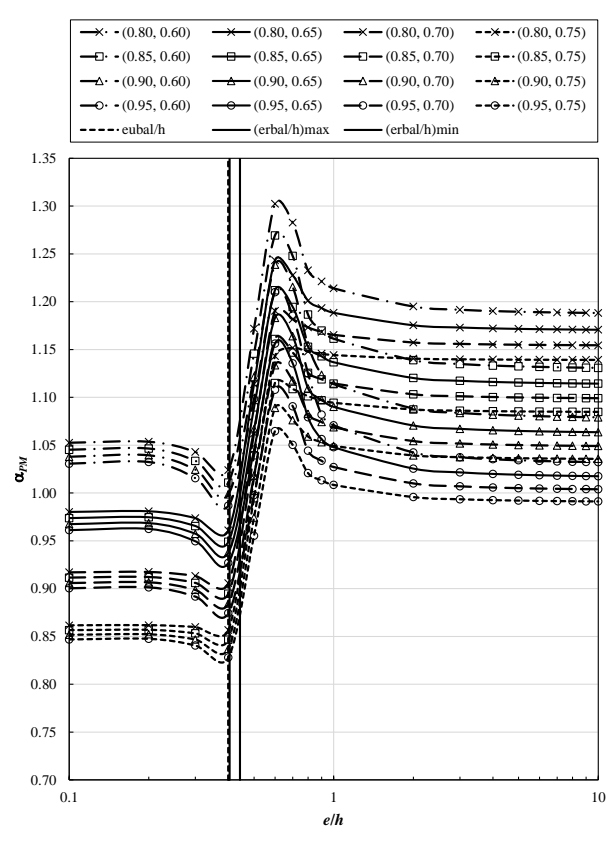

(a)

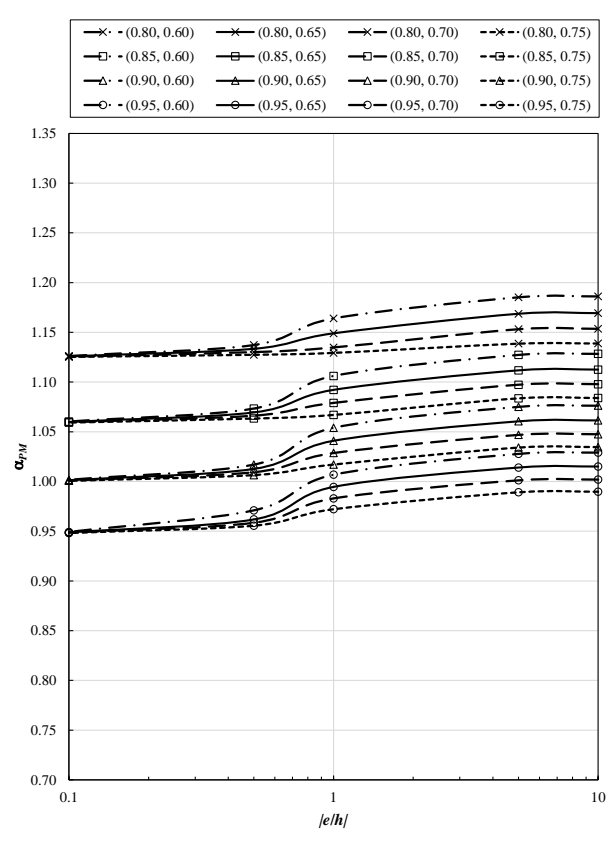

(b)

Figure A.18: Design combined flexural and axial strength ratios, $\alpha_{P M}$, for Column Section 3 and Property Combination 3: (a) $e / h>0$; (b) $e / h<0$ 


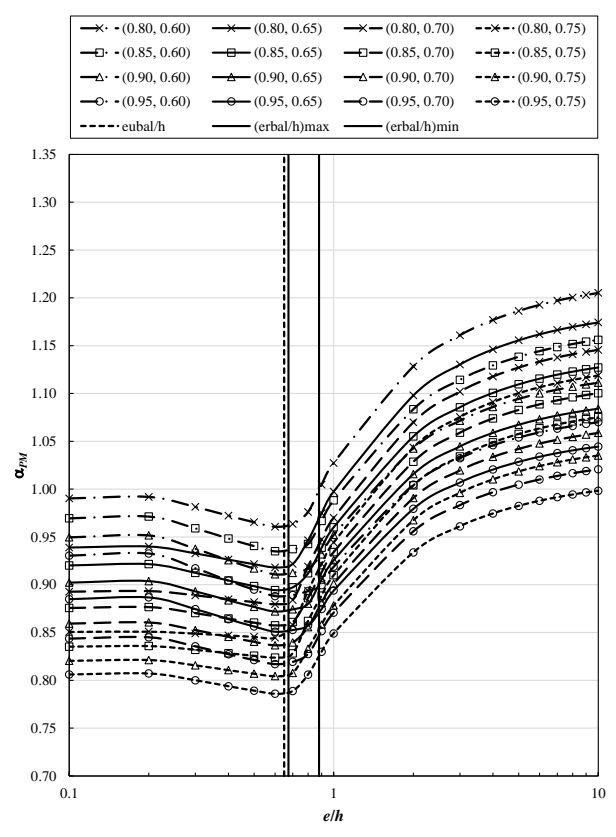

(a)

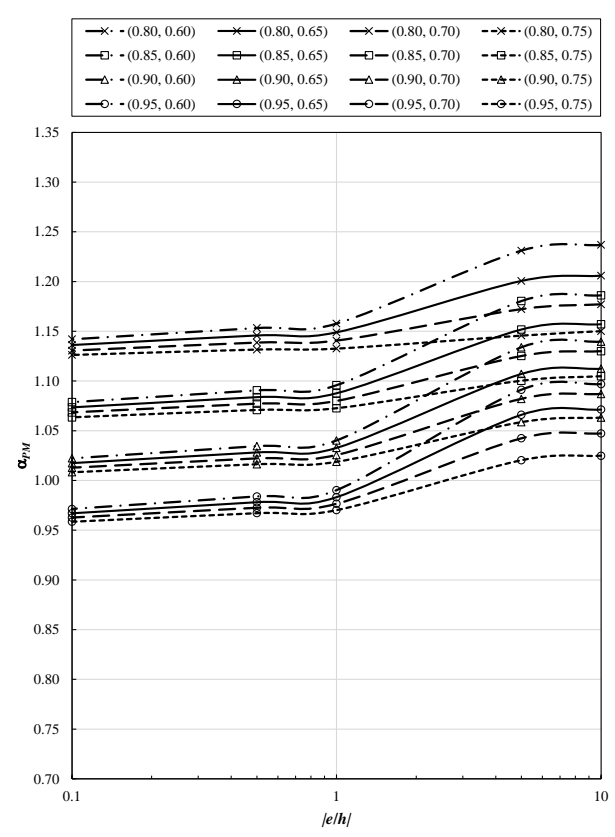

(b)

Figure A.19: Design combined flexural and axial strength ratios, $\alpha_{P M}$, for Column Section 3 and Property Combination 4: (a) $e / h>0$; (b) $e / h<0$

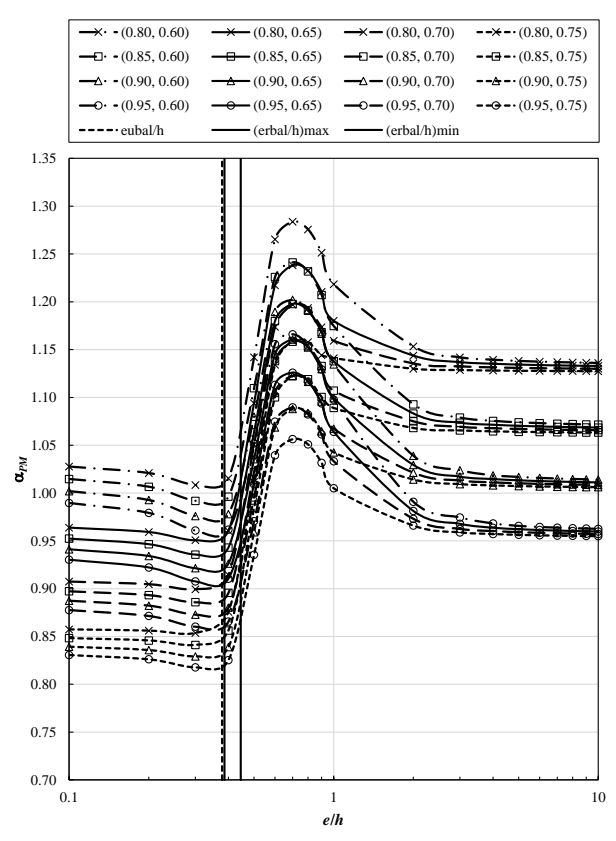

(a)

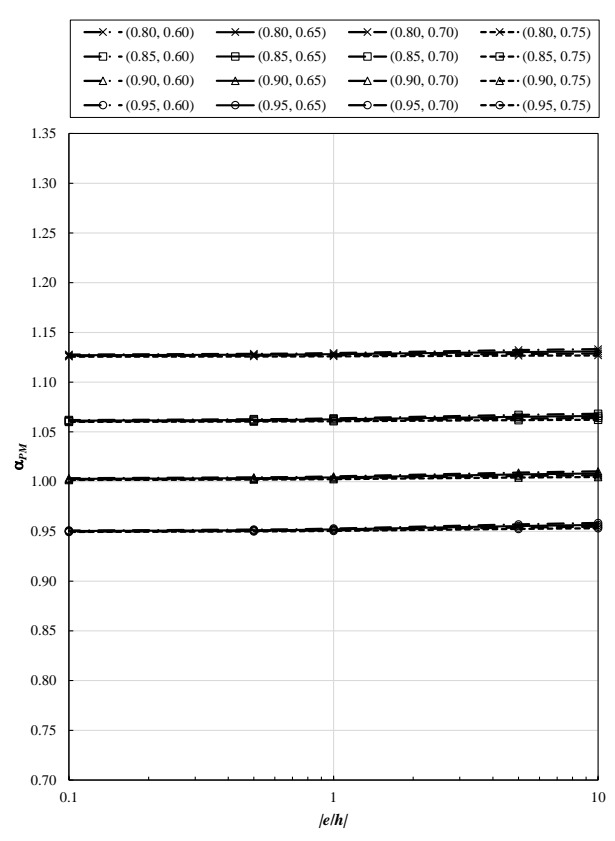

(b)

Figure A.20: Design combined flexural and axial strength ratios, $\alpha_{P M}$, for Column Section 3 and Property Combination 5: (a) $e / h>0$; (b) $e / h<0$ 


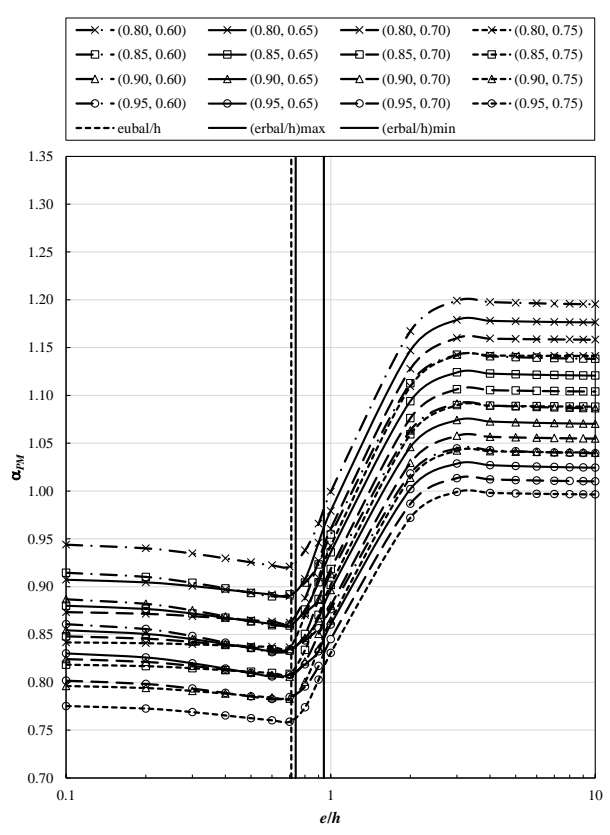

(a)

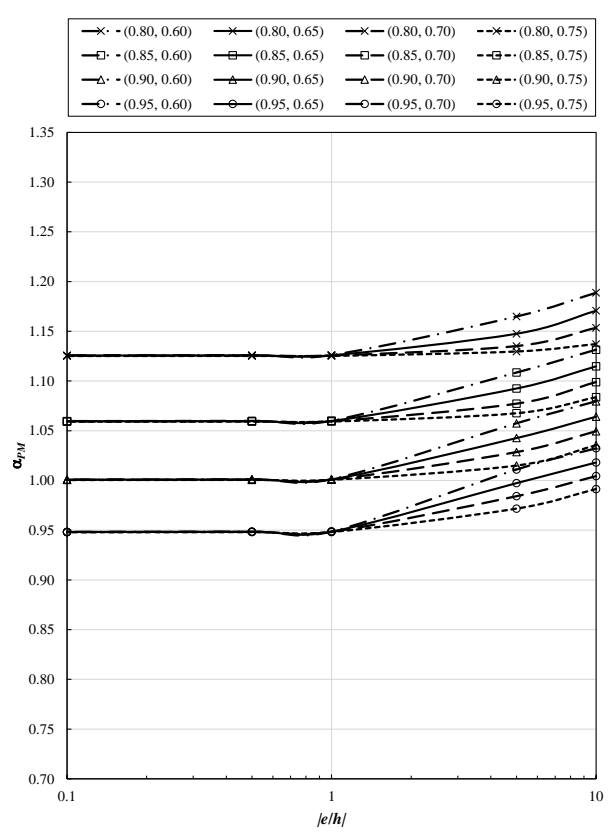

(b)

Figure A.21: Design combined flexural and axial strength ratios, $\alpha_{P M}$, for Column Section 3 and Property Combination 6: (a) $e / h>0$; (b) $e / h<0$

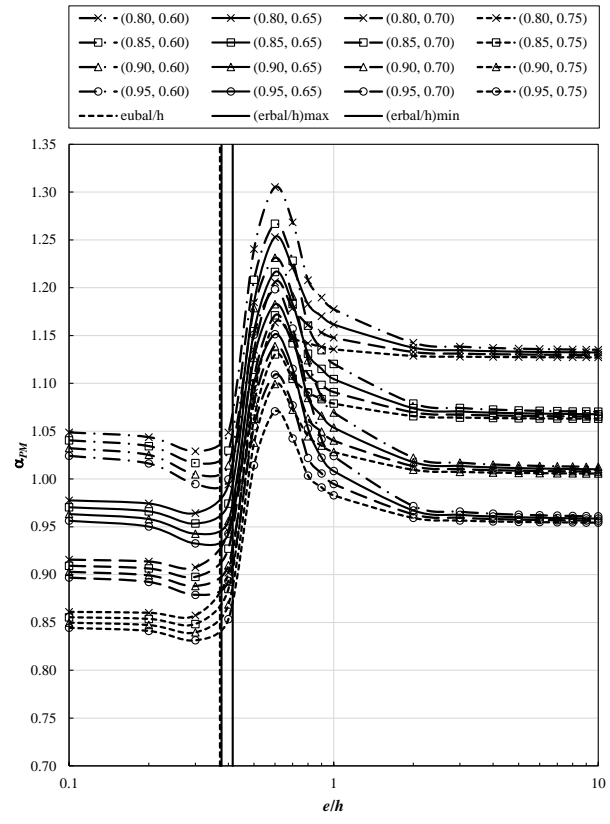

(a)

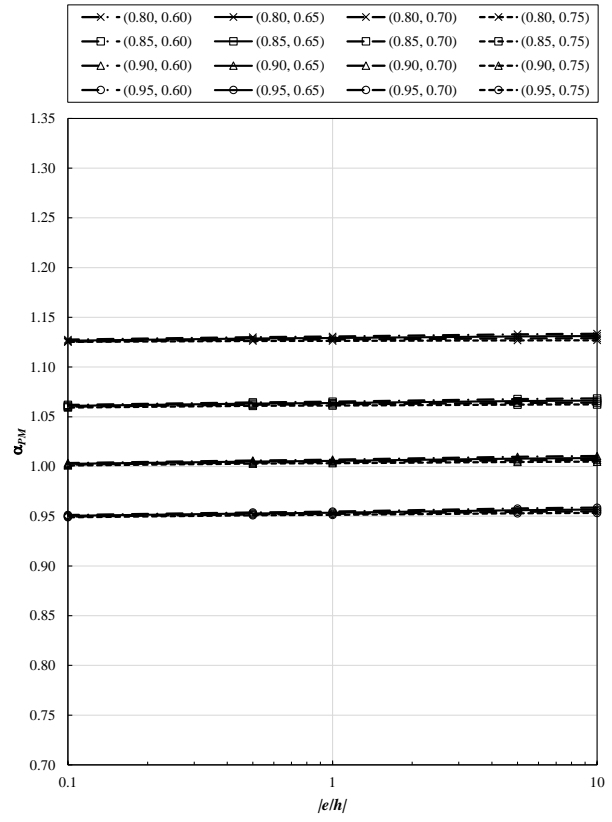

(b)

Figure A.22: Design combined flexural and axial strength ratios, $\alpha_{P M}$, for Column Section 3 and Property Combination 7: (a) $e / h>0$; (b) $e / h<0$ 


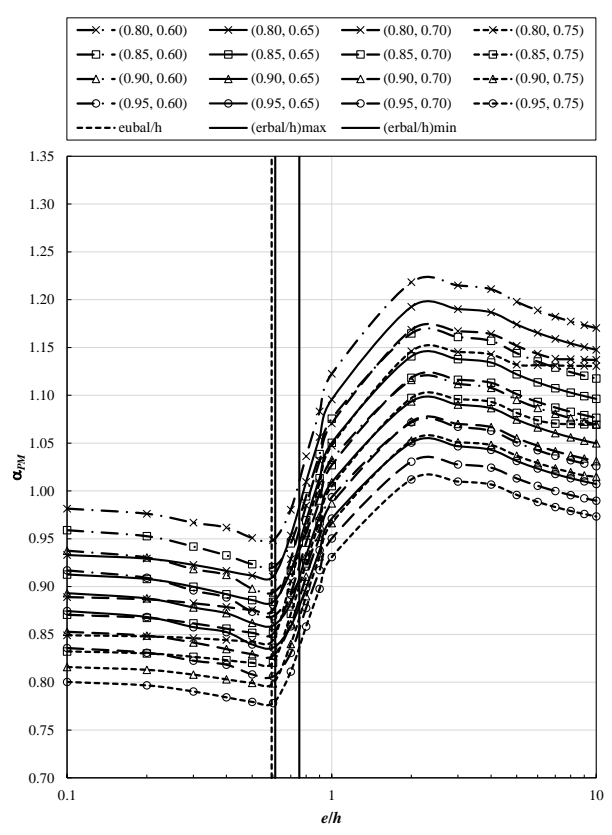

(a)

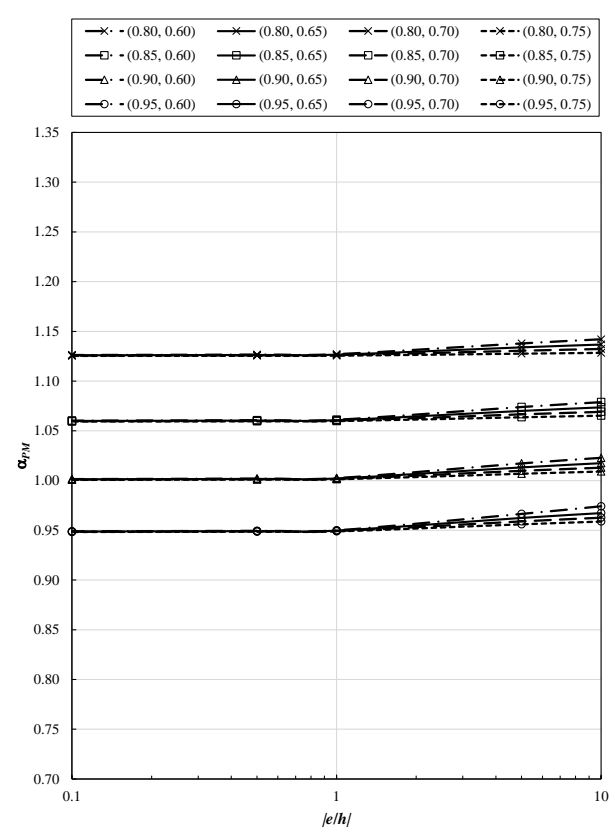

(b)

Figure A.23: Design combined flexural and axial strength ratios, $\alpha_{P M}$, for Column Section 3 and Property Combination 8: (a) $e / h>0$; (b) $e / h<0$

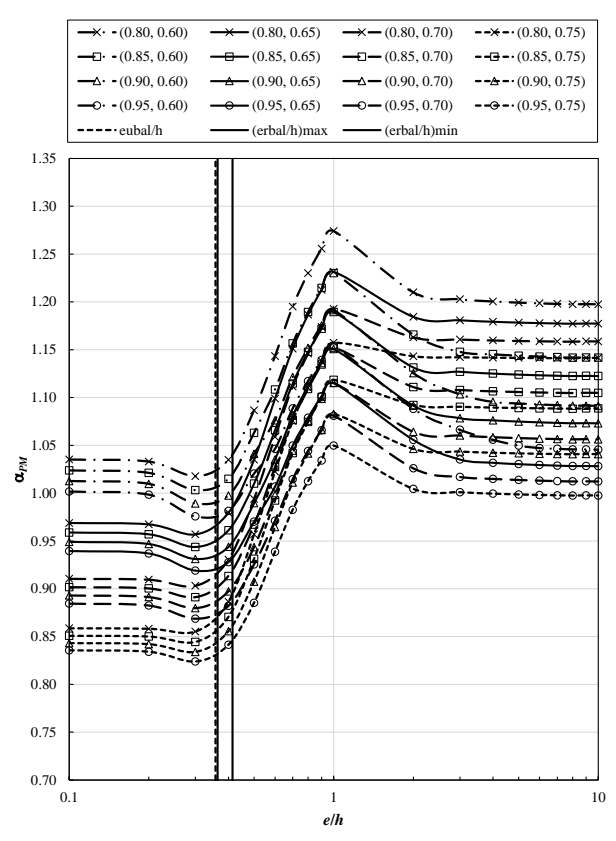

(a)

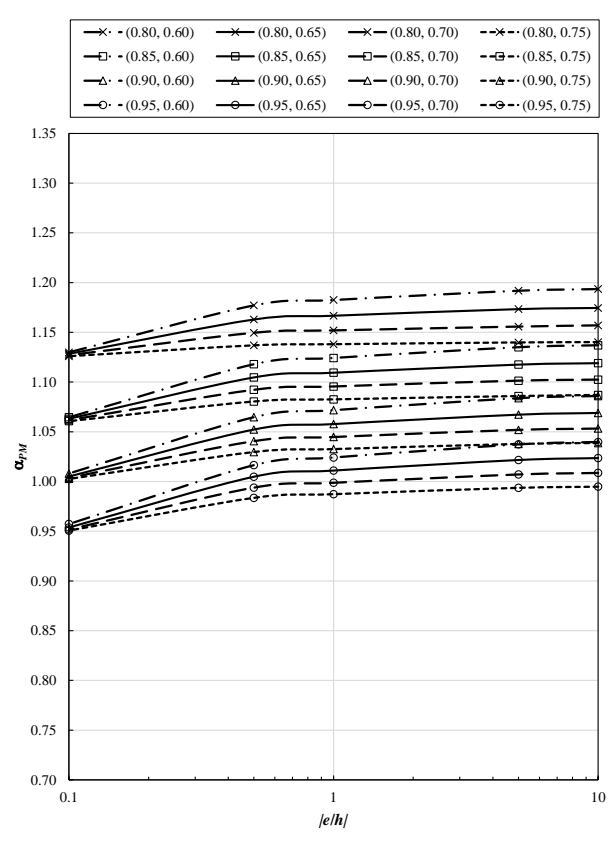

(b)

Figure A.24: Design combined flexural and axial strength ratios, $\alpha_{P M}$, for Column Section 4 and Property Combination 1: (a) $e / h>0$; (b) $e / h<0$ 


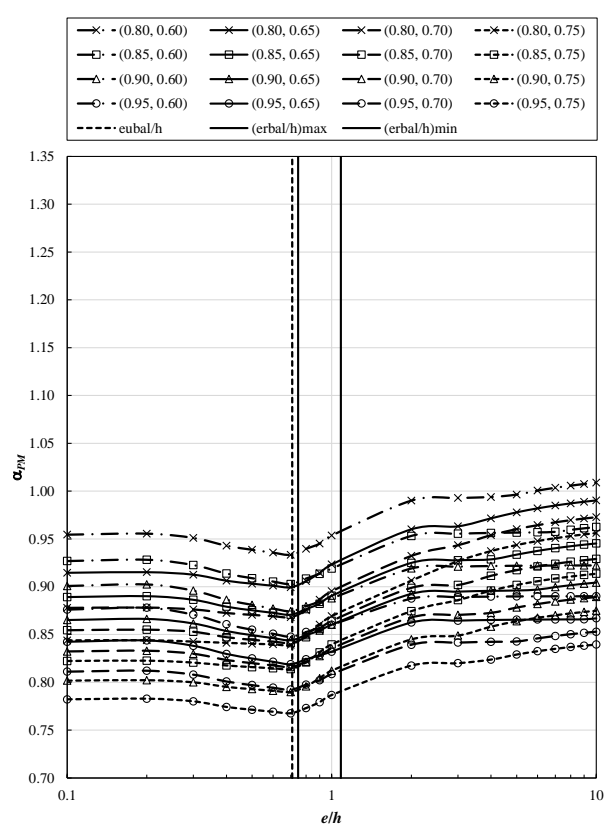

(a)

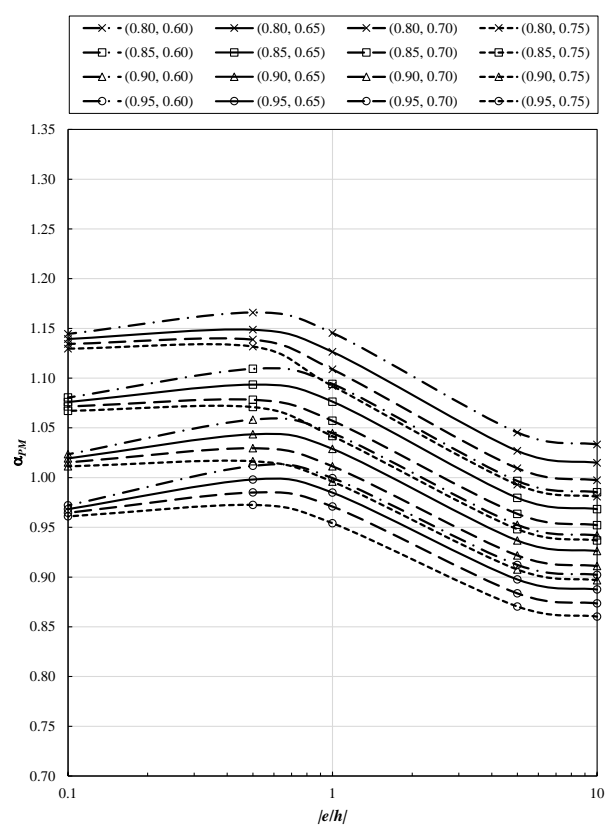

(b)

Figure A.25: Design combined flexural and axial strength ratios, $\alpha_{P M}$, for Column Section 4 and Property Combination 2: (a) $e / h>0$; (b) $e / h<0$

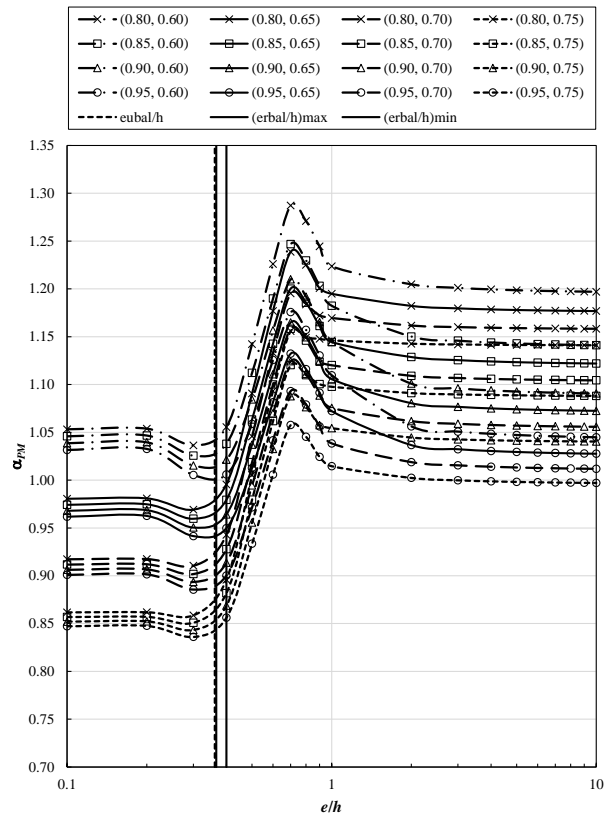

(a)

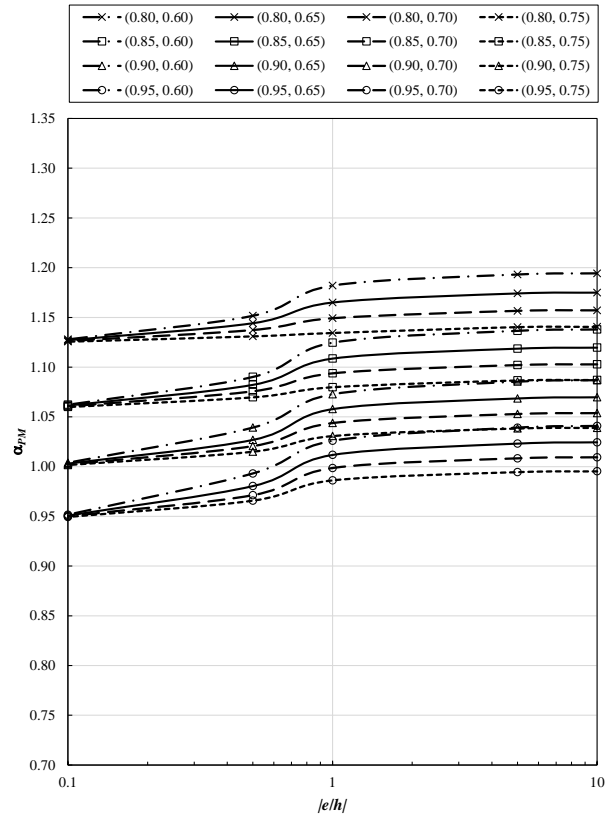

(b)

Figure A.26: Design combined flexural and axial strength ratios, $\alpha_{P M}$, for Column Section 4 and Property Combination 3: (a) $e / h>0$; (b) $e / h<0$ 


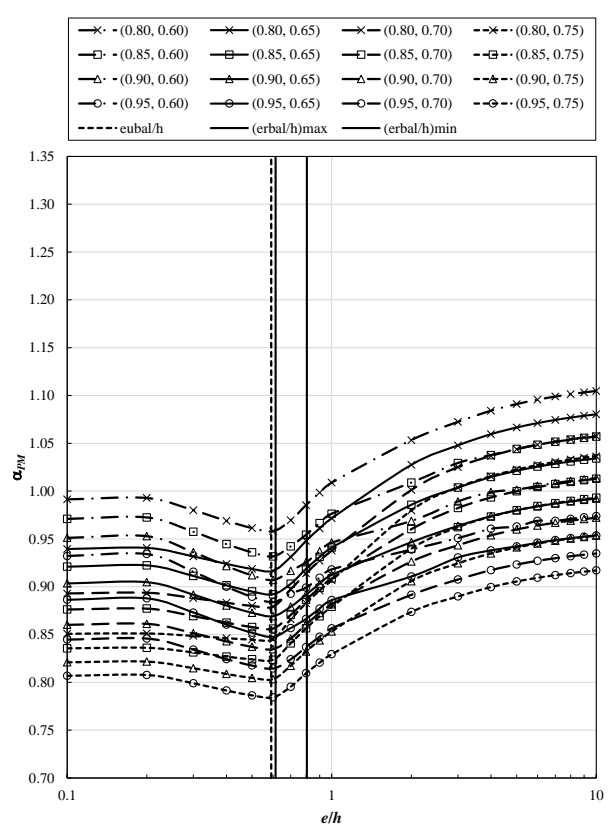

(a)

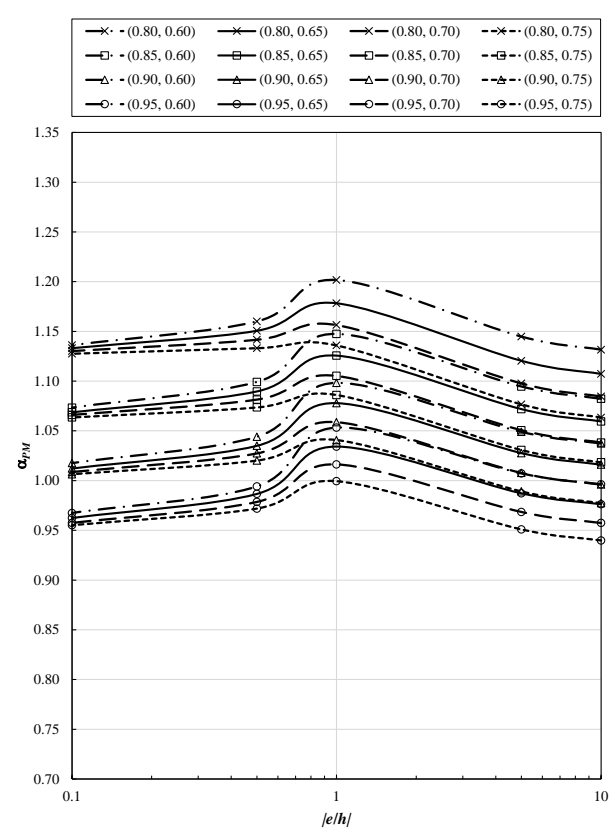

(b)

Figure A.27: Design combined flexural and axial strength ratios, $\alpha_{P M}$, for Column Section 4 and Property Combination 4: (a) $e / h>0$; (b) $e / h<0$

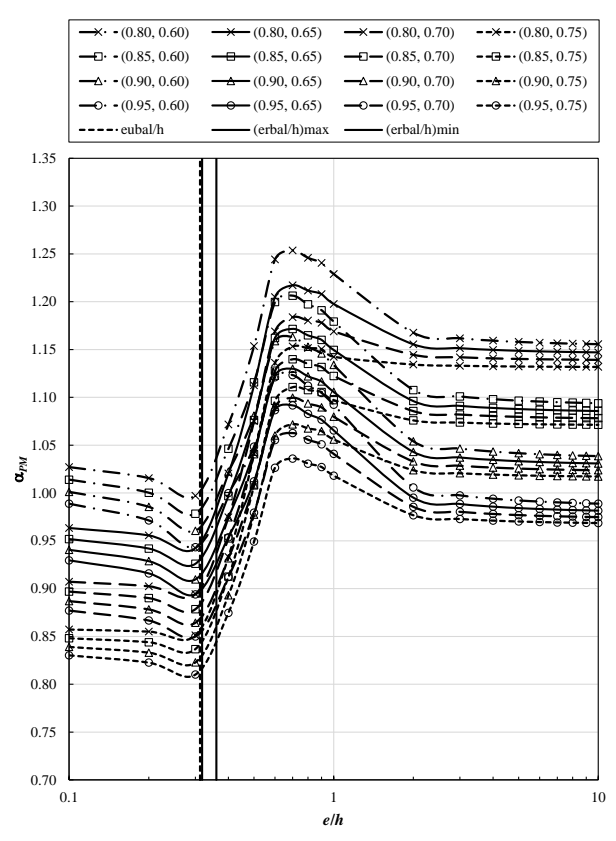

(a)

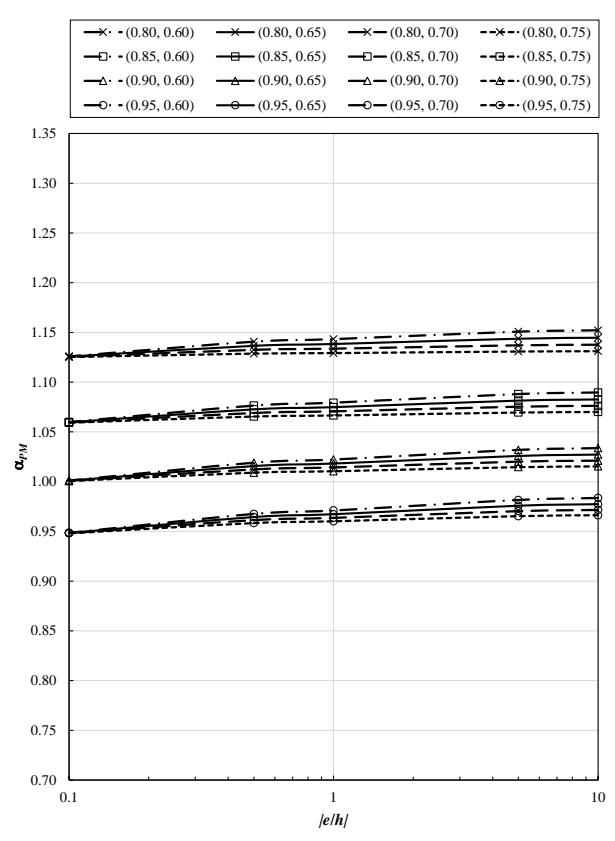

(b)

Figure A.28: Design combined flexural and axial strength ratios, $\alpha_{P M}$, for Column Section 4 and Property Combination 5: (a) $e / h>0$; (b) $e / h<0$ 


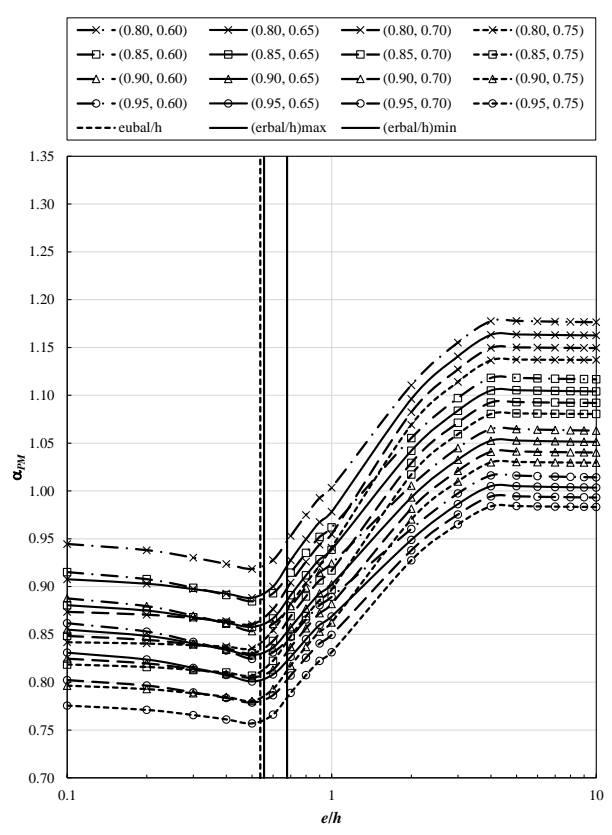

(a)

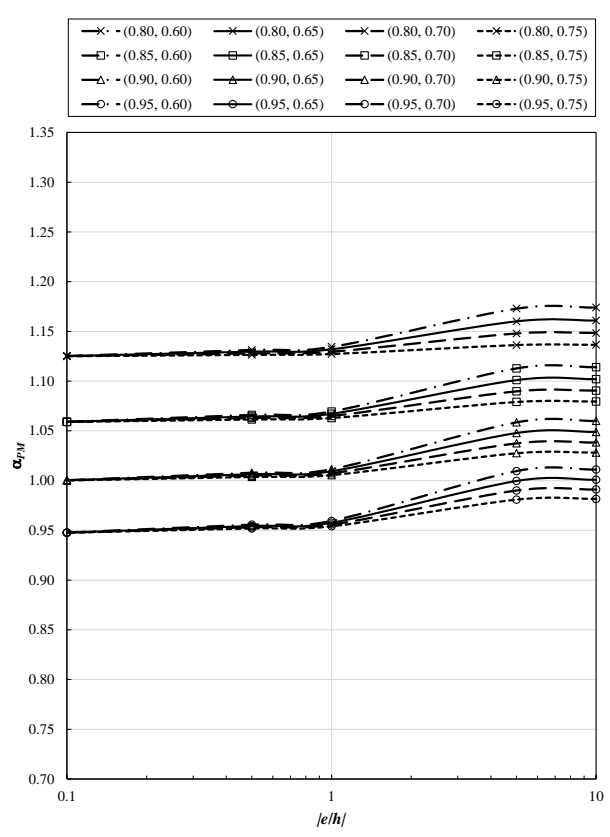

(b)

Figure A.29: Design combined flexural and axial strength ratios, $\alpha_{P M}$, for Column Section 4 and Property Combination 6: (a) $e / h>0$; (b) $e / h<0$

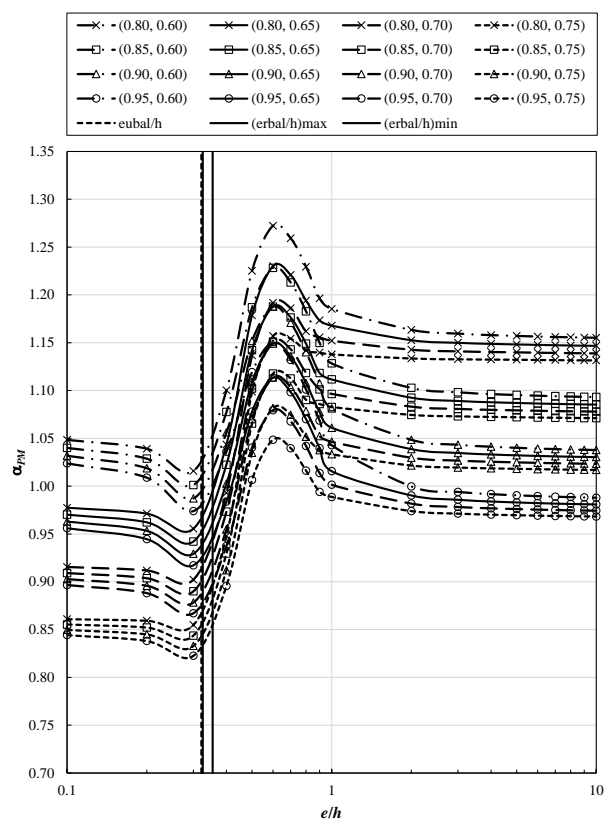

(a)

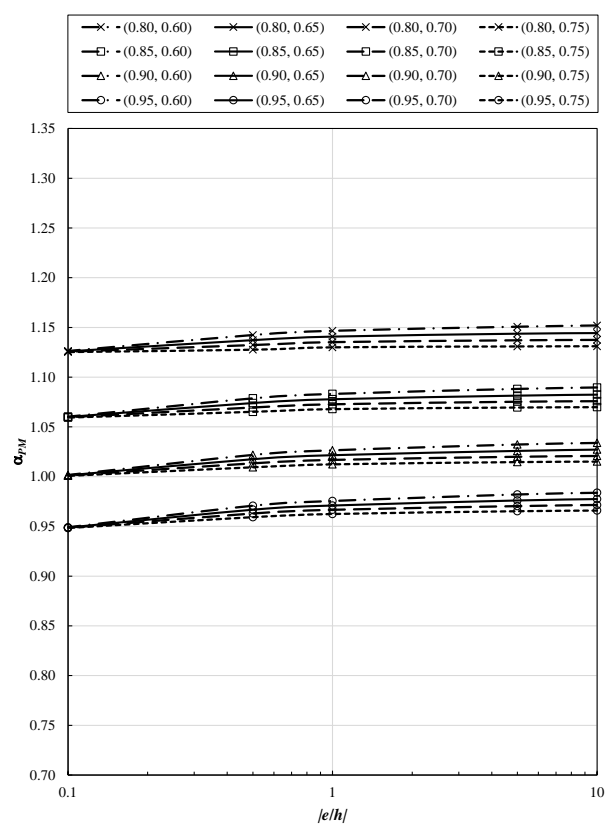

(b)

Figure A.30: Design combined flexural and axial strength ratios, $\alpha_{P M}$, for Column Section 4 and Property Combination 7: (a) $e / h>0$; (b) $e / h<0$ 


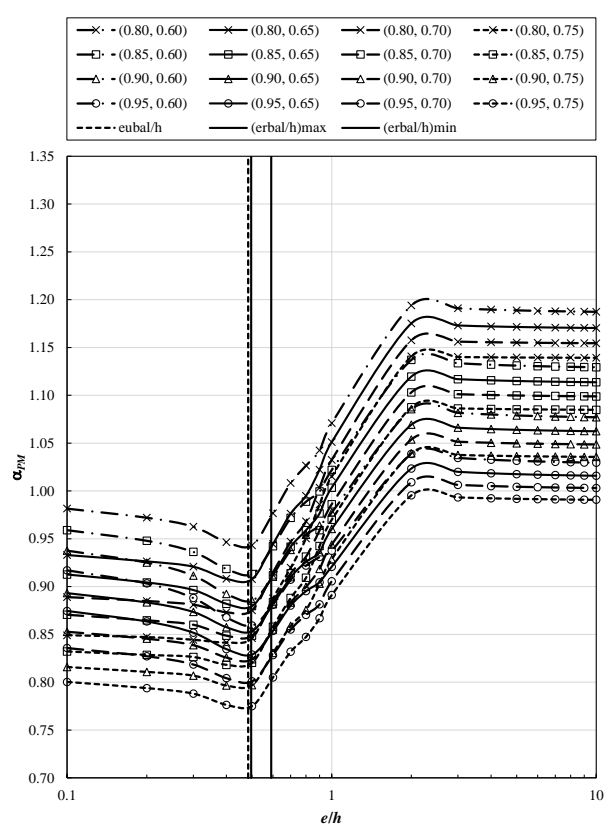

(a)

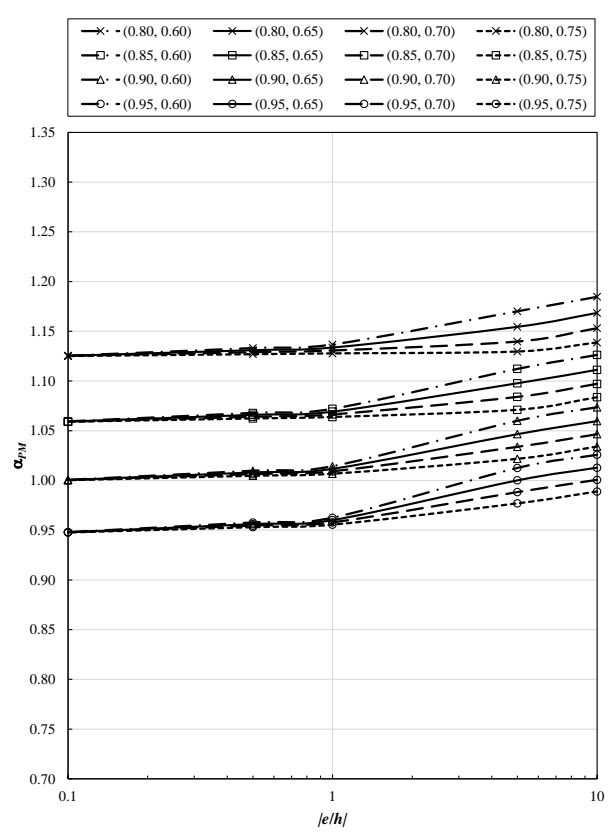

(b)

Figure A.31: Design combined flexural and axial strength ratios, $\alpha_{P M}$, for Column Section 4 and Property Combination 8: (a) $e / h>0$; (b) $e / h<0$

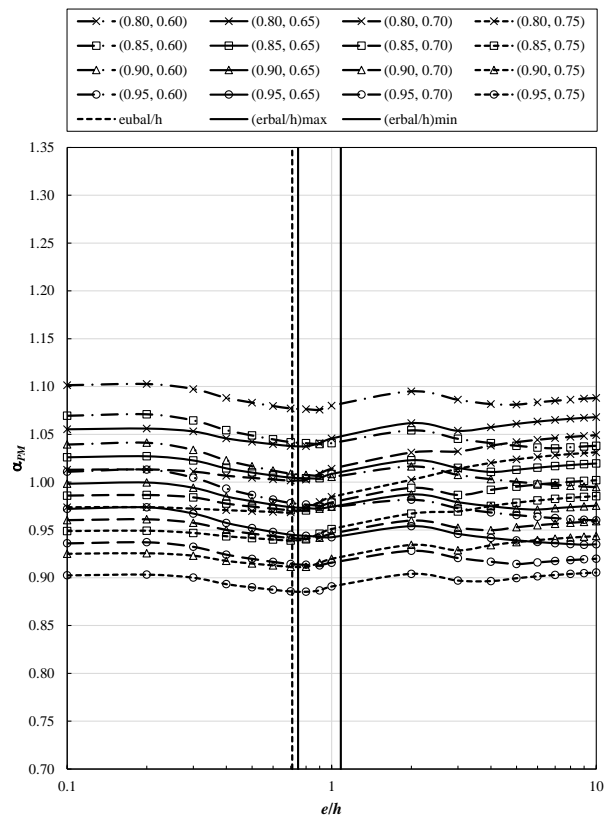

(a)

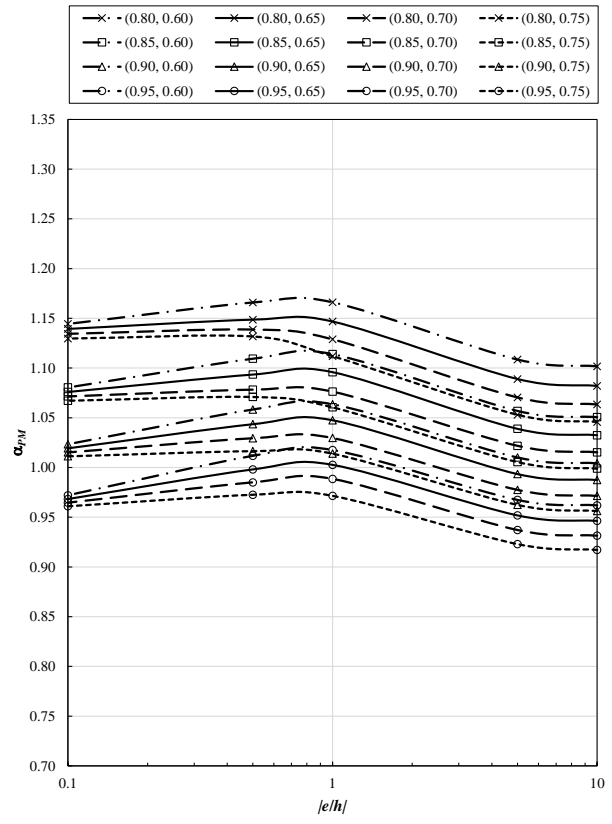

(b)

Figure A.32: Design combined flexural and axial strength ratios, $\alpha_{P M}$, for Column Section 5 and Property Combination 2: (a) $e / h>0$; (b) $e / h<0$ 


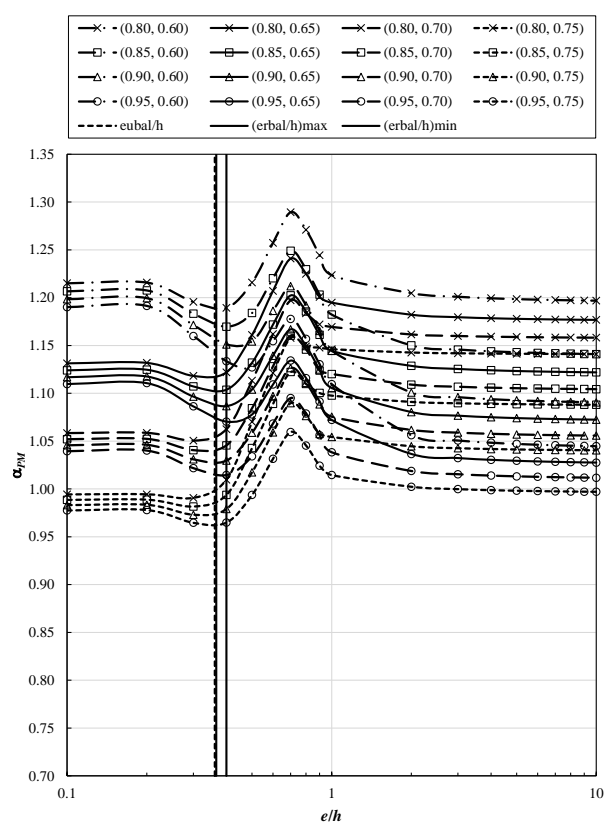

(a)

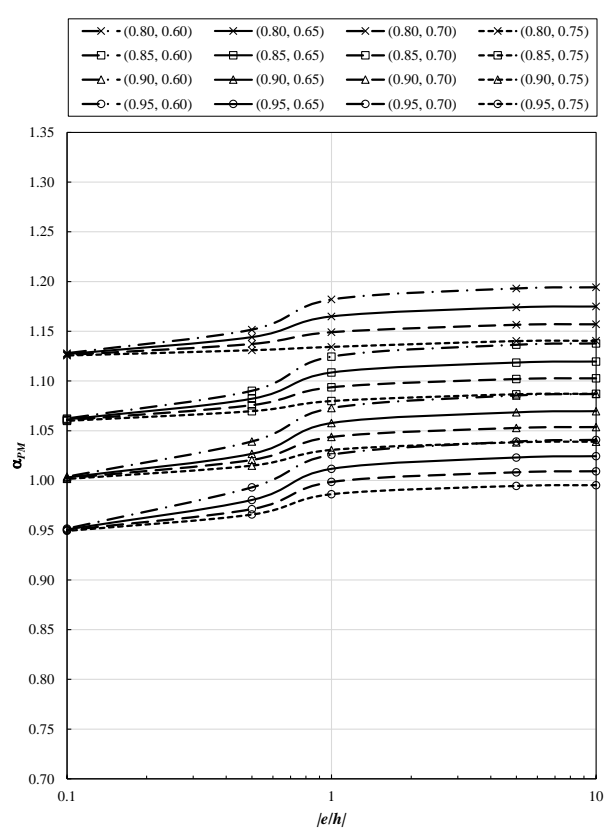

(b)

Figure A.33: Design combined flexural and axial strength ratios, $\alpha_{P M}$, for Column Section 5 and Property Combination 3: (a) $e / h>0$; (b) $e / h<0$

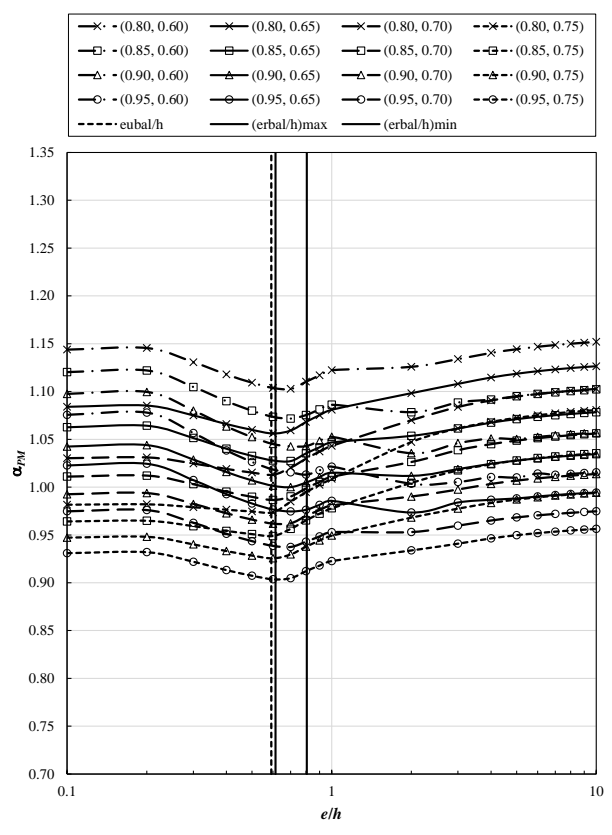

(a)

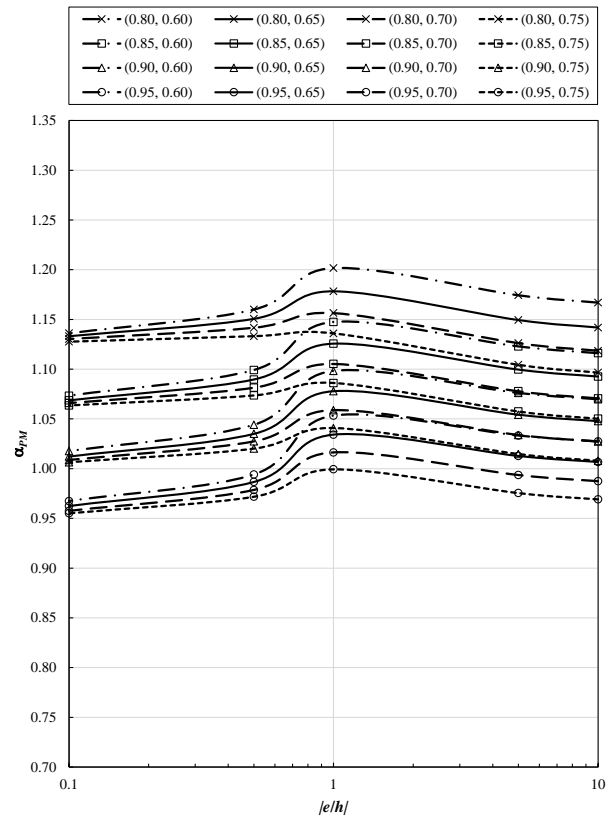

(b)

Figure A.34: Design combined flexural and axial strength ratios, $\alpha_{P M}$, for Column Section 5 and Property Combination 4: (a) $e / h>0$; (b) $e / h<0$ 


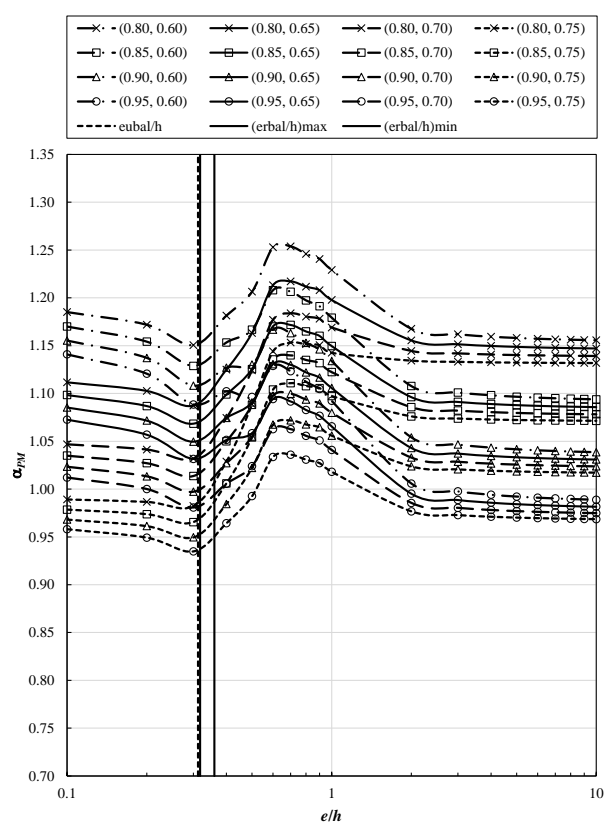

(a)

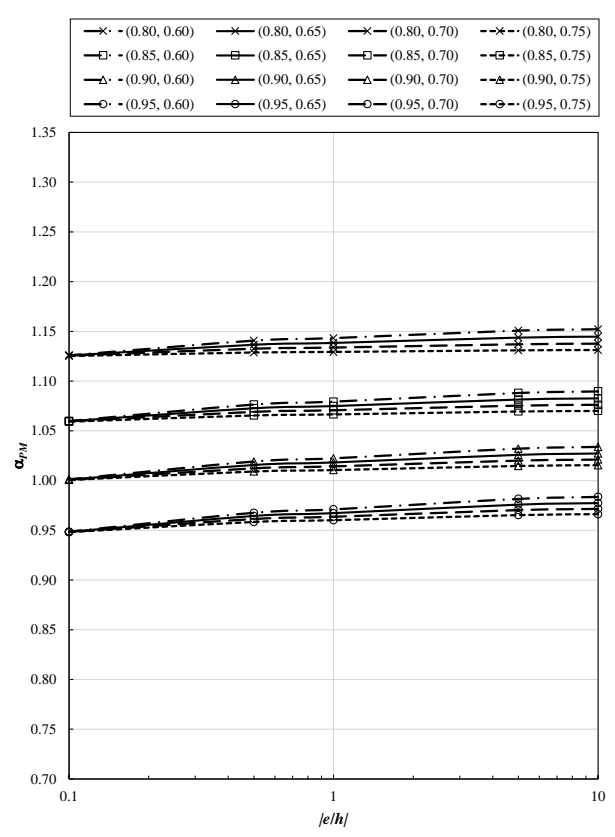

(b)

Figure A.35: Design combined flexural and axial strength ratios, $\alpha_{P M}$, for Column Section 5 and Property Combination 5: (a) $e / h>0$; (b) $e / h<0$

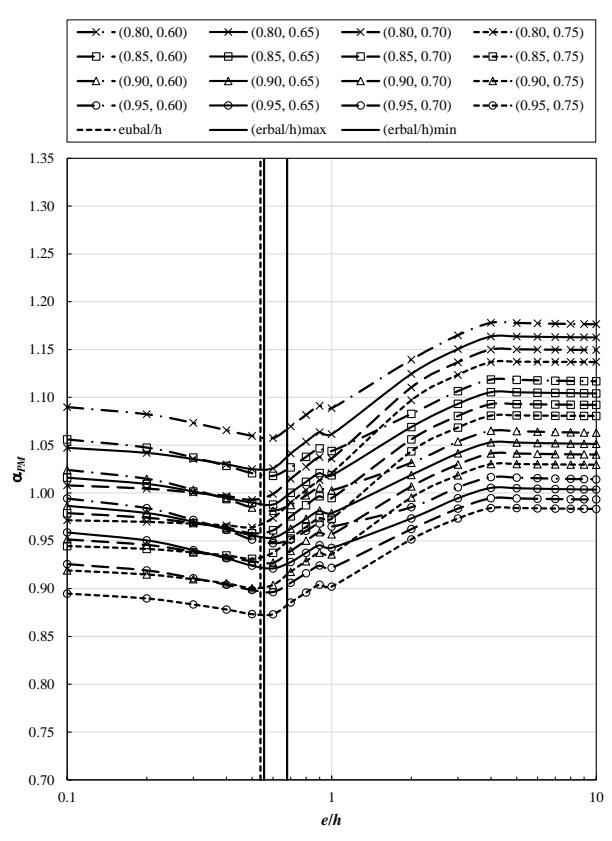

(a)

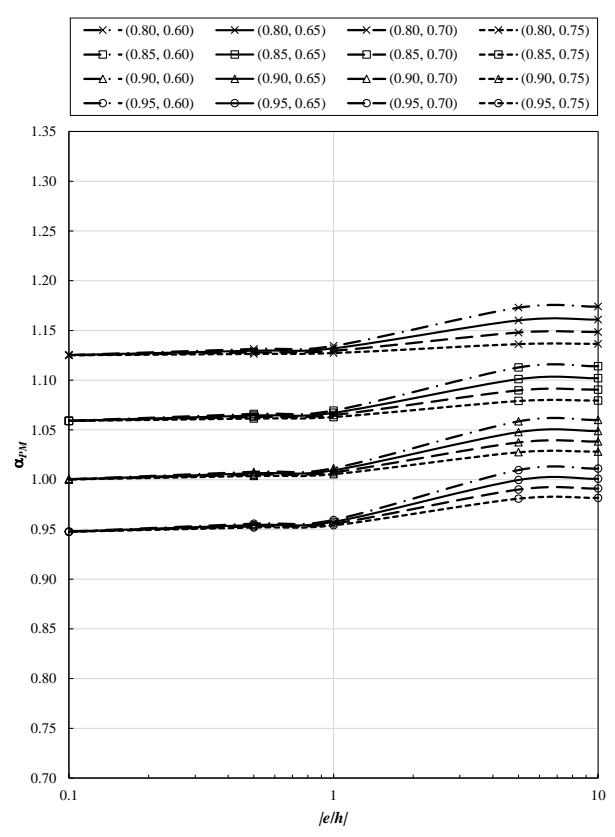

(b)

Figure A.36: Design combined flexural and axial strength ratios, $\alpha_{P M}$, for Column Section 5 and Property Combination 6: (a) $e / h>0$; (b) $e / h<0$ 


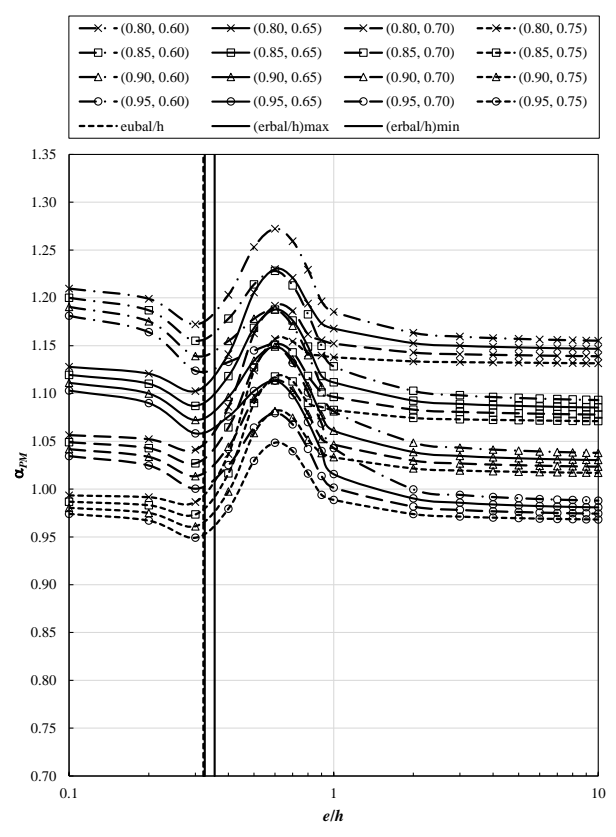

(a)

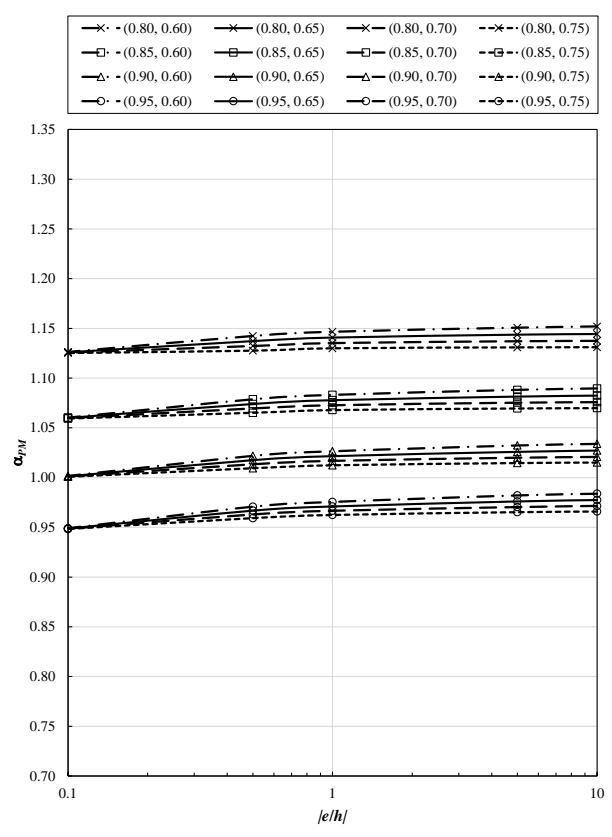

(b)

Figure A.37: Design combined flexural and axial strength ratios, $\alpha_{P M}$, for Column Section 5 and Property Combination 7: (a) $e / h>0$; (b) $e / h<0$

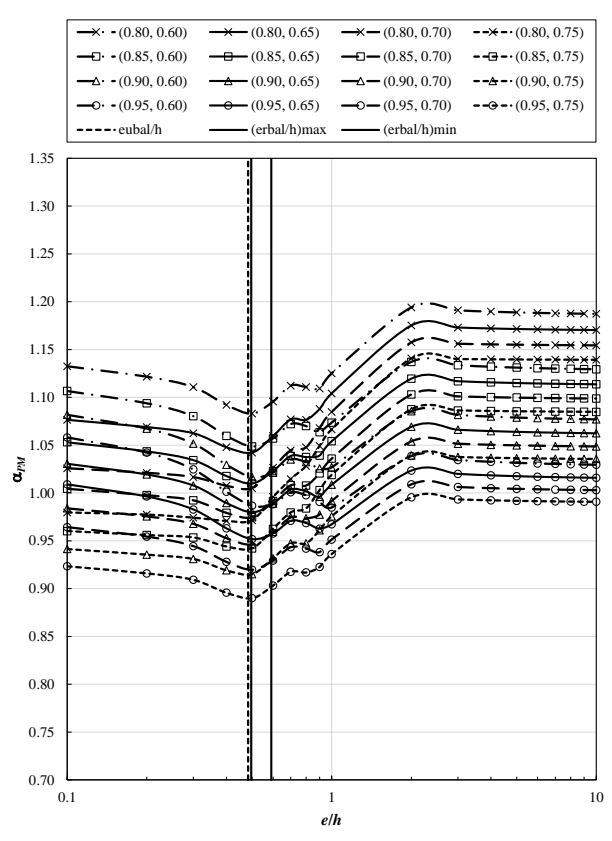

(a)

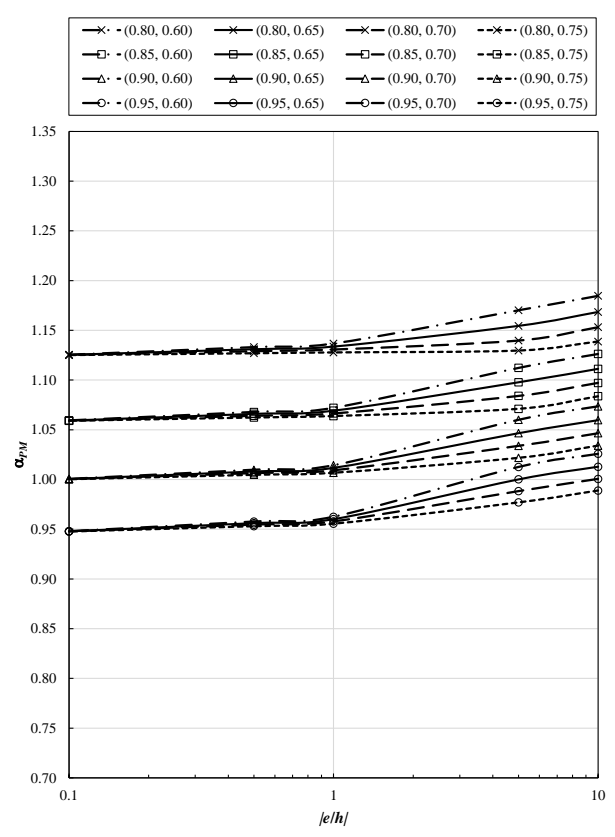

(b)

Figure A.38: Design combined flexural and axial strength ratios, $\alpha_{P M}$, for Column Section 5 and Property Combination 8: (a) $e / h>0$; (b) $e / h<0$ 


\section{A.1 Codes}

The Matlab (Version R2016b; The Mathworks, Inc. 2016) codes used to obtain design combined flexural and axial strength ratios, $\alpha_{P M}$, corresponding to specific $e / h$ values for Column Section 1 (square section with three bars in each face) and Column Section 5 (spirally reinforced circular section with eight bars evenly distributed around the perimeter), two representative cross sections, are as follows:

\section{A.1.1 Notation}

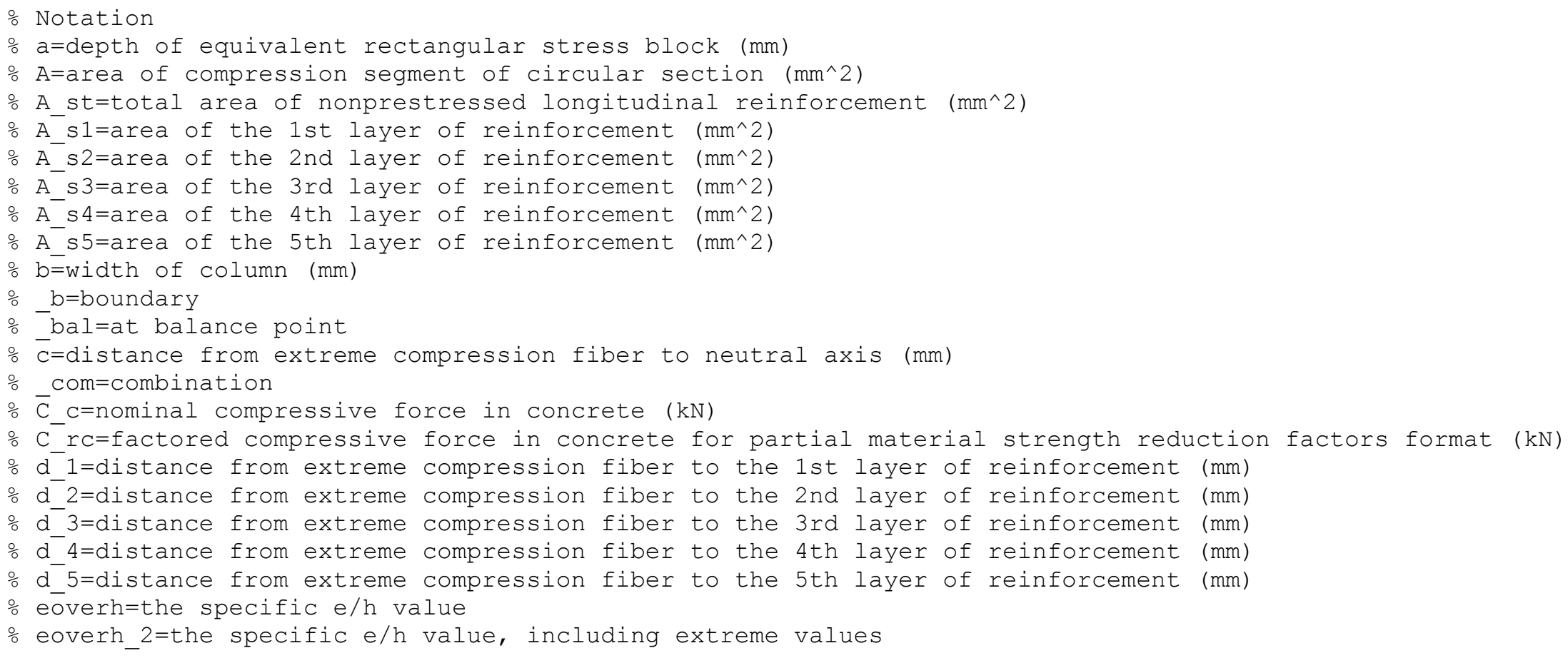


o e r=design eccentricity for partial material strength reduction factors format (m)

o e u=design eccentricity for ACI 318-14 (m)

: E_s=modulus of elasticity of reinforcement (MPa)

$\mathrm{f}_{-} \mathrm{C}=$ specified compressive strength of concrete (MPa)

f_sl=stress in the lst layer of reinforcement (MPa)

$\mathrm{f}_{-} \mathrm{s} 2=$ stress in the 2 nd layer of reinforcement (MPa)

$\mathrm{f}$ s $3=$ stress in the 3 rd layer of reinforcement (MPa)

f_s4=stress in the 4th layer of reinforcement (MPa)

f-s5=stress in the 5 th layer of reinforcement (MPa)

f_y=specified yield strength for nonprestressed reinforcement (MPa)

\% F rsl=factored force in the 1st layer of reinforcement for partial material strength reduction factors format $(\mathrm{kN})$

\% F_rstefactored force in the 2nd layer of reinforcement for partial material strength reduction factors

format $(\mathrm{kN})$

F_rs3=factored force in the 3rd layer of reinforcement for partial material strength reduction factors

format $(\mathrm{kN})$

: F rstefactored force in the 4th layer of reinforcement for partial material strength reduction factors

format $(\mathrm{kN})$

F rs5=factored force in the 5th layer of reinforcement for partial material strength reduction factors

format $(\mathrm{kN})$

\% F_sl=nominal force in the 1st layer of reinforcement ( $\mathrm{kN})$

$\div \mathrm{F}$ s2=nominal force in the 2nd layer of reinforcement ( $\mathrm{kN})$

\% F_s3=nominal force in the 3rd layer of reinforcement (kN)

: F s4=nominal force in the 4th layer of reinforcement ( $\mathrm{kN})$

F_s5=nominal force in the 5 th layer of reinforcement (kN)

o $\mathrm{h}=$ overall depth of column (mm)

\% hovere=the specific h/e value

o hovere_r=h/e_r, where e_r=design eccentricity for partial material strength reduction factors format

o hovere_u=h/e_u, where e_u=design eccentricity for ACI 318-14 (m)

o hovere_2=the specific h/e value, including extreme values

o M n=nominal flexural strength (kN.m)

- M r=design flexural strength for partial material strength reduction factors format (kN.m)

-

$\circ$ pro=property

: $\mathrm{P}=$ nominal axial strength $(\mathrm{kN})$

- P-nt=nominal axial tensile strength $(\mathrm{kN})$

$\circ \mathrm{P} o=$ nominal axial strength at zero eccentricity $(\mathrm{kN})$

\% P_r=design axial strength for partial material strength reduction factors format (kN) 
\% P_rmax=maximum design axial compressive strength for partial material strength reduction factors format $(\mathrm{kN})$

P_rodesign axial strength at zero eccentricity for partial material strength reduction factors format $(\mathrm{kN})$

P_rt=design axial tensile strength for partial material strength reduction factors format (kN)

$\mathrm{s}_{-}=$sutscript

$s=$ sort

sam=samples

$\% \bar{Z}=r a t i o$ of strain in extreme tension layer of reinforcement to yield strain

\% alpha_PM=design combined flexural and axial strength ratio, equal to design combined flexural and axial $\frac{\circ}{0}$

gamma=ratio of distance between outer layers of reinforcement in column to overall column depth

o epsilon_sl=strain in the 1st layer of reinforcement

o epsilon_s2=strain in the 2nd layer of reinforcement

opsilon s3=strain in the 3rd layer of reinforcement

opsilon_s4=strain in the 4th layer of reinforcement

opsilon s5=strain in the 5 th layer of reinforcement

opsilon_y=yield strain of reinforcement

\% angle theta= angle theta, angle used to calculate compression segment of circular column

o rho g=total reinforcement ratio, equal to ratio of total longitudinal reinforcement area to crosssectional area of column

phi=strength reduction factor in ACI 318-14

- phi_c=partial material strength reduction factor for concrete

- phis=partial material strength reduction factor for reinforcing steel

o phi sc=a pair of partial material strength reduction factors

o phi $\bar{M}$ n=design flexural strength in ACI 318-14 (kN.m)

\% phiP ${ }^{-}$=design axial strength in ACI 318-14 (kN)

\% phiP_nmax=maximum design axial compressive strength in ACI 318-14 (kN)

\% phip nt=design axial tensile strength in ACI 318-14 (kN)

o phiP_o=design axial strength at zero eccentricity in ACI $318-14$ (kN) 


\section{A.1.2 Column Section 1}

\section{A.1.2.1 Code 1-Design Combined Flexural and Axial Strength Ratios, $\alpha_{P M}$}

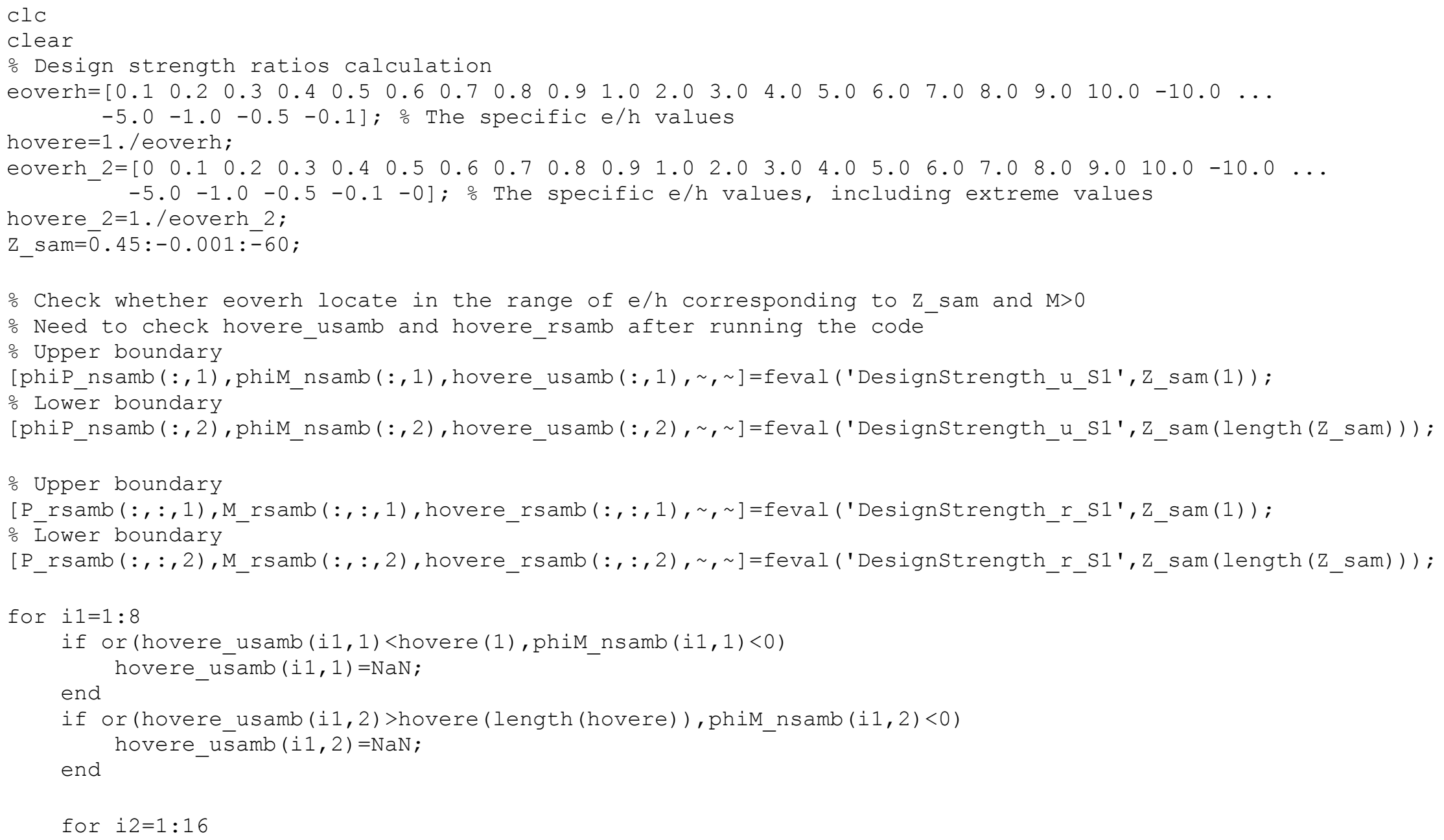




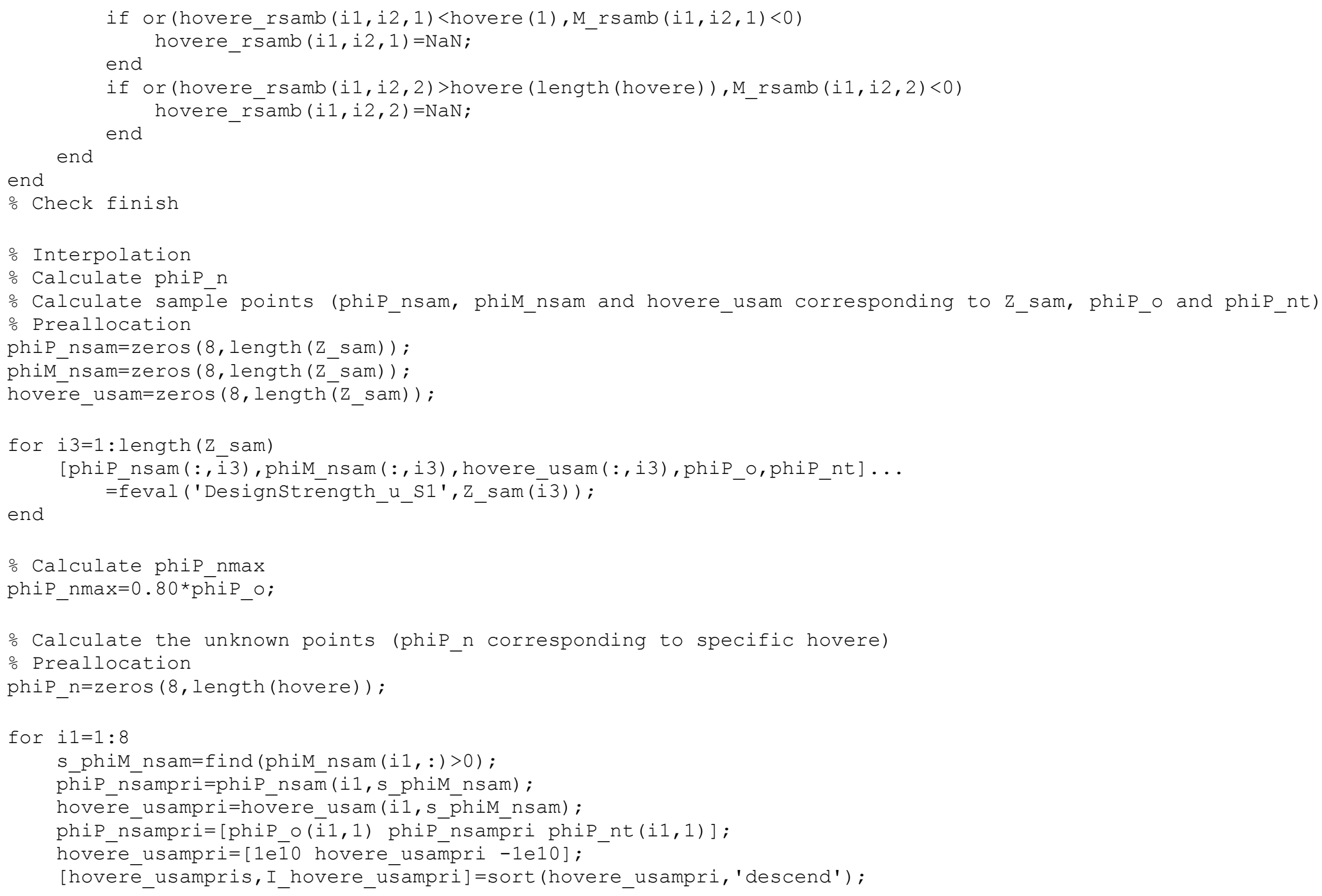


phip_n(i1, :)=interpl (hovere_usampris,phiP_nsampri (I_hovere_usampri), hovere, 'linear') ; s_phìP nmax=find (phiP_n (i,$\overline{:})>\operatorname{phiP} \operatorname{nmax}(i \overline{1}, 1))$;

end

phiP_n (i1,s_phiP_nmax $\overline{)}=$ phiP_nmax $(i \overline{1}, 1)$;

\% phiP $n$ includes phiP nmax and phiP nt

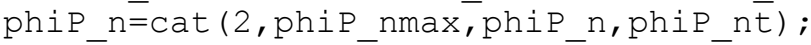

o Calculate P_r

o Calculate sāmple points (P_rsam, M_rsam and hovere_rsam corresponding to Z_sam, P_ro and P_rt)

- Preallocation

P_rsam=zeros $\left(8,16\right.$, length $\left.\left(Z \_s a m\right)\right)$;

M rsam=zeros $(8,16$, length (Z sam));

hovere_rsam=zeros $\left(8,16\right.$, length $\left(z_{-}\right.$sam $\left.)\right)$;

for i $3=1:$ length $(Z$ sam) end

[P_rsam $(:,:, i \overline{3}), M$ rsam $(:,:, i 3)$, hovere_rsam $(:,:$,i3), P_ro, P_rt] =feval ('DesignStrength_r_S1', z_sam(i3)) ;

o Calculate P_rmax

$\mathrm{P}_{\text {_rmax }}=0.80 * \mathrm{P}_{\text {_ro }}$;

o Permute the 2nd and 3rd dimensions for P rsam, M rsam, hovere rsam, $\mathrm{P}$ ro, $\mathrm{P}$ rt and $\mathrm{P}$ rmax

P_rsam=permute (P_rsam, [1,3,2]) ;

M_rsam=permute (M_rsam, $[1,3,2])$;

hovere rsam=permute (hovere rsam, $[1,3,2])$;

P_ro=pērmute (P_ro, $[1,3,2])$;

P rt $=$ permute (P rt, $[1,3,2])$;

$\mathrm{P}_{\text {_rmax }}$-permute $\left(\mathrm{P}_{\text {rmax }},[1,3,2]\right)$;

o Calculate the unknown points (P_r corresponding to specific hovere)

- Preallocation

P_r=zeros ( 8 , length (hovere), 16$)$;

for $i 1=1: 8$

for $i 2=1: 16$ 
S_M_rsam=find (M_rsam (i1, : ,i2)>0);

P rsampri=P rsam(i1,s M rsam,i2);

hōvere rsampri=hovere rsam(i1, s M rsam,i2);

P rsampri=[P ro(i1, 1, i2) P rsampri P rt(i1, 1,i2)];

hōvere rsampri=[1e10 hoverēe rsampri - -1e10];

[hoverēersampris, I_hovere_rs̄ampri] =sort (hovere_rsampri, 'descend' );

P r (i1, : i2) =interpl (hovere rsampris, P rsampri (I hovere rsampri), hovere, 'linear') ;

$S_{-} P_{-} r m a x=f i n d\left(P_{-} r(i 1,:, i 2)>P_{2} \operatorname{rmax}(i 1,1, i 2)\right)$;

end

P_rT(i1,s_P_rmax, $i 2)=P \operatorname{rmax}(i \overline{1}, 1, i 2)$;

end

$\circ \mathrm{P} r$ includes $\mathrm{P}$ rmax and $\mathrm{P}$ rt

P r $=$ cat (2, P rmax, P r, P rt);

\% Check whether the sign of phiP $n\left(\begin{array}{ll}P & r\end{array}\right)$ is identical with the sign of eoverh

ㅇ (Need to check the results aftēer calculation)

for $i 1=1: 8$

for i $4=1+1$ : length (eoverh_2) -1

if $\operatorname{sign}($ eoverh_2(i4) $) \sim=\operatorname{sign}(\operatorname{phiP} n(i 1, i 4))$

phiP_n $(i 1, i 4)=\mathrm{NaN}$;

end

end

for $i 1=1: 8$

for $i 2=1: 16$

for $i 4=1+1$ : length (eoverh 2)-1

if sign(eoverh_2(i4) $)^{\sim}=\operatorname{sign}\left(P_{-} r(i 1, i 4, i 2)\right)$

P_r (i1, i4, i2) $=\mathrm{NaN}$;

end

end

end

end

- Calculate limited balance points

Z_bal=-1; 


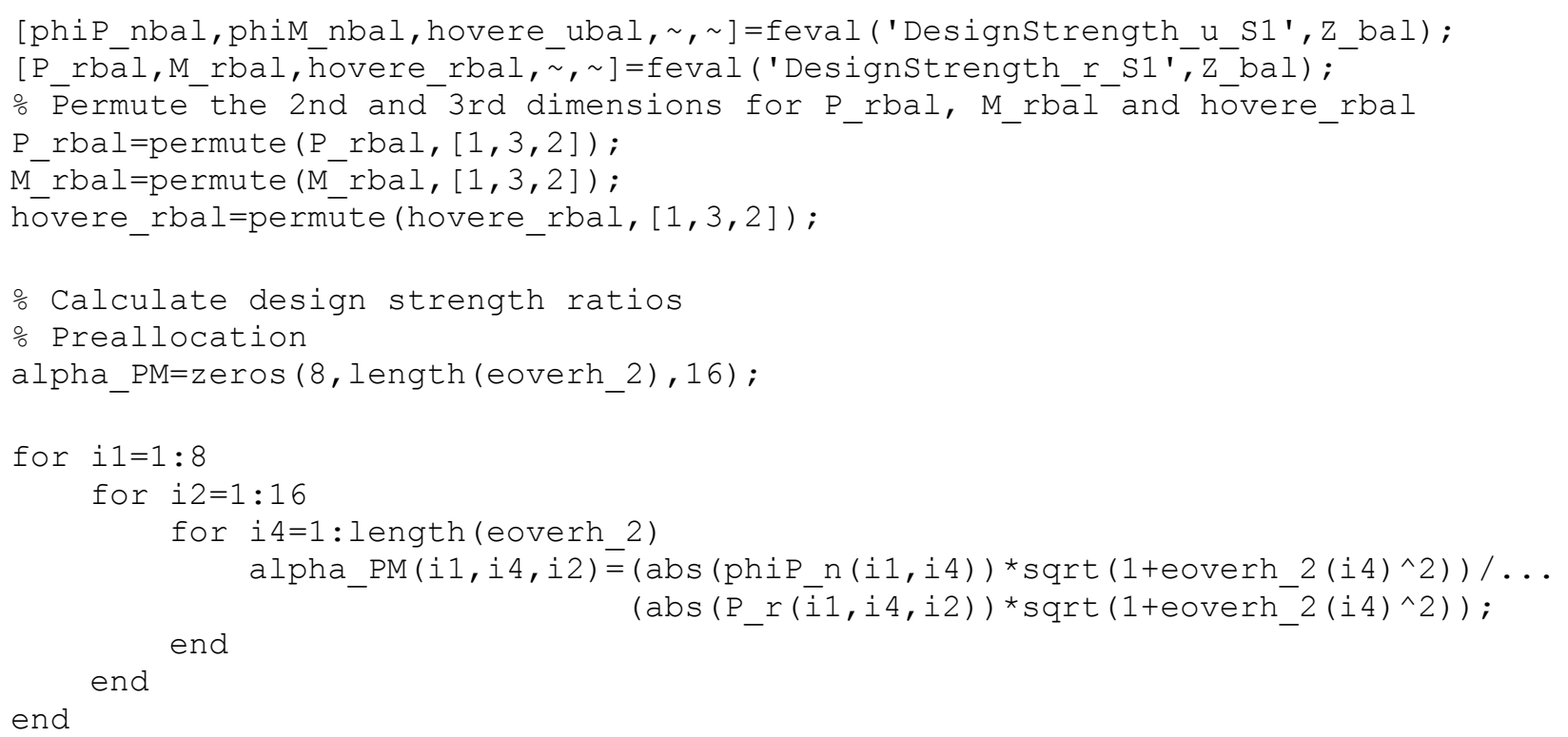

save alpha_PM_S1.mat phiP_n P_r alpha_PM hovere_ubal hovere_rbal

\section{A.1.2.2 Code 2-Function of Design Strengths for ACI 318-14}

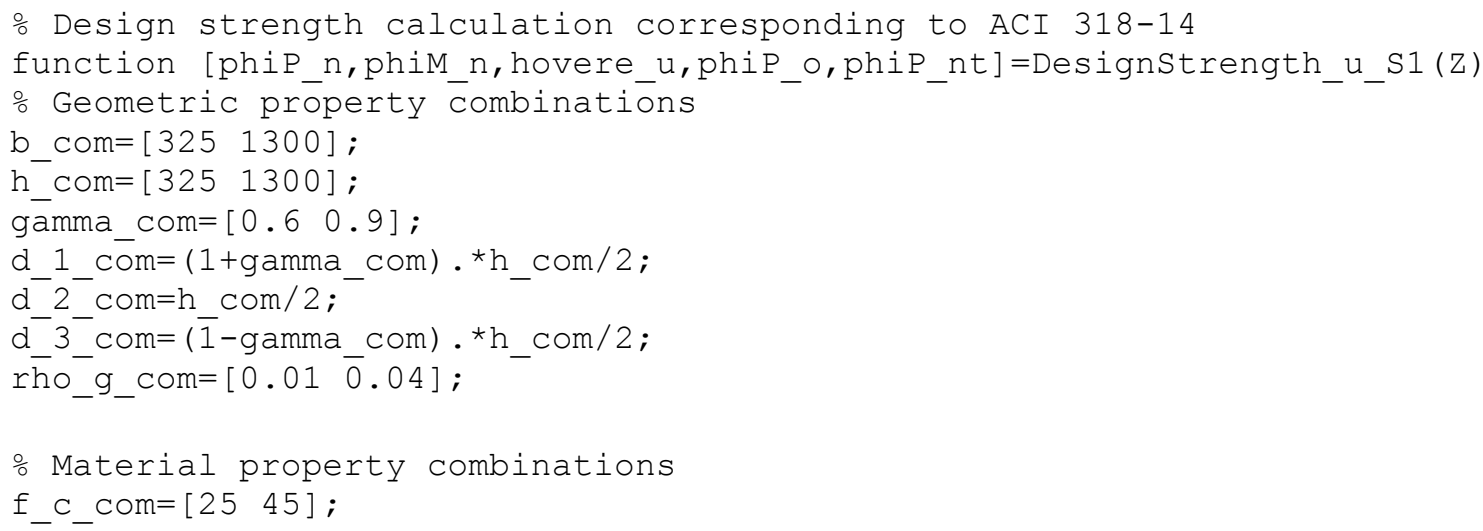


beta_1_com $=\left[0.850 .85-0.05 *\left(f_{-}{ }_{-} \_\right.\right.$com $\left.\left.(2)-28\right) / 7\right]$;

f_y $=420$;

$E-S=200000$

epsilon_y=f_y/E_s;

- Summarize property combinations in one matrix

pro_com $=\left[\mathrm{b} \_\mathrm{com}(1) \mathrm{h} \_\mathrm{com}(1)\right.$ gamma_com(1) d_1_com(1) d_2 ${ }_{-}$com(1) d_3_com(1) f_c_com(1) beta_1_com(1) ... rho_g_com $(\overline{1})$

b_com(1) h_com(1) gamma_com(1) d_1_com(1) d_2_com(1) d_3_com(1) f_c_com(1) beta_1_com(1) ... r⿳亠口冋o_g_com $(\overline{2})$;

b_com(1) h_com(1) gamma_com(1) d_1_com(1) d_2 2 com(1) d_3_com(1) f_c_com(2) beta_1_com(2) ... rho_g_com $(\overline{1})$;

b_com (1) h_com(1) gamma_com(1) d_1_com(1) d_2_com(1) d_3_com(1) f_c_com(2) beta_1_com(2) ... rh̄o $g \operatorname{com}(\overline{2})$;

b_com(2) h_com(2) gamma_com(2) d_1_com(2) d_2 ${ }_{-}$com(2) d_3_com(2) f_c_com(1) beta_1_com(1) ... rho g com (1);

b_com(2) h_com(2) gamma_com(2) d_1_com(2) d_2_com(2) d_3_com(2) f_c_com(1) beta_1_com(1) ... rho $g \operatorname{com}(\overline{2})$;

b_com(2) h_com(2) gamma_com(2) d_1_com(2) d_2_com(2) d_3_com(2) f_c_com(2) beta_1_com(2) ... rho_g_com $(\overline{1})$;

b_com(2) h_com(2) gamma_com(2) d_1_com(2) d_2 2 com(2) d_3_com(2) f_c_com(2) beta_1_com(2) ... rho_g_com $(\overline{2})]$;

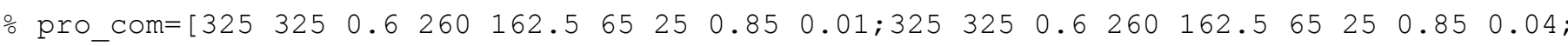

$\therefore \quad 3253250.6260162 .5 \quad 65 \quad 450.73 \quad 0.01 ; 325 \quad 325 \quad 0.6 \quad 260162.5 \quad 65 \quad 45 \quad 0.73 \quad 0.04 ;$

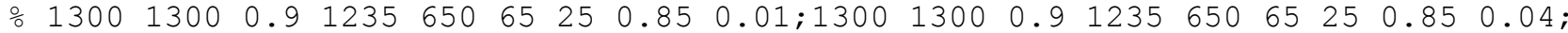

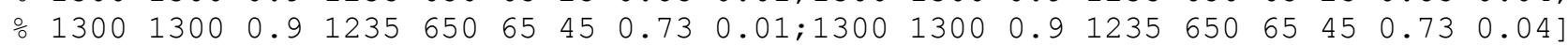

- Preallocation

$\mathrm{b}=\operatorname{zeros}(8,1)$;

$\mathrm{h}=\operatorname{zeros}(8,1)$;

gamma $=$ zeros $(8,1)$

d_1 $1=\operatorname{zeros}(8,1)$;

d- $2=\operatorname{zeros}(8,1)$;

d $3=\operatorname{zeros}(8,1)$;

$\mathrm{f}_{-}^{-} \mathrm{c}=\operatorname{zeros}(8,1)$;

beta $1=\operatorname{zeros}(8,1)$; 
rho_g=zeros $(8,1)$;

A $s t=z \operatorname{eros}(8,1)$;

A_s $1=\operatorname{zeros}(8,1)$;

A_s $2=\operatorname{zeros}(8,1)$;

A s $3=\operatorname{zeros}(8,1)$;

$\mathrm{C}=$ zeros $(8,1)$;

$\mathrm{a}=\operatorname{zeros}(8,1)$;

epsilon_sl=zeros $(8,1)$;

epsilon $s 2=\operatorname{zeros}(8,1)$;

epsilon $s 3=\operatorname{zeros}(8,1)$;

f_sl=zeros $(8,1)$;

f $s 2=\operatorname{zeros}(8,1)$;

f_s $3=\operatorname{zeros}(8,1)$;

C $\mathrm{C}=$ zeros $(8,1)$;

$\mathrm{F} s 1=\operatorname{zeros}(8,1)$;

F_s2=zeros $(8,1)$;

$\mathrm{F} s 3=\operatorname{zeros}(8,1)$;

$\mathrm{P}_{-}^{-} \mathrm{n}=\operatorname{zeros}(8,1)$;

$\mathrm{M} n=\operatorname{zeros}(8,1)$;

phi=zeros $(8,1)$;

phiP_n=zeros $(8,1)$;

phim n=zeros $(8,1)$;

phiP_o=zeros $(8,1)$;

phip nt $=$ zeros $(8,1)$;

e $u=$ zeros $(8,1)$;

hovere_u=zeros $(8,1)$;

for $i 1=1: 8$

ㅇ Properities

$\mathrm{b}(i 1,1)=$ pro_com $(i 1,1)$;

$h(i 1,1)=$ pro $\operatorname{com}(i 1,2)$;

gamma $(i 1,1)=$ pro_com $(i 1,3)$;

d_1 $(i 1,1)=$ pro_com $(i 1,4)$;

d $2(i 1,1)=\operatorname{pro} \operatorname{com}(i 1,5)$;

d_3 $(i 1,1)=$ pro_com $(i 1,6)$;

f $c(i 1,1)=\operatorname{pro} \operatorname{com}(i 1,7)$;

bēta_1 (i1, 1) = pro_com $(i 1,8)$; 
rho_g $(i 1,1)=$ pro_com $(i 1,9)$;

A st $(i 1,1)=$ rho $g(i 1,1) * b(i 1,1) * h(i 1,1)$;

A_s1 $(i 1,1)=3 * A \_s t(i 1,1) / 8$;

A $\mathrm{s} 2(i 1,1)=\mathrm{A}$ st $(i 1,1) / 4$;

A_s3 $(i 1,1)={ }^{*}{ }^{*}$ __st $(i 1,1) / 8$;

- Calculation process

- $Z=$ ? input of function

- Calculate c

$c(i 1,1)=\left(0.003 /\left(0.003-z * e p s i l o n \_y\right)\right) * d \_1(i 1,1)$;

- Calculate a

$\mathrm{a}(i 1,1)=$ beta_1 $(i 1,1){ }^{*} \mathrm{c}(i 1,1)$;

o Compare a with $h$

if $a(i 1,1)>h(i 1,1)$

$\mathrm{a}(i 1,1)=\mathrm{h}(i 1,1)$;

end

o Calculate epsilon_s1, epsilon_s2, epsilon_s3, f_s1, f_s2, and f_s3

epsilon_sl $(i 1,1)=Z *$ epsilon_y;

epsilon s2 $(i 1,1)=0.003 *(c(i 1,1)-d 2(i 1,1)) / c(i 1,1)$;

epsilon_s3 $(i 1,1)=0.003 *\left(c(i 1,1)-d \_3(i 1,1)\right) / c(i 1,1)$;

f_sl $(i 1,1)=e p s i l o n \_s 1(i 1,1) * E \_s$;

f $s 2(i 1,1)=e p s i l o n s 2(i 1,1) * \mathrm{E} s$;

f_s3 $(i 1,1)=e p s i l o n \_s 3(i 1,1) * E_{-}^{-} s$;

o Compare f_s1, f_s2 and f_s3 with +-f_y

if $f \mathrm{sl}(i 1,1)>f \mathrm{y}$

$\bar{f}_{\text {_t }} \mathrm{s}(\mathrm{i} 1,1)=\overline{\mathrm{f}}_{-} \mathrm{y}$;

elseiff $f(i 1,1)<-f y$

$f_{-} \mathrm{s}^{-}(\mathrm{i} 1,1)=-\mathrm{f}_{-} \mathrm{y}$;

end

if f_s2 $(i 1,1)>f_{-} y$

$$
\text { f_s } 2(i 1,1)=\bar{f} y \text {; }
$$

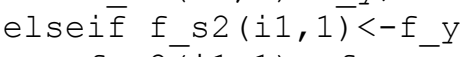

$f_{-} s 2 \overline{(i 1,1)}=-f_{-} y$;

end 


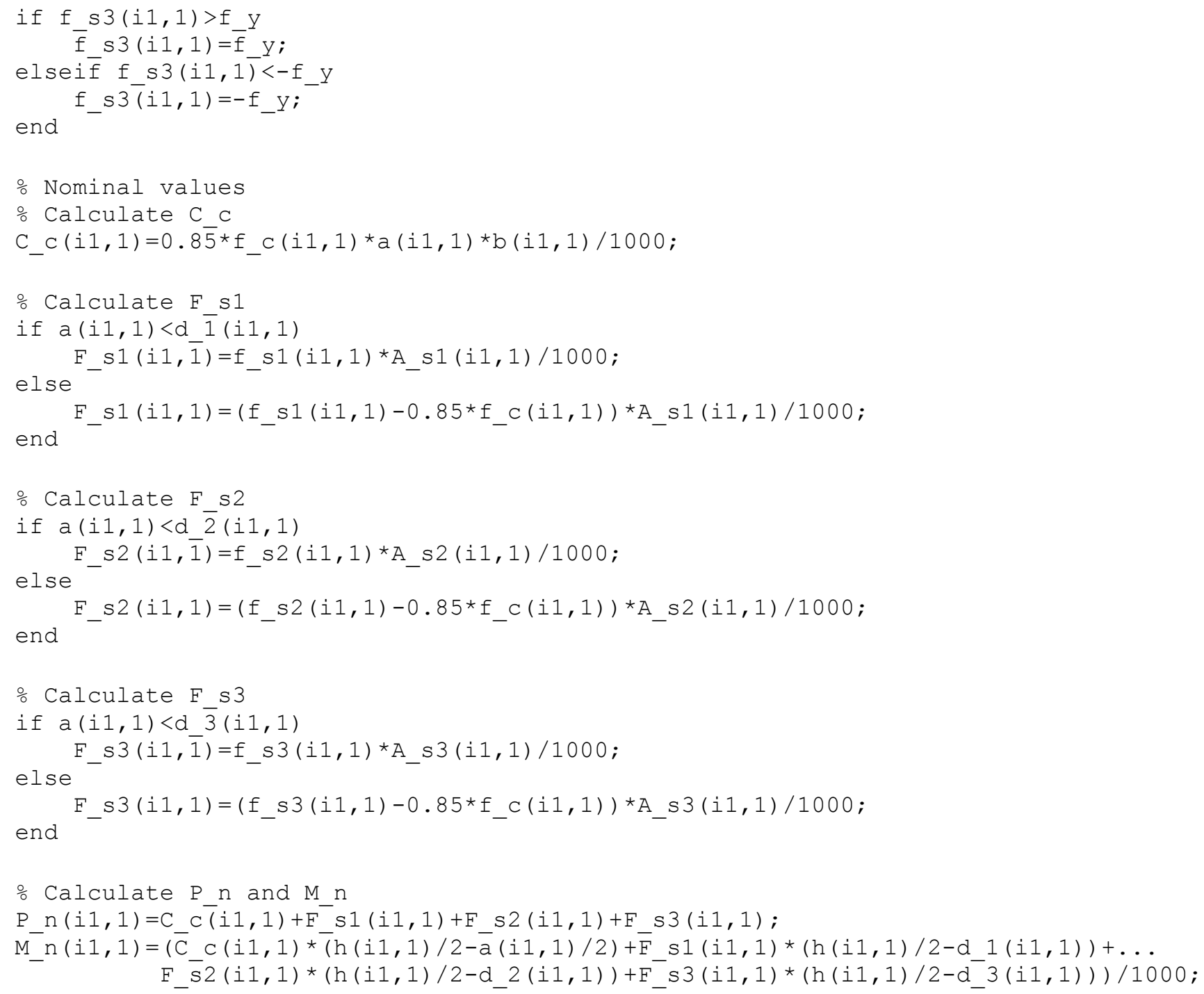




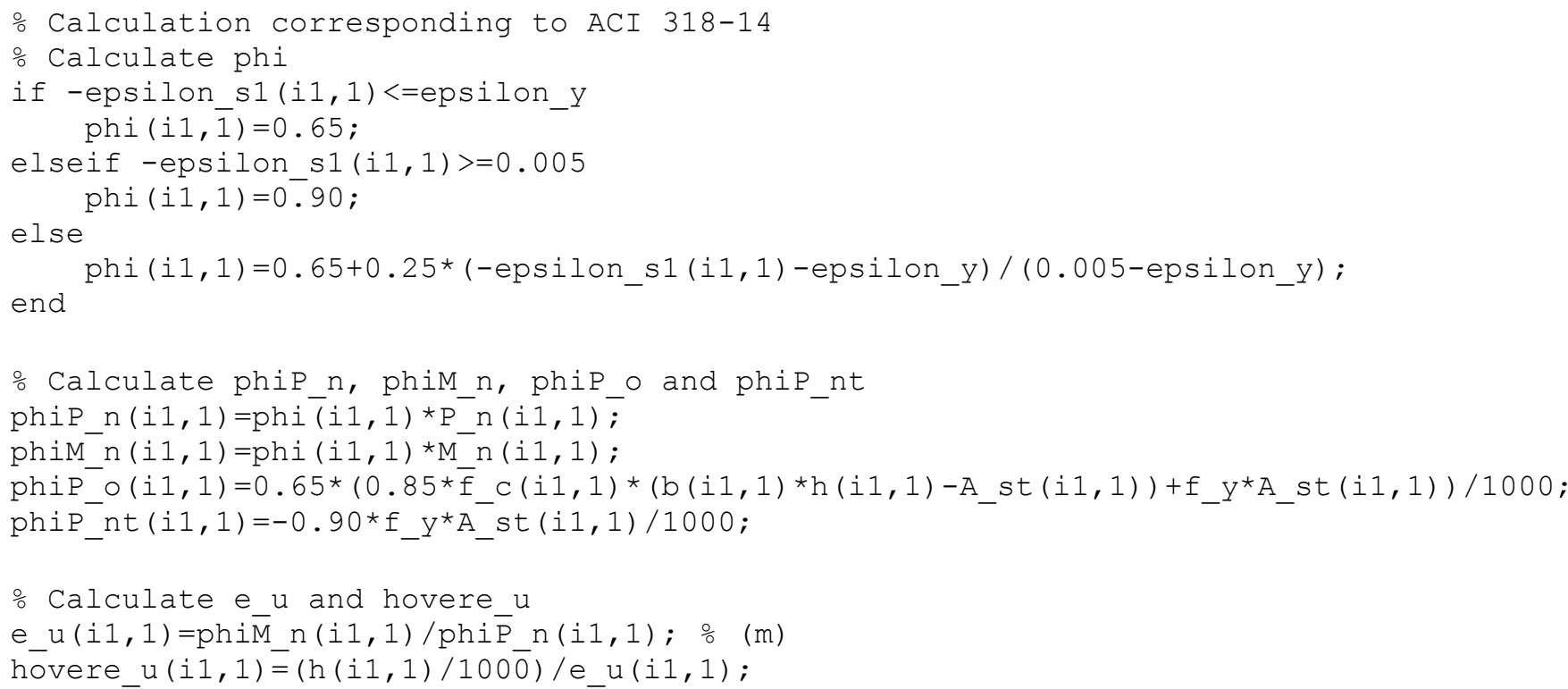

\section{A.1.2.3 Code 3-Function of Design Strengths for Partial Material Strength Reduction Factors}

o Design strength calculation corresponding to partial strength reduction factors

function [P $r, M r$, hovere $r, P$ ro, P rt] =Designstrength r S1 (Z)

\% Geometric property combinations

b_com $=\left[\begin{array}{ll}325 & 1300\end{array}\right]$;

$\mathrm{h} \mathrm{com}=\left[\begin{array}{ll}325 & 1300\end{array}\right]$;

gamma_com $=\left[\begin{array}{lll}0.6 & 0.9\end{array}\right]$;

d $1 \mathrm{com}=(1+$ gamma $\mathrm{com}) \cdot{ }^{*} \mathrm{~h} \mathrm{com} / 2$;

d_2_com=h_com/2;

d 3 - $c o m=(\overline{1}-$ gamma com $) . *$ h_com $/ 2$;

rho_g_com $=\left[\begin{array}{ll}0.01 & 0.04\end{array}\right]$;

o Material property combinations 


\title{
f_c_com=[ $\left[\begin{array}{ll}25 & 45\end{array}\right]$;
}

beta_1_com $=\left[\begin{array}{ll}0.85 & 0.85-0.05 *\left(f \_c \_c o m(2)-28\right) / 7\end{array}\right]$;

$\mathrm{f} \_\mathrm{y}=\overline{4} 2 \overline{0}$;

$\mathrm{E}-\mathrm{S}=200000$;

epsilon_y=f_y/E_s;

o Summarize property combinations in one matrix

pro_com=[b_com(1) h_com(1) gamma_com(1) d_1_com(1) d_2_com(1) d_3_com(1) f_c_com(1) beta_1_com(1) ... rho_g_com $(\overline{1})$;

b_com $(1)$ h_com(1) gamma_com(1) d_1_com(1) d_2_com(1) d_3_com(1) f_c_com(1) beta_1_com(1) ... rho_g_com $(\overline{2})$;

b_com(1) h_com(1) gamma_com(1) d_1_com(1) d_2 2 com(1) d_3_com(1) f_c_com(2) beta_1_com(2) ... rho $g \operatorname{com}(\overline{1})$;

b_com(1) h_com(1) gamma_com(1) d_1_com(1) d_2_com(1) d_3_com(1) f_c_com(2) beta_1_com(2) ... rho_g_com $(\overline{2})$;

b_com(2) h_com(2) gamma_com(2) d_1_com(2) d_2 2 com(2) d_3_com(2) f_c_com(1) beta_1_com(1) ... rho_g_com $(\overline{1})$;

b_com (2) h_com(2) gamma_com(2) d_1_com(2) d_2_com(2) d_3_com(2) f_c_com(1) beta_1_com(1) ... rho_g_com $(\overline{2})$;

b_com(2) h_com(2) gamma_com(2) d_1_com(2) d_2_com(2) d_3_com(2) f_c_com(2) beta_1_com(2) ... rh̄o_g_com $(\overline{1})$;

b_com(2) h_com(2) gamma_com(2) d_1_com(2) d_2_com(2) d_3_com(2) f_c_com(2) beta_1_com(2) ... r⿳亠口冋o_g_com $(\overline{2})]$;

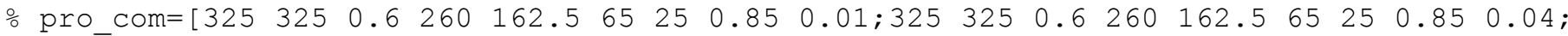

$\begin{array}{llllllllllllllllllll}\circ & 325 & 325 & 0.6 & 260 & 162.5 & 65 & 45 & 0.73 & 0.01 ; 325 & 325 & 0.6 & 260 & 162.5 & 65 & 45 & 0.73 & 0.04 ;\end{array}$

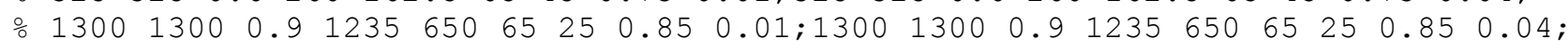

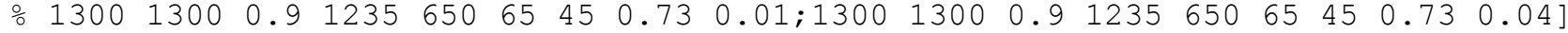

\author{
o Preallocation \\ $\mathrm{b}=\operatorname{zeros}(8,1) ;$ \\ $\mathrm{h}=\mathrm{zeros}(8,1)$; \\ gamma $=$ zeros $(8,1)$; \\ d_ $1=\operatorname{zeros}(8,1)$; \\ d $2=\operatorname{zeros}(8,1)$; \\ d_ $3=\operatorname{zeros}(8,1)$; \\ $\mathrm{f}_{-} \mathrm{c}=\operatorname{zeros}(8,1)$;
}


beta_1=zeros $(8,1)$;

rho $g=$ zeros $(8,1)$;

A st $=z \cos (8,1)$;

A $s 1=\operatorname{zeros}(8,1)$;

A s $2=\operatorname{zeros}(8,1)$;

A_s $3=\operatorname{zeros}(8,1)$;

$\mathrm{C}=\operatorname{zeros}(8,1)$;

$\mathrm{a}=$ zeros $(8,1)$;

epsilon $s 1=\operatorname{zeros}(8,1)$

epsilon_s $2=$ zeros $(8,1)$;

epsilon_s $3=\operatorname{zeros}(8,1)$;

f $\mathrm{s} 1=\operatorname{zeros}(8,1)$;

f_s $2=\operatorname{zeros}(8,1)$;

f $\mathrm{s} 3=\operatorname{zeros}(8,1)$;

C rc $=$ zeros $(8,16)$;

F_rs $1=\operatorname{zeros}(8,16)$;

Frs $2=\operatorname{zeros}(8,16)$

F_rs3=zeros $(8,16)$;

P_r $=$ zeros $(8,16)$;

M r zeros $(8,16)$;

P_ro=zeros $(8,16)$;

P rt $=$ zeros $(8,16)$;

e_r $\operatorname{zeros}(8,16)$;

hovere $r=$ zeros $(8,16)$;

for $i 1=1: 8$

을 Properities

b $(i 1,1)=$ pro_com $(i 1,1)$,

$\mathrm{h}(i 1,1)=\operatorname{pro} \operatorname{com}(i 1,2)$;

gamma $(i 1,1)=$ pro_com $(i 1,3)$;

d $1(i 1,1)=\operatorname{pro} \operatorname{com}(i 1,4)$;

$d_{-} 2(i 1,1)=$ pro_com $(i 1,5)$;

d $3(i 1,1)=$ pro_com $(i 1,6)$;

f $c(i 1,1)=\operatorname{pro} \operatorname{com}(i 1,7)$;

bèta_1 $(i 1,1)=\overline{p r o}$ com $(i 1,8)$;

rho $g(i 1,1)=$ pro $\operatorname{com}(i 1,9)$;

A_st $(i 1,1)=r h o \_\bar{g}(i 1,1) * b(i 1,1) * h(i 1,1)$; 
A_s1 $(i 1,1)=3 * A \_s t(i 1,1) / 8$;

A s2 $(i 1,1)=A$ st $(i 1,1) / 4$;

A_s3 $(i 1,1)=3{ }^{\star} A \_s t(i 1,1) / 8$;

o Calculation process

\% $\mathrm{Z}=$ ? input of function

- Calculate c

$c(i 1,1)=\left(0.003 /\left(0.003-z * e p s i l o n \_y\right)\right) * d \_1(i 1,1)$;

o Calculate a

$\mathrm{a}(\mathrm{i} 1,1)=$ beta_1 $(i 1,1){ }^{*} \mathrm{C}(\mathrm{i} 1,1)$;

\% Compare a with h

if $\mathrm{a}(\mathrm{i} 1,1)>\mathrm{h}(\mathrm{i} 1,1)$

end

$$
\mathrm{a}(\mathrm{i} 1,1)=\mathrm{h}(\mathrm{i} 1,1) \text {; }
$$

o Calculate epsilon_s1, epsilon_s2, epsilon_s3, f_s1, f_s2, and f_s3

epsilon_sl $(i 1,1)=\mathrm{z} * \bar{e} p s i l o n \_y$;

epsilon s2 (i1, 1) =0.003* (c (i1, 1) -d 2(i1, 1))/c (i1, 1);

epsilon_s3 $(i 1,1)=0.003 *\left(c(i 1,1)-d_{-} 3(i 1,1)\right) / c(i 1,1)$;

f $\mathrm{s} 1(i 1,1)=e p s i l o n s 1(i 1,1) \star E s$;

f $\mathrm{s} 2(i 1,1)=e p s i l o n$ s2 $(i 1,1) * \mathrm{E} s$;

$\mathrm{f}_{-}^{-} \mathrm{s} 3(\mathrm{i} 1,1)=e \mathrm{silon}{ }_{-}^{-} \mathrm{s} 3(\mathrm{i} 1,1) \mathrm{E}_{-}^{-} \mathrm{s}$;

Compare f_s1, f_s2 and f_s3 with $+-f_{-} y$

if $f$ sl $(i 1, \overline{1})>f \quad y$

$\bar{f} s 1(i 1,1)=\bar{f} y$;

elseif f_sl(i1,1)<-f y

end

$$
\left.f_{-} s \overline{(i 1}, 1\right)=-f_{-} y \text {; }
$$

if $f_{-} s 2(i 1,1)>f_{-} y$

$$
\bar{f}_{-} s 2(i 1,1)=\bar{f} \_y \text {; }
$$

elseiff $f 2(i 1,1)<-f y$

$$
\text { f_s } 2 \overline{(i 1}, 1)=-f_{-} y \text {; }
$$

end

if f_s3(i1, 1)>f_y 
f_s3 $(i 1,1)=f \_y$;

elseif $f$ s3 (i1, 1)<-f_y

end

$$
\text { f_s3 }(i 1,1)=-f_{-} y \text {; }
$$

- Calculation corresponding to partial strength reduction factors

\% Partial strength reduction factor combinations

$\therefore$ phi_s $=\left[\begin{array}{llll}0.80 & 0.85 & 0.90 & 0.95\end{array}\right]$;

$\therefore$ phi $\mathrm{C}=\left[\begin{array}{llll}0.60 & 0.65 & 0.70 & 0.75\end{array}\right] ;$

phi_s $\bar{c}=\left[\begin{array}{lllll}0.80 & 0.60 ; 0.80 & 0.65 ; 0.80 & 0.70 ; 0.80 & 0.75 ;\end{array}\right.$ $0.850 .60 ; 0.850 .65 ; 0.850 .70 ; 0.850 .75$

$0.900 .60 ; 0.900 .65 ; 0.900 .70 ; 0.900 .75 ;$

$0.950 .60 ; 0.950 .65 ; 0.950 .70 ; 0.950 .75]$;

for $i 2=1: 16$

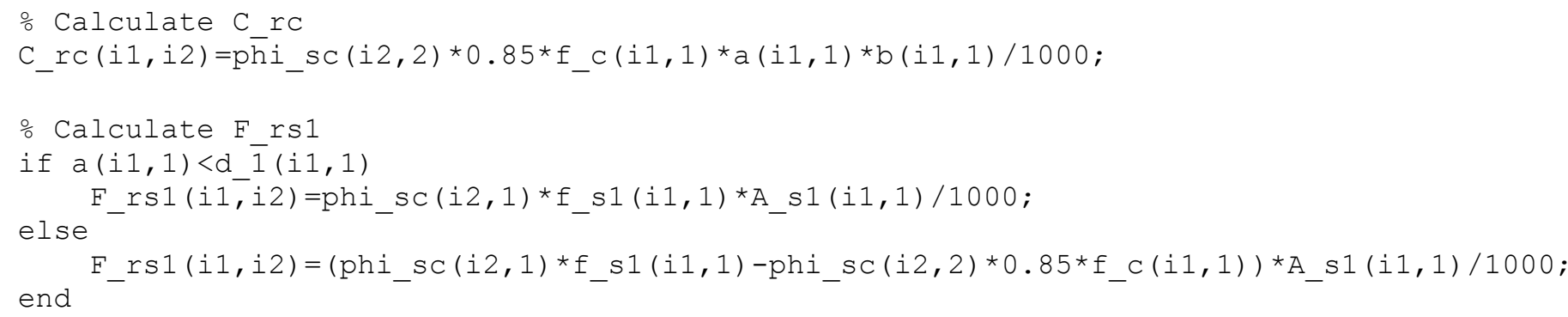


end

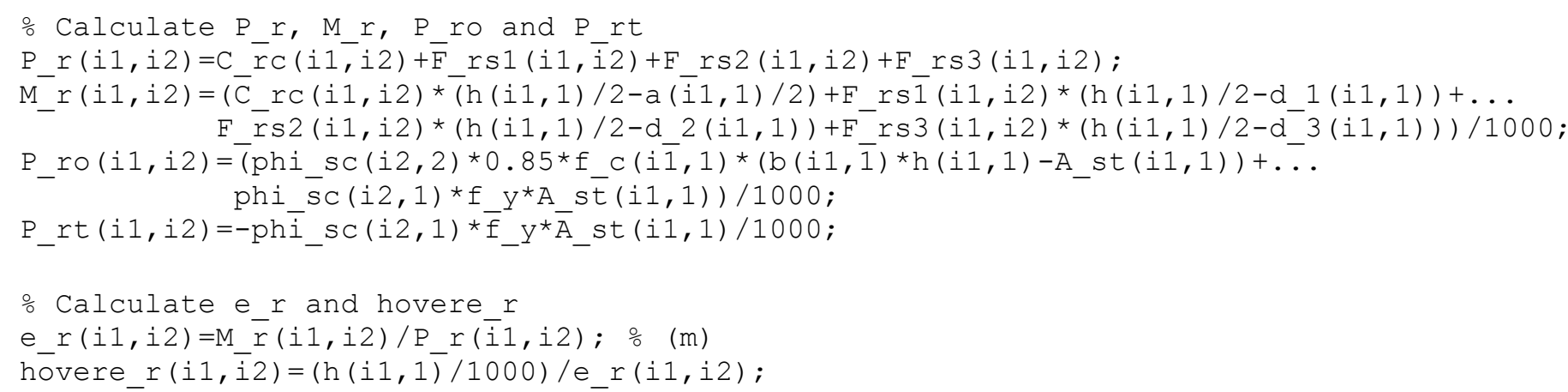

end

\section{A.1.3 Column Section 5}

\section{A.1.3.1 Code 1-Design Combined Flexural and Axial Strength Ratios, $\alpha_{P M}$} $\mathrm{ClC}$

clear

\% Design strength ratios calculation

eoverh=[ $\begin{array}{llllllllllllllllllllllllll}0.1 & 0.2 & 0.3 & 0.4 & 0.5 & 0.6 & 0.7 & 0.8 & 0.9 & 1.0 & 2.0 & 3.0 & 4.0 & 5.0 & 6.0 & 7.0 & 8.0 & 9.0 & 10.0 & -10.0 & \ldots\end{array}$ $-5.0-1.0-0.5-0.1] ; \div$ The specific e/h values

hovere=1. / eoverh;

eoverh_2=[ $\begin{array}{llllllllllllllllllllllllll}0 & 0.1 & 0.2 & 0.3 & 0.4 & 0.5 & 0.6 & 0.7 & 0.8 & 0.9 & 1.0 & 2.0 & 3.0 & 4.0 & 5.0 & 6.0 & 7.0 & 8.0 & 9.0 & 10.0 & -10.0 & \ldots\end{array}$ $-5.0-1.0-0.5-0.1-0]$; $\frac{0}{0}$ The specific e/h values, including extreme values hovere $2=1$. / eoverh 2 ;

Z $\mathrm{sam}=\overline{0} .30:-0.001:-35$;

\% Check whether eoverh locate in the range of e/h corresponding to $Z$ sam and $M>0$

\% Need to check hovere_usamb and hovere_rsamb after running the code

\% Upper boundary

[phiP_nsamb $(:, 1)$,phiM_nsamb $(:, 1)$, hovere_usamb $(:, 1), \sim, \sim]=$ feval ('DesignStrength_u_S5', z_sam (1) ) ; 


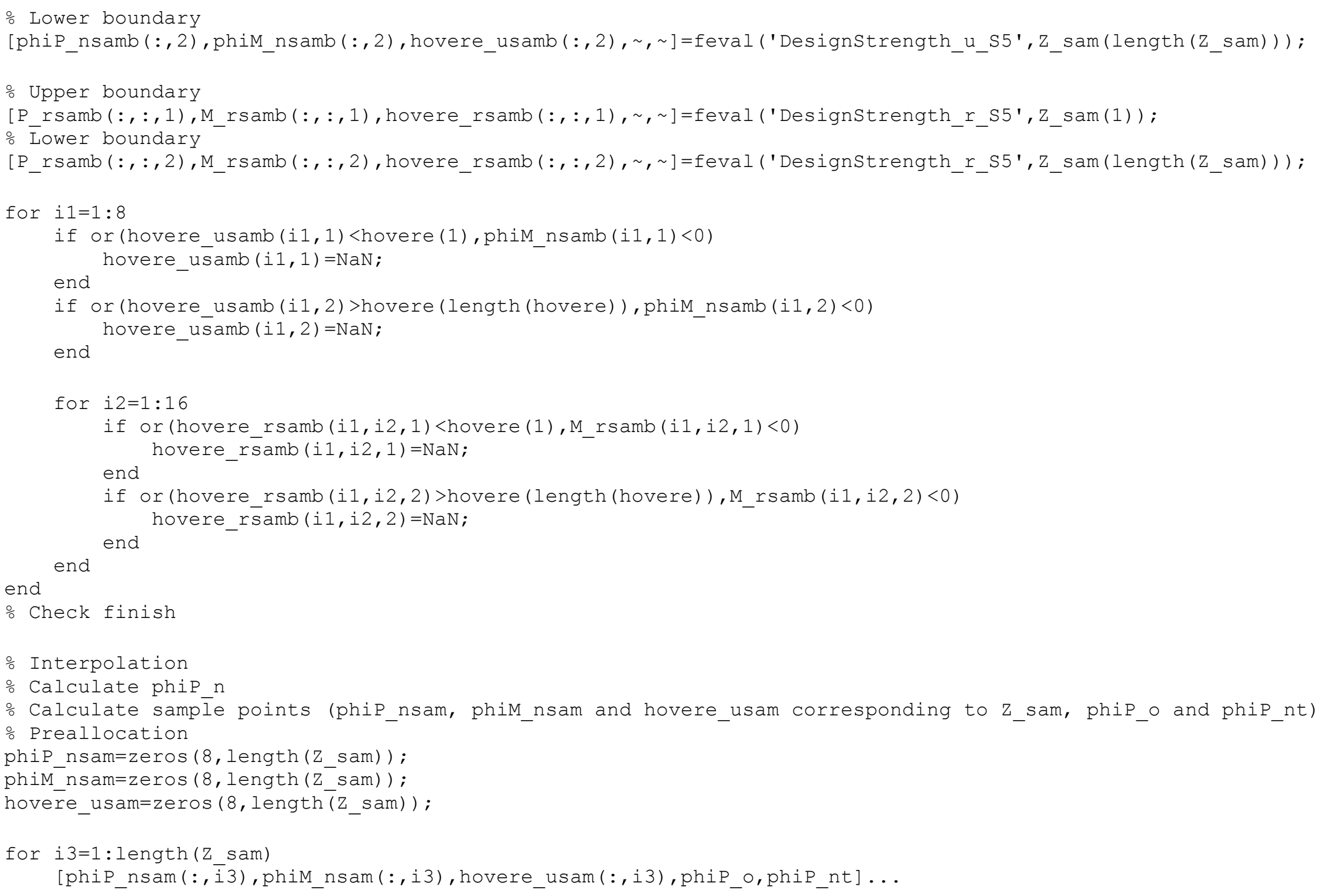


end

=feval ('Designstrength_u_S5',z_sam(i3));

\% Calculate phip nmax

phiP_nmax $=0.85 *$ phiP_o;

- Calculate the unknown points (phip $n$ corresponding to specific hovere)

o Preallocation

phiP_n=zeros (8, length (hovere));

for $i 1=1: 8$

s phim nsam=find (phim nsam (i1, :) >0);

phip nsampri=phip nsam(i1,s_phiM_nsam);

hovere_usampri=hovere_usam(îi,s_phim_nsam);

phiP nsampri=[phiP o(i1,1) phiP nsampri phip nt $(i 1,1)]$;

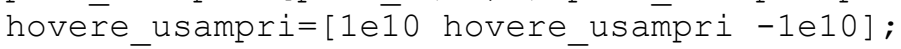

[hovere usampris, I hovere usampri]=sort (hovere usampri, 'descend') ;

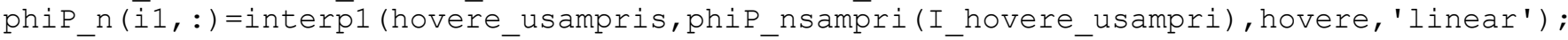

s_phì nmax=find (phiP n $(i \overline{1}, \overline{:})>\operatorname{phiP} \operatorname{nmax}(i \overline{1}, 1))$;

end

phiP $n(i 1, s$ phiP $n m a x)=p h i P \operatorname{nmax}(i 1,1)$

\% phiP_n includes phiP_nmax and phiP_nt

phiP_n=cat (2,phiP_nmax,phiP_n,phiP_nt) ;

- Calculate $\mathrm{P}$ r

o Calculate sämple points (P_rsam, M_rsam and hovere_rsam corresponding to Z_sam, P_ro and P_rt)

o Preallocation

P_rsam=zeros $\left(8,16\right.$, length $\left.\left(Z \_s a m\right)\right)$

M-rsam=zeros $\left(8,16\right.$, length $\left(z^{-}\right.$sam $\left.)\right)$;

hovere rsam=zeros $(8,16$, length ( $Z$ sam $))$;

for i $3=1$ : length ( $Z$ sam)

end

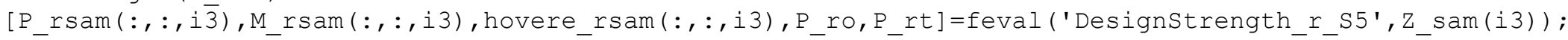


o Calculate P_rmax

P_rmax $=0.85 *$ P_ro;

\% Permute the 2nd and 3rd dimensions for P rsam, M rsam, hovere rsam, $\mathrm{P}$ ro, $\mathrm{P}$ rt and $\mathrm{P}$ rmax P_rsam=permute ( $\left.P_{\text {_rsam, }}[1,3,2]\right)$;

M rsam=permute (M rsam, $[1,3,2])$;

hovere_rsam=permute (hovere_rsam, [1,3,2]);

P ro=permute $(\mathrm{P} r o,[1,3,2])$;

P rt $=$ permute (P rt, $[1,3,2])$;

$\mathrm{P}_{-}{ }_{\text {rmax }}=\operatorname{permute}\left(\mathrm{P}_{\text {rmax }},[1,3,2]\right)$;

o Calculate the unknown points (P_r corresponding to specific hovere)

\% Preallocation

P_r=zeros (8, length (hovere), 16);

for $i 1=1: 8$

for $i 2=1: 16$

S_M_rsam=find (M_rsam $(i 1,:, i 2)>0)$;

P r r sampri=P rsam (i1, s M rsam,i2);

hovere rsampri=hovere rsam(i1, s M rsam,i2);

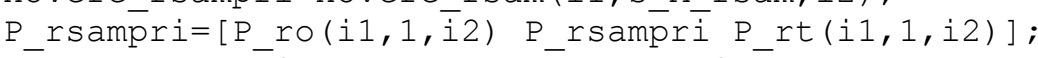

hovere rsampri=[1e10 hovere rsampri -1e10];

[hoverēersampris, I hovere rśsampri] =sort (hovere rsampri, 'descend') ;

P_r (i1,, ,i2) =interp 1 (hovere_rsampris, P_rsampri(I_hovere_rsampri), hovere, 'linear') ;

$S \mathrm{P} r \max =f i n d(\mathrm{P} r(i 1,:, i 2)>\mathrm{P} \operatorname{rmax}(i 1,1, i 2))$

$\mathrm{P}_{-}{ }_{\mathrm{r}}\left(\mathrm{i} 1, \mathrm{~s}_{-} \mathrm{P}_{-} \mathrm{rmax}, \mathrm{i} 2\right)=\mathrm{P}_{\text {rmax }}(i \overline{1}, 1, i 2)$;

end

end

$\div$ P_r includes P_rmax and P rt

P_r $=$ cat (2, P_rmax, P_r, P_rt);

\% Check whether the sign of phiP $n\left(\begin{array}{ll}P & r\end{array}\right)$ is identical with the sign of eoverh

ㅇ (Need to check the results after calculation)

for $i 1=1: 8$

for i $4=1+1$ : length (eoverh 2) -1

if sign (eoverh_2(i4) $) \sim=\operatorname{sign}\left(\operatorname{phiP} \_n(i 1, i 4)\right)$ 
end

phiP_n (i1, i4) $=\mathrm{NaN}$;

end

end

for $i 1=1: 8$

for $i 2=1: 16$

for i4=1+1: length (eoverh 2) -1

if sign(eoverh $2(i 4)) \sim=\operatorname{sign}(\mathrm{P} r(i 1, i 4, i 2))$ $\mathrm{P}_{-} r(\mathrm{i} 1, i 4, i \overline{2})=\mathrm{NaN}$;

end

$$
\text { end }
$$

end

end

- Calculate limited balance points

Z_bal $=-1$;

[phiP nbal, phiM nbal, hovere ubal, , ] =feval ('DesignStrength u S5', Z bal);

[P_rbāl, M_rbal, hovere_rbal, $\sim$, ] =feval ('DesignStrength_r_S5',z-bal);

\% Permute the 2nd and 3 rd dimensions for $\mathrm{P}$ rbal, M rbăl and hovere rbal

P rbal=permute (P_rbal, $[1,3,2])$;

M_rbal =permute (M_rbal, $[1,3,2])$;

hovere rbal=permute (hovere rbal, $[1,3,2])$;

\% Calculate design strength ratios

\% Preallocation

alpha $\mathrm{PM}=$ zeros $(8$, length (eoverh 2), 16);

for $i 1=1: 8$

for $i 2=1: 16$

for i $4=1$ : length (eoverh 2 )

alpha_PM(i1,i4,i2)=(abs (phiP_n(i1,i4))*sqrt $(1+$ eoverh_2(i4)^2))/..

end

$\left(\operatorname{abs}\left(P_{-} r(\bar{i} 1, i 4, i 2)\right) * \operatorname{sqrt}\left(1+\operatorname{eoverh}{ }^{-} 2(i 4)^{\wedge} 2\right)\right)$; 
save alpha_PM_S5.mat phiP_n P_r alpha_PM hovere_ubal hovere_rbal

\section{A.1.3.2 Code 2-Function of Design Strengths for ACl 318-14}

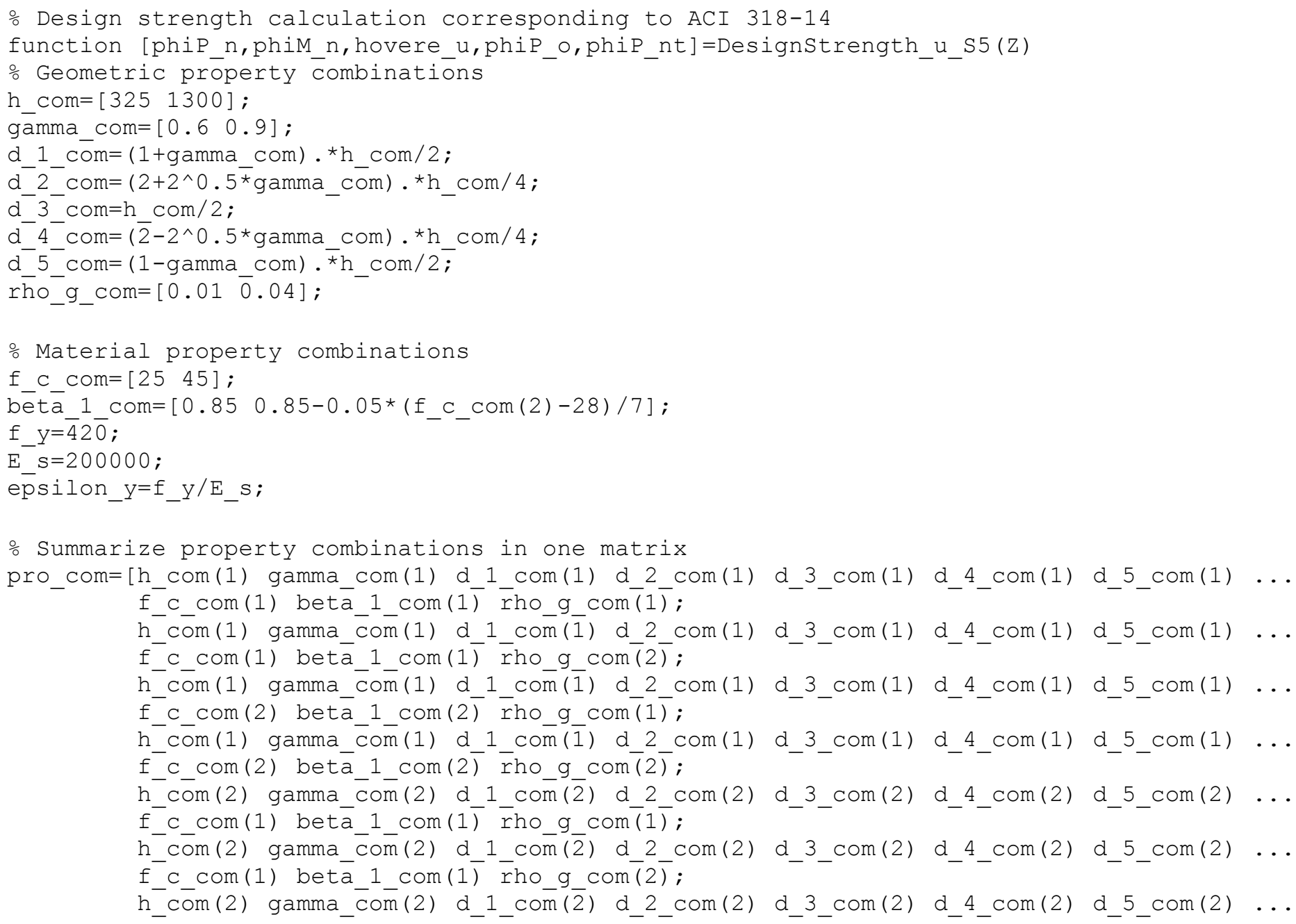


f_c_com (2) beta_1_com(2) rho_g_com(1) ;

h_com (2) gamma_com(2) d_1_com(2) d_2_com(2) d_3_com(2) d_4_com(2) d_5_com(2) ...

f_c_com (2) betā_1_com(2) $\bar{r}$ rho_g_com (2) ] ;

\% Preallocation

$\mathrm{h}=\mathrm{zeros}(8,1) ;$

gamma $=$ zeros $(8,1)$;

d_ $1=\operatorname{zeros}(8,1)$;

d $2=\operatorname{zeros}(8,1)$;

d- $3=\operatorname{zeros}(8,1)$;

d $4=\operatorname{zeros}(8,1)$;

d_ $5=\operatorname{zeros}(8,1)$;

f $c=\operatorname{zeros}(8,1)$;

beta $1=$ zeros $(8,1)$

rho_g $\bar{g}=\operatorname{zeros}(8,1)$;

A $s t=z e r o s ~(8,1)$;

A_s $1=\operatorname{zeros}(8,1)$;

A s $2=\operatorname{zeros}(8,1)$;

A s $3=\operatorname{zeros}(8,1)$;

A_s $4=\operatorname{zeros}(8,1)$;

A s $5=\operatorname{zeros}(8,1)$;

$\mathrm{C}=$ zeros $(8,1)$;

$\mathrm{a}=\operatorname{zeros}(8,1)$;

epsilon s1=zeros $(8,1)$

epsilon_s $2=\operatorname{zeros}(8,1)$;

epsilon $s 3=\operatorname{zeros}(8,1)$;

epsilon_s $4=\operatorname{zeros}(8,1)$;

epsilon_s $5=\operatorname{zeros}(8,1)$;

f $\mathrm{s} 1=$ zeros $(8,1)$;

f_s $2=\operatorname{zeros}(8,1)$;

f $\mathrm{s} 3=\operatorname{zeros}(8,1)$;

f $s 4=\operatorname{zeros}(8,1)$;

f s $5=\operatorname{zeros}(8,1)$;

angle theta $=$ zeros $(8,1)$.

$A=\operatorname{zeros}(8,1)$;

C $\mathrm{C}=$ zeros $(8,1)$;

F_s $1=\operatorname{zeros}(8,1)$; 


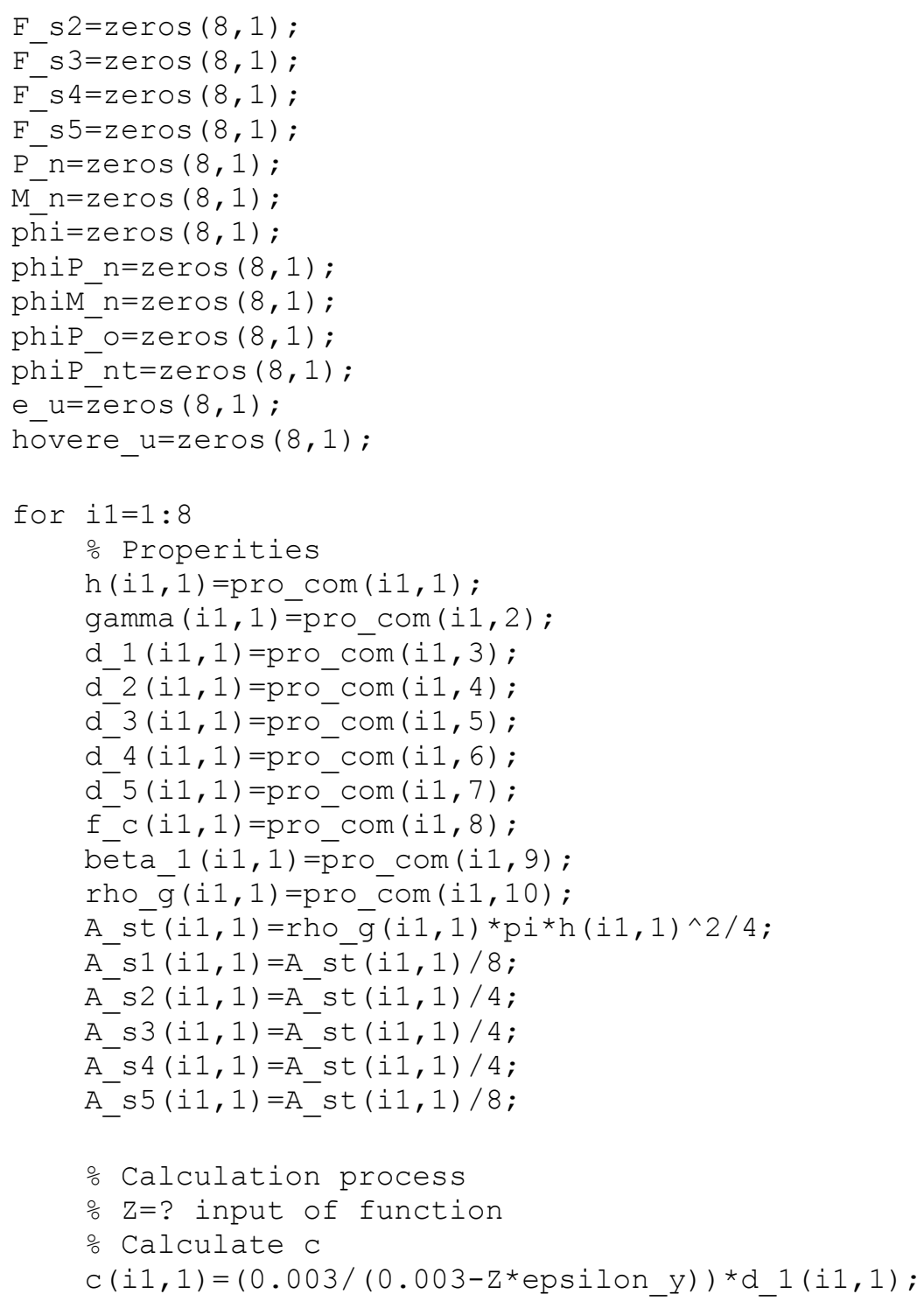


- Calculate a

$\mathrm{a}(\mathrm{i} 1,1)=\operatorname{beta} 1(\mathrm{i} 1,1){ }^{*} \mathrm{C}(\mathrm{i} 1,1)$;

o Compare a with $h$

if $\mathrm{a}(\mathrm{i} 1,1)>\mathrm{h}(\mathrm{i} 1,1)$

$$
\mathrm{a}(\mathrm{i} 1,1)=\mathrm{h}(\mathrm{i} 1,1) \text {; }
$$

end

\% Calculate epsilon s1, epsilon s2, epsilon s3, epsilon s4, epsilon s5, \% f s1, f s2, f s3, f_s4 and f_s5

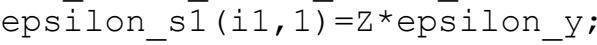

epsilon s2 $(i 1,1)=0.003 *(c(i 1,1)-d 2(i 1,1)) / c(i 1,1)$;

epsilon_s3 $(i 1,1)=0.003 *(c(i 1,1)-d-3(i 1,1)) / c(i 1,1)$;

epsilon ${ }^{-} \mathrm{s} 4(i 1,1)=0.003 *\left(\mathrm{c}(i 1,1)-\mathrm{d}^{-} 4(i 1,1)\right) / \mathrm{c}(i 1,1)$;

epsilon s5 $(i 1,1)=0.003 *(c(i 1,1)-d 5(i 1,1)) / c(i 1,1)$;

f_sl $(i 1,1)=e p s i l o n \_s 1(i 1,1) * E \_s$;

f $\mathrm{s} 2(i 1,1)=e$ silon $\mathrm{s} 2(\mathrm{i} 1,1) * \mathrm{E} \mathrm{s}$;

f_s3 $(i 1,1)=e p s i l o n \_s 3(i 1,1) * E_{-}^{-} s$;

f-s4 $(i 1,1)=e p s i l o n$ s4 $(i 1,1) * \mathrm{E}^{-} \mathrm{s}$;

f_s5 $(i 1,1)=e p s i l o n \_s 5(i 1,1) * E \_s$;

o Compare f_s1, f_s2, f_s3, f_s4 and f_s5 with +-f_y if f_sl $(i 1, \overline{1})>f_{-} y$$$
\bar{f} \_s 1(i 1,1)=\bar{f} \_y \text {; }
$$

elseif́f_sl(i1,1)<-f_y

$\left.f_{-}{ }^{-} \overline{(i 1}, 1\right)=-f_{-} y$;

end

if $f_{-} s 2(i 1,1)>f_{-} y$

$\bar{f} \mathrm{~s} 2(i 1,1)=\bar{f}_{-} \mathrm{y}$;

elseif f_s2 $(i 1,1)<-f \_y$

end

$$
f_{-} \mathrm{s} \overline{(i 1,1)}=-\mathrm{f} \_\mathrm{y} \text {; }
$$

if $\mathrm{f} \mathrm{s} 3(i 1,1)>\mathrm{f} y$

$\overline{\mathrm{f}} \mathrm{s} 3(\mathrm{i} 1,1)=\overline{\mathrm{f}} \mathrm{y}$;

elseiff $f 3(i 1,1)<-f$ y

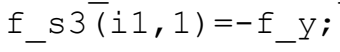


end

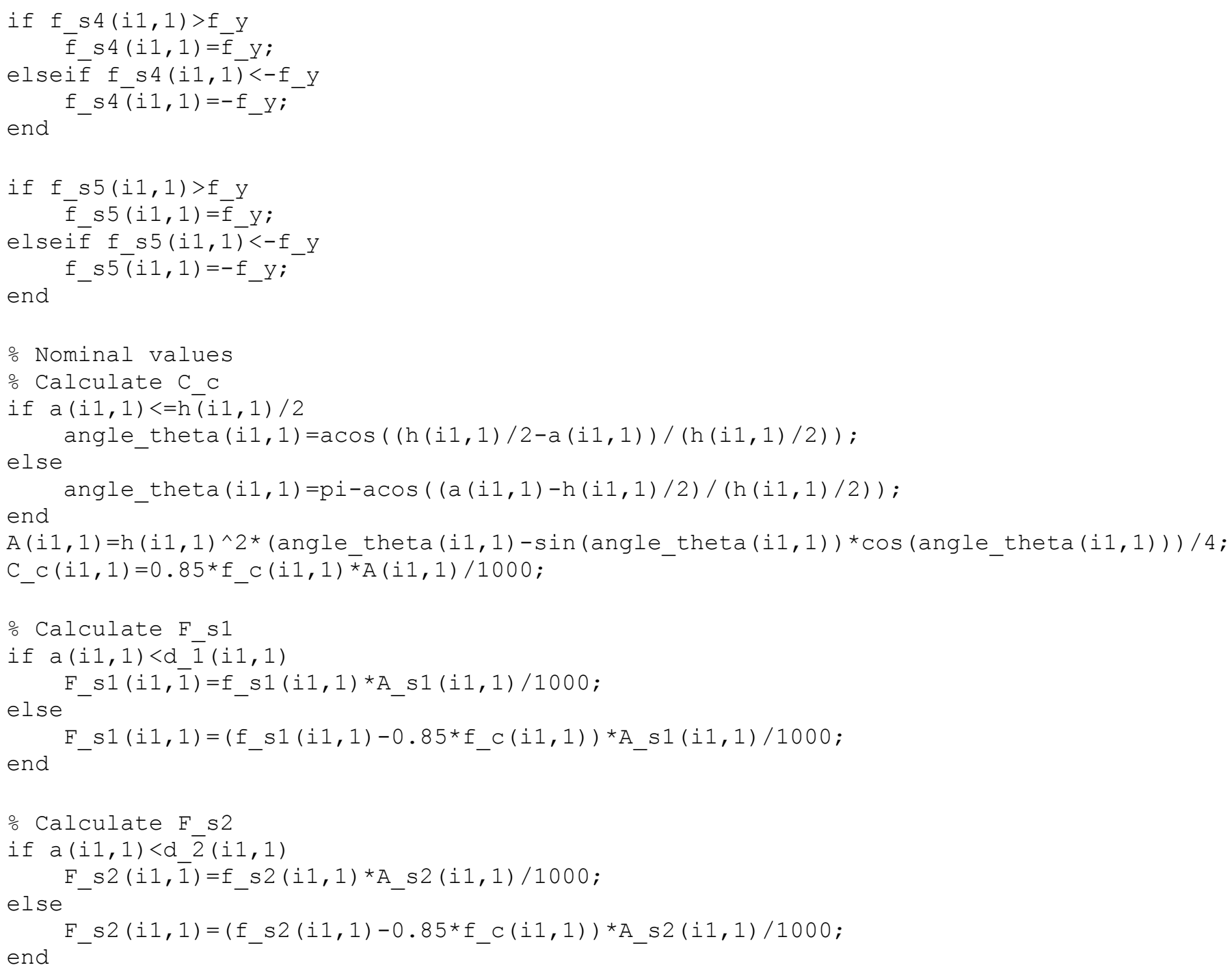




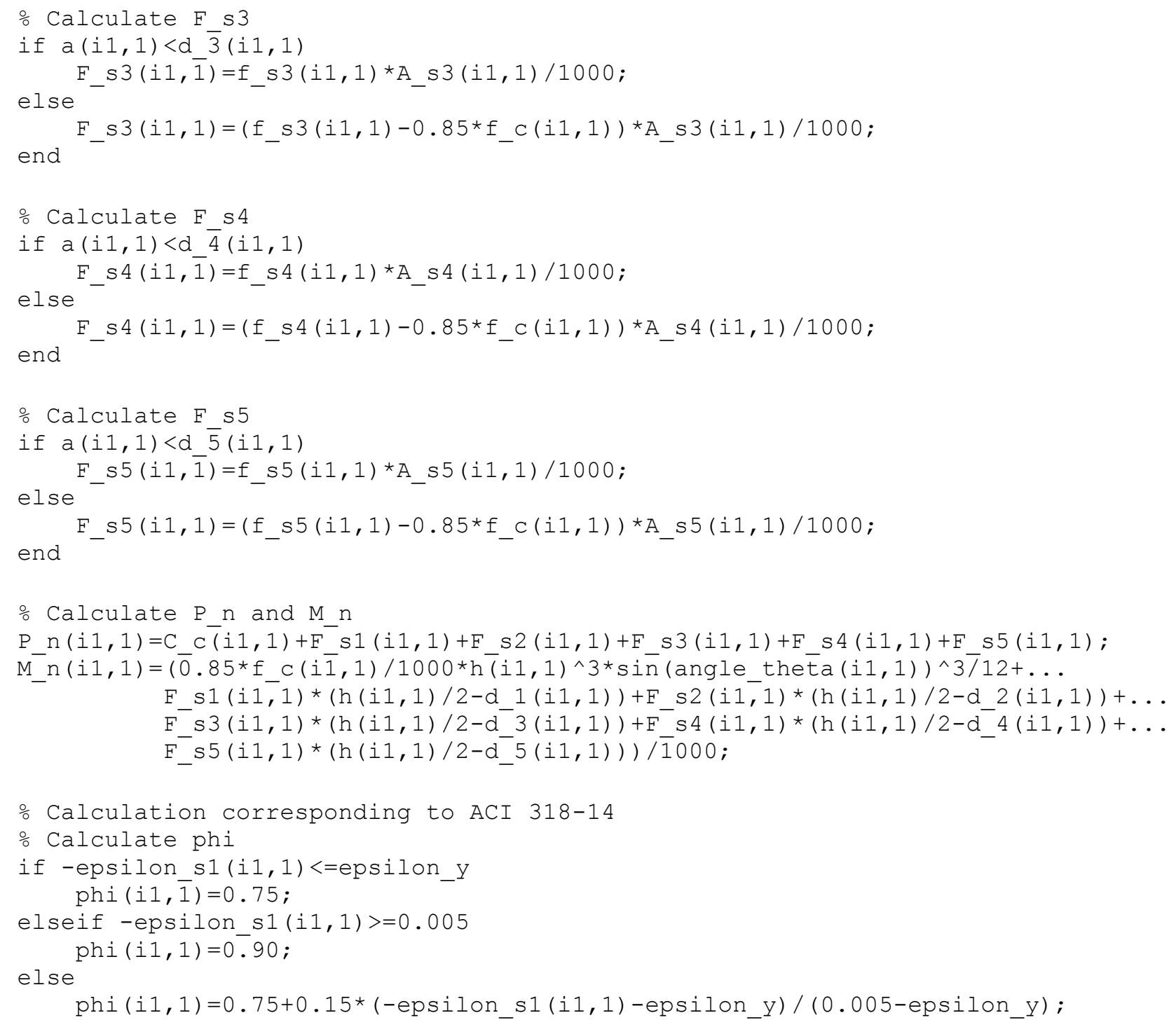


end

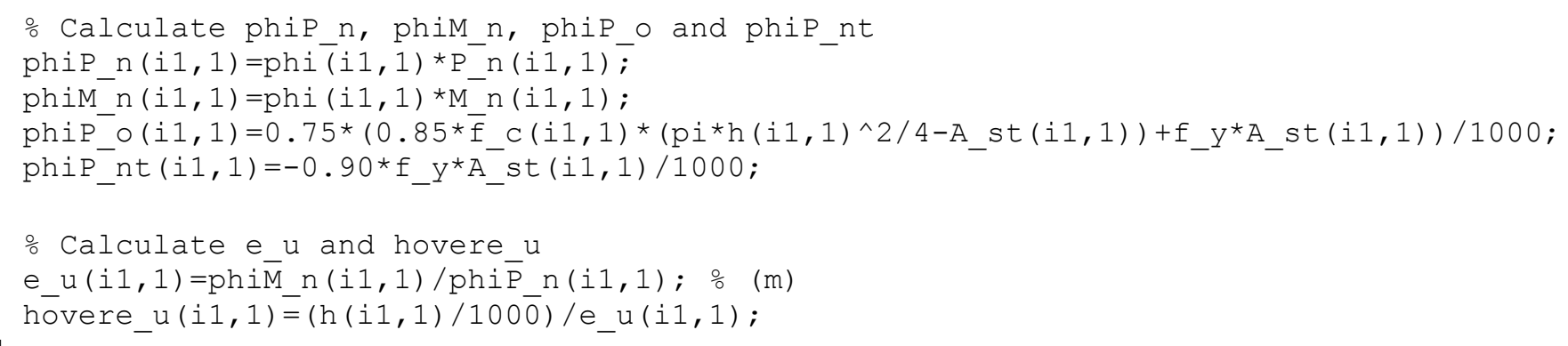

end

\section{A.1.3.3 Code 3-Function of Design Strengths for Partial Material Strength Reduction Factors}

o Design strength calculation corresponding to partial strength reduction factors

function [P_r,M_r, hovere_r, P_ro, P_rt] =DesignStrength_r_S5 (Z)

o Geometric property combinations

h_com $=\left[\begin{array}{ll}325 & 1300\end{array}\right]$;

gämma_com $=\left[\begin{array}{lll}0.6 & 0.9\end{array}\right]$;

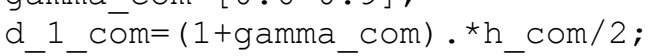

d $2 \mathrm{com}=\left(2+2^{\wedge} 0.5^{\star} \mathrm{gamma} \mathrm{com}\right) \cdot{ }^{\star} \mathrm{h} \mathrm{com} / 4$;

d_ 3 - $\mathrm{com}=\mathrm{h} \_\mathrm{com} / 2$;

d-4_com $=(\overline{2}-2 \wedge 0.5 *$ gamma com $) .{ }^{*} \mathrm{~h} \mathrm{com} / 4$;

d_5_com $=(1-$ gamma_com $) \cdot{ }^{\star}$ h_com $/ 2$;

rho_g_com $=\left[\begin{array}{lll}0.01 & \overline{0} .04\end{array}\right]$;

\% Material property combinations

f_c_com=[25 45] ;

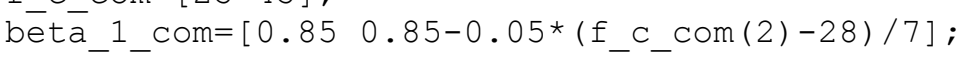

f $\mathrm{y}=\overline{4} 2 \overline{0}$;

E_s=200000;

epsilon_y=f_y/E_s;

o Summarize property combinations in one matrix

pro_com $=\left[\mathrm{h} \_\mathrm{com}(1)\right.$ gamma_com(1) d_1_com(1) d_2_com(1) d_3_com(1) d_4_com(1) d_5_com(1) ... 


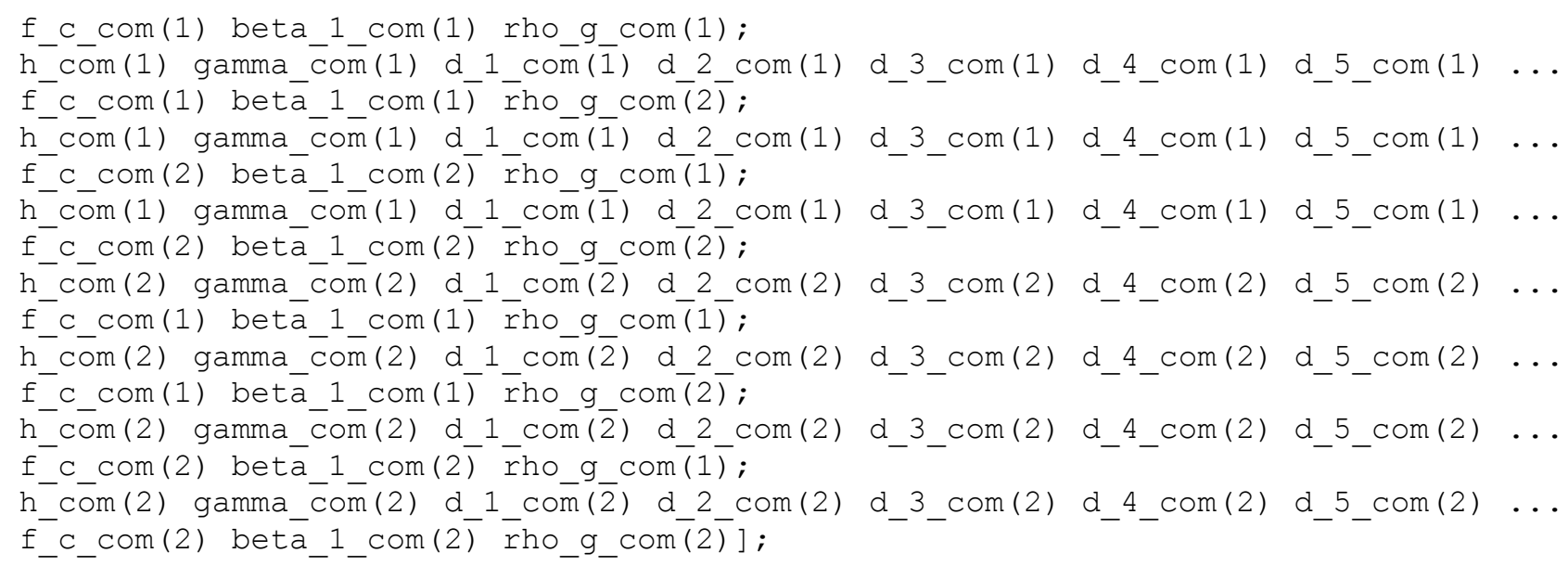

- Preallocation

$\mathrm{h}=\operatorname{zeros}(8,1)$;

gamma $=$ zeros $(8,1)$;

d_1 $=\operatorname{zeros}(8,1)$;

d $2=\operatorname{zeros}(8,1)$;

d_ $3=\operatorname{zeros}(8,1)$;

d- $4=\operatorname{zeros}(8,1)$;

d $5=\operatorname{zeros}(8,1)$;

$\mathrm{f}_{-}^{-} \mathrm{c}=\operatorname{zeros}(8,1)$;

beta $1=\operatorname{zeros}(8,1)$

rho_ $\bar{g}=\operatorname{zeros}(8,1)$;

A st $\bar{t}=\operatorname{zeros}(8,1)$;

A $s 1=z \operatorname{eros}(8,1)$;

A_s $2=\operatorname{zeros}(8,1)$;

A $s 3=\operatorname{zeros}(8,1)$;

A_s $4=\operatorname{zeros}(8,1)$;

A s $5=\operatorname{zeros}(8,1)$;

$C=$ zeros $(8,1)$;

$\mathrm{a}=\operatorname{zeros}(8,1)$;

epsilon sl=zeros $(8,1)$;

epsilon_s $2=\operatorname{zeros}(8,1)$; 
epsilon_s $3=\operatorname{zeros}(8,1)$; epsilon $s 4=$ zeros $(8,1)$. epsilon_s $5=\operatorname{zeros}(8,1)$; f_sl=zeros $(8,1)$;

f $s 2=\operatorname{zeros}(8,1)$;

f_s $3=\operatorname{zeros}(8,1)$;

f $s 4=\operatorname{zeros}(8,1)$;

f_s $5=\operatorname{zeros}(8,1)$;

angle theta $=$ zeros $(8,1)$;

$A=\operatorname{zeros}(8,1)$

C_rc $=$ zeros $(8,16)$;

Frsi=zeros $(8,16)$;

Frrs $=$ zeros $(8,16)$;

Frs $3=$ zeros $(8,16)$;

Frs $4=$ zeros $(8,16)$.

F_rs $5=\operatorname{zeros}(8,16)$;

P $r=z e r o s ~(8,16)$;

M_r $=\operatorname{zeros}(8,16)$;

P ro=zeros $(8,16)$;

P rt $=$ zeros $(8,16)$

$e^{-} r=\operatorname{zeros}(8,16)$;

hovere $r=$ zeros $(8,16)$;

for $i 1=1: 8$

\% Properities

h $(i 1,1)=\operatorname{pro} \operatorname{com}(i 1,1)$;

gamma $(i 1,1)=$ pro_com $(i 1,2)$;

d_1 $(i 1,1)=$ pro_com $(i 1,3)$;

d $2(i 1,1)=\operatorname{pro} \operatorname{com}(i 1,4)$;

d_3 $(i 1,1)=$ pro_com $(i 1,5)$;

d $4(i 1,1)=\operatorname{pro} \operatorname{com}(i 1,6)$;

d_5 $(i 1,1)=$ pro_com $(i 1,7)$;

$\mathrm{f}^{-} \mathrm{C}(\mathrm{i} 1,1)=$ pro_com $(i 1,8)$;

beta $1(i 1,1)=\overline{p r o} \operatorname{com}(i 1,9)$;

rho_g $(i 1,1)=$ pro_com $(i 1,10)$;

A st $(i 1,1)=r h o g(i 1,1) * p i * h(i 1,1)^{\wedge} 2 / 4$;

A_s1 $(i 1,1)=$ A_st $(i 1,1) / 8$; 
A_s2 $(i 1,1)=$ A_st $(i 1,1) / 4$;

A s3 $(i 1,1)=$ A st $(i 1,1) / 4$;

$A^{-} \mathrm{S} 4(i 1,1)=A^{-} \operatorname{st}(i 1,1) / 4$;

A_s5 $(i 1,1)=A_{-}$st $(i 1,1) / 8$;

- Calculation process

$\therefore \mathrm{Z}=$ ? input of function

- Calculate c

$c(i 1,1)=\left(0.003 /\left(0.003-Z^{*}\right.\right.$ epsilon y $\left.)\right) * d 1(i 1,1)$;

- Calculate a

$\mathrm{a}(i 1,1)=$ beta $1(i 1,1){ }^{*} \mathrm{C}(i 1,1)$;

\% Compare a with h

if $\mathrm{a}(\mathrm{i} 1,1)>\mathrm{h}(\mathrm{i} 1,1)$

$\mathrm{a}(\mathrm{i} 1,1)=\mathrm{h}(\mathrm{i} 1,1)$;

end

\% Calculate epsilon_s1, epsilon_s2, epsilon_s3, epsilon_s4, epsilon_s5,

$\circ \mathrm{f} s 1, \mathrm{f} 2 \mathrm{f} \mathrm{f} 3, \mathrm{f} \mathrm{s} 4$ and $\mathrm{f} \mathrm{s} 5$

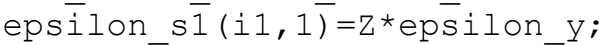

epsilon s2 $(i 1,1)=0.003 *(c(i 1,1)-d 2(i 1,1)) / c(i 1,1)$;

epsilon s3 $(i 1,1)=0.003 *(\mathrm{c}(i 1,1)-\mathrm{d} 3(i 1,1)) / \mathrm{c}(i 1,1)$;

epsilon_s4 $(i 1,1)=0.003 *\left(c(i 1,1)-d^{-} 4(i 1,1)\right) / c(i 1,1)$;

epsilon s5 $(i 1,1)=0.003 *(c(i 1,1)-d 5(i 1,1)) / c(i 1,1)$;

f_sl $(i 1,1)=e p s i l o n \_s 1(i 1,1) * E \_s$;

f $s 2(i 1,1)=e p s i l o n s 2(i 1,1){ }^{*} \mathrm{~s}$

f $s 3(i 1,1)=e p s i l o n s 3(i 1,1) * E s$

f_s4 $(i 1,1)=e p s i l o n \_s 4(i 1,1) * E_{-}^{-}$;

f $\mathrm{s} 5(i 1,1)=e p s i l o n s 5(i 1,1) * E s$

o Compare f_s1, f_s2, f_s3, f_s4 and f_s5 with +-f_y

if f_sl $(i 1, \overline{1})>f_{-} y$

$$
\text { f } s 1(i 1,1)=\bar{f} y \text {; }
$$

elseif f_sl(i1, 1)<-f_y

end

if $f_{-} s 2(i 1,1)>f_{-} y$ 
f_s2 $(i 1,1)=f \_y$;

elseif f $s 2(i 1,1)<-f \quad y$

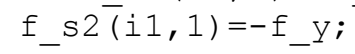

end

if f_s3 $(i 1,1)>f_{-} y$

f_s $3(i 1,1)=\bar{f}_{-} y$;

elseiff_s3 $(i 1,1)<-f \_y$

$\left.f_{-} 3 \overline{(i 1}, 1\right)=-f_{-} y$;

end

if $f_{-} s 4(i 1,1)>f_{y} y$

$$
\text { f_s } 4(i 1,1)=\bar{f} y \text {; }
$$

elseiff f_s4 (i1, 1)<-f_y

$$
\text { f_s } 4 \overline{(i 1}, 1)=-f_{-} y \text {; }
$$

end

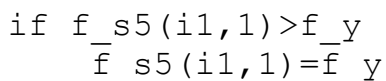

elseif f_s5 (i1, 1)<-f_y

end

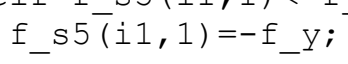

- Calculation corresponding to partial strength reduction factors

- Partial strength reduction factor combinations

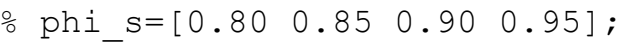

$\therefore$ phi $c=\left[\begin{array}{llll}0.60 & 0.65 & 0.70 & 0.75\end{array}\right]$;

phi_s $\bar{c}=\left[\begin{array}{lllll}0.80 & 0.60 ; 0.80 & 0.65 ; 0.80 & 0.70 ; 0.80 & 0.75 ;\end{array}\right.$ $0.850 .60 ; 0.850 .65 ; 0.850 .70 ; 0.850 .75 ;$

$0.900 .60 ; 0.900 .65 ; 0.900 .70 ; 0.900 .75 ;$

$0.950 .60 ; 0.950 .65 ; 0.950 .70 ; 0.950 .75] ;$

for $i 2=1: 16$

\% Calculate C rc

if $\mathrm{a}(i 1,1)<=\mathrm{h}(i 1,1) / 2$

angle theta $(i 1,1)=\operatorname{acos}((h(i 1,1) / 2-a(i 1,1)) /(h(i 1,1) / 2))$ else 

end

F_rs5 $(i 1, i 2)=\left(p h i \_s c(i 2,1) * f \_s 5(i 1,1)-p h i \_s c(i 2,2) * 0.85 * f \_c(i 1,1)\right) * A \_s 5(i 1,1) / 1000 ;$

\% Calculate P_r, M_r, P_ro and P_rt

P_r $(i 1, i 2)=C \_\bar{r} C(i 1, i 2)+\bar{F} \_r s 1(i 1, \bar{i} 2)+F \_r s 2(i 1, i 2)+F \_r s 3(i 1, i 2)+F \_r s 4(i 1, i 2)+F \_r s 5(i 1, i 2)$;

$\mathrm{M}-\mathrm{r}(\mathrm{i} 1, \mathrm{i} 2)=\left(\overline{\mathrm{ph}} \mathrm{sc}(\mathrm{i} 2,2) \star 0.85 * \mathrm{f} \mathrm{C}(\mathrm{i} 1, \overline{1}) / 1000 * \mathrm{~h}(\mathrm{i} 1, \overline{1}) \wedge 3 * \sin (\operatorname{angle} \operatorname{theta}(\mathrm{i} 1,1) \overline{1})^{\wedge} 3 / 12+\ldots\right.$

F_rsi $(i 1, i 2) *\left(h(i 1,1) / 2-d_{-} 1(i 1,1)\right)+F_{-} r s 2(i 1, i 2) *\left(h(i 1,1) / 2-d_{-} 2(i 1,1)\right)+\ldots$

$\mathrm{F}^{-} \operatorname{rs} 3(i 1, i 2) *\left(\mathrm{~h}(i 1,1) / 2-\mathrm{d}^{-} 3(i 1,1)\right)+\mathrm{F}^{-} \operatorname{rs} 4(i 1, i 2) *\left(\mathrm{~h}(i 1,1) / 2-\mathrm{d}^{-} 4(i 1,1)\right)+\ldots$

F-rs5 (i1, i2)*(h $\left.\left.(i 1,1) / 2-d_{-}^{-} 5(i 1,1)\right)\right) / \overline{1} 000$

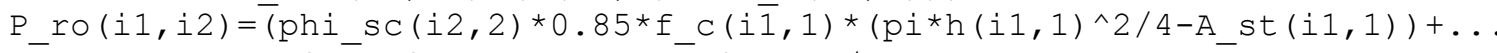

phi $\overline{s c}(i 2,1) * f y * A s \bar{t}(i 1,1)) / 1000$;

P_rt $(i 1, i 2)=-p h \bar{i} \_s c(i 2,1) * \bar{f} \_y * \bar{A} \_s t(i 1,1) / 1000$;

- Calculate e_r and hovere_r

e_r $(i 1, i 2)=M_{-} \bar{r}(i 1, i 2) / P_{r} r(\bar{i} 1, i 2) ;$ 응

hovere_r $(i 1, \bar{i} 2)=(h(i 1,1) / 1000) / e \_r(i 1, i 2)$;

end

end 


\section{Appendix B-Supplementary Information for Chapter 3}

\section{B.1 Supplemtentary Information for Concrete Compressive Strength}

Table B. 2 shows bias coefficients and coefficients of variation of $F_{1}$, for cast-in-place and precast concrete. The weighted average computed based on the data reported by Nowak and Szerszen (2003) has a bias coefficient of 1.238 and a coefficient of variation of 0.127 for cast-in-place concrete and a bias coefficient of 1.217 and a coefficient of variation 0.131 for precast concrete. Bartlett (2007) assumed $F_{1}$ for cast-in-place concrete has a bias coefficient of 1.27 and a coefficient of variation of 0.122 .

Table B.3 shows the bias coefficients and coefficients of variation of $F_{2}$ for cast-in-place and precast concrete (Bartlett 2007). Table B.4 shows the bias coefficients and coefficients of variation of $F_{i-p}$ for cast-in-place and precast concrete (Bartlett and MacGregor 1999).

Table B.5 shows statistical parameters for in-situ concrete compressive strength reported by Ellingwood et al. (1980). The values in this table intend to account for $F_{1}, F_{2}, F_{i-p}$ and $F_{r}$. There is no distinction between cast-in-place or precast concrete reported. 
Table B.1: Statistical parameters for geometric properties

\begin{tabular}{|c|c|c|c|c|}
\hline Item & Source & Comment & & \\
\hline Slabs & & & $\begin{array}{l}\text { Mean } \\
(\mathrm{mm})\end{array}$ & $\begin{array}{l}\sigma \\
(\mathrm{mm})\end{array}$ \\
\hline \multirow[t]{2}{*}{$h$} & Ellingwood et al. 1980 & 1696 Swedish slabs & Nominal+0.76 & 11.94 \\
\hline & & 99 slabs & Nominal+5.33 & 6.60 \\
\hline$d$ & Ellingwood et al. 1980 & $\begin{array}{l}\text { One-way slab, } \\
\text { bottom bars }\end{array}$ & Nominal-3.30 & 8.89 \\
\hline \multirow{4}{*}{$\begin{array}{l}\text { Beams } \\
b \\
h\end{array}$} & & & $\begin{array}{l}\text { Mean } \\
(\mathrm{mm})\end{array}$ & $\begin{array}{l}\sigma \\
(\mathrm{mm})\end{array}$ \\
\hline & Ellingwood et al. 1980 & Stem width & Nominal +2.54 & 3.81 \\
\hline & Ellingwood et al. 1980 & 108 beams & Nominal-3.05 & 6.35 \\
\hline & & 24 beams & Nominal +20.57 & 13.97 \\
\hline \multicolumn{2}{|l|}{ Columns } & & $\begin{array}{l}\text { Mean } \\
(\mathrm{mm})\end{array}$ & $\begin{array}{l}\sigma \\
(\mathrm{mm})\end{array}$ \\
\hline$b, h$ & Ellingwood et al. 1980 & Rectangular & Nominal+1.52 & 6.35 \\
\hline$h$ & Mirza and MacGregor 1979 & Circular & Nominal & 4.76 \\
\hline \multirow{3}{*}{$\begin{array}{l}\text { Slabs, Be } \\
d\end{array}$} & ams and Columns & & $\begin{array}{l}\text { Mean } \\
(\mathrm{mm})\end{array}$ & $\begin{array}{l}\sigma \\
(\mathrm{mm})\end{array}$ \\
\hline & ACI Committee 3182014 & $d \leq 203 \mathrm{~mm}$ & Nominal & 4.76 \\
\hline & & $d>203 \mathrm{~mm}$ & Nominal & 6.35 \\
\hline \multicolumn{2}{|c|}{ Reinforcement } & & $\delta$ & $V$ \\
\hline$A_{s}$ & Nowak and Szerszen 2003 & - & 1.0 & 0.015 \\
\hline
\end{tabular}


Table B.2: Statistical parameters for $F_{1}$

\begin{tabular}{lllll}
\hline Source & Comment & $n$ & $\delta$ & $V$ \\
\hline Nowak and & Cast-in-place concrete, $f_{c}{ }^{\prime}=20.7 \mathrm{MPa}$ & 88 & 1.35 & 0.102 \\
Szerszen 2003 & Cast-in-place concrete, $f_{c}{ }^{\prime}=24.1 \mathrm{MPa}$ & 25 & 1.21 & 0.079 \\
& Cast-in-place concrete, $f_{c}{ }^{\prime}=27.6 \mathrm{MPa}$ & 116 & 1.235 & 0.145 \\
& Cast-in-place concrete, $f_{c}{ }^{\prime}=31.0 \mathrm{MPa}$ & 28 & 1.14 & 0.042 \\
& Cast-in-place concrete, $f_{c}{ }^{\prime}=34.5 \mathrm{MPa}$ & 30 & 1.15 & 0.058 \\
& Cast-in-place concrete, $f_{c}{ }^{\prime}=41.3 \mathrm{MPa}$ & 30 & 1.12 & 0.042 \\
& Mean & & 1.238 & 0.127 \\
& Precast concrete, $f_{c}{ }^{\prime}=34.5 \mathrm{MPa}$ & 330 & 1.38 & 0.120 \\
& Precast concrete, $f_{c}{ }^{\prime}=37.9 \mathrm{MPa}$ & 26 & 1.19 & 0.101 \\
& Precast concrete, $f_{c}{ }^{\prime}=41.3 \mathrm{MPa}$ & 493 & 1.16 & 0.090 \\
& Precast concrete, $f_{c}{ }^{\prime}=44.8 \mathrm{MPa}$ & 325 & 1.14 & 0.081 \\
& Mean & & 1.217 & 0.131 \\
& Cast-in-place concrete, $f_{c}{ }^{\prime}=25-45 \mathrm{MPa}$ & 85 & 1.27 & 0.122 \\
\hline
\end{tabular}

Note: $n$, number of samples.

Table B.3: Statistical parameters for $F_{2}$

\begin{tabular}{llll}
\hline Source & Comment & $\delta$ & $V$ \\
\hline Bartlett 2007 & Cast-in-place concrete & 1.03 & 0.113 \\
& Precast concrete & 0.95 & 0.133 \\
\hline
\end{tabular}

Table B.4: Statistical parameters for $F_{i-p}$

\begin{tabular}{llll}
\hline Source & Comment & $\delta$ & $V$ \\
\hline Bartlett and MacGregor 1999 & Cast-in-place concrete & 1.0 & 0.130 \\
& Precast concrete & 1.0 & 0.103 \\
\hline
\end{tabular}


Table B.5: Statistical parameters for in-situ concrete compressive strength

\begin{tabular}{lllll}
\hline Source & Comment & $\begin{array}{l}\text { Mean } \\
(\mathrm{MPa})\end{array}$ & $\delta$ & $V$ \\
\hline Ellingwood et al. 1980 & $f_{c}^{\prime}=21 \mathrm{MPa}$ & 19.3 & 0.92 & 0.18 \\
& $f_{c}^{\prime}=28 \mathrm{MPa}$ & 23.7 & 0.85 & 0.18 \\
& $f_{c}^{\prime}=35 \mathrm{MPa}$ & 28.2 & 0.81 & 0.15 \\
\hline
\end{tabular}

Table B.6: Statistical parameters for $f_{y}=420 \mathrm{MPa}$

\begin{tabular}{|c|c|c|c|c|c|}
\hline Source & Bar size & $\begin{array}{l}\text { Mean yield } f_{y} \\
(\mathrm{MPa})\end{array}$ & $n$ & $\delta$ & $V$ \\
\hline Nowak and & No.3 $(9.5 \mathrm{~mm})$ & 496.1 & 72 & 1.20 & 0.04 \\
\hline \multirow[t]{9}{*}{ Szerszen 2003} & No.4 (12.5mm) & 473.3 & 79 & 1.145 & 0.065 \\
\hline & No.5 (15.5mm) & 465.1 & 116 & 1.125 & 0.04 \\
\hline & No.6 (19mm) & 476.1 & 38 & 1.15 & 0.05 \\
\hline & No.7 (22mm) & 481.6 & 29 & 1.165 & 0.05 \\
\hline & No.8 (25mm) & 473.7 & 36 & 1.145 & 0.05 \\
\hline & No.9 (28mm) & 475.7 & 28 & 1.15 & 0.05 \\
\hline & No.10 (31mm) & 470.2 & 5 & 1.14 & 0.04 \\
\hline & No.11 (34.5mm) & 473.7 & 13 & 1.145 & 0.035 \\
\hline & Recommended & & & 1.145 & 0.05 \\
\hline $\begin{array}{l}\text { Ellingwood et al. } \\
1980\end{array}$ & - & 472.5 & - & 1.125 & 0.098 \\
\hline
\end{tabular}


Table B.7: Means and standard deviations of reliability indices for moment using partial material strength reduction factors, $\beta_{M r}$, for $f_{c}^{\prime}=45 \mathrm{MPa}$ and $\rho=0.003-0.005$

\begin{tabular}{ccccccccccc}
\hline & & \multicolumn{10}{c}{$\phi_{c}$} \\
\cline { 3 - 10 }$w_{L} / w_{D}$ & $\phi_{s}$ & Mean & $\sigma$ & Mean & $\sigma$ & Mean & $\sigma$ & Mean & $\sigma$ \\
\cline { 2 - 10 } 0.5 & 0.80 & 3.938 & 0.023 & 3.924 & 0.019 & 3.911 & 0.016 & 3.900 & 0.013 \\
& 0.85 & 3.568 & 0.025 & 3.552 & 0.021 & 3.539 & 0.018 & 3.527 & 0.015 \\
& 0.90 & 3.219 & 0.027 & 3.202 & 0.023 & 3.188 & 0.019 & 3.176 & 0.016 \\
& 0.95 & 2.889 & 0.030 & 2.872 & 0.025 & 2.857 & 0.021 & 2.844 & 0.018 \\
& & & & & & & & & \\
1.5 & 0.80 & 3.695 & 0.017 & 3.683 & 0.014 & 3.672 & 0.011 & 3.663 & 0.009 \\
& 0.85 & 3.391 & 0.019 & 3.378 & 0.016 & 3.367 & 0.013 & 3.357 & 0.010 \\
& 0.90 & 3.104 & 0.021 & 3.091 & 0.018 & 3.079 & 0.015 & 3.069 & 0.012 \\
& 0.95 & 2.834 & 0.023 & 2.820 & 0.019 & 2.808 & 0.016 & 2.797 & 0.013 \\
\hline
\end{tabular}

Table B.8: Means and standard deviations of reliability indices for moment using partial material strength reduction factors, $\beta_{M r}$, for $f_{c}^{\prime}=45 \mathrm{MPa}$ and $\rho=0.006-0.010$

\begin{tabular}{cccccccccc}
\hline & & \multicolumn{10}{c}{$\phi_{c}$} \\
\cline { 3 - 10 }$w_{L} / w_{D}$ & $\phi_{s}$ & Mean & $\sigma$ & Mean & $\sigma$ & Mean & $\sigma$ & Mean & $\sigma$ \\
\cline { 3 - 10 } 0.5 & 0.80 & 4.042 & 0.042 & 4.012 & 0.035 & 3.985 & 0.030 & 3.963 & 0.025 \\
& 0.85 & 3.679 & 0.045 & 3.647 & 0.038 & 3.619 & 0.032 & 3.595 & 0.027 \\
& 0.90 & 3.339 & 0.049 & 3.304 & 0.041 & 3.275 & 0.035 & 3.249 & 0.030 \\
& 0.95 & 3.018 & 0.053 & 2.982 & 0.045 & 2.950 & 0.038 & 2.923 & 0.032 \\
& & & & & & & & & \\
1.5 & 0.80 & 3.770 & 0.030 & 3.745 & 0.025 & 3.724 & 0.020 & 3.705 & 0.017 \\
& 0.85 & 3.474 & 0.034 & 3.447 & 0.028 & 3.424 & 0.023 & 3.405 & 0.019 \\
& 0.90 & 3.196 & 0.037 & 3.167 & 0.031 & 3.143 & 0.026 & 3.122 & 0.021 \\
& 0.95 & 2.934 & 0.040 & 2.904 & 0.034 & 2.878 & 0.029 & 2.856 & 0.024 \\
\hline
\end{tabular}


Table B.9: Means and standard deviations of reliability indices for moment using partial material strength reduction factors, $\beta_{M r}$, for $f_{c}^{\prime}=45 \mathrm{MPa}$ and $\rho=0.011-0.018$

\begin{tabular}{ccccccccccc}
\hline & & \multicolumn{10}{c}{$\phi_{c}$} \\
\cline { 3 - 10 }$w_{L} / w_{D}$ & $\phi_{s}$ & Mean & $\sigma$ & Mean & $\sigma$ & Mean & $\sigma$ & Mean & $\sigma$ \\
\cline { 2 - 10 } 0.5 & 0.80 & 4.214 & 0.061 & 4.155 & 0.050 & 4.104 & 0.040 & 4.061 & 0.032 \\
& 0.85 & 3.868 & 0.069 & 3.805 & 0.056 & 3.751 & 0.046 & 3.704 & 0.037 \\
& 0.90 & 3.545 & 0.076 & 3.478 & 0.063 & 3.420 & 0.052 & 3.371 & 0.042 \\
& 0.95 & 3.243 & 0.084 & 3.171 & 0.070 & 3.110 & 0.058 & 3.057 & 0.048 \\
& & & & & & & & & \\
1.5 & 0.80 & 3.896 & 0.045 & 3.847 & 0.036 & 3.806 & 0.028 & 3.771 & 0.022 \\
& 0.85 & 3.615 & 0.052 & 3.563 & 0.042 & 3.519 & 0.033 & 3.482 & 0.026 \\
& 0.90 & 3.353 & 0.058 & 3.298 & 0.048 & 3.251 & 0.039 & 3.211 & 0.031 \\
& 0.95 & 3.107 & 0.065 & 3.049 & 0.054 & 2.999 & 0.044 & 2.956 & 0.036 \\
\hline
\end{tabular}

Table B.10: Means and standard deviations of reliability index ratios for shear, $\beta_{V u} / \beta_{V r}$, for $f_{c}^{\prime}=45 \mathrm{MPa}$ and $\rho_{t}=0.001-0.010$

\begin{tabular}{cccccccccc}
\hline & & \multicolumn{8}{c}{$\phi_{c}$} \\
\cline { 3 - 10 }$w_{L} / w_{D}$ & $\phi_{s}$ & Mean & $\sigma$ & Mean & $\sigma$ & Mean & $\sigma$ & Mean & $\sigma$ \\
\cline { 3 - 10 } 0.5 & 0.80 & 0.967 & 0.042 & 0.991 & 0.032 & 1.015 & 0.022 & 1.041 & 0.011 \\
& 0.85 & 1.007 & 0.053 & 1.031 & 0.044 & 1.057 & 0.034 & 1.083 & 0.023 \\
& 0.90 & 1.048 & 0.066 & 1.074 & 0.057 & 1.100 & 0.046 & 1.128 & 0.036 \\
& 0.95 & 1.092 & 0.080 & 1.118 & 0.070 & 1.146 & 0.060 & 1.174 & 0.049 \\
& & & & & & & & & \\
1.5 & 0.80 & 0.969 & 0.039 & 0.991 & 0.030 & 1.014 & 0.020 & 1.037 & 0.010 \\
& 0.85 & 1.006 & 0.049 & 1.029 & 0.040 & 1.052 & 0.031 & 1.076 & 0.021 \\
& 0.90 & 1.044 & 0.061 & 1.067 & 0.052 & 1.091 & 0.042 & 1.116 & 0.032 \\
& 0.95 & 1.083 & 0.073 & 1.107 & 0.064 & 1.132 & 0.054 & 1.158 & 0.044 \\
\hline
\end{tabular}




$$
\begin{aligned}
& \rightarrow \times-(0.80,0.60) \quad \longrightarrow(0.80,0.65) \quad \longrightarrow \times-(0.80,0.70) \quad--x-\cdot(0.80,0.75) \\
& \longrightarrow \cdot-(0.85,0.60) \quad \square(0.85,0.65) \quad \longrightarrow-(0.85,0.70) \quad--\square-\cdot(0.85,0.75) \\
& \rightarrow \cdot-(0.90,0.60) \quad \longrightarrow(0.90,0.65) \quad \longrightarrow-(0.90,0.70) \quad--\Delta-\cdot(0.90,0.75) \\
& \longrightarrow \cdot(0.95,0.60) \quad 0(0.95,0.65) \quad \longrightarrow(0.95,0.70) \quad-\Theta-\cdot(0.95,0.75) \\
& \text { … } \cdots \text { ACI } 318-14
\end{aligned}
$$

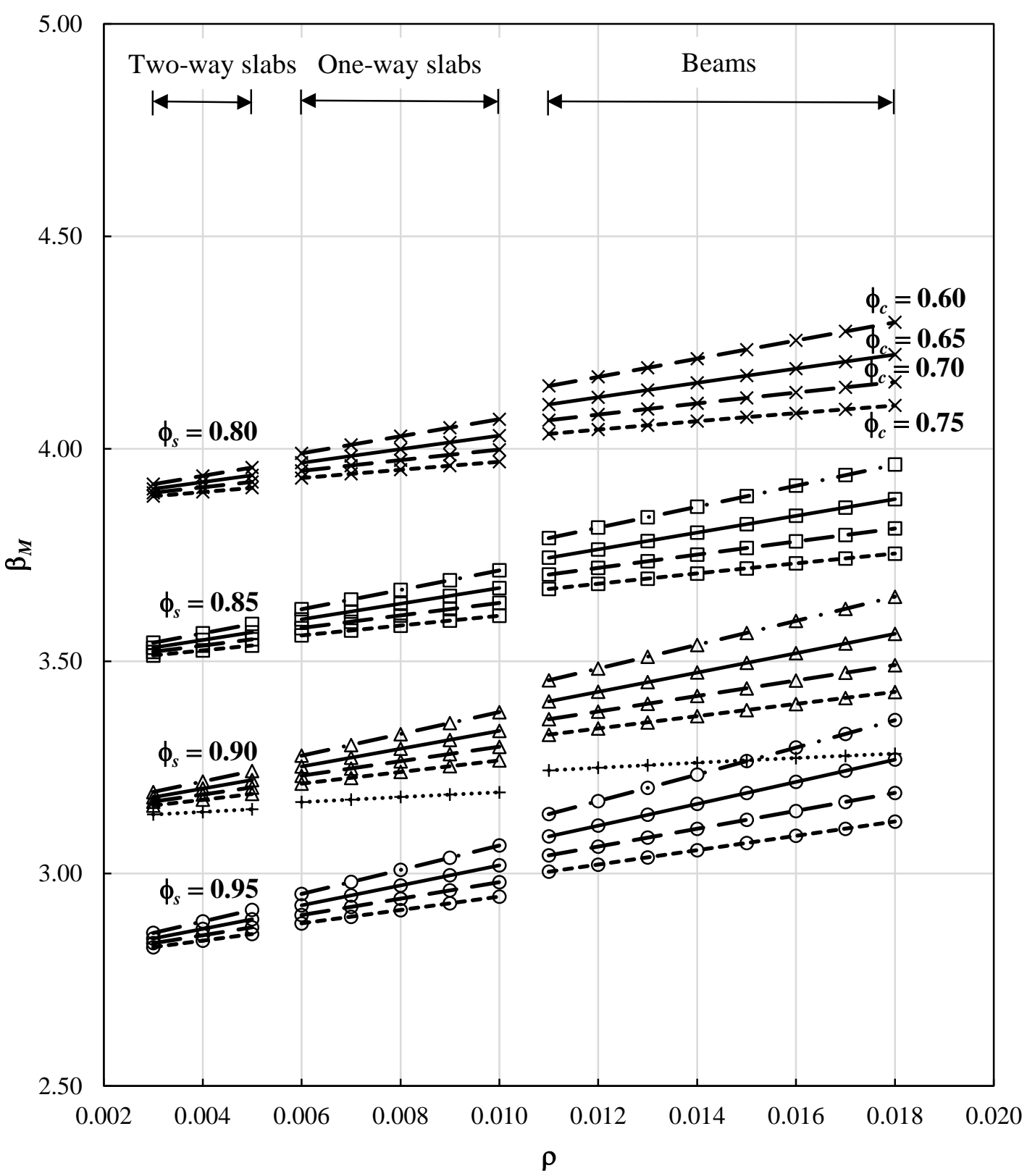

Figure B.1: Reliability indices for moment, $\beta_{M}$, for $f_{c}{ }^{\prime}=45 \mathrm{MPa}, w_{L} / w_{D}=0.5, \rho=0.003-$ 0.018 , and constant coefficients of variation for $d$ 


\begin{tabular}{|c|c|c|c|}
\hline$\longrightarrow \times \cdot-(0.80,0.60)$ & $\longrightarrow \leftarrow(0.80,0.65)$ & $\longrightarrow x-(0.80,0.70)$ & $--x-\cdot(0.80,0.75)$ \\
\hline$\longrightarrow \cdot-(0.85,0.60)$ & $\square(0.85,0.65)$ & $\square-(0.85,0.70)$ & $--\square-・(0.85,0.75)$ \\
\hline$\longrightarrow \cdot-(0.90,0.60)$ & $\triangle(0.90,0.65)$ & $\triangle-(0.90,0.70)$ & $--\Delta-\cdot(0.90,0.75)$ \\
\hline$\longrightarrow \cdot-(0.95,0.60)$ & $\longrightarrow(0.95,0.65)$ & $\longrightarrow-(0.95,0.70)$ & $--\Theta-\cdot(0.95,0.75)$ \\
\hline - ACI 318-14 & & & \\
\hline
\end{tabular}

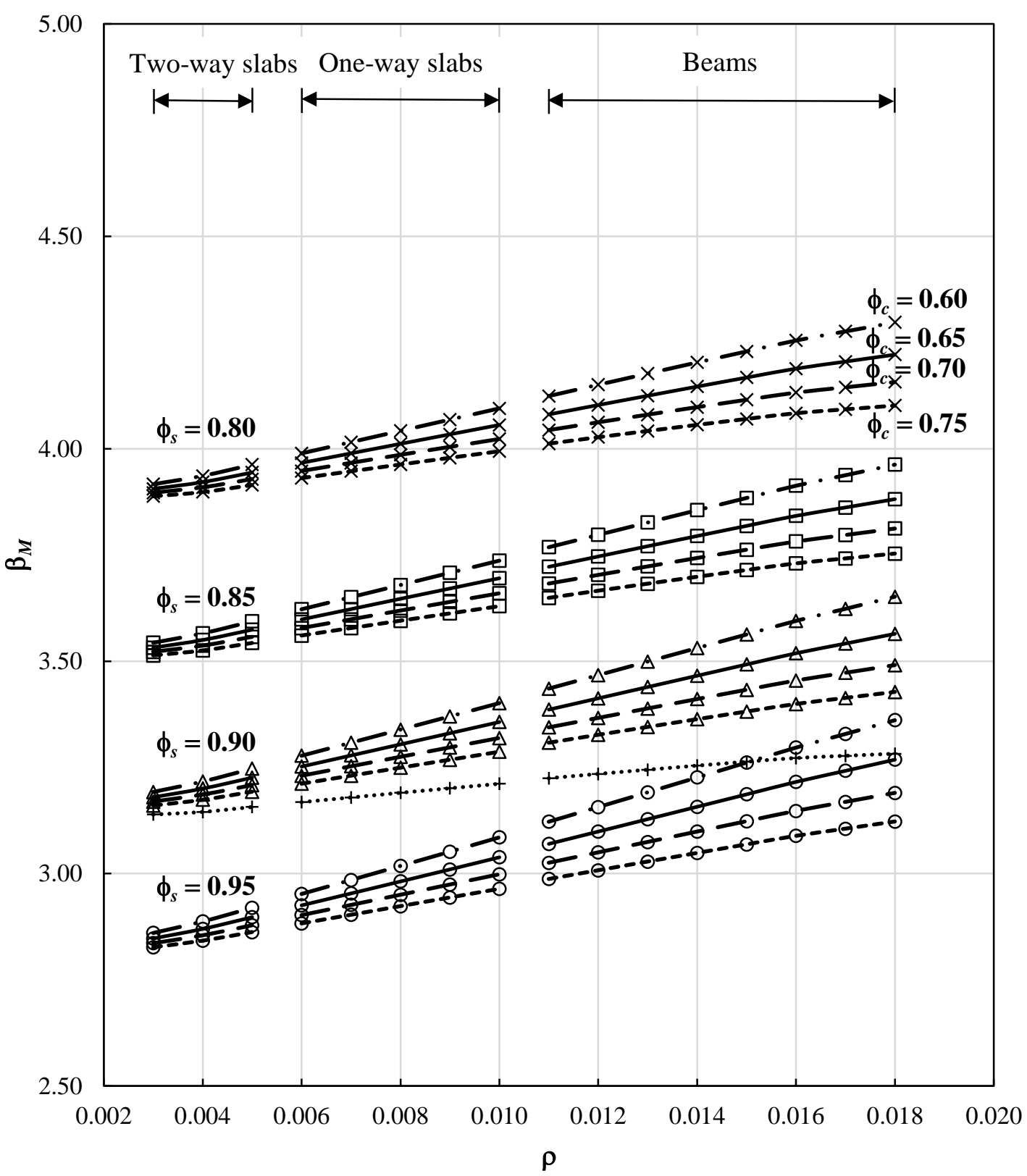

Figure B.2: Reliability indices for moment, $\beta_{M}$, for $f_{c}{ }^{\prime}=45 \mathrm{MPa}, w_{L} / w_{D}=0.5, \rho=0.003-$ 0.018 , and linear coefficients of variation for $d$ 


$$
\begin{aligned}
& \rightarrow \times \cdot-(0.80,0.60) \quad \longrightarrow(0.80,0.65) \quad \longrightarrow \times-(0.80,0.70) \quad--x-\cdot(0.80,0.75) \\
& \longrightarrow \cdot-(0.85,0.60) \quad \square(0.85,0.65) \quad \longrightarrow-(0.85,0.70) \quad--\square-\cdot(0.85,0.75) \\
& \rightarrow \cdot-(0.90,0.60) \quad \longrightarrow(0.90,0.65) \quad \longrightarrow-(0.90,0.70) \quad--\Delta-\cdot(0.90,0.75) \\
& \longrightarrow \cdot(0.95,0.60) \quad 0(0.95,0.65) \quad \longrightarrow(0.95,0.70) \quad-\Theta-\cdot(0.95,0.75)
\end{aligned}
$$

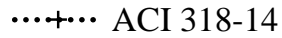

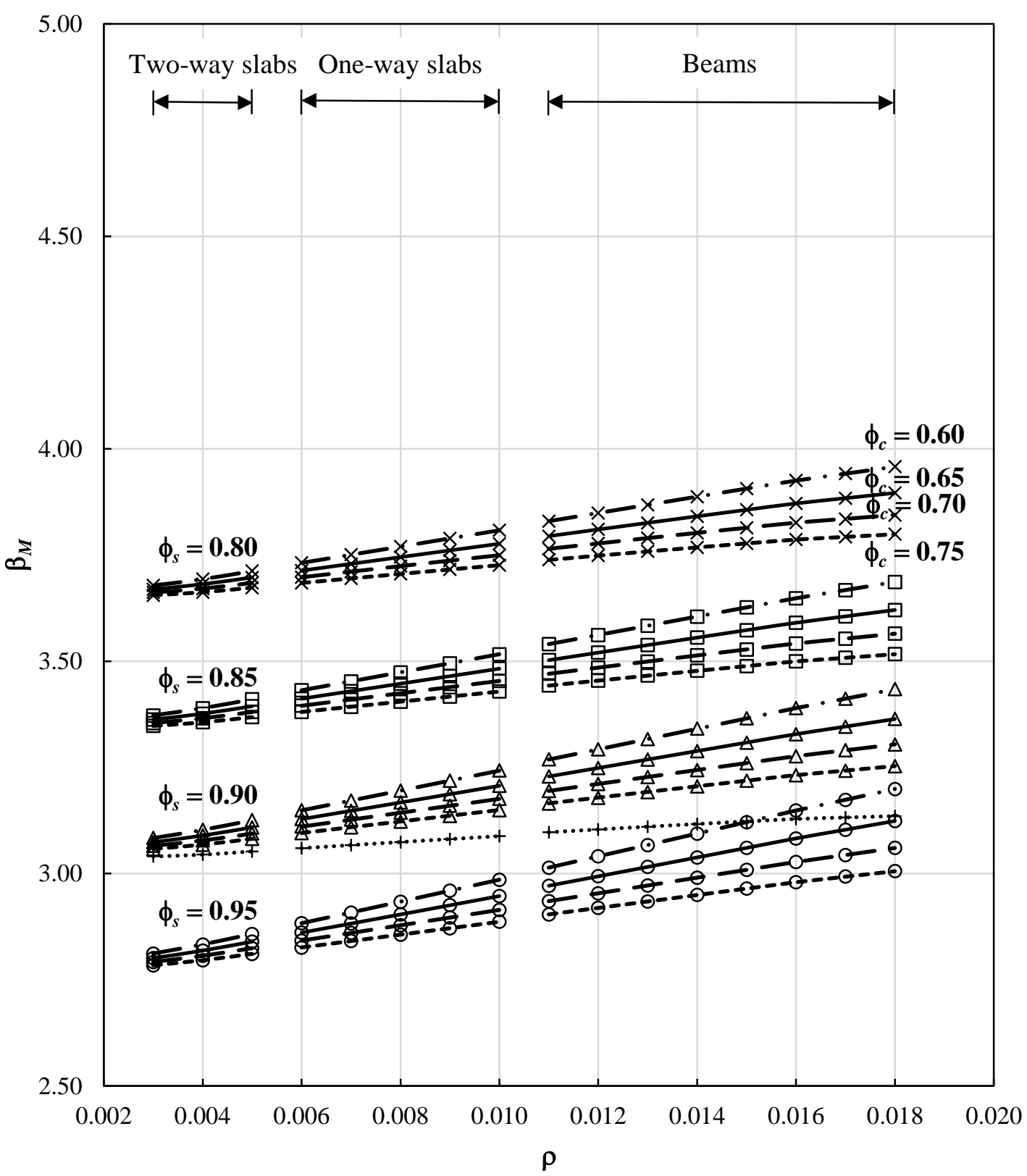

Figure B.3: Reliability indices for moment, $\beta_{M}$, for $f_{c}{ }^{\prime}=45 \mathrm{MPa}, w_{L} / w_{D}=1.5, \rho=0.003-$ 0.018 , and linear coefficients of variation for $d$ 


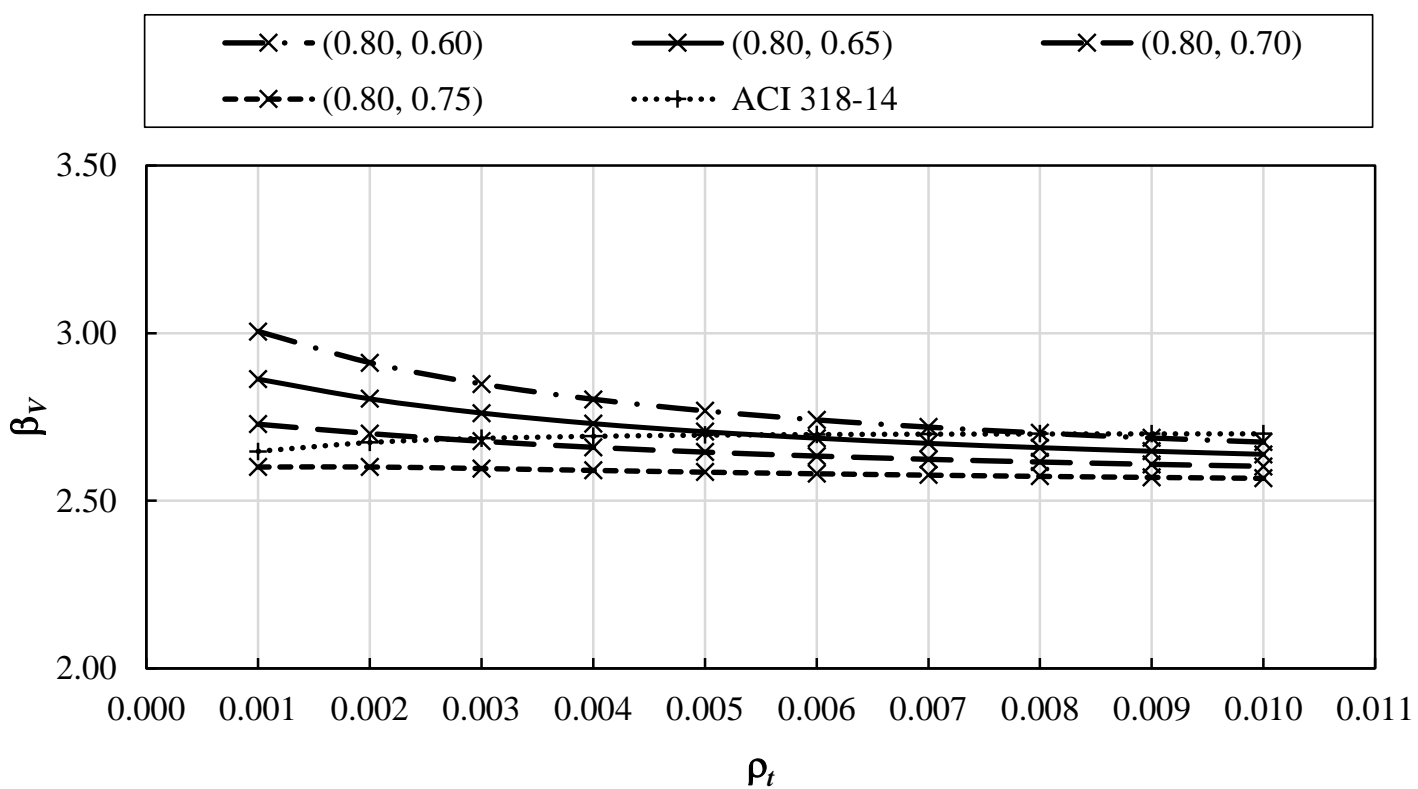

(a)

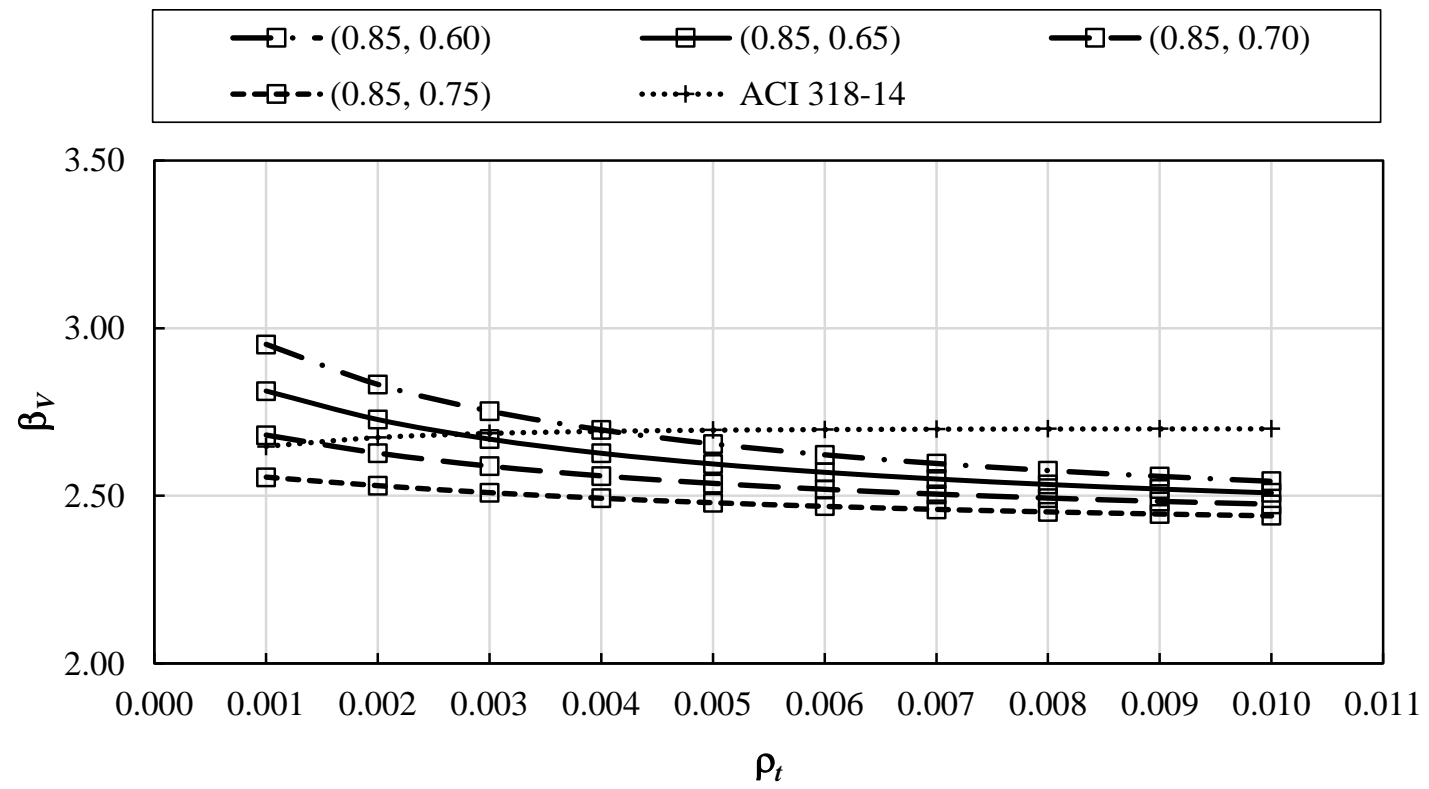

(b) 


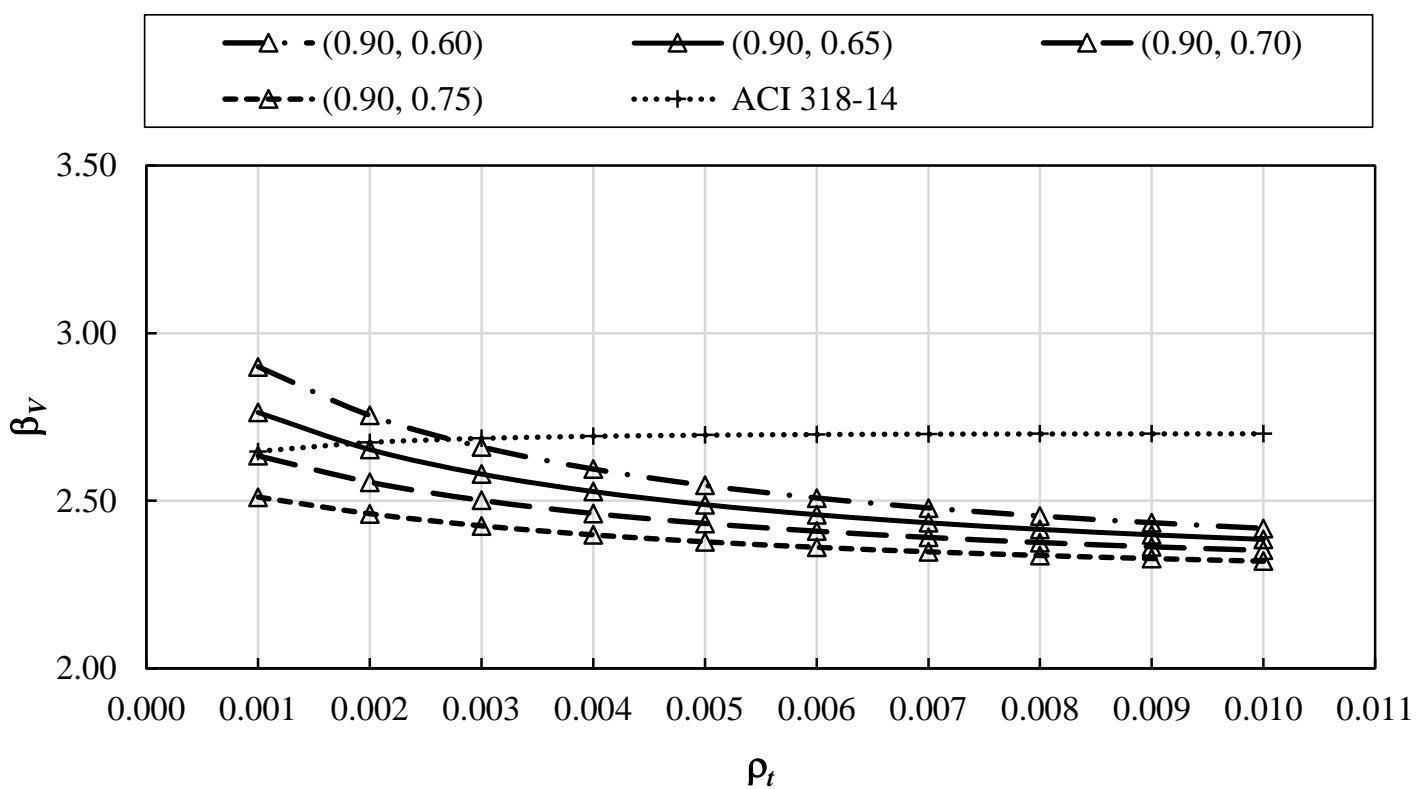

(c)

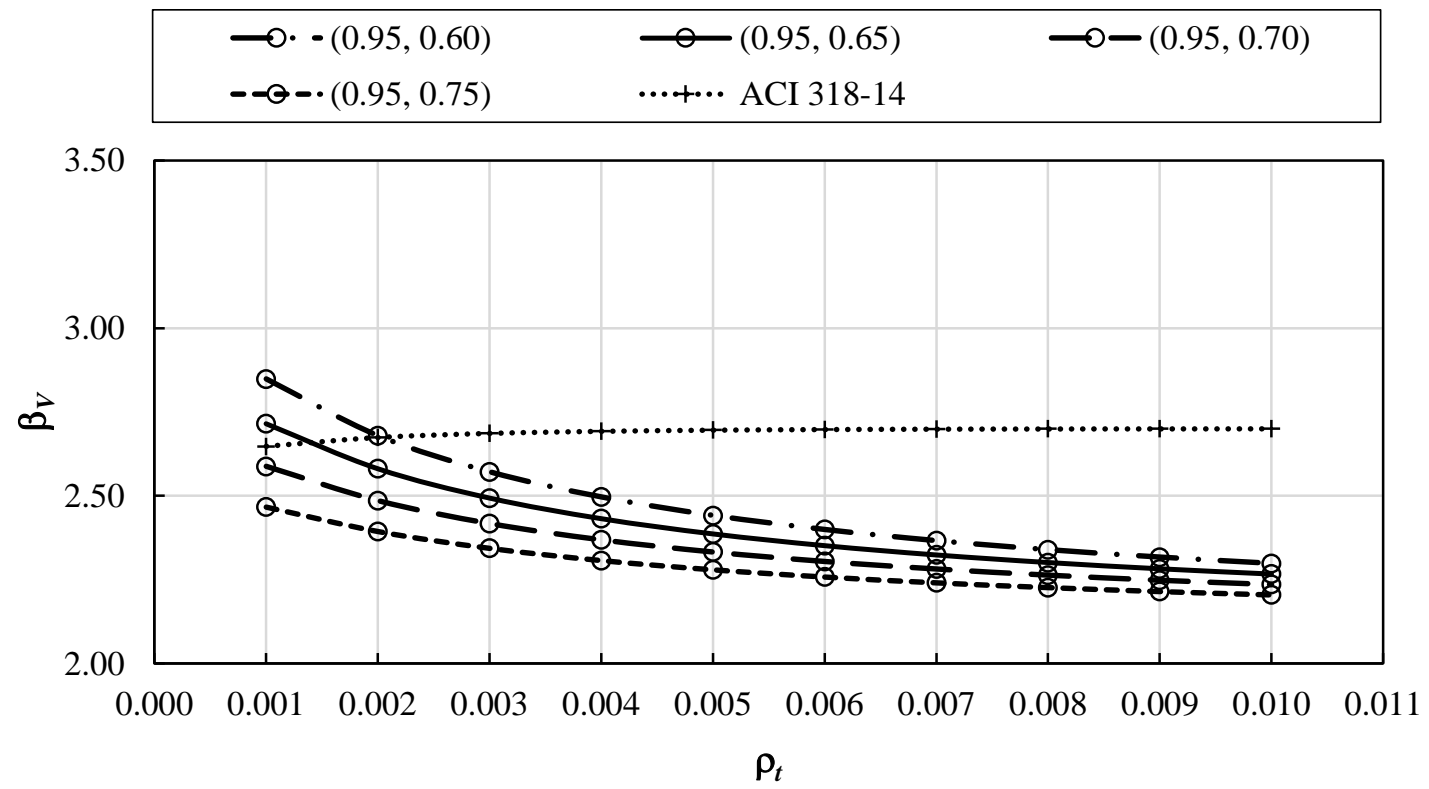

(d)

Figure B.4: Reliability indices for shear, $\beta_{V}$, for $f_{c}{ }^{\prime}=45 \mathrm{MPa}, w_{L} / w_{D}=0.5$, and $\rho_{t}=$ 0.001-0.010: (a) $\phi_{s}=0.80$; (b) $\phi_{s}=0.85$; (c) $\phi_{s}=0.90$; (d) $\phi_{s}=0.95$ 


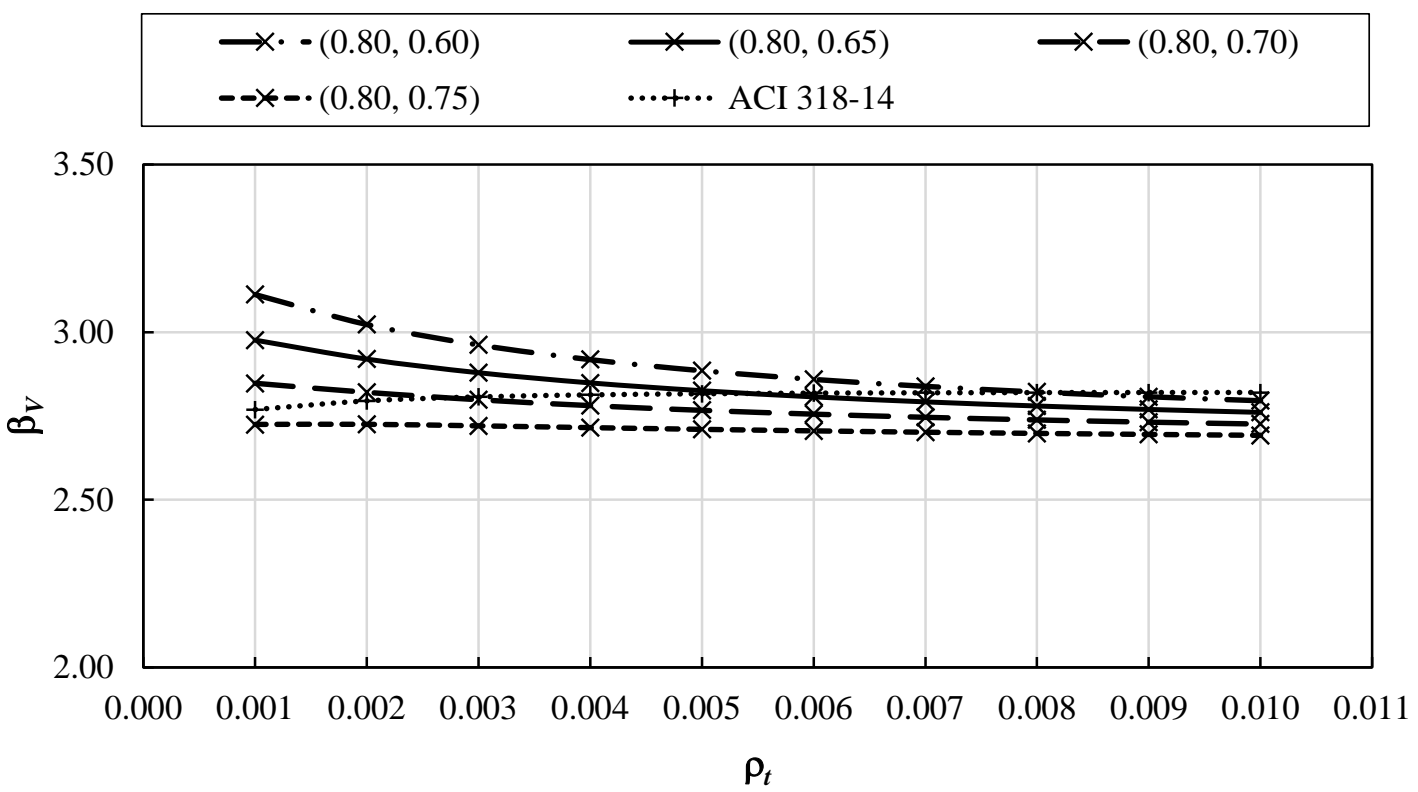

(a)

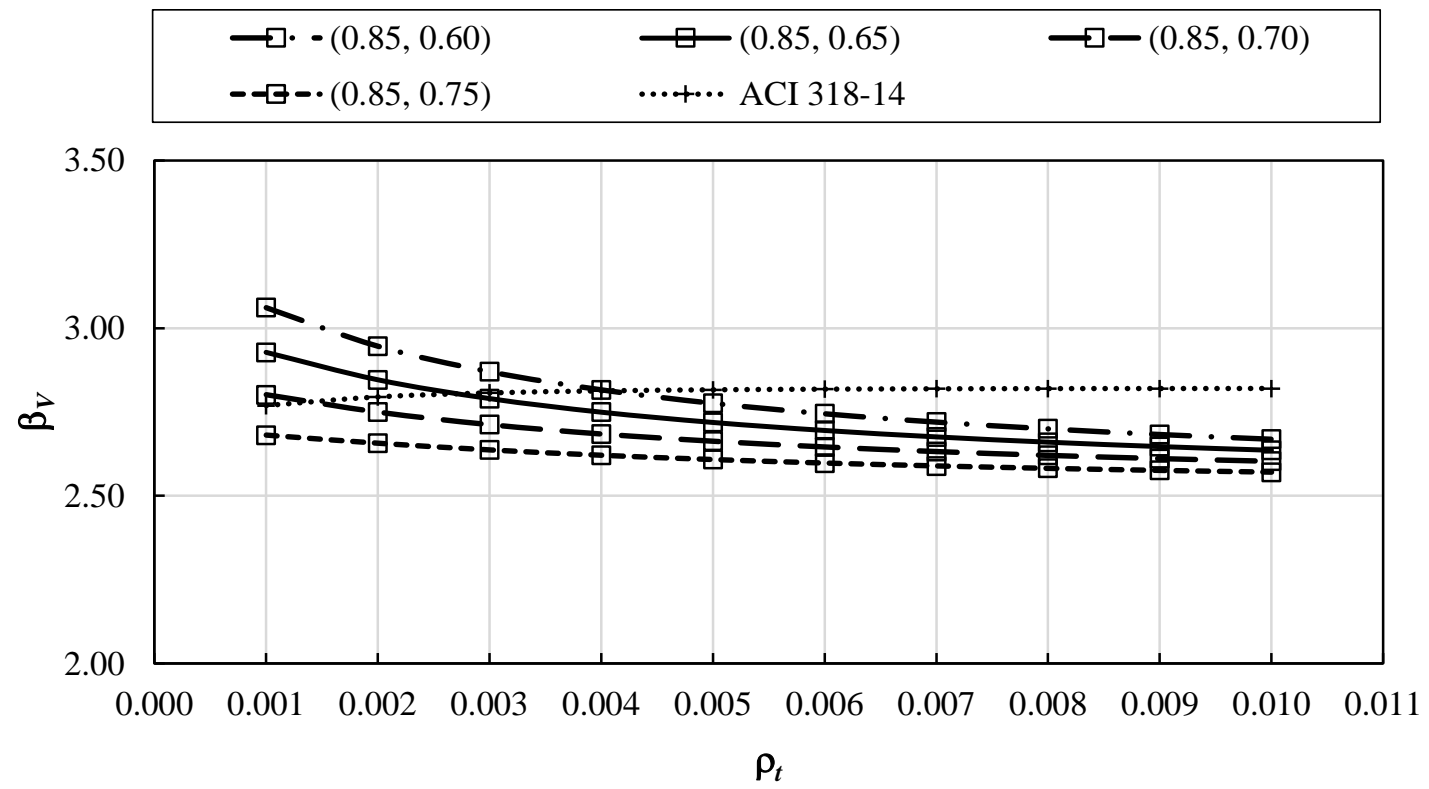

(b) 


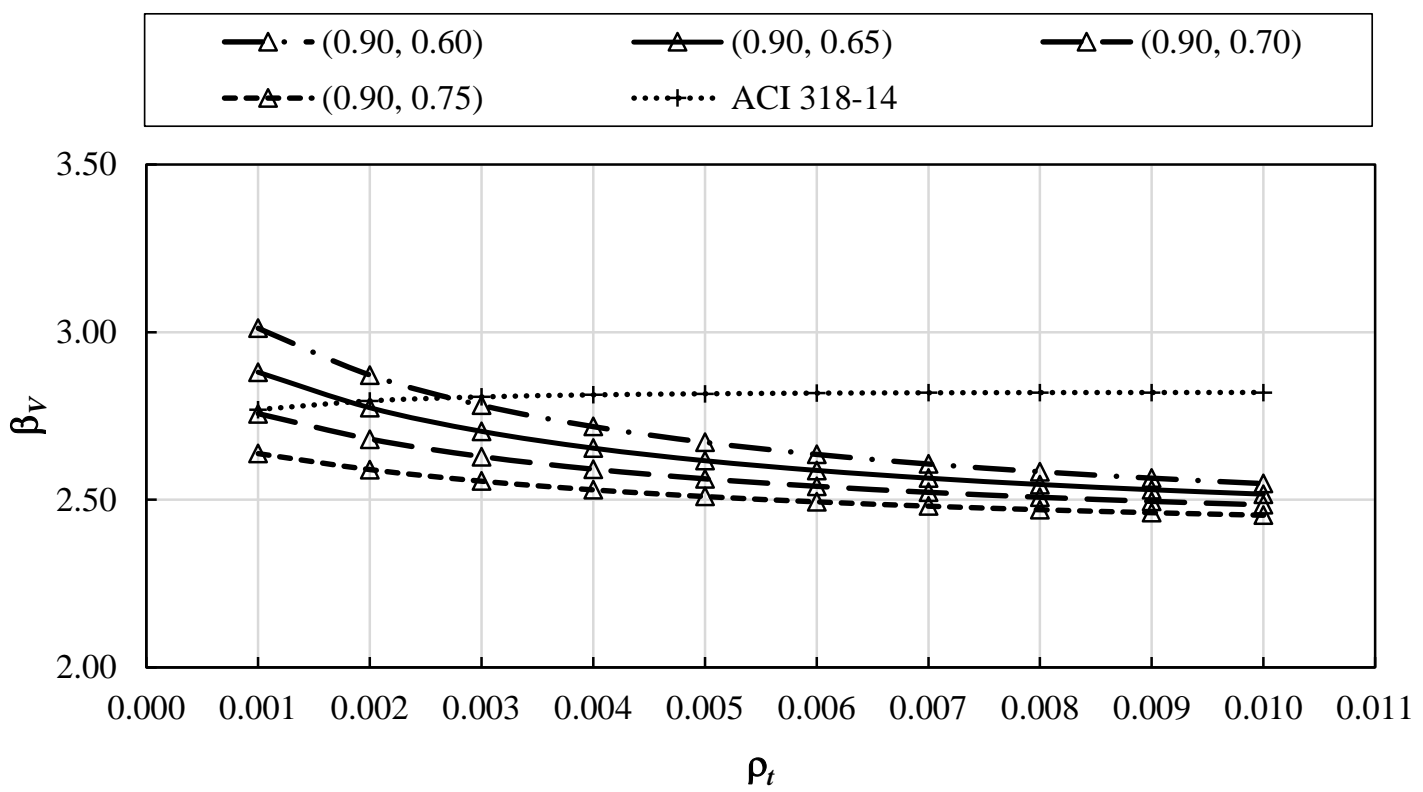

(c)

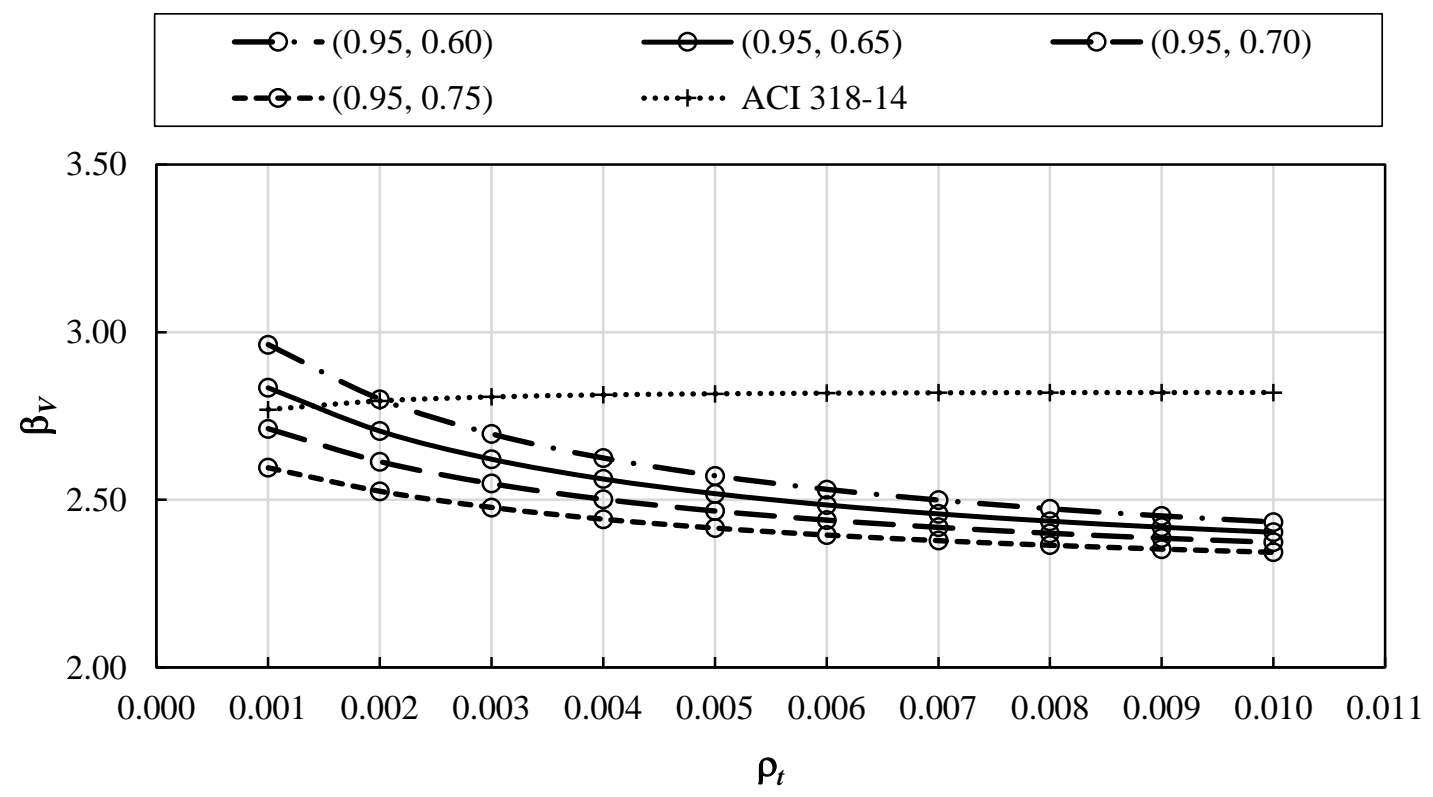

(d)

Figure B.5: Reliability indices for shear, $\beta_{V}$, for $f_{c}^{\prime}=45 \mathrm{MPa}, w_{L} / w_{D}=1.5$, and $\rho_{t}=$ 0.001-0.010: (a) $\phi_{s}=0.80$; (b) $\phi_{s}=0.85$; (c) $\phi_{s}=0.90$; (d) $\phi_{s}=0.95$ 


\section{Appendix C-Supplementary Information for Chapter 4}

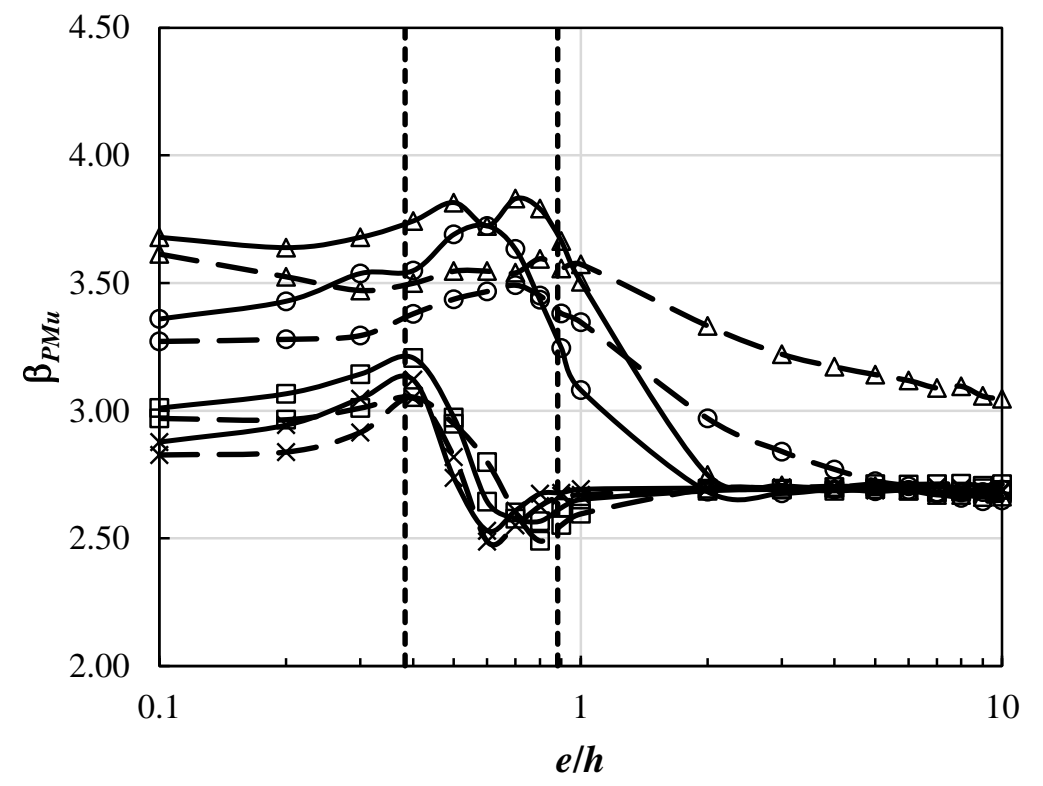

$$
\begin{aligned}
& \square-(0.6,25 \mathrm{MPa}, 1 \%) \\
& \triangle-(0.6,25 \mathrm{MPa}, 4 \%) \\
& \longrightarrow \times(0.6,45 \mathrm{MPa}, 1 \%) \\
& \longrightarrow-(0.6,45 \mathrm{MPa}, 4 \%) \\
& \text { —— }(0.9,25 \mathrm{MPa}, 1 \%) \\
& \triangle(0.9,25 \mathrm{MPa}, 4 \%) \\
& \longrightarrow(0.9,45 \mathrm{MPa}, 1 \%) \\
& \longrightarrow(0.9,45 \mathrm{MPa}, 4 \%) \\
& \text {-- - }(\text { eubal/h)max }
\end{aligned}
$$

(a)

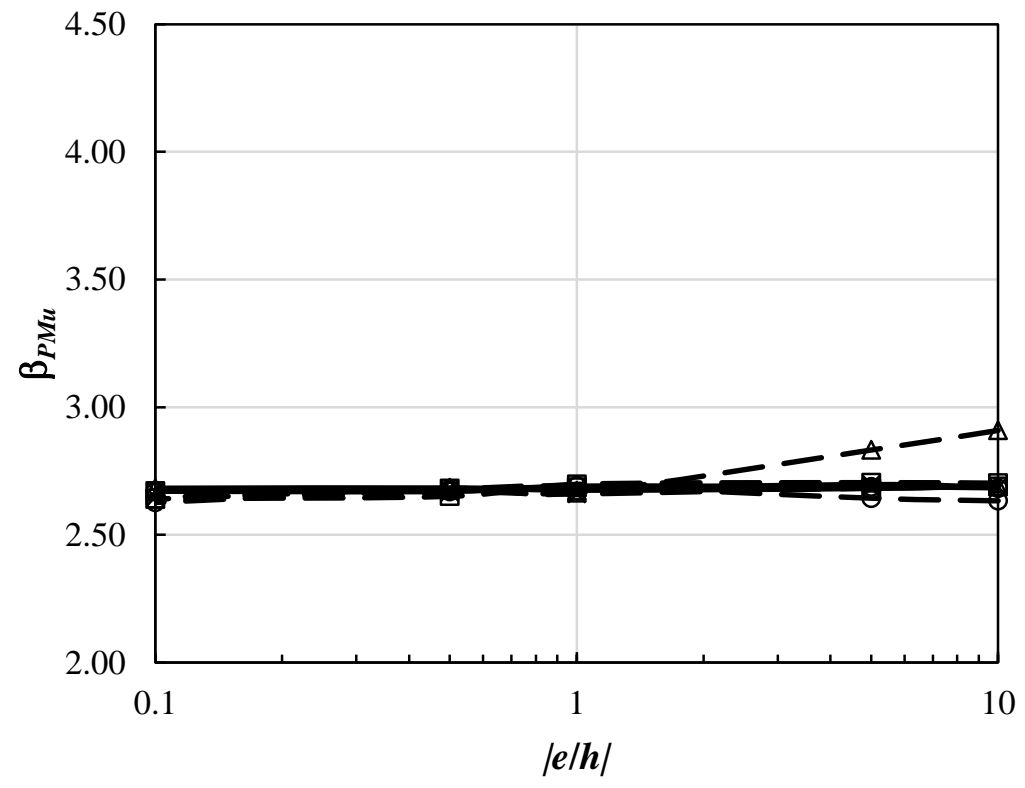

$$
\begin{aligned}
& \longrightarrow-(0.6,25 \mathrm{MPa}, 1 \%) \\
& \rightarrow-(0.6,25 \mathrm{MPa}, 4 \%) \\
& \rightarrow-(0.6,45 \mathrm{MPa}, 1 \%) \\
& \rightarrow-(0.6,45 \mathrm{MPa}, 4 \%) \\
& \longrightarrow-(0.9,25 \mathrm{MPa}, 1 \%) \\
& \longrightarrow-(0.9,25 \mathrm{MPa}, 4 \%) \\
& \longrightarrow-(0.9,45 \mathrm{MPa}, 1 \%) \\
& \square-(0.9,45 \mathrm{MPa}, 4 \%)
\end{aligned}
$$

(b)

Figure C.1: Reliability indices for combined moment and axial force using strength reduction factors in ACI 318-14, $\beta_{P M u}$, for Column Section 1 and $L / D=1.5$ : (a) $e / h>0$;

$$
\text { (b) } e / h<0
$$




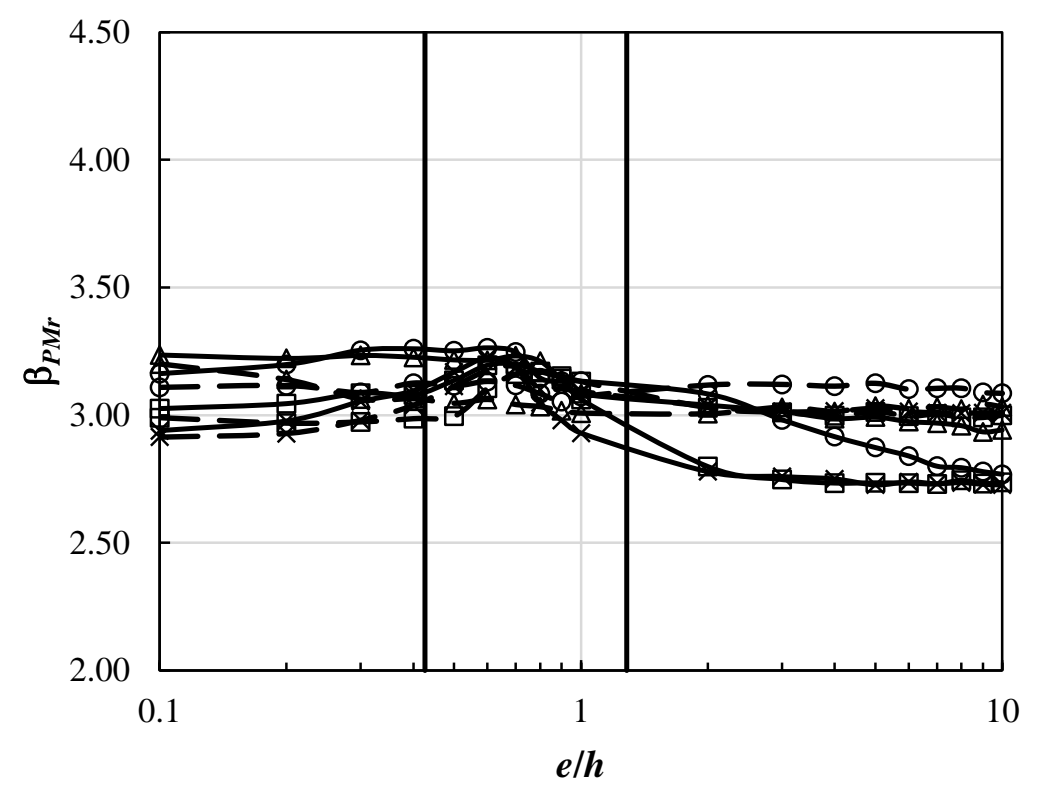

$$
\begin{aligned}
& \longrightarrow \text { (0.6, } 25 \mathrm{MPa}, 1 \%) \\
& \longrightarrow-(0.6,25 \mathrm{MPa}, 4 \%) \\
& \longrightarrow-(0.6,45 \mathrm{MPa}, 1 \%) \\
& \longrightarrow-(0.6,45 \mathrm{MPa}, 4 \%) \\
& \square(0.9,25 \mathrm{MPa}, 1 \%) \\
& \triangle(0.9,25 \mathrm{MPa}, 4 \%) \\
& \longrightarrow(0.9,45 \mathrm{MPa}, 1 \%) \\
& \text { - }(0.9,45 \mathrm{MPa}, 4 \%) \\
& \text { - (erbal/h)max }
\end{aligned}
$$

(a)

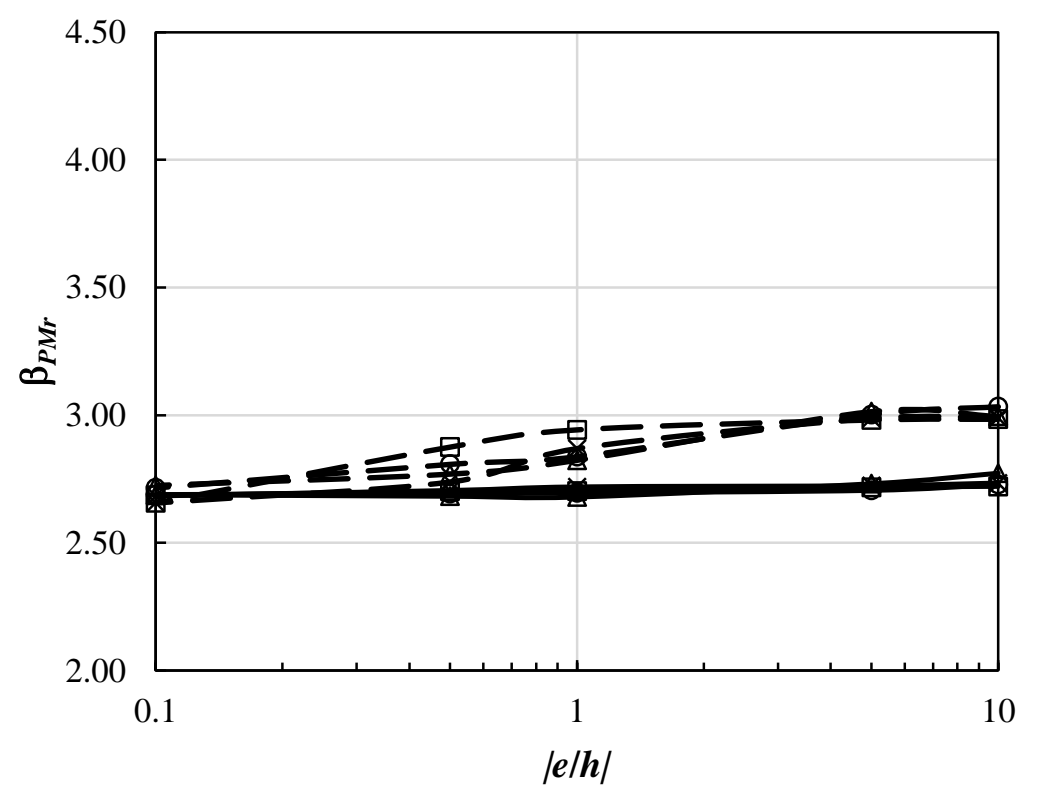

$$
\begin{aligned}
& \longrightarrow(0.6,25 \mathrm{MPa}, 1 \%) \\
& \rightarrow \triangle(0.6,25 \mathrm{MPa}, 4 \%) \\
& \rightarrow-(0.6,45 \mathrm{MPa}, 1 \%) \\
& \longrightarrow \text { - }(0.6,45 \mathrm{MPa}, 4 \%) \\
& \longrightarrow(0.9,25 \mathrm{MPa}, 1 \%) \\
& \triangle(0.9,25 \mathrm{MPa}, 4 \%) \\
& \longrightarrow(0.9,45 \mathrm{MPa}, 1 \%) \\
& \text { - }(0.9,45 \mathrm{MPa}, 4 \%)
\end{aligned}
$$

(b)

Figure C.2: Reliability indices for combined moment and axial force, $\beta_{P M r}$, corresponding to $\phi_{s}=0.90$ and $\phi_{c}=0.60$, for Column Section 1 and $L / D=1.5$ : (a) $e / h>0$; (b) $e / h<0$ 

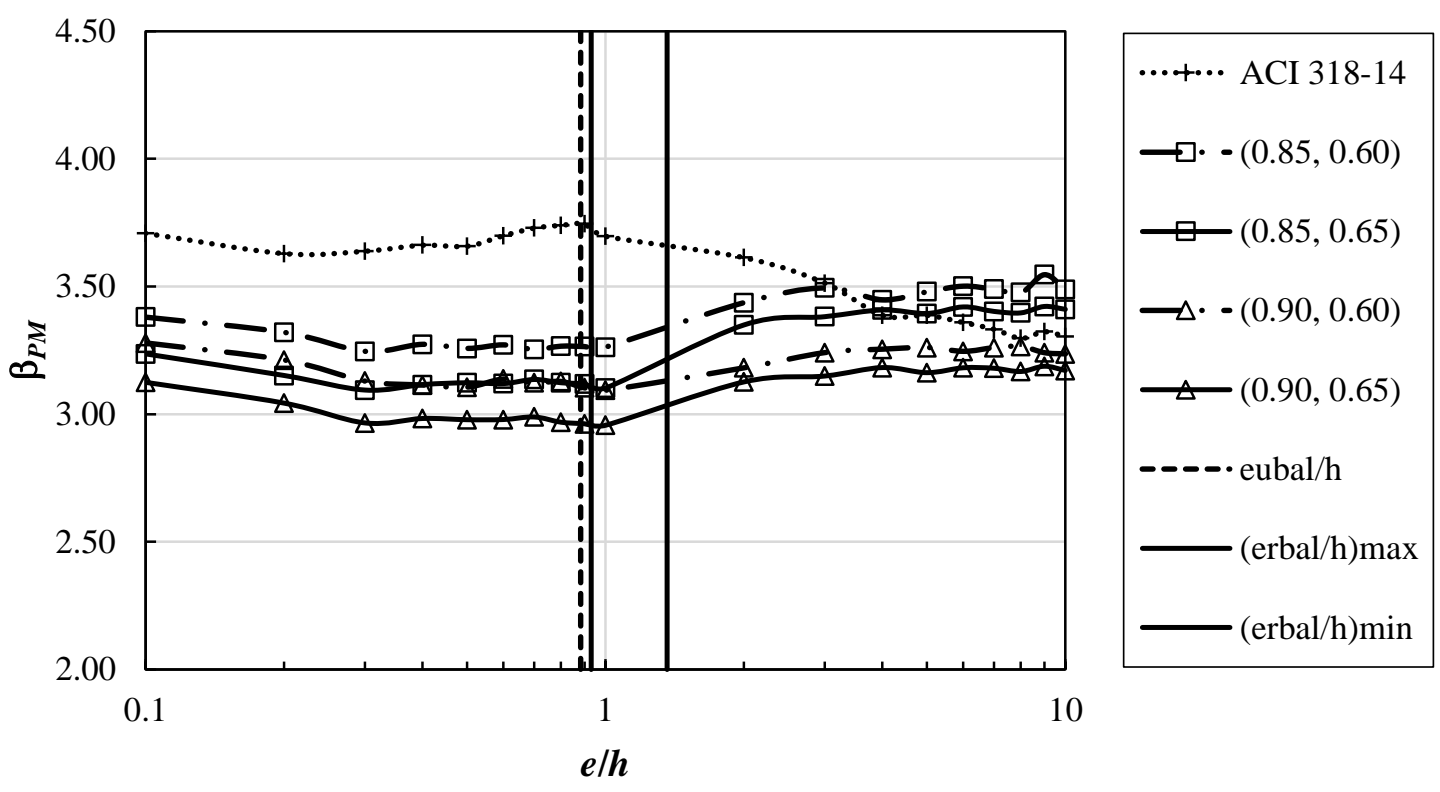

(a)

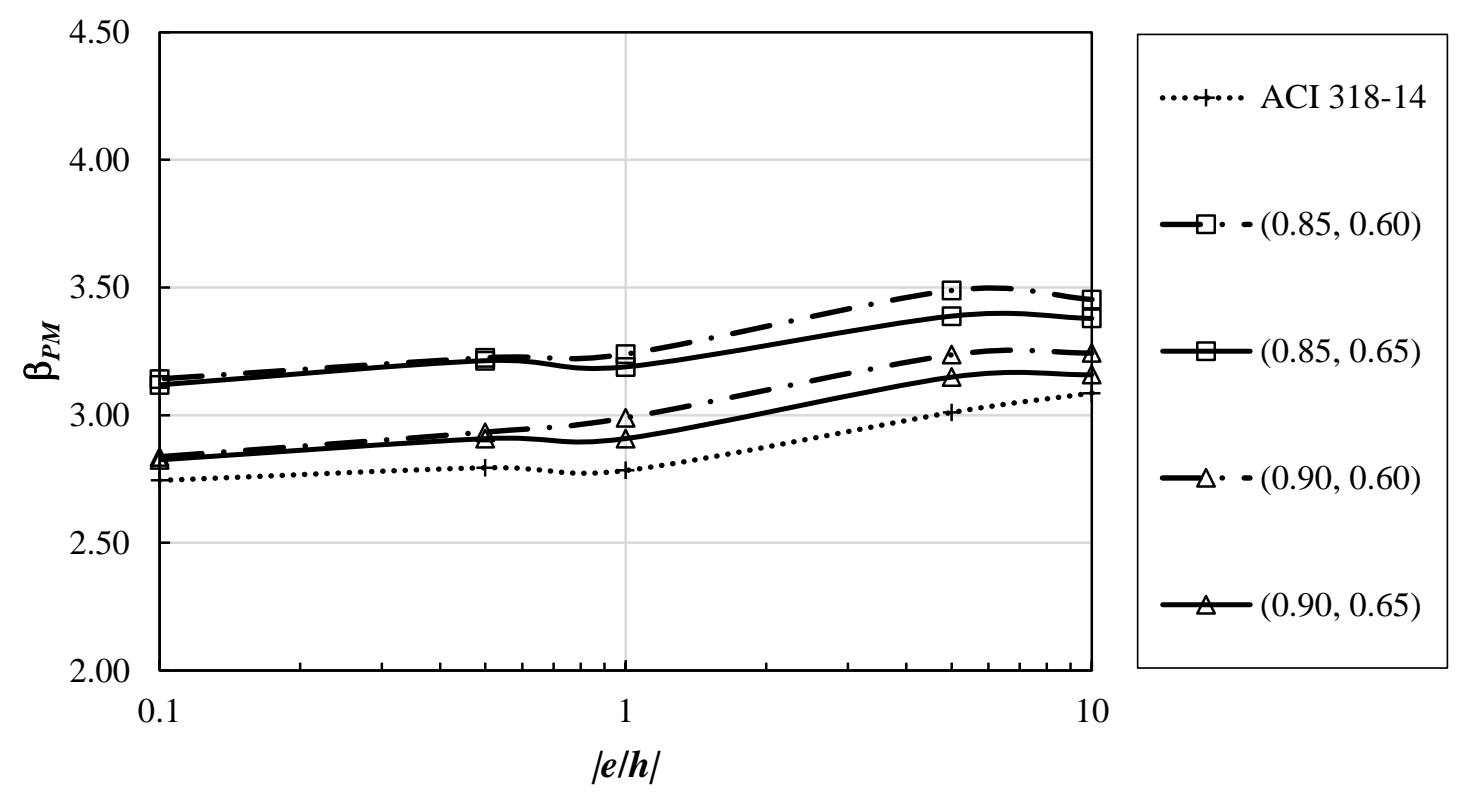

(b)

Figure C.3: Reliability indices for combined moment and axial force, $\beta_{P M}$, for Column Section 1, Property Combination 2, and $L / D=0.5$ : (a) $e / h>0$; (b) $e / h<0$ 

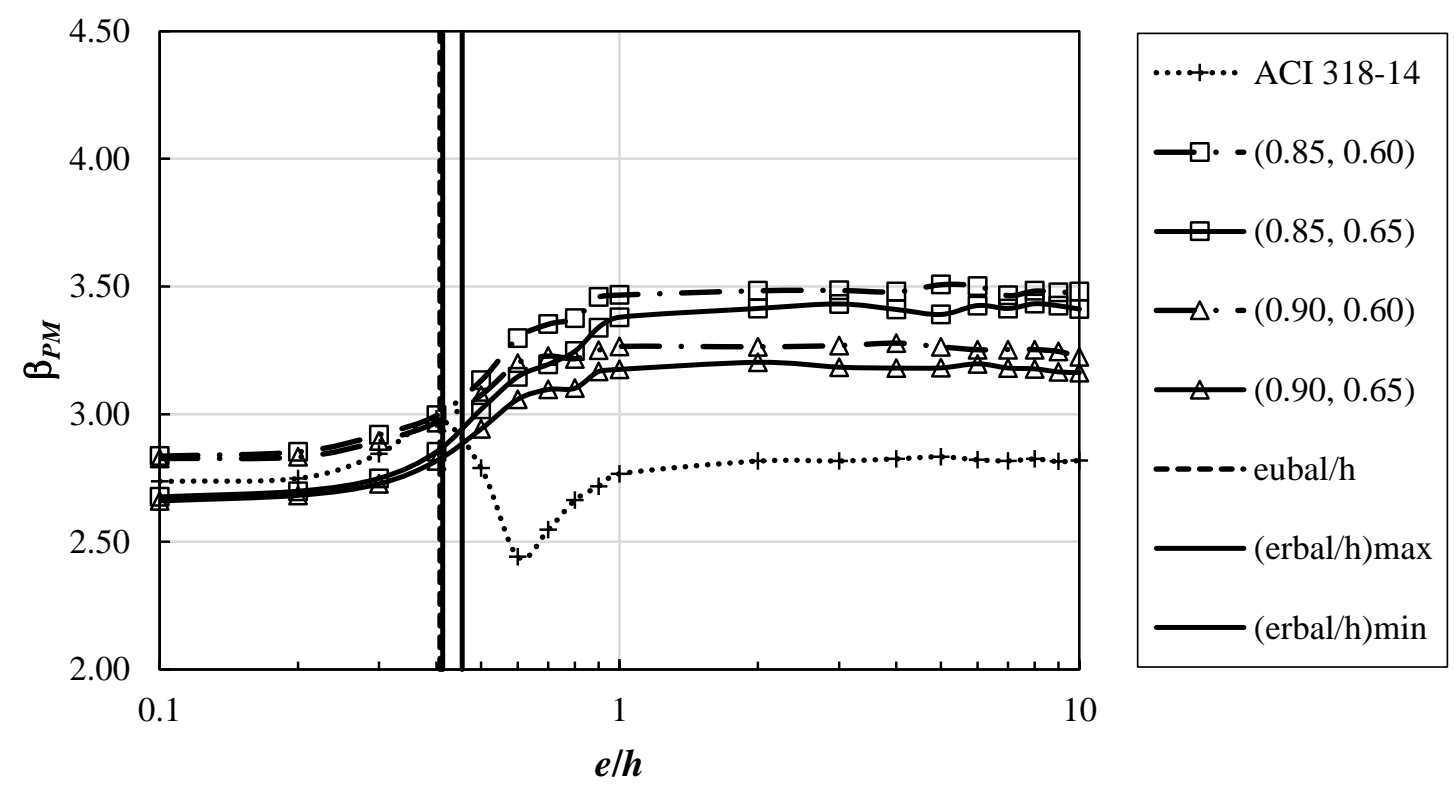

(a)

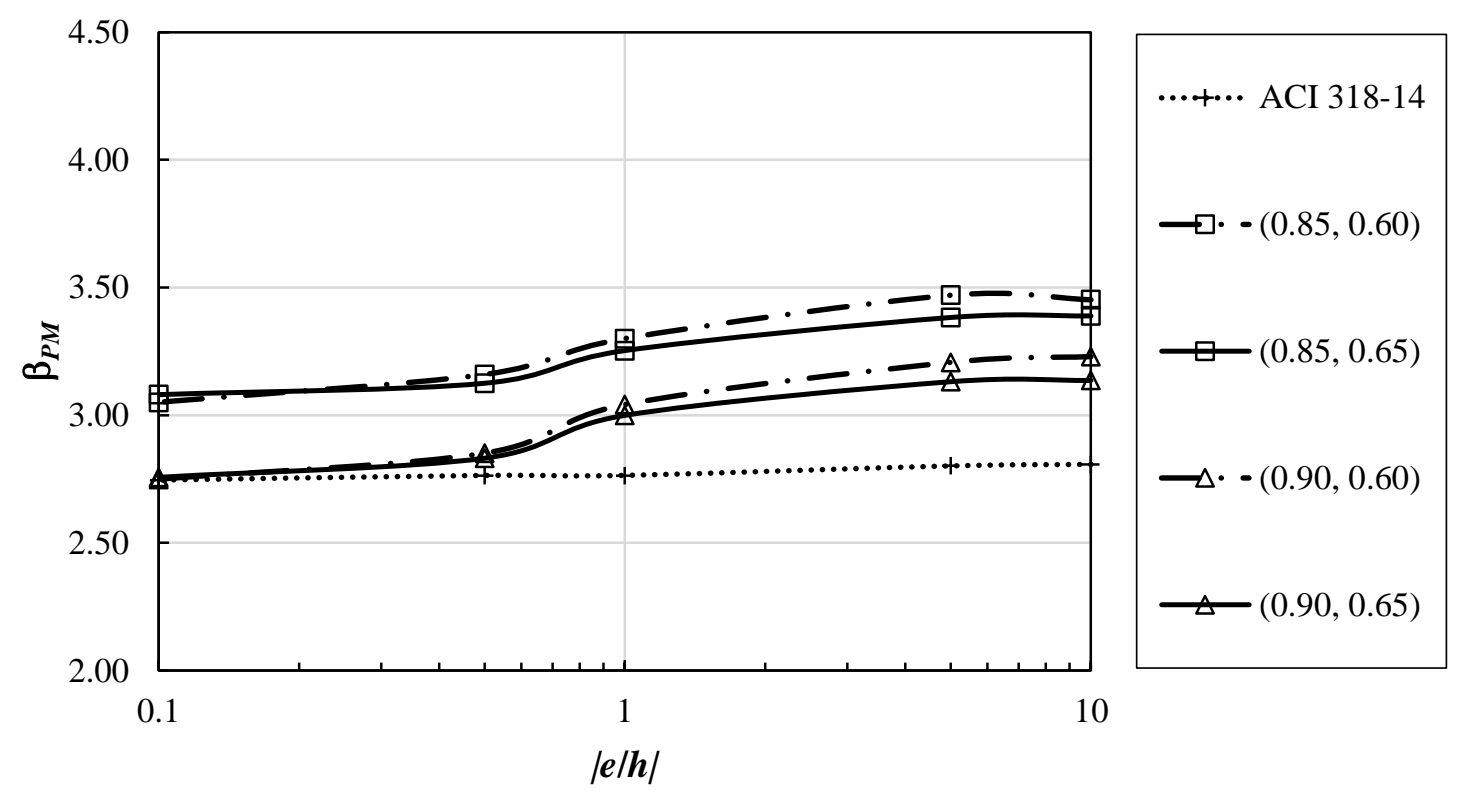

(b)

Figure C.4: Reliability indices for combined moment and axial force, $\beta_{P M}$, for Column Section 1, Property Combination 3, and $L / D=0.5$ : (a) $e / h>0$; (b) $e / h<0$ 

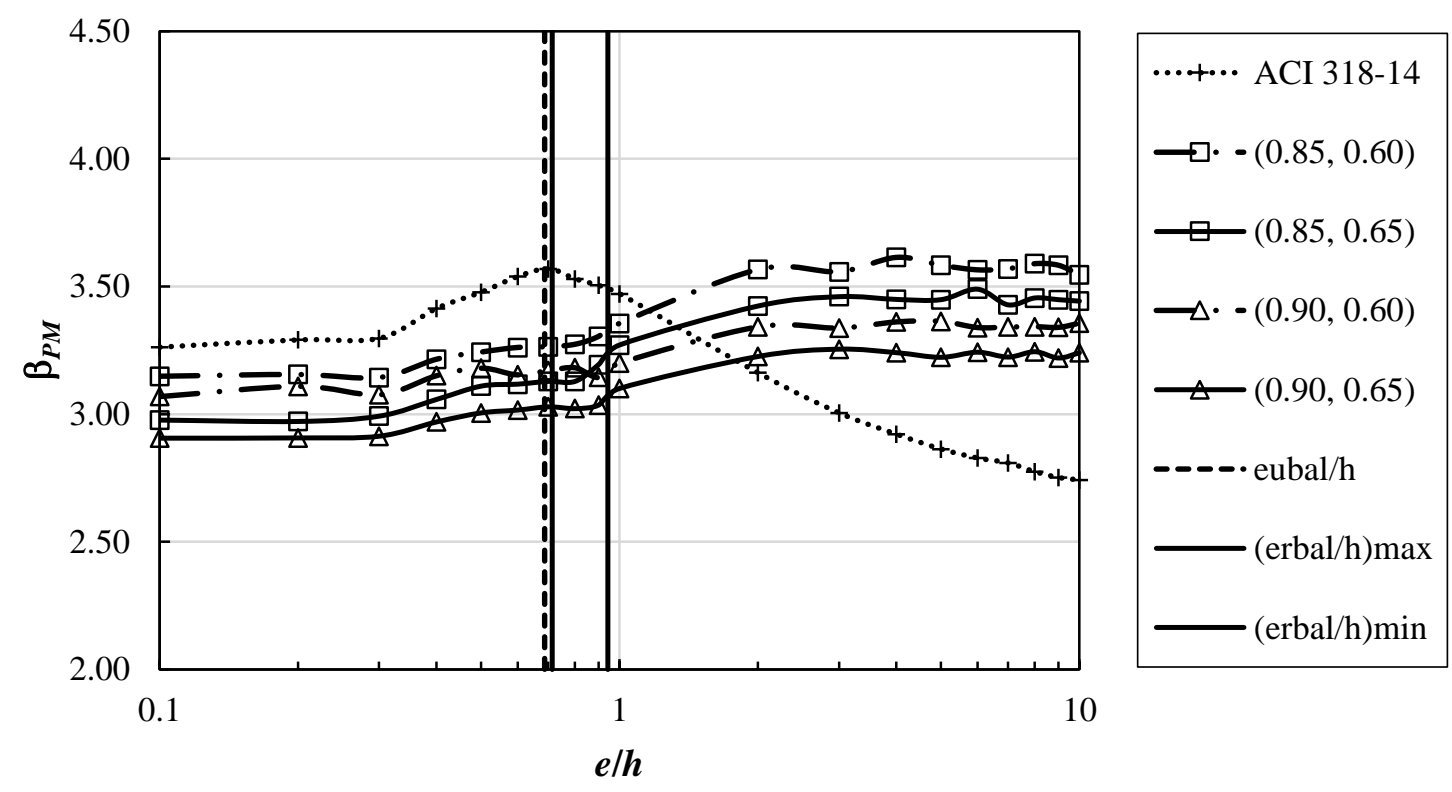

(a)
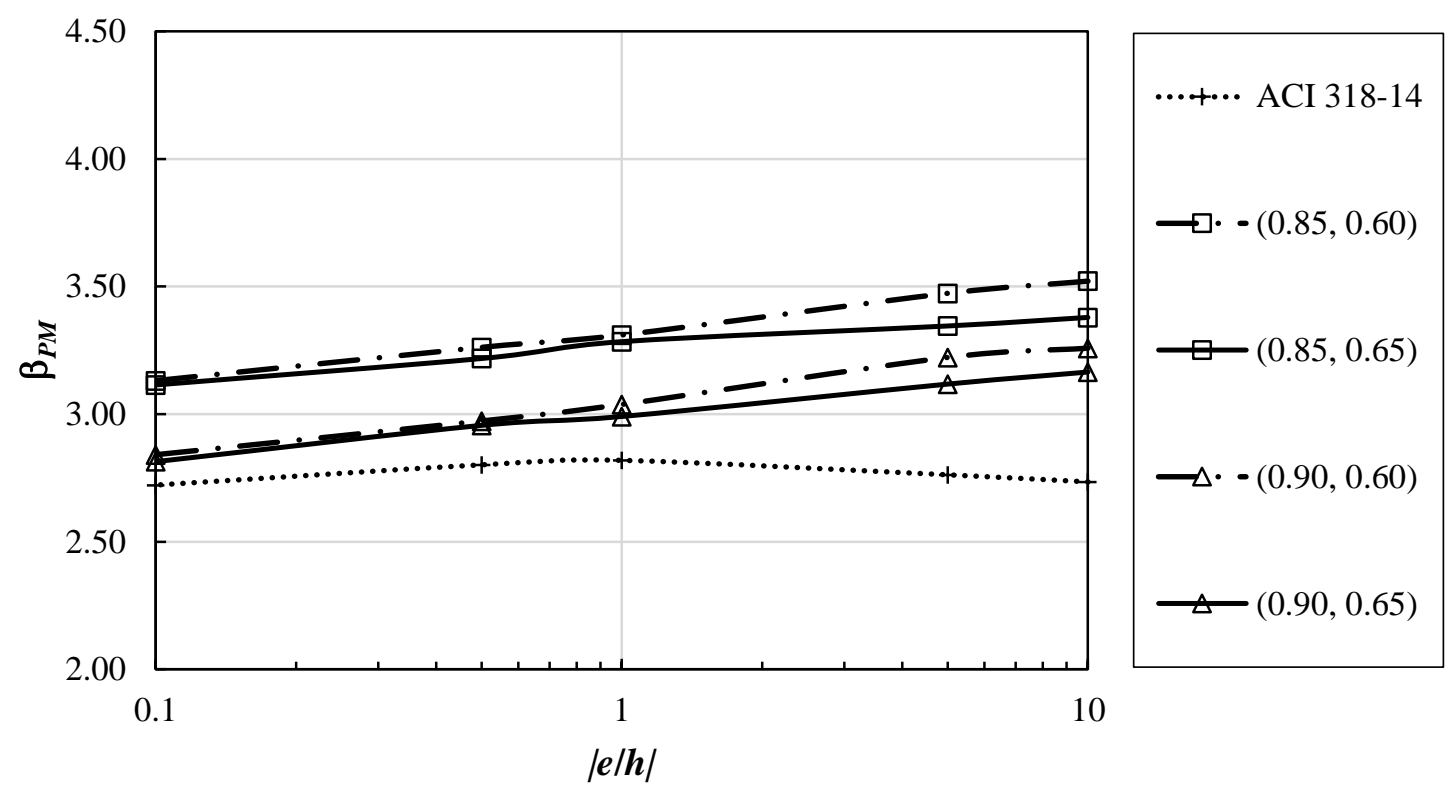

(b)

Figure C.5: Reliability indices for combined moment and axial force, $\beta_{P M}$, for Column Section 1, Property Combination 4, and $L / D=0.5$ : (a) $e / h>0$; (b) $e / h<0$ 

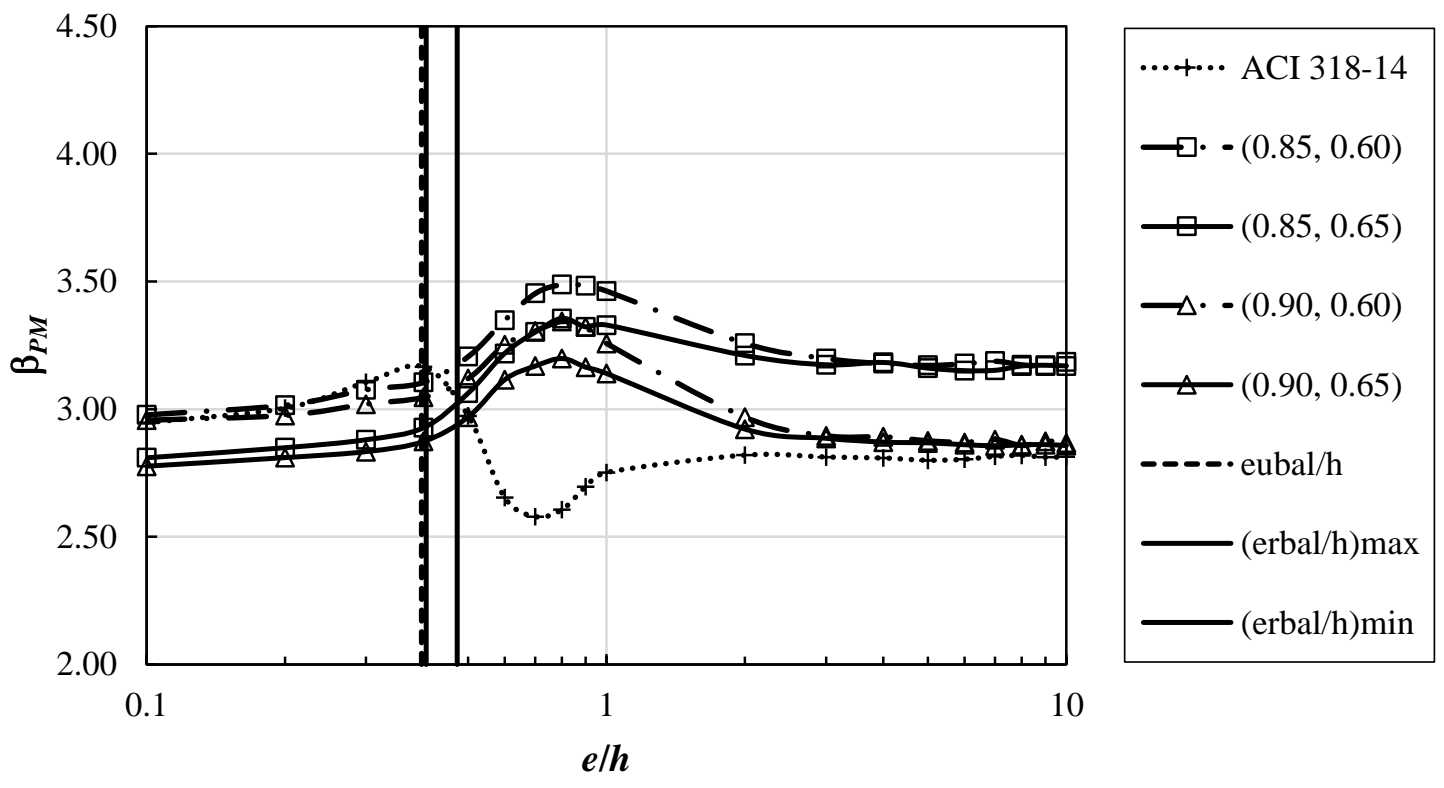

(a)
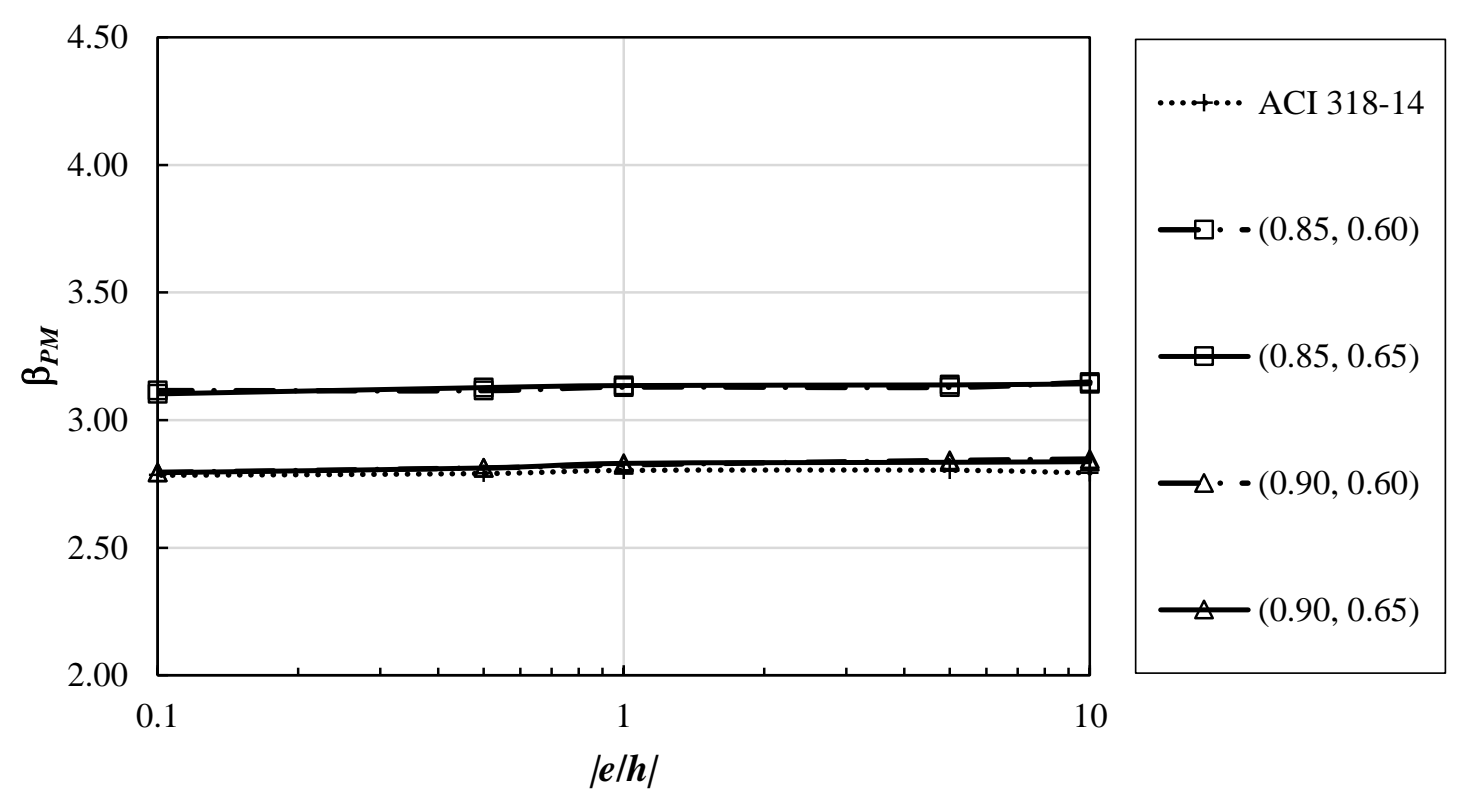

(b)

Figure C.6: Reliability indices for combined moment and axial force, $\beta_{P M}$, for Column Section 1, Property Combination 5, and $L / D=0.5$ : (a) $e / h>0$; (b) $e / h<0$ 

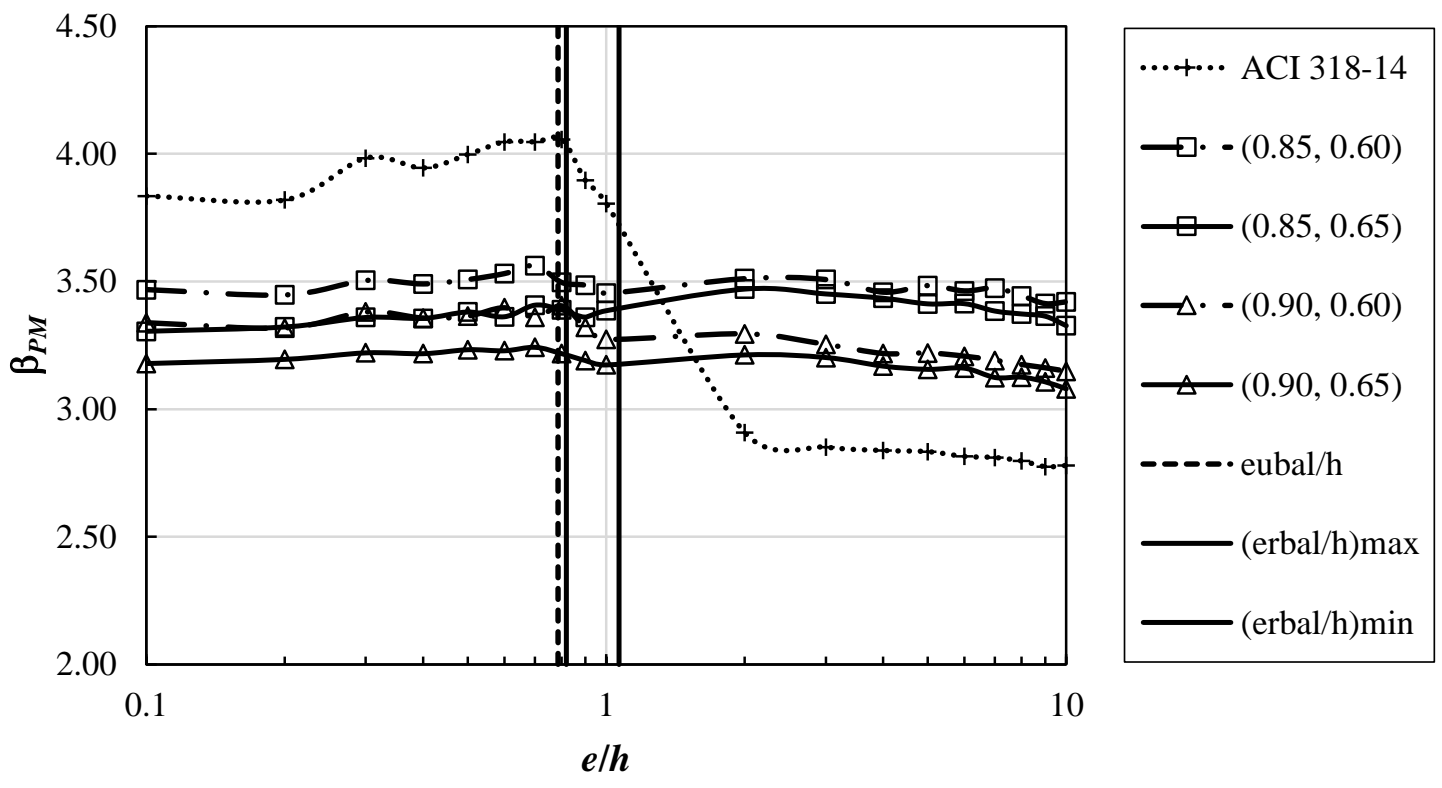

(a)
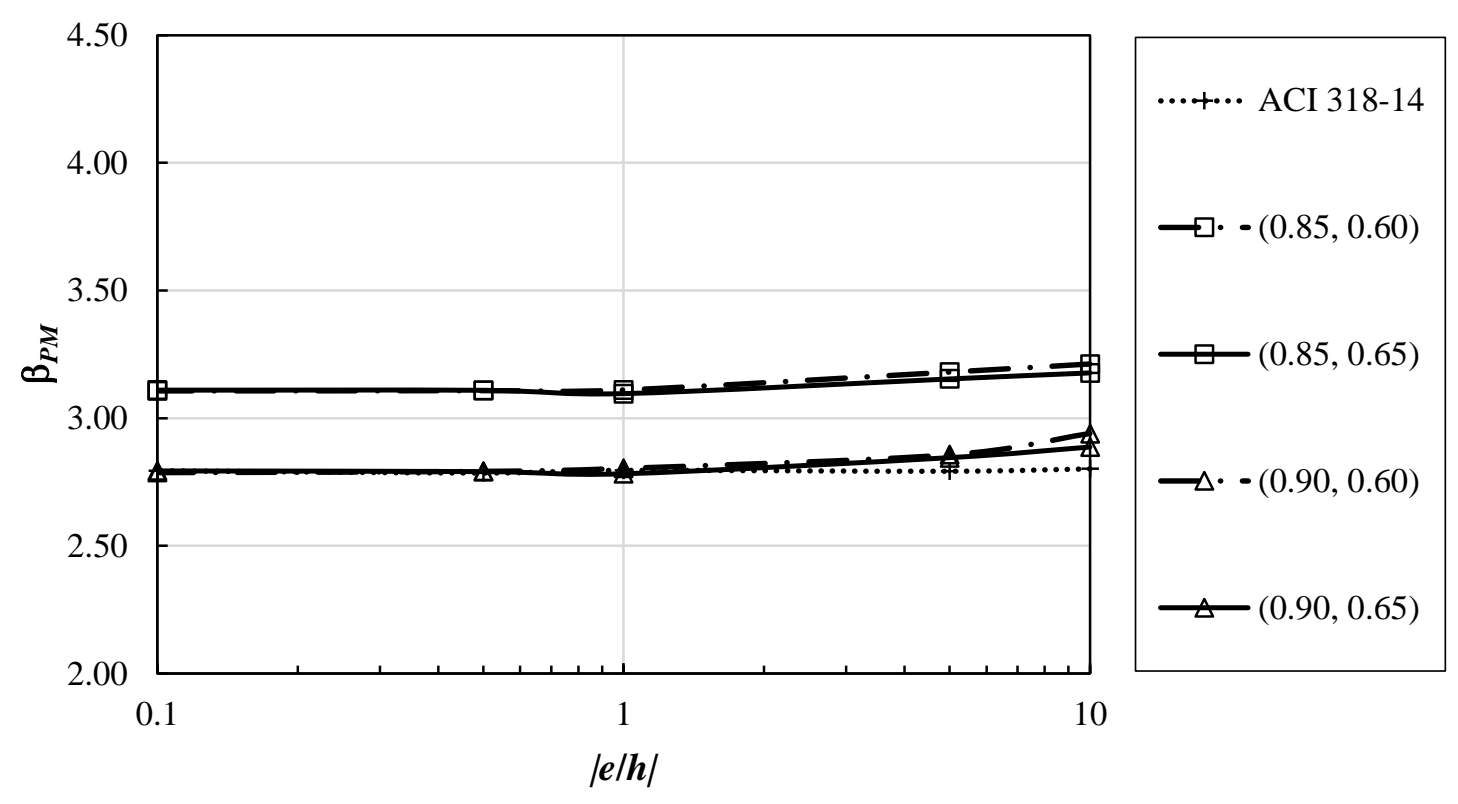

(b)

Figure C.7: Reliability indices for combined moment and axial force, $\beta_{P M}$, for Column Section 1, Property Combination 6, and $L / D=0.5$ : (a) $e / h>0$; (b) $e / h<0$ 

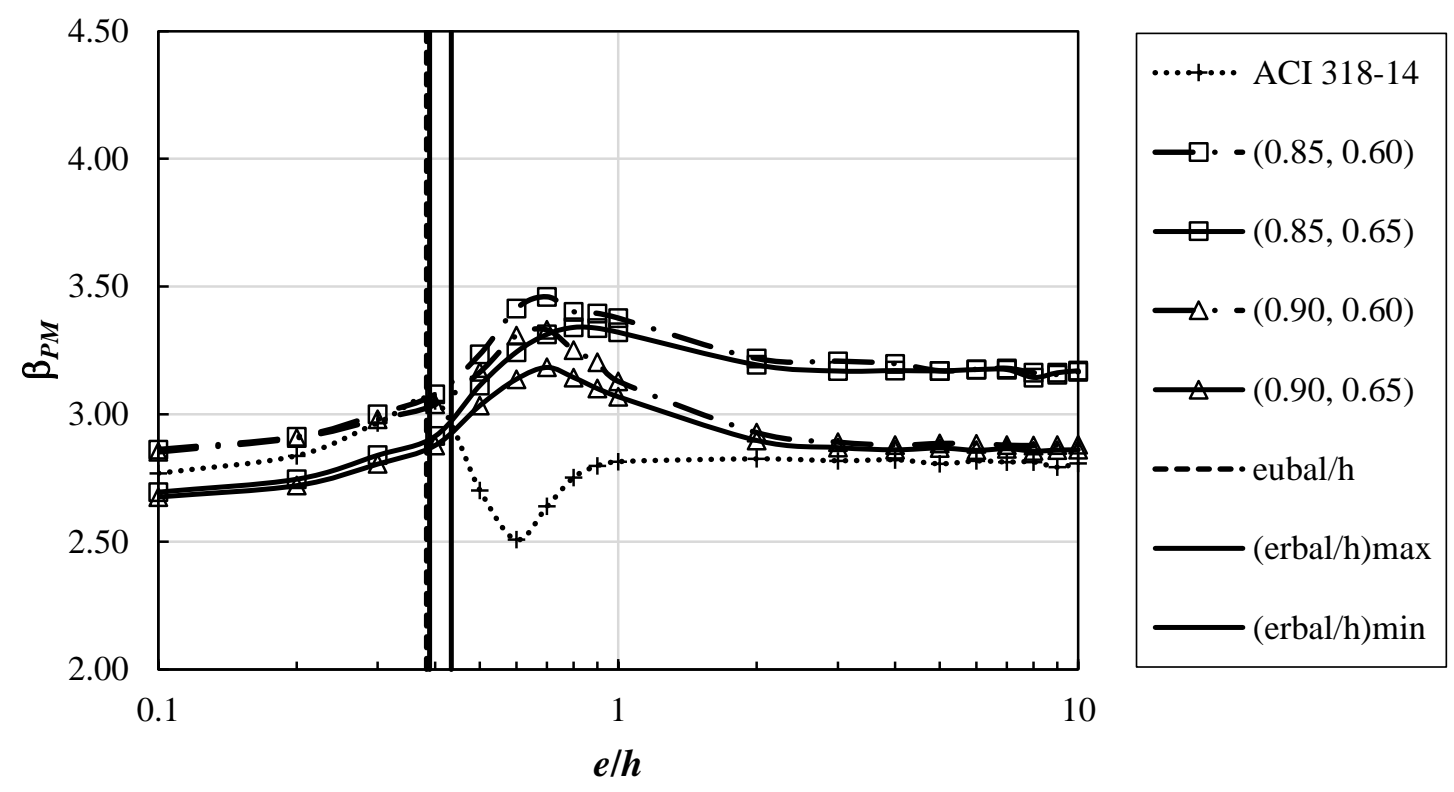

(a)
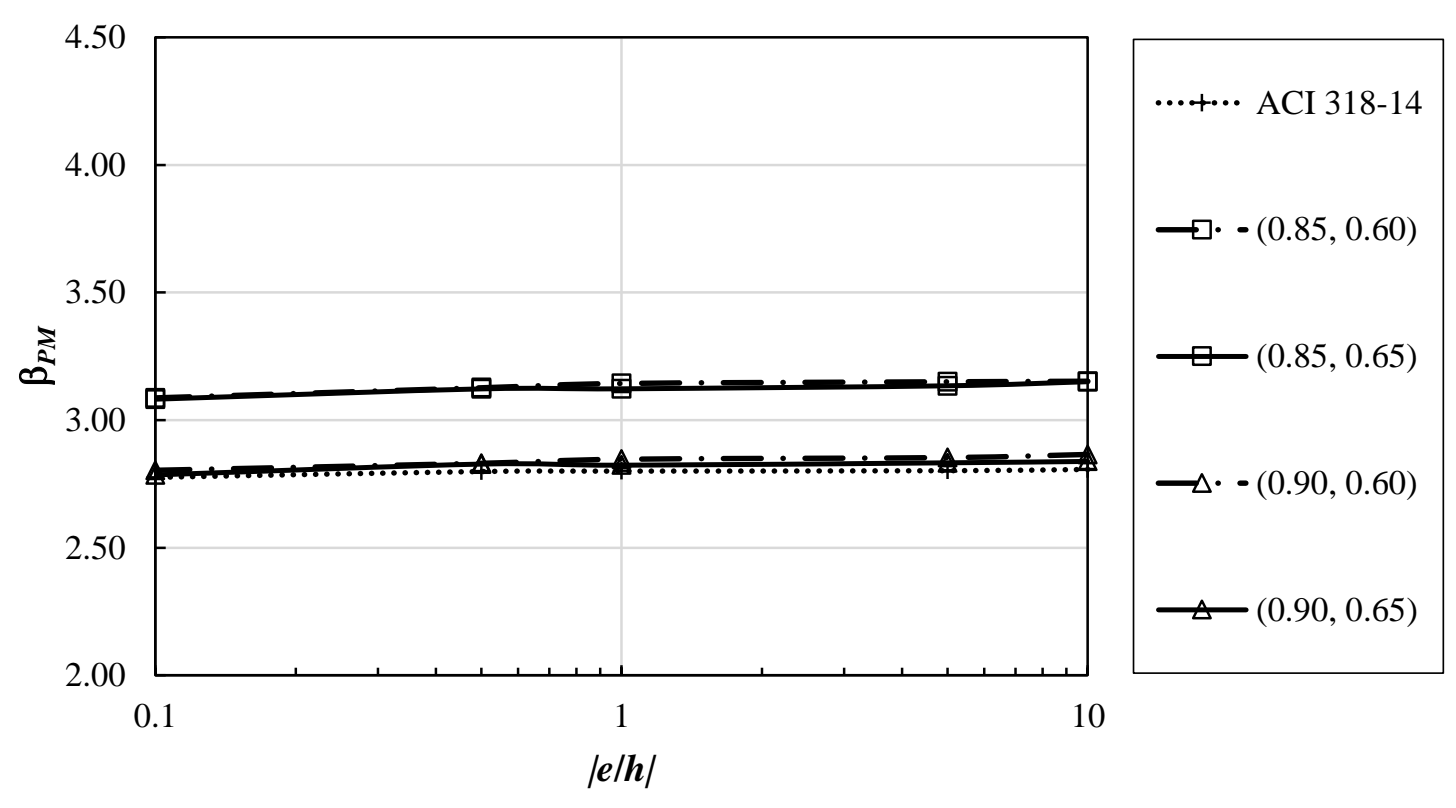

(b)

Figure C.8: Reliability indices for combined moment and axial force, $\beta_{P M}$, for Column Section 1, Property Combination 7, and $L / D=0.5$ : (a) $e / h>0$; (b) $e / h<0$ 

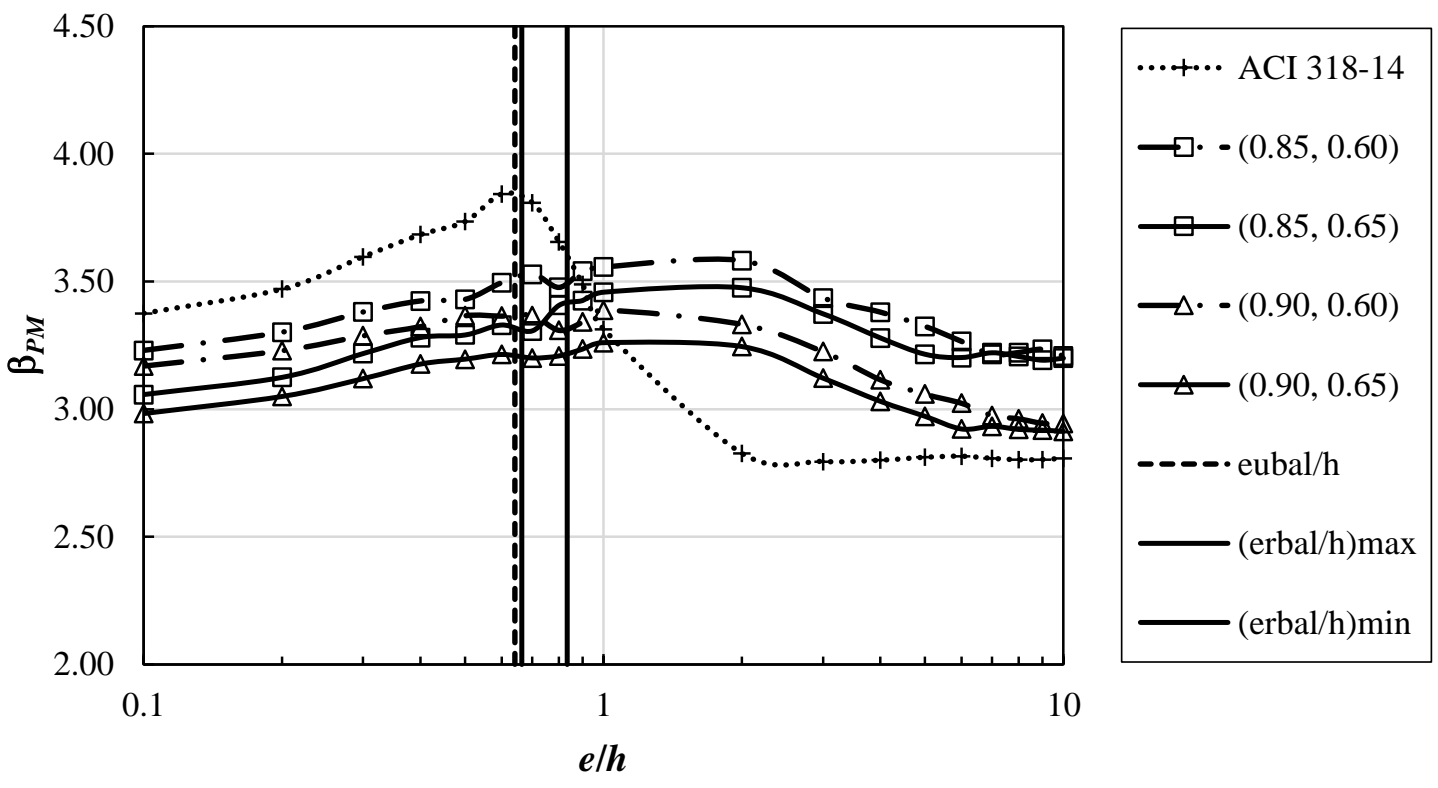

(a)
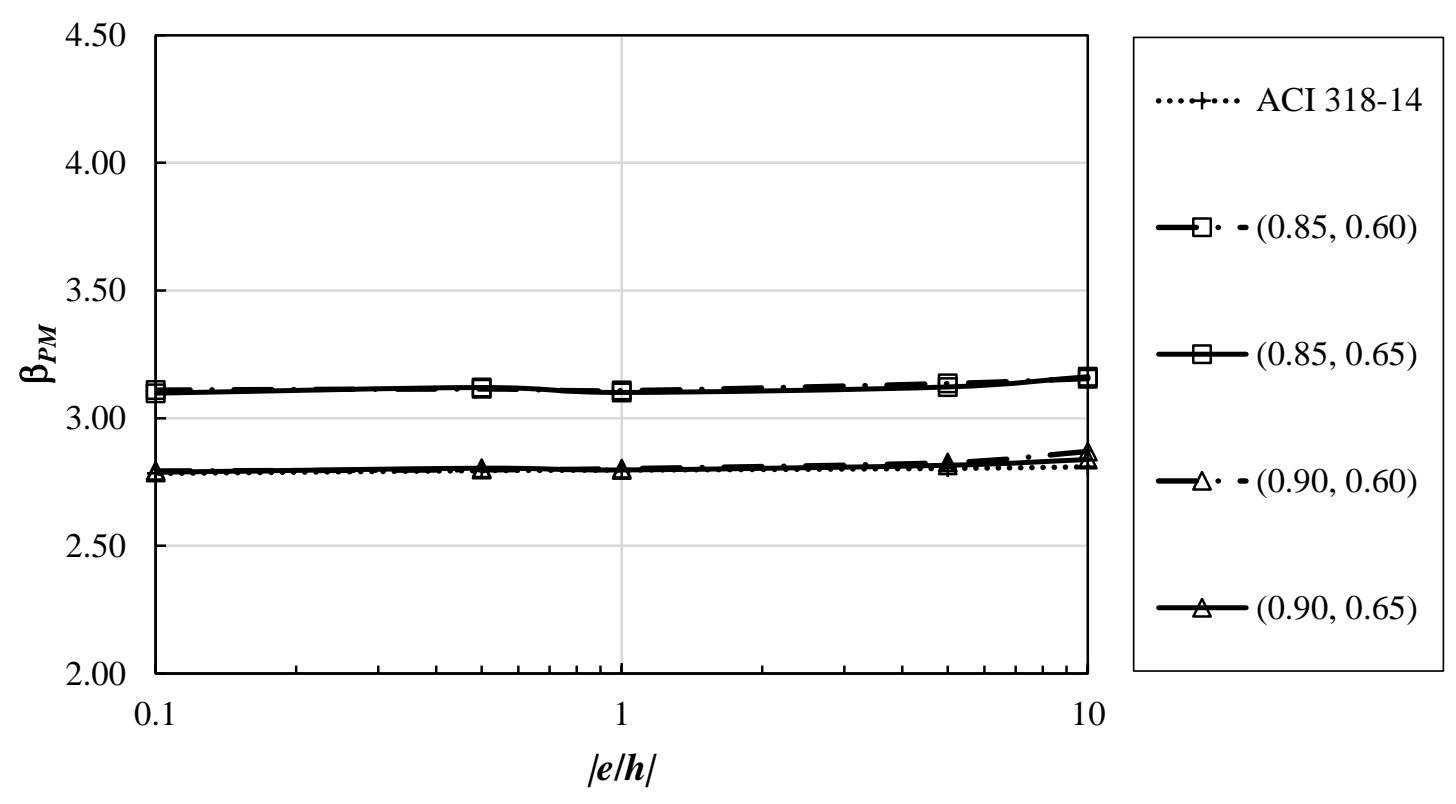

(b)

Figure C.9: Reliability indices for combined moment and axial force, $\beta_{P M}$, for Column Section 1, Property Combination 8, and $L / D=0.5$ : (a) $e / h>0$; (b) $e / h<0$ 

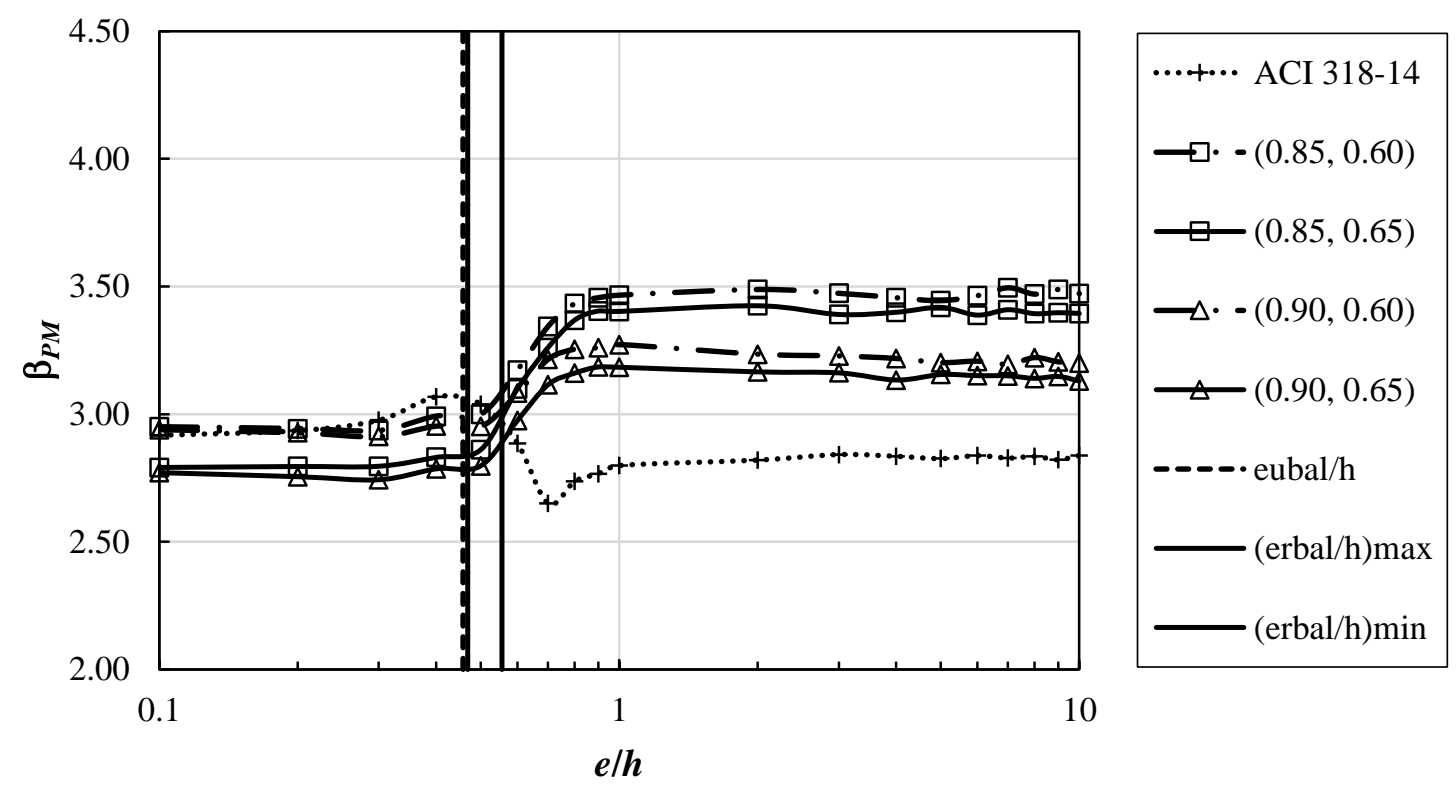

(a)
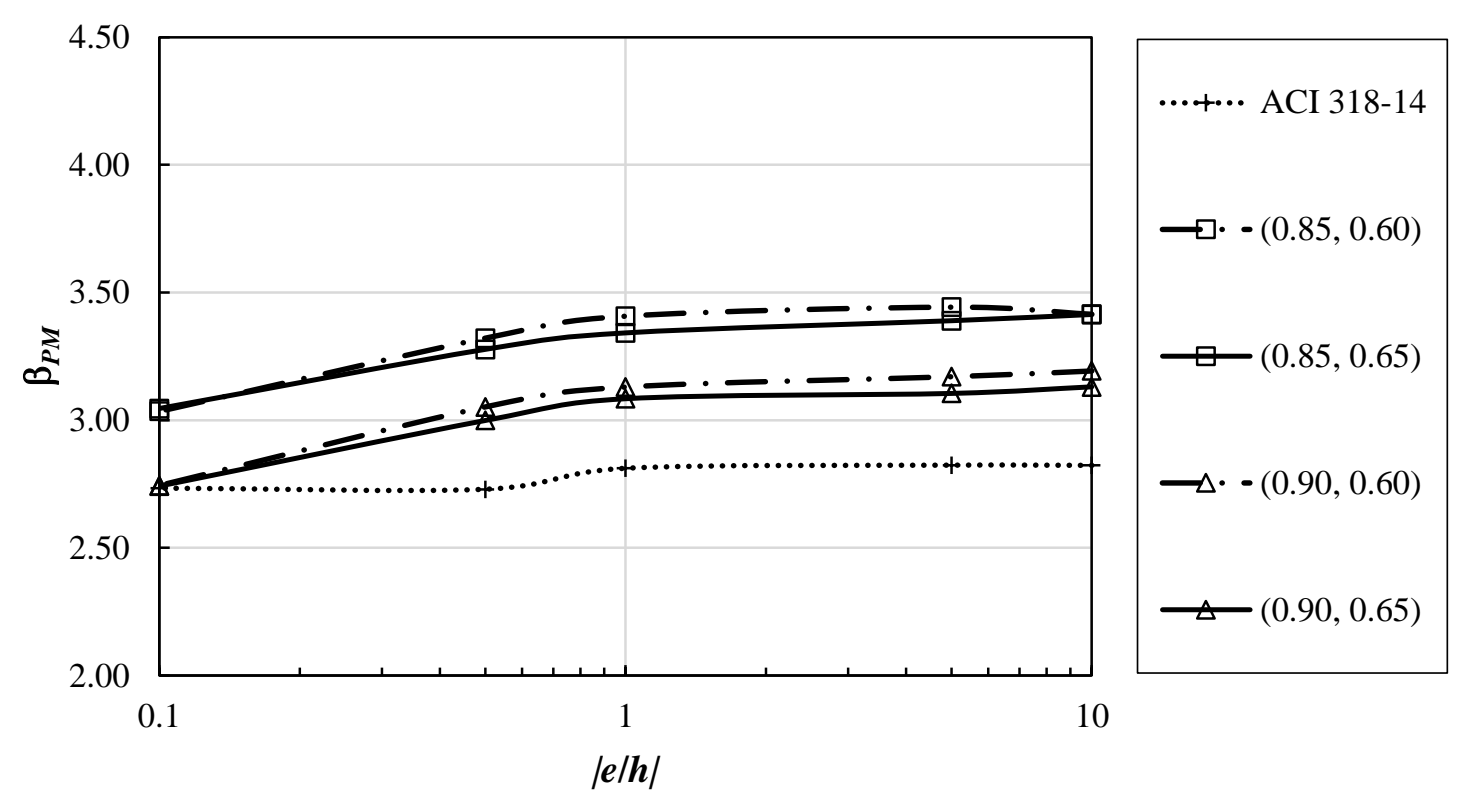

(b)

Figure C.10: Reliability indices for combined moment and axial force, $\beta_{P M}$, for Column Section 2, Property Combination 1, and $L / D=0.5$ : (a) $e / h>0$; (b) $e / h<0$ 

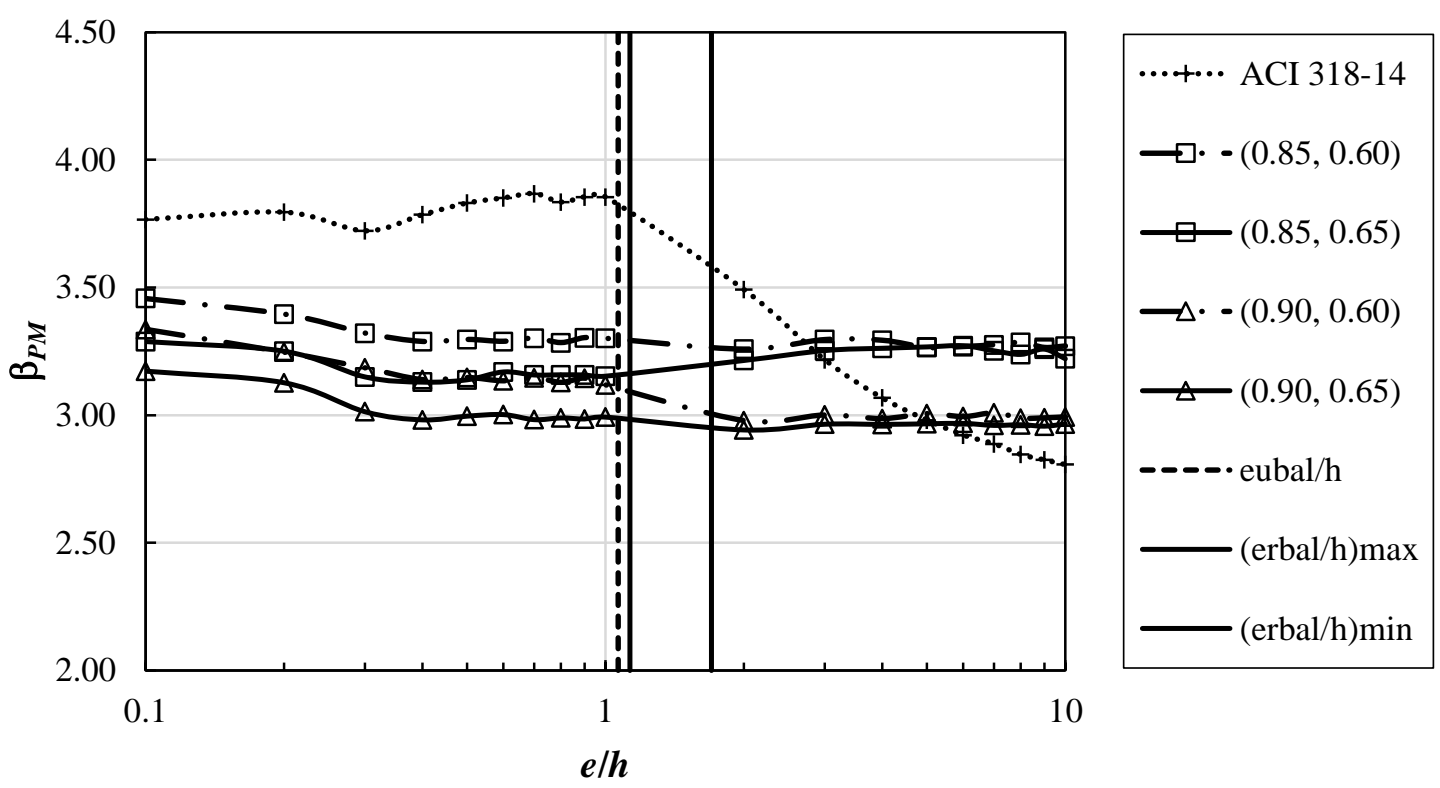

(a)
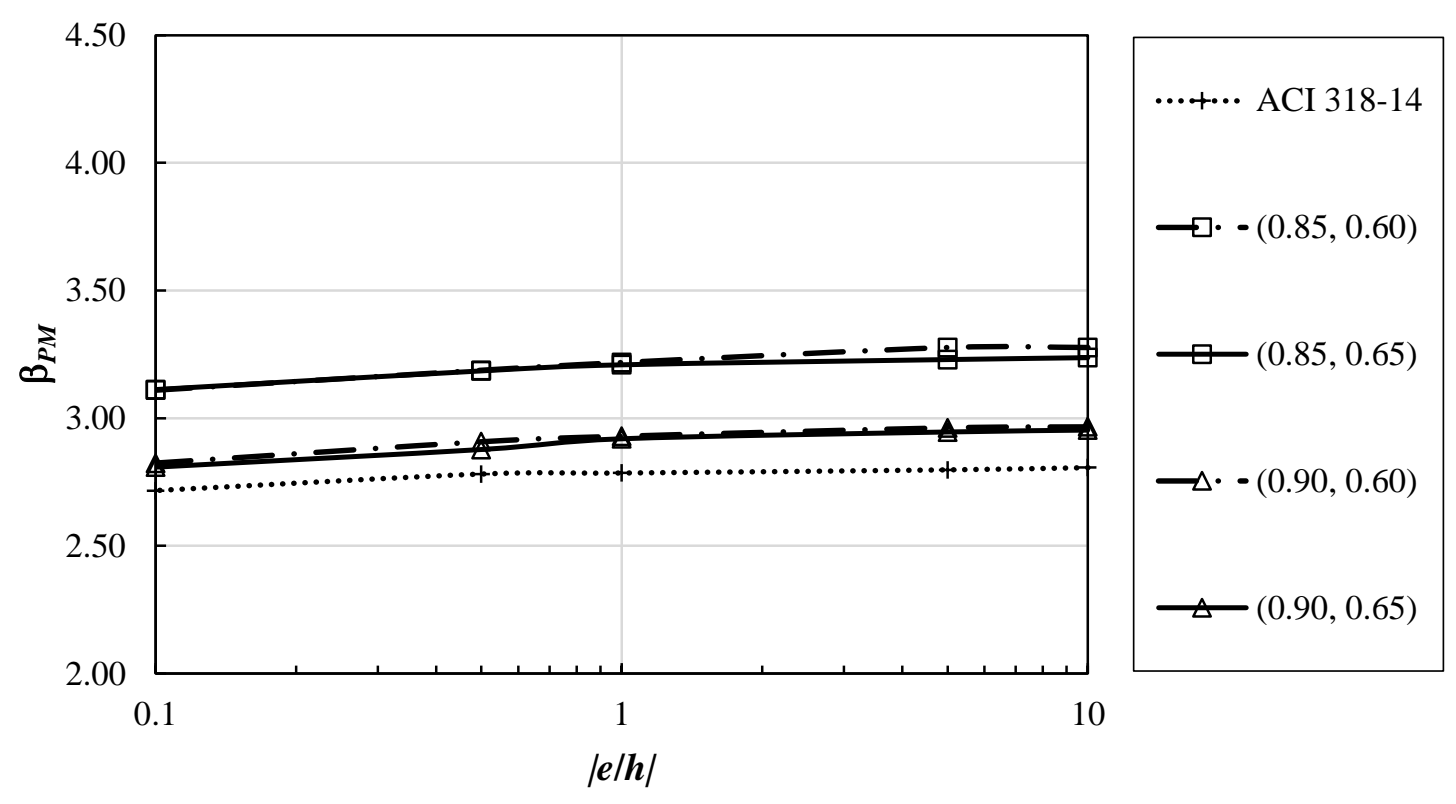

(b)

Figure C.11: Reliability indices for combined moment and axial force, $\beta_{P M}$, for Column Section 2, Property Combination 2, and $L / D=0.5$ : (a) $e / h>0$; (b) $e / h<0$ 

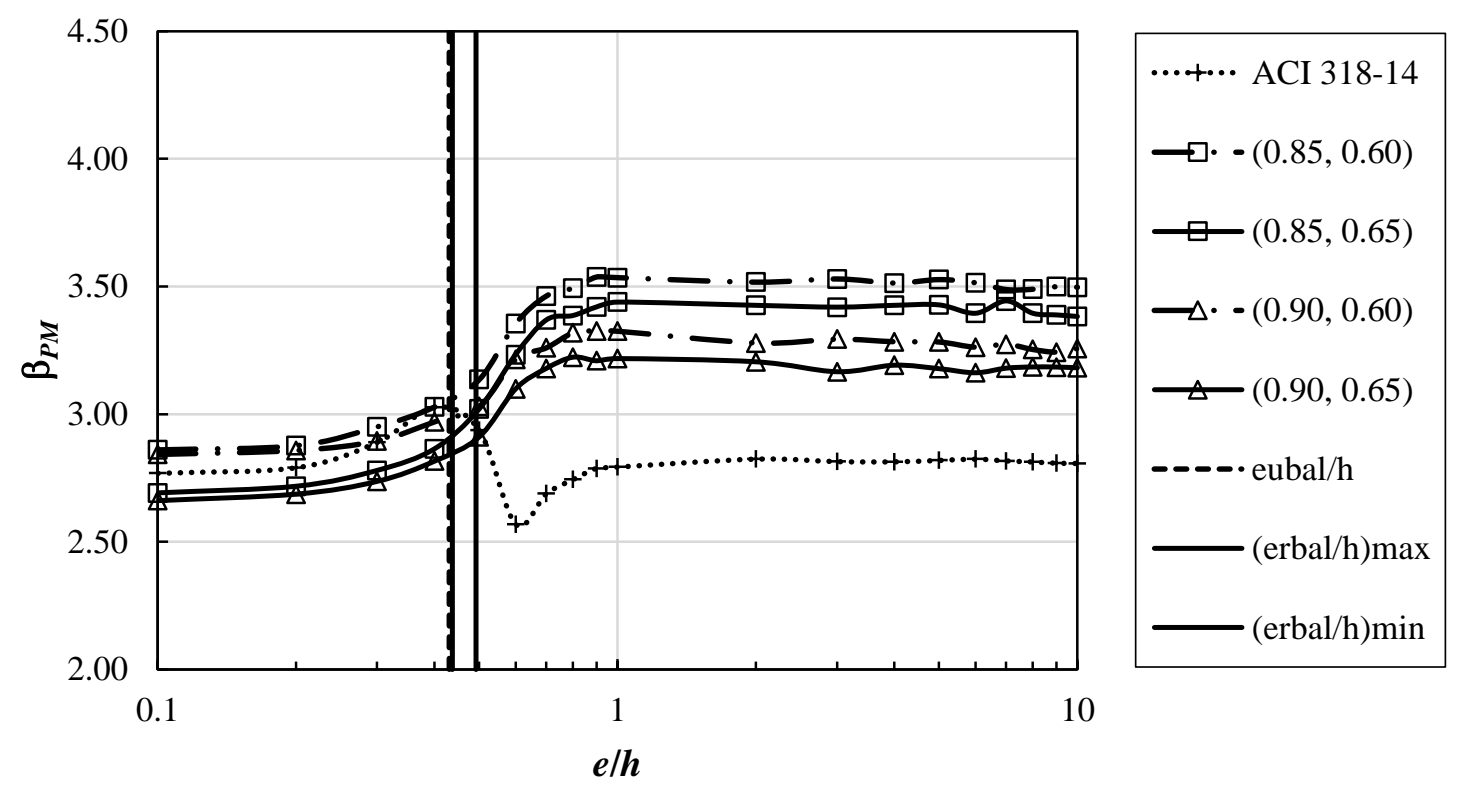

(a)
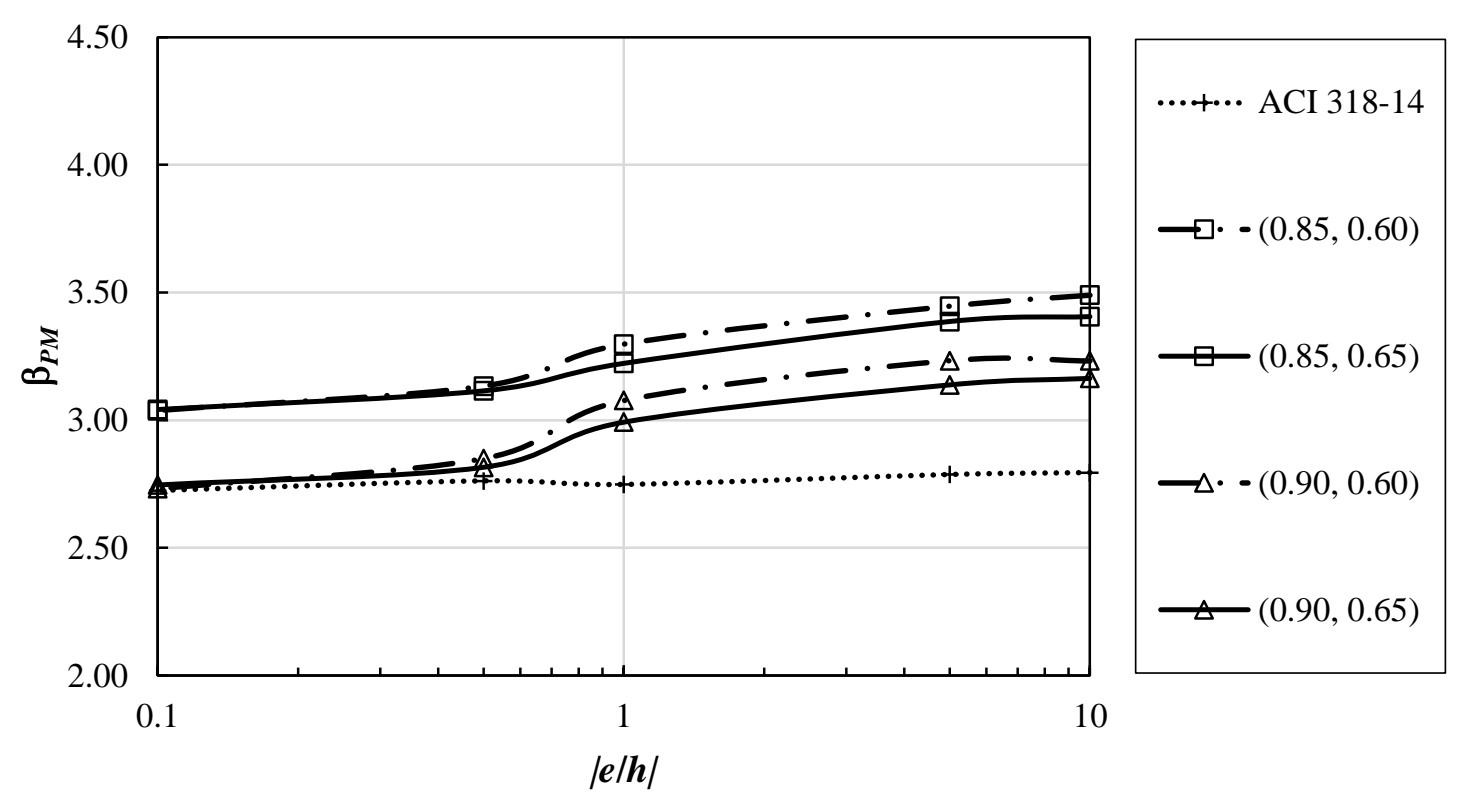

(b)

Figure C.12: Reliability indices for combined moment and axial force, $\beta_{P M}$, for Column Section 2, Property Combination 3, and $L / D=0.5$ : (a) $e / h>0$; (b) $e / h<0$ 

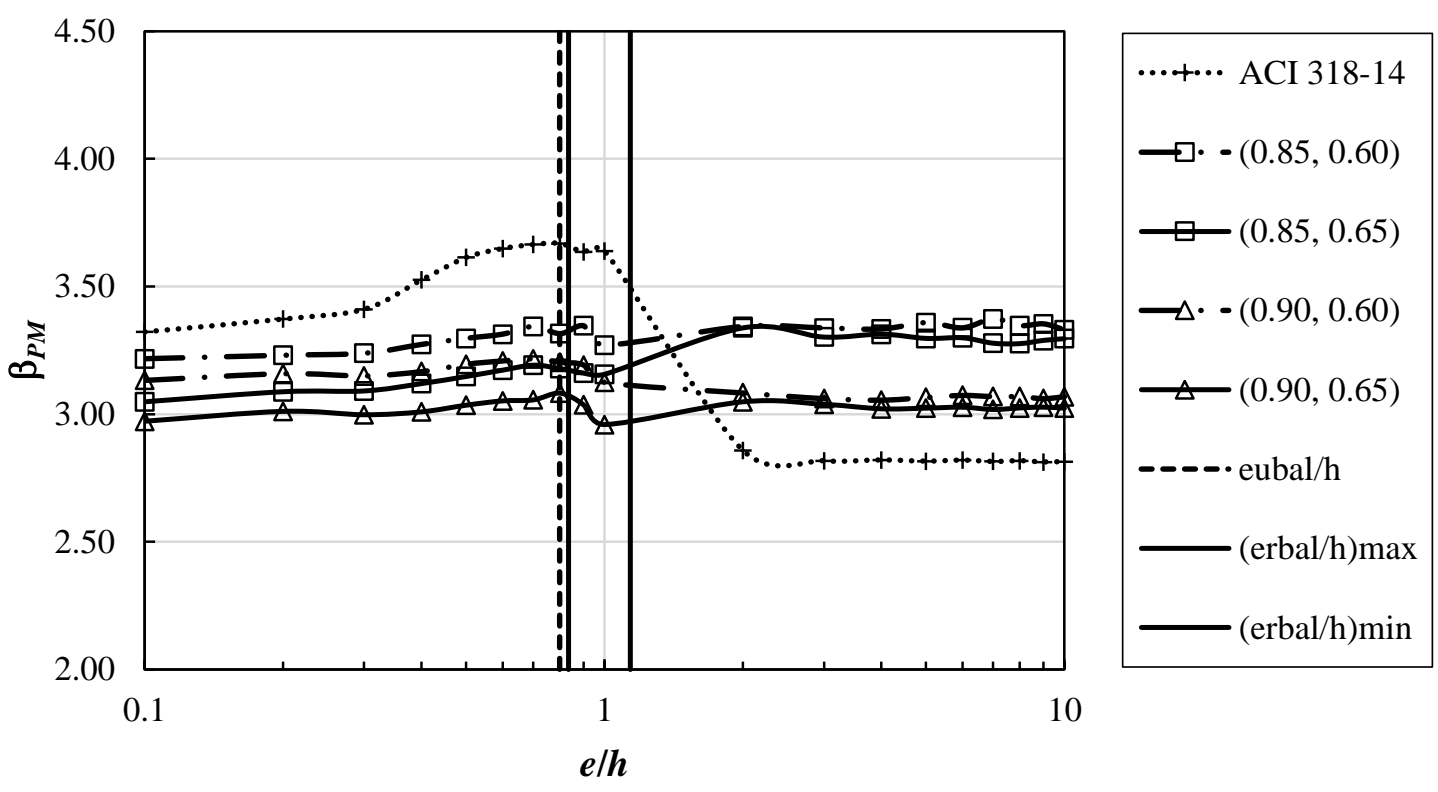

(a)
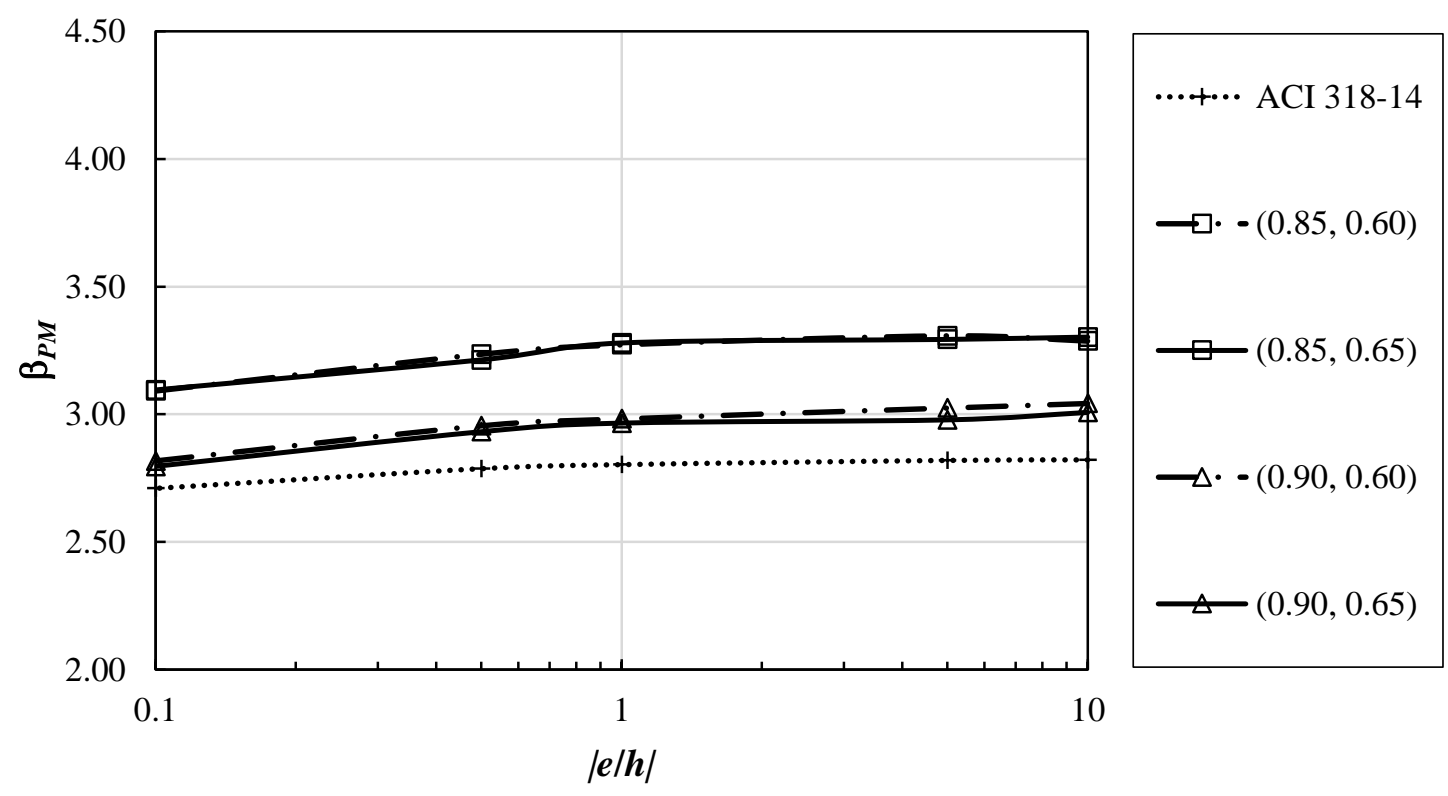

(b)

Figure C.13: Reliability indices for combined moment and axial force, $\beta_{P M}$, for Column Section 2, Property Combination 4, and $L / D=0.5$ : (a) $e / h>0$; (b) $e / h<0$ 

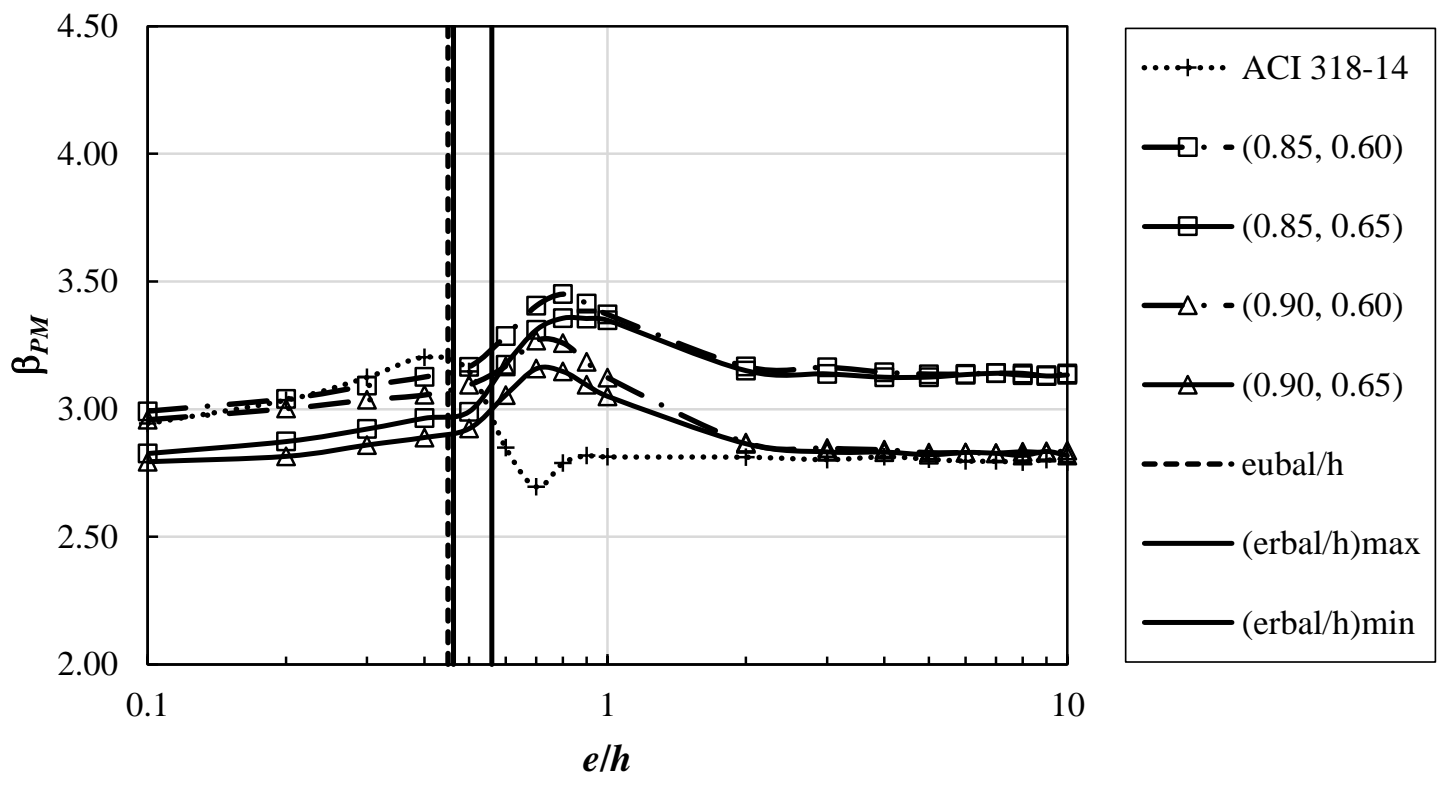

(a)
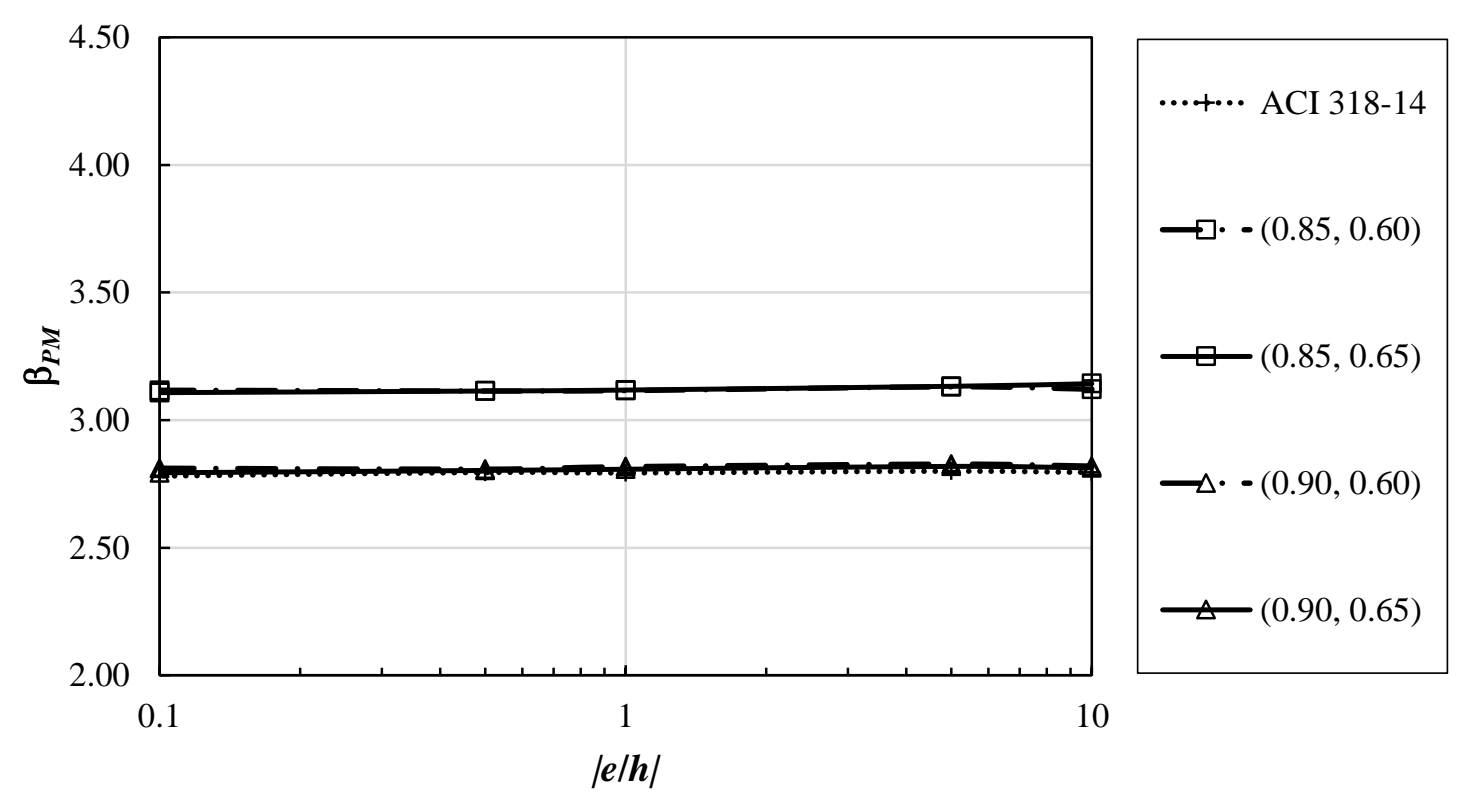

(b)

Figure C.14: Reliability indices for combined moment and axial force, $\beta_{P M}$, for Column Section 2, Property Combination 5, and $L / D=0.5$ : (a) $e / h>0$; (b) $e / h<0$ 

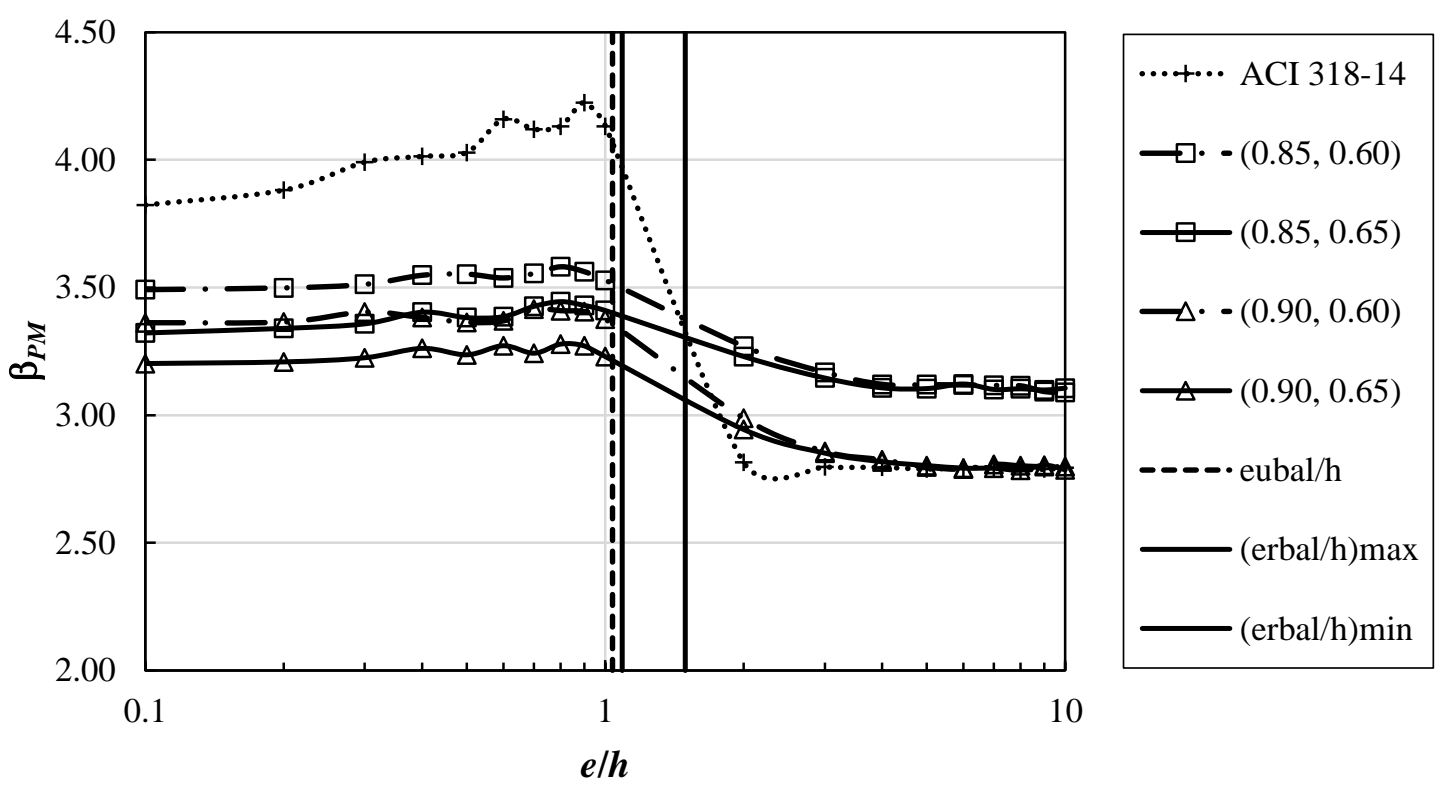

(a)
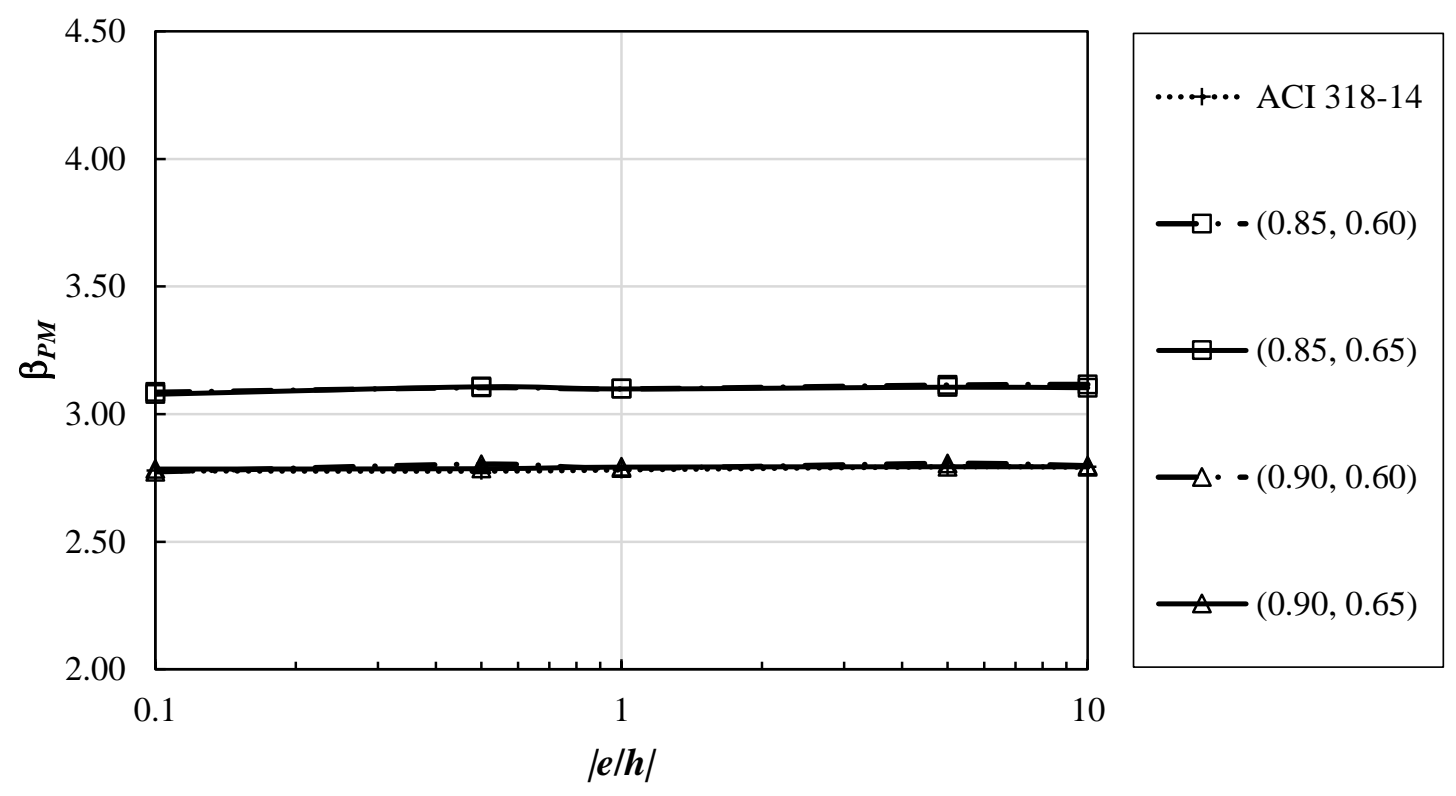

(b)

Figure C.15: Reliability indices for combined moment and axial force, $\beta_{P M}$, for Column Section 2, Property Combination 6, and $L / D=0.5$ : (a) $e / h>0$; (b) $e / h<0$ 

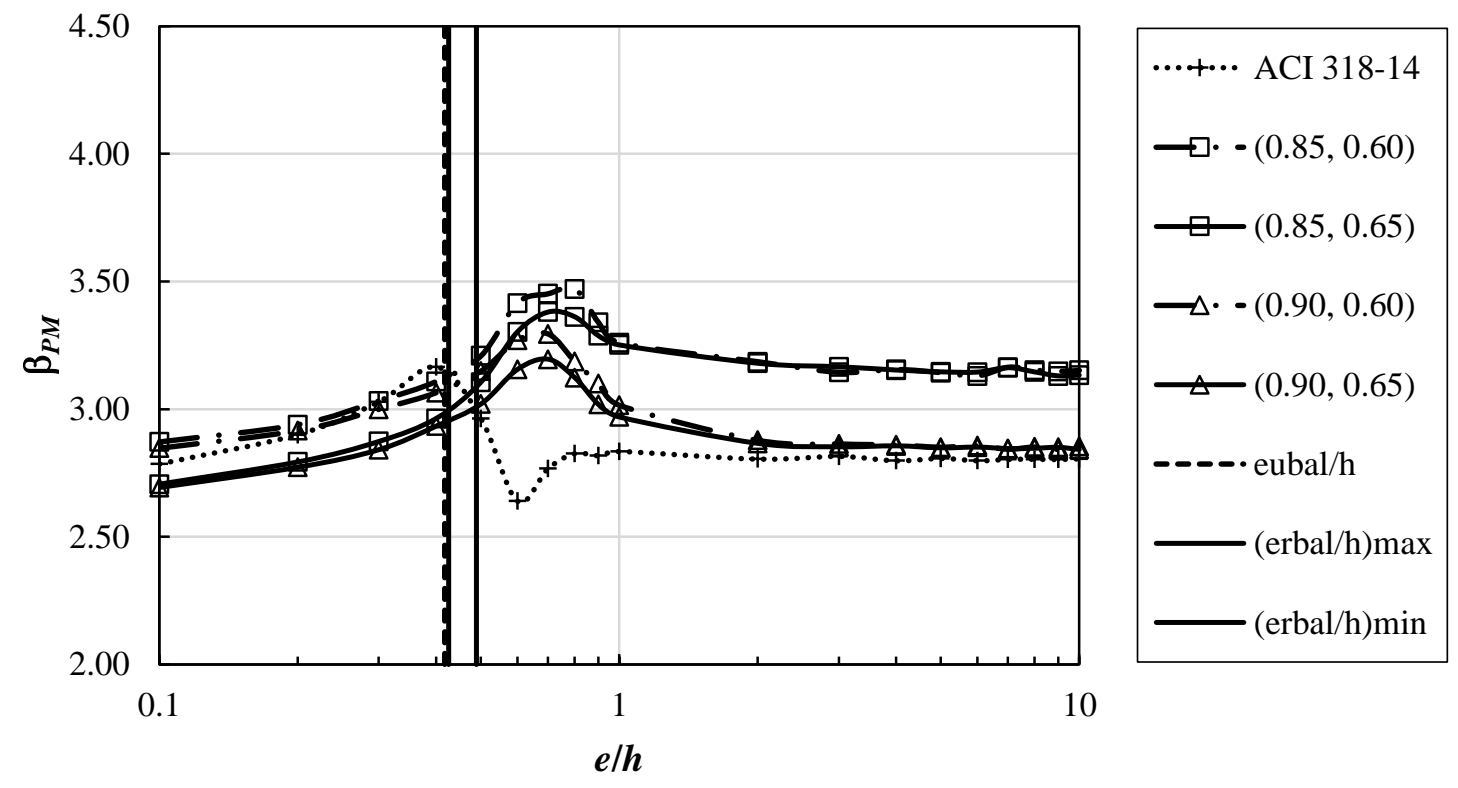

(a)
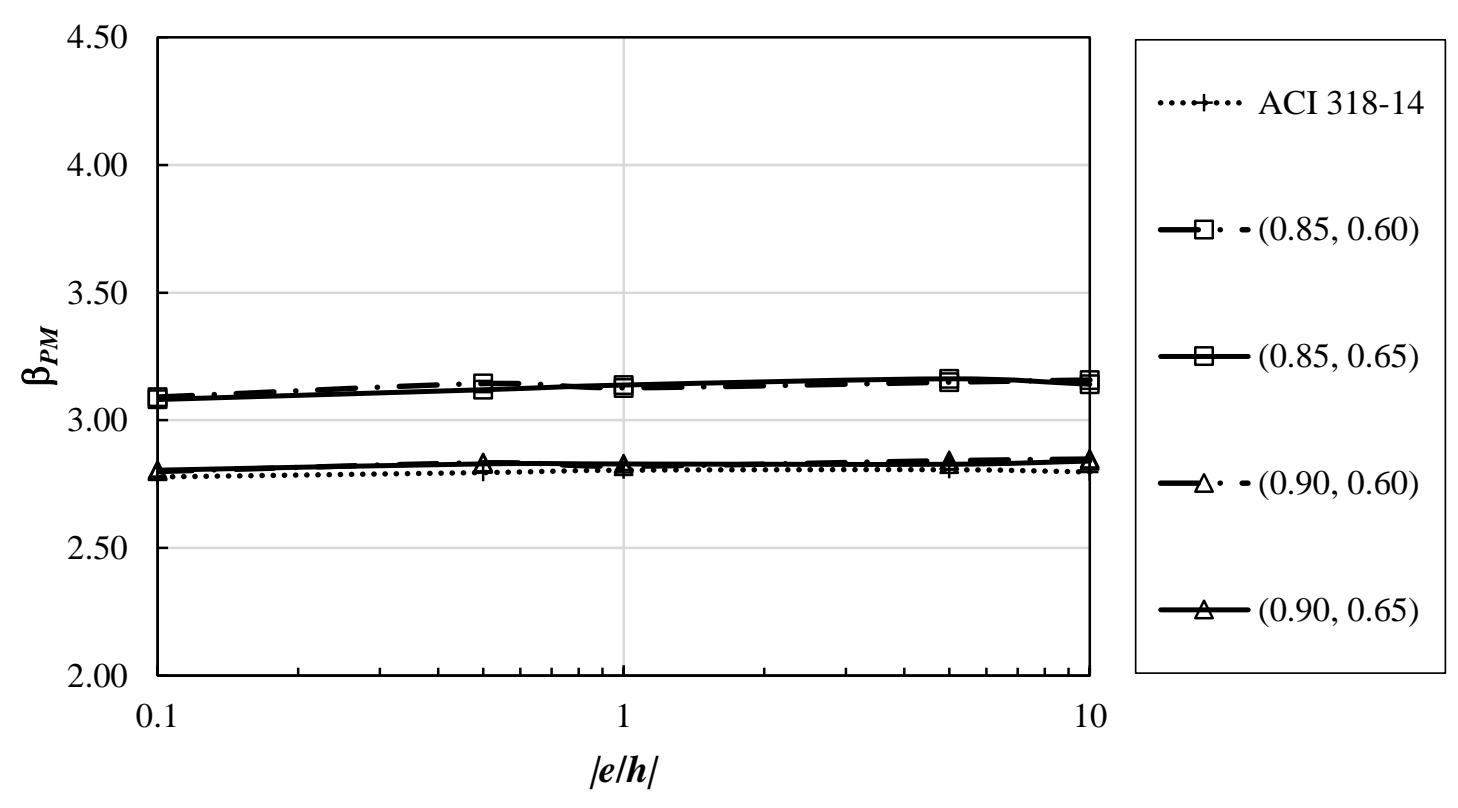

(b)

Figure C.16: Reliability indices for combined moment and axial force, $\beta_{P M}$, for Column Section 2, Property Combination 7, and $L / D=0.5$ : (a) $e / h>0$; (b) $e / h<0$ 

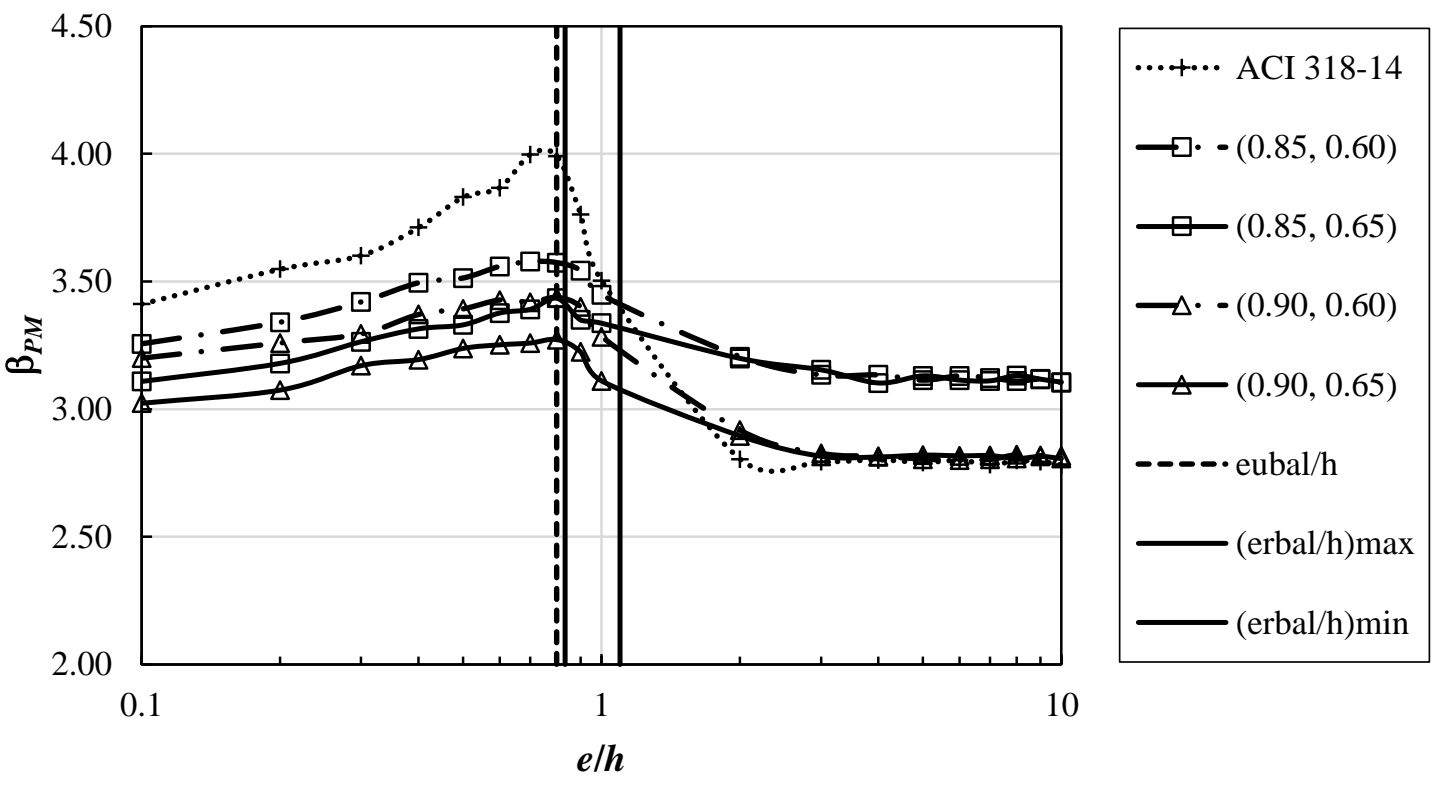

(a)
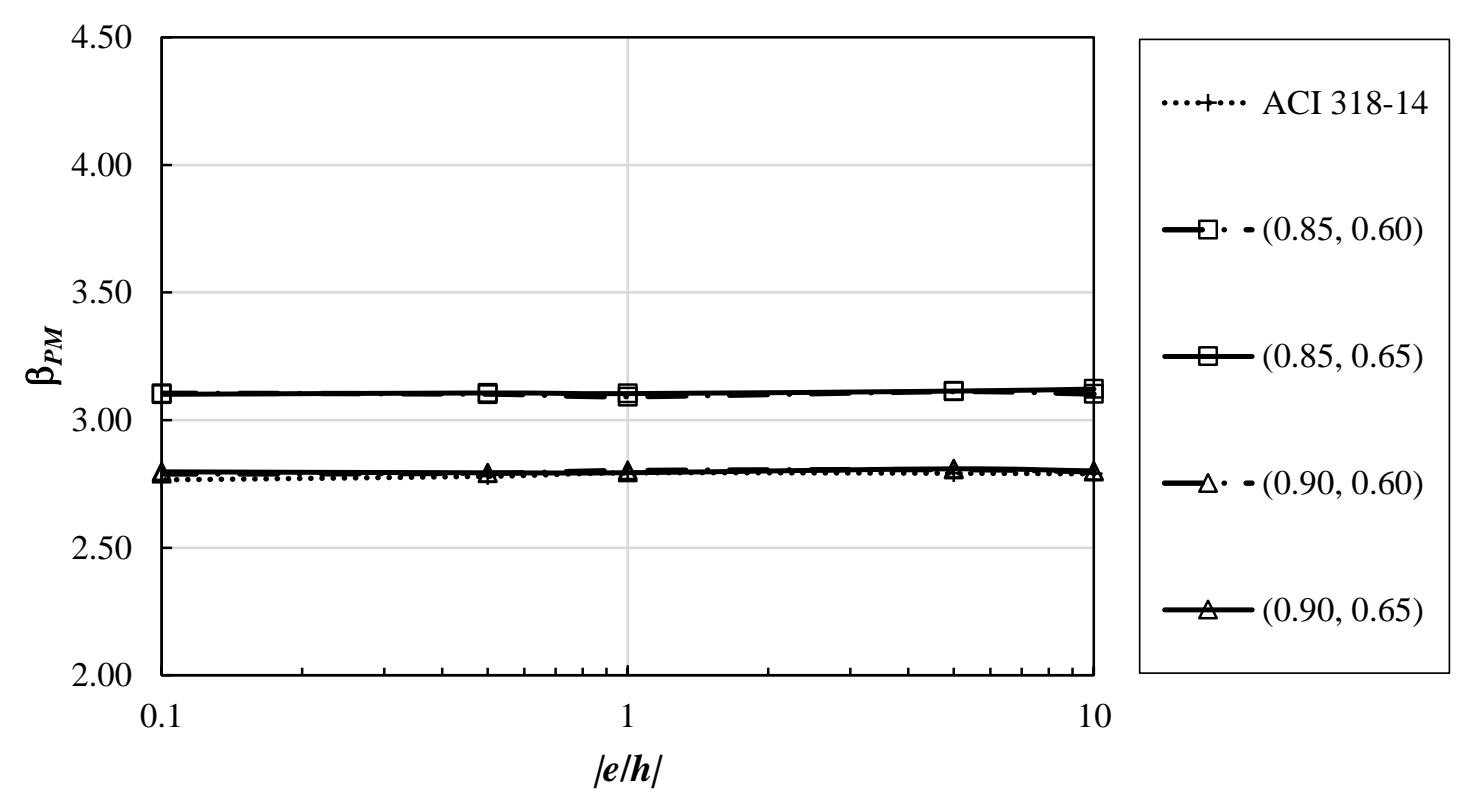

(b)

Figure C.17: Reliability indices for combined moment and axial force, $\beta_{P M}$, for Column Section 2, Property Combination 8 , and $L / D=0.5$ : (a) $e / h>0$; (b) $e / h<0$ 

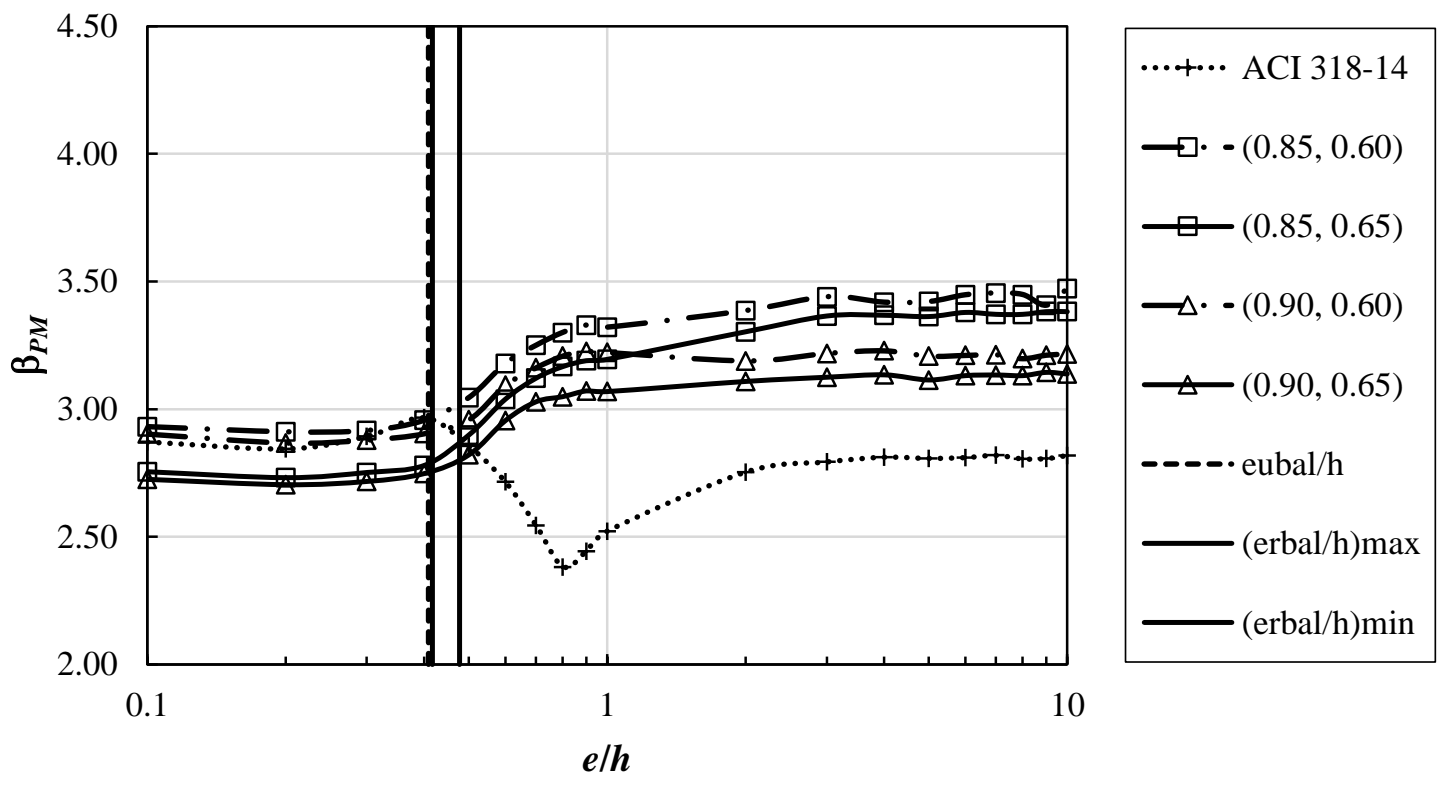

(a)
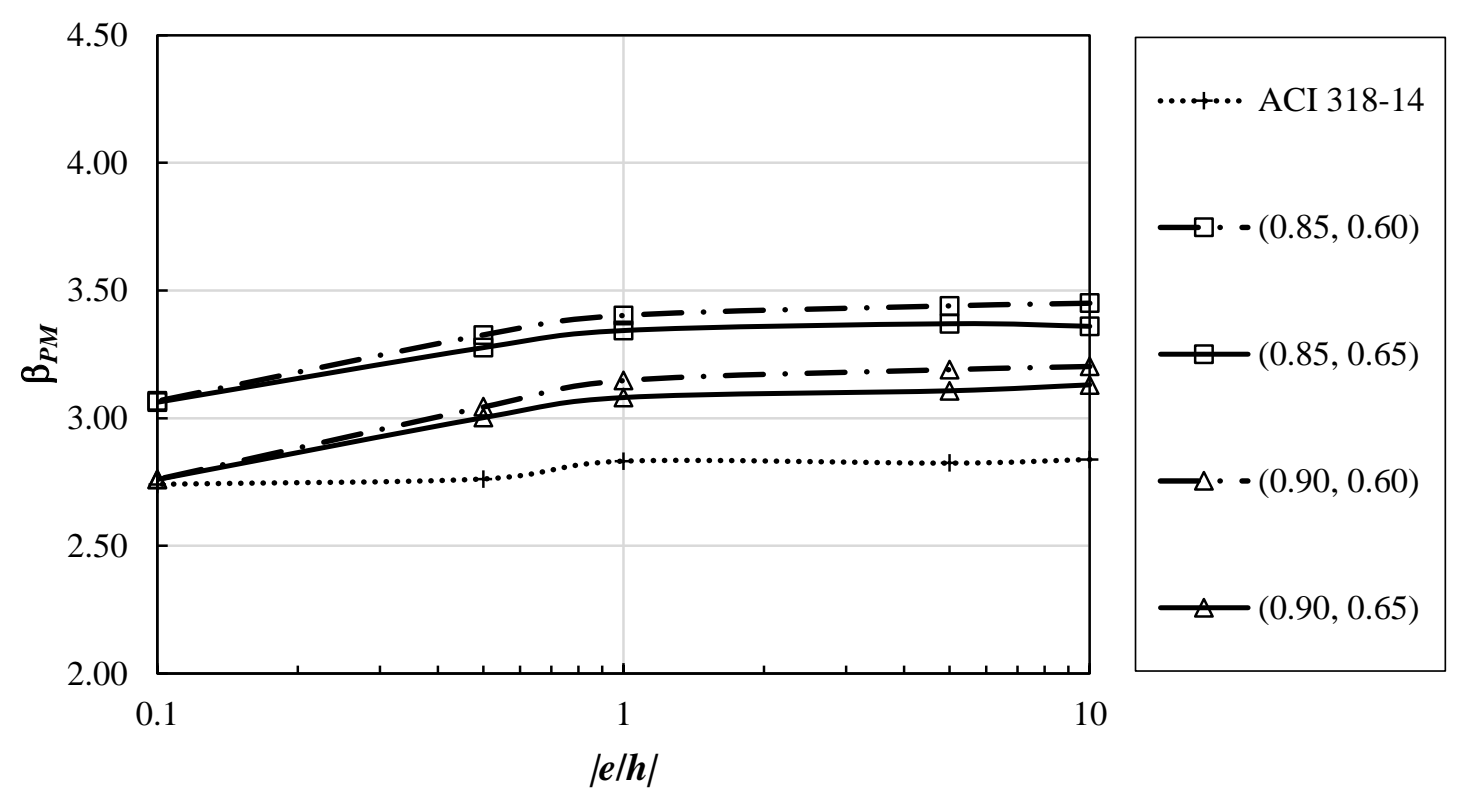

(b)

Figure C.18: Reliability indices for combined moment and axial force, $\beta_{P M}$, for Column Section 3, Property Combination 1, and $L / D=0.5$ : (a) $e / h>0$; (b) $e / h<0$ 

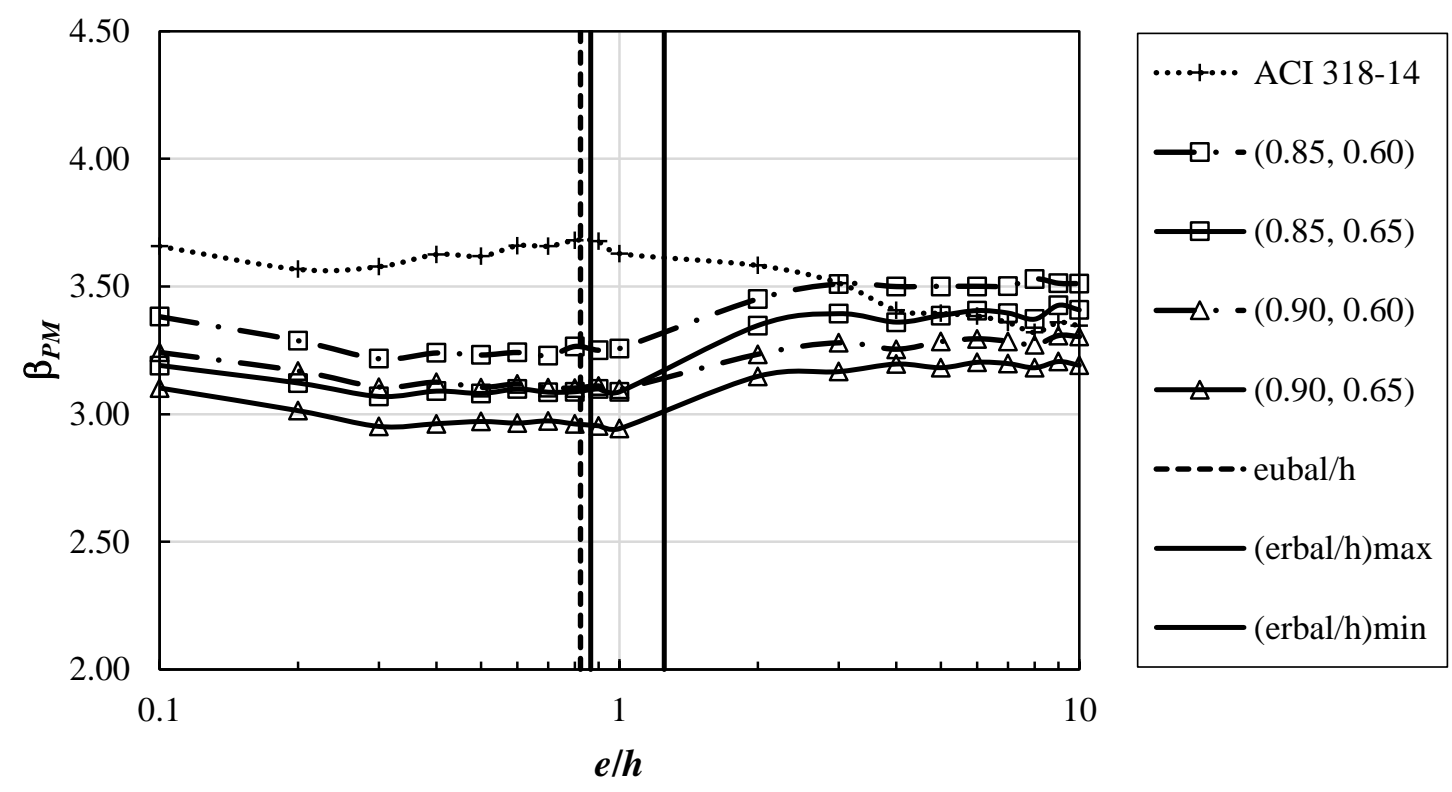

(a)

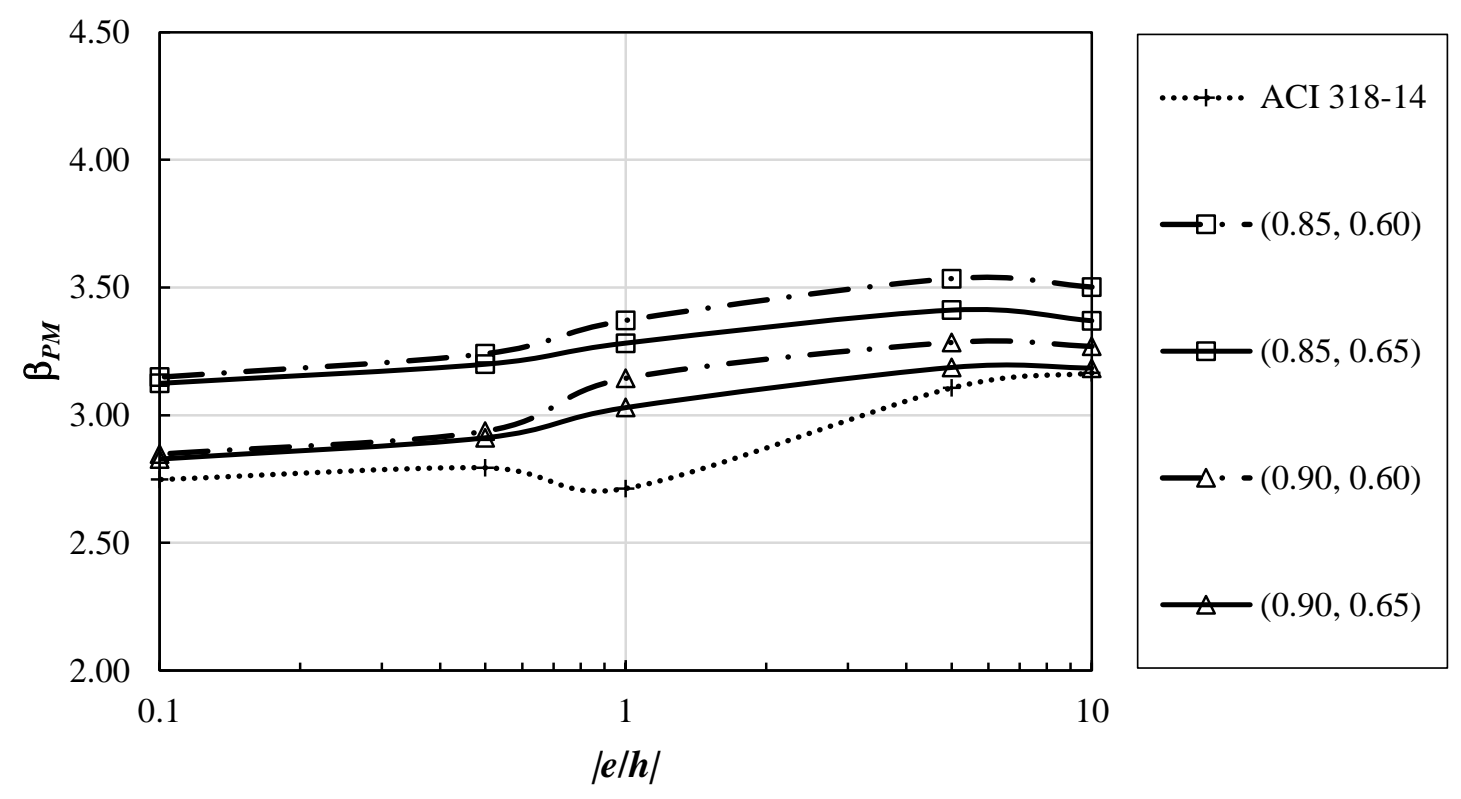

(b)

Figure C.19: Reliability indices for combined moment and axial force, $\beta_{P M}$, for Column Section 3, Property Combination 2, and $L / D=0.5$ : (a) $e / h>0$; (b) $e / h<0$ 

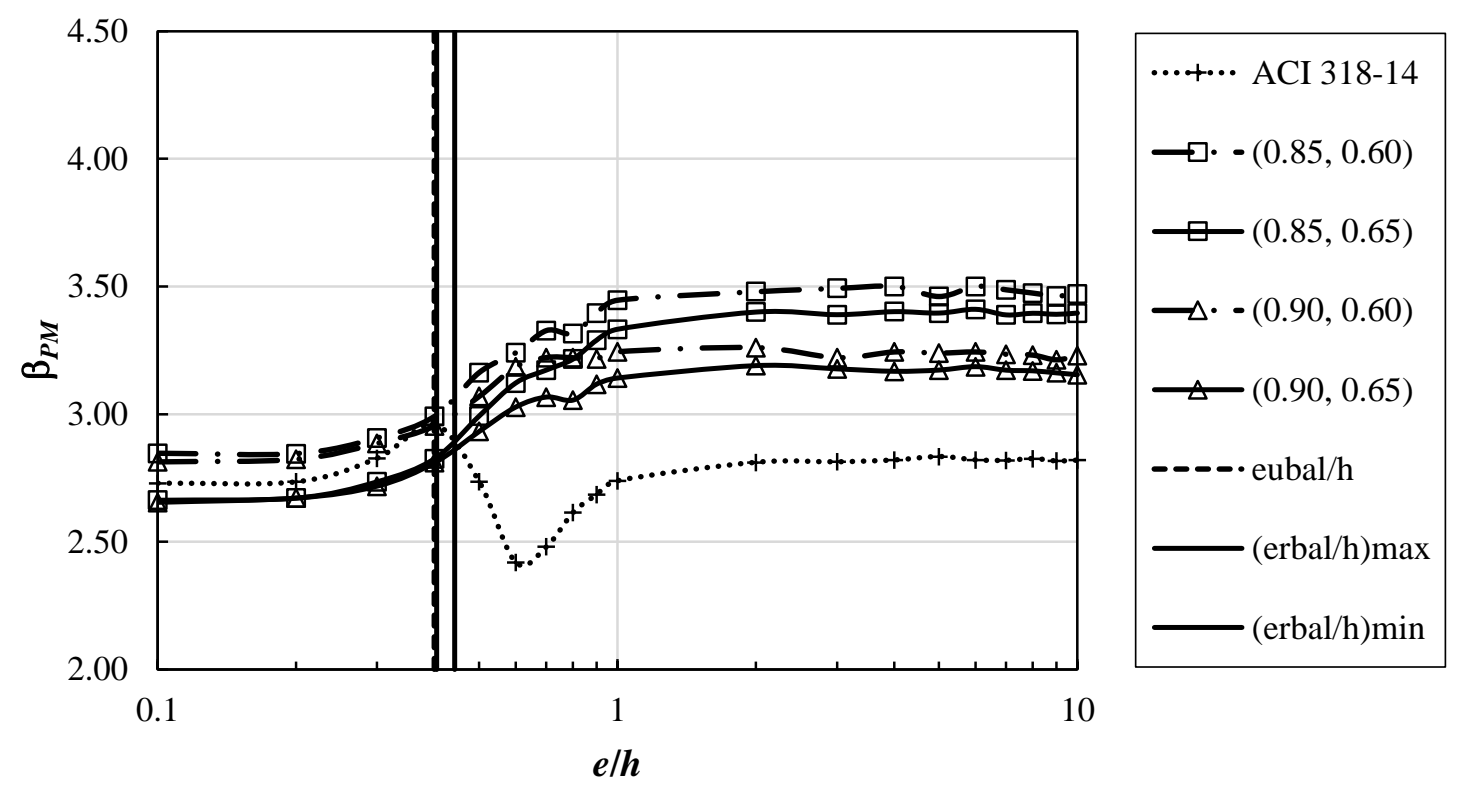

(a)

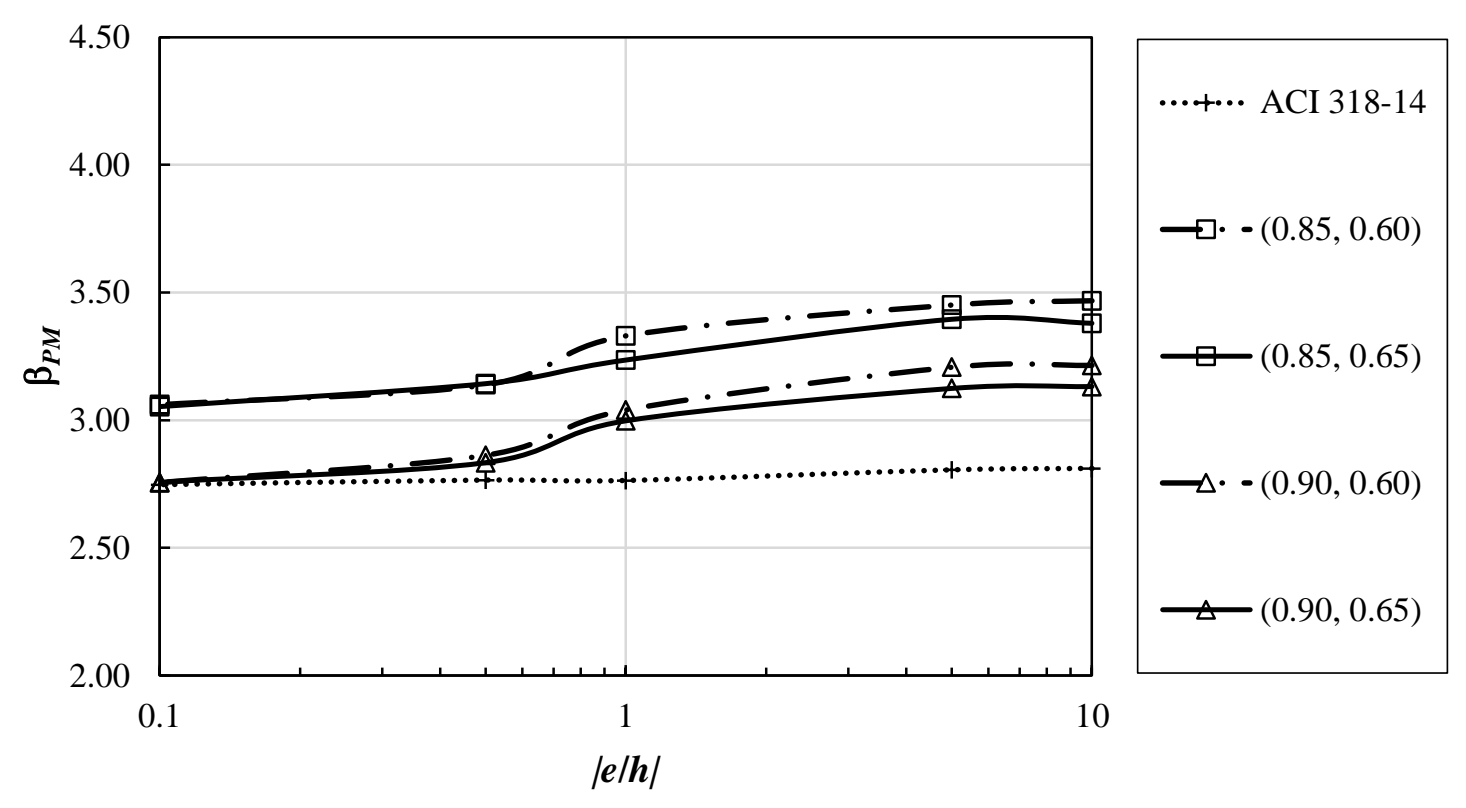

(b)

Figure C.20: Reliability indices for combined moment and axial force, $\beta_{P M}$, for Column Section 3, Property Combination 3, and $L / D=0.5$ : (a) $e / h>0$; (b) $e / h<0$ 

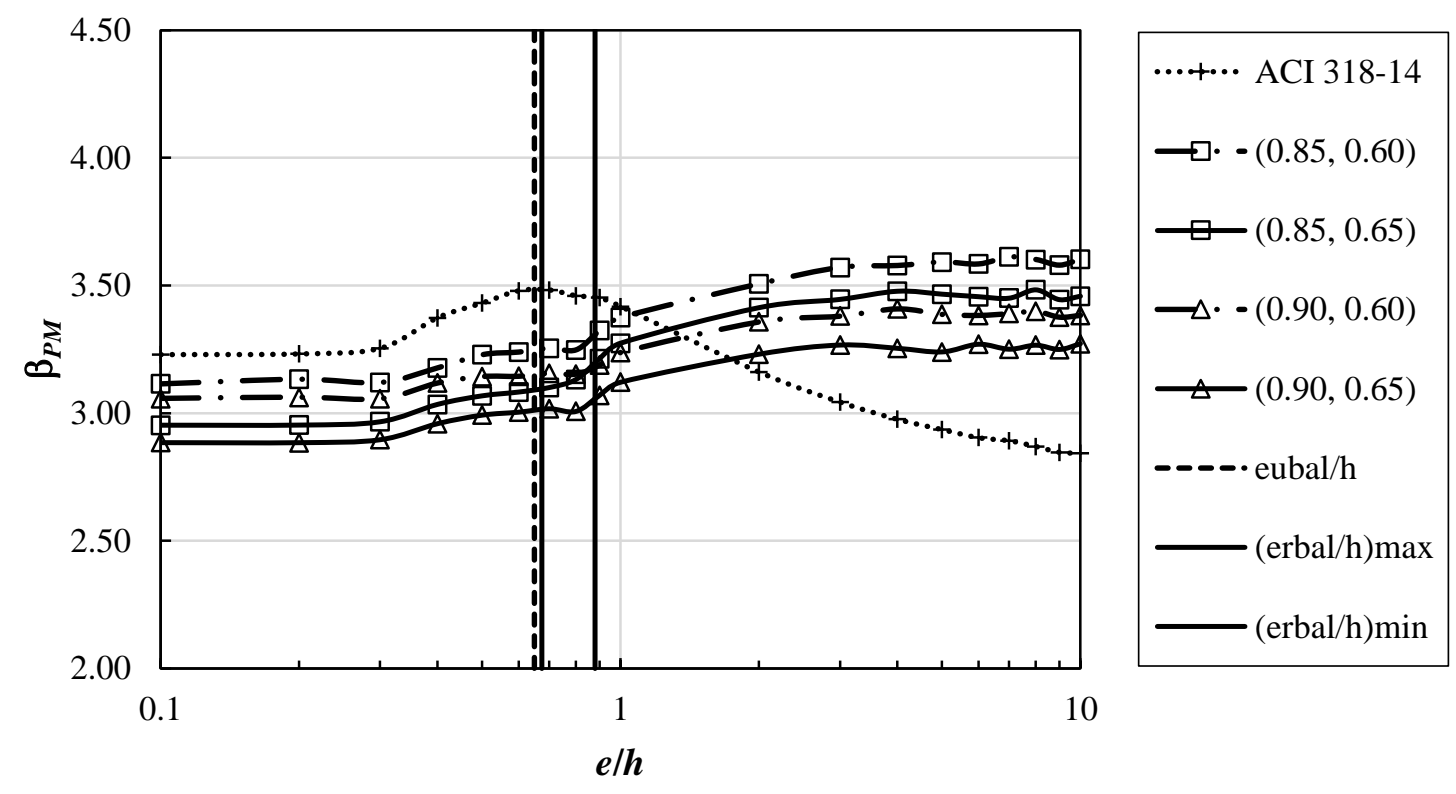

(a)

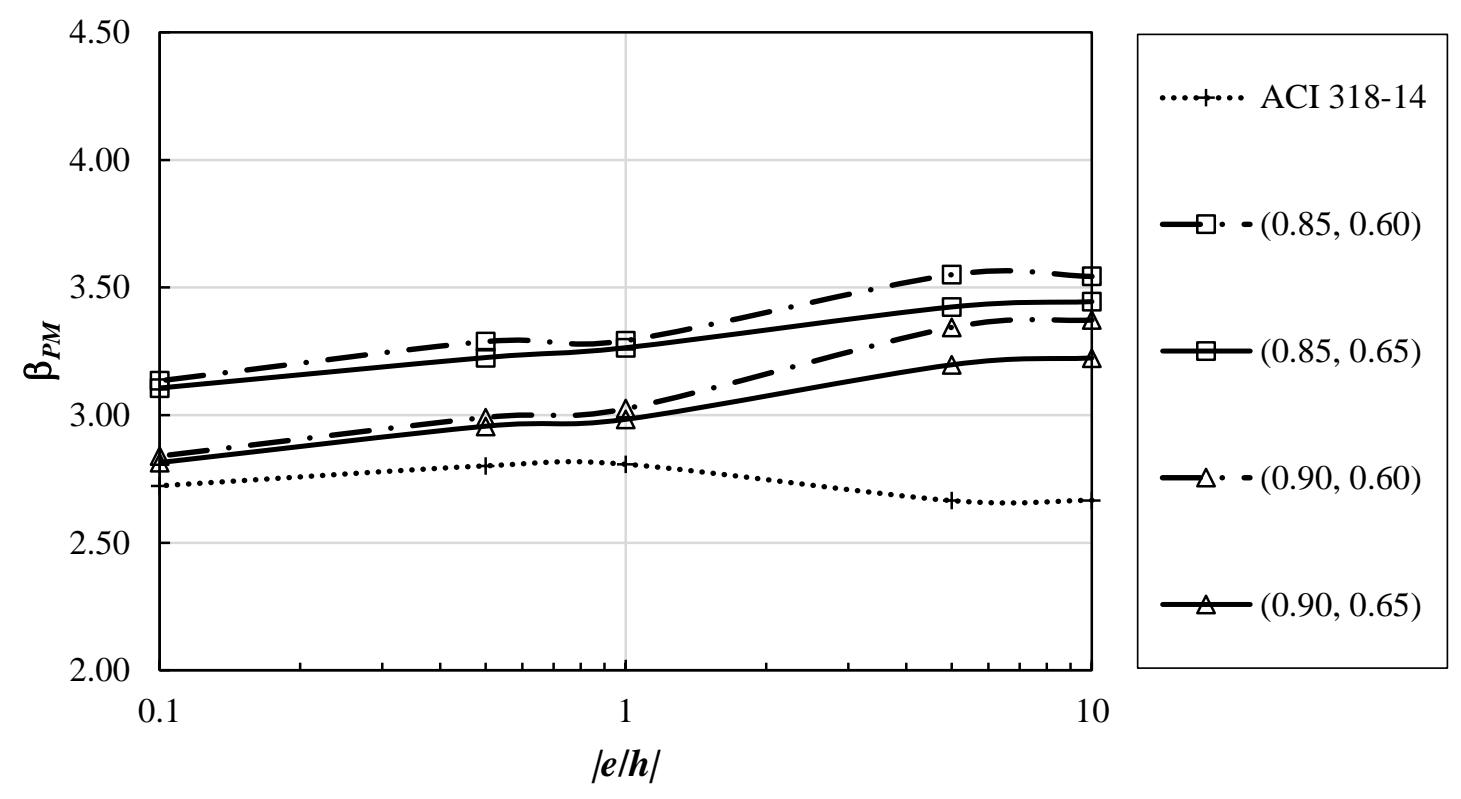

(b)

Figure C.21: Reliability indices for combined moment and axial force, $\beta_{P M}$, for Column Section 3, Property Combination 4, and $L / D=0.5$ : (a) $e / h>0$; (b) $e / h<0$ 

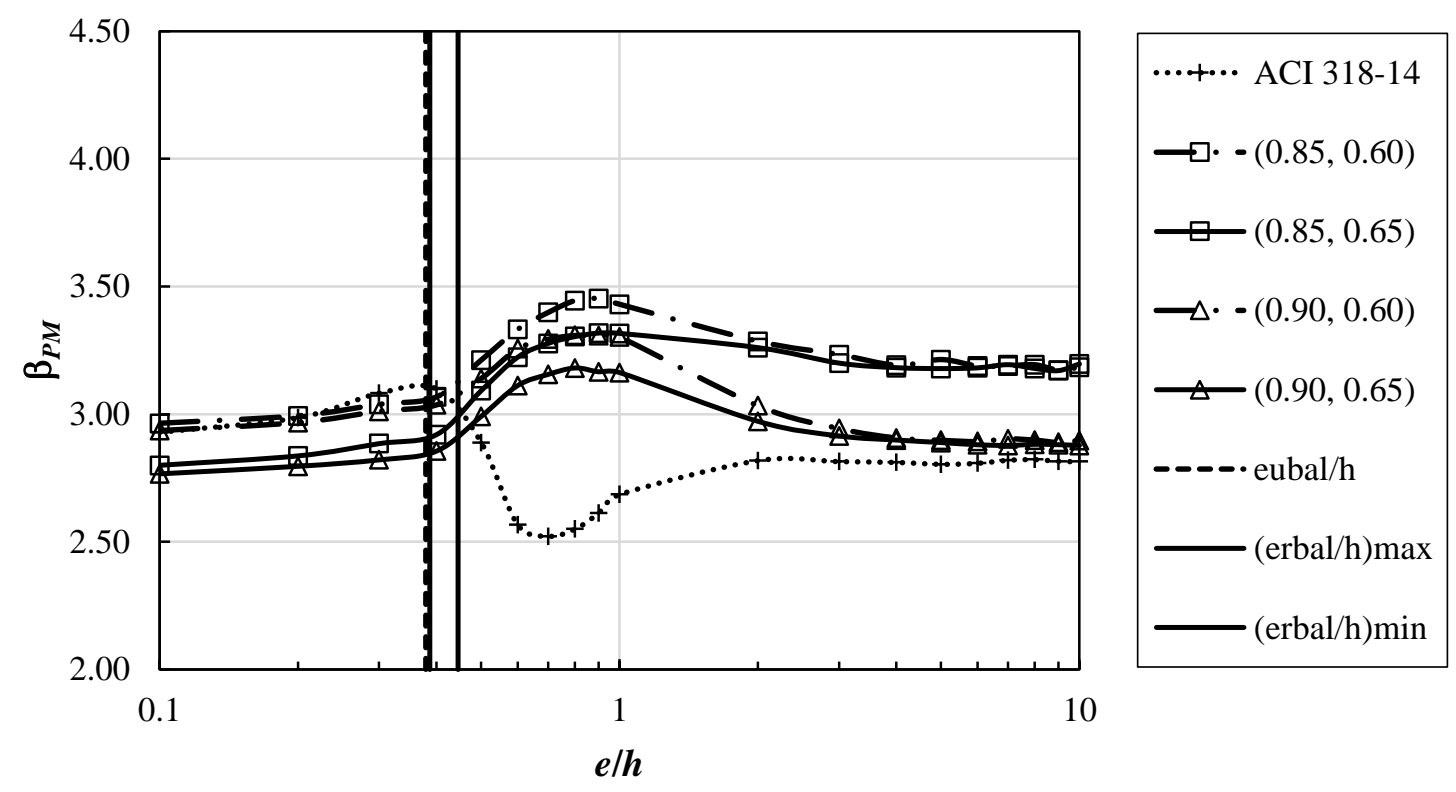

(a)
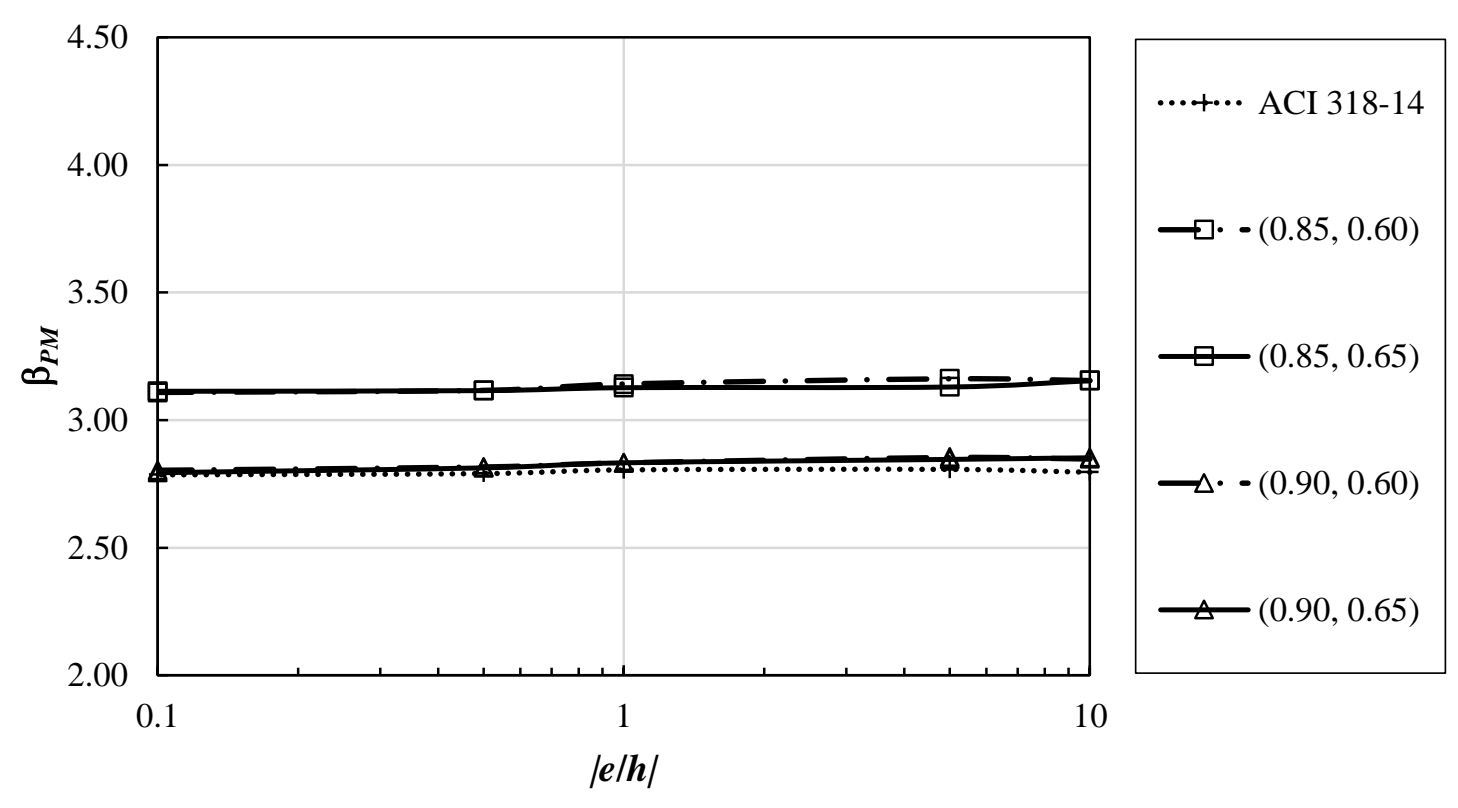

(b)

Figure C.22: Reliability indices for combined moment and axial force, $\beta_{P M}$, for Column Section 3, Property Combination 5, and $L / D=0.5$ : (a) $e / h>0$; (b) $e / h<0$ 

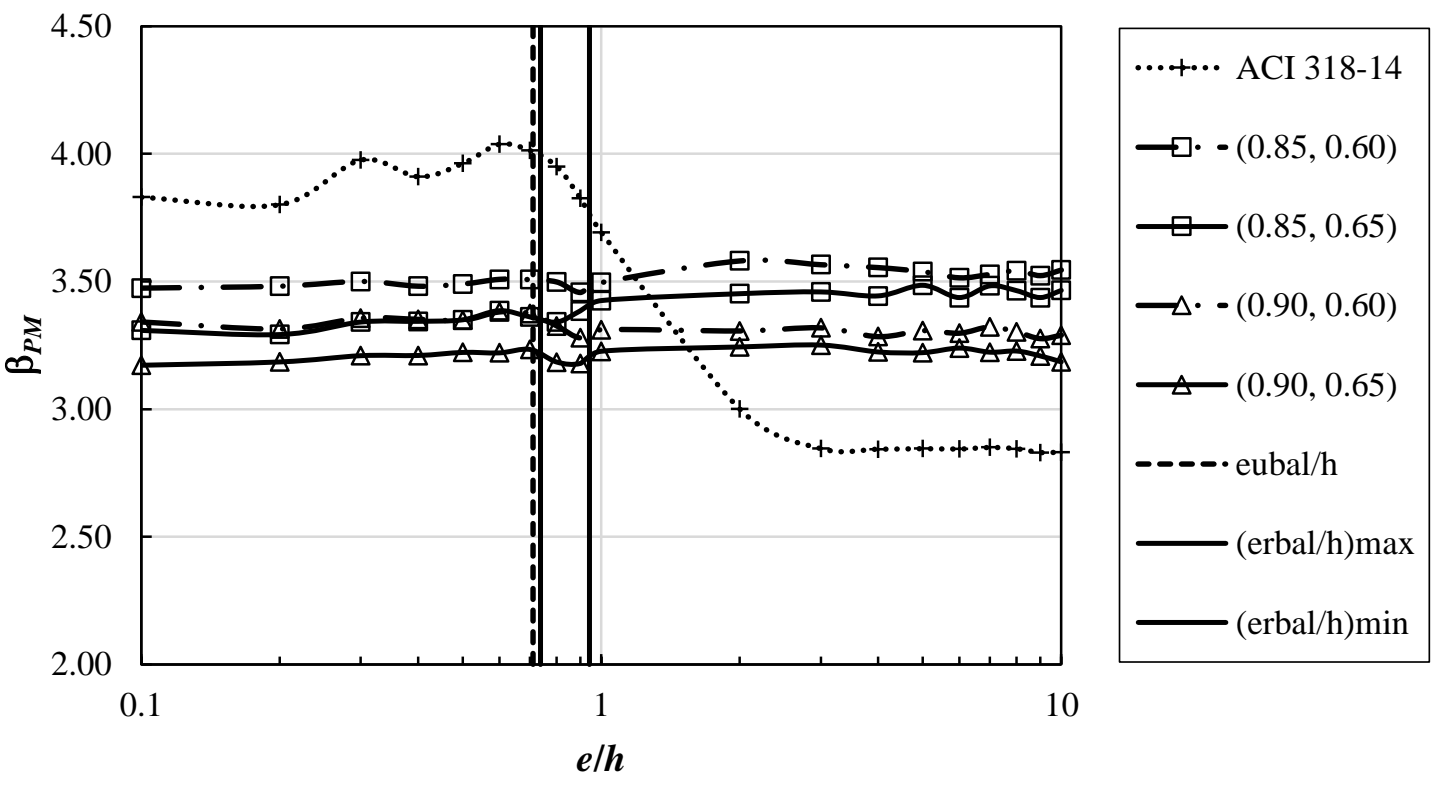

(a)
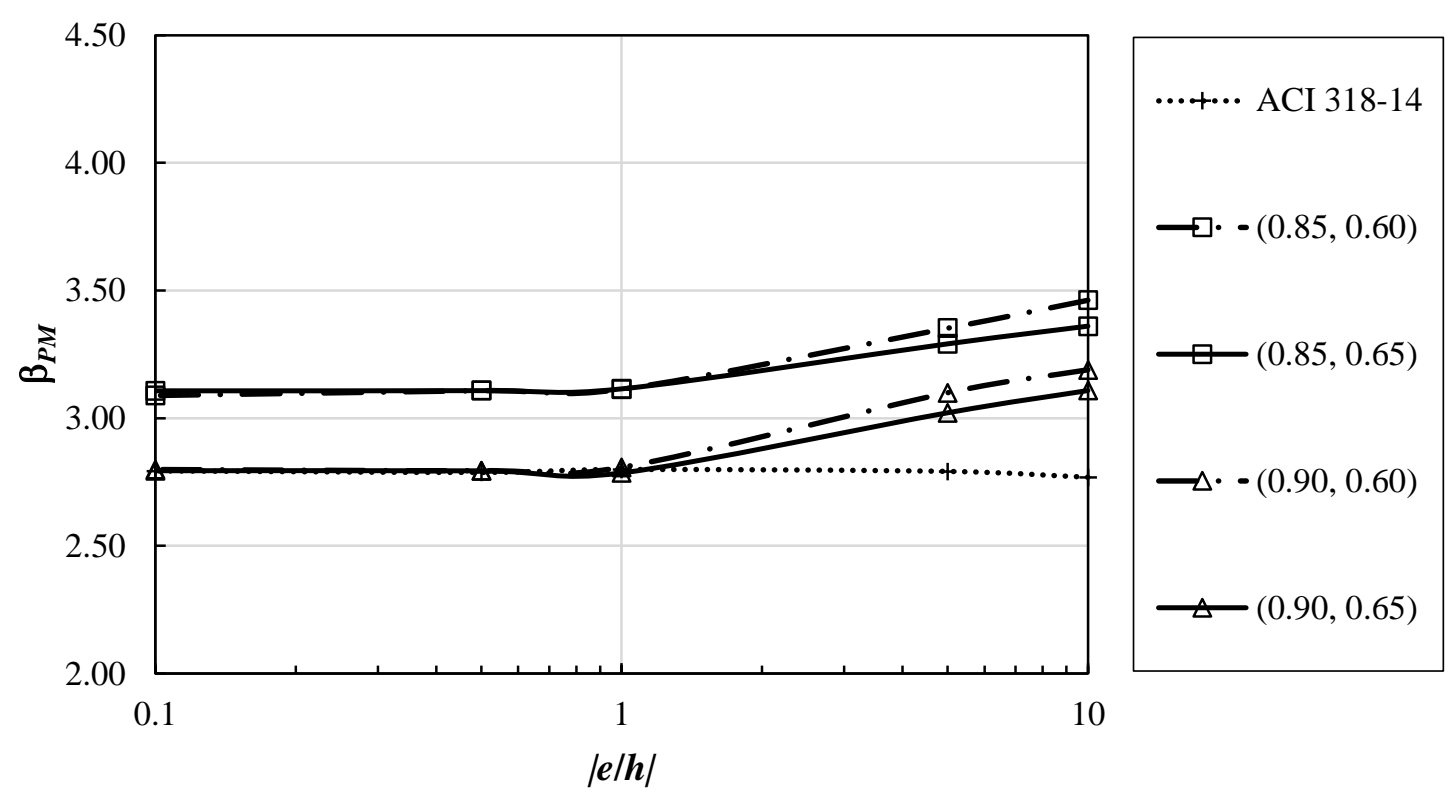

(b)

Figure C.23: Reliability indices for combined moment and axial force, $\beta_{P M}$, for Column Section 3, Property Combination 6, and $L / D=0.5$ : (a) $e / h>0$; (b) $e / h<0$ 

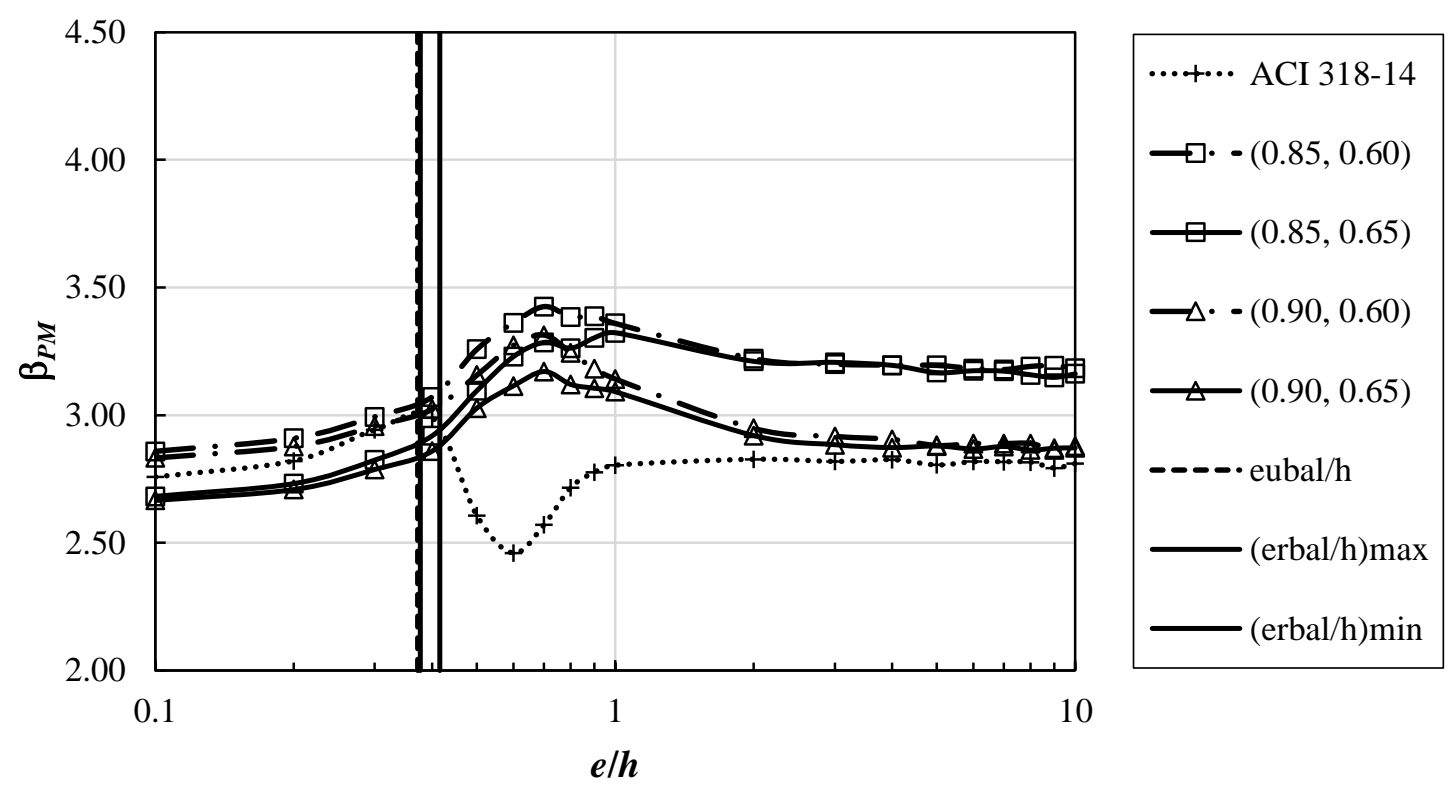

(a)
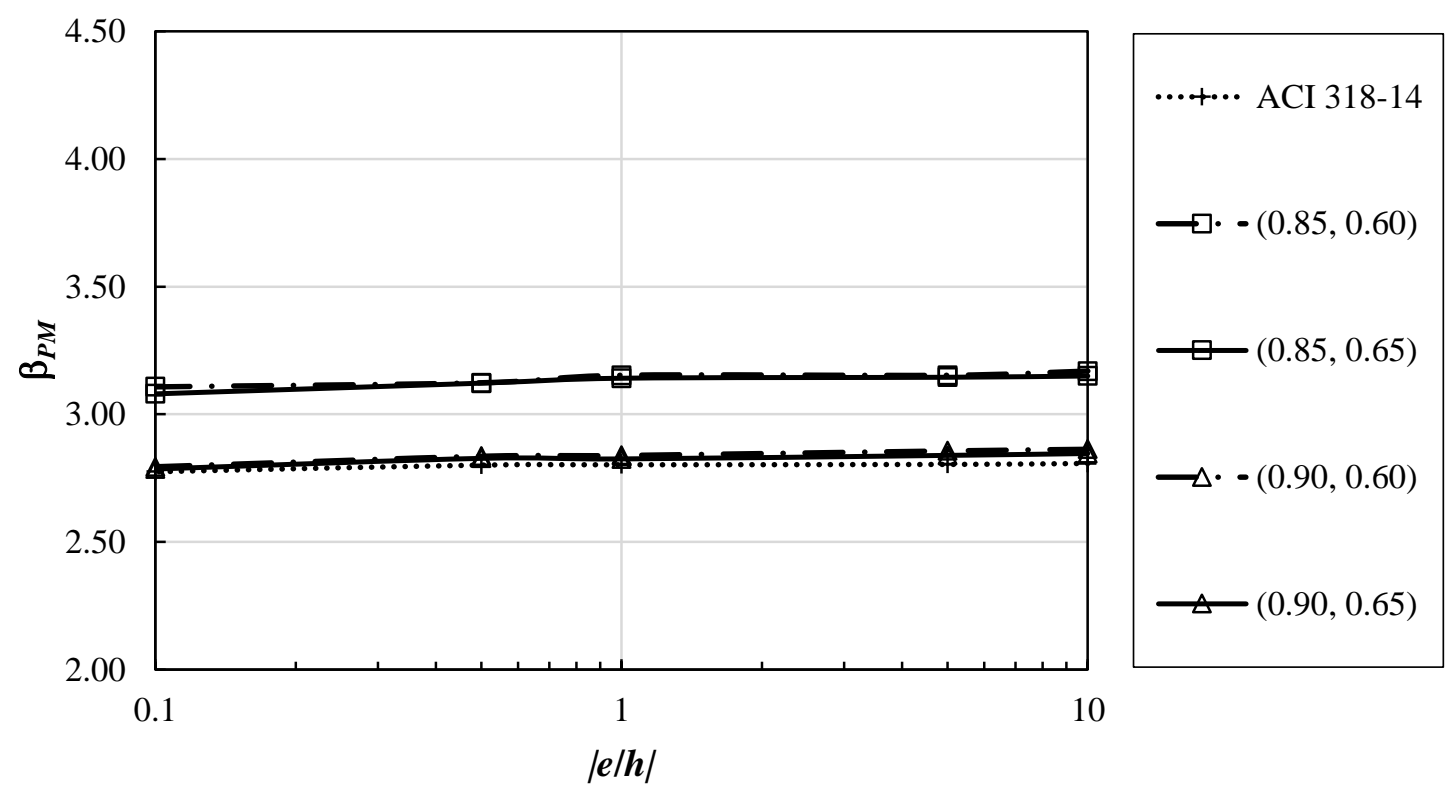

(b)

Figure C.24: Reliability indices for combined moment and axial force, $\beta_{P M}$, for Column Section 3, Property Combination 7, and $L / D=0.5$ : (a) $e / h>0$; (b) $e / h<0$ 

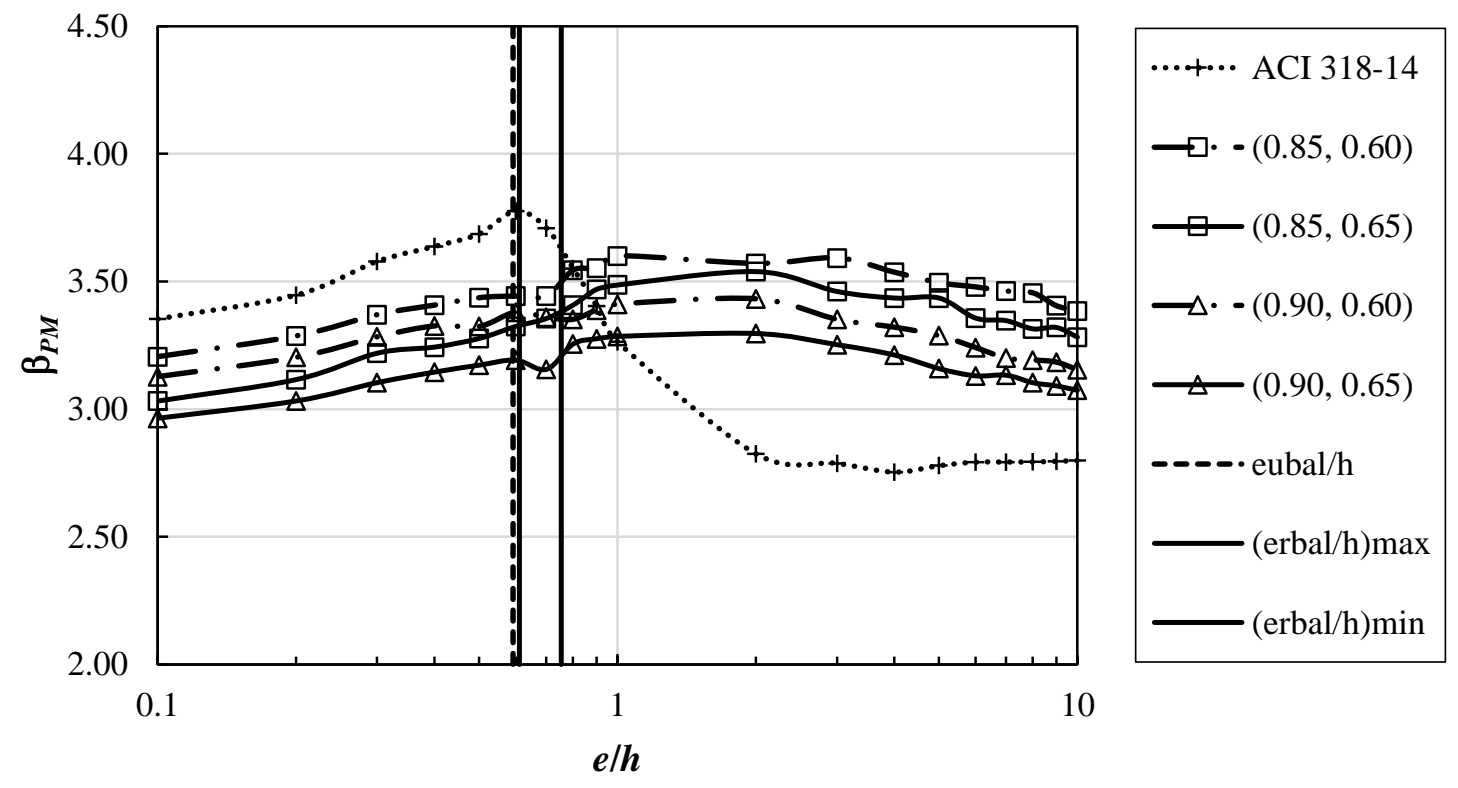

(a)
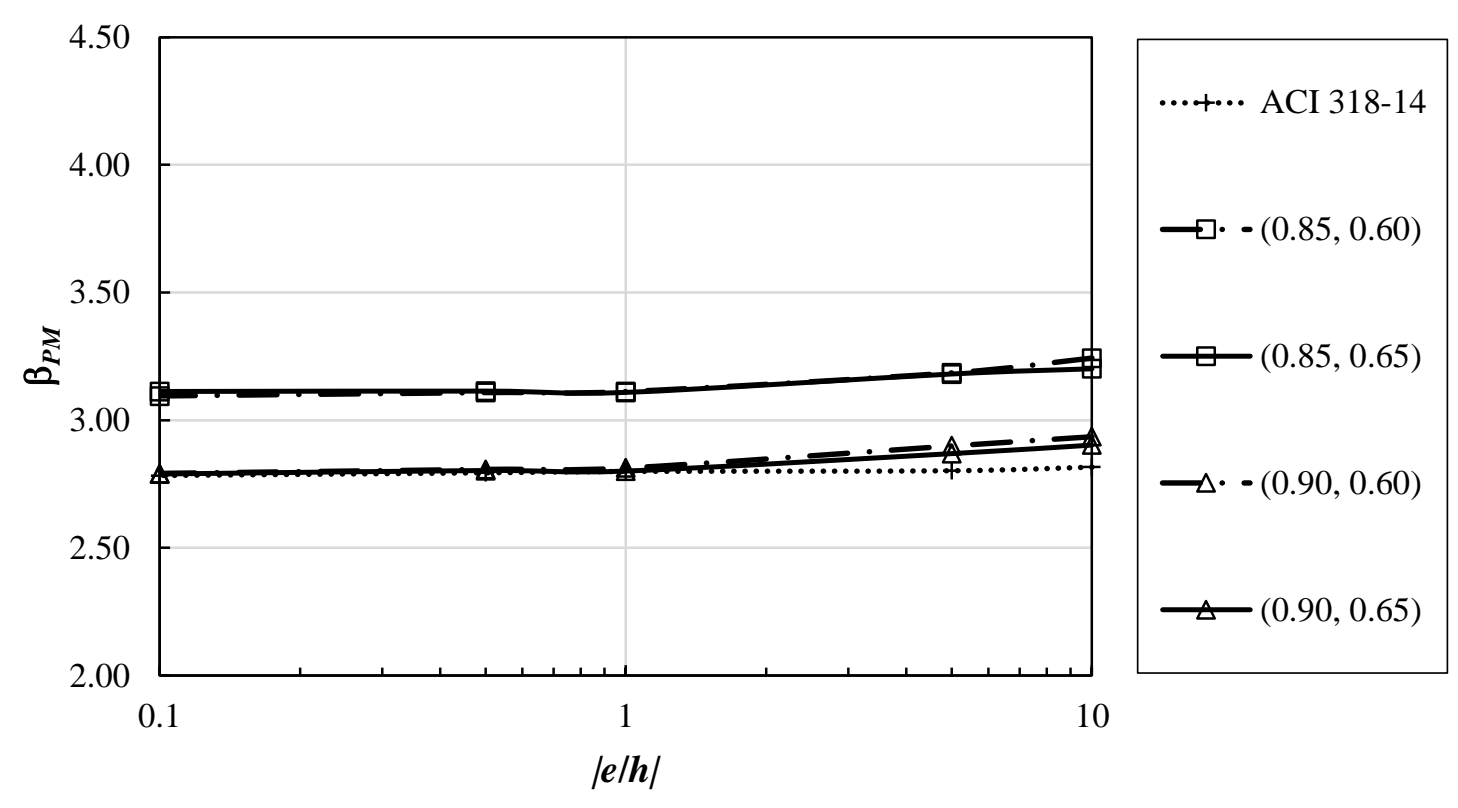

(b)

Figure C.25: Reliability indices for combined moment and axial force, $\beta_{P M}$, for Column Section 3, Property Combination 8, and $L / D=0.5$ : (a) $e / h>0$; (b) $e / h<0$ 

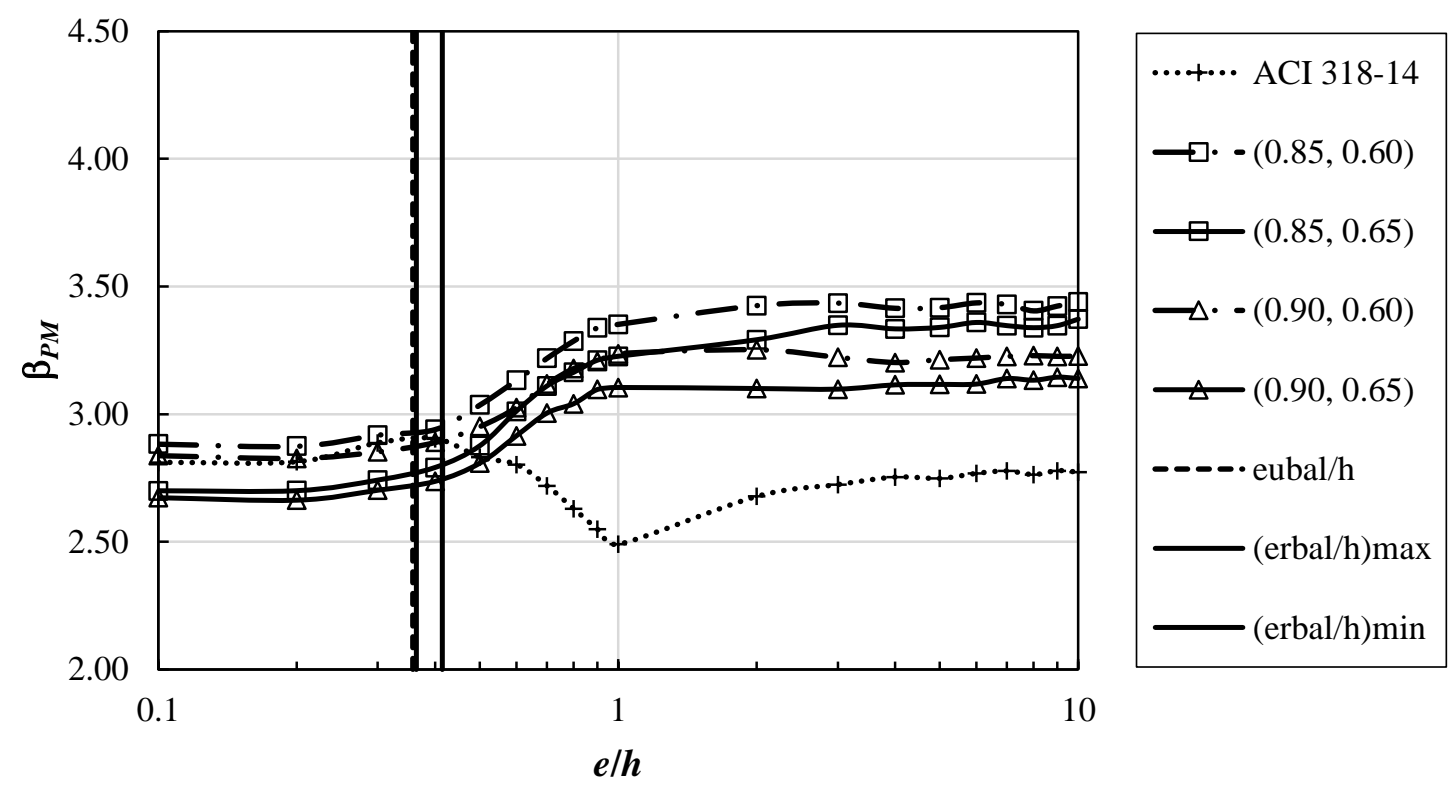

(a)
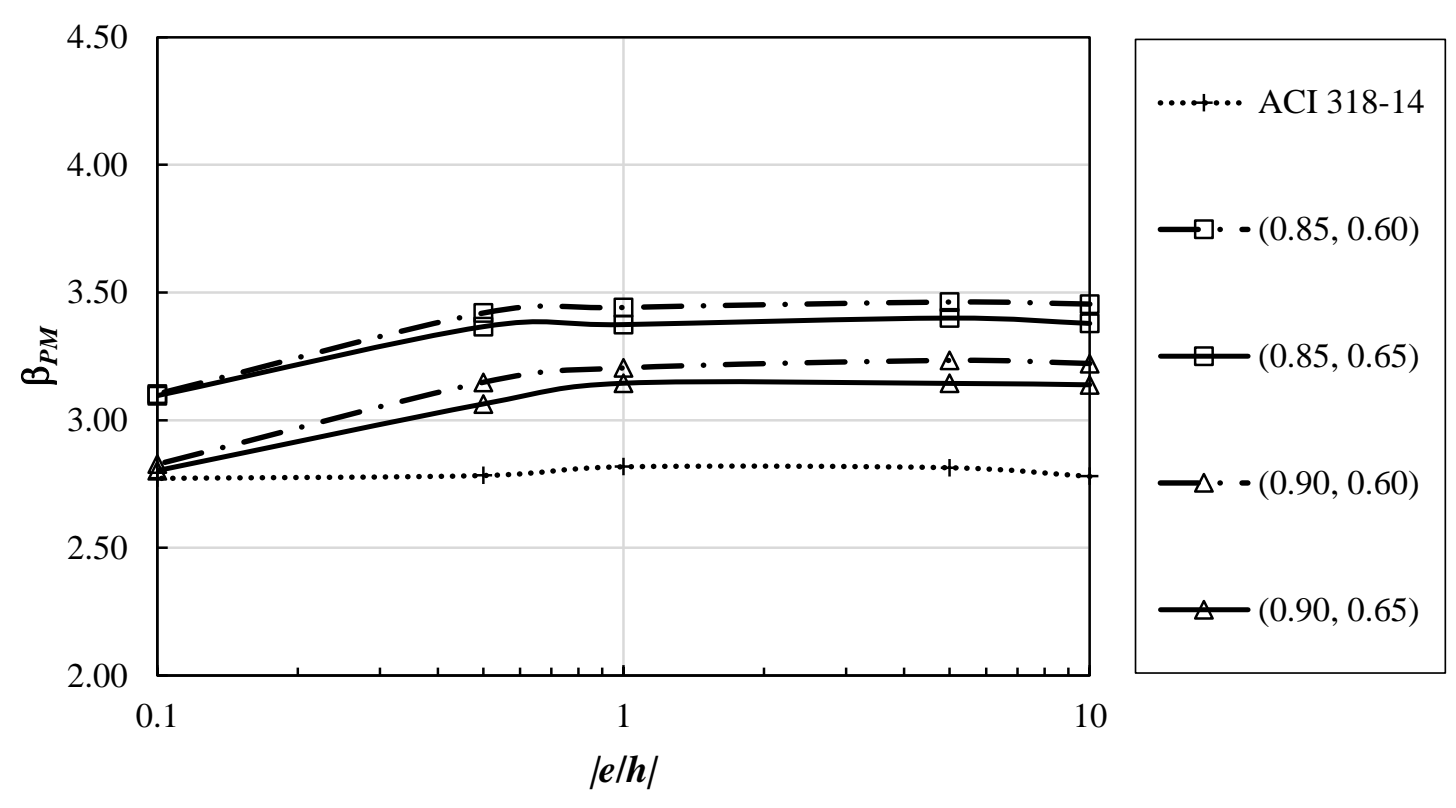

(b)

Figure C.26: Reliability indices for combined moment and axial force, $\beta_{P M}$, for Column Section 4, Property Combination 1, and $L / D=0.5$ : (a) $e / h>0$; (b) $e / h<0$ 

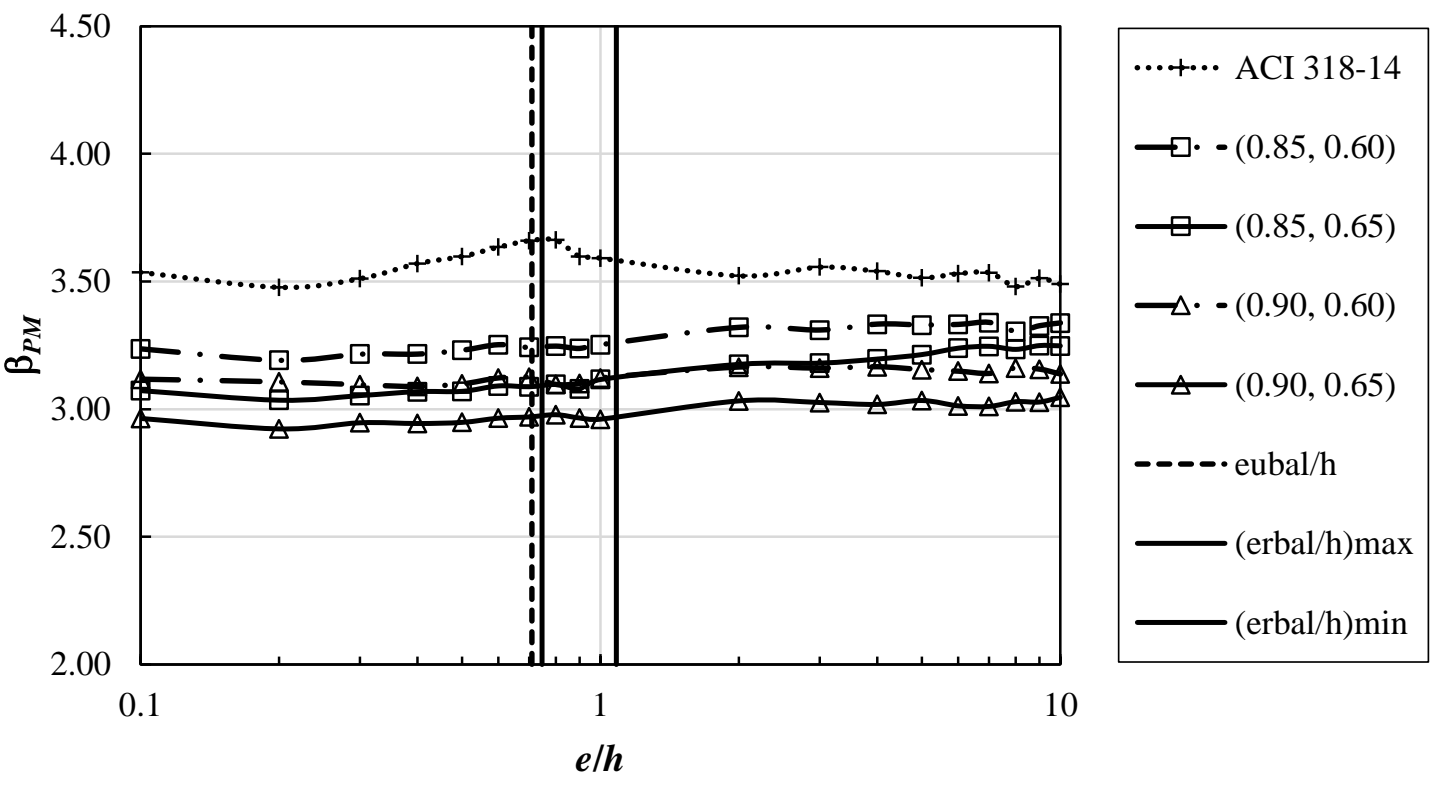

(a)
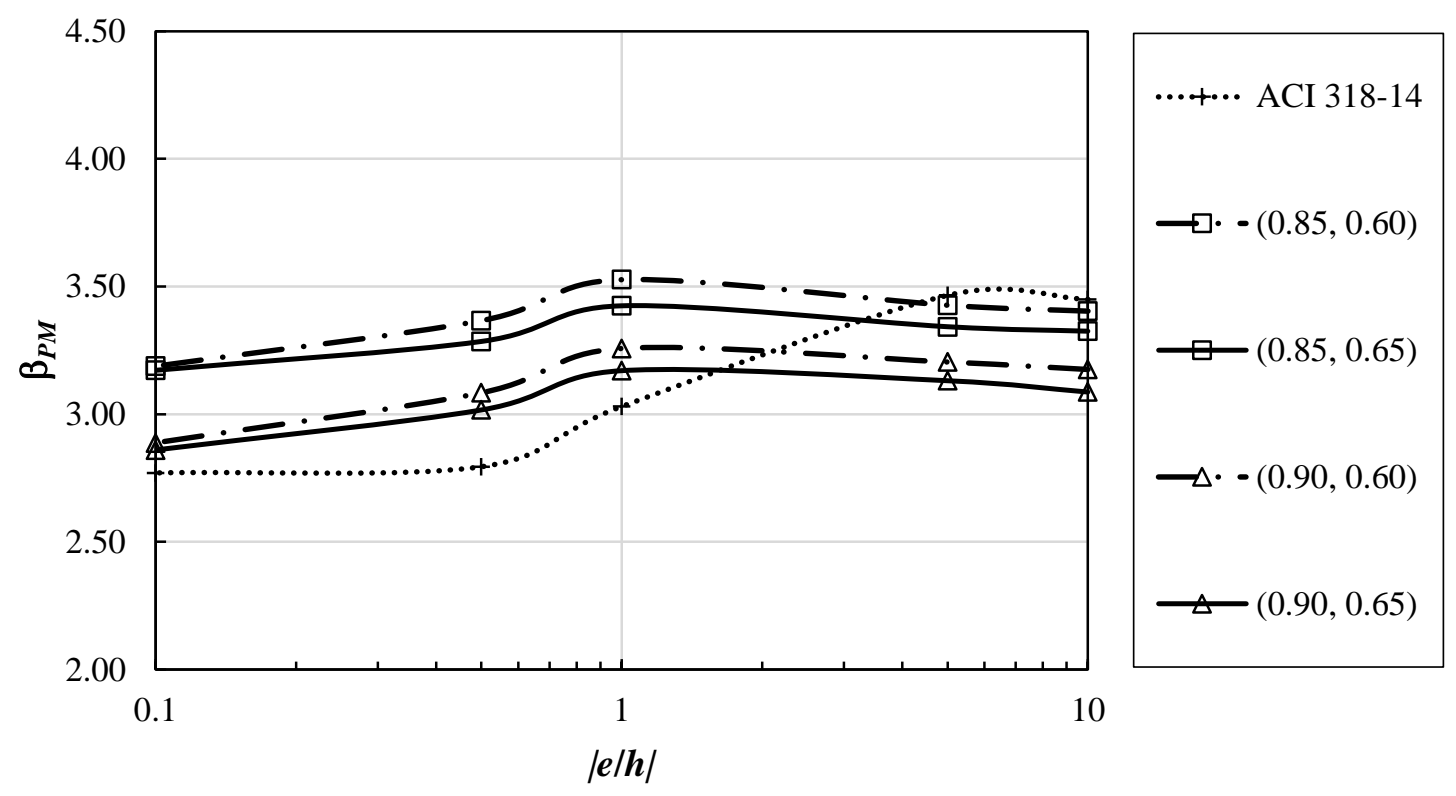

(b)

Figure C.27: Reliability indices for combined moment and axial force, $\beta_{P M}$, for Column Section 4, Property Combination 2, and $L / D=0.5$ : (a) $e / h>0$; (b) $e / h<0$ 

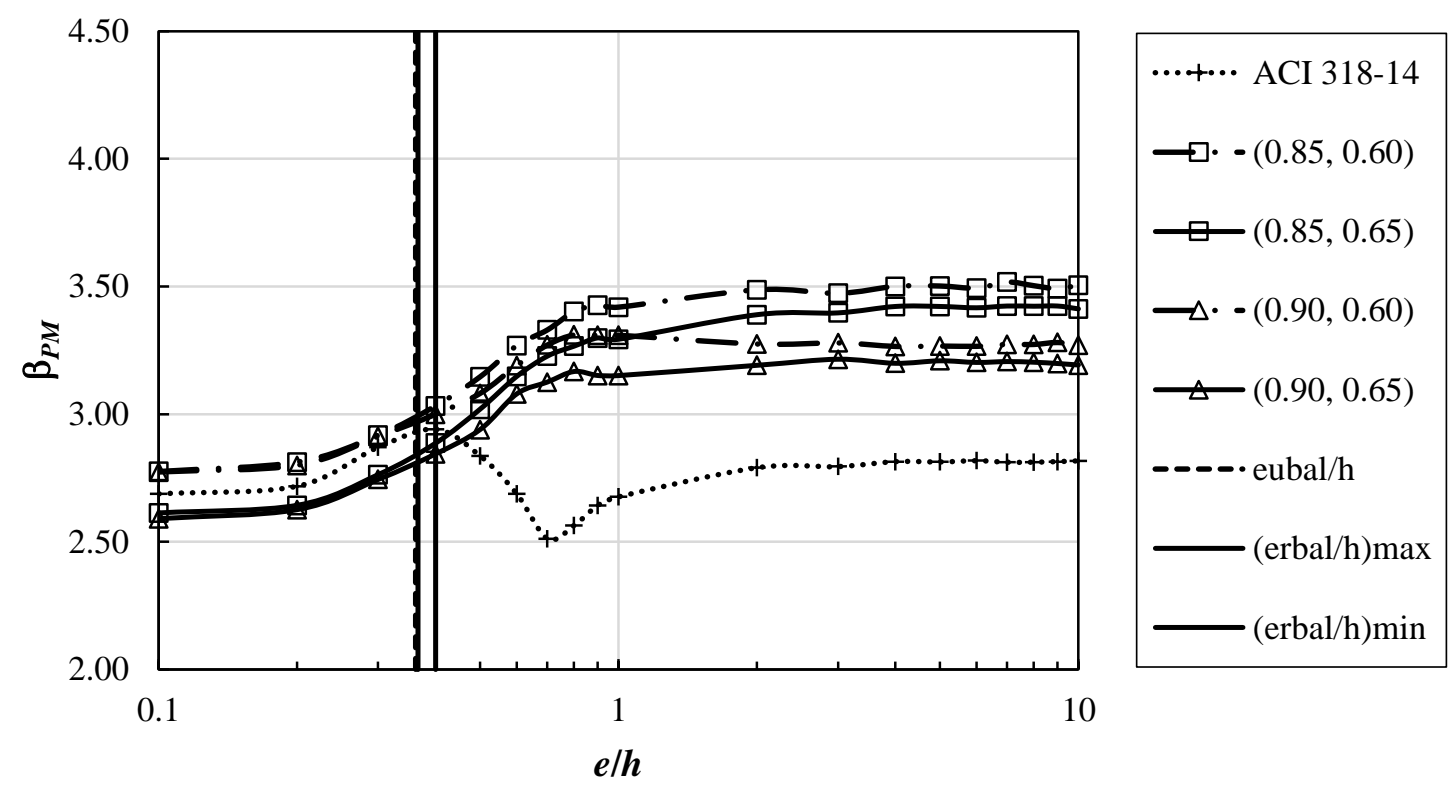

(a)
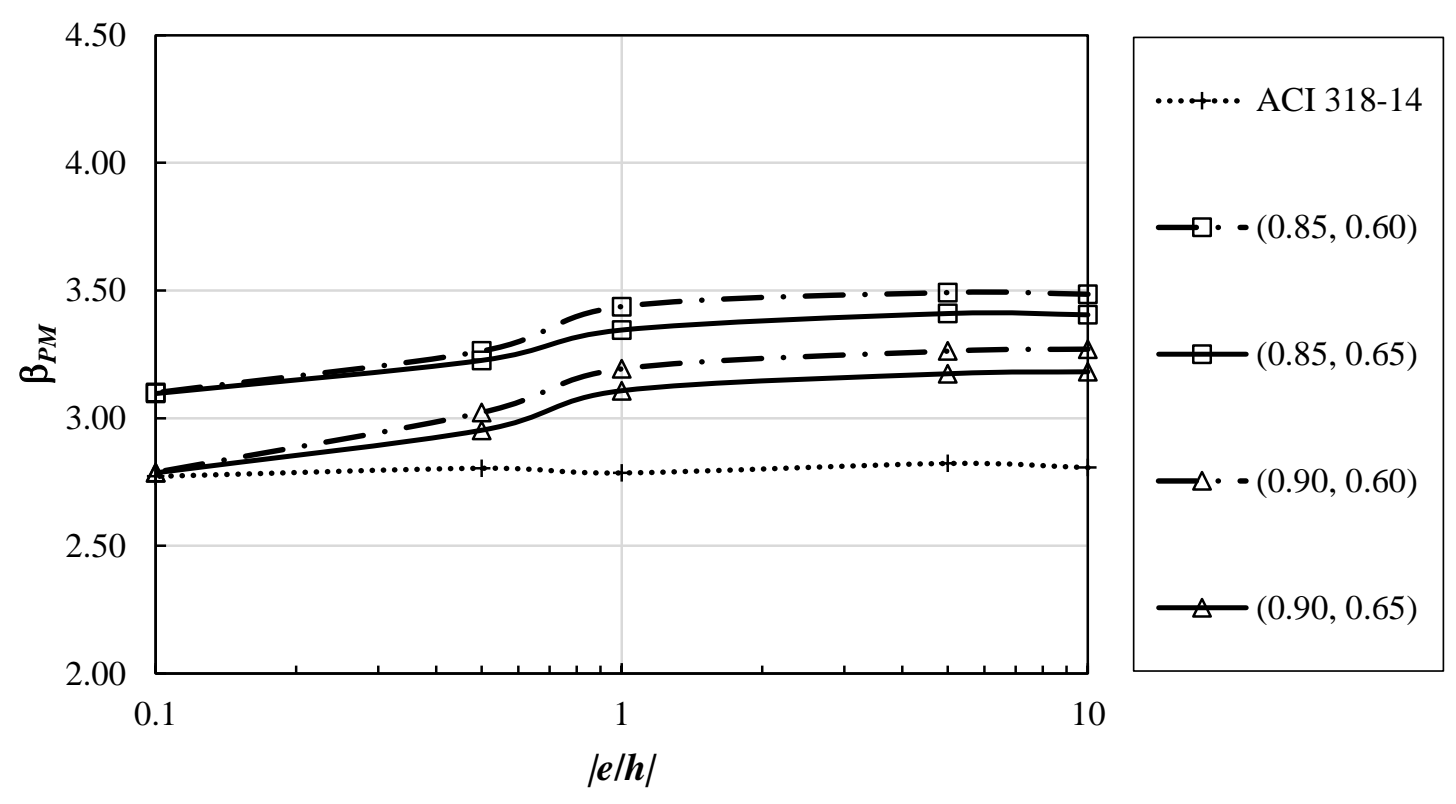

(b)

Figure C.28: Reliability indices for combined moment and axial force, $\beta_{P M}$, for Column Section 4, Property Combination 3, and $L / D=0.5$ : (a) $e / h>0$; (b) $e / h<0$ 

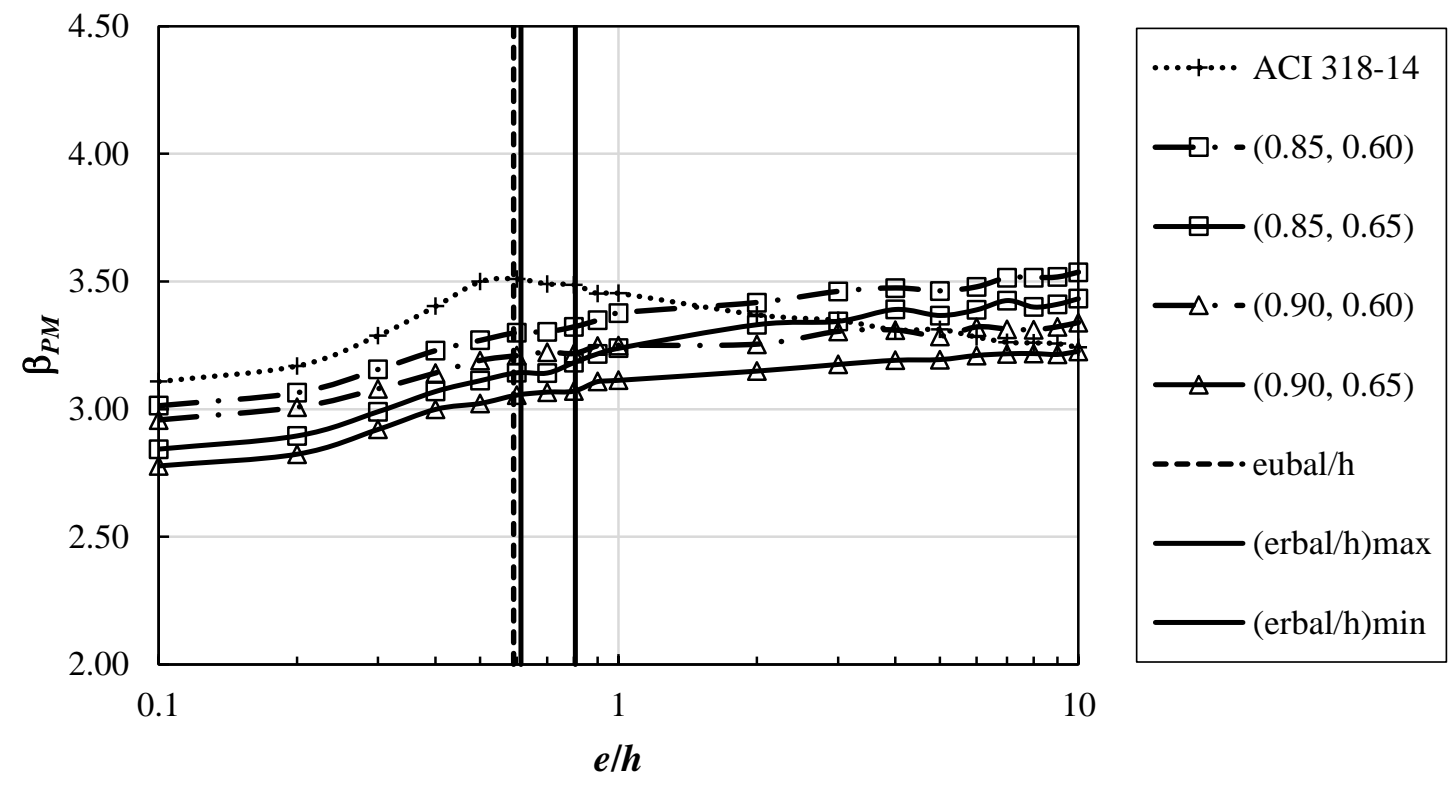

(a)
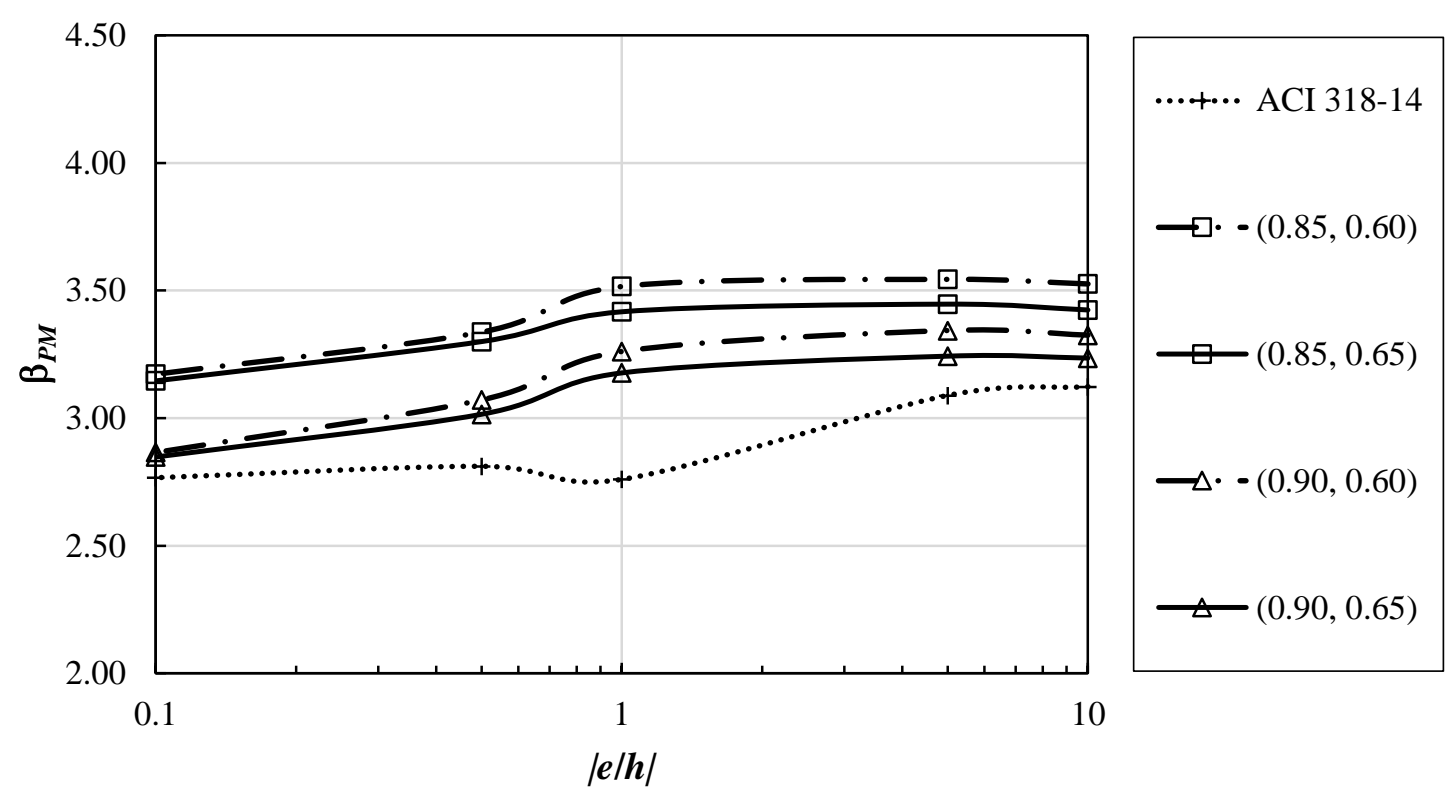

(b)

Figure C.29: Reliability indices for combined moment and axial force, $\beta_{P M}$, for Column Section 4, Property Combination 4, and $L / D=0.5$ : (a) $e / h>0$; (b) $e / h<0$ 

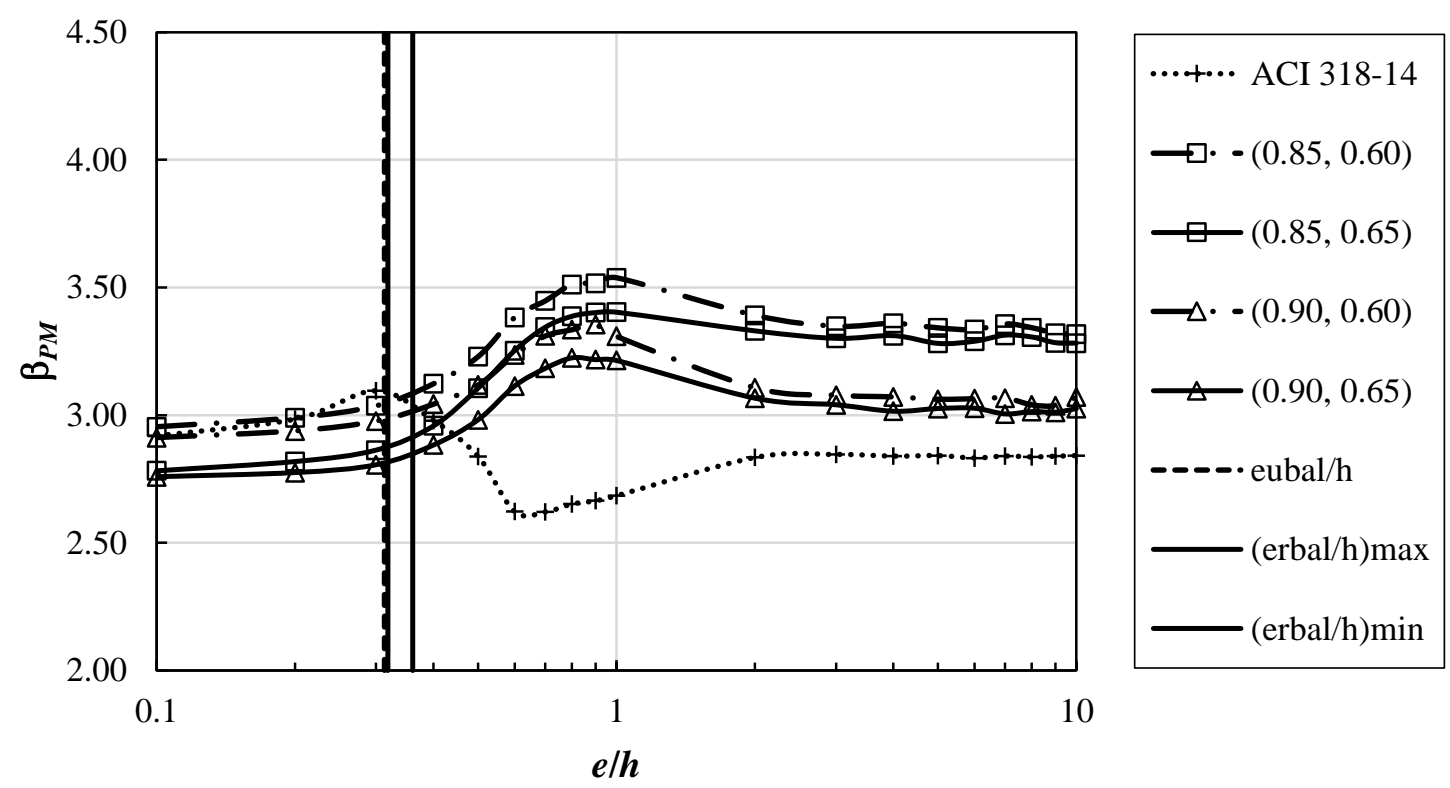

(a)
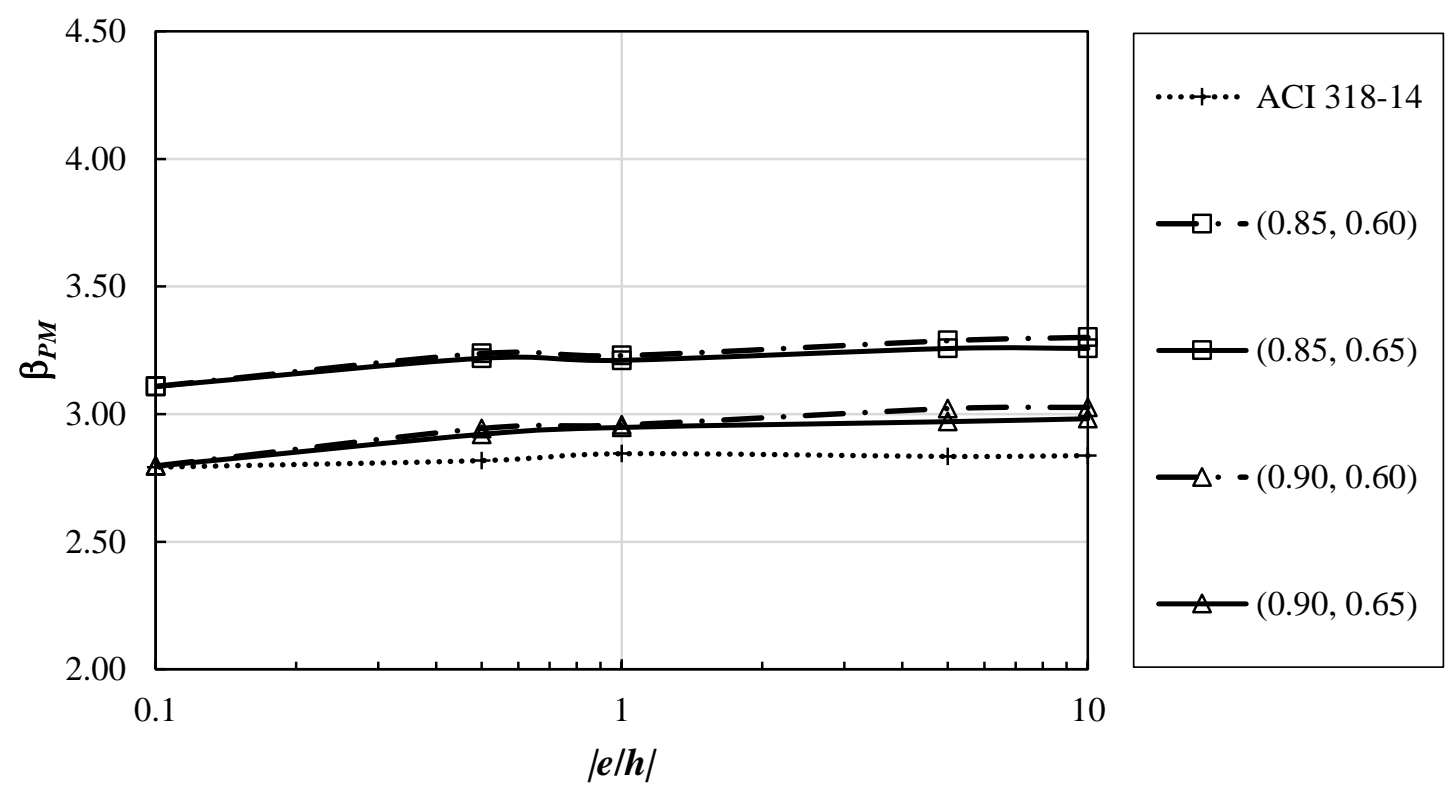

(b)

Figure C.30: Reliability indices for combined moment and axial force, $\beta_{P M}$, for Column Section 4, Property Combination 5, and $L / D=0.5$ : (a) $e / h>0$; (b) $e / h<0$ 

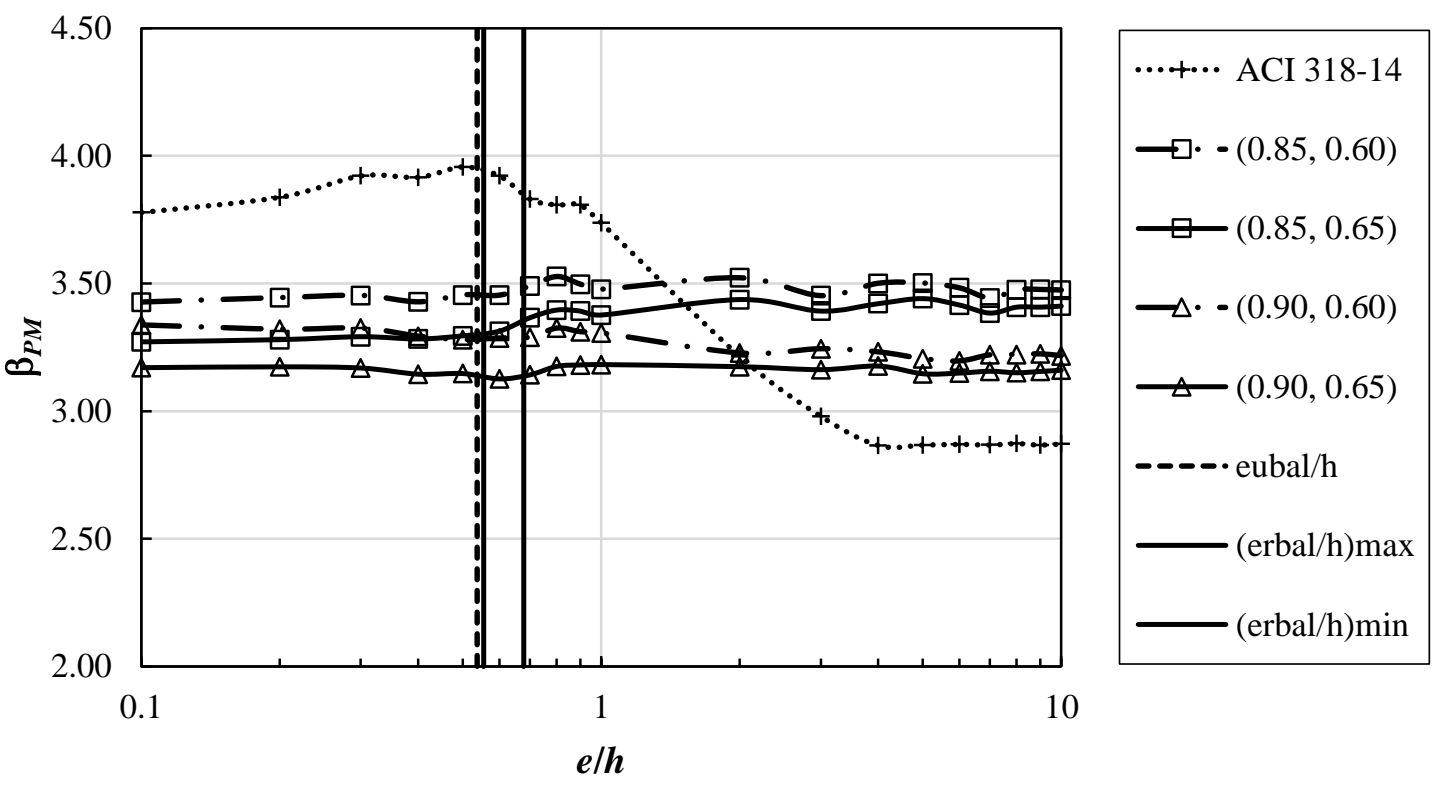

(a)
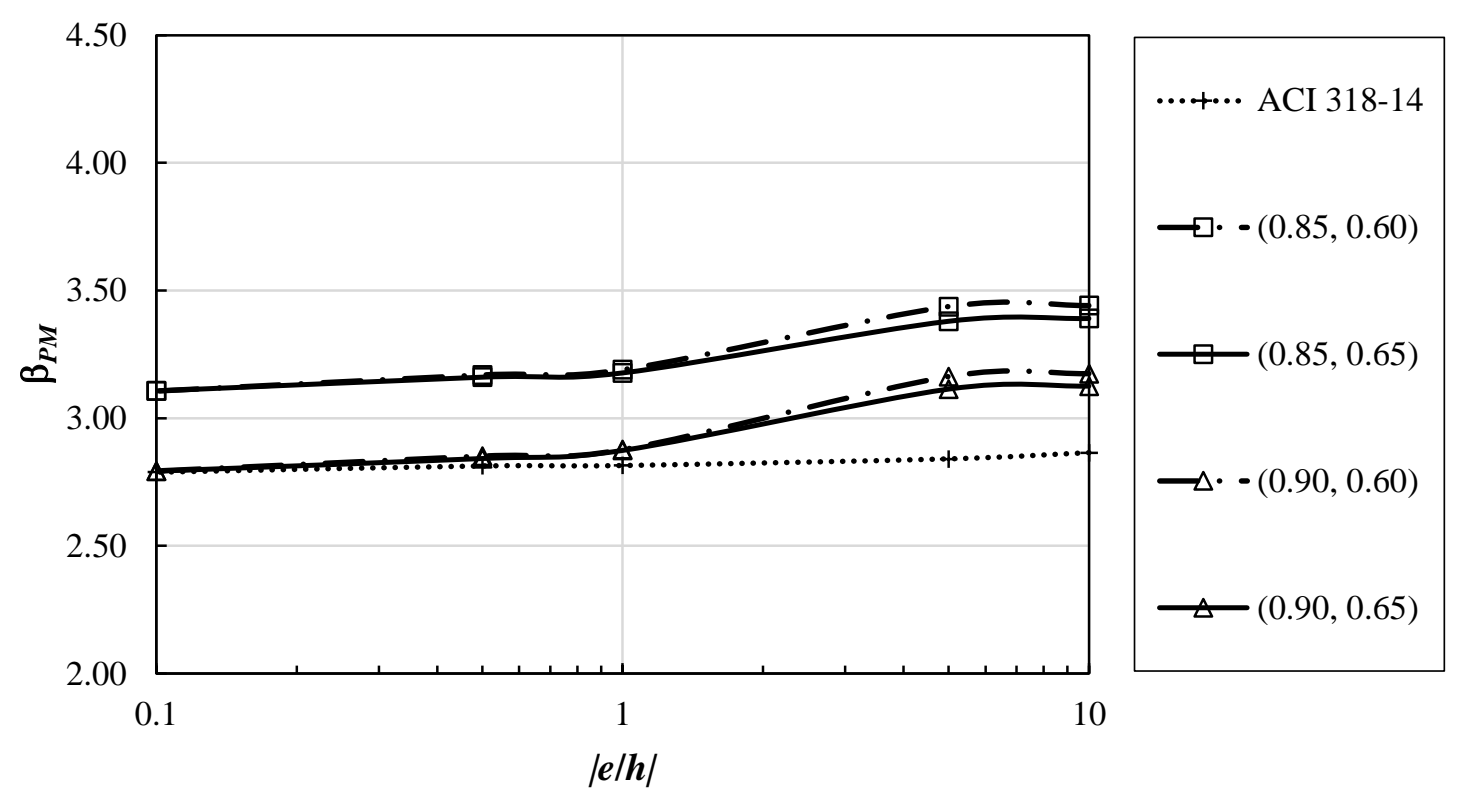

(b)

Figure C.31: Reliability indices for combined moment and axial force, $\beta_{P M}$, for Column Section 4, Property Combination 6, and $L / D=0.5$ : (a) $e / h>0$; (b) $e / h<0$ 

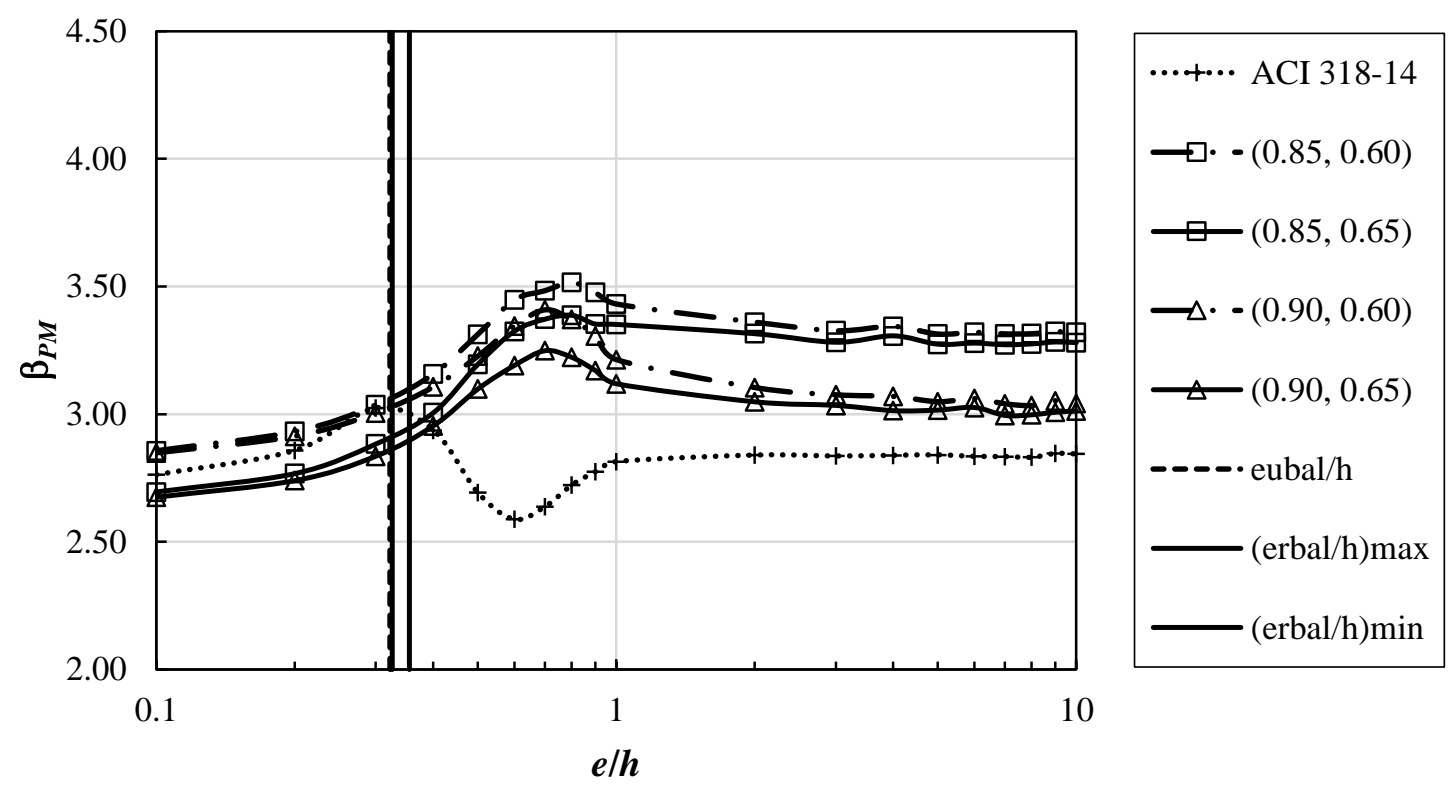

(a)
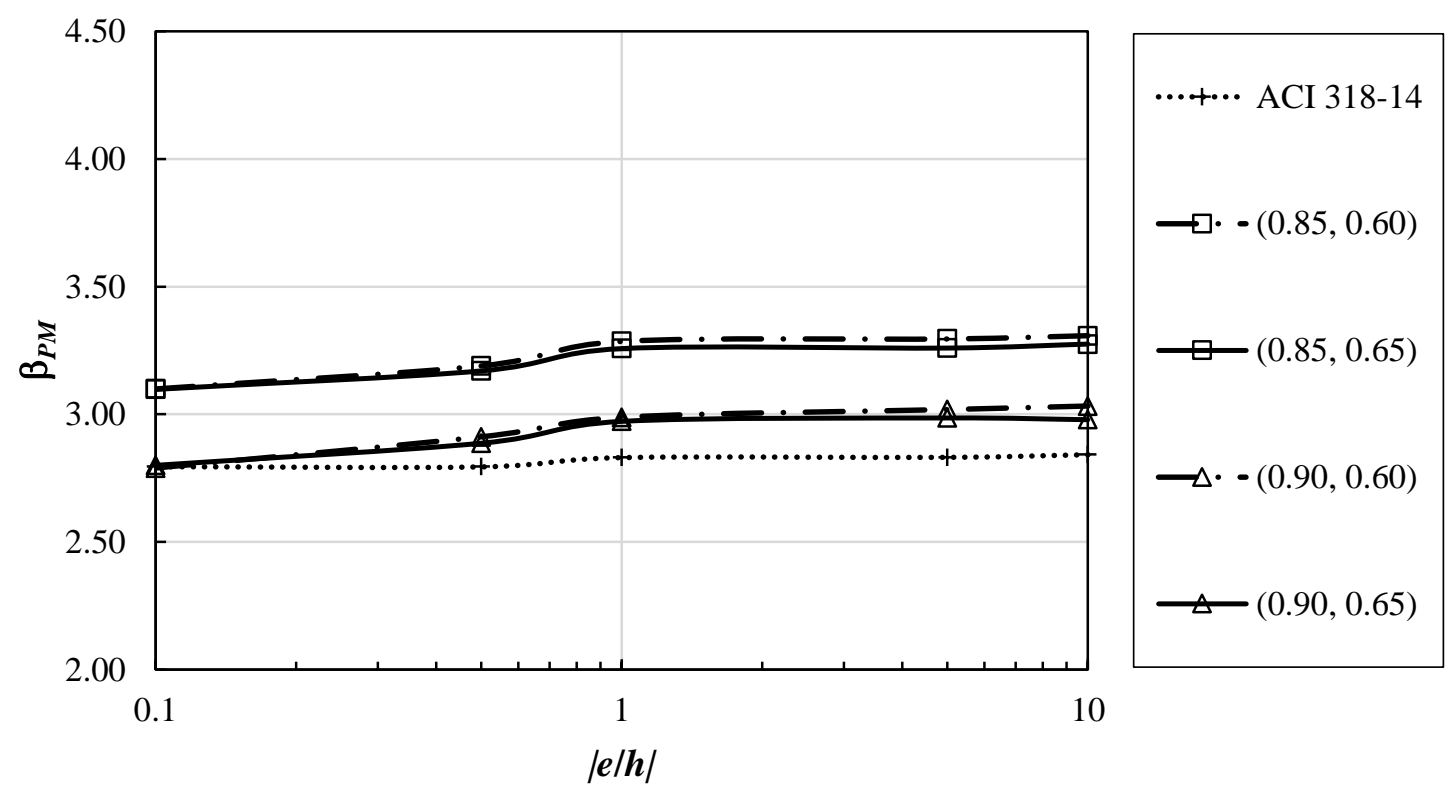

(b)

Figure C.32: Reliability indices for combined moment and axial force, $\beta_{P M}$, for Column Section 4, Property Combination 7, and $L / D=0.5$ : (a) $e / h>0$; (b) $e / h<0$ 

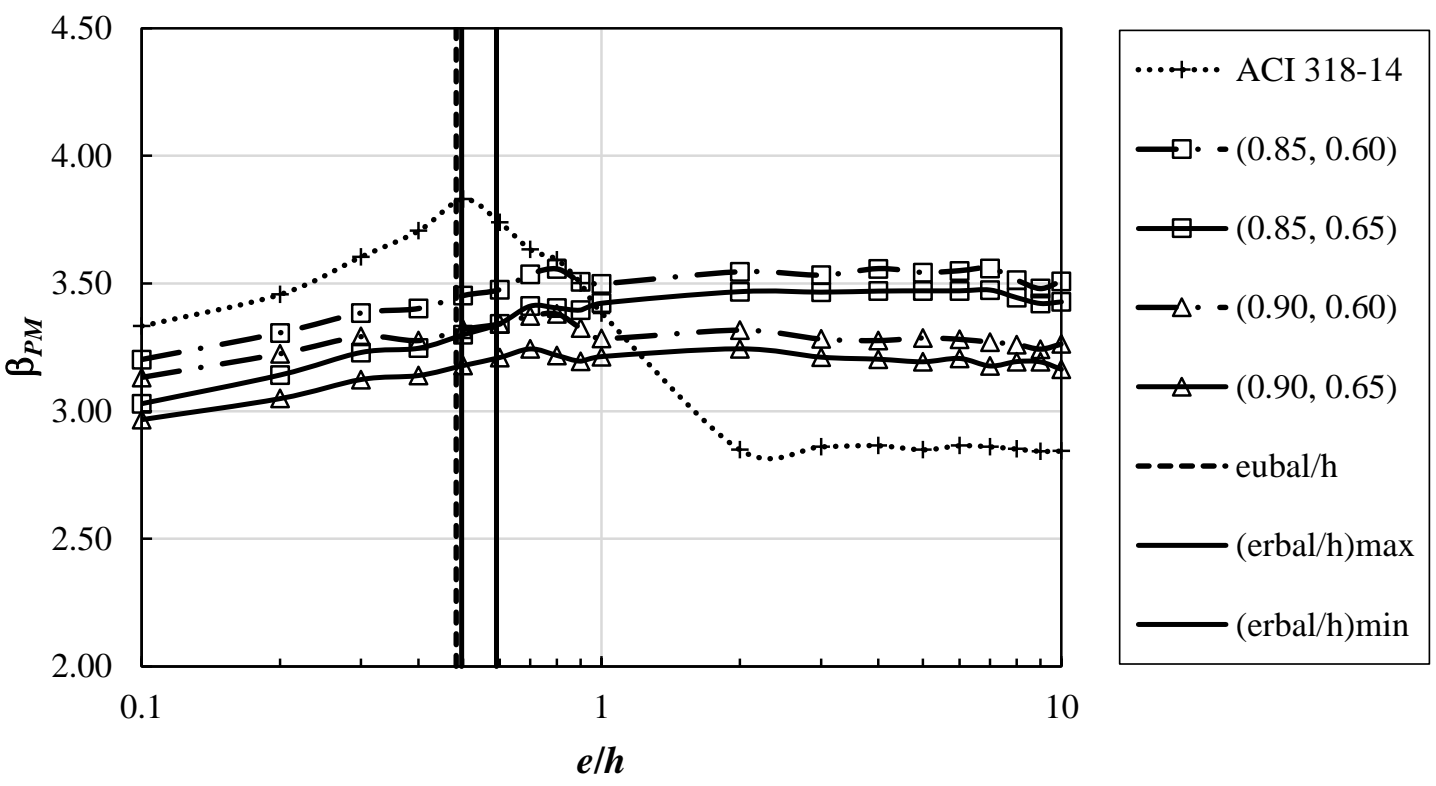

(a)
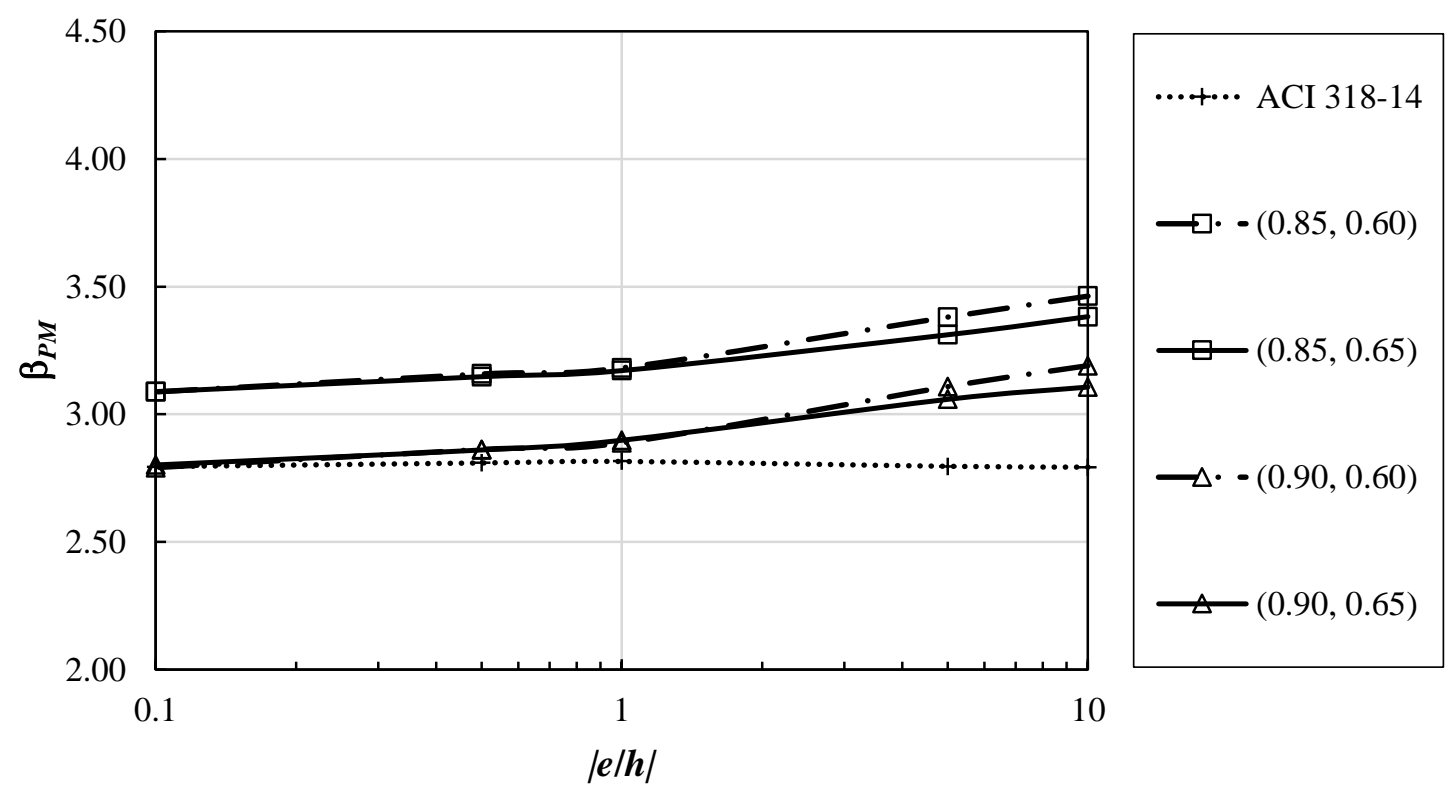

(b)

Figure C.33: Reliability indices for combined moment and axial force, $\beta_{P M}$, for Column Section 4, Property Combination 8, and $L / D=0.5$ : (a) $e / h>0$; (b) $e / h<0$ 

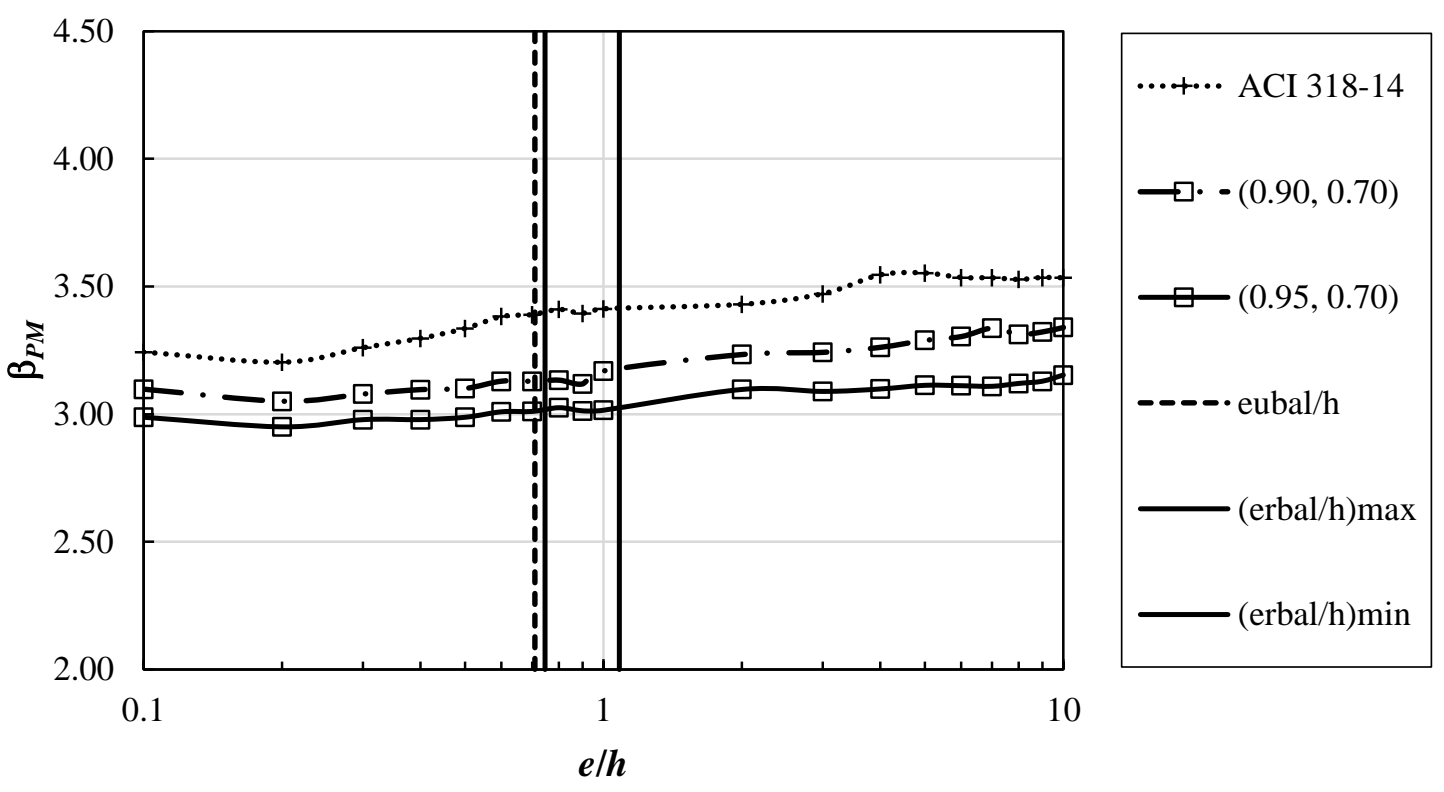

(a)

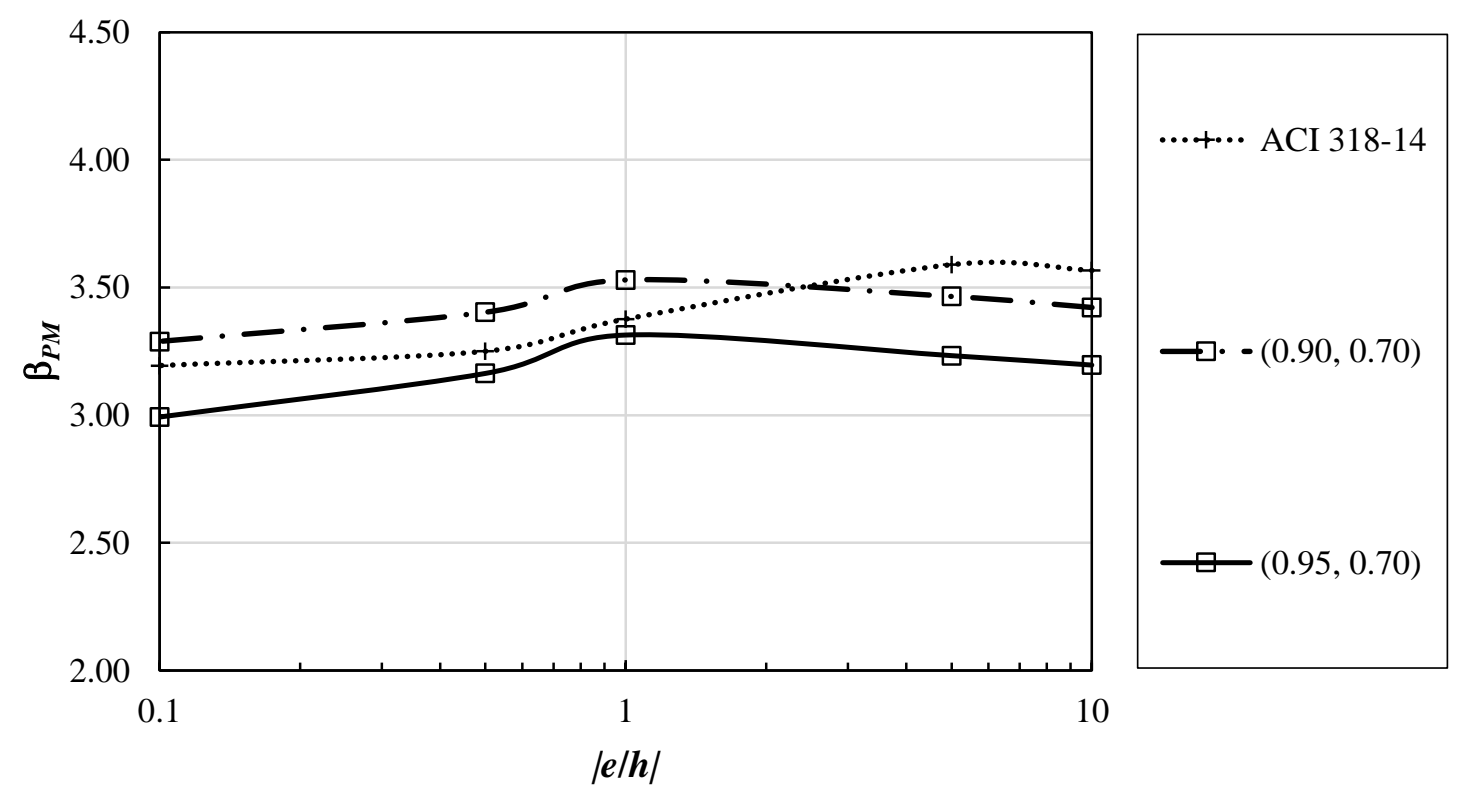

(b)

Figure C.34: Reliability indices for combined moment and axial force, $\beta_{P M}$, for Column Section 5, Property Combination 2, and $L / D=0.5$ : (a) $e / h>0$; (b) $e / h<0$ 

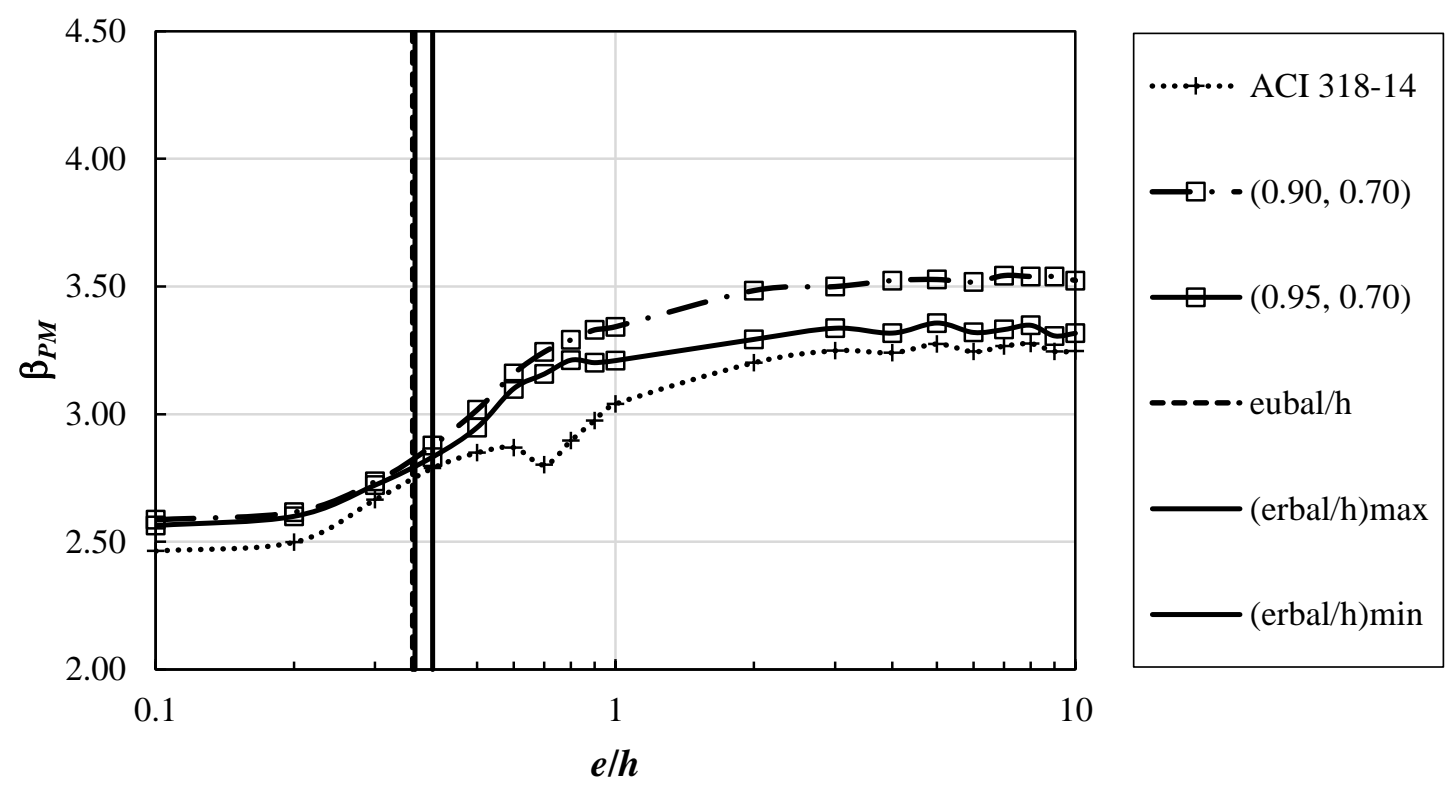

(a)

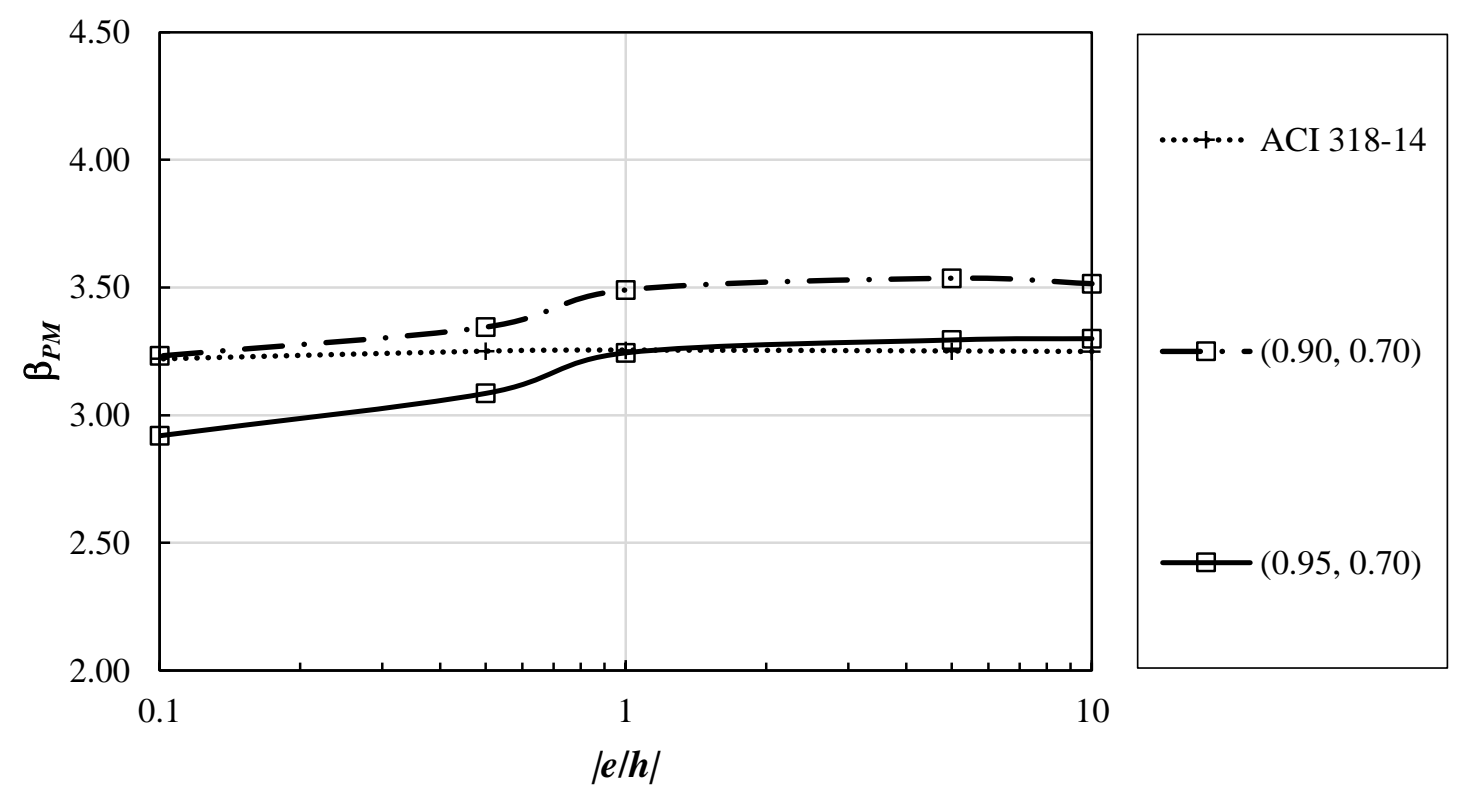

(b)

Figure C.35: Reliability indices for combined moment and axial force, $\beta_{P M}$, for Column Section 5, Property Combination 3, and $L / D=0.5$ : (a) $e / h>0$; (b) $e / h<0$ 

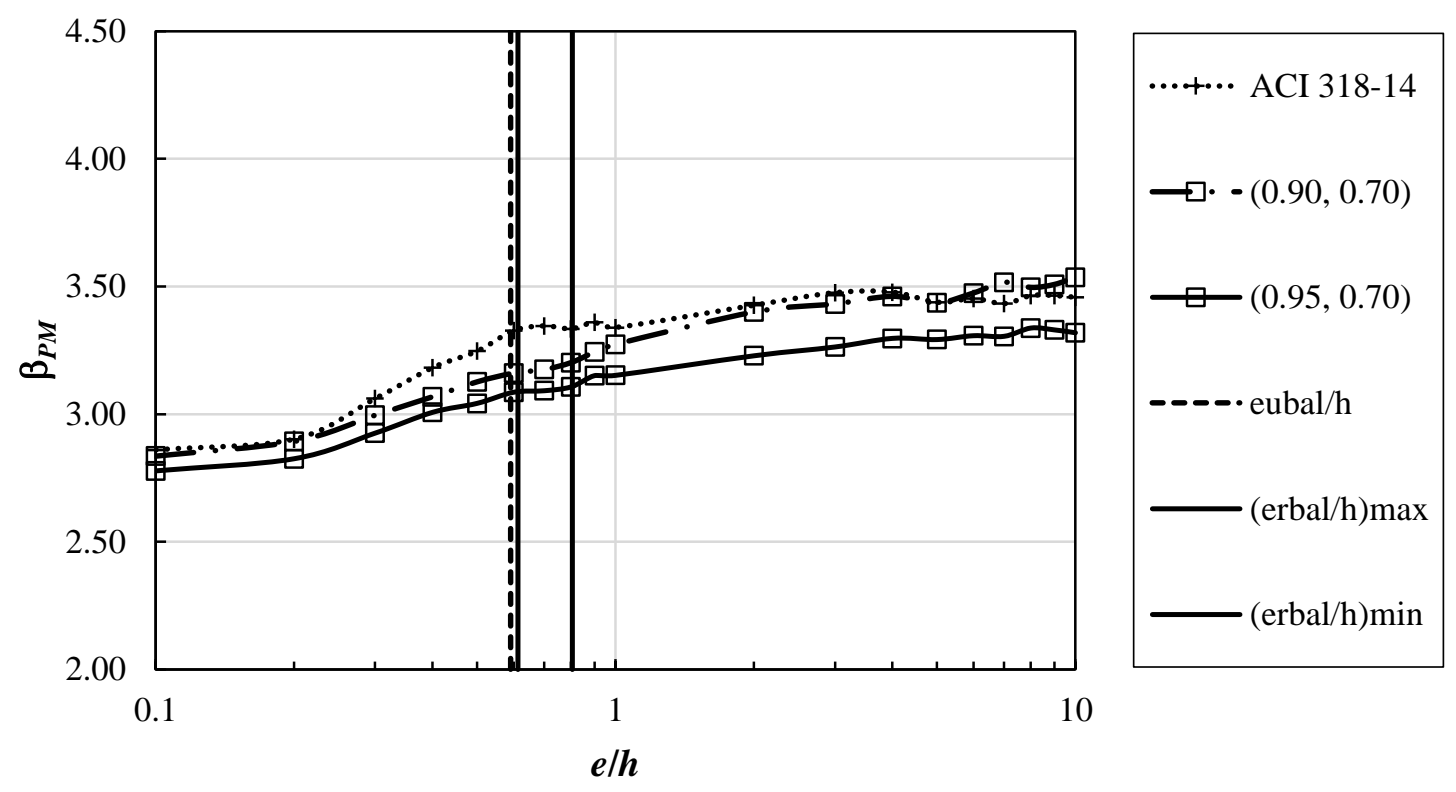

(a)

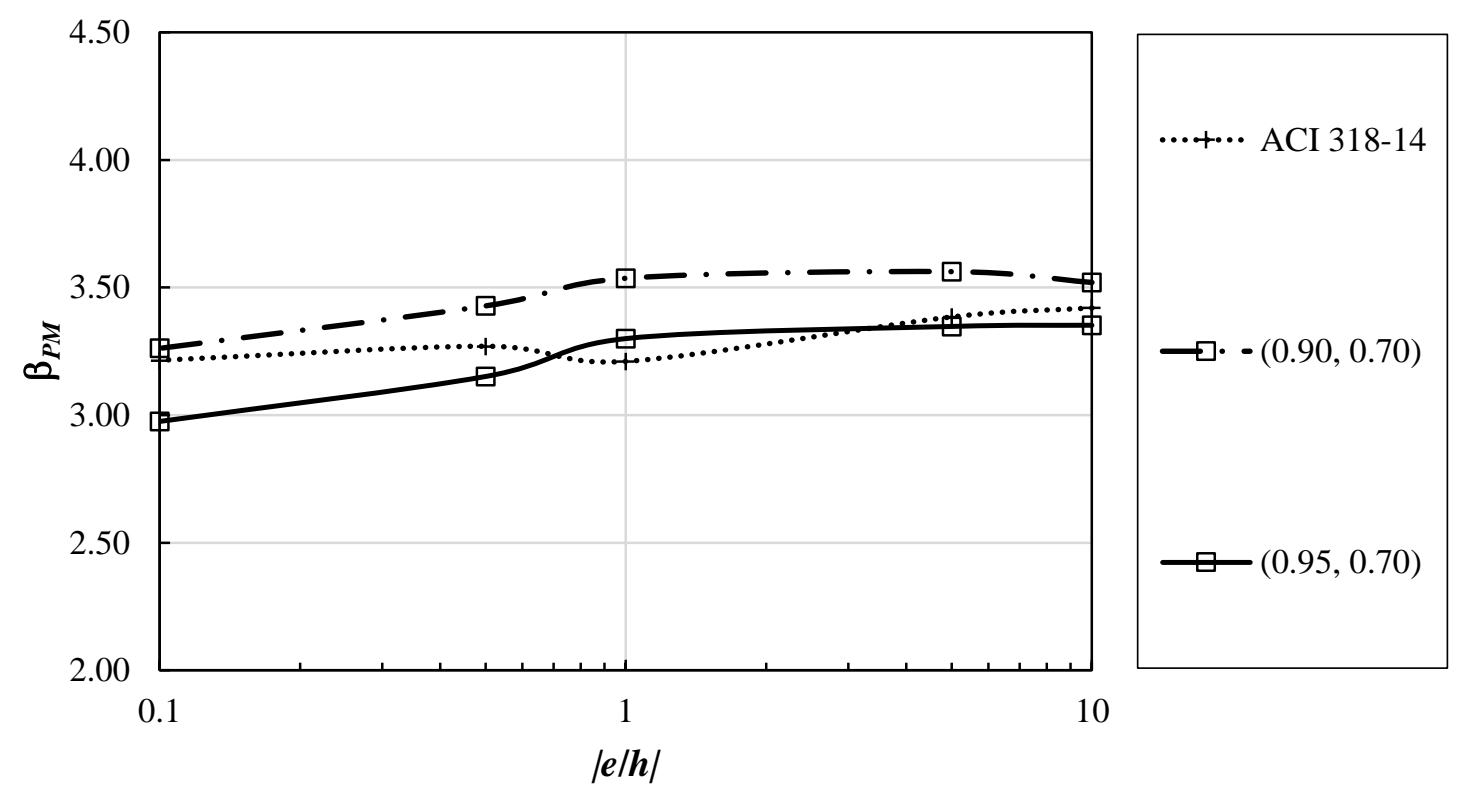

(b)

Figure C.36: Reliability indices for combined moment and axial force, $\beta_{P M}$, for Column Section 5, Property Combination 4, and $L / D=0.5$ : (a) $e / h>0$; (b) $e / h<0$ 

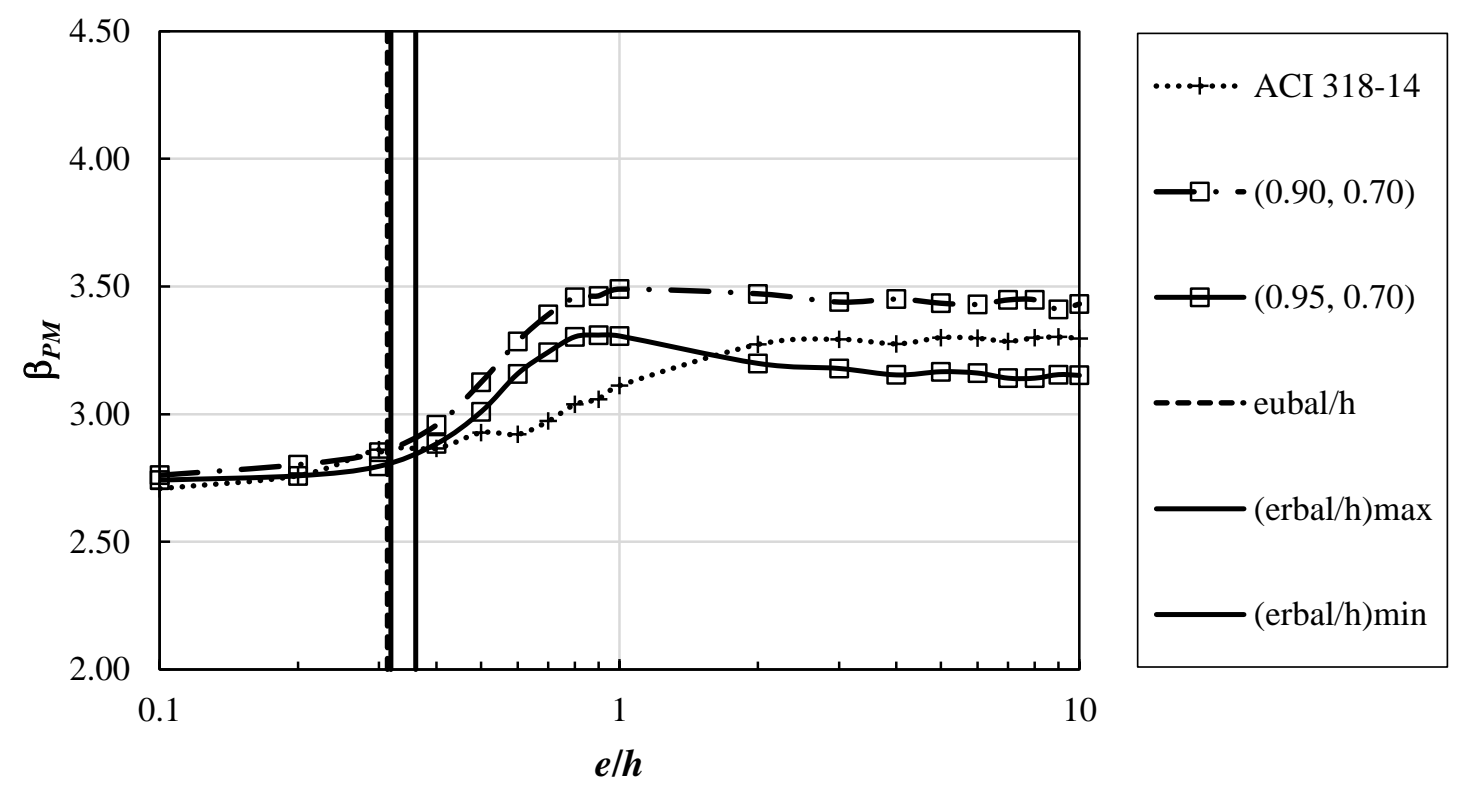

(a)

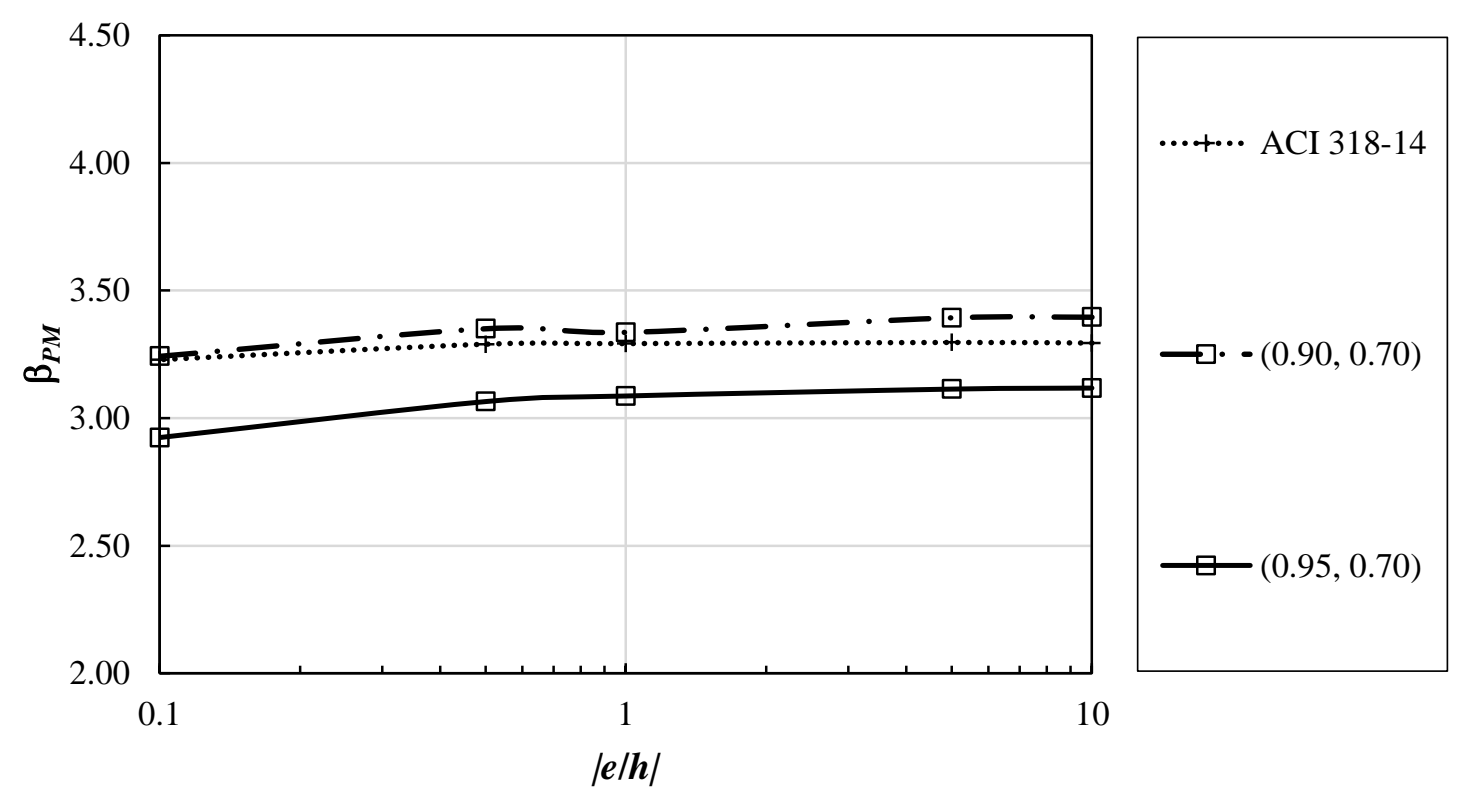

(b)

Figure C.37: Reliability indices for combined moment and axial force, $\beta_{P M}$, for Column Section 5, Property Combination 5, and $L / D=0.5$ : (a) $e / h>0$; (b) $e / h<0$ 

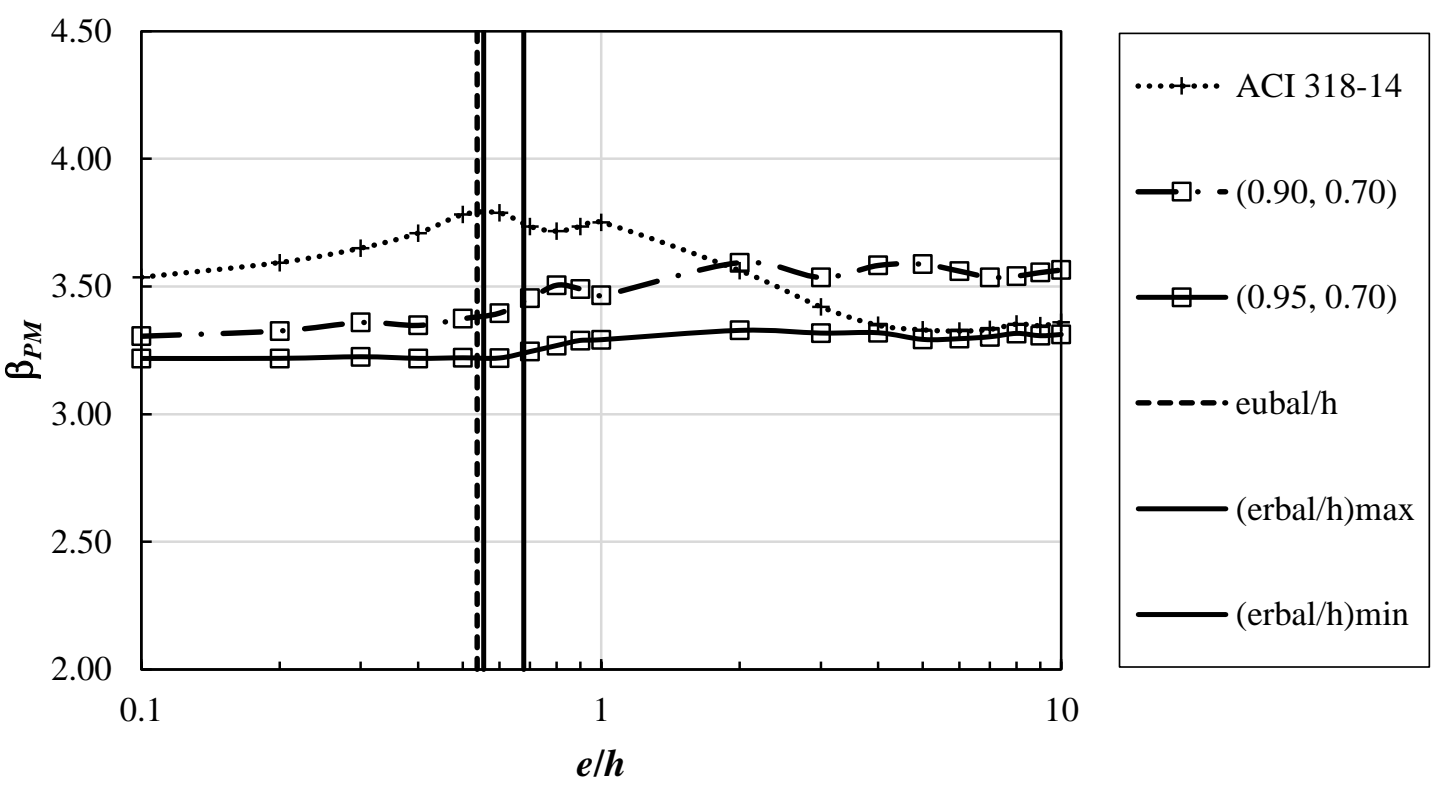

(a)

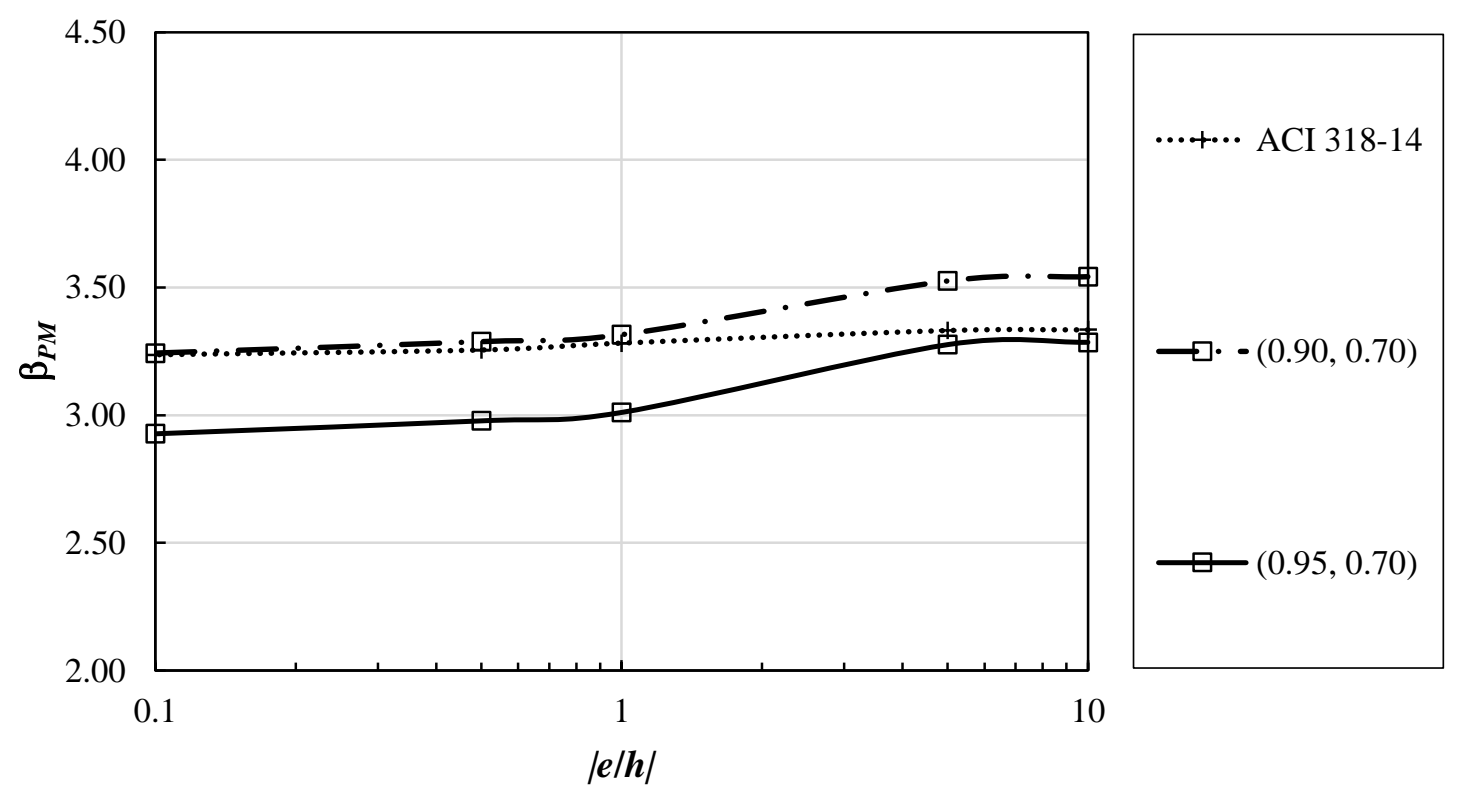

(b)

Figure C.38: Reliability indices for combined moment and axial force, $\beta_{P M}$, for Column Section 5, Property Combination 6, and $L / D=0.5$ : (a) $e / h>0$; (b) $e / h<0$ 

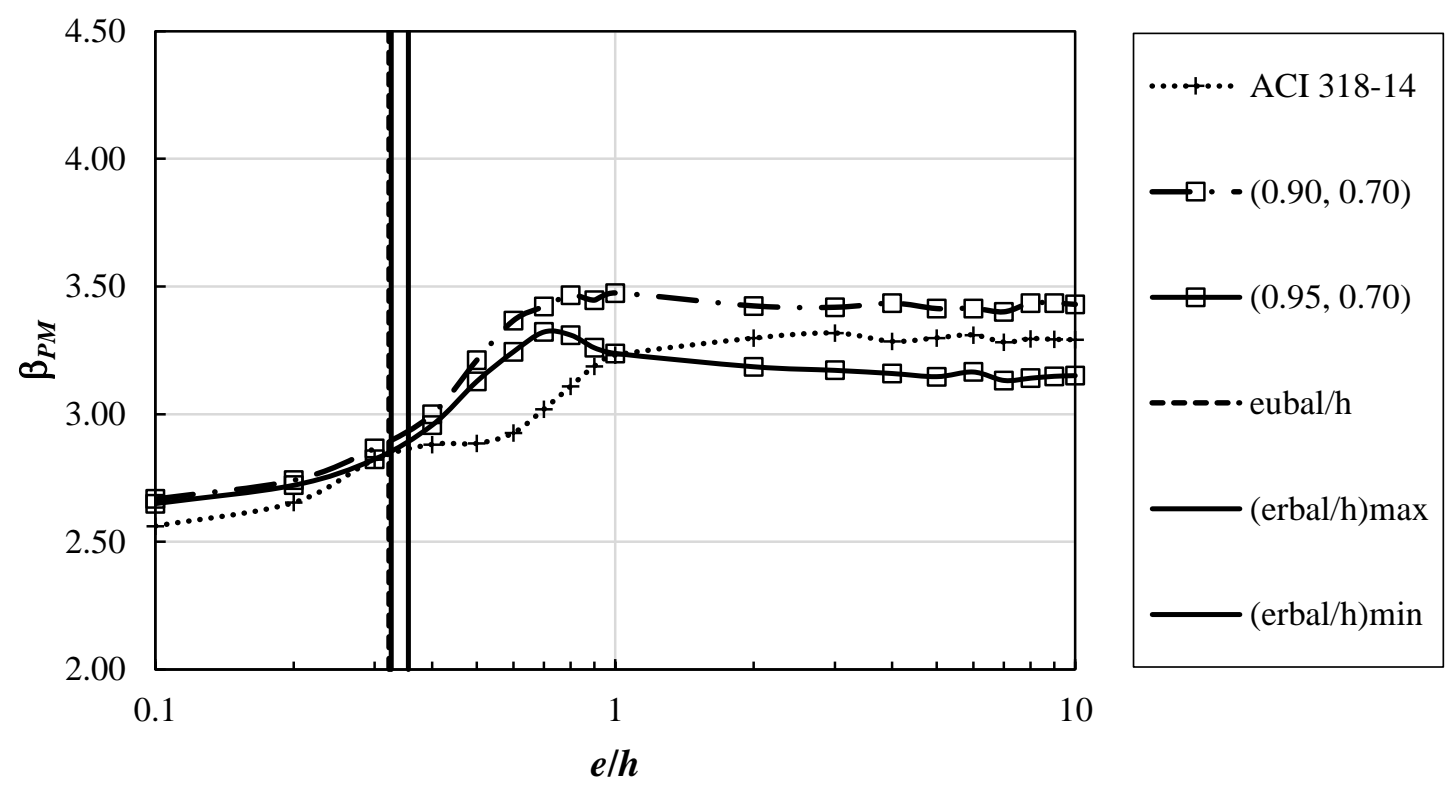

(a)
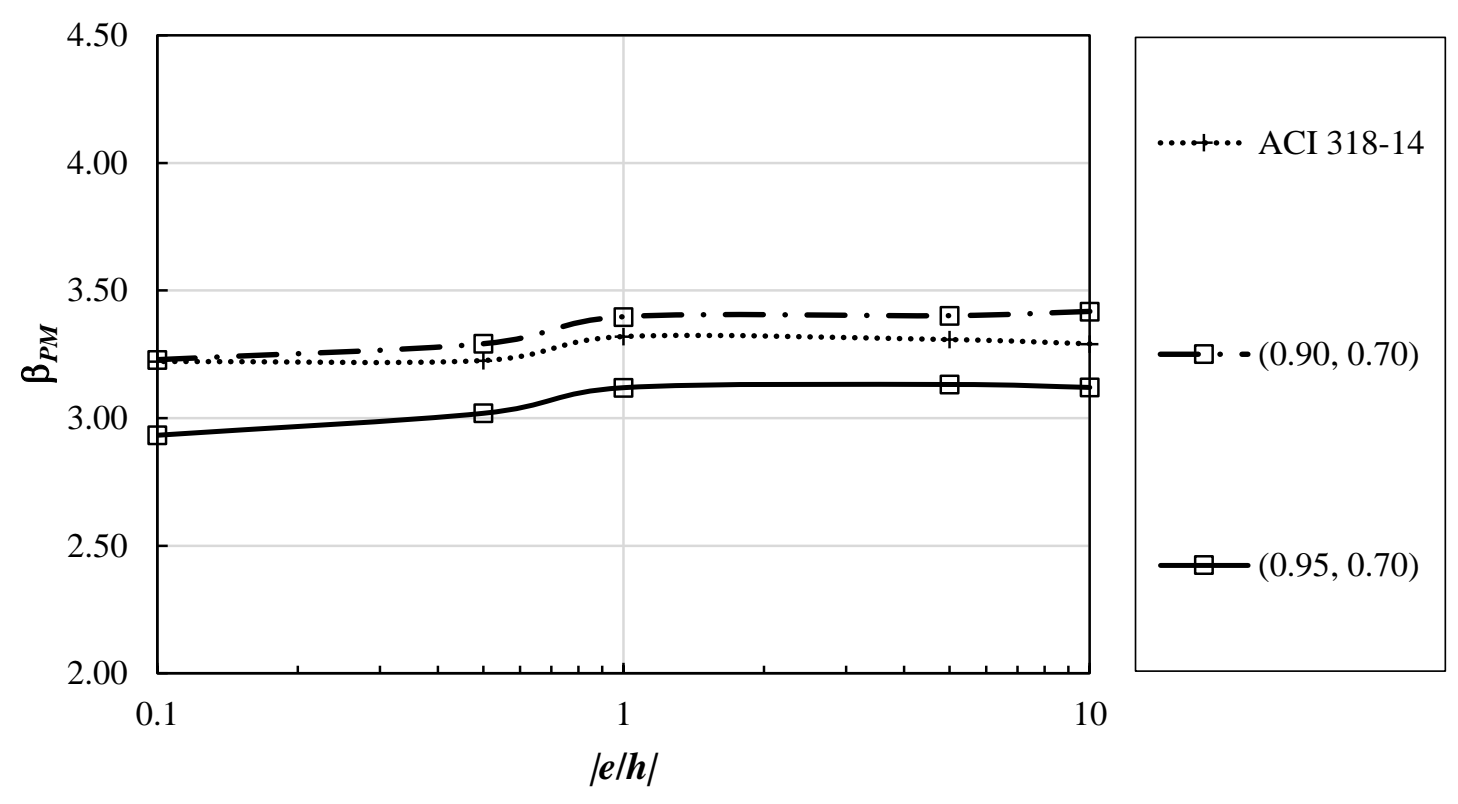

(b)

Figure C.39: Reliability indices for combined moment and axial force, $\beta_{P M}$, for Column Section 5, Property Combination 7, and $L / D=0.5$ : (a) $e / h>0$; (b) $e / h<0$ 

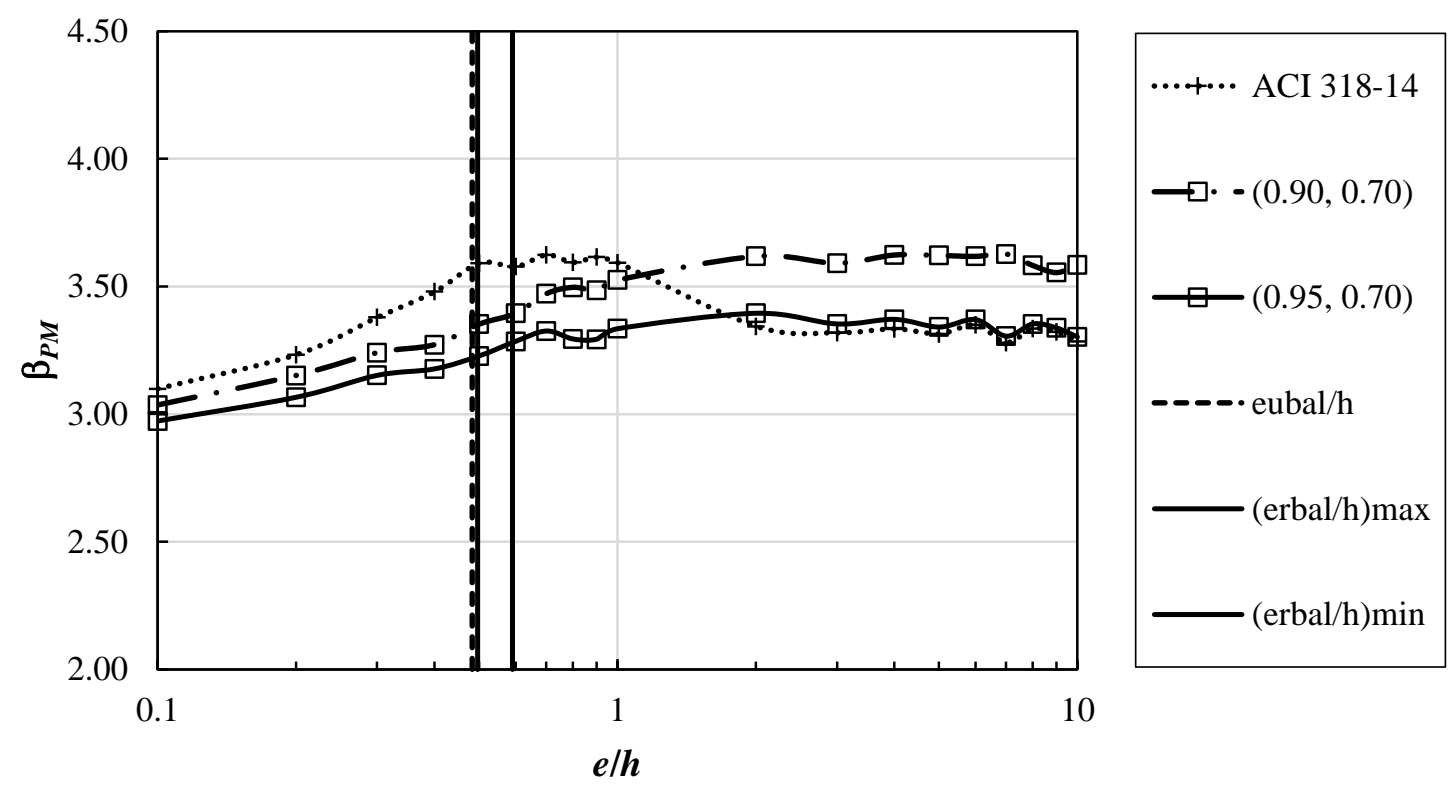

(a)

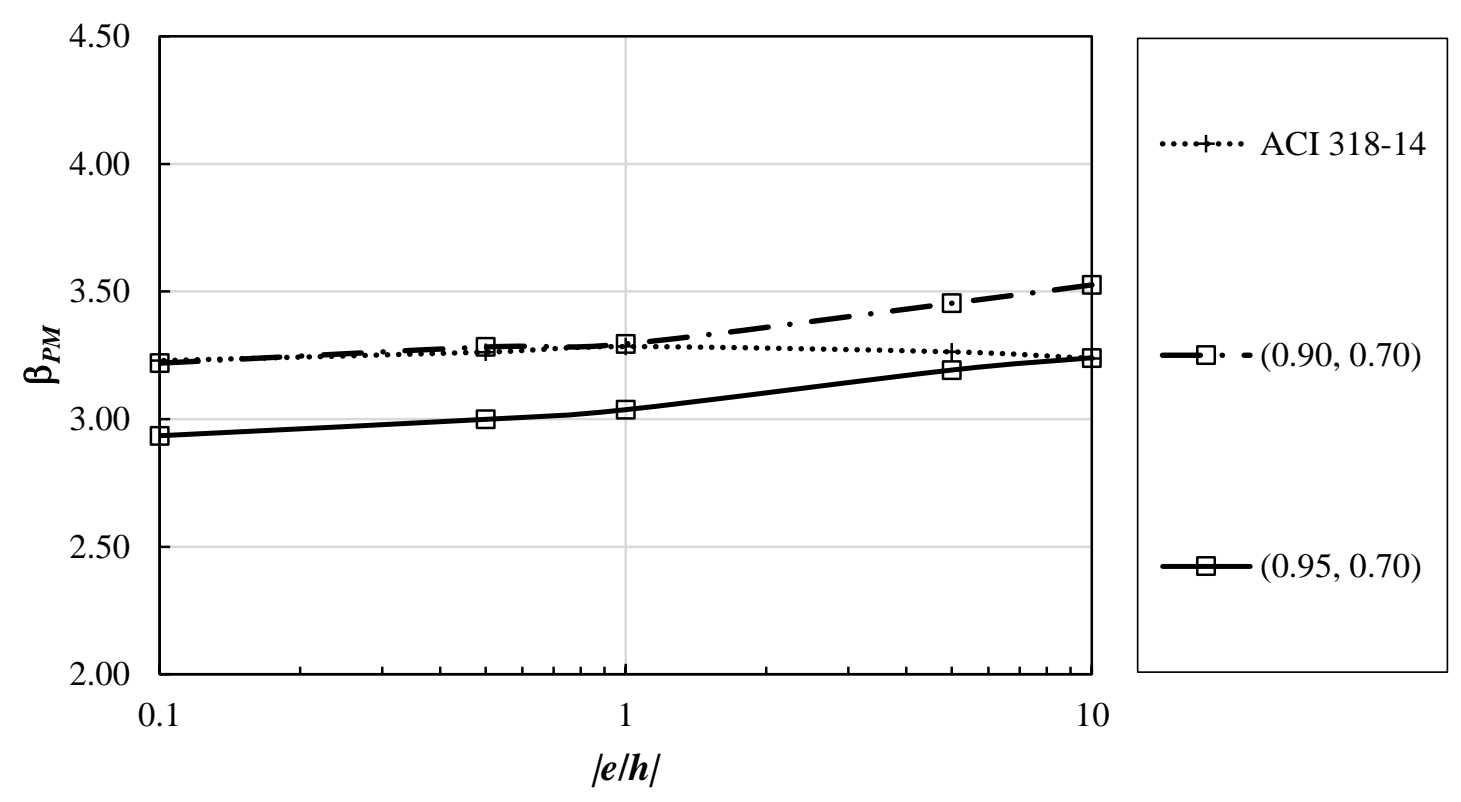

(b)

Figure C.40: Reliability indices for combined moment and axial force, $\beta_{P M}$, for Column Section 5, Property Combination 8, and $L / D=0.5$ : (a) $e / h>0$; (b) $e / h<0$ 


\section{C.1 Codes}

The Matlab (Version R2016b; The Mathworks, Inc. 2016) codes used to calculate reliability indices for combined moment and axial force, $\beta_{P M}$, for Column Section 1 (square section with three bars in each face) and Column Section 5 (spirally reinforced circular section with eight bars evenly distributed around the perimeter), two representative cross sections, are as follows:

The calculation needs to refer the results saved in alpha_PM_S1.mat and alpha_PM_S5.mat, which are presented in Appendix A.

\section{C.1.1 Notation}

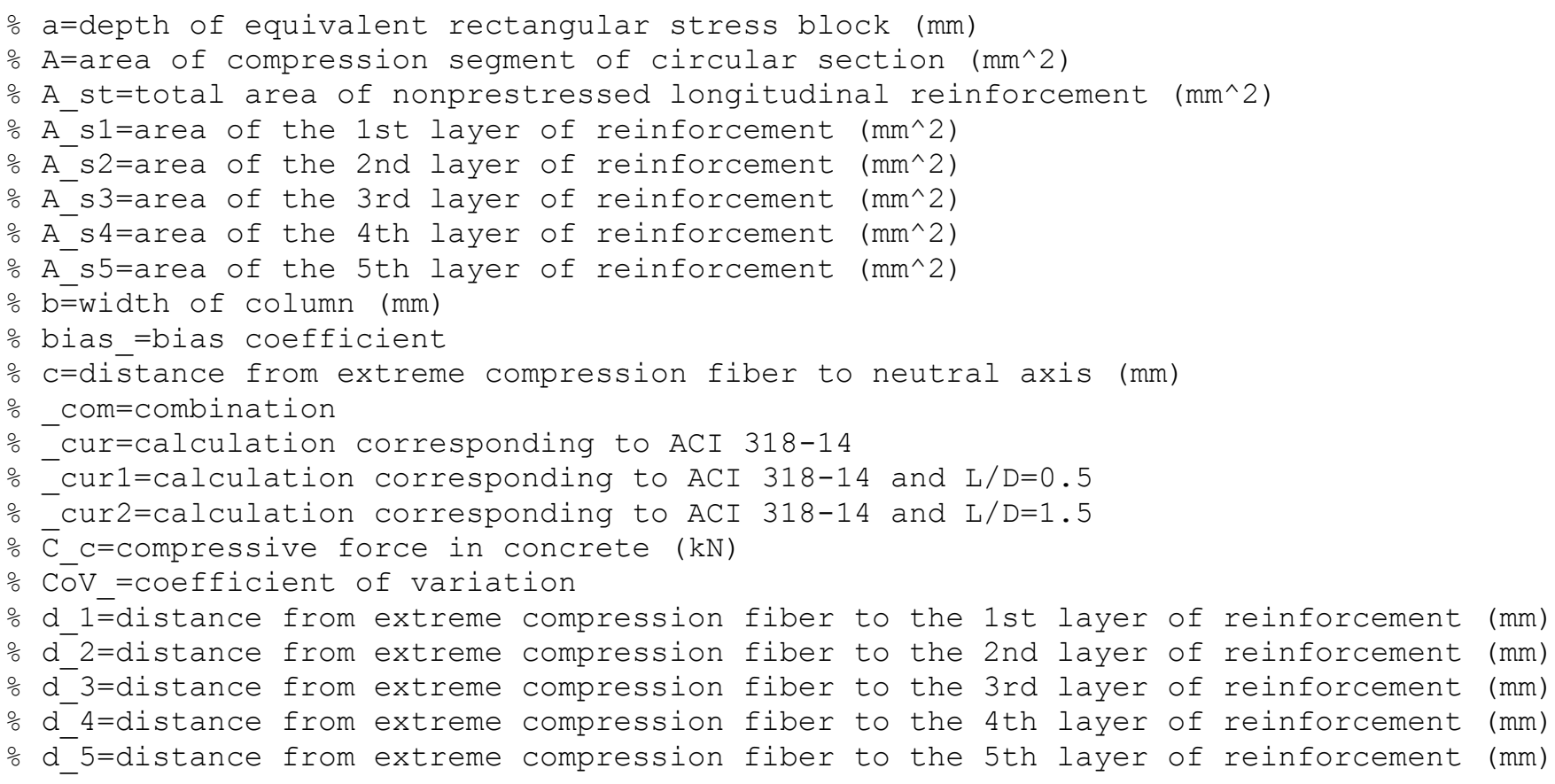


을 dead load

$\div$ e=eccentricity $(\mathrm{mm})$

\% eoverh=the specific e/h value

o eoverh_2=the specific e/h value, including extreme values

: E_s=modulus of elasticity of reinforcement (MPa)

: $\mathrm{f}^{-} \mathrm{C}=$ specified compressive strength of concrete (MPa)

$\mathrm{f} \mathrm{sl}=\mathrm{stress}$ in the 1st layer of reinforcement (MPa)

f_s2=stress in the 2nd layer of reinforcement (MPa)

$\mathrm{f}^{-} \mathrm{s} 3=$ stress in the $3 \mathrm{rd}$ layer of reinforcement (MPa)

f_s4=stress in the 4 th layer of reinforcement (MPa)

$\mathrm{f}^{-} \mathrm{s} 5=\mathrm{stress}$ in the 5 th layer of reinforcement (MPa)

f $y=s p e c i f i e d ~ y i e l d ~ s t r e n g t h$ for nonprestressed reinforcement (MPa)

F_sl=force in the lst layer of reinforcement (kN)

$\mathrm{F}^{-} \mathrm{s} 2$ =force in the $2 \mathrm{nd}$ layer of reinforcement (kN)

F s3=force in the 3rd layer of reinforcement (kN)

- F_s4=force in the 4 th layer of reinforcement (kN)

$\% \mathrm{~F} 5$ =force in the 5 th layer of reinforcement (kN)

o $=$ limit state function

$\div \mathrm{h}=$ overall depth of column (mm)

응 hovereh/e

은 simulated value

$\circ \bar{k} \mathrm{f}=\mathrm{value}$ to count numbers of failure

들ive load

o LoverD=ratio of live load to dead load, L/D

mean $=$ mean

M=flexural strength (kN.m)

: $\mathrm{n}=$ numbers of simulation in one subset

- neg=negative

$\circ \bar{n}$ f=numbers of failure

N=total numbers of simulation

- pri=prime

o pro=property

- pro=calculation corresponding to partial material strength reduction factors

\% prol=calculation corresponding to partial material strength reduction factors and L/D=0.5

pro2=calculation corresponding to partial material strength reduction factors and L/D=1.5

$\div \overline{\mathrm{P}}=\mathrm{axial}$ strength $(\mathrm{kN})$

\% P f=probability of failure

: P_max=maximum axial compressive strength $(\mathrm{kN})$ 
- P_o=axial strength at zero eccentricity (kN)

\% P r=design axial strength for partial material strength reduction factors format (kN)

- Prof=professional factor

o $P_{-} t=a x i a l$ tensile strength $(\mathrm{kN})$

\% $r \bar{n} \_n=s t a n d a r d$ normally distributed random number

orn_u=standard uniformly distributed random number

$\% \mathrm{~s}=$ sutscript

s $=$ sort

- sam=samples

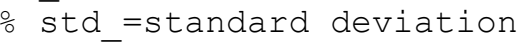

\% T_D=factor to account for transformation from dead load to dead load effect

\% $\mathrm{T}$ L=factor to account for transformation from live load to live load effect

\% $\mathrm{Z}=$ ratio of strain in extreme tension layer of reinforcement to yield strain

\% alpha_=dispersion parameter for Gumbel distribution

o beta_PMrl=reliability index for combined moment and axial force obtained using partial material strength reduction factors and $\mathrm{L} / \mathrm{D}=0.5$

\% beta_PMr2=reliability index for combined moment and axial force obtained using partial material strength $\frac{\circ}{0}$

o beta_PMul=reliability index for combined moment and axial force obtained using strength reduction factors $\frac{\circ}{\circ}$

o beta $\mathrm{PMu}$ =reliability index for combined moment and axial force obtained using strength reduction factors ㄴ in ACI 318-14 and $\mathrm{L} / \mathrm{D}=1.5$

o beta 1 =factor relating depth of equivalent rectangular compressive stress block to depth of neutral axis

gamma=ratio of distance between outer layers of reinforcement in column to overall column depth

opsilon_sl=strain in the 1st layer of reinforcement

opsilon s2=strain in the 2 nd layer of reinforcement

opsilon_s3=strain in the 3rd layer of reinforcement

opsilon s4=strain in the 4th layer of reinforcement

opsilon_s5=strain in the 5th layer of reinforcement

opsilon ${ }^{-}$y=yield strain of reinforcement

\% angle theta= angle theta, angle used to calculate compression segment of circular column

\% mu_méan of the assocriated normal distribution for lognormal distribution

- mu =location parameter for Gumbel distribution

orho_g=total reinforcement ratio, equal to ratio of total longitudinal reinforcement area to cross-

\% sectional area of column

\% sigma =standard deviation of the assocriated normal distribution for lognormal distribution

o phi_sc=a pair of partial material strength reduction factors 
\% phiP_n=design axial strength in ACI 318-14 (kN)

\section{C.1.2 Column Section 1}

\section{C.1.2.1 Code 1-Reliability Indices for $\mathrm{ACl} 318-14$ and $L D=0.5$}

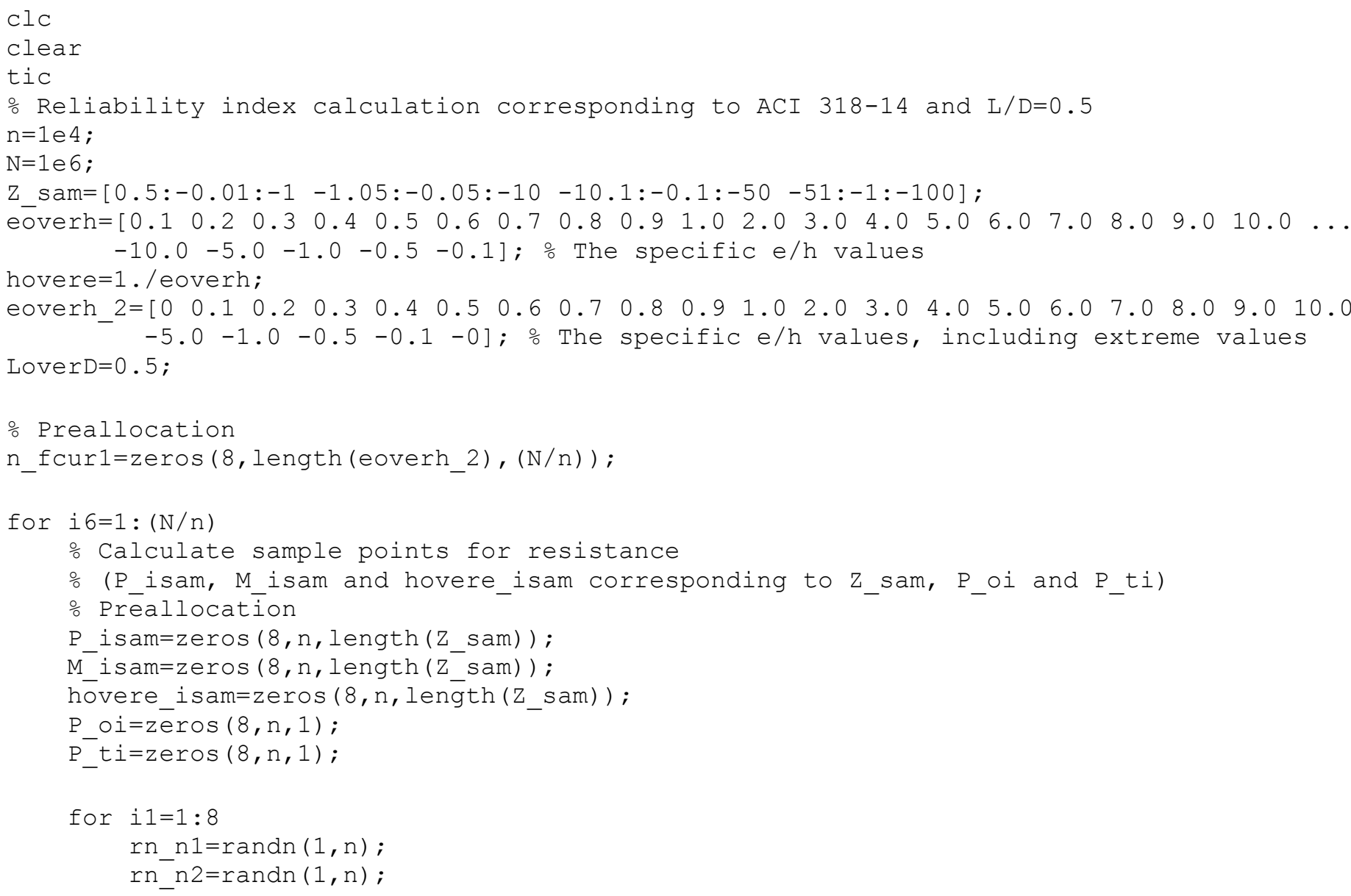




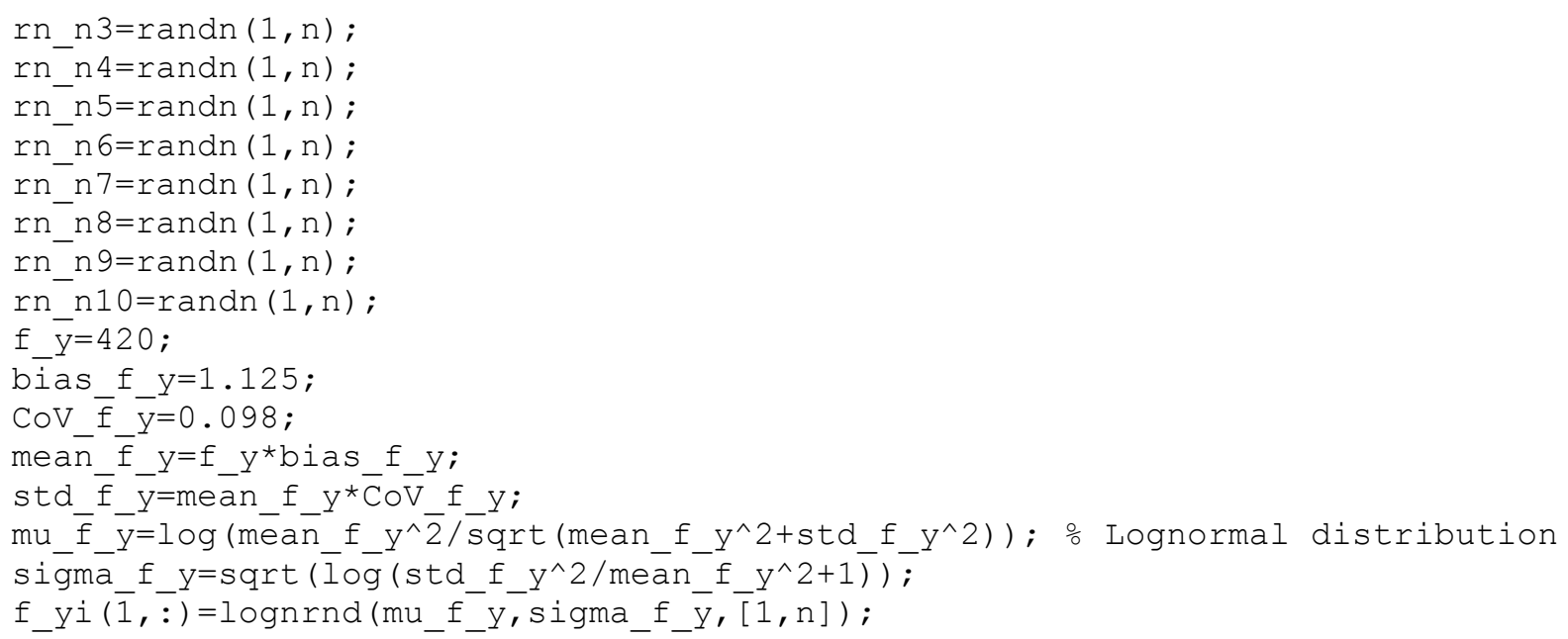

\% Calculate load effect

ㄴ Lover $\mathrm{D}=0.5$; $\frac{\circ}{0}$ Defined previously 
[D_cur1i,L_cur1i,T_Di,T_Li]=feval ('LoadEffectSim_cur_S1',LoverD, n) ;

- Interpolation

- Calculate the unknown points ( $\mathrm{P}$ i)

o Preallocation

$\mathrm{P}_{\text {_ilueros }}(8$, length (hovere), n);

for $i 1=1: 8$

for $i 5=1: n$

S_M_isam=find (M_isam $(i 1,:$, i5) $>0)$

P iśampri=P isam(i1,s M isam,i5);

hovere_isampri=hovere_isam(i1,s_M_isam,i5);

P isampri=[P oi(i1,1,i5) P isampri P ti(i1,1,i5)]:

hōvere_isamp $\bar{r} i=[1 e 10$ hoverēeisampri -

[hovere isampris, I hovere_isampri]=sort (hovere isampri, 'descend') ;

P i (i1,, , i5) =interpl (hovere isampris, P isampri(I hovere isampri), hovere, 'linear');

S_P_maxi=find ( $\left.P_{-} i(i 1,:, i 5)>\bar{P} \operatorname{maxi}(i 1,1, i 5)\right)$;

end

$\mathrm{P} i(i 1, \mathrm{~s} P \max i, i 5)=\mathrm{P} \operatorname{maxi}(i \overline{1}, 1, i 5)$;

end

o $\mathrm{P} i$ includes $\mathrm{P}$ maxi and $\mathrm{P}$ ti

$P_{-} i=\operatorname{cat}\left(2, P_{-}\right.$maxi, $\left.P_{-} i, P_{-} t i\right)$;

Limit state function and numbers of failure

- Preallocation

9 curli=zeros $(8$, length (eoverh 2$), \mathrm{n})$;

k_fcurli=zeros $(8$, length (eoverh_2), n) ;

for i4=1: length (eoverh 2)

g curli(:,i4, : ) ...

$=\left(\operatorname{abs}\left(P_{-} i(:, i 4,:)\right){ }^{\star} \operatorname{sqrt}\left(1+\right.\right.$ eoverh_2 $\left.\left.(i 4)^{\wedge} 2\right)\right) . / \ldots$

(abs (D curli(:,i4,:)*T Di+L curli (:,i4,:)*T Li)*sqrt (1+eoverh 2(i4)^2));

\% T_Di, T_Lì are numbers, not vectors

end

s g curli=find $(\log (g$ curli $)<0)$;

k_fourli(s_g_curli)=1; 
end

$n \_f \operatorname{cur} 1(:,:, i 6)=\operatorname{sum}\left(k_{f} f \operatorname{cur} 1 i, 3\right) ;$

\% Probability of failure

P f curl=sum (n_four 1,3$) / N$;

\% Reliability index

beta_PMul=-norminv (P_fcur1, 0,1$)$;

toc

save beta_PMul_S1 n_fcurl P_fcurl beta_PMul

\section{C.1.2.2 Code 2-Reliability Indices for $\mathrm{ACI} 318-14$ and $L D=1.5$}

ClC

clear

tic

o Reliability index calculation corresponding to ACI 318-14 and L/D=1.5

$\mathrm{n}=1 \mathrm{e} 4$;

$\mathrm{N}=1$ e 6 ;

Z_sam $=[0.5:-0.01:-1-1.05:-0.05:-10-10.1:-0.1:-50-51:-1:-100]$;

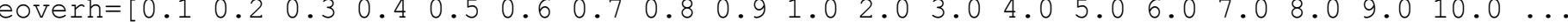

$-10.0-5.0-1.0-0.5-0.1] ; \%$ The specific e/h values

hovere=1./eoverh;

eoverh $2=\left[\begin{array}{lllllllllllllllllllllllllllll}0 & 0.1 & 0.2 & 0.3 & 0.4 & 0.5 & 0.6 & 0.7 & 0.8 & 0.9 & 1.0 & 2.0 & 3.0 & 4.0 & 5.0 & 6.0 & 7.0 & 8.0 & 9.0 & 10.0 & -10.0 & \ldots\end{array}\right.$ Lover $\mathrm{D}=1.5$ $-5.0-1.0-0.5-0.1-0] ;$ o The specific e/h values, including extreme values

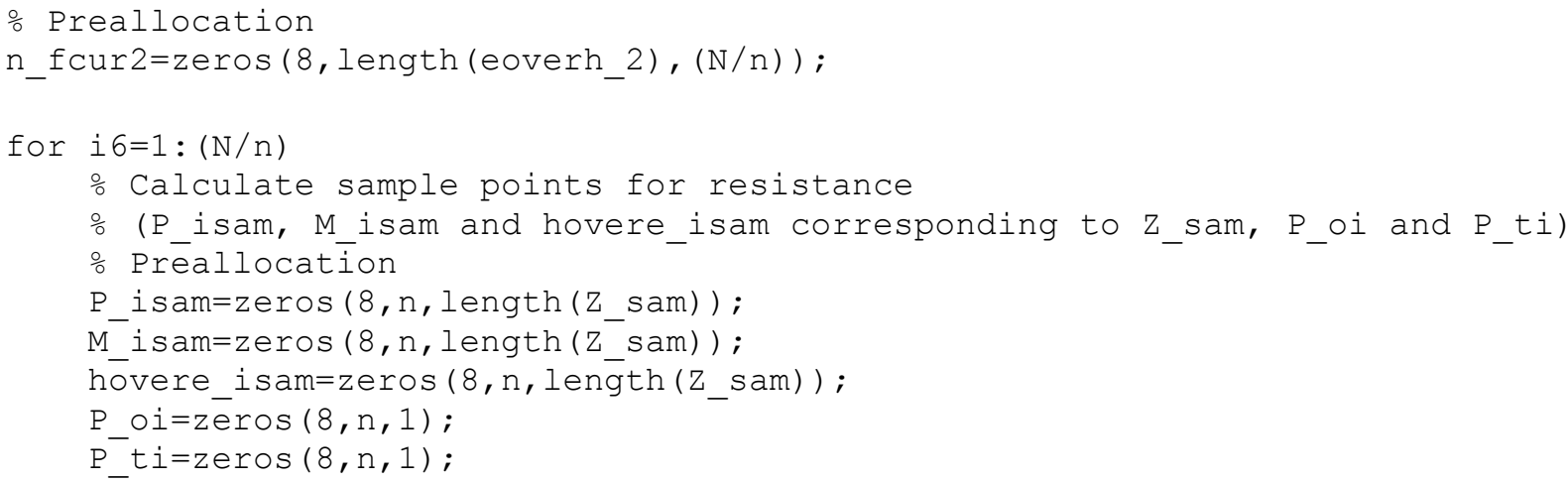


for $i 1=1: 8$

rn_n $1=\operatorname{randn}(1, \mathrm{n})$;

$r n \_n 2=\operatorname{randn}(1, n)$;

rn_n $3=\operatorname{randn}(1, \mathrm{n})$;

rn $\mathrm{n} 4=\operatorname{randn}(1, \mathrm{n})$;

rn_n5=randn $(1, n)$;

$\mathrm{rn}^{-} \mathrm{n} 6=\operatorname{randn}(1, \mathrm{n})$;

rn_n $7=\operatorname{randn}(1, \mathrm{n})$;

$\mathrm{rn} \_\mathrm{n} 8=\operatorname{randn}(1, \mathrm{n})$;

$\mathrm{rn} n 9=\operatorname{randn}(1, \mathrm{n})$;

rn_n10=randn $(1, \mathrm{n})$;

$f_{-} \bar{y}=420$;

bias $f y=1.125$;

$\mathrm{CoV}_{-} \overline{\mathrm{f}}_{-\mathrm{y}}=0.098$;

meañ $\bar{f}_{-}=f \_y^{*}$ bias $f \quad y$;

std_f ${ }_{-} \bar{y}=m e a n \_f \_y * \overline{C o} \bar{V}_{-} f_{-} y$;

mu $\bar{f} \bar{y}=\log \left(\operatorname{mean} f \_y^{\wedge} \overline{2} / \overline{s q r t}\left(\right.\right.$ mean $\left.\left.f \_y^{\wedge} 2+\operatorname{std} f \_y^{\wedge} 2\right)\right)$;

sigma_f_y=sqrt $\left(\log \left(\right.\right.$ std_f_${ }^{\prime} \mathrm{y}^{\wedge} 2 /$ mean_f $\left.\left.\_\mathrm{y}^{\wedge} 2+1\right)\right)$;

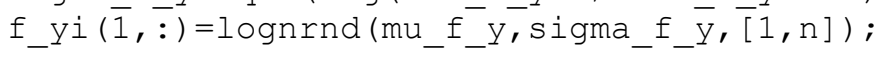

for $i 3=1$ : length $\left(Z_{-}\right.$sam)

[P isam(i1, :,i3), M isam(i1, :,i3), hovere isam(i1, :,i3), P oi (i1, :, 1), P ti (i1, :, 1)] . .

=feval ('ResistānceSim_S1',i1,rn_n1, rn_n2,rn_n3,rn_n $\overline{4}, r n \_n 5, r n \_n 6, r n \_n 7, r n \_n 8, r n \_n 9, \ldots$ rn_n10,f_yi,z_sam(i3));

end

- Calculate P_maxi

P_maxi=0.80*P_oi;

- Permute the 2nd and 3rd dimensions

P_isam=permute ( $\left.P_{\text {_isam, }}[1,3,2]\right)$;

M_isam=permute (M_isam, $[1,3,2])$;

hovere_isam=permüte (hovere_isam, $[1,3,2])$;

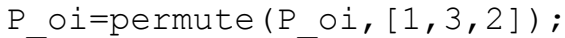

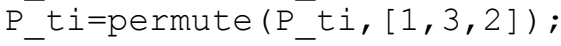


P_maxi=permute (P_maxi, $[1,3,2])$;

Calculate load effect

\% Lover $=1.5 ;$ o Defined previously

[D_cur2i,L_cur2i,T_Di,T_Li]=feval ('LoadEffectSim_cur_S1', LoverD, n) ;

- Interpolation

o Calculate the unknown points (P_i)

- Preallocation

P_i=zeros ( 8 , length (hovere), n);

for $i 1=1: 8$

for $i 5=1: n$

$\mathrm{S}$ M isam=find $(\mathrm{M}$ isam $(i 1,:$, i5) $>0)$;

P isampri=P isam(i1,s M isam,i5).

hōvere isampri=hovere $i \bar{s}$ am (i1, s_M isam, i5);

P isampri=[P oi(i1, 1, í5) P isampri P ti(i1,1,i5)];

hovere isampri=[1e10 hovere isampri -1e10];

[hoverē isampris, I hovere is sampri]=sort (hovere isampri, 'descend') ;

P i (i1, : i5)=interpl (hovere isampris, P isampri(I hovere isampri), hovere, 'linear');

S_P maxi=find ( $P_{-} i(i 1,:$, i5) $>\bar{P} \operatorname{maxi}(i 1,1, \overline{1} 5))$;

end

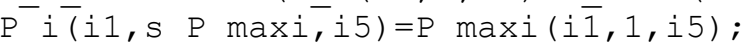

end

O $\mathrm{P}$ i includes $\mathrm{P}$ maxi and $\mathrm{P}$ ti

$P_{-} i=\operatorname{cat}\left(2, P_{-}\right.$maxi, $\left.P_{-} i, P_{-} t i\right)$;

o Limit state function and numbers of failure

- Preallocation

g cur2i=zeros (8, length (eoverh 2), n);

k_four2i=zeros $(8$, length (eoverh_2), n) ;

for i $4=1:$ length (eoverh_2)

g cur2i $(:, i 4,:) \ldots$

$=\left(\operatorname{abs}\left(P_{-} i(:, i 4,:)\right){ }^{\star} \operatorname{sqrt}\left(1+\right.\right.$ eoverh_2 $\left.\left.(i 4)^{\wedge} 2\right)\right) . / \ldots$ 


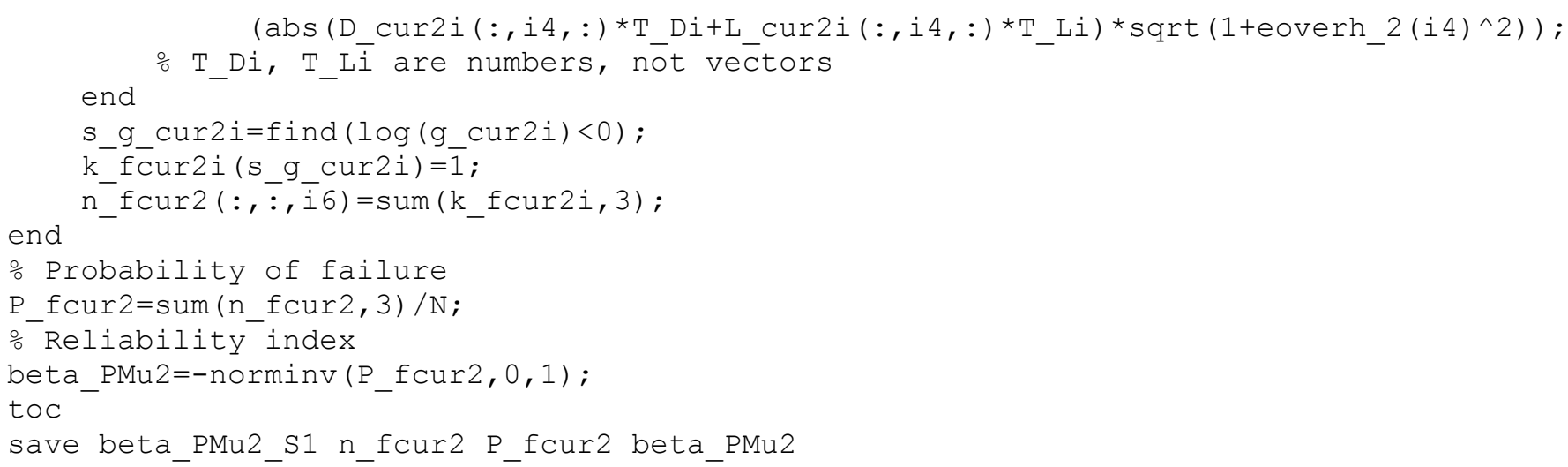

\section{C.1.2.3 Code 3-Reliability Indices for Partial Material Strength Reduction Factors and $L / D=0.5$}

$\mathrm{ClC}$

clear

tic

o Reliability index calculation corresponding to partial strength reduction factors and L/D=0.5 $\mathrm{n}=1 \mathrm{e} 4$; 
- Preallocation

$\mathrm{P}$ isam=zeros ( $8, \mathrm{n}$, length ( $\mathrm{Z}$ sam) ) ;

$\mathrm{M}^{-}$isam=zeros $\left(8, \mathrm{n}\right.$, length $\left(\mathrm{z}_{-}^{-}\right.$sam $\left.)\right)$;

hōvere isam=zeros $(8, n, \operatorname{leng}$ th $(\mathrm{z}$ sam $))$;

$\mathrm{P}$ oi=zeros $(8, \mathrm{n}, 1)$;

$\mathrm{P}^{-}$ti=zeros $(8, \mathrm{n}, 1)$;

for $i 1=1: 8$

rn $\mathrm{n} 1=\operatorname{randn}(1, \mathrm{n})$

$r \mathrm{n}-\mathrm{n} 2=\operatorname{randn}(1, \mathrm{n})$;

rn $\mathrm{n} 3=\operatorname{randn}(1, \mathrm{n})$;

rn_n $4=\operatorname{randn}(1, \mathrm{n})$;

$r n-n 5=\operatorname{randn}(1, \mathrm{n})$;

rn $\mathrm{n} 6=\operatorname{randn}(1, \mathrm{n})$;

rn_n $7=\operatorname{randn}(1, \mathrm{n})$;

$\mathrm{rn} n 8=\operatorname{randn}(1, \mathrm{n})$;

rn_n $9=\operatorname{randn}(1, \mathrm{n})$;

rn n $10=\operatorname{randn}(1, \mathrm{n})$;

f $\bar{y}=420$;

bias_f_y=1.125;

$\mathrm{CoV} \overline{\mathrm{f}} \overline{\mathrm{y}}=0.098$;

meañ $\bar{f}_{-} y=f_{-}{ }^{*}$ bias_f_y;

std_ $\bar{f} \_\bar{y}=m e \bar{n} \_f \_y * \bar{C} \circ \bar{V} \_f \_y$;

mu $\bar{f} \bar{y}=\log \left(\operatorname{mean} f y^{\wedge} \overline{2} / \overline{s q r t}\left(\right.\right.$ mean $\left.\left.f y^{\wedge} 2+\operatorname{std} f y^{\wedge} 2\right)\right)$; $\frac{0}{\circ}$ Lognormal distribution

sigma_f_y=sqrt $(\bar{l} \circ \bar{g}($ std_f_y^2/meān_f_y^2+1) $) \overline{;}$

f yi $(\overline{1}, \overline{:})=\operatorname{lognrnd}(\mathrm{mu} f \bar{y}, \operatorname{sigma} \mathrm{f} \overline{\mathrm{y}}, \overline{[} \overline{1}, \mathrm{n}])$;

for i3=1: length (Z sam)

$\left[P_{-} i \operatorname{sam}(i 1,:, \bar{i} 3), M_{-} i \operatorname{sam}(i 1,:, i 3), \operatorname{hovere} i \operatorname{sam}(i 1,:, i 3), P_{-}\right.$oi $\left.(i 1,:, 1), P_{-} t i(i 1,:, 1)\right] \ldots$ feval ('ResistanceSim S1',i1, rn n1, rn $n 2, r n n 3, r n n 4, r n n 5, r n n 6, r n n 7, r n n 8, r n n 9, \ldots$

end rn_n10,f_yi,z_sam(i3));

end

- Calculate $\mathrm{P}$ maxi

$P_{-} \operatorname{maxi}=0.80 * \mathrm{P}_{-} \mathrm{oi}$; 
\% Permute the 2nd and 3rd dimensions

$P$ isam=permute ( $P$ isam, $[1,3,2])$;

M_isam=permute $\left(\mathrm{M}^{-}\right.$isam, $\left.[1,3,2]\right)$;

hovere_isam=permute (hovere_isam, $[1,3,2])$;

P_oi=permute (P_oi, $[1,3,2])$;

$\mathrm{P}_{-}^{-} \mathrm{ti}=\operatorname{permute}\left(\mathrm{P}_{-}^{-} \mathrm{t} i,[1,3,2]\right)$;

$\mathrm{P}$ maxi=permute $\overline{(\mathrm{P}} \max i,[1,3,2])$;

- Calculate load effect

o Lover $\mathrm{D}=0.5$; \% Defined previously

\% s phi sc \% Defined previously

[D_prolī,L_proli,T_Di,T_Li]=feval ('LoadeffectSim_pro_S1',LoverD, s_phi_sc, n) ;

ㅇterpolation

o Calculate the unknown points (P_i)

- Preallocation

$\mathrm{P}_{\text {_i }}$ zeros $(8$, length (hovere), n);

for $i 1=1: 8$

for $i 5=1: n$

S_M_isam=find (M_isam $(i 1,:$, i5) $>0)$;

$\mathrm{P}$ isampri=P isam(i1,s M isam, i5);

hovere_isampri=hovere_isam(i1,s_M_isam,i5);

P_isampri=[P_oi(i1, 1, í5) P_isamprí P ti(i1,1,i5)];

hovere isampri=[1e10 hovere isampri -1e10];

[hoverēeisampris, I_hovere_isampri]=sort (hovere_isampri, 'descend') ;

P i (i1, : i5)=interpl (hovere isampris, P isampri(I hovere isampri), hovere, 'linear');

S P maxi=find(P i(i1, : i5) $>\bar{P} \operatorname{maxi}(i 1,1, i 5))$;

end

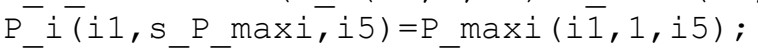

end

$\circ P_{-} i$ includes $P_{-}$maxi and $P_{-} t i$

$P_{-} i=\operatorname{cat}\left(2, P_{-}\right.$maxi, $\left.P_{-} i, P_{-} t i\right)$;

\% Limit state function and numbers of failure

o Preallocation 


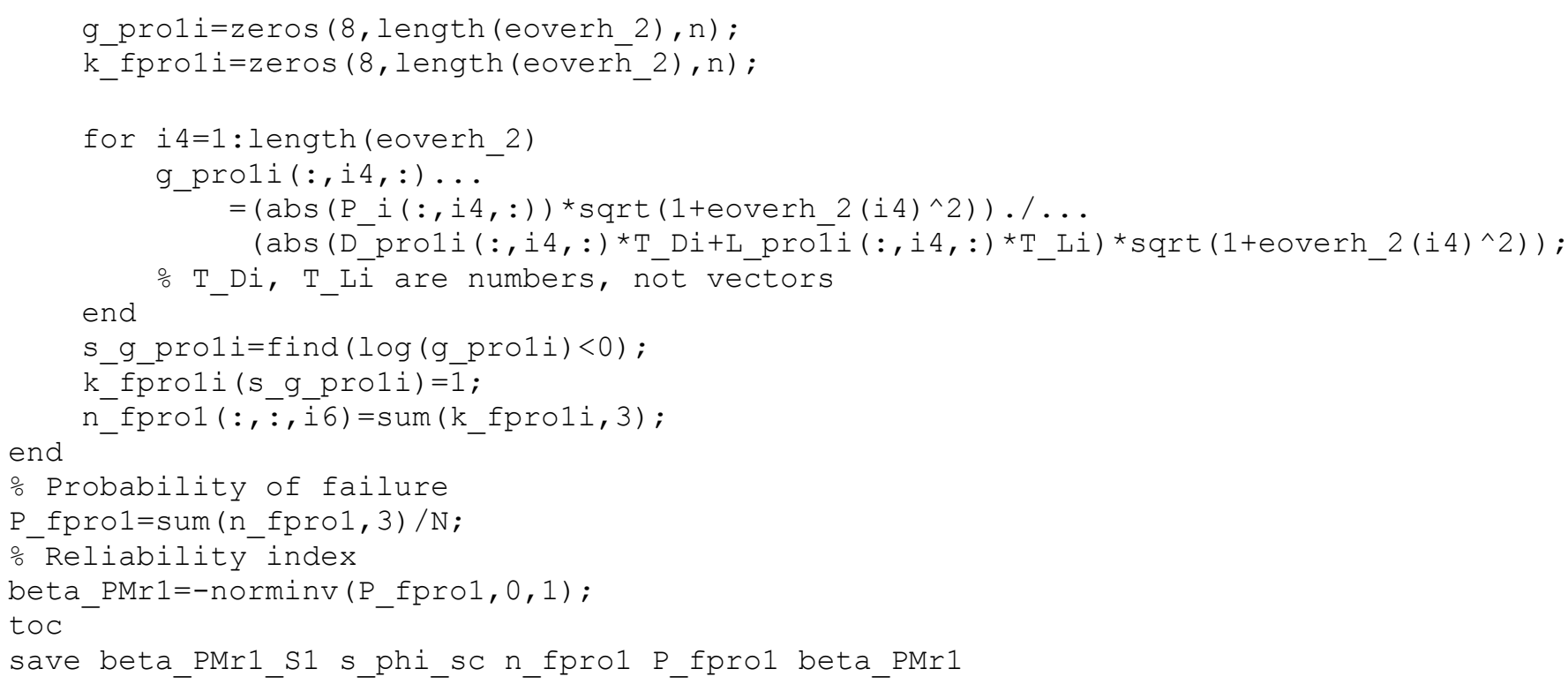

\section{C.1.2.4 Code 4-Reliability Indices for Partial Material Strength Reduction Factors and $L / D=1.5$} clc

clear

tic

- Reliability index calculation corresponding to partial strength reduction factors and L/D=1.5 $\mathrm{n}=1 \mathrm{e} 4$;

$\mathrm{N}=1$ e 6 ;

Z_sam $=[0.5:-0.01:-1-1.05:-0.05:-10-10.1:-0.1:-50-51:-1:-100]$

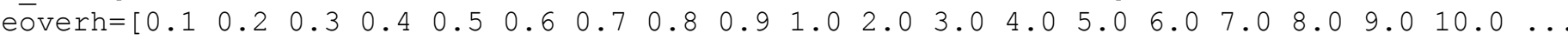
$-10.0-5.0-1.0-0.5-0.1] ;$ 응

hovere=1. / eoverh;

eoverh $2=\left[\begin{array}{lllllllllllllllllllllllllllll}0 & 0.1 & 0.2 & 0.3 & 0.4 & 0.5 & 0.6 & 0.7 & 0.8 & 0.9 & 1.0 & 2.0 & 3.0 & 4.0 & 5.0 & 6.0 & 7.0 & 8.0 & 9.0 & 10.0 & -10.0 & \ldots\end{array}\right.$ Lover $\mathrm{D}=1.5$

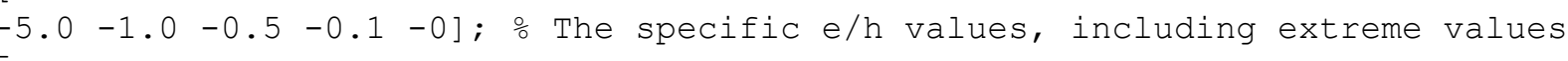


o Preallocation

n_fpro2=zeros $(8$, length (eoverh_2), (N/n));

for i $6=1:(\mathrm{N} / \mathrm{n})$

o Calculate sample points for resistance

( $\mathrm{P}$ isam, $\mathrm{M}$ isam and hovere isam corresponding to $\mathrm{Z}$ sam, $\mathrm{P}$ oi and $\mathrm{P}$ ti)

o Preallocation

$\mathrm{P}$ isam $=$ zeros $(8, \mathrm{n}$, length $(\mathrm{Z}$ sam $))$;

$\mathrm{M}$ isam=zeros $(8, \mathrm{n}$, length $(\mathrm{Z}$ sam $))$;

hōvere_isam $=$ zeros $\left(8, n, \operatorname{leng}\right.$ th $\left.\left(z \_s a m\right)\right)$;

$\mathrm{P}$ oi=zeros $(8, \mathrm{n}, 1)$;

P_ti=zeros $(8, \mathrm{n}, 1)$;

for $i 1=1: 8$

$r n \_n 1=\operatorname{randn}(1, \mathrm{n})$

$\mathrm{rn} \mathrm{n} 2=\operatorname{randn}(1, \mathrm{n})$;

rn_n $3=\operatorname{randn}(1, \mathrm{n})$

rn $\mathrm{n} 4=\mathrm{randn}(1, \mathrm{n})$.

rn_n $5=\operatorname{randn}(1, \mathrm{n})$;

rn $n 6=\operatorname{randn}(1, n)$

$\mathrm{rn} \mathrm{n} 7=\operatorname{randn}(1, \mathrm{n})$

rn_n $8=\operatorname{randn}(1, \mathrm{n})$;

rn $\mathrm{n} 9=\operatorname{randn}(1, \mathrm{n})$;

$r n-n 10=\operatorname{randn}(1, \mathrm{n})$;

f $\bar{y}=420$;

bias f $y=1.125$;

CoV_f_y $=0.098$;

mean $f y=f y^{\star}$ bias $f y$;

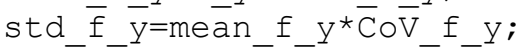

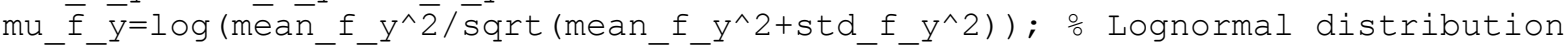

sigma f $y=\operatorname{sqrt}\left(\log \left(\right.\right.$ std $f y^{\wedge} 2 /$ mean $\left.\left.f y^{\wedge} 2+1\right)\right)$;

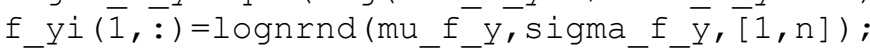

for $i 3=1:$ length $(Z$ sam)

[P isam(i1, : i3), M isam(i1, : i3), hovere isam(i1, : i3), P oi (i1, : 1), P ti(i1, : , 1)] ...

=feval ('Resistāncesim_s1',i1,rn_n1, rn_n2,rn_n3,rn_n $\overline{4}, r n \_n 5, r n \_n 6, r n \_n 7, r n \_n 8, r n \_n 9, \ldots$ rn n10, f yi, z sam(i3)); 
end

end

- Calculate $\mathrm{P}$ maxi

$P_{-} \operatorname{maxi}=0.80 * \mathrm{P}_{-}^{-}$oi

- Permute the 2nd and 3rd dimensions

$\mathrm{P}$ isam=permute $(\mathrm{P}$ isam, $[1,3,2])$;

$\mathrm{M}^{-}$isam=permute $\left(\mathrm{M}^{-}\right.$isam, $\left.[1,3,2]\right)$;

hovere isam=permute (hovere isam, $[1,3,2])$;

$\mathrm{P}$ oi=permute (P_oi, $[1,3,2])$;

$\mathrm{P}_{-}^{-} \mathrm{ti}=\operatorname{permute}\left(\mathrm{P}_{-}^{-} \mathrm{t} i,[1,3,2]\right)$;

$\mathrm{P}$ maxi=permute $\overline{(P} \operatorname{maxi},[1,3,2])$;

\% Calculate load effect

\% LoverD=1.5; \% Defined previously

\% s phi sc Defined previously

[D_pro2i, L_pro2i, T_Di,T_Li]=feval ('LoadEffectSim_pro_S1', LoverD, s_phi_sc, n) ;

ㅇterpolation

- Calculate the unknown points ( $P_{-}$i)

o Preallocation

P_i=zeros $(8$, length (hovere), n);

for $i 1=1: 8$

for $i 5=1: n$

S_M_isam=find (M_isam $(i 1,:$, i5) $>0)$;

$\mathrm{P}$ is sampri=P isam(i1, s M isam, i5);

hōvere isamp̄i=hovere isam(i1,s M isam,i5);

P_isampri=[P_oi(i1, $1, \dot{i} 5)$ P_isamprí P_ti(i1, 1, i5)] ;

hovere isampri=[1e10 hovere isampri -1e10];

[hoverétisampris, I_hovere_isampri]=sort (hovere_isampri, 'descend');

P i (i1, $\bar{i}, i 5)=i n t e r \bar{p} 1$ (hovere isampris, P isampri (I hovere isampri), hovere, 'linear') ;

S_P_maxi=find ( $P_{-} i(i 1,:$, i5) $>\bar{P} \operatorname{maxi}(i 1,1, i 5))$;

end

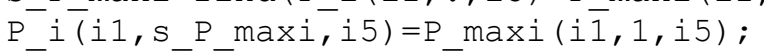

end 


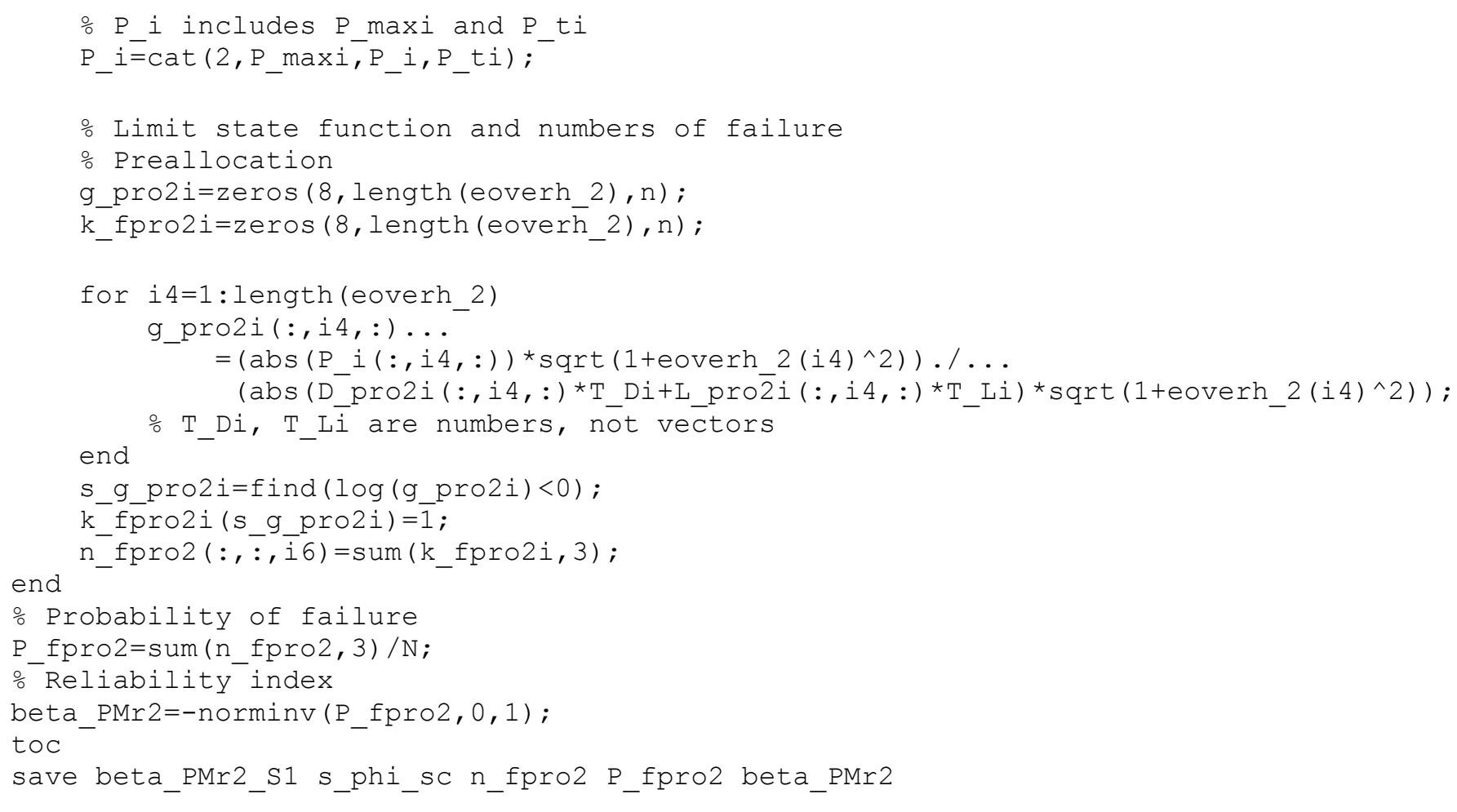

\section{C.1.2.5 Code 5-Function of Simulated Resistances}

- Resistance simulation

function [P_i,M_i, hovere_i,P_oi,P_ti]=ResistanceSim_S1 (i1,rn_n1,rn_n2,rn_n3,rn_n4,rn_n5,rn_n6,rn_n7,... rñn8,rn_n9,rn_n10, f_yi, z)

\% Nominal value combinations

\% Geometric property combinations

b_com $=\left[\begin{array}{ll}325 & 1300\end{array}\right]$;

$\mathrm{h}-\mathrm{com}=\left[\begin{array}{ll}325 & 1300\end{array}\right]$

gämma_com $=\left[\begin{array}{lll}0.6 & 0.9\end{array}\right]$;

d_1_com=(1+gamma_com $).{ }^{*}$ h_com/2 ; 
d_2_com $=$ h_com $/ 2$;

d_3_com $=\left(\overline{1}-g a m m a \_c o m\right) \cdot{ }^{*}$ _com/2;

rho_g_com $=\left[\begin{array}{lll}0.01 & 0.04\end{array}\right]$;

- Material property combinations

f $C$ COm=[25 45];

- ${ }^{-} \mathrm{y}=420$; $\%$ Defined in beta

E_s $=200000$;

o Summarize property combinations in one matrix

pro_com=[b_com(1) h_com(1) gamma_com(1) d_1_com(1) d_2_com(1) d_3_com(1) f_c_com(1) rho_g_com(1) ;

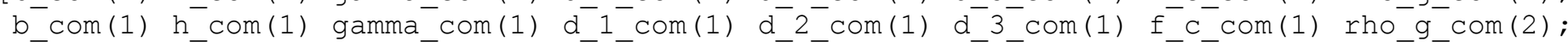
b com(1) h com(1) gamma com(1) d 1 com(1) d 2 com(1) d 3 com(1) f c com(2) rho g com(1): b_com (1) h_com(1) gamma_com(1) d_1_com(1) d_2_com(1) d_3_com(1) f_c_com(2) rho_g_com(2); $\mathrm{b}$ com (2) h com(2) gamma com(2) d 1 com(2) d 2 com(2) d 3 com(2) f c com(1) rho g com(1); $\mathrm{b}$ com (2) h com(2) gamma com(2) d 1 com(2) d 2 com(2) d 3 com(2) f c com(1) rho g com(2); b_com (2) h_com(2) gamma_com(2) d_1_com(2) d_2_com(2) d_3_com(2) f_c_com(2) rho_g_com(1);

b_com (2) h_com(2) gamma_com(2) d_1_com(2) d_2_com(2) d_3_com(2) f_c_com(2) rho_g_com(2)];

\% pro $\mathrm{com}=\left[\begin{array}{lllllllllllllllll}325 & 325 & 0.6 & 260 & 162.5 & 65 & 25 & 0.01 ; 325 & 325 & 0.6 & 260 & 162.5 & 65 & 25 & 0.04 ; ;\end{array}\right.$

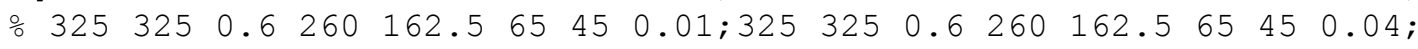

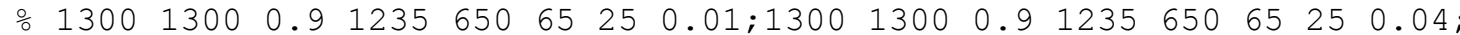

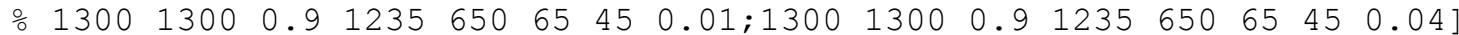

Professional factor

Prof $=1$

\% Nominal values

Geometric properties

b $(1,1)=$ pro_com $(i 1,1)$;

$\mathrm{h}(1,1)=\operatorname{pro} \operatorname{com}(i 1,2)$

o $\operatorname{gamma}(1, \overline{1})=$ pro_com $(i 1,3)$;

d $1(1,1)=\operatorname{pro} \operatorname{com}(i 1,4)$;

d $2(1,1)=\operatorname{pro} \operatorname{com}(i 1,5)$;

d $3(1,1)=$ pro_com $(i 1,6)$;

rho $g(1,1)=$ pro $\operatorname{com}(i 1,8)$;

A_st $(1,1)=r h o \_\bar{g}(1,1) * b(1,1) * h(1,1)$; 
A_s $1(1,1)=3 * A \_s t(1,1) / 8$;

A s $2(1,1)=A$ st $(1,1) / 4$;

A_s3 $(1,1)=3{ }^{\star}$ A_st $(1,1) / 8$;

o Material properties

f $\mathrm{c}(1,1)=$ pro $\operatorname{com}(i 1,7)$;

\% f_y=420; \%-Defined in beta

을 $=200000$; Defined previously

\% Professional factor

응 Prof=1; 응 Defined previously

o Statistical parameters (Bias coefficient and CoV)

- Geometric properties

\% bias b $\%$ Use mean directly

\% CoV_- ${ }_{-} \%$ Use standard deviation directly

bias_h $\%$ Use mean directly

$\circ \mathrm{CoV} \overline{\mathrm{h}} \div$ Use standard deviation directly

\% bias gamma $=1$; $\frac{\circ}{0}$ Deterministic

o CoV_gamma $=0$;

bias_d_1=1;

$\mathrm{Co} \overline{\mathrm{V}} \overline{\mathrm{d}} 1$ \% Use standard deviation directly

bias d 2=1;

$\circ \mathrm{Co} \overline{\mathrm{V}} \overline{\mathrm{d}}_{-} 2$ \% Use standard deviation directly

bias_d_3 3 ;

\% Co $\bar{V} \_\bar{d} \_3$ o Use standard deviation directly

o bias_rho_g=1; $\%$ Deterministic

ㅇ Cov $\overline{r h}$. $\bar{g}=0$;

bias_A_s $=1.0$; 
CoV_A_s $=0.015$;

O Material properties

bias $f \mathrm{c}=1.15$.

CoV_f ${ }_{-} \bar{C}=0.211$;

\% bias_f_y $=1.125$; $\%$ Defined in beta

$\therefore \mathrm{CoV}_{-} \overline{\mathrm{y}}_{\mathrm{y}}=0.098 ; \%$ Defined in beta

ㅁ bias E $\mathrm{s}=1$; $\frac{\circ}{\circ}$ Deterministic

$\circ$ CoV_E ${ }_{-}{ }_{S}=0$;

\% Professional factor

bias Prof $=1.00$;

CoV_Prof $=0.08$;

\% Statistical parameters (Mean and Standard deviation)

\% Geometric properties

mean_b $(1,1)=b(1,1)+1.52$;

std_b $(1,1)=6.35$;

mean $\mathrm{h}(1,1)=\mathrm{h}(1,1)+1.52$;

std_h $(1,1)=6.35$;

mean_d_1 $(1,1)=d \_1(1,1) *$ bias_d_1 ;

std_d_ $\overline{1}(1,1)=6 . \overline{3} 5$;

mean_d_2 $(1,1)=d \_2(1,1) *$ bias_d_2 ;

if $i 1<=4$

else

std_d_2 $(1,1)=4.76$;

end

std_d_2 $(1,1)=6.35$;

mean d $3(1,1)=$ d $3(1,1) *$ bias d 3 ;

std_d_ $\overline{3}(1,1)=4 . \overline{7} 6$; 
mean_A_s1 $(1,1)=$ A_s1 $(1,1) *$ bias_A_s;

std_A_ $\overline{\mathrm{s}} 1(1,1)=\operatorname{mean} \bar{A}_{-} \mathrm{s} 1(1,1) * \overline{\mathrm{C}} \mathrm{o} \bar{V}_{-} \mathrm{A}_{-} \mathrm{s}$;

mean $\bar{A}$ s2 $(1,1)=A$ s $\overline{2}(\overline{1}, 1) *$ bias $A \bar{s}$;

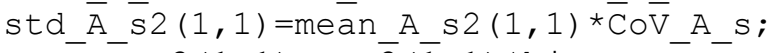

mean $\overline{\mathrm{A}} \mathrm{s} 3(1,1)=\mathrm{A} s \overline{3}(\overline{1}, 1) *$ bias $\mathrm{A} \overline{\mathrm{s}}$;

std_A $\bar{A} \_\bar{s} 3(1,1)=$ meān_A_s3 $(1,1) * \bar{C} \circ \bar{V} \_A \_s$;

- Material properties

mean_f_c $(1,1)=f \_c(1,1) *$ bias_f $c$;

std_e ${ }_{-} \bar{c}(1,1)=$ mean_f_c $(1,1) * \bar{C} 0 \bar{V} \_f \_c$;

\% mean_f_y=f_y*bias_f_y; $\%$ Defined in beta

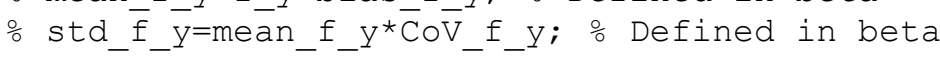

o Professional factor

mean Prof=Prof*bias Prof;

std_Prof=mean_Prof* $\bar{C}$ ov_Prof;

o Simulation

\% Geometric properties

b_i $(1,:)=$ mean_b $(1,1)+\operatorname{std} b(1,1) * r n \_n 1 ;$ o Normal distribution

$h^{-} i(1,:)=$ mean $^{-} h(1,1)+\operatorname{std}^{-} h(1,1) * r n-n 2$; Normal distribution

o- gamma $i\left(1,1 \overline{)}=\operatorname{gamma}\left(1,1 \overline{)}\right.\right.$; $\frac{\circ}{0}$ Deterministic

d_1i $(1, \overline{:})=$ mean_d_1 $(1,1)+$ std_d_1 $(1,1) * r n \_n 3 ;$ Normal distribution

d $2 i(1,:)=$ mean d $2(1,1)+$ std d $2(1,1)$ *rn n 4 ; Normal distribution

d_3i $(1,:)=$ mean_d_3 $(1,1)+$ std_d_3 $(1,1) * r n-n 5 ;$ 을 Normal distribution

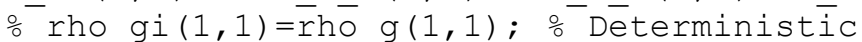

A_sli $(1,:)=$ mean_A_sl $(1,1)+$ std_A_s1 $(1,1) * r n \_n 6 ; \%$ Normal distribution

A_s2i $(1,:)=$ mean_A_s2 $(1,1)+$ std_A_s2 $(1,1) * r n \_n 7$; $\%$ Normal distribution

A s3i $(1,:)=$ mean A s3 $(1,1)+$ std A s3 $(1,1) * r n$ n8; $\frac{\circ}{\circ}$ Normal distribution

․ Material properties

$f_{-} c i(1,:)=$ mean_f_c $(1,1)+$ std_f_c $(1,1) * r n \_n 9 ;$ o Normal distribution

beta $1 i(1,:)=0.85-0.05 *$ (f $\mathrm{ci}(\overline{1},:)-28) / 7$;

s_beta_1_28=find (f_ci $(1,: \overline{)}<=28)$; 
beta_1i(1, s_beta_1_28)=0.85;

s beta 1 56=find (f $\mathrm{ci}(1,:)>=56)$;

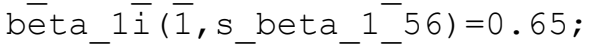

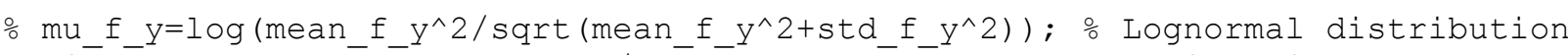

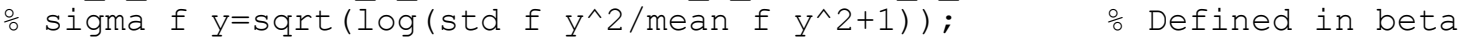

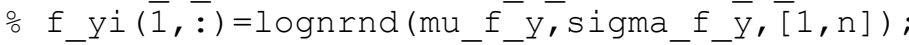

E si=E s; 응 Deterministic

epsilon_yi $(1,:)=f \_y i(1,:) / E_{\text {_tsi }}$

- Professional factor

Prof_i $(1,:)=$ mean_Proftstd_Prof*rn_n10; $\%$ Normal distribution

\% Resistance calculation

- Calculate $\mathrm{c}$ i

c_i $(1,:)=\left(0.003 . /\left(0.003-z^{*}\right.\right.$ epsilon_yi $\left.\left.(1,:)\right)\right) .{ }^{*} d_{-} 1 i(1,:)$;

$\circ$ Calculate a_i

a $i(1,:)=$ beta $1 i(1,:) \cdot{ }^{*} \mathrm{C} i(1,:)$;

o- Compare a_i with $\mathrm{h}_{-}{ }^{-}$

s a $i=f i n d(a$ i $(1,:)>h$ i $(1,:))$;

a_i $\left(1, s_{-} a_{-} i\right)=h_{-} i\left(1, s_{-} a_{-} i\right)$;

o Calculate epsilon_sli, epsilon_s2i, epsilon_s3i, f_sli, f_s2i and f_s3i epsilon_sli $(1,:)=z^{*}$ epsilon_yi $(1, \overline{:})$;

epsilon s $2 i(1,:)=0.003 *(\mathrm{c} i(1,:)-d 2 i(1,:)) . / c i(1,:)$;

epsilon_s3i $(1,:)=0.003 *\left(c_{-}^{-} i(1,:)-d_{-}^{-} 3 i(1,:)\right) . / c_{-}^{-} i(1,:)$;

f $\operatorname{sil}(1,:)=e p s i l o n \operatorname{sil}(1,:) * \mathrm{E}$ si

f_s2i $(1,:)=$ epsilon_s2i $(1,:) * E$ si;

f_s3i $(1,:)=e p s i l o n \_s 3 i(1,:) * E_{-}^{-}$si

o Compare f_sli, f_s $2 i$ and f_s3i with +-f_yi

s_f_sliu=find(f_sli(1,:)>f_yi $(1,:))$; $\frac{o}{0}$ Upper boundary, f_yi

f_sili(1,s_f_sliu $)=f \_y i\left(1, s_{-} f \_s l i u\right)$;

s f slil=find(f sli(1,:)<-f yi $(1,:))$; $\frac{\circ}{\circ}$ Lower boundary, -f yi

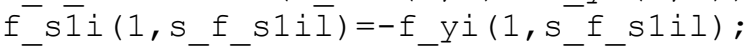


s_f_s2iulfind(f_s2i(1,:)>f_yi( $1,:))$; O Upper boundary, f_yi f $\mathrm{s} \overline{2} i(1, \mathrm{~s} f \mathrm{~s} 2 i \bar{u})=f$ yi $(1, \mathrm{~s} f \mathrm{~s} 2 i u)$;

s_f_s2il=find(f_s2i(1,:)<-f_yi $(1,:))$; $\%$ Lower boundary, -f_yi

$f_{-}{ }^{-} \overline{2} i\left(1, s \_f \_s 2 i \bar{l}\right)=-f \_y i\left(1, s \_f \_s 2 i l\right)$;

s_f_s3iu=find(f_s3i(1,:)>f_yi(1,:)); O Upper boundary, f_yi

f_s3i(1,s_f_s $3 i \bar{u})=f$ yi $\left(1, s_{f}\right.$ s $\left.3 i u\right)$;

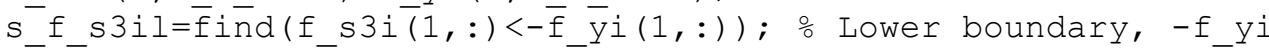

$f_{-}^{-} \overline{3} i\left(1, s_{-} f_{-} s 3 i \bar{l}\right)=-f \_y i\left(1, s_{-}{ }_{-} s 3 i l\right)$;

o Calculate C ci

C_ci $(1,:)=0.8 \overline{5} * f_{-} c i(1,:) \cdot{ }^{*} a+i(1,:) \cdot{ }^{*} b_{-} i(1,:) / 1000$;

o Calculate F_sii

F_sli $(1,:)=\left(f_{-}^{-} s 1 i(1,:)-0.85{ }^{\prime}{ }_{-}\right.$ci $\left.(1,:)\right) \cdot{ }^{* A}$ _sli $(1,:) / 1000$;

S_F_sli=find $\left(\bar{a}_{-} i(1,:)<d_{-}{ }_{1} i(1, \bar{l})\right)$;

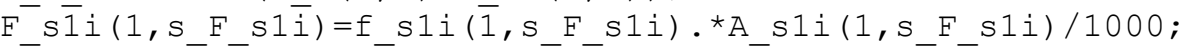

\% Calculate F s2i

F_s2i $(1,:)=\left(f \_s 2 i(1,:)-0.85 * f \_c i(1,:)\right) .{ }^{*}$ A_s2i $(1,:) / 1000$;

s_F_s2i=find $\left(\bar{a}_{-} i(1,:)<d_{-} 2 i(1, \bar{i})\right)$;

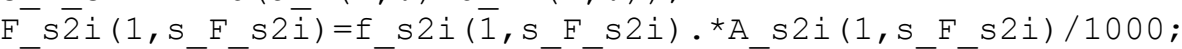

- Calculate F s3i

F_s3i $(1,:)=\left(f_{-}^{-}\right.$3i $\left.(1,:)-0.85 * f \_c i(1,:)\right) .{ }^{*}$ A_s3i $(1,:) / 1000$;

s_F_s3i=find $\left(\bar{a}_{-} i(1,:)<d_{-} 3 i(1, \overline{:})\right)$;

F_s3i(1,s_F_s3i) $=f$ s3i( $\overline{1}, s_{-}$F_s3i).*A_s3i(1,s_F_s3i)/1000;

- Calculate $\mathrm{P} i$ and $\mathrm{M} i, \mathrm{P}$ oi and $\mathrm{P}$ ti

P_i $(1,:)=\operatorname{Prof}_{-}^{-} i(1,:) \cdot \bar{\star}\left(C_{-} C \bar{i}(1,:)+F_{-} \bar{s}_{1} i(1,:)+F_{-} s 2 i(1,:)+F_{-} s 3 i(1,:)\right)$;

$\operatorname{Mi}(1,:)=\operatorname{Prof} i(1,:) . *(\mathrm{C} \mathrm{Ci}(1,:) . *(\mathrm{~h} i(1,:) / 2-\mathrm{a} i(1,:) / 2)+\mathrm{F} \operatorname{sil}(1,:) . *(\mathrm{~h} i(1,:) / 2-\mathrm{d} 1 \mathrm{i}(1,:))+\ldots$

F_s2 $\left.\left.\bar{i}(1,:) \cdot{ }^{*}\left(h \_\bar{i}(1,:) / 2-d \_2 \bar{i}(1,:)\right)+F \_s \overline{3} i(1,:) \cdot *\left(h+\bar{i}(1,:) / 2-d \_3 i \overline{(1},:\right)\right)\right) / 100 \overline{0}$

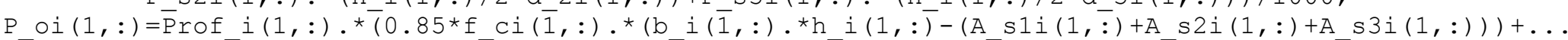

f_yi $(1,:) \cdot *($ A_s1i $(1, \overline{:})+$ A_s2i $(1,: \overline{)}+$ A_s3i( $1, \overline{:}))) / 1000$;

P_ti $(1,:)=-\overline{P r o f} i(1,:) .{ }^{\star} f_{-} y i(1,:) .{ }^{\star}\left(A_{-} \operatorname{si}(1,:)+A_{-} s 2 i(1,:)+A_{-} s 3 i(1,:)\right) / 1000$; 
\% Calculate eccentricities, e_i, and hovere_i e_i $(1,:)=M_{i} i(1,:)$. / $P_{-} i(1,:) ; \bar{\circ}(\mathrm{m})$

hovere $i(1,:)=(h(1,1) / 1000) . / e i(1,:)$; $\frac{\circ}{0}$ Use nominal $h$

end

\section{C.1.2.6 Code 6-Function of Simulated Load Effects and Nominal Values Based on ACI 318-14}

o Load effect simulation

\% (nominal loads calculation is based on design strengths corresponding to ACI 318-14)

function [D curi, L curi, T Di, T Li]=LoadEffectSim cur S1 (LoverD, n)

o Load design strengths

load alpha_PM_S1.mat phiP_n

\% Nominal values

$\div$ Loads

$\therefore$ LoverD $=\left[\begin{array}{ll}0.5 & 1.5\end{array}\right]$; $\%$ Input of funtion

D cur=abs (phiP n)/(1.2+1.6*LoverD); $\%$ Use absolute values

L_cur=LoverD*D_cur;

o Transformations from load to load effect

$\mathrm{T} D=1$;

$\mathrm{T}_{-} \mathrm{L}=1$;

- Statistical parameters (Bias coefficient and CoV)

ㄴoads

bias $D=1.05$;

$\mathrm{COV}_{-} \overline{\mathrm{D}}=0.10$;

bias $\mathrm{L}=1.00$;

$\mathrm{COV} \mathrm{L}=0.25$;

- Transformations from load to load effect

o bias_T_D $=1$; $\frac{\circ}{\circ}$ Effect is accounted in D

$\mathrm{COV}$ T $\overline{\mathrm{D}}=0$; 
$\circ$ bias_T_L=1; $\frac{\circ}{\circ}$ Effect is accounted in $\mathrm{L}$

$\therefore \mathrm{COV}_{-} \overline{\mathrm{T}}_{-} \overline{\mathrm{L}}_{\mathrm{C}} \mathrm{O}$;

\% Statistical parameters (Mean and Standard deviation)

o Loads

mean D cur=D cur*bias D;

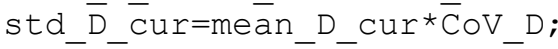

mean_L_cur=L_cur*bias_L;

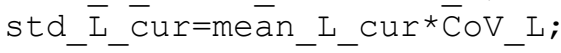

\% Transformations from load to load effect

$\div \mathrm{T}$ D Effect is accounted in D

$\div T^{-} L$ Effect is accounted in I

\% Simulation

- Preallocation

D_curi=zeros $\left(8, \operatorname{size}\left(D_{-} \operatorname{cur}, 2\right), \mathrm{n}\right)$;

alpha L cur=zeros $(8, \operatorname{size}(\mathrm{D} \operatorname{cur}, 2))$;

mu_L_cur $=$ zeros $\left(8, \operatorname{size}\left(D_{-}\right.\right.$cur, 2$\left.)\right)$;

L_curi=zeros $\left(8, \operatorname{size}\left(D_{\text {_cur }}, 2\right), \mathrm{n}\right)$;

for $i 1=1: 8$

for i $4=1$ :size (D_cur, 2)

rn n $11=\operatorname{rand} \bar{n}(1,1, n) ;$ o standard normally distributed random numbers

rn $u l=r a n d(1,1, n)$; $\frac{\circ}{\circ}$ Standard uniformly distributed random numbers

Dead Loads

D_curi(i1,i4,:)=mean_D_cur(i1,i4)+std_D_cur(i1,i4)*rn_n11; \% Normal distribution

o Live Loads

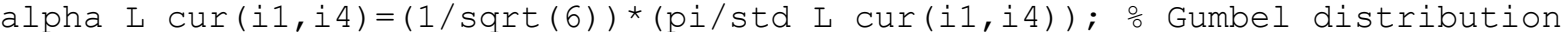

mu_L_cur $(i 1, i 4)=$ mean_L_cur $(i 1, i 4)-0.57 \overline{7} 2 \bar{T}$ alpha_L_cur $(i 1, i 4)$;

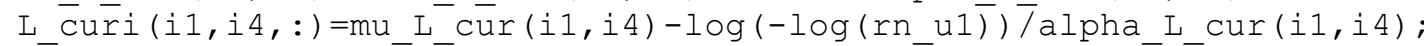


S_negcur=find (phiP_n $(1,:)<0)$;

$\mathrm{D}$ curi(:, s negcur, :) =-D curi (:, s negcur, :); 응 Negative values indicate tension

L_curi (:,s_negcur, : )=-L_curi (:, s_negcur, : ) ;

\% Transformations from load to load effect

$\mathrm{T}$ Di=T D; $\frac{\circ}{0}$ Effect is accounted in D

$\mathrm{T}^{-} \mathrm{Li}=\mathrm{T}_{-}^{-} \mathrm{L} ; \%$ Effect is accounted in $\mathrm{L}$

end

\section{C.1.2.7 Code 7-Function of Simulated Load Effects and Nominal Values Based on Partial Material Strength Reduction Factors}

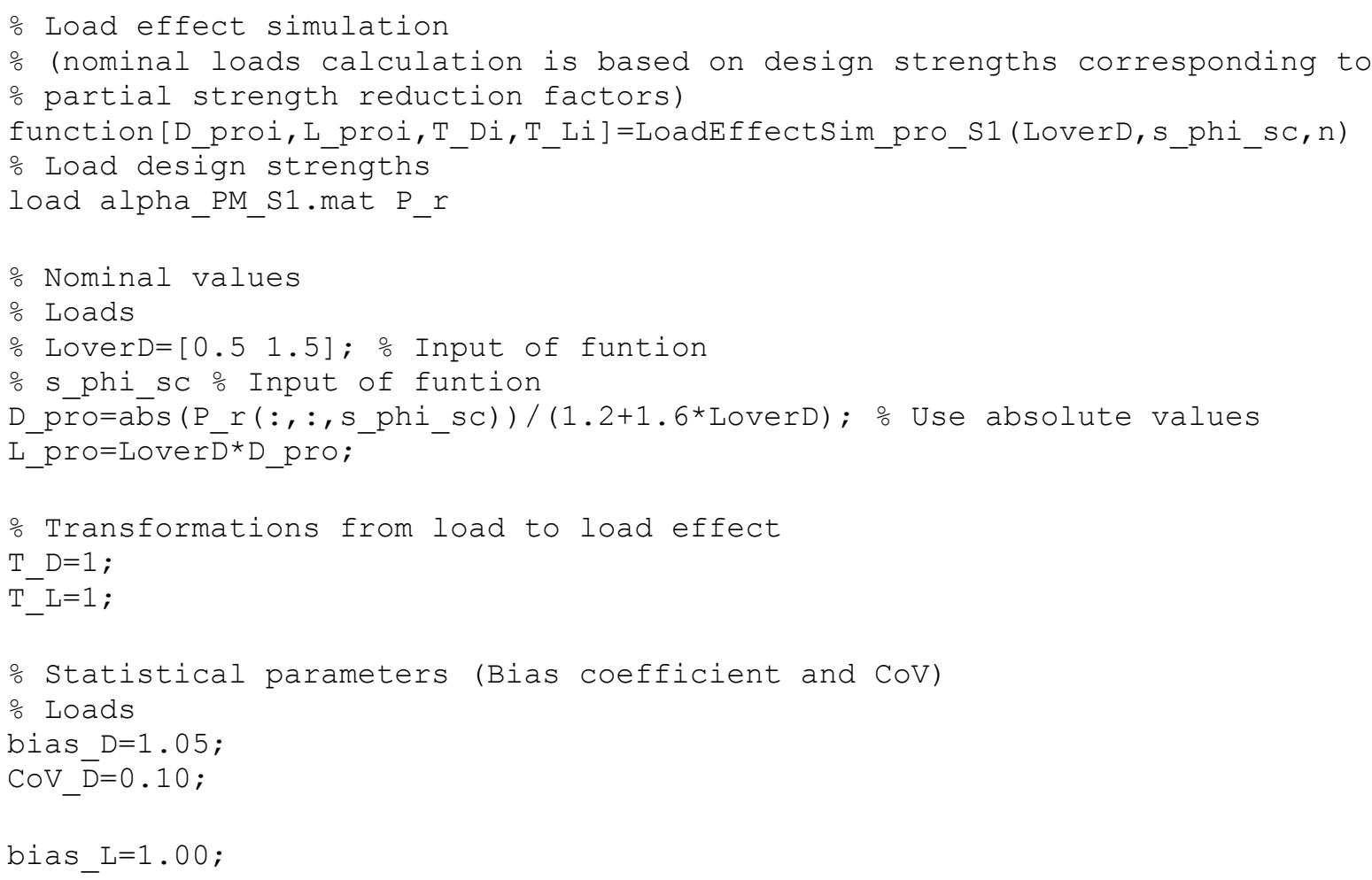


COV_L $=0.25$;

\% Transformations from load to load effect

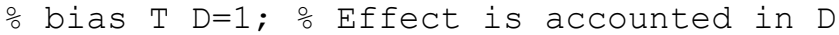

$\circ$ CoV_T ${ }_{-} \bar{D}=0$;

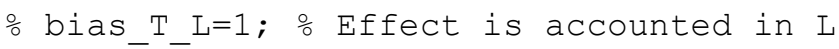

$\circ \mathrm{CoV}_{-}{ }_{-}{ }_{-} \overline{\mathrm{L}}=0$;

\% Statistical parameters (Mean and Standard deviation)

ㄴoads

mean D pro=D pro*bias D;

std_D_pro=mean_D_pro* $\bar{C} \circ V_{-}$;

mean_L_pro=L_pro*bias_L;

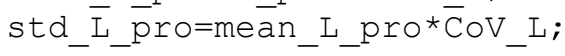

\% Transformations from load to load effect

$\div$ T D Effect is accounted in $D$

$\div T_{-}^{-}$E Effect is accounted in $L$

- Simulation

- Preallocation

D_proi=zeros (8,size (D_pro, 2), n);

alpha L pro=zeros $(8, \operatorname{size}(\mathrm{D}$ pro, 2$))$;

mu_L_pro $=\operatorname{zeros}\left(8, \operatorname{size}\left(D \_p r o, 2\right)\right)$;

L_proi=zeros (8, size (D_pro, 2), n);

for $i 1=1: 8$

for i4=1:size (D_pro, 2)

rn_nll=randn $(1,1, n)$; $\frac{\circ}{\circ}$ Standard normally distributed random numbers

rn $u 1=r a n d(1,1, n)$; $\frac{o}{\circ}$ Standard uniformly distributed random numbers

\% Dead Loads

D_proi $(i 1, i 4,:)=$ mean_D_pro(i1,i4)+std_D_pro(i1,i4)*rn_n11; \% Normal distribution 


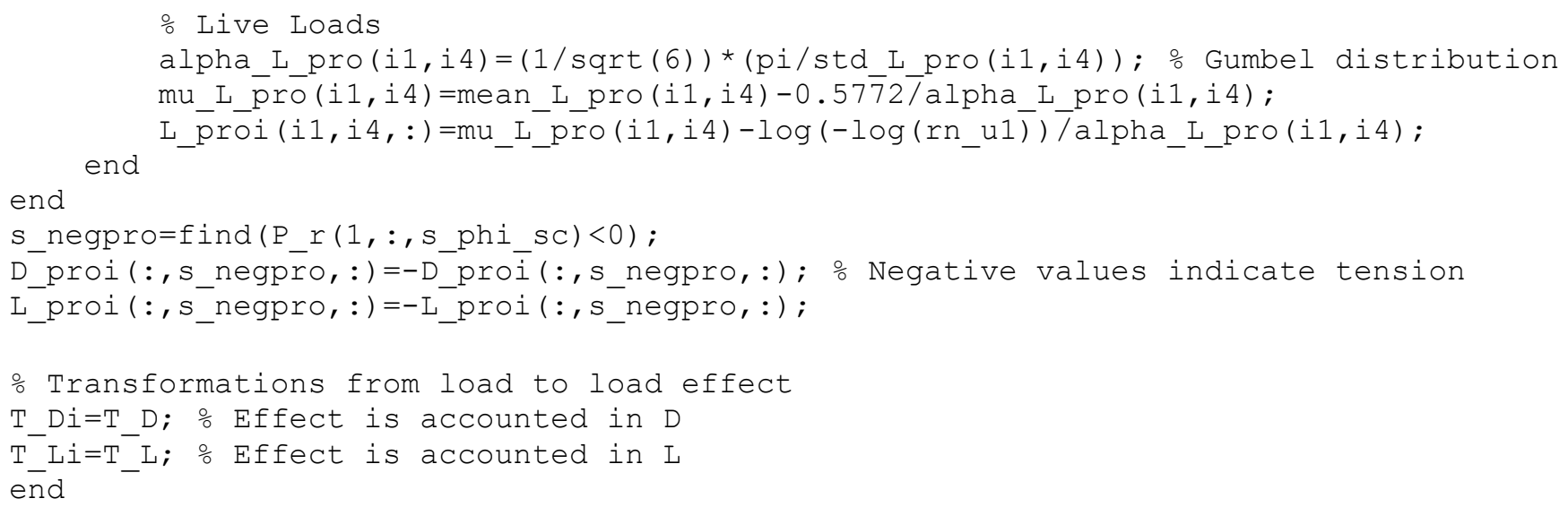

\section{C.1.3 Column Section 5}

\section{C.1.3.1 Code 1-Reliability Indices for $\mathrm{ACl} 318-14$ and $L / D=0.5$}

ClC

clear

tic

o Reliability index calculation corresponding to ACI 318-14 and L/D=0.5 $\mathrm{n}=1 \mathrm{e} 4$;

$\mathrm{N}=1 \mathrm{e} 6$;

Z_sam $=[0.5:-0.01:-1-1.05:-0.05:-10-10.1:-0.1:-50-51:-1:-100]$;

eoverh $=\left[\begin{array}{lllllllllllllllllllllllllllll}0.1 & 0.2 & 0.3 & 0.4 & 0.5 & 0.6 & 0.7 & 0.8 & 0.9 & 1.0 & 2.0 & 3.0 & 4.0 & 5.0 & 6.0 & 7.0 & 8.0 & 9.0 & 10.0 & \ldots\end{array}\right.$ $-10.0-5.0-1.0-0.5-0.1] ; \%$ The specific e/h values

hovere $=1$. / eoverh;

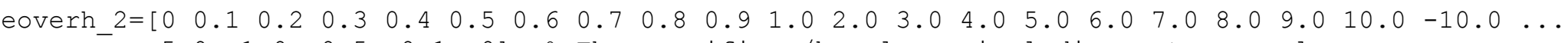
Lover $\mathrm{D}=0.5$ $-5.0-1.0-0.5-0.1-0] ;$ o The specific e/h values, including extreme values

- Preallocation

n_fcurl=zeros $(8$, length (eoverh_2), (N/n)); 
for $i 6=1:(\mathrm{N} / \mathrm{n})$

- Calculate sample points for resistance

을

o Préallocation

P isam=zeros $(8, \mathrm{n}$, length (Z_sam) );

$M_{-}^{-}$isam=zeros $\left(8, n\right.$, length $\left(z_{-}^{-}\right.$sam $\left.)\right)$;

hovere isam=zeros $(8, \mathrm{n}$, length $(Z$ sam $))$ :

P_oi=zéros $(8, \mathrm{n}, 1)$;

P_ti=zeros $(8, n, 1)$;

for $i 1=1: 8$

rn_n $1=\operatorname{randn}(1, \mathrm{n})$;

rn_n2=randn $(1, n)$;

$r n$ n $3=r a n d n ~(1, n)$;

rn_n $4=\operatorname{randn}(1, n)$;

rn $\mathrm{n} 5=\operatorname{randn}(1, \mathrm{n})$

rn_n6=randn $(1, n)$;

rn n $7=\operatorname{randn}(1, \mathrm{n})$;

$r n$ n $8=\operatorname{randn}(1, n)$;

rn_n9=randn $(1, n)$;

rn $\mathrm{n} 10=\operatorname{randn}(1, \mathrm{n})$;

rn_n11=randn $(1, n)$;

rn_n12=randn $(1, n)$;

rn $\mathrm{n} 13=\operatorname{randn}(1, \mathrm{n})$;

$f_{-} \bar{y}=420$;

bias $f y=1.125$;

$\mathrm{CoV}_{\overline{\mathrm{f}}} \overline{\mathrm{y}}=0.098$;

meañ $\bar{f} \_y=f \_y * b i a s \_f \_y$;

std_f_y $=m e a n \_f \_{ }^{*} \bar{C} \circ V_{-} f \_y$;

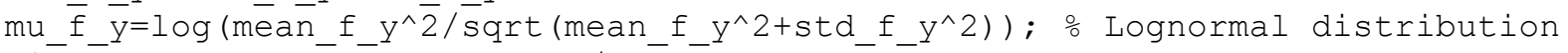

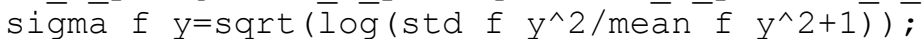

$f_{-} y i(\overline{1}, \overline{:})=\operatorname{logn} r n d\left(m_{-} f_{-} y, \operatorname{sigma}{ }_{-} \_\bar{y},[1, n]\right)$;

for i3=1: length $(Z$ sam $)$

[P_isam(i1, : i 3$),$ M isam(i1, :,i3), hovere isam(i1, :,i3), P oi (i1, : , 1), P ti (i1, :, 1) ] . .

=feval ('ResistanceSim_S5', i1,rn_n1, $r \mathrm{rn} n 2, r n \_n 3, r n \_n 4, r n \_n 5, r n \_n 6, r n \_n 7, r n \_n 8, r n \_n 9, \ldots$ rn_n10,rn_n11,rn_n12,rn_n13,f_yi,z_sam(i3)); 
end

end

\% Calculate $\mathrm{P}$ maxi

P_maxi $=0.85 * P_{-}^{-}$oi;

- Permute the 2nd and 3rd dimensions

$\mathrm{P}$ isam=permute ( $\mathrm{P}$ isam, $[1,3,2])$;

$M_{-}$is sam=permute $\left(M_{-}\right.$isam, $\left.[1,3,2]\right)$;

hovere isam=permute (hovere isam, $[1,3,2])$;

P oi=permute (P_oi, $[1,3,2])$;

$\mathrm{P}_{-} \mathrm{ti}=\operatorname{permute}\left(\mathrm{P}_{-} \mathrm{ti},[1,3,2]\right)$;

$\mathrm{P}$ maxi=permute $(\mathrm{P} \operatorname{maxi},[1,3,2])$;

\% Calculate load effect

o Lover $\mathrm{D}=0.5$; $\frac{\circ}{\circ}$ Defined previously

[D_curli,L_curli,T_Di,T_Li]=feval ('LoadEffectSim_cur_S5' , LoverD, n) ;

- Interpolation

- Calculate the unknown points ( $P_{-} i$ )

- Preallocation

P_i=zeros $(8$, length (hovere), n);

for $i 1=1: 8$

for $i 5=1: n$

S_M_isam=find (M_isam $(i 1,:$, i5) $>0)$;

P_isampri=P_isam(i1, s_M_isam,i5);

hovere isampri=hovere isam(i1, s M isam,i5):

P isampri=[P oi (i1, 1, $\bar{i} 5)$ P isampri $\mathrm{P}$ ti(i1, 1, i5)];

hovere isampri=[1e10 hovere isampri -le10];

[hovere isampris, I hovere isampri]=sort (hovere isampri, 'descend');

P_i(il,, i5) =interpi (hovere_isampris, P_isampri(I_hovere_isampri), hovere, 'linear');

${ }^{-} \mathrm{P} \operatorname{maxi}=\mathrm{find}(\mathrm{P} i(\mathrm{i} 1$, :,$i 5)>\overline{\mathrm{P}} \operatorname{maxi}(i 1,1, i 5)$ )

$P_{-} i\left(i 1, s_{-} P_{-} m a x i, i 5\right)=P_{-} \operatorname{maxi}(i \overline{1}, 1, i 5)$;

end 


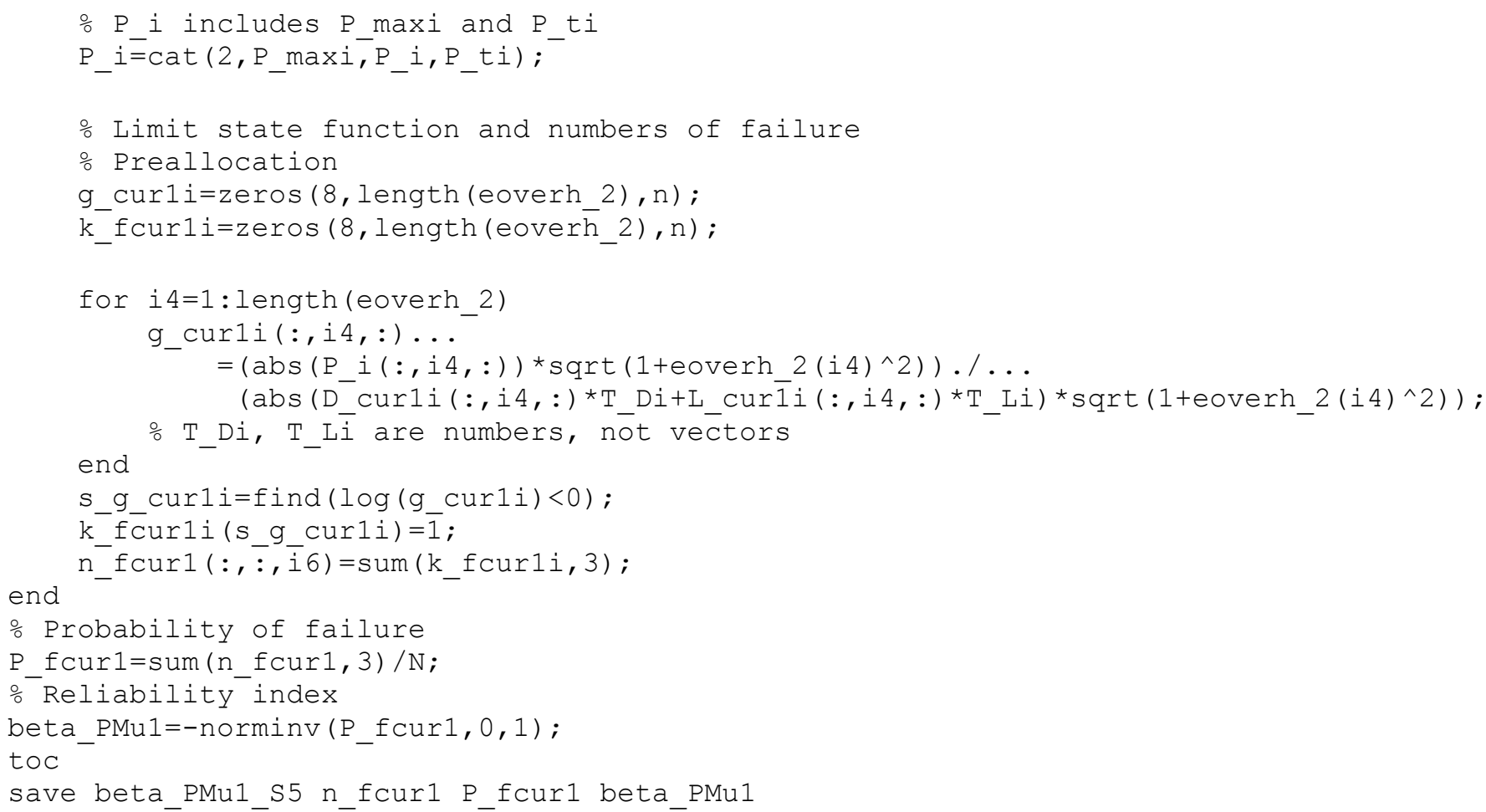

\section{C.1.3.2 Code 2-Reliability Indices for $\mathrm{ACI} 318-14$ and $L / D=1.5$}

ClC

clear

tic

o Reliability index calculation corresponding to ACI 318-14 and L/D=1.5

$\mathrm{n}=1 \mathrm{e} 4$;

$\mathrm{N}=1$ e 6 ;

Z_sam $=[0.5:-0.01:-1-1.05:-0.05:-10-10.1:-0.1:-50-51:-1:-100]$;

éverh $=\left[\begin{array}{llllllllllllllllllllllllll}0.1 & 0.2 & 0.3 & 0.4 & 0.5 & 0.6 & 0.7 & 0.8 & 0.9 & 1.0 & 2.0 & 3.0 & 4.0 & 5.0 & 6.0 & 7.0 & 8.0 & 9.0 & 10.0 & \ldots\end{array}\right.$ $-10.0-5.0-1.0-0.5-0.1] ; \%$ The specific e/h values 
hovere=1. / eoverh;

eoverh_2 $=\left[\begin{array}{llllllllllllllllllllllllllll}0 & 0.1 & 0.2 & 0.3 & 0.4 & 0.5 & 0.6 & 0.7 & 0.8 & 0.9 & 1.0 & 2.0 & 3.0 & 4.0 & 5.0 & 6.0 & 7.0 & 8.0 & 9.0 & 10.0 & -10.0 & \ldots\end{array}\right.$ $-5.0-1.0-0.5-0.1-0]$; $\frac{0}{0}$ The specific e/h values, including extreme values

Lover $=1.5$

- Preallocation

n_fcur2=zeros $(8$, length (eoverh_2), (N/n));

for $i 6=1:(\mathrm{N} / \mathrm{n})$

\% Calculate sample points for resistance

o (P_isam, M_isam and hovere_isam corresponding to Z_sam, P_oi and P_ti)

o Preallocation

P isam=zeros $(8, \mathrm{n}$, length ( $\mathrm{Z}$ sam) );

$\mathrm{M}^{-}$isam $=\operatorname{zeros}\left(8, \mathrm{n}\right.$, length $\left(\mathrm{z}^{-}\right.$sam $\left.)\right)$;

hovere isam=zeros $(8, \mathrm{n}$, length (Z_sam) );

$\mathrm{P}$ oi=zeros $(8, \mathrm{n}, 1)$;

$\mathrm{P}_{-}^{-} \mathrm{ti}=\operatorname{zeros}(8, \mathrm{n}, 1)$;

for $i 1=1: 8$

rn $n 1=r a n d n(1, n)$;

$r \mathrm{n}$-n2 $=\operatorname{randn}(1, \mathrm{n})$;

rn $\mathrm{n} 3=\operatorname{randn}(1, \mathrm{n})$

rn_n $4=\operatorname{randn}(1, \mathrm{n})$;

$r n+n 5=\operatorname{randn}(1, \mathrm{n})$

rn $\mathrm{n} 6=\operatorname{randn}(1, \mathrm{n})$;

rn_n $7=\operatorname{randn}(1, \mathrm{n})$;

$\mathrm{rn} \mathrm{n} 8=\operatorname{randn}(1, \mathrm{n})$

$r n \_n 9=\operatorname{randn}(1, \mathrm{n})$;

$r n \_n 10=\operatorname{randn}(1, \mathrm{n})$;

rn $\mathrm{n} 11=\operatorname{randn}(1, \mathrm{n})$

rn_n12=randn $(1, \mathrm{n})$;

rn $\mathrm{n} 13=\operatorname{randn}(1, \mathrm{n})$;

$f_{-y}=420$;

bias f $y=1.125$;

CoV $\bar{f} \bar{y}=0.098$;

meañ $\bar{f}_{-} y=f \_y * b i a s \_f \_y ;$

std $\bar{f} \bar{y}=m e \overline{a n} f y^{\star} \bar{C} \circ \bar{V} f y ;$ 
mu_f_y=log (mean_f_y^2/sqrt (mean_f_y^2+std_f_y^2)); 응 Lognormal distribution sigma f $y=\operatorname{sqrt}\left(\log \left(\right.\right.$ std $f y^{\wedge} 2 /$ mean $\left.\left.f y^{\wedge} 2+1\right)\right)$

f_yi $\left.(\overline{1}, \overline{:})=\operatorname{lognrnd}\left(\mathrm{mu}_{-} \mathrm{f}_{-} \mathrm{y}, \mathrm{sigma} \mathrm{f}_{-} \overline{\mathrm{y}}, \overline{[} \overline{1}, \mathrm{n}\right]\right)$;

for i3=1: length $(Z$ Zsam)

[P isam(i1, : i3), M isam(i1, : i3), hovere isam(i1, : i3), P oi (i1, : 1), P ti (i1, : 1)] ...

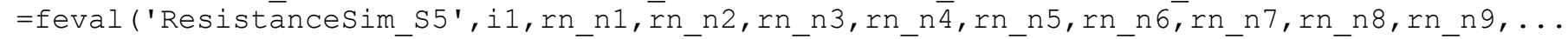
rn_n10,rn_n11,rn_n12,rn_n13,f_yi,z_sam(i3));

end

end

o Calculate P_maxi

$P \operatorname{maxi}=0.85 * \mathrm{P} \_i$

\% Permute the 2nd and 3rd dimensions

$P_{\text {_isam }}$ permute $\left(\mathrm{P}_{-}\right.$isam, $\left.[1,3,2]\right)$;

$\mathrm{M}^{-}$isam=permute $\left(\mathrm{M}^{-}\right.$isam, $\left.[1,3,2]\right)$;

hovere_isam=permute (hovere_isam, $[1,3,2])$;

P_oi=permute (P_oi, $[1,3,2])$;

P ti=permute (P ti, $[1,3,2])$;

$\mathrm{P}_{-}$maxi=permute (P_maxi, $\left.[1,3,2]\right)$;

- Calculate load effect

ㄴover $\mathrm{D}=1.5$; $\frac{\circ}{0}$ Defined previously

[D_cur2i,L_cur2i,T_Di, T_Li]=feval ('LoadEffectSim_cur_S5 ', LoverD, n) ;

- Interpolation

- Calculate the unknown points ( $P_{-}$i)

o Preallocation

P_i=zeros $(8$, length (hovere), n);

for $i 1=1: 8$

for $i 5=1: n$

S_M_isam=find (M_isam $(i 1,:$, i5) $>0)$;

$\mathrm{P}$ isampri=P isam(i1, S M isam,i5);

hovere_isamp̄i=hovere_is̄am(i1,s_M_isam,i5); 


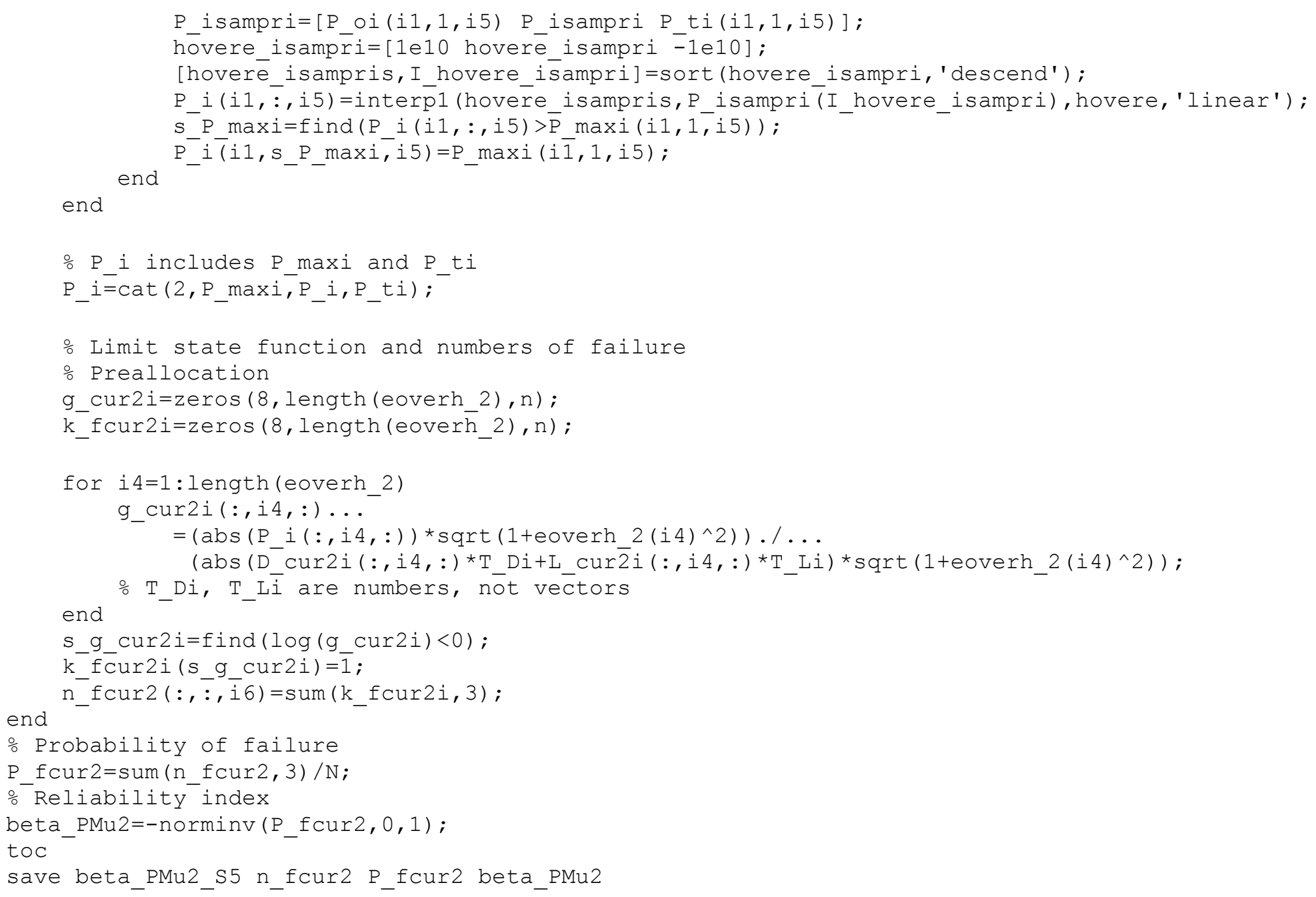

C.1.3.3 Code 3-Reliability Indices for Partial Material Strength Reduction Factors and $L / D=0.5$ 
clear

tic

\% Reliability index calculation corresponding to partial strength reduction factors and L/D=0.5

$\mathrm{n}=1 \mathrm{e} 4$;

$\mathrm{N}=1 \mathrm{e} 6$;

Z_sam $=[0.5:-0.01:-1-1.05:-0.05:-10-10.1:-0.1:-50-51:-1:-100]$;

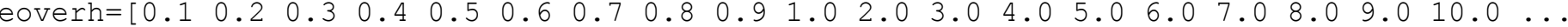

$-10.0-5.0-1.0-0.5-0.1] ; \div$ The specific e/h values

hovere=1./eoverh;

eoverh_2 $=\left[\begin{array}{llllllllllllllllllllllll}0 & 0.1 & 0.2 & 0.3 & 0.4 & 0.5 & 0.6 & 0.7 & 0.8 & 0.9 & 1.0 & 2.0 & 3.0 & 4.0 & 5.0 & 6.0 & 7.0 & 8.0 & 9.0 & 10.0 & -10.0 & \ldots\end{array}\right.$ $-5.0-1.0-0.5-0.1-0]$; $\frac{\circ}{0}$ The specific e/h values, including extreme values

Lover $\mathrm{D}=0.5$

S_phi_sC=15;

o Preallocation

n fprol=zeros $(8$, length (eoverh 2$),(\mathrm{N} / \mathrm{n}))$;

for $i 6=1:(\mathrm{N} / \mathrm{n})$

- Calculate sample points for resistance

\% (P_isam, M_isam and hovere_isam corresponding to Z_sam, P_oi and P_ti)

o Préallocation

P isam=zeros $(8, \mathrm{n}$, length $(Z$ _sam $))$;

$\mathrm{M}$ isam=zeros $(8, \mathrm{n}$, length ( $\mathrm{Z}$ sam $))$;

hovere_isam=zeros $\left(8, \mathrm{n}\right.$, length $\left.\left(Z_{\text {__sam }}\right)\right)$;

P_oi=zeros $(8, \mathrm{n}, 1)$;

P_ti=zeros $(8, n, 1)$;

\section{for $i 1=1: 8$}

$\mathrm{rn} n 1=\operatorname{randn}(1, \mathrm{n})$;

$r n n 2=r a n d n(1, n)$;

rn_n $3=\operatorname{randn}(1, \mathrm{n})$;

rn $\mathrm{n} 4=\operatorname{randn}(1, \mathrm{n})$;

rn_n $5=\operatorname{randn}(1, \mathrm{n})$;

$r n-n 6=\operatorname{randn}(1, n)$;

rn $\mathrm{n} 7=\operatorname{randn}(1, \mathrm{n})$.

rn_n $8=\operatorname{randn}(1, \mathrm{n})$;

rn $n 9=\operatorname{randn}(1, n)$; 
rn_n10=randn $(1, \mathrm{n})$;

rn n $11=$ randn $(1, n)$;

rn_n12=randn $(1, \mathrm{n})$;

rn $\mathrm{n} 13=\operatorname{randn}(1, \mathrm{n})$;

$\mathrm{f}_{-\bar{y}}=420$;

bias_f_y $=1.125$;

$\mathrm{CoV} \overline{\mathrm{f}} \overline{\mathrm{y}}=0.098$;

mean_f_y $\bar{f}_{-}{ }^{*}{ }^{\star}$ bias_f_y;

std_f $\overline{\mathrm{y}}=$ mean_f_y ${ }^{*} \overline{\mathrm{C}} \circ \overline{\mathrm{V}}{ }_{\mathrm{f}} \mathrm{y}$;

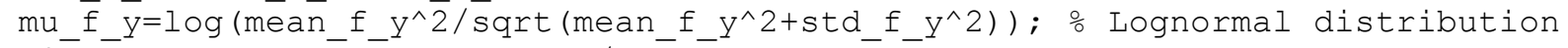

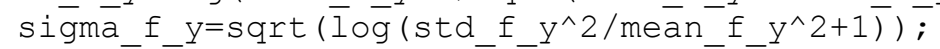

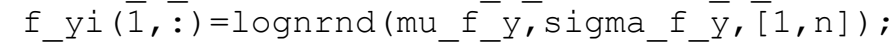

for i3=1: length (Z_sam)

[P_isam(i1, : i i3), M_isam(i1, : i3), hovere_isam(i1, : i3), P_oi $\left.(i 1,:, 1), P_{-} t i(i 1,:, 1)\right] \ldots$

=feval ('Resistāncesim_s5',il,rn_n1, rn_n2, rn_n3,rn_n4, rn_n5, rn_n6, rn_n7, rn_n8,rn_n9, . . .

end

\section{end} rn_n10,rn_n11,rn_n12,rn_n13,f_zyi,z_sam(i3)) ;

o Calculate P maxi

$\mathrm{P} \operatorname{maxi}=0.85 * \mathrm{P}^{-} \mathrm{oi}$;

- Permute the 2nd and 3rd dimensions

$\mathrm{P}$ isam=permute ( $\mathrm{P}$ isam, $[1,3,2])$;

$\mathrm{M}^{-}$isam=permute $\left(\mathrm{M}^{-}\right.$isam, $\left.[1,3,2]\right)$;

hovere isam=permute (hovere isam, $[1,3,2])$;

P_oi=permute (P_oi, $[1,3,2])$;

$\mathrm{P}^{-}$ti=permute $\left(\mathrm{P}^{-} \mathrm{ti},[1,3,2]\right)$;

P_maxi=permute (P_maxi, $[1,3,2])$;

- Calculate load effect

ㄴ Lover $D=0.5$; 응 Defined previously

\% s_phi_sc 응 Defined previously

[D_prolī,L_proli,T_Di,T_Li]=feval ('LoadEffectSim_pro_S5 ', LoverD,s_phi_sc, n) ;

ㅇterpolation 
- Calculate the unknown points ( $\mathrm{P}_{-}$i)

- Preallocation

P_i=zeros ( 8 , length (hovere), n);

for $i 1=1: 8$

for $i 5=1: n$

S_M_isam=find (M_isam (i1, : i5) >0);

P_isampri=P_isam(i1, s_M_isam, i5);

hovere isampri=hovere isam(i1, s M isam, i5);

P_isampri=[P_oi(i1, 1, í5) P_isampri $\bar{i}$ P_ti(i1, 1,i5) ];

hovere isampri=[1e10 hoverēe isampri -1e10];

[hovere_isampris, I_hovere_isampri] =sort (hovere_isampri, 'descend');

P_i (i1,, , i5)=interp̄ (hovere_isampris, P_isampri(I_hovere_isampri), hovere, 'linear');

S_P maxi=find ( $P_{-} i(i 1,:$, i5) $>\bar{P} \operatorname{maxi}(i 1,1, i 5))$;

end

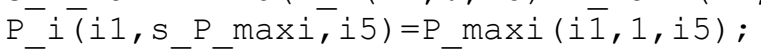

end

\% $P_{-} i$ includes $P_{-}$maxi and $P_{-} t i$

$P_{-} i=\operatorname{cat}\left(2, P_{-} \max i, P_{-} i, P_{-} t i\right)$;

Limit state function and numbers of failure

o Preallocation

9_proli=zeros $(8$, length (eoverh_2), n) ;

$\mathrm{k}$-fproli=zeros $(8$, length (eoverh_ 2$), \mathrm{n})$;

for i $4=1$ : length (eoverh_2)

g_proli(:,i4,:) ...

$=\left(\operatorname{abs}\left(P_{i} i(:, i 4,:)\right) * \operatorname{sqrt}\left(1+\right.\right.$ eoverh $\left.\left.2(i 4)^{\wedge} 2\right)\right) . / \ldots$

(abs (D_proli(:,i4,:)*T_Di+L_proli(: ,i4,:)*T_Li)*sqrt $(1+$ eoverh_2 (i4)^2));

end

․ T_Di, T_Li are numbers, not vectors

s_g_proli=find $(\log ($ g_proli) $<0)$;

k_fproli(s_g_proli) $=1$; end

n_fprol $(:, \bar{i}, \bar{i} 6)=\operatorname{sum}\left(k_{\text {f fproli }}, 3\right)$;

o Probability of failure 
P_fprol=sum(n_fpro1, 3) /N ;

o Reliability index

beta_PMr1=-norminv (P_fpro1, 0,1);

toc

save beta_PMr1_S5 s_phi_sc n_fpro1 P_fprol beta_PMr1

\section{C.1.3.4 Code 4-Reliability Indices for Partial Material Strength Reduction Factors and $L / D=1.5$}

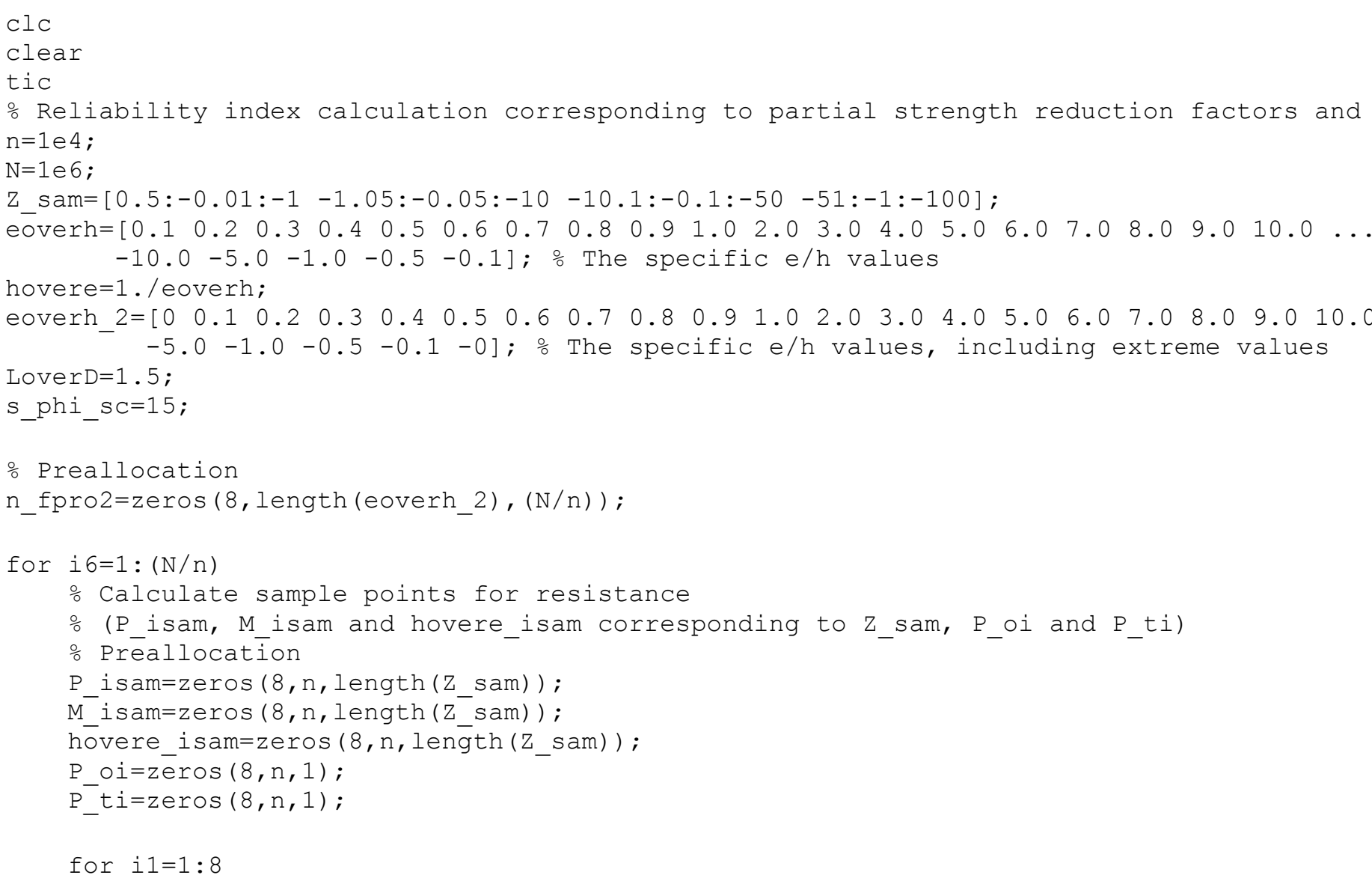




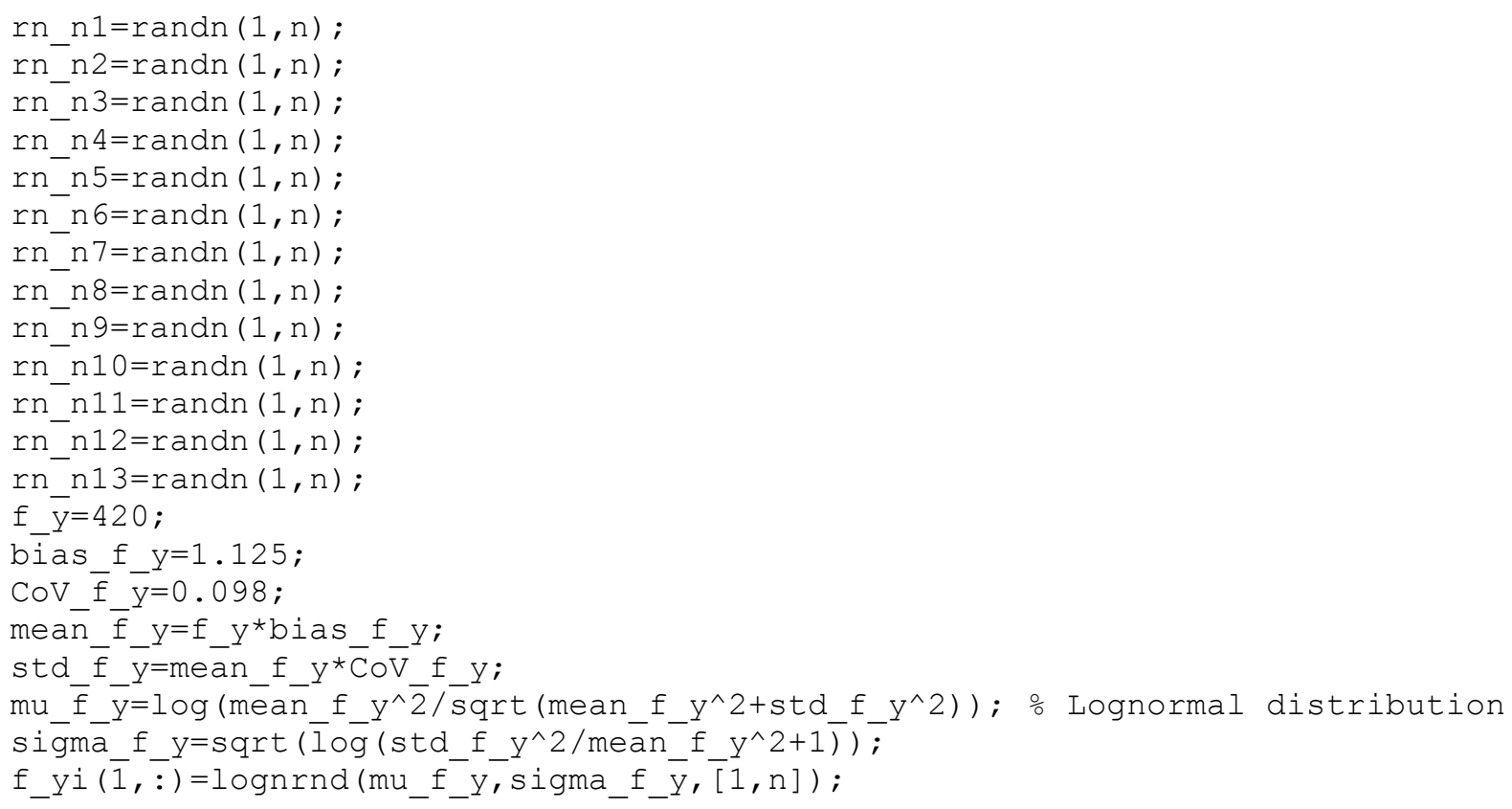

- Calculate $\mathrm{P}$ maxi

$\mathrm{P}_{\text {maxi }}=0.85 * \mathrm{P}_{\text {_oi }}$;

\% Permute the 2nd and 3rd dimensions

$\mathrm{P}$ isam=permute $\left(\mathrm{P}_{-}\right.$isam, $\left.[1,3,2]\right)$;

$M$ isam=permute (M isam, $[1,3,2])$;

hovere_isam=permüte (hovere_isam, $[1,3,2])$;

P_oi=pērmute (P_oi, $[1,3,2])$; 
P_ti=permute (P_ti, $[1,3,2])$;

P_maxi=permute (P_maxi, $[1,3,2])$;

- Calculate load effect

ㄴ Lover $\mathrm{D}=1.5$; $\frac{\circ}{\circ}$ Defined previously

\% s phi sc Defined previously

[D_pro2i, L_pro2i,T_Di,T_Li]=feval ('LoadEffectSim_pro_S5',LoverD, s_phi_sc, n) ;

O Interpolation

- Calculate the unknown points (P_i)

- Preallocation

P_i=zeros $(8$, length (hovere), n) ;

for $i 1=1: 8$

for $i 5=1: n$

S_M_isam=find (M_isam (i1, : i5) $>0)$;

$\mathrm{P}$ is $\bar{s}$ ampri=P isam(i1, s M isam, i5):

hovere_isampri=hovere_isam(i1,s_M_isam,i5);

P_isampri=[P_oi(i1, 1, i5) P_isamprí P ti(i1, 1,i5)] ;

hovere isampri=[1e10 hovere isampri-1e10];

[hoverēisampris, I_hovere_isampri]=sort (hovere_isampri, 'descend');

$\mathrm{P}$ i (i1, : i5)=interp1 (hovere isampris, P isampri(I hovere isampri), hovere, 'linear') ;

$S$ P maxi=find(P_i(i1, : i5) $>\bar{P} \operatorname{maxi}(i 1,1, i 5))$;

end

P_i(i1,s_P_maxi, 15$)=P_{\text {maxi }}(i \overline{1}, 1, i 5)$;

end

$\circ P_{-} i$ includes $P_{-}$maxi and $P_{-} t i$

P_i=cat (2,P_maxi,P_i,P_ti);

\% Limit state function and numbers of failure

- Preallocation

g pro2i=zeros ( 8 , length (eoverh 2), n);

k_fpro2i=zeros $(8$, length (eoverh_2), n) ;

for i4=1:length (eoverh_2) 
g_pro2i(:,i4,:) ...

$=\left(\operatorname{abs}\left(P_{i} i(:, i 4,:)\right) * \operatorname{sqrt}(1+\right.$ eoverh $\left.2(i 4) \wedge 2)\right) . / \ldots$

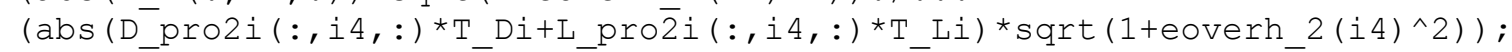

end

\% T Di, T L $\bar{i}$ are numbers, not vectors

s_g pro2i=find $(\log (g$ pro2i) $<0)$;

k_fpro2i (s_g_pro2i) $=1$;

end

$n_{-} \operatorname{fpro2}(:,, i, i 6)=\operatorname{sum}\left(k_{-} \operatorname{fpro} 2 i, 3\right)$;

- Probability of failure

P_fpro2=sum (n_fpro2,3)/N

: Reliability index

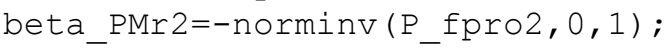

toc

save beta_PMr2_S5 s_phi_sc n_fpro2 P_fpro2 beta_PMr2

\section{C.1.3.5 Code 5-Function of Simulated Resistances}

- Resistance simulation

function [P_i,M_i, hovere_i, P_oi,P_ti]=ResistanceSim_S5 (i1,rn_n1,rn_n2,rn_n3,rn_n4,rn_n5,rn_n6,rn_n7,... $\left.r \bar{n} \_n 8, r n \_n 9, r n \_n 10, r n \_n \overline{1} 1, r n \_n 12, r n \_n 13, f_{-}^{-} y i, z\right)$

- Nominal value combinations

\% Geometric property combinations

h $\mathrm{com}=\left[\begin{array}{ll}325 & 1300\end{array}\right]$;

gamma_com $=\left[\begin{array}{lll}0.6 & 0.9\end{array}\right]$;

d_1_com $=(1+$ gamma_com $) . *$ h_com $/ 2$;

d $2 \mathrm{com}=\left(2+2^{\wedge} 0.5^{\star} \mathrm{gamma} \mathrm{com}\right) \cdot{ }^{\star} \mathrm{h} \mathrm{com} / 4$.

d_ 3 - $c o m=h \_c o m / 2$;

d $4{ }^{-} \mathrm{com}=\left(\overline{2}-2 \wedge 0.5{ }^{*} \mathrm{gamma} \mathrm{com}\right) .{ }^{*} \mathrm{~h} \mathrm{com} / 4$;

d_5_com $=(1-\mathrm{gamma} c \mathrm{com}) \cdot{ }^{\overline{ }} \mathrm{h} \_\mathrm{com} / 2 \overline{\text {; }}$

rho_g_com $=\left[\begin{array}{lll}0.01 & 0.04\end{array}\right]$;

- Material property combinations

f $\mathrm{C} C \mathrm{com}=\left[\begin{array}{ll}25 & 45\end{array}\right]$;

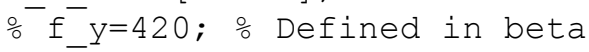

E $S=200000$; 
o Summarize property combinations in one matrix

pro_com $=\left[\mathrm{h} \_\right.$com (1) gamma_com(1) d_1_com(1) d_2_com(1) d_3_com(1) d_4_com(1) d_5_com(1) ... f_c_com (1) rho_g_com (1)

h_com(1) gamma_com(1) d_1_com(1) d_2_com(1) d_3_com(1) d_4_com(1) d_5_com(1) ... f_c_com (1) rho_g_com (2);

h_com (1) gamma_com(1) d_1_com(1) d_2_com(1) d_3_com(1) d_4_com(1) d_5_com(1) ... f_c_com (2) rho_g_com (1);

h_com (1) gamma_com(1) d_1_com(1) d_2_com(1) d_3_com(1) d_4_com(1) d_5_com(1) ... $\mathrm{f}^{-} \mathrm{C} \operatorname{com}(2) \mathrm{rho}^{-} \mathrm{g} \operatorname{com}(2)$;

h_com (2) gamma_com(2) d_1_com(2) d_2_com(2) d_3_com(2) d_4_com(2) d_5_com(2) ... f_c_com (1) rho_g_com (1) $\overline{\text {; }}$

h_com (2) gamma_com(2) d_1_com(2) d_2_com(2) d_3_com(2) d_4_com(2) d_5_com(2) ... f_c_com (1) rho_g_com (2) ;

h_com(2) gamma_com(2) d_1_com(2) d_2_com(2) d_3_com(2) d_4_com(2) d_5_com(2) ... f_c_com (2) rho_g_com (1);

$\mathrm{h}$-com (2) gamma_com(2) d_1_com(2) d_2_com(2) d_3_com(2) d_4_com(2) d_5_com(2) ...

f_c_com (2) rho_g_com (2)] ;

o Professional factor

Prof $=1$;

\% Nominal values

\% Geometric properties

$\mathrm{h}(1,1)=$ pro_com $(\mathrm{i} 1,1)$;

o $\operatorname{gamma}(1, \overline{1})=\operatorname{pro} \operatorname{com}(i 1,2)$;

d $1(1,1)=$ pro_com $(i 1,3)$;

d_2 $(1,1)=$ pro_com $(i 1,4)$;

d $3(1,1)=$ pro com $(i 1,5)$;

$\mathrm{d}^{-} 4(1,1)=$ pro_com $(i 1,6)$;

d $5(1,1)=$ pro $\operatorname{com}(i 1,7)$;

rho $g(1,1)=$ pro_com $(i 1,9)$;

A_st $(1,1)=r h o \_\bar{g}(1,1) * \mathrm{pi} * \mathrm{~h}(1,1)^{\wedge} 2 / 4$;

A $\sin (1,1)=A$ st $(1,1) / 8$;

$A_{-} s 2(1,1)=A_{-}$st $(1,1) / 4$;

A_s3 $(1,1)=$ A_st $(1,1) / 4$

A $s 4(1,1)=A$ st $(1,1) / 4$;

A_s $5(1,1)=A$ _st $(1,1) / 8$; 


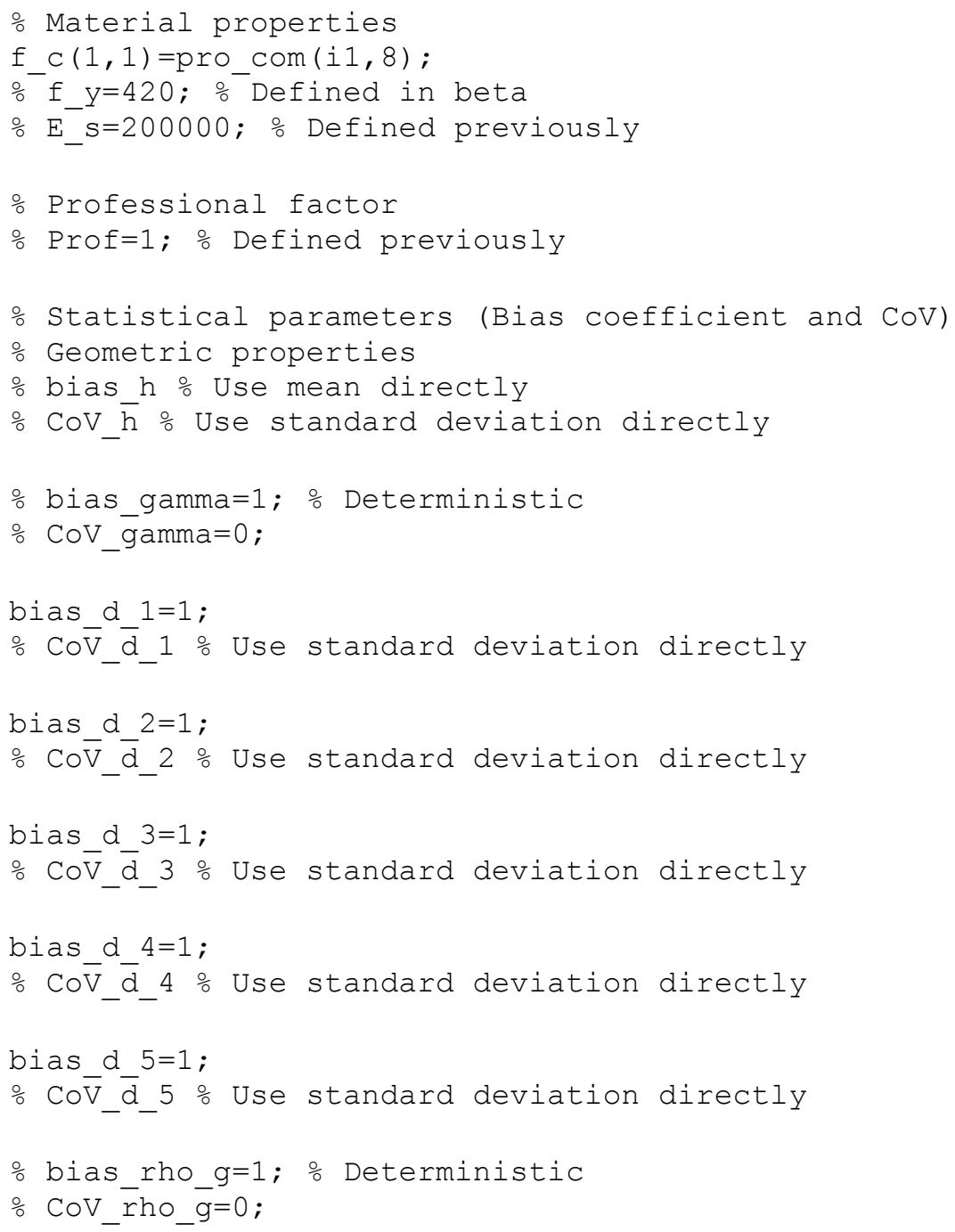




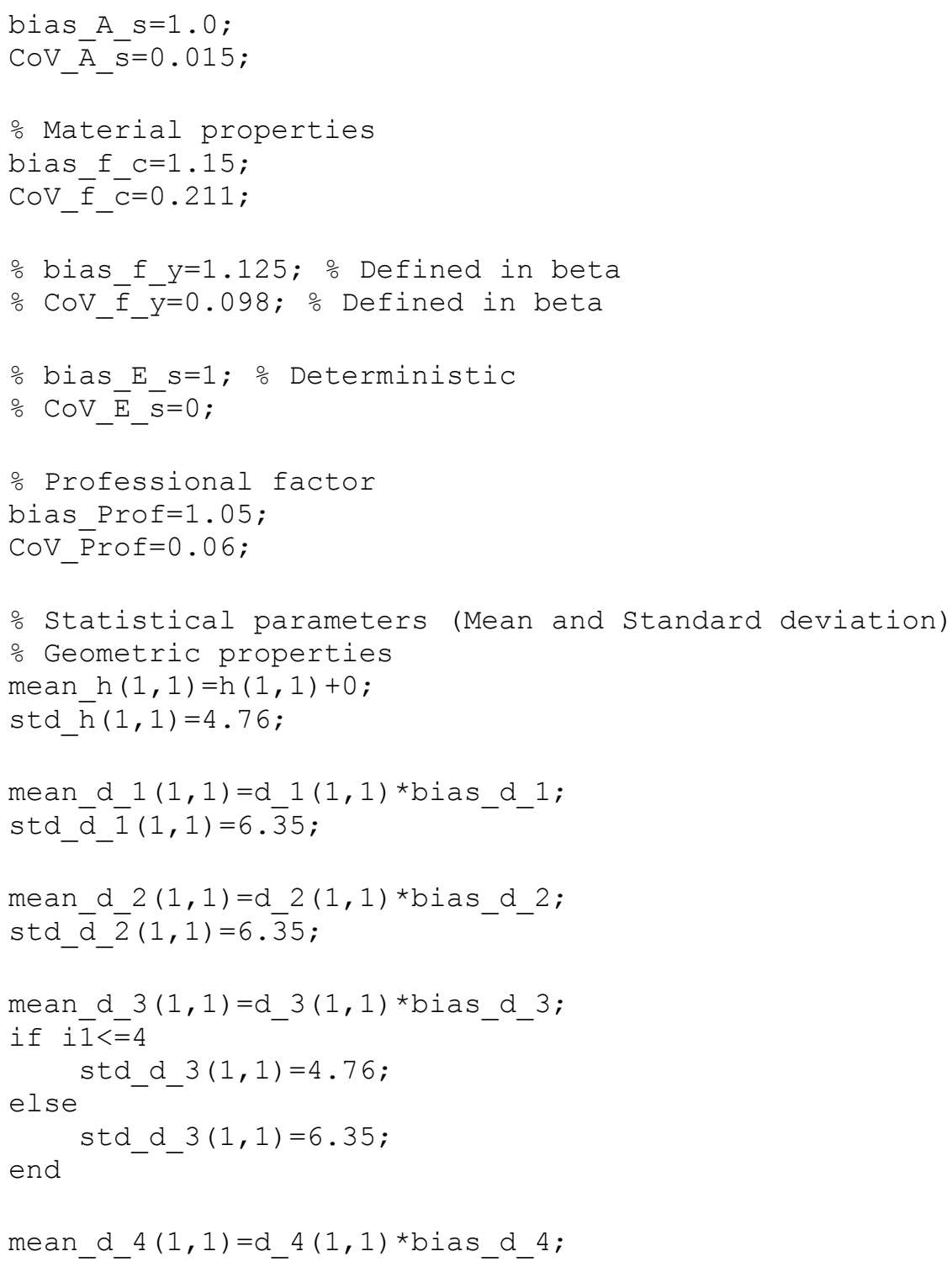


if $i 1<=4$

else

std_d_4 $(1,1)=4.76$;

end

std_d_4 $(1,1)=6.35$;

mean_d_5 $(1,1)=d \_5(1,1) *$ bias_d_5;

std_d ${ }_{-} \overline{5}(1,1)=4 . \overline{7} 6$;

mean A s $1(1,1)=A \_s 1(1,1) *$ bias_A_s;

std_A_ $\bar{A} \_1(1,1)=m e a n_{-} A_{-} s 1(1,1) * \bar{C} \circ \bar{V} \_A \_s ;$

mean_ $\bar{A} \_s 2(1,1)=A \_s \overline{2}(\overline{1}, 1) *$ bias_A_ $s$;

std_ $\bar{A} \_s 2(1,1)=$ mean_A_s2 $(1,1) * \bar{C} \circ \bar{V} \bar{V}_{-}{ }_{-} s$;

mean_ $\bar{A} \_s 3(1,1)=A \_s \overline{3}(\overline{1}, 1) *$ bias_A_s $\bar{s}$;

std_ $\bar{A} \_\bar{s} 3(1,1)=$ meàn_A_s $3(1,1) * \bar{C} \circ \bar{V} \_A \_s$;

mean_A_s4 $(1,1)=A_{-} s 4(\overline{1}, 1) *$ bias_A_s;

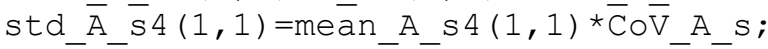

mean $\bar{A} s 5(1,1)=A$ s $5(1,1) *$ bias $A$ s;

std_ $\bar{A} \_s 5(1,1)=$ mean__A_s5 $(1,1) * \bar{C} \circ \bar{V} \_$A_s ;

\% Material properties

mean $f \mathrm{c}(1,1)=\mathrm{f} \mathrm{c}(1,1) *$ bias $\mathrm{f} c$;

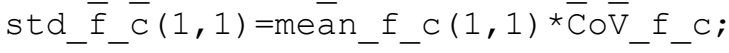

\% mean_f_y=f_y*bias_f_y; $\%$ Defined in beta

o std $\bar{f}_{-} \bar{y}=m e a n_{-}{ }_{-}{ }^{*}{ }^{*} \bar{C} \circ \bar{V}_{-}{ }_{-} \_y ; \circ$ Defined in beta

- Professional factor

mean_Prof=Prof*bias_Prof;

std_Prof $=$ mean_Prof $* \bar{C} \circ V_{-}$Prof;

\% Simulation

\% Geometric properties

$h \_i(1,:)=$ mean_h $(1,1)+$ std_h $(1,1) * r n \_n 1 ; \%$ Normal distribution

o- gamma $i(1,1)=\operatorname{gamma}(1,1)$; $\frac{\circ}{0}$ Deterministic

d_1i $(1, \overline{:})=$ mean_d_1 $(1,1)+$ std_d_1 $(1,1) * r n \_n 2$; $\%$ Normal distribution 
d_2i $(1,:)=$ mean_d_2 $(1,1)+$ std_d_2 $(1,1) * r n \_n 3 ; \%$ Normal distribution d $3 i(1,:)=$ mean d $3(1,1)+$ std d $3(1,1) * r n n 4$; 0 Normal distribution d $4 i(1,:)=$ mean d $4(1,1)+$ std d $^{-} 4(1,1) * r n-n 5$; $\%$ Normal distribution d $5 i(1,:)=$ mean d $5(1,1)+$ std_d_5 $(1,1) * r n \_n 6$; $\circ$ Normal distribution o-rho_gi $(1,1)=\overline{r h o} g(1,1)$; $\frac{0}{0}$ Deterministic

A_s1i $(1,:)=$ mean_A_s1 $(1,1)+$ std_A_s1 $(1,1) * r n \_n 7$; $\%$ Normal distribution A s2i $(1,:)=$ mean A s2 $(1,1)+$ std A s2 $(1,1)$ * rn n8; 응 Normal distribution

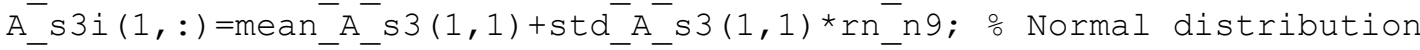
A s4i $(1,:)=$ mean A s4 $(1,1)+\operatorname{std}^{-} \mathrm{A} s 4(1,1) * \mathrm{rn} n 10$; $\%$ Normal distribution A_s5i $(1,:)=$ mean_A_s5 $(1,1)+$ std_A_s5 $(1,1) *$ rn_n11; \% Normal distribution

\section{\% Material properties}

f $c i(1,:)=$ mean $f C(1,1)+\operatorname{std} f c(1,1) * r n n 12$; Normal distribution beta $1 i(1,:)=0.8 \overline{5}-0.05 *$ (f $\mathrm{c} \bar{i}(\overline{1},:)-28) / 7 \bar{i}$

s_beta_1_28=find (f_ci $(1,: \overline{)}<=28)$;

beta $1 \bar{i}(\overline{1}$, s beta $1-28)=0.85$;

s_beta_1_56=find $\left.\mathrm{f}_{-}^{-} \mathrm{ci}(1, \mathrm{e})>=56\right)$;

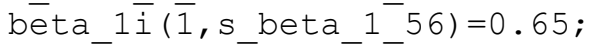

\% mu_f_y=log (mean_f_y^2/sqrt (mean_f_y^2+std_f_y^2)); \% Lognormal distribution

o sigma_f_y=sqrt $(\bar{l} \circ \bar{g}($ std_f_y^2/mean_f_y^2+1) $)$;

o Defined in beta

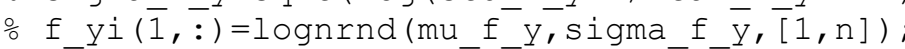

E si=E s; 응 Deterministic

epsilon yi $(1,:)=f$ yi $(1,:) / E$ si

- Professional factor

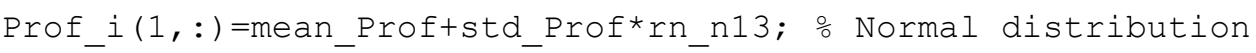

- Resistance calculation

- Calculate c i

c_i $(1,:)=\left(0.0 \overline{0} 3 . /\left(0.003-z^{*}\right.\right.$ epsilon_yi $\left.\left.(1,:)\right)\right) . \star d \_1 i(1,:)$;

o Calculate a i

a_i $(1,:)=$ beta_1i( $1,:) \cdot{ }^{\star} c_{-} i(1,:)$;

o- Compare a i with $h$ i

s a $i=f i n d(a$ i $(1,:)>h$ i $(1,:))$;

$\left.a_{-} i \overline{(} 1, s_{-} a_{-} i\right) \overline{=}{ }_{-} i\left(1, s_{-} \bar{a}_{-} i\right)$; 
\% Calculate epsilon_sli, epsilon_s2i, epsilon_s3i, epsilon_s4i, epsilon_s5i,

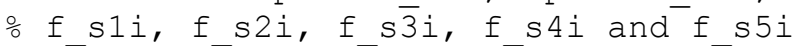

epsilon sīi $(1,:)=\bar{Z} *$ epsilon yi $(1,:)$;

epsilon_s2i $(1,:)=0.003 *\left(c_{-} \bar{i}(1,:)-d_{-} 2 i(1,:)\right) . / c_{-} i(1,:)$;

epsilon s3i $(1,:)=0.003 *(\mathrm{c} i(1,:)-d 3 i(1,:)) . / c i(1,:)$;

epsilon_s4i $(1,:)=0.003 *\left(c_{-}^{-} i(1,:)-d_{-}^{-} 4 i(1,:)\right) . / c_{-}^{-} i(1,:)$;

epsilon_s5i( $1,:)=0.003 *\left(c_{-}^{-} i(1,:)-d-5 i(1,:)\right) . / c_{-}^{-} i(1,:)$;

f $\operatorname{sil}(1,:)=e p s i l o n \operatorname{sil}(1,:) * \mathrm{E}$ si $;$

f_s2i $(1,:)=e p s i l o n \_s 2 i(1,:) * E_{-}^{-}$si;

f $\mathrm{s} 3 i(1,:)=e p s i l o n$ s3i $(1,:) * E$ si

f_s4i $(1,:)=$ epsilon_s4i $(1,:) * \mathrm{E}^{-} \mathrm{si}$;

f_s5i $(1,:)=$ epsilon_s5i( $1,:) * E_{-}$si;

o Compare f s1i, f s2i, f s3i, f s4i and f s5i with +-f yi

s_f_sliu=find(f_slì $\left.(1,:)>\bar{f} \_y i(1, \bar{l})\right)$; o Upper boundary, $\bar{f} \_y i$

f_sili(1,s_f_sliu $)=f \_y i\left(1, s_{-}\right.$f_sliu);

s f slil=find(f sli(1,:)<-f yi(1,:)); \% Lower boundary, -f yi

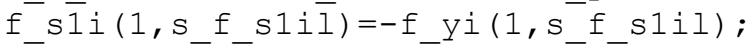

s_f_s2iu=find(f_s2i(1,:)>f_yi(1,:)) ; O Upper boundary, f_yi

$\mathrm{f}^{-} \mathrm{s} \overline{2} i(1, \mathrm{~s} f \mathrm{~s} 2 \mathrm{i} \bar{u})=\mathrm{f} y i\left(1, \mathrm{~s}^{-} \mathrm{f}\right.$ s $\left.2 i u\right)$;

s_f_s $2 i l=\bar{f} i \bar{n} d\left(f \_s 2 i(1,:)<-\bar{f} \_\bar{y}(1,:)\right)$; o Lower boundary, -f_yi

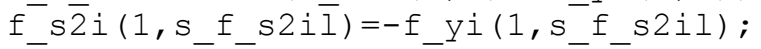

s f s3iu=find(f s3i(1,:)>f yi(1,:)) ; o Upper boundary, f yi

f- $\mathrm{s} \overline{3} i(1, \mathrm{~s} f \mathrm{~s} 3 i \bar{u})=\mathrm{f} y i(1, \mathrm{~s}$ f s3iu);

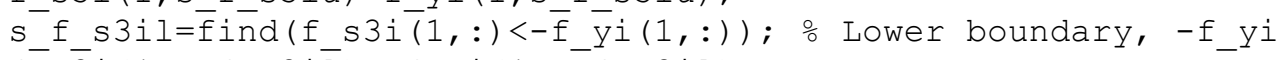

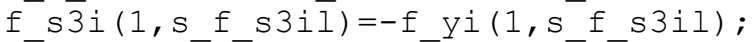

s f s4iu=find(f s4i(1,:)>f yi(1,:)); 告 Upper boundary, f yi

f_s $\overline{4} i\left(1, s_{-} f_{-} 4 i \bar{u}\right)=f \_y i\left(1, s_{-} f_{-} 4 i u\right)$;

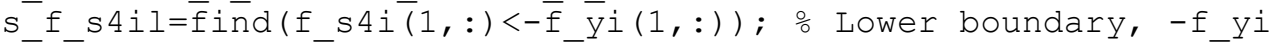

f_s4i(1,s_f_s4il)=-f_yi(1,s_f_s4il);

s_f_s5iu=find(f_s5i(1,:)>f_yi(1,:)) ; 응 Upper boundary, f_yi 
f_s5i(1,s_f_s5iu)=f_yi(1,s_f_s5iu);

s_f_s5il=find(f_s5i(1,:)<-f_yi(1,:)); 응 Lower boundary, -f_yi

f_s $\overline{5} i\left(1, s_{-} f_{-} 5 i \bar{l}\right)=-f_{\text {_yi }}\left(1, \mathrm{~s}_{-} \mathrm{f}_{-} \mathrm{s} 5 \mathrm{il}\right)$;

o Calculate C_ci

angle theta $i \overline{(1,:)}) \operatorname{acos}((\mathrm{h} i(1,:) / 2-a i(1,:)) . /(h i(1,:) / 2))$;

s_a_ipri=fiñd(a_i(1,:)>h_i $(1,:) / 2)$;

angle theta $i(1$, s a ipri) $=p i-a c o s((a) i(1, s$ a ipri) -hi(1,s a ipri)/2)./(hi(1,s a ipri)/2));

A $i(1,:)=h \bar{i}(1,:) \cdot{ }^{\wedge} \overline{2}$. ${ }^{*}(\operatorname{angle}$ theta $i \overline{(1,}:)-\bar{s} i \bar{n}(\operatorname{angle}$ theta $\bar{i}(\overline{1},:))$. ${ }^{*} \cos (\operatorname{ang} \overline{l e}$ theta $\left.i(1,:))\right) / 4 ;$

$C_{-}^{-} \mathrm{Ci}(1,:)=\overline{0} .85 * \mathrm{f}_{-} \mathrm{C} i(1,:) \cdot{ }^{\star A} \mathrm{~A}_{-} \overline{\mathrm{i}}(1,:) \overline{1} 1000$

닫late F_sli

F sli $(1,:)=(f \operatorname{sli}(1,:)-0.85 * f \operatorname{ci}(1,:)) . * A \operatorname{sli}(1,:) / 1000$

S_F_sli=find ( $\bar{a} \_i(1,:)<$ d_li $\left.(1, \overline{:})\right)$;

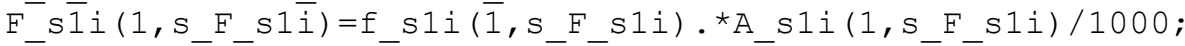

- Calculate F s2i

F_s2i $(1,:)=\left(f \_s 2 i(1,:)-0.85 * f \_c i(1,:)\right) \cdot{ }^{*} A \_s 2 i(1,:) / 1000$;

S_F_s2i=find $\left(a_{-} i(1,:)<d_{-} 2 i(1,:)\right)$;

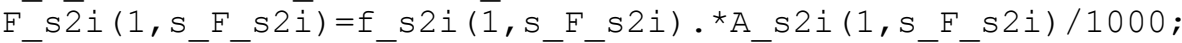

을 Calate F s3i

F_s3i $(1,:)=\left(f_{-}^{-} s 3 i(1,:)-0.85 * f \_c i(1,:)\right) \cdot{ }^{*}$ A_s3i $(1,:) / 1000$;

S F s3i=find (a i $(1,:)<d$ 3i $(1,:))$;

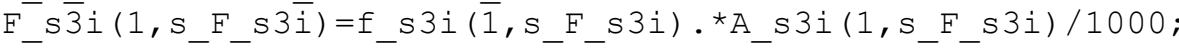

- Calculate F s4i

F s4i(1,:) $=(f-s 4 i(1,:)-0.85 * f$ ci $(1,:)) .{ }^{\star A} s 4 i(1,:) / 1000$ i

S F s4i=find ( $\bar{a}$ i $(1,:)<\mathrm{d} 4 i(1, \overline{:}))$;

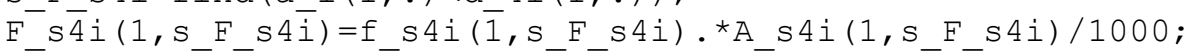

o Calculate F s5i

F s5i $(1,:)=(f \operatorname{s5i}(1,:)-0.85 * f \operatorname{ci}(1,:))$. ${ }^{\star A} \operatorname{s5i}(1,:) / 1000$;

S_F_s5i=find ( $\left.\bar{a}_{-} i(1,:)<d_{-} 5 i(1, \overline{:})\right)$;

F_s $\overline{5} i\left(1, s \_F \_s 5 \bar{i}\right)=f \_s 5 i\left(\overline{1}, s_{-}\right.$F_s5i). ${ }^{\star}$ A_s5i(1,s_F_s5i)/1000; 
o Calculate $P_{-} i$ and $M_{-} i, P_{-} \circ i$ and $P_{-} t i$

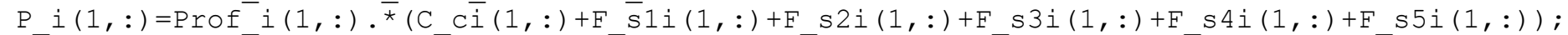

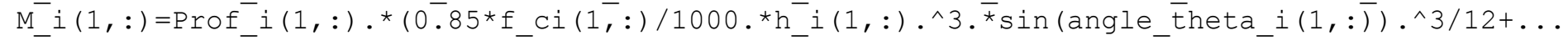

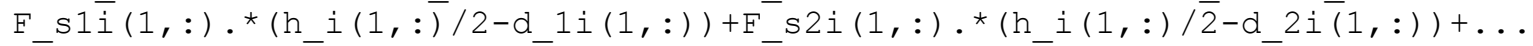
F_s3i $(1,:) . *\left(h \_i(1,:) / 2-d_{-} 3 i(1,:)\right)+F_{-} s 4 i(1,:) . *\left(h-i(1,:) / 2-d_{-} 4 i(1,:)\right)+\ldots$

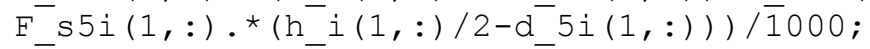

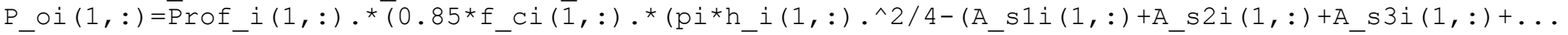
A_s $4 \bar{i}(1,:)+$ A_s $5 i(1,: \overline{)}))+\ldots$

f yi $(1,:){ }^{*}(\bar{A} \operatorname{si}(1,:)+A \operatorname{si} 2 i(1,:)+A \operatorname{s} 3 i(1,:)+A$ s $4 i(1,:)+A$ s $\left.5 i(1,:))\right) / 1000$;

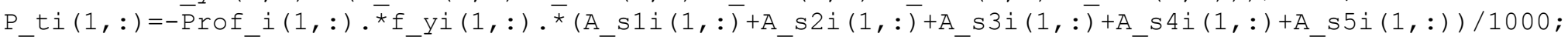

o Calculate eccentricities, e_i, and hovere_i

e $i(1,:)=\mathrm{M} i(1,:) . / \mathrm{P} i(1,:) ; \overline{\frac{o}{0}}(\mathrm{~m})$

hovere_i $(1,:)=(h(1,1) / 1000)$. /e_i $(1,:)$; o Use nominal h

end

\section{C.1.3.6 Code 6-Function of Simulated Load Effects and Nominal Values Based on ACI 318-14}

ㄴoad effect simulation

\% (nominal loads calculation is based on design strengths corresponding to ACI 318-14)

function [D curi, L curi, T Di, T Li]=LoadEffectSim cur S5 (LoverD, n)

o Load design strēngths

load alpha_PM_S5.mat phiP_n

\% Nominal values

\% Loads

$\circ$ LoverD $=\left[\begin{array}{ll}0.5 & 1.5\end{array}\right]$; \% Input of funtion

D cur=abs (phiP n)/(1.2+1.6*LoverD); \% Use absolute values

L_cur=LoverD*D_cur;

\% Transformations from load to load effect

$\mathrm{T} D=1$;

$\mathrm{T}_{-}^{-} \mathrm{L}=1$;

\% Statistical parameters (Bias coefficient and CoV)

o Loads

bias_D=1.05; 
CoV_D $=0.10$

bias $\mathrm{L}=1.00$;

$\operatorname{CoV} \overline{\mathrm{L}}=0.25$;

\% Transformations from load to load effect

o bias_T_D $=1$; $\frac{\circ}{\circ}$ Effect is accounted in $D$

$\circ \mathrm{COV}_{-}{ }_{\mathrm{T}}-\overline{\mathrm{D}}=0$;

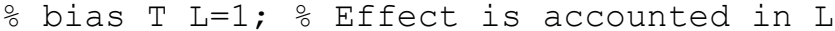

$\circ \mathrm{COV}_{-} \overline{\mathrm{T}}{ }_{-} \overline{\mathrm{L}}=0$;

\% Statistical parameters (Mean and Standard deviation)

o Loads

mean_D_cur=D_cur*bias_D;

std_D_cur=mean_D_cur ${ }^{*} \overline{\mathrm{C}} \mathrm{OV} \_$D;

mean L cur $=\mathrm{L}$ cur*bias L;

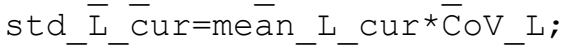

- Transformations from load to load effect

- T D Effect is accounted in D

$\circ \mathrm{T}^{-} \mathrm{L}$ Effect is accounted in $\mathrm{L}$

\% Simulation

- Preallocation

D curi=zeros ( 8 , size (D cur, 2), n);

al̄pha_L_cur=zeros $\left(8\right.$, size $\left(D_{-}\right.$cur, 2));

mu L cur=zeros $(8, \operatorname{size}(\mathrm{D}$ cur, 2$))$;

L_curi=zeros $\left(8, \operatorname{size}\left(D_{-} \operatorname{cur}, 2\right), \mathrm{n}\right)$;

for $i 1=1: 8$

for i $4=1$ :size (D_cur, 2)

rn $\mathrm{n} 14=\mathrm{randn}(1,1, \mathrm{n})$; $\%$ Standard normally distributed random numbers

$r n \_u l=r a n d(1,1, n)$; $\%$ Standard uniformly distributed random numbers 


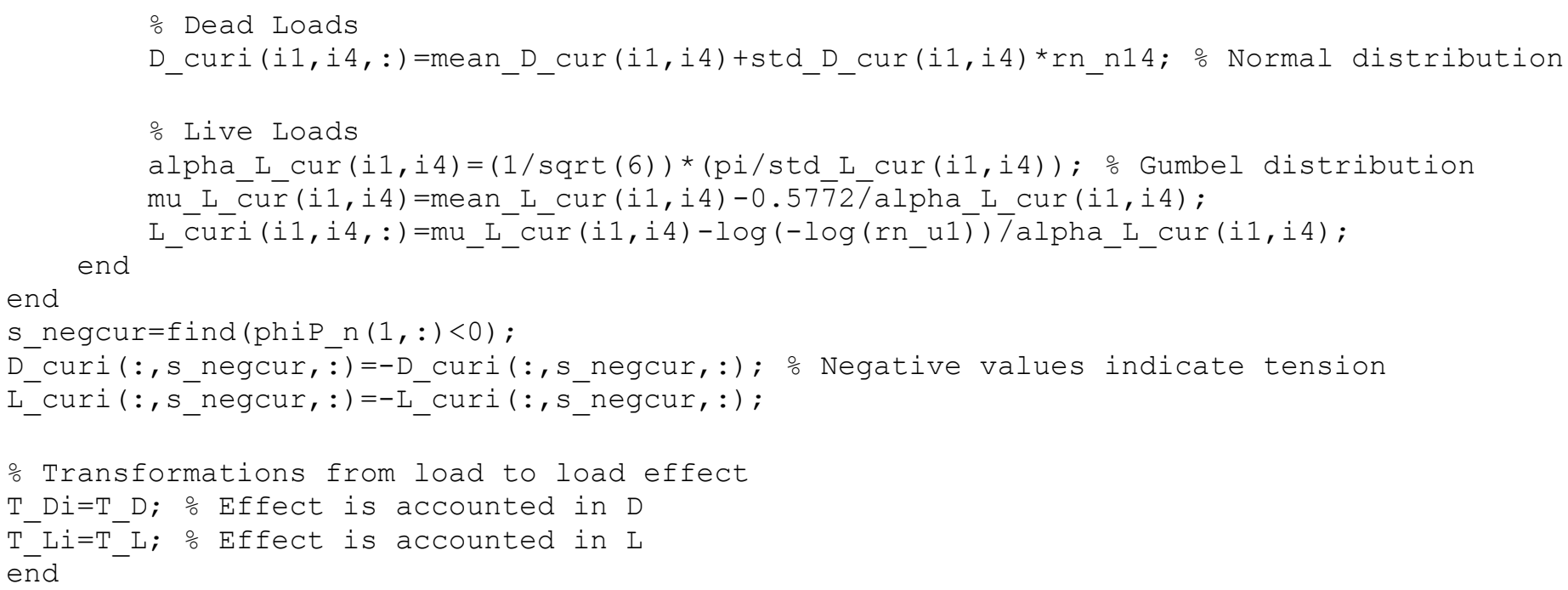

C.1.3.7 Code 7-Function of Simulated Load Effects and Nominal Values Based on Partial Material Strength Reduction Factors

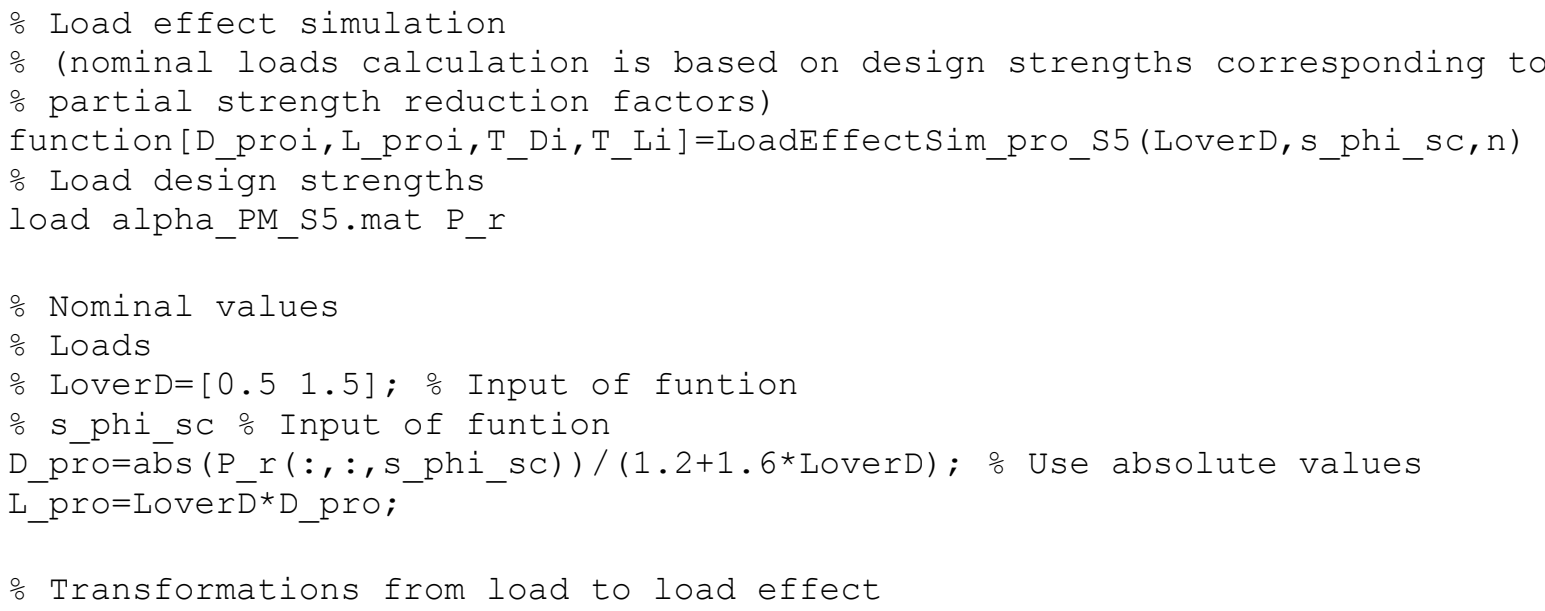


$\mathrm{T}-\mathrm{D}=1$;

T_ ${ }^{-}=1$;

- Statistical parameters (Bias coefficient and CoV)

o Loads

bias $\mathrm{D}=1.05$;

$\mathrm{CoV}_{-} \overline{\mathrm{D}}=0.10$;

bias_L $=1.00$;

CoV_ $\overline{\mathrm{L}}=0.25$;

\% Transformations from load to load effect

$\therefore$ bias $\mathrm{T} D=1$; $\frac{\circ}{0}$ effect is accounted in $\mathrm{D}$

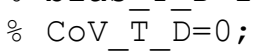

\% bias_T_L=1; $\%$ Effect is accounted in L

$\div \mathrm{COV}_{-}{ }_{\mathrm{T}} \mathrm{L}=0$;

o Statistical parameters (Mean and Standard deviation)

o Loads

mean D pro=D pro*bias D;

std_D_pro=mean_D_pro ${ }^{*} \bar{C} O V \_D$;

mean_L_pro=L_pro*bias_L;

std_L_pro=mean_L_pro* $\mathrm{CoV}$ _L ;

\% Transformations from load to load effect

$\div$ T_D Effect is accounted in D

$\circ \mathrm{T}^{-} \mathrm{L}$ Effect is accounted in $\mathrm{L}$

- Simulation

o Preallocation

D_proi=zeros $\left(8, \operatorname{size}\left(D \_p r o, 2\right), n\right)$;

alpha_L_pro=zeros $\left(8\right.$, size $\left.\left(D \_p r o, 2\right)\right)$;

mu_L_pro=zeros $\left(8, \operatorname{size}\left(\mathrm{D} \_\right.\right.$pro, 2$\left.)\right)$;

L proi=zeros $(8$, size (D pro, 2), n) 
for $i 1=1: 8$

for i4=1:size (D_pro, 2)

rn n $14=r a n d n(1,1, n)$; O Standard normally distributed random numbers

rn_ul=rand $(1,1, n)$; o standard uniformly distributed random numbers

ㅁ Dead Loads

D_proi $(i 1, i 4,:)=$ mean_D_pro $(i 1, i 4)+$ std_D_pro(i1,i4)*rn_n14; $\%$ Normal distribution

․ Live Loads

alpha_L_pro $(i 1, i 4)=(1 / \operatorname{sqrt}(6)) *\left(p i / s t d \_L \_p r o(i 1, i 4)\right)$; $\frac{\circ}{0}$ Gumbel distribution

mu L pro (i1,i4)=mean L pro(i1,i4) $-0.57 \overline{7} 2 /$ alpha L pro(i1,i4);

L_proi $(i 1, i 4,:)=$ mu_L_pro $(i 1, i 4)-\log \left(-\log \left(r n \_u 1\right)\right)$ /alpha_L_pro(i1,i4);

end end

s_negpro=find $\left(P_{-} r(1,:\right.$, s_phi_sC $\left.)<0\right)$;

D proi $(:, s$ negpro, $:)=-D$ proi $\left(:, s\right.$ negpro, : ); $\frac{\circ}{\circ}$ Negative values indicate tension

L_proi (:,s_negpro, :) =-L_proi (:, s_negpro, : );

\% Transformations from load to load effect

T_Di=T_D; $\%$ Effect is accounted in D

$T_{-}{ }_{-}{ }_{1}=T_{-}{ }_{-}$; $\div$Effect is accounted in $\mathrm{L}$

end 


\section{Curriculum Vitae}

Name:

Post-secondary Education and Degrees:

\section{Honours and} Awards:

Related Work Experience
Tong Zhang

Beijing Jiaotong University

Beijing, China

2010-2014 B.Eng.

The University of Western Ontario

London, Ontario, Canada

2014-2017 M.E.Sc.

Beijing Jiaotong University First-Place Scholarship 2012

Beijing Jiaotong University Second-Place Zhijin Scholarship 2012

Beijing Jiaotong University Excellent Student Award 2012

Beijing Jiaotong University Second-Place Scholarship 2013

Western Graduate Research Scholarship 2015-2016

Teaching Assistant

The University of Western Ontario 2015-2016 